\title{
Komposita-Erstglieder \\ Untersuchungen zur anaphorischen Zugänglichkeit
}

\author{
Dissertation \\ zur Erlangung des philosophischen Doktorgrades \\ an der Philosophischen Fakultät der \\ Georg-August-Universität Göttingen
}

\author{
vorgelegt von \\ Stefanie Rößler \\ aus Merseburg (Saale)
}

Göttingen 2017 
1. Gutachterin: Prof. Dr. Anke Holler

2. Gutachter: Prof. Dr. Markus Steinbach

Tag der mündlichen Prüfung: 13.03.2018 


\section{Abstract}

The first constituent of a nominal compound (N1) is usually assumed to be an anaphoric island (e.g. Lapointe 1979, Postal 1969). Due to lexical integrity it seems to be inaccessible for pronominal anaphora and can only be accessed via bridging (cf. (1-a)). However, there are exceptions to this rule (cf. (1-b)).

a. *Max is a wild-animal hunter but Pete only kills domesticated ones. (Postal 1969:215)

b. The rocket launch had to be delayed because of some unexpected problems with its fuel tanks. (Coulmas 1988)

The degree of compositionality (cf. Coulmas 1988, Ward et al. 1991) and pragmatic licensing (cf. McKoon et al. 1993, Wunderlich 1986) are two approaches that may explain these exceptions. The thesis at hand investigates the influence of discourse-independent, distinct conceptual (i) and grammatical (ii) factors on N1-accessibility.

For conceptual factors (i), the world knowledge about the denotatum of the N1 is considered to be influencing the level of activation. In line with this assumption an animate $\mathrm{N} 1$ is supposed to have an advantage in comparison with an inanimate N1 (e.g. dog bowl vs. metal bowl). In addition, the concept combination of the $\mathrm{N} 1$ and the N2 was investigated. There is a group of properties influencing the accessibility of the N1 by influencing the conceptual focus of the compound. The semantic relation (e.g. Levi 1978) is one of them, e.g. the relation HAVE focuses more on the N1 than FOR (e.g. can stock vs. can opener). Driven by the diverse semantic relations, the more generalized property of the spatio-temporal contiguity (STC) of the constituents is taken into account, meaning that a conceptually present N1 is more accessible than an absent one (e.g. car accident vs. car insurance).

The difference between root and synthetic compounds is understood as the crucial structural condition (ii). In line with the framework of Distributed Morphology (cf. Halle/Marantz 1993. Harley 2009) it is claimed that the N1 has a different categorial and therefore referential status depending on the structure of the compound. This predicts a higher degree of accessibility in the case of synthetic than in the case of root compounds (e.g. roof greening vs. roof garden).

A series of three psycholinguistic experiments was conducted in German to investigate these theoretical considerations. The first experiment was a sentence completion task. Participants were asked to complete a sentence fragment which starts with a pronoun, its antecedent being the N1 of a compound in a preceding sentence. The compounds were manipulated for the factors ANIMACY and RELATION. The hypothesis was that there will be more references to the N1 under the conditions 'animate' and 'HAVE' than under the conditions 'inanimate' and 'FOR'. The analysis descriptively shows tendencies in predicted directions. For ANIMACY there is a main effect, and then there are indications of an 
interaction, namely that the RELATION has a stronger influence under the inanimate condition.

The second experiment was conducted along the same lines as Experiment 1 but with the factors STC and STRUCTURE. The hypothesis was that there will be more references to the N1 under the conditions 'present' and 'synthetic compound'. The analysis showed a main effect for STC. The effect of STRUCTURE goes against the prediction.

Experiment 3 used eye tracking to investigate the more subtle structural factor during online resolution processing. Participants were asked to read small discourses where N1reference was established with a pronoun and disambiguated through the context. In agreement with the initial prediction on the advantage of N1 in synthetic compounds, an effect on the first-pass fixation duration and the total reading time of the anaphora was found. The influence of the STC was also confirmed.

The experiments investigated a range of factors that have-to the best of my knowledge-not yet been tested systematically. The data highlight the interplay of different kinds of factors that have to be integrated by processing models. The effect of subtle structural factors might be buried below world knowledge factors, and can only be unearthed by finegrained online processing measures. That makes the possibility of N1-reference a gradual phenomenon influenced by a multitude of scalar factors which cannot be captured by a categorial concept like lexical integrity. Furthermore, the effect on the first-pass fixation duration in the eye tracking experiment indicates the establishment of a discourse referent by the N1. This finding gives insight into the process of parsing and allows an interpretation of the N1-reference similar to direct anaphora, since no pragmatic licensing like bridging or coercion could have taken place at this point. 


\section{Inhaltsverzeichnis}

Abstract iii

1. Einleitung 1

1.1. Komposita und die (Un-)Zugänglichkeit ihrer Erstglieder . . . . . . . . . . . . . 1

1.2. Relevanz für die linguistische Theoriebildung . . . . . . . . . . . . . . . . . 4

1.3. Aufbau und Ziel der Arbeit . . . . . . . . . . . . . . . . . . . 5

\begin{tabular}{ll}
\hline 2. Einordnung des Phänomens in die Theorielandschaft & $\mathbf{7}$
\end{tabular}

2.1. Anaphorische Inseln und lexikalische Integrität . . . . . . . . . . . . . . . . . 7

2.2. Komposita in der Grammatiktheorie . . . . . . . . . . . . . . . . . 13

2.2.1. Definition und Klassifikation . . . . . . . . . . . . . . . . . . 14

2.2.2. Charakteristika von Komposita . . . . . . . . . . . . . . 16

2.2.3. Morphosyntax von Komposita . . . . . . . . . . . . . . . . . . 24

2.2.4. Semantik von Komposita. . . . . . . . . . . . . . . . . . . . . . . . . 27

2.3. Komposita in der Verarbeitungstheorie . . . . . . . . . . . . . . . . . . . . . . . . . . . . . . . . . . .

2.3.1. Kenngrößen der Verarbeitungstheorie . . . . . . . . . . . . . . . 33

2.3.2. Modelle des lexikalischen Zugriffs . . . . . . . . . . . . . . . . . . . . . . . 40

2.3.3. Interpretationsmechanismen . . . . . . . . . . . . . . . . . . . . . . . . . . . . 41

2.3.4. Zentrale Verarbeitungsfaktoren . . . . . . . . . . . . . . . . . . . . . 44

2.3.5. Die Rolle des N1 bei der Komposita-Verarbeitung. . . . . . . . . . . 50

2.4. Anaphorische Pronomen als Zugänglichkeitsmarker . . . . . . . . . . . . 52

2.4.1. Pronominale Diskursanaphern . . . . . . . . . . . . . . . 52

2.4.2. Direkte vs. indirekte Anaphern . . . . . . . . . . . . . . . . . . 54

2.4.3. Prozess der Anaphernresolution. . . . . . . . . . . . . . . . . . . . . . . 58

2.5. Verwandte Phänomene . . . . . . . . . . . . . . . . . . 60

2.5.1. Phrasenkomposita . . . . . . . . . . . . . . . . 6 60

$2.5 .2 . \quad$ Univerbierungen . . . . . . . . . . . . . . . . . 62

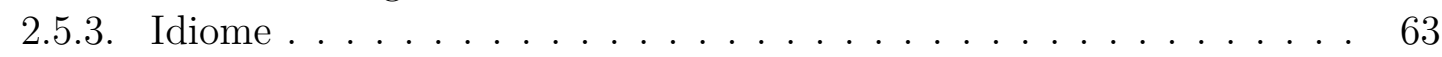

$2.5 .4 . \quad$ Klammerparadoxie . . . . . . . . . . . . . . . 63

2.5.5. Genitiv-Attribute . . . . . . . . . . . . . . . . . . . . . . . . . . 65

2.6. Stand der Forschung . . . . . . . . . . . . . . . . 66

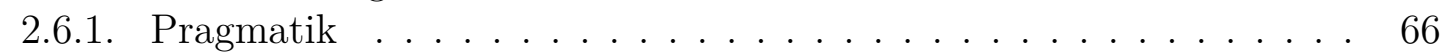

2.6.2. Transparenz und Kompositionalität . . . . . . . . . . . . . . . . . . . 67

2.6.3. Weitere Ansätze . . . . . . . . . . . . . . . . . . . . 69

2.7. Zusammenfassung und Zielsetzung . . . . . . . . . . . . . . . . . 70

$\begin{array}{ll}\text { 3. Konzeptuelle Faktoren } & 73\end{array}$

3.1. Allgemeine Vorüberlegungen . . . . . . . . . . . . . . . . . . . . . . . . 73

3.2. Konzeptuelles und grammatisches N1-Wissen . . . . . . . . . . . . . . . . 74

3.2.1. Lebenswelteffekt . . . . . . . . . . . . . . . . . . . . 74

3.2.2. Wahrnehmungseffekt . . . . . . . . . . . . . . . . . . 77 
3.2.3. Unikalität . . . . . . . . . . . . . . . . . . . . . . . . . 79

3.3. Aspekte der Konzeptkombination . . . . . . . . . . . . . . . . 80

3.3.1. Vorüberlegungen . . . . . . . . . . . . . . . . . 80

3.3.2. Semantische Relation . . . . . . . . . . . . . . . . . . . . 81

3.3.3. Konzeptueller Fokus . . . . . . . . . . . . . . . . . . . 83

3.3.4. Raum-zeitliche Kontiguität (RZK) . . . . . . . . . . . . . . . . . . . . . . . . . . . . . . 85

3.3.5. Interaktion der Teilkonzepte . . . . . . . . . . . . . . . . . 86

3.3.6. Verarbeitungseffekte . . . . . . . . . . . . . . . 87

3.3.7. Nuklearität . . . . . . . . . . . . . . . . . . . . . . . . . 88

3.4. Experiment 1: Animatheit und Relation . . . . . . . . . . . . . . . 89

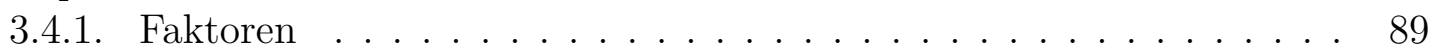

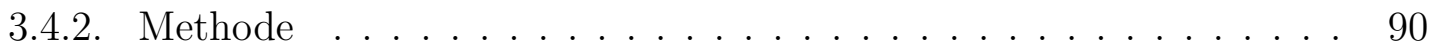

3.4.3. Material . . . . . . . . . . . . . . . . . . . . . 91

3.4.4. Hypothesen . . . . . . . . . . . . . . . . . . . . 93

3.4.5. Durchführung . . . . . . . . . . . . . . . . . . . . 93

3.4.6. Auswertung . . . . . . . . . . . . . . . . . . . . . . . . 95

3.4.7. Interpretation und Diskussion . . . . . . . . . . . . . . 100

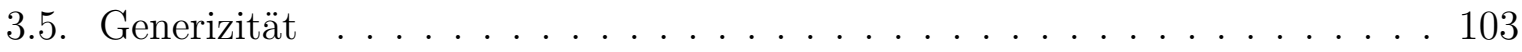

3.6. Zwischenfazit . . . . . . . . . . . . . . . . . . 107

4. Strukturaspekte 109

4.1. Beschreibungsansätze . . . . . . . . . . . . . . . . . . . . . . . . . . . 109

4.1.1. Lexikalistische Ansätze . . . . . . . . . . . . . . . . . . . . . . . . . 109

4.1.2. Syntaktische Ansätze . . . . . . . . . . . . . . . . . . 111

4.1.3. Hybride Ansätze . . . . . . . . . . . . . . . . . . . . . . . . . . . . . 112

4.1.4. Zusammenschau . . . . . . . . . . . . . . . . . . . . . . . . . . . . . 113

4.2. Strukturaufbau der Komposita-Arten . . . . . . . . . . . . . . . . . . . 114

4.2.1. Wurzelkomposita . . . . . . . . . . . . . . . . . . . . . 114

4.2.2. Rektionskomposita . . . . . . . . . . . . . . . . . . 114

4.2.3. DM-Ansatz für Komposita . . . . . . . . . . . . . . . . . . . . . 127

4.2.4. Modellierung fürs Deutsche . . . . . . . . . . . . . . . . . . . . . 130

4.3. $\quad$ Argumente für Unterschiede in der N1-Zugänglichkeit . . . . . . . . . . . . . . 134

4.3.1. Argumentstatus und Kategorisierung . . . . . . . . . . . . . . . . . 134

4.3.2. VP in Rektionskomposita . . . . . . . . . . . . . . . . . . . 136

4.3.3. Referenzialität . . . . . . . . . . . . . . . . . . . . . . 138

4.3.4. Weitere Evidenz . . . . . . . . . . . . . . . . . . . . . . . 141

4.3.5. Weitere Formen von Komposita . . . . . . . . . . . . . . . . . . . . 144

4.4. Zwischenfazit . . . . . . . . . . . . . . . . . . . . . . . . . . 149

5. Experiment 2: RZK und Struktur 151

5.1. Faktoren . . . . . . . . . . . . . . . . . . . . . 151

5.2. Methode . . . . . . . . . . . . . . . . . . . 152

5.3. Material . . . . . . . . . . . . . . . . . . . . 152

5.3.1. Komposita-Auswahl. . . . . . . . . . . . . . . . . . 152

$5.3 .2 . \quad$ Item-Erstellung . . . . . . . . . . . . . . . . . . . . . . 154

5.3.3. Filler und Kontroll-Items. . . . . . . . . . . . . . . . . . . . 157

5.4. Hypothesen . . . . . . . . . . . . . . . . . . . . . . . 158

$5.5 . \quad$ Durchführung . . . . . . . . . . . . . . . . . . . . . . 159

5.5.1. Ablauf . . . . . . . . . . . . . . . . . . . . 159 
5.5 .2$. Technik . . . . . . . . . . . . . . . . . . . . 159

5.5.3. Versuchspersonen . . . . . . . . . . . . . . . . . 160

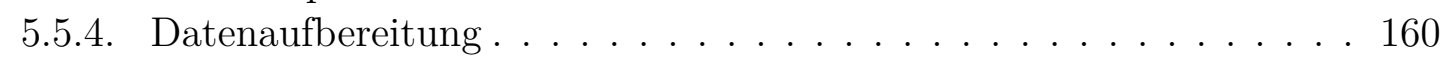

5.6. Auswertung . . . . . . . . . . . . . . . . . . . . 161

5.6.1. Deskriptive Statistik . . . . . . . . . . . . . . . . . 161

$5.6 .2 . \quad$ Inferenzstatistik $\ldots \ldots \ldots \ldots \ldots \ldots$

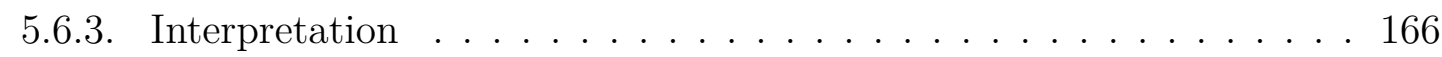

5.7. Weitere Analysen . . . . . . . . . . . . . . . . . . . . 166

5.7.1. Post-hoc-Analyse des Struktur-Effekts . . . . . . . . . . . . . . . . 167

5.7 .2$. Genus . . . . . . . . . . . . . . . . . . . 168

$5.7 .3 . \quad$ Spezifizität . . . . . . . . . . . . . . . . . . . . . . . 169

5.7 .4$. Zählbarkeit . . . . . . . . . . . . . . . . . . . . . 170

$5.7 .5 . \quad$ Nominalisierungsart . . . . . . . . . . . . . . . . . . . . . . . 171

5.7.6. Validierung des Designs . . . . . . . . . . . . . . . . . 171

5.8. Interpretation und Diskussion . . . . . . . . . . . . . . 176

$\begin{array}{ll}\text { 6. Experiment 3: Struktur } & 179\end{array}$

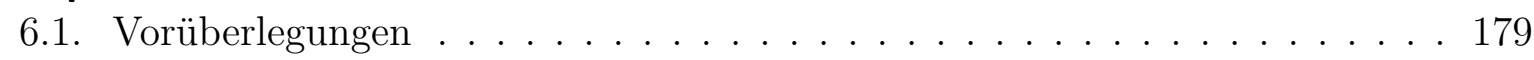

6.1.1. Faktor . . . . . . . . . . . . . . . . . . . . . . . 179

$6.1 .2 . \quad$ Komposita-Auswahl . . . . . . . . . . . . . . . . . . . . 180

6.2. Experiment 3a: Satzergänzungsaufgabe . . . . . . . . . . . . . . . . . . 182

6.2.1. Methode . . . . . . . . . . . . . . . . . . . 182

$6.2 .2 . \quad$ Material . . . . . . . . . . . . . . . . . . . . . . 182

6.2 .3$. Hypothesen . . . . . . . . . . . . . . . . . . . 183

6.2 .4$. Durchführung . . . . . . . . . . . . . . . . . . . . . . . . 183

$6.2 .5 . \quad$ Auswertung . . . . . . . . . . . . . . . . . . . . . . 185

6.2.6. Weitere Analysen . . . . . . . . . . . . . . . . . . 188

6.2.7. Interpretation und Diskussion . . . . . . . . . . . . . . . . . 199

6.3. Experiment 3b: Kohärenzrating . . . . . . . . . . . . . . . . . . . . . 200

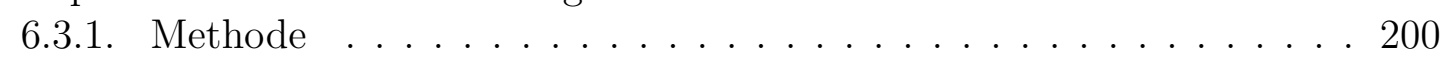

6.3 .2$. Material . . . . . . . . . . . . . . . . . . . . 200

6.3.3. Hypothesen . . . . . . . . . . . . . . . . . . . 203

6.3.4. Durchführung . . . . . . . . . . . . . . . . . . . . . . . 204

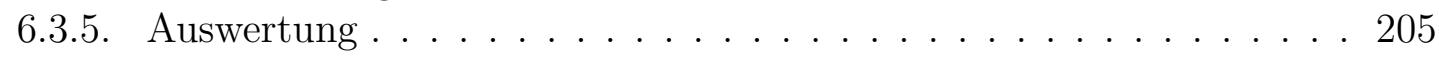

6.3.6. Interpretation und Diskussion . . . . . . . . . . . . . . . . . . 208

6.4. Experiment 3c: Eye-Tracking . . . . . . . . . . . . . . . . . . . 208

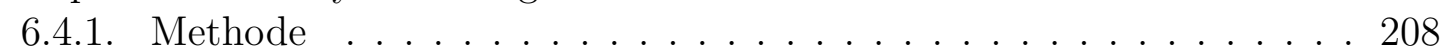

6.4 .2$. Material . . . . . . . . . . . . . . . . . . . . . . 208

$6.4 .3 . \quad$ Hypothesen . . . . . . . . . . . . . . . . . . . . . . . . . . . . . . . . . . . . . . . . . . . . . . . . . . . . .

$6.4 .4 . \quad$ Durchführung . . . . . . . . . . . . . . . . . . . . . . . . 213

6.4 .5$. Auswertung . . . . . . . . . . . . . . . . . . . . . . 215

6.4.6. Interpretation und Diskussion . . . . . . . . . . . . . . . . . 240

6.4.7. Weitere Analysen . . . . . . . . . . . . . . . . . . . . . . . 242

6.5. Zusammenfassung der drei Experimente $(3 \mathrm{a}, \mathrm{b}, \mathrm{c})$. . . . . . . . . . . . . 261

6.5.1. Zusammenfassung der Ergebnisse. . . . . . . . . . . . . . . . . 261

6.5.2. Evaluation des Materials . . . . . . . . . . . . . . . . . 262

7. Übergreifende Diskussion 265

7.1. Lexikalische Integrität revisited . . . . . . . . . . . . . . . . . . . 265 
7.2. Einflussfaktoren für die N1-Zugänglichkeit . . . . . . . . . . . . . . . . . . 267

7.2.1. Arten von Faktoren . . . . . . . . . . . . . . . . . . . . 267

7.2.2. Interaktion von Faktoren . . . . . . . . . . . . . . . . 270

7.3. Implikationen für die Grammatiktheorie . . . . . . . . . . . . . . . . . . . . 271

7.3.1. Morphosyntax von Komposita . . . . . . . . . . . . . . . . . . . 271

7.3.2. Semantik von Komposita . . . . . . . . . . . . . . . . . . . . . . . 272

7.4. Implikationen für die Verarbeitungstheorie . . . . . . . . . . . . . . . . . . . 274

7.5. Implikationen für die Anaphorik . . . . . . . . . . . . . . . . . . . 275

7.6. Ausblick . . . . . . . . . . . . . . . . . . . . . . . . 277

$\begin{array}{ll}\text { 8. Schlussbemerkung } & 281\end{array}$

\begin{tabular}{ll}
\hline Literaturverzeichnis & 285
\end{tabular}

Weitere Quellen 299

\begin{tabular}{ll}
\hline Tabellenverzeichnis & 301
\end{tabular}

\begin{tabular}{|ll}
\hline Abbildungsverzeichnis & 307
\end{tabular}

\begin{tabular}{ll}
\hline Abkürzungen & 311
\end{tabular}

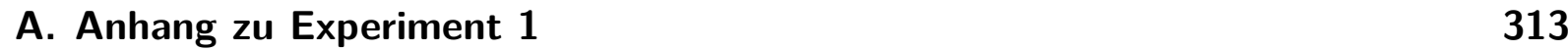

A.1. Komposita . . . . . . . . . . . . . . . . . . . . 313

A.2. Items . . . . . . . . . . . . . . . . . . . . . . . 314

\begin{tabular}{ll}
\hline B. Anhang zu Experiment 2 & 317
\end{tabular}

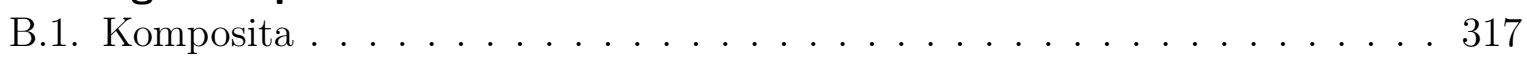

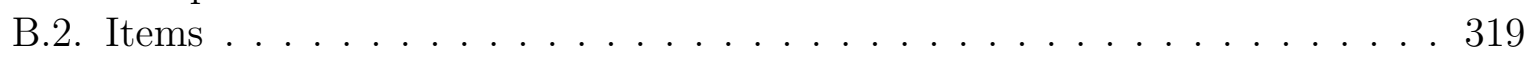

B.2.1. Stimuli . . . . . . . . . . . . . . . . . . . . . 319

B.2.2. Kontroll-Items . . . . . . . . . . . . . . . . . . . . 324

B.3. Weitere Plots . . . . . . . . . . . . . . . . . . . 326

\begin{tabular}{ll}
\hline C. Anhang zu Experiment 3 & 329
\end{tabular}

C.1. Komposita . . . . . . . . . . . . . . . . . . . . . . 329

C.2. Anhang zu Experiment 3a . . . . . . . . . . . . . . . . . . 331

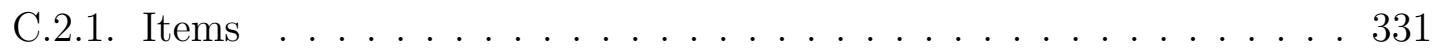

C.2.2. Weitere Plots . . . . . . . . . . . . . . . . . . . 335

C.2.3. Auswertungs-Output: Bsp. aus R 3.2.0 . . . . . . . . . . . . . . . 337

C.3. Anhang zu Experiment 3b . . . . . . . . . . . . . . . . . . 338

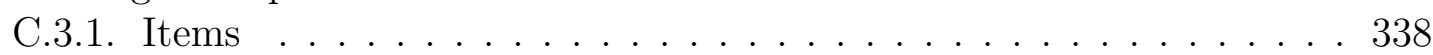

C.3.2. Weiteres Material . . . . . . . . . . . . . . . . . . . . . 348

C.3.3. Weitere Plots . . . . . . . . . . . . . . . . . . . . 351

C.3.4. Auswertungs-Output: Bsp. aus R 3.2.0 . . . . . . . . . . . . . . . . . 352

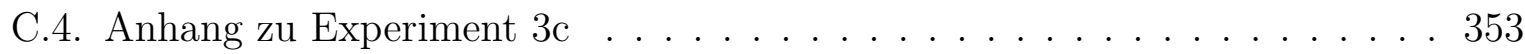

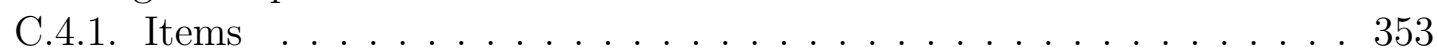

C.4.2. Weitere Plots . . . . . . . . . . . . . . . . . . 354

C.4.3. Auswertungs-Output: Bsp. aus R 3.2.0 . . . . . . . . . . . . 356

\begin{tabular}{ll}
\hline Danksagung & 359
\end{tabular} 


\section{Einleitung}

Manche deutschen Wörter sind so lang, dass man sie nur aus der Ferne ganz sehen kann. $[\ldots]$

„Freundschaftsbezeigungen“

„Dilettantenaufdringlichkeiten“

„Stadtverordnetenversammlungen“"

Dies sind keine Wörter, es sind Umzüge sämtlicher Buchstaben des Alphabets. Und sie kommen nicht etwa selten vor. Wo man auch immer eine deutsche Zeitung aufschlägt, kann man sie majestätisch über die Seite marschieren sehen [...]

Das Wörterbuch muss irgendwo eine Grenze ziehen, daher lässt es diese Sorte von Wörtern aus, und zwar mit Recht, denn diese langen Dinger sind wohl kaum rechtmäßige Wörter, sondern vielmehr Wortkombinationen, deren Erfinder man hätte umbringen sollen. Es sind zusammengesetzte Wörter ohne Bindestrich. Die einzelnen Wörter, die zu ihrem Aufbau benutzt wurden, stehen im Wörterbuch, allerdings sehr verstreut. Man kann sich also das Material Stück um Stück zusammensuchen und auf diese Weise schließlich auf die Bedeutung stoßen, aber es ist eine mühselige Plackerei. [...]

Mark Twain: Die schreckliche deutsche Sprache

(Original 1880, Übersetzung 2009)

\subsection{Komposita und die (Un-)Zugänglichkeit ihrer Erstglieder}

Aufgrund ihrer hohen Produktivität und ihrer Auffälligkeit im Schriftbild erscheinen Komposita nicht nur für Mark Twain als Besonderheit der deutschen Sprache. Dabei handelt es sich bei der Komposition sogar über die Grenzen der indoeuropäischen Sprachfamilie hinaus um den wohl produktivsten Wortbildungstyp.

Ihr Kennzeichen ist, dass mindestens zwei bekannte Ausdrücke zu einem neuen kombiniert werden, der gegenüber den Input-Ausdrücken über einen semantischen Mehrwert verfügt. Dieser Mehrwert kann gelernt sein, z.B. die speziellen konstruktiven Eigenschaften, über die ein Hühnerstall verfügt. Er kann auch spontan assoziiert werden, z.B. dass es sich bei einem Zettelnagel vermutlich um eine kreative Aufbewahrungslösung handelt. Diese Möglichkeit macht die Komposition zu einer höchst ökonomischen Form der Wortschatzerweiterung.

Für die Linguistik ergeben sich daraus eine ganze Reihe von Fragen: Welchen Status haben die Konstituenten von Komposita, wie sind sie miteinander verbunden und wie entsteht der semantische Mehrwert?

Durch die Kombination bekannter Einzelmorpheme suggerieren Komposita Transparenz und Verständlichkeit. Gleichzeitig sind sie semantisch unterspezifiziert, was sie für die Theorie der Sprachverarbeitung interessant macht. Für die Grammatik ergeben sich v.a. durch die Verortung an der Schnittstelle zwischen Morphologie und Syntax zahlreiche Fragen, die u.a. Spencer (1991) und Scalise/Vogel (2010) aufgreifen. 
Compounds are particularly interesting linguistic constructions for a number of reasons. First, they constitute an anomaly among grammatical constructions because they are ,words', but they at the same time exhibit a type of ,internal syntax'. This syntax, furthermore, is somewhat ,invisible: (Scalise/Vogel 2010 2)

Komposita bilden keine homogene Ausdrucksklasse, sondern unterscheiden sich untereinander sowohl semantisch (z.B. exozentrisch vs. endozentrisch) als auch strukturell (z.B. Wurzel- vs. Rektionskomposita) und verarbeitungstechnisch (z.B. Ad-hoc-Komposita vs. Lexikalisierungen). Bei nominalen Komposita (NN) besteht zudem zwischen der ersten Konstituente (N1) und der zweiten Konstituente (N2) eine Asymmetrie bzgl. eines Großteils ihrer grammatischen Eigenschaften. Dazu gehört u.a., dass dem N2 die Eigenschaft der Festlegung der grammatischen Kategorie und des Denotats zugeschrieben wird. Dies lässt sich bspw. in (1) beobachten: Der Ausdruck Hühnerstall bezeichnet semantisch einen Stall und auch die morphosyntaktischen Eigenschaften Genus (m) und Numerus (Pl) werden von der zweiten Konstituente an das gesamte Kompositum übertragen. Dem N1 hingegen kommt eine saturierende oder modifizierende Funktion zu 11Daraus lässt sich ableiten, dass die erste Konstituente über keine unabhängigen grammatischen Eigenschaften verfügt oder diese zumindest nicht zum Tragen kommen.

$$
\text { die Hühner }+ \text { der Stall } \rightarrow \text { der Hühnerstall }
$$

Aus diesem Mangel an morphosyntaktischen Eigenschaften der ersten Konstituente - des Erstglieds - lässt sich wiederum schlussfolgern, dass auch keine Kongruenz zwischen einem Erstglied und Pronomen hergestellt werden kann. Daher besteht die verbreitete Annahme, dass die Erstglieder von nominalen Komposita unzugänglich für pronominale Anaphern sind (z.B. bei Dressler (2007), Postal (1969), Schwarz (2000), Spencer (1991), Wunderlich (1986)). Illustriert wird diese These durch zahlreiche Beispiele des Deutschen wie unter (2) aber auch des Englischen wie unter (3) und weiterer rechtsköpfiger Sprachen.

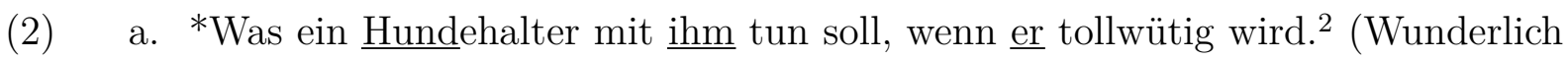
1986:221)

b. *Atomwaffengegener haben gegen ihre Lagerung in Europa protestiert. (Coulmas 1988:318)

c. Paul ist nun in einem Eislaufkurs. *Das geschieht nächsten Mittwoch auf eigene Gefahr. (Härtl 2015:171)

(3) a. *Max is a wild-animal hunter but Pete only kills domesticated ones. (Postal 1969 .215)

b. * Divorce lawyers often experience it themselves. (Levi 1978:72)

c. ${ }^{*}$ Truck-drivers fill them up with Arco Diesel. (Sproat 1985:326)

Diese Unzugänglichkeit der ersten Konstituente von Komposita wird oft mit dem Begriff der „anaphorischen Insel“ (vgl. Postal 1969) bezeichnet und unter Berufung auf die lexikalische Integrität als Wortkriterium herangezogen, dazu ausführlicher in Kapitel 2.1.

\footnotetext{
${ }^{1}$ Näheres zu den grammatischen Eigenschaften von Komposita siehe Kapitel 2.2.2

${ }^{2} \mathrm{Im}$ Folgenden werden Antezedenten und andere Formen von Zielen anaphorischer Bezugnahme sowie die entsprechenden Anaphern unabhängig von den Hervorhebungen im Original durch Unterstreichungen kenntlich gemacht.
} 
Beobachtungen des Sprachgebrauchs zeigen nun, dass der anaphorische Bezug auf die erste Konstituente von Komposita eine seltene, aber dennoch produktive Form der Sprachverwendung ist. Während der Beschäftigung mit dem Phänomenbereich konnten die Beispiele (4) - (7) als Zeugnisse authentischen Sprachgebrauchs gesammelt werden.

(4) Kira Dorn: Lupo, kannst du dir vorstellen, dass jemand die Bombe in deinem Garten vergraben hat?

Lupo: $\quad$ Ein Rosenhasser? Die tun doch niemandem was.

(Tatort vom 05.02.2017 (Min. 09:27))

[... das Fenster ist offen und es ziehen die ganze Zeit Croissant-Gerüche von dem Bäcker unter uns zu mir hoch. Ich mag die ja nicht mal, aber appetitanregend ist es halt trotzdem.

(Hörbeleg)

(6) [.. ] ich möchte mich bei Ihnen ganz herzlich für das Afrikabuch bedanken. Es ist wunderschön, genau so, wie ich es in Erinnerung habe.

(private Postkarte)

(7) Verkäuferin: Wir haben diese Woche ein Berliner-Angebot!

Kundin: Oh ja, dann nehme ich davon noch zwei.

(Hörbeleg beim Bäcker)

Für das Englische führt u.a. Coulmas (1988) akzeptable Fälle wie (8) auf.

(8) The rocket launch had to be delayed because of some unexpected problems with its fuel tanks.

Derartige Belege zeigen, dass ein pronominaler Erstglied-Bezug unter bestimmten Bedingungen akzeptabel sein kann. Gleichzeitig wird der Bedarf illustriert, sich mit den speziellen Eigenschaften von Komposita im Allgemeinen und dem anaphorischen Potenzial von Erstgliedern im Besonderen auseinander zu setzen.

Bei Fragen der anaphorischen Zugänglichkeit wird zumeist von verschiedenen Graden an Akzeptabilität ausgegangen. Auch die hier aufgeführten Fälle legen dies nahe. Hierfür scheint bereits auf den ersten Blick ein komplexes Zusammenspiel von vielfältigen sprachlichen und kognitiven Aspekten wie Diskurs- und Informationsstruktur, syntaktischer Umgebung und Eigenschaften der Komposita bzw. ihrer Konstituenten verantwortlich zu sein.

Diese Arbeit beschäftigt sich mit den Bedingungen für Erstglied-Zugänglichkeit; genauer gesagt, mit den Bedingungen für die Akzeptabilität der pronominal-anaphorischen Bezugnahme. Dabei wird nach den Voraussetzungen für die Zugänglichkeit gefragt, nach Unterschieden zwischen verschiedenen Komposita und nach den wirkenden grammatischen und kognitiven Prinzipien. Der Fokus liegt dabei nicht auf der syntaktischen Umgebung, sondern auf den spezifischen Eigenschaften der Komposita selbst. 


\subsection{Relevanz für die linguistische Theoriebildung}

Bei der Auseinandersetzung mit dem anaphorischen Potenzial von Komposita-Erstgliedern sind verschiedene Subdisziplinen der Linguistik involviert. Im Gegenzug ermöglicht der Phänomenbereich ebenso wertvolle Einsichten in diese.

Im Bereich der Grammatik spielen Fragen nach der Notwendigkeit von Kongruenz eine Rolle, ebenso wie die Bedeutung semantischer Relationen. Das für diese Arbeit zentrale Gebiet der Grammatik ist die Morphosyntax. Die postulierte Erstglied-Unzugänglichkeit wird bei Definitionsversuchen für Komposita oftmals als Wortkriterium herangezogen (vgl. Kap. 2.2.2). Dem liegt die Annahme zugrunde, dass Wörter gemäß dem Prinzip der lexikalischen Integrität referenziell opak sind. Werden Komposita als Wörter betrachtet, müssen deren Konstituenten ebenfalls referenziell opak sein. Die Eigenschaft, referenziell zu sein, ist jedoch Voraussetzung für eine anaphorische Wiederaufnahme (vgl. Spencer 1991:42). Die Gültigkeit dieser Interpretation von lexikalischer Integrität und ihr Status als Wortkriterium sollen in dieser Arbeit zur Diskussion gestellt werden. Die Annahme gradueller Erstglied-Zugänglichkeit stellt zudem die Trennung zwischen Morphologie und Syntax in Frage und eröffnet die Forderung nach einer neuen Modellierung der internen Struktur von Komposita. Im Rahmen des Frameworks der Distribuierten Morphologie (vgl. u.a. Marantz 1997) wird hierfür in Kapitel 4 ein Versuch unternommen.

Fragen nach Zugänglichkeit, nach Akzeptabilitätsgraden und dem Aktivationsniveau der Konstituenten stammen aus dem Bereich der Sprachverarbeitung und sind somit in der Psycholinguistik zu verorten. Hierbei steht der Einfluss verschiedener Arten von Konzeptwissen zur Diskussion; ebenso Parsing-Modelle und die Verarbeitung von Komposita im Allgemeinen (z.B. Libben et al. 2003, Zwitserlood 1994). In diesem Zusammenhang dienen pronominale Anaphern als Werkzeug, um Aktivationsunterschiede und Zugänglichkeit sichtbar zu machen.

Diese Arbeit beschäftigt sich ausschließlich mit der Akzeptabilität von Diskursanaphern, weshalb die Diskurspragmatik als drittes Beschäftigungsfeld relevant ist und Einflussgrößen wie Kontextwissen oder Informationsstruktur bereit hält. Im Bereich der Anaphorik gilt es den Erstglied-Bezug in das Kontinuum zwischen direkter und indirekter Anapher einzuordnen. Eine Einordnung des Phänomens als indirekte Anapher erklärt den Bezug über Inferenzziehung und löst ihn somit von morphosyntaktischen Beschränkungen. Allerdings gilt allgemein auch für die indirekte Anaphorik, dass Pronomen nicht als Anaphern zur Verfügung stehen (vgl. Schwarz 2000). Darüber hinaus ist im Fall von Komposita das Erstglied an der Textoberfläche morphosyntaktisch realisiert und daher nicht im klassischen Sinne indirekt. Das Phänomen der skalaren Erstglied-(Un-)Zugänglichkeit ist somit auch für die Anaphorik interessant.

Zur Eingrenzung des Themengebietes beschränkt sich diese Arbeit auf endozentrische NN-Komposita des Deutschen (und aufgrund der Literaturlage z.T. des Englischen). Sie fokussiert Verarbeitungsunterschiede, die in den Komposita selbst begründet liegen und die anaphorische Zugänglichkeit beeinflussen. Annahmen der Syntax-Theorie basieren auf der Generativen Grammatik im Chomsky'schen Sinne. Nur solche Grammatikmodelle werden in Betracht gezogen. 


\subsection{Aufbau und Ziel der Arbeit}

Ziel dieser Arbeit ist es, das Phänomen der graduellen Erstglied-(Un-)Zugänglichkeit und dessen Umstände zu beschreiben und zu analysieren. Dabei ist das erste Ziel ein Überblick über die Natur der verschiedenen Arten von Einflussgrößen. Bereits erschlossene Faktoren werden diskutiert, dazu gehört der Grad an Transparenz der Komposition sowie diskurspragmatische Eigenschaften. Weitere und v.a. komposita-inhärente Faktoren sollen identifiziert, experimentell evaluiert und analysiert werden. Der Schwerpunkt liegt auf fundierter, empirischer Evidenz und nicht auf einer erschöpfenden Zusammenstellung. Dabei herrscht ein psycholinguistischer Blickwinkel vor. Im Zentrum stehen konzeptuelle und strukturelle Aspekte der Komposition sowie deren Interaktion. Je nach Art von Faktoren gilt es zu hinterfragen, inwiefern sich aus dem Einfluss des Faktors Einsichten in die Komposita-Verarbeitung und die Anaphernresolution ableiten lassen und welche Aspekte bei einer grammatischen Analyse berücksichtigt werden müssen. Die Analyse des anaphorischen Potenzials von Komposita-Erstgliedern soll einen Beitrag zur linguistischen Analyse von Komposita leisten und dabei die verschiedenen linguistischen Subdisziplinen verzahnen. Anaphern dienen hierbei primär als Werkzeug, um die Zugänglichkeit messbar zu machen. Sekundär jedoch auch als Analysegegenstand, z.B. in Bezug auf Verwendungsmöglichkeiten anaphorischer Pronomen.

In Kapitel 2 soll zunächst der Phänomenbereich abgesteckt werden. Dazu gehört die Betrachtung der Komposita als Ausdrucksklasse mit ihren spezifischen Eigenschaften und Annahmen zu ihrer Verarbeitung. Anschließend folgt eine Einordnung in die Anaphorik und die relevanten psycholinguistischen Größen, z.B. generelle Annahmen zur Zugänglichkeit. Schließlich werden verwandte Phänomene wie die Klammerparadoxie und Idiome angerissen und der Stand der Forschung zur Erstglied-Zugänglichkeit zusammengetragen. Kapitel 3 widmet sich den konzeptuellen Faktoren. Zum einen wird der Einfluss des Denotatwissens des Erstglieds beleuchtet, um darin Eigenschaften zu identifizieren, die die Zugänglichkeit beeinflussen können. Zum anderen wird die Interaktion der Konzepte von Erst- und Zweitglied bei der Etablierung einer Konzeptkombination untersucht, bzw. deren Auswirkungen auf die Erstglied-Zugänglichkeit. Daran anschließend wird ein erstes Experiment berichtet, welches Faktoren aus beiden Faktorgruppen bzgl. ihres Einflusses untersucht. Abschließend wird die Generizität als Faktor diskutiert.

Der Strukturaufbau verschiedener Komposita-Arten ist das Thema von Kapitel 4. Zunächst werden die zentralen Unterschiede zwischen Rektions- und Wurzelkomposita diskutiert. Anschließend erfolgt eine Diskussion des referenziellen Status des Erstglieds in den beiden Komposita-Arten und eine Modellierung im Rahmen des Frameworks der Distribuierten Morphologie. Anhand dieses Ansatzes wird dafür argumentiert, dass das Erstglied im Fall von Rektionskomposita zugänglicher ist.

In Kapitel 5 wird ein zweites Experiment berichtet, welches einen konzeptuellen Faktor in Verbindung mit dem strukturellen Faktor auf seinen Einfluss hin überprüft.

Das dritte Experiment widmet sich dem strukturellen Faktor und wird in Kapitel 6 berichtet. Neben der in allen drei Experimenten verwendeten Satzergänzungsaufgabe zur Erhebung von Daten der Offline-Verarbeitung, erweitert Experiment 3 mittels Eye-Tracking das Daten-Spektrum auf Belege der Online-Verarbeitung.

Die vorliegende Arbeit betrachtet sowohl grammatische als auch außersprachliche Faktoren der Erstglied-Zugänglichkeit und macht sich hierfür psycholinguistisches Instrumentarium zu Nutze. In diesem Sinne ist sie auch als Versuch eines Brückenschlags zwischen 
Grammatik und Kognition zu betrachten. Neben konkreten Erkenntnissen zu den Umständen der Erstglied-Zugänglichkeit sucht diese Arbeit nach neuen Erkenntnissen zur Verarbeitung von Komposita sowie für deren linguistische Analyse. Theoretische Implikationen liefern Hypothesen für Verarbeitungsunterschiede. Evidenz für letztere ermöglicht wiederum die Rückbindung an die linguistische Theorie. 


\section{Einordnung des Phänomens in die Theorielandschaft}

\subsection{Anaphorische Inseln und lexikalische Integrität}

Im Allgemeinen gilt ein N1 als unzugänglich für pronominale Anaphern. In der Literatur werden Komposita daher oft als ,anaphorische Inseln` bezeichnet. Bereits 1969 definierte Paul Postal eine anaphorische Insel ganz allgemein als:

[...] a sentence part which cannot contain an anaphoric element whose antecedent lies outside of the part in question and which cannot contain the antecedent structure for anaphoric elements lying outside. (Postal 1969 205)

Darauf aufbauend formuliert er als Prinzip A:

Lexical items are anaphoric islands with respect to outbound anaphora involving coreferential pronouns. $($ Postal $1969: 207))^{1}$

Unter , lexical items' versteht Postal monomorphemische Ausdrücke. Die Blockierung von Bedeutungsbestandteilen illustriert er u.a. anhand von (1). Die in der lexikalischen Semantik von orphan enthaltenen Eltern sind für die Anapher them nicht zugänglich.

(1) *Max is an orphan and he deeply misses them. (Postal 1969:206)

Gleiches gelte nun nach Prinzip A' auch für ,derivatives', wozu Postal komplexe Formen wie Ableitungen (vgl. (2) aus Postal (1969:213f)) und Komposita (vgl. (6-b) aus Postal (1969.215)) zählt.

(2) a. * McCarthyites are now puzzled by his intentions.

b. * blue-eyed people should conceal them with dark glasses

(3) a. Max hunts for wild animals but Pete only kills domesticated ones.

b. *Max is a wild-animal hunter but Pete only kills domesticated ones.

\footnotetext{
${ }^{1}$ Unter einer ,outbound anaphora' versteht er „the relation between a chunk, part of which is interpreted as antecedent, and some anaphor outside of that chunk" (Postal 1969 206), in Abgrenzung zu ,inbound anaphora', bei der die Anapher Teil eines Chunks ist und das Antezedens außerhalb steht. Derartige Fälle sind jedoch weder im Englischen noch im Deutschen produktiv und werden hier deshalb nicht berücksichtigt.
} 
In diesem Sinne sind für Postal alle Ausdrück mit Wortstatus - monomorphemische Ausdrücke, Ableitungen und Komposita - gleichermaßen anaphorische Insel, da sie die anaphorische Wiederaufnahme von Bedeutungsteilen nicht zulassen. Diese Gemeinsamkeit von monomorphemischen Ausdrücken und Komposita spiegele sich darin wider, dass die Anaphern in (4-a) und (4-b) gleichermaßen ungrammatisch sind (vgl. Postal 1969:226). In (4-a) liegt dabei ein monomorphemischer Ausdruck für das Fleisch eines Tieres vor, in (4-b) ein Kompositum.

(4) a. *The best pork comes from young ones.

b. *The best wombatmeat comes from young ones.

Aufbauend auf Postals Beobachtungen beschreiben auch andere Autor*inn*en Komposita als anaphorische Inseln. So kontrastiert Levi (1978:72) in den Beispielen (5) - (8) grammatische Bezüge auf Konstituenten von Phrasen mit ungrammatischen Bezügen auf Konstitutenten von Komposita.

(5) a. Lawyers dealing in divorce often experience it themselves.

b. ${ }^{*}$ Divorce lawyers often experience it themselves.

(6) a. Engineers specializing in electricity have to study it for years.

b. * Electrical engineers have to study it for years.

(7) a. Drivers of trucks spend days at a time in them.

b. ${ }^{*}$ Truck drivers spend days at a time in them.

(8) a. Criticizing by parents may alienate their children.

b. * Parental criticizing may alienate their children.

Sproat (1985:326) subsummiert mit Bezug auf (9), „It seems in general [...] that parts of words cannot be referred to by pronouns $[\ldots]^{\text {“ }}$

(9) a. Drivers of trucks fill them up with Arco Diesel.

b. * Truck-drivers fill them up with Arco Diesel.

Für das Deutsche lassen sich ähnliche Aussagen finden.2 Coulmas definiert das Phänomen der anaphorischen Insel wie folgt:

Anaphorische Inseln sind Satzteile, deren Elemente weder als Antezedens einer Anapher fungieren können, noch selbst anaphorisch auf ein außerhalb dieser Satzteile stehendes Antezedens bezogen sein können. (Coulmas 1988 317)

\footnotetext{
${ }^{2}$ Die Gültigkeit des Inselstatus für Komposita wird sowohl für das Englische als auch für das Deutsche angenommen. Es scheint sich demnach um ein übersprachliches, jedoch um kein allgemeingültiges Phänomen, z.B. im Japanischen ist ein Bezug auf einzelne Teile mit ihrerseits kompositabildenden Anaphern möglich (vgl. Coulmas 1988 333). Es stellt sich somit die Frage, welches die kritischen Parameter einer Sprache sind, die einen Inselstatus hervrobringen können.
} 
Demzufolge gelten alle Konstituenten komplexer Einheiten auf Wortebene als unzugänglich für pronominale Anaphern. Coulmas stimmt mit Postal überein, dass es unerheblich sei, ob diese ausschließlich semantisch komplex (z.B. (10-a)) oder morphologisch komplex im Sinne von Derivation (10-b) und (10-c) oder Komposition (10-d) und (10-e) sind (Coulmas 1988:318ff) $\mathrm{S}^{3}$

(10) a. *Carla ist Mutter geworden und Marlene hat auch eins gekriegt.

b. *Esperantisten schwören auf die Vorzüge dieser Sprache.

c. *Die Blondine hat es sich gefärbt.

d. *Atomwaffengegner haben gegen ihre Lagerung in Europa protestiert.

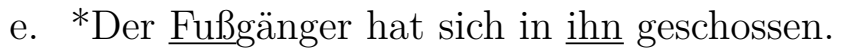

Ähnliche Aussagen lassen sich auch bei Schwarz (2000) und Dressler (2007) finden. Schwarz definiert eine anaphorische Insel als:

sprachliche[n] Ausdruck, bei dem man sich nicht mit einem Pronomen anaphorisch auf den in der semantischen Repräsentation enthaltenen, aber nicht explizit genannten Bedeutungsteil (bzw. Referenten) beziehen kann [...] Eine Insel, weil kein ,Landweg' und keine ,Brücke“ vorhanden ist, um eine textuelle Verbindungsmöglichkeit zu gestatten. (Schwarz 2000 124f)

Allerdings gilt es an dieser Stelle zu fragen, inwiefern die erste Konstituente von Komposita nicht explizit genannt ist. Der Umstand, dass es sich nicht um ein selbstständiges Wort handelt, macht die Realisierung einer Form meiner Auffassung nach nicht weniger explizit.

Bei der Eigenschaft eines Kompositums, eine anaphorische Insel zu sein, handelt es sich um eine deskriptive Beobachtung; es bleibt die Frage, worin dieser Status begründet liegt.

Postal (1969.219) beschreibt einen grammatikinternen Transformations-Mechanismus, der die Konstituenten unzugänglich werden lässt. Die zugrundeliegende semantische Struktur sei im Fall von (11-a) und (11-b) identisch und auch das Wortbildungsmuster sei gleich (vgl. (12)).
a. wombatmeat as [MEAT] from [WOMBAT]
b. pork as $[\mathrm{MEAT}]$ from $[\mathrm{PIG}]$
a. [WOMBAT][MEAT]
b. $[\mathrm{PIG}][\mathrm{MEAT}]$

Lediglich bei der lexikalischen Einsetzung (lexical insertion) unterschieden sich die beiden Ausdrücke dahingehend, wie viel des Ausdrucks durch ein einzelnes Morphem ersetzt wird. Die Grenzen der anaphorischen Inseln würden demnach vor der lexikalischen Einsetzung gesetzt und die beiden Ausdrucksarten verhielten sich gleich (vgl. Postal 1969:225). Diese Annahme setzt meines Erachtens voraus, dass Komposita als Ganzes gespeichert und eingesetzt werden. Diese Annahme wirft weitreichende Probleme auf, denen in Kapitel 2.3 nachgegangen wird.

\footnotetext{
${ }^{3}$ Coulmas erwähnt allerdings, dass die Gründe hier unterschiedlicher Natur seien.
} 
Eine in der einschlägigen Literatur verbreitete Begründung für das Phänomen der anaphorischen Inseln ist eine generellere Eigenschaft, die Wörtern zugeschrieben wird: Die lexikalische Integrität. Dazu formuliert der Morphologe Andrew Spencer:

[...] we cannot refer to the tea of teapot using an anaphor because words tend to be anaphoric islands. Anaphoric islandhood is a special case of a more general property of words: lexical integrity. The general pattern is for no syntactic process to be allowed to refer exclusively to part of words. ${ }^{4}$ (Spencer 1991:42)

Auch bei anderen Autor*inn*en findet sich als Erläuterung zur Lexical Integrity Hypothesis (LIH), dass kein syntaktischer Prozess ausschließlich lexikalische Strukturen involvieren darf (vgl. Lapointe 1979, Pesetsky 1979). Ähnliche Vorhersagen macht die Atomizitätshypothese, derzufolge Wörter auf der Ebene der phrasalen Syntax und Semantik Atome sind. Diese atomaren Einheiten verfügen zwar über spezifische Eigenschaften, jedoch nicht über eine interne Struktur, weshalb die Konstituenten dieser Einheiten nicht für die Syntax relevant sein können (vgl. Di Sciullo/Williams 1987) . $^{5}$ Mit der Autonomie-Hypothese macht Wunderlich (1986) ähnliche Aussagen für das Deutsche:

Die Autonomie-Hypothese besagt, daß die Bestandteile eines Worts syntaktischen Regeln nicht zugänglich sind. Für Bewegungsregeln und syntaktische Modifikation gilt dies fast uneingeschränkt. Bis auf einige, vielleicht pragmatisch zu erklärende Freiheiten gilt dies auch für die anaphorischen Beziehungen, soweit es sich nicht um referenzielle Anaphern handelt, die keinen syntaktischen Regeln folgen (nur vielleicht syntaktisch beschränkt sind). (Wunderlich 1986 225)

Aus dieser Annahme lässt sich im Umkehrschluss ableiten, dass, wenn der Status von Komposita als anaphorische Inseln auf lexikalischer Integrität beruht, anaphorische Beziehungen als rein syntaktische Prozesse aufzufassen sind. Dabei bleibt unklar, welche Annahmen für Diskursanaphern gelten. Eine ähnliche Überlegung dazu findet sich bei Wunderlich:

\begin{abstract}
Wenn Syntax und Lexikon autonom sind und die Anaphernregel primär syntaktisch bedingt ist, dann sind Bestandteile von Wörtern für die Anaphernregel nicht zugänglich: Wörter sind als anaphorische Inseln zu betrachten. Sollte sich aber herausstellen, daß Wörter nicht strikte anaphorische Inseln sind, gilt entweder das Autonomieprinzip nicht strikt oder die Anaphernregel ist nicht ausschließlich syntaktisch (sondern evtl. semantisch oder pragmatisch) bedingt. (Wunderlich 1986 219)
\end{abstract}

Eine andere Frage, die sich hierbei stellt, ist: Wenn keine anaphorische Referenz Teile von Wörtern involvieren darf, müsste dies nicht nur für die Erstglieder, sondern auch für die Zweitglieder in isolierter Form gelten. Anhand von Beispielen wie (13) beschreibt Levi (1977) diese Form der Bezugnahme jedoch als möglich.

a. State taxes were higher than municipal ones.

b. Steam irons need more maintenance than those that iron dry.

Im Fall von Komposita mit adjektivischem Erstglied zeigt Schäfer (2013) entsprechende Möglichkeit auch fürs Deutsche (vgl. (14)).

\footnotetext{
${ }^{4}$ Hervorhebungen wie im Original.

${ }^{5}$ Ähnliche Voraussagen machen auch die Bracketing Erasure Convention nach Pesetsky $(197934)$, die Strong Lexicalist Hypothesis (vgl. Booij/van Haaften 1988, Di Sciullo/Williams 1987, Lieber 1983. Selkirk 1982) und die Word Structure Autonomy Condition (vgl. Selkirk|1982:70).
} 
a. Ich liebe Großstädte, in kleinen gehe ich ein.

b. Ich liebe $\left[\right.$ Groß $\left.[\text { städte }]_{i}\right]$, in kleinen $\mathrm{PRO}_{i}$ gehe ich ein.

Ortner/Ortner 1984:39) bewerten Fälle wie (15) vor allem als Phänomen der gesprochenen Sprache, da sie vom Standpunkt strenger Norm als falsch zu bewerten seien.

Ich trank Flaschenbier, weil sie kein anderes hatten.

Das Phänomen der lexikalischen Integrität bedarf demnach einer differenzierten Betrachtung. Hierzu liefert eine Definition von Stephen Anderson (1992) einen Ansatz.

Principle of Lexical Integrity:

The syntax neither manipulates nor has access to the internal structure of words. Anderson 1992 )

Auf die Formulierung von Anderson Bezug nehmend, setzt sich Geert Booij (2009b) mit den beiden darin enthaltenen Arten von Interaktion zwischen Morphologie und Syntax auseinander: (i) Die Manipulation von Teilen der wortinternen Struktur und (ii) die Zugänglichkeit der wortinternen Struktur.

Die Annahme (i) buchstabiert Booij dergestalt aus, dass keine syntaktische Bewegung, kein Aufsplitten und keine Flexion im Sinne von Zuweisung struktureller Kasus bei Wortteilen möglich ist. Ein Gegenargument gegen das Verbot des Aufsplittens wäre die Trennbarkeit von Partikelverben. Dies entkräftet Booij, indem er Partikelverben am Beispiel des Niederländischen den Wortstatus abspricht (vgl. Booij 2009b:86). Wortinterne flektierte Formen seien entweder Teil einer wortinternen Phrase (vgl. Phrasenkomposita in Kap. 2.5.1), die in sich grammatisch sein muss, oder inhärent flektiert. Im Gegensatz zu kontextueller Flexion sei die inhärente Flexion nicht durch die Syntax gesteuert (vgl. Booij 2009b:89). Aus diesem Grund hält Booij an der Gültigkeit von (i) fest. Anders verhält es sich mit (ii): Bestandteile von Wörtern scheinen zwar nicht manipulierbar, jedoch können sie die konkrete Realisierung einer Wortform beeinflussen, wie es die Flexion der Adjektive bei der Klammerparadoxie zeigt (vgl. Kap. 2.5.4). Darüber hinaus führt Booij unter typologischem Zugang weitere Beispiele konstruktionsabhängiger Morphologie an. In diesem Sinne scheint die wortinterne Struktur sichtbar zu sein für syntaktische Regeln, Regeln der semantischen Interpretation und auch der Phonologie (vgl. Booij 2009b:90f). Booij schlussfolgert daraus, dass die Schnittstelle zwischen Morphologie und Syntax dergestalt sein muss, „that the syntax may have to see word-internal morphological structure“ (Booij 2009b 94). Aufgrund dessen sei das Prinzip der lexikalischen Integrität neu zu formulieren bzw. zu restringieren: Die Bewegung und Aufspaltung von Wortteilen sowie die Zuweisung morphosyntaktischer Eigenschaften durch die Syntax sei, wie schon bei Anderson formuliert, nicht möglich (vgl. Booij 2009b:97). Komplexe Wörter hingegen hätten eine interne Struktur, die für die Regeln der Grammatik sichtbar, bzw. zugänglich sein kann.

Da das Prinzip der lexikalischen Integrität oftmals als Wortkriterium fungiert, muss auch dies neu diskutiert werden. Für Booij ist nicht die lexikalische Integrität an sich das entscheidende Wortkriterium, sondern die relative Kohäsion. Ein Wort bildet eine kohäsivere Einheit als eine Phrase, was sich wiederum darin äußert, dass sich Wörter nicht manipulieren lassen. Gleichzeitig handelt es sich um eine relative Eigenschaft, die eben auch Einschränkungen und Ausnahmen zulässt (vgl. Booij 2009b:97). 
Booijs Standpunkt lässt sich so zusammenfassen, dass der Kern der lexikalischen Integrität darin liegt, dass Wörter nicht syntaktisch manipuliert werden können und der zentrale Unterschied zwischen Wörtern und Phrasen im Grad der Kohäsion zwischen den Konstituenten liegt (vgl. Booij 2009b:98). Die generelle Möglichkeit von Ausnahmen betont Booij explizit: „Lexical Integrity as the prohibition on syntactic manipulation of word-internal constituents is not an absolute universal, but rather the default situation." (Booij 2009b: 98)

Booijs Ansatz lässt sich mit der Annahme gradueller N1-Zugänglichkeit vereinbaren, da hier zwar die anaphorische Zugänglichkeit von Wortteilen Bedingung ist, jedoch keine Manipulation dieser. Gleichzeitig ist die Zugänglichkeit von Komposita-Konstituenten gegenüber der Zugänglichkeit phrasaler Konstituenten eingeschränkt. Der von Booij beschriebene Unterschied in der Kohäsion wird dem Phänomen in dieser Hinsicht gerecht. Ob es sich um ein Manipulationsverbot oder nur eine -beschränkung handelt, gilt es empirisch zu hinterfragen. Auch die Trennung zwischen inhärenter und kontextueller Flexion ist diskutabel. Meiner Ansicht nach sagt der Flexionsauslöser selbst nichts über den Status der Realisierung als syntaktische Form aus. Die Zugänglichkeit wortinterner Struktur hält Booij generell für möglich. Sämtliche Beispiele Booijs sind typologischer Natur. Ein Ziel der vorliegenden Arbeit ist es, die Diskussion auch durch experimentelle Befunde zu befruchten.

Das Prinzip der lexikalischen Integrität nun dergestalt zu verkürzen, dass die syntaktische Manipulation wortinterner Strukturen nicht möglich ist, deren Zugänglichkeit hingegen schon, wäre zu kurz gegriffen und auch nicht im Sinne Booijs. Vielmehr nehme ich an, dass das Verbot der syntaktischen Manipulation wortinterner Strukturen der stärkere Teil des Prinzips ist, die Zugänglichkeit der schwächere. Ausnahmen lassen sich für beide Regeln finden und werden im weiteren Verlauf dieses Kapitels diskutiert. Darauf aufbauend ist die Idee der relativen Kohäsion als Wortkriterium mit meinen Beobachtungen vereinbar. Das Prinzip der lexikalischen Integrität fasse ich im Folgenden nach wie vor im Sinne Andersons und anderer Autor*inn*en als Kombination beider Regeln auf.

Die generelle Annahme, dass lexikalische Integrität die Ursache für die Unzugänglichkeit von Erstgliedern ist, bringt zwei weitere Probleme mit sich: Zunächst kann sie nicht der ausschließliche Grund für die Ungrammatikalität der genannten Beispiele sein, da in einigen Fällen auch andere grammatische Regeln verletzt werden, was an sich bereits Ungrammatikalität bewirkt, z.B. die Verletzung von Bindungsprinzipien. Darüber hinaus setzt die Annahme der Existenz eines Prinzips wie der lexikalischen Integrität eine lexikalistische Grammatiktheorie voraus. Andernfalls wäre ein Grenzziehung zwischen Morphologie und Syntax irrelevant. Unter der Voraussetzung eines rein syntaktischen Ansatzes müssen andere Prinzipien herangezogen werden, um die Ungrammatikalität von anaphorischen N1-Bezügen zu begründen.

Ein Vertreter eines syntaktischen Ansatzes ist Richard Sproat (1985). In Bezug auf die Position Postals expliziert er, dass pork und wombatmeat nicht gleich zu behandeln seien. Im Fall von pork handele es sich um keine morphologisch komplexe Struktur und Elemente der semantischen Repräsentation seien per se für die Syntax unzugänglich. Der Status von pork als anaphorischer Insel sei demnach trivial. Im Fall von wombatmeat als morphologisch komplexem Wort sei die Lage jedoch anders. Hier argumentiert er rein syntaktisch mit der referenziellen Opazität von Erstgliedern. 
In fact, I shall argue that it follows trivially from the fact that the morphology deals essentially exclusively in non-maximal projections, and given the reasonable assumption that only referential expressions - i.e. maximal projections - can serve as antecedents to pronouns, Anaphoric Islandhood is to be expected. (Sproat 1985:328f)

Die syntaktische Argumentationslinie basiert auf der Annahme, dass Erstglieder generell nicht maximal und damit nicht referenziell sind. Als Voraussetzung für Referenzialität und die Möglichkeit der pronominalen Wiederaufnahme wird die Anwesenheit eines Antezedens in Form einer NP postuliert, d.h. einer maximalen Projektion mit Argumentstatus. An der Position eines N1 könne dies nicht vorliegen (vgl. Levi 1978, Schwarz 2000, Sproat 1985). Ausdruck der mangelnden Referenzialität von Erstgliedern sei, dass sie keine spezifischen Objekte denotieren (vgl. Olsen 2012, Spencer 1991).

For instance, neither student nor film in student film society serve to pick out any specific student or film. This is why these non-heads can be used attributively. In this respect, constituents of compounds differ from consituents of sentences. (Spencer 1991 312)

Hierbei ist jedoch anzumerken, dass es auch nicht-referierende NPn gibt. So denotiert Löwe in (16-a) keinen spezifischen Löwen, sondern den Prototyp. Im Gegensatz dazu gibt es durchaus spezifische Erstglieder. So denotiert das N1 Löwe in (16-b) ein spezielles Individuum der Gattung, nämlich den einen Löwen, der im Zoo gesehen wurde.
a. Der Löwe gilt als König der Tiere.
b. Das Löwenbild, das du gestern im Zoo geschossen hast, ist wirklich gut ge- lungen.

Was ist vor diesem Hintergrund nun unter dem Begriff der anaphorischen Insel zu verstehen? Handelt es sich um eine grammatische Beschränkung, spielen hierbei die lexikalische Integrität und Annahmen zu speziellen N1-Eigenschaften wie Nicht-Flektierbarkeit und Nicht-Referenzialität eine entscheidende Rolle. Begegnet man dem Phänomen jedoch auf Diskursebene, zeichnet sich ein anderes Bild: Die eingangs aufgeführten Beispiele authentischer Sprache zeigen, dass ein N1-Bezug anscheinend durch lineare Distanz, Satzgrenzen und den pragmatischen Kontext möglich werden kann. Hier scheint die Zugänglichkeit weniger eine Frage der Grammatik als der Verarbeitung zu sein.

Die zentralen Ansatzpunkte für die folgenden Analysen sind demnach grammatische Beschränkungen und Faktoren der kognitiven Verarbeitung. Die Grundlagen hierfür sollen in den folgenden Abschnitten gelegt werden. Zunächst werden die grammatischen Eigenschaften von Komposita näher beleuchtet (Kap. 2.2) und anschließend die relevanten Annahmen zur Verarbeitung von Komposita und ihren Konstituenten reflektiert (Kap. 2.3). Als dritter Baustein des Phänomens des N1-Bezugs gilt es die anaphorischen Prozesse näher zu betrachten (Kap. 2.4).

\subsection{Komposita in der Grammatiktheorie}

Zunächst gilt es Komposita von einfachen Wörtern auf der einen und von Phrasen auf der anderen Seite abzugrenzen und zu definieren sowie nach den spezifischen Eigenschaften der Konstituenten und ihrer linguistischen Analyse zu fragen. 


\subsubsection{Definition und Klassifikation}

\section{Definition}

Die Komposition ist das produktivste Wortbildungsmuster des Deutschen (vgl. Klos 2011: 298). An der Schnittstelle zwischen Morphologie und Syntax lassen sich Komposita wie Phrasen als komplexe, (vorrangig) rechtsköpfige Strukturen beschreiben, die aus kleineren Einheiten bestehen und deren Gesamtbedeutung über Teilbedeutungen erschließbar ist (vgl. Klos 2011:17). In Abgrenzung zur Phrasenbildung stehen am Ende der Komposition Wörter als Ergebnis, so definiert Bauer: „the formation of a new lexeme by adjoining two or more lexemes" (Bauer 2003:40). Eine weitere klassische Definition stammt von Susan Olsen:

Composition (also: compounding; Germ.: Komposition, Zusammensetzung) is one of the major processes available in language for the formation of new words. It denotes the combining of two free forms or stems to form a new complex word referred to as a compound (Germ.: Kompositum, Zusammensetzung). (Olsen|2000 897)

Eine zentrale Funktion der Komposition ist die Sprachökonomie. Komposita ermöglichen die effiziente Benennung neuer Konzepte ohne weitere Erläuterung, wie z.B. beim eingangs erwähnten Zettelnagel. Bei Erben (2000:67) heißt es, sie bieten eine „bequeme Möglichkeit der knappen umrißhaft andeutenden Benennung“. Ihre Effizienz gründet darin, dass sie den Wortschatz erweitern und dabei auf bekanntes lexikalisches Material zurückgreifen können (vgl. Klos 2011:292). Die ,einfache' Verbindung zweier Ausdrücke sei oft weniger aufwändig als komplexe syntaktische Verbindungen. Komposita spezifizieren, kategorisieren, typisieren und haben z.T. auch eine stilistische Funktion (vgl. Klos 2011:17).

\section{Klassifikation}

Bei der Klassifikation von Komposita können unterschiedliche Klassifikationskriterien angelegt werden. Eine Möglichkeit ist die Klassifikation anhand der involvierten Wortarten (siehe (17) nach Meibauer (2007:48f)). Die große Produktivität und Formenvielfalt in der Komposition resultiert letztlich auch daraus, dass nahezu alle Wortarten, Buchstaben, Konfixe, syntaktische Fügungen etc. in einem Kompositum auftreten können (vgl. Dudengrammatik RN 1093 (Wermke et al. 2009:717)).

$$
\begin{array}{ll}
\text { a. } & \mathrm{N} \rightarrow \mathrm{N}+\mathrm{N}(\text { z.B. Holz+haus) } \\
\text { b. } & \mathrm{N} \rightarrow \mathrm{A}+\mathrm{N} \text { (z.B. Rot+licht) } \\
\text { c. } & \mathrm{N} \rightarrow \mathrm{V}+\mathrm{N} \text { (z.B. Web+stuhl) } \\
\text { d. } & \mathrm{N} \rightarrow \mathrm{P}+\mathrm{N} \text { (z.B. Vor+geschmack) } \\
\text { e. } & \mathrm{A} \rightarrow \mathrm{N}+\mathrm{A} \text { (z.B. haut+freundlich) } \\
\text { f. } & \mathrm{A} \rightarrow \mathrm{A}+\mathrm{A} \text { (z.B. alt+klug) } \\
\text { g. } & \mathrm{A} \rightarrow \mathrm{V}+\mathrm{A} \text { (z.B. rutsch+fest) } \\
\text { h. } & \mathrm{V} \rightarrow \mathrm{N}+\mathrm{V} \text { (z.B. rad+fahren) } \\
\text { i. } & \mathrm{V} \rightarrow \mathrm{A}+\mathrm{V} \text { (z.B. froh+locken) } \\
\text { j. } & \mathrm{V} \rightarrow \mathrm{V}+\mathrm{V} \text { (z.B. schwing+schleifen) } \\
\text { k. } & {[\ldots]}
\end{array}
$$


Für diese Arbeit ist v.a. der Kompositionstyp N+N (17-a) von Interesse, d.h. Komposita mit einem nominalen Erstglied (N1) und einem nominalen Zweitglied (N2).

Ein weiteres mögliches Klassifikationskriterium orientiert sich an der Position des semantischen Kopfs und basiert auf der Sanskrit-Grammatik. Dabei wird zwischen endozentrischen Komposita (Sanskrit ,tatpurusha'), exozentrischen Komposita (Sanskrit ,bahvrihi') und Kopulativkomposita (Sanskrit ,dvandva') unterschieden (vgl. Olsen 2000:908). Bei endozentrischen Komposita befindet sich der semantische Kopf innerhalb des Kompositums, z.B. denotiert Computertisch einen Tisch. Bei exozentrischen befindet sich der Kopf außerhalb des Kompositums und muss inferiert werden, z.B. denotiert Schreihals keinen Hals, sondern einen schreienden Menschen (vgl. Dressler 2007:33). Kopulativkomposita bilden semantisch eine gleichwertige Kombination aus Erst- und Zweitglied, z.B. denotiert Dichter-Sänger einen Menschen, der sowohl Dichter ist als auch Sänger. Im Deutschen ist die Unterteilung zwischen endo- und exozentrischen Komposita weniger zentral, da letztere kaum produktiv sind. Bei den attestierten Fällen handelt es sich meist um possessiv-metonymische Bezeichnungen für Menschen, wie Schreihals oder Dummkopf. Im Englischen existiert zusätzlich die Form der exozentrischen Imperative, z.B. pickpocket (vgl. Härtl 2015:161).

Eine andere Möglichkeit der Klassifizierung liegt auf der Ebene der strukturellen Fügung. Die zentrale Unterteilung innerhalb der Nominalkomposita besteht hierbei zwischen Determinativ- oder Wurzelkomposita 6 und Rektionskomposita (so bei Meibauer 2007, Scalise/Bisetto 2009, Spencer 1991). Der Unterschied zwischen Wurzel- und Rektionskomposita liegt darin, dass Wurzelkomposita aus zwei nominalen Wurzeln zusammengesetzt sind, z.B. Dach+garten. Der Kopf von Rektionskomposita ist hingegen deverbal, z.B. bei Dach+begrünung. Das N1 kann in diesem Fall eine Argumentstelle des Verbs einnehmen.

Andere Klassifizierungsmodelle vereinen wiederum mehrere Kriterien. Eine Übersicht für das Englische findet sich in Scalise/Bisetto (vgl. 2009:39f). Das Kriterium für die Klassifikation von Scalise/Bisetto (vgl. 2009:45) in (18) ist die Art, auf die der Kopf das N1 selegiert. Dabei fassen die Autor*inn*en unter „Subordinate“ Komposita mit sich komplementierenden Konstituenten wie apron string (endozentrisch) oder pickpocket (exozentrisch). Unter „Attributive“ fassen sie die Fälle, in denen die Komposita-Konstituenten in einem attributiven Verhältnis zueinander stehen, z.B. blackbird (endozentrisch) oder greybeard (exozentrisch). „Coordinate“ umfasst die Fälle, in denen vorrangig konjunktive Koordination zwischen den Konstituenten vorliegt, z.B. poet doctor, bzw. Austria-Hungary (vgl. dazu auch Scalise/Vogel (2010:7)).

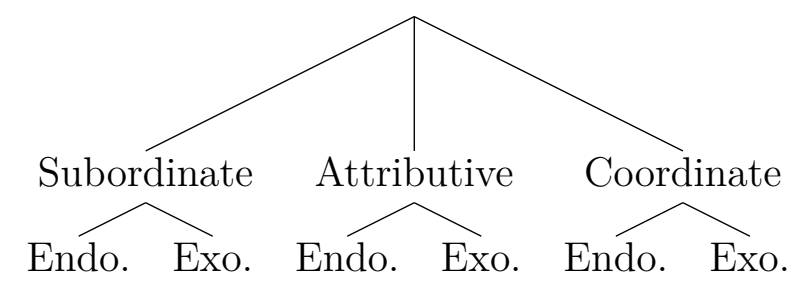

\footnotetext{
${ }^{6} \mathrm{Da}$ auch bei Rektionskomposita ein Determinationsverhältnis vorliegt, verwende ich zur Abgrenzung den Ausdruck ,Wurzelkomposita“ und lehne mich dabei an den Englischen Terminus ,root compound an.
} 
Darüber hinaus unterbreiten Scalise/Bisetto (vgl. 2009:50) einen Vorschlag für ein intermediäres Level, worin die Kategorie „Subordinate“ in Komposita mit nominalem und verbalem Kern unterteilt wird. Die Kategorie „Attributive“ wird in „attributive“ und „appositive" unterteilt. Aufgrund der Vermischung der Beschreibungsebenen sowie der z.T. kaum eindeutig vorzunehmenden Einordnung einiger Fälle, erscheint mir diese Einteilung nicht optimal. Auch Spencer (vgl. 2011:494) erachtet diese Aufteilung als uneindeutig und vor allem die Trennung zwischen „Appositive“ und „Subordinate“ als unscharf. Eine ähnliche Einteilung, die jedoch weitere, differenzierende Unterebenen enthält, findet sich bei Lieber (vgl. 2009:358ff). Weitere mögliche Klassifikationskriterien sind z.B. Transparenz oder Relationalität (vgl. Dressler 2007:43f).

Für das Phänomen der Erstglied-Zugänglichkeit ist die Wahl eines Klassifikationsmodells insofern von Interesse, als dass eine Abgrenzung erfolgen muss, da nicht alle Annahmen gleichsam für alle Arten von Komposita gültig sind. Vor diesem Hintergrund beschränken sich die weiteren Ausführungen auf Fälle endozentrischer NN-Determinativkomposita (vorrangig Wurzel- und Rektionskomposita) entsprechend der Einteilung nach Olsen $(2000)$ und Reinhard (2001). Diese Entscheidung basiert darauf, dass Determinativkomposita im Deutschen die produktivste Form von Komposita sind, wobei NN-Komposita besonders häufig vertreten sind (vgl. Erben 2000:62f) und sich somit gut für die empirische Arbeit eignen.

\subsubsection{Charakteristika von Komposita}

\section{Charakteristika des Gesamtkompositums}

Komposita sind von Natur aus (zumindest potenziell) ambig, da ihre Teile durch eine unterspezifizierte Relation miteinander verbunden sind (dazu z.B. Dressler (2007:36) und Olsen (2000:898)). So kann es sich beim Denotat des Kompositums Kuchenteller um einen Teller mit Kuchen, einen Teller für Kuchen oder einen Teller mit einem darauf abgebildeten Kuchen handeln. ,Unterspezifiziert' bedeutet hier, dass stets zumindest theoretisch verschiedene Konstruktionsbedeutungen, d.h. Relationen zwischen den Konstituenten möglich sind, auch wenn es zumeist eine starke Präferenz für eine Lesart gibt (vgl. dazu Meineke 1991:52f). So denotiert Filtertüte konsensuell einen Kaffeefilter. In entsprechendem situativen Kontext kann jedoch auch eine Tüte mit Zigarettenfiltern das Denotat sein. Die verschiedenen Arten von semantischen Relationen werden in Kapitel 2.2.4 thematisiert.

Das Verhältnis der Konstituenten ist des Weiteren zumeist dadurch charakterisiert, dass ein N2 durch ein N1 näher spezifiziert, bzw. modifiziert wird. Typisch für Komposita ist subsektive Modifikation, also Teilmengenbildung,7Dank dieser Eigenschaft können sie zur Denotation von Kontrasten genutzt werden, z.B. zur Unterscheidung von Teekanne und Kaffeekanne (vgl. Gagné/Spalding 2007:146). Weitere Arten der Modifikation werden in Kapitel 3.3 beschrieben.

Eine weitere typische Eigenschaft ist die Unvertauschbarkeit der Konstituenten (vgl. Meineke 1991:45). So denotiert Tellerkuchen etwas anderes als Kuchenteller. Anhand dieses

\footnotetext{
${ }^{7}$ Siehe dazu auch die Dudengrammatik RN 1095 (Wermke et al.|2009 718).
} 
Beispiels lässt sich auch das u.a. für deutsche und englische Komposita geltende KopfRechts-Prinzip 8 illustrieren: Das Denotat eines Determinativkompositums ist immer vom Typ N2, d.h. das Kompositum Kuchenteller denotiert eine Entität der Gattung Teller und das Kompositum Tellerkuchen eine Entität der Gattung Kuchen. Dabei reichert das N1 das N2 semantisch mit zusätzlichen Merkmalen an (vgl. Klos 2011:287).

Trotz der intuitiven Unterscheidbarkeit zwischen Komposita und Phrasen ist diese Einteilung auf theoretischer Ebene alles andere als trivial. Neben der Rechtsköpfigkeit teilen sich die beiden Strukturen auch die Eigenschaft der Binarität und der Rekursivität (vgl.(19)) . Eine weitere Gemeinsamkeit scheint darin zu liegen, dass es sich bei den Konstituenten zumeist um formal selbstständige Wörter handelt (z.B. Ortner/Ortner 1984) 9

$$
\begin{array}{ll}
\text { a. } & \mathrm{N}+\mathrm{N}(\mathrm{z} . \mathrm{B} . \text { Sand }+ \text { kuchen }) \\
\text { b. } & (\mathrm{N}+\mathrm{N})+\mathrm{N}(\mathrm{z} . \mathrm{B} . \text { Sandkuchen+teller }) \\
\text { c. } & ((\mathrm{N}+\mathrm{N})+\mathrm{N})+\mathrm{N} \text { (z.B. Sandkuchenteller+porzellan) }
\end{array}
$$

Als Wortkriterium wird häufig die bereits diskutierte lexikalische Integrität herangezogen (u.a. Böer et al. 2012, Giegerich 2006). Unabhängig von den angeführten Kritikpunkten zur lexikalischen Integrität an sich, gibt es auch Argumente gegen ihre Gültigkeit als Wortkriterium. Ausgehend von der Annahme, dass es sich bei Komposita um Wörter handelt, stellt Haspelmath (2011) fest, dass diese im Englischen i.d.R. durch ein Leerzeichen getrennt werden und dadurch an der Oberfläche formal als zwei Wörter erscheinen. Auf der anderen Seite gibt es im Deutschen phrasale Konstruktionen, die ebenfalls anaphorische Inseln sind, z.B. Univerbierung wie (20) (vgl. Kap. 2.5.2).

*Ich fahre Fahrrad, wenn es nicht kaputt ist.

Ein weiteres Kriterium zu Identifikation von Komposita ist ihre Untrennbarkeit. Es zeigt sich, dass Komposita im Gegensatz zu Phrasen im Satz nur als Ganzes verschoben werden können (vgl. Meineke 1991:32). Allerdings unterliegen Nominalphrasen hier ebenfalls Beschränkungen. Für das Kriterium der Untrennbarkeit spricht, dass Komposita nur im Ganzen modifiziert werden können. Im Gegensatz zu Phrasen wie (21-a) kann weder das N1 (vgl. (21-b) noch das N2 (vgl. (21-c)) separat modifiziert werden (vgl. dazu Olsen (2000:898) oder Meineke (1991:36)). Eine Ausnahme hierfür bildet die Klammerparadoxie (vgl. Kap. 2.5.4).
a. ein frischer Teller für Kuchen/ein Teller für frischen Kuchen
b. *ein [frischer Kuchen]teller
c. *ein Kuchen[frischerteller]

Auch im Betonungsmuster liegen Unterschiede: Sowohl im Deutschen als auch im Englischen liegt der Hauptakzent des Kompositums auf der schweren Silbe der ersten Konstituente (dazu Olsen (2000:898) oder Meineke (1991:28)). Punske (vgl. 2016:383) lehnt

\footnotetext{
${ }^{8}$ Bei der Position des Kopfs handelt es sich um einen sprachspezifischen Parameter. In den germanischen Sprachen sind Komposita rechtsköpfig, in den romanischen Sprachen befindet sich der Kopf links (vgl. Booij 2009a 210).

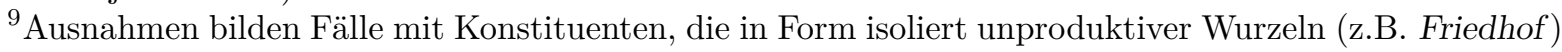
oder ganzer Phrasen (z.B. Der-schöne-Rheingau-Laberei) vorliegen (vgl. Dressler 2007:39).
} 
dies als Wortkriterium für das Englische ab. Mit (22-a) führt er Beispiele mit finalem (also phrasalem) Akzent auf. Im Deutschen ist der Hauptakzent auf dem N2 zumindest in kontrastiver Funktion möglich (vgl. (22-b)). Das Betonungsmuster allein kann somit ebenfalls nicht als entscheidendes Kriterium dienen.

a. apple pie, nylon rope, stone castle, steel beam, duck soup

b. Du sollst nicht die KuchenPLATTE, sondern den KuchenTELLER benutzen.

In Bezug auf orthografische Eigenschaften existiert das Kriterium der Zusammenschreibung ${ }^{10}$ (vgl. Ortner/Ortner 1984). Dies wird allerdings von Lieber/Štekauer (2009:8) nicht als Wortkriterium, sondern als Reflex auf die gesprochene Sprache bewertet.

Gemäß Böer et al. (vgl. 2012.71) verfügen Komposita aufgrund ihres höheren Grads an Markiertheit über eine bessere Memorisierbarkeit als Phrasen. Auch verfügten Komposita über einen höheren Grad an Diskurssalienz, sowie über unterschiedliche kognitive Status (vgl. Böer et al. 2012:71ff). Derartige Kriterien können jedoch aufgrund fehlender unmittelbarer Überprüfbarkeit nicht als direkte Evidenz dienen.

Neben diesen formalen Kriterien finden sich u.a. bei Bücking (2010) Bemühungen der funktionalen Abgrenzung zwischen Phrase und Kompositum. Die typische Verwendung von Komposita ist nach Bücking (2010:254) die (potenzielle) Benennung oder Kategorisierung eines zugrundeliegenden Konzepts, während Phrasen eine Beschreibung liefern, z.B. ein Hochhaus vs. ein hohes Haus (dazu auch Olsen (2000:898f)). Diese Unterscheidung scheint auf einen Großteil der Fälle zuzutreffen, jedoch nicht alle Fälle von Komposita abzudecken. Für die Fälle in (23)] scheint die Unterscheidung zu gelten: Während ein Teller für Kuchen in (23-a) einen beliebigen Teller beschreibt, der lediglich in Größe und Tiefe für einen Kuchen geeignet sein muss, benennt Kuchenteller in (23-b) eine spezielle Art von Teller mit dem konkreten Verwendungszweck der Kuchenaufbewahrung. In (24) hingegen ist der Funktionsunterschied weniger deutlich. Die Eigenschaft ein Hausbesetzer zu sein in (24-b) unterschiedet sich von der Tätigkeit des Hausbesetzens in (24-a) lediglich darin, dass ersteres habituell und letzteres aktuell geschieht. Unter Verwendung eines disambiguierenden Temporaladverbials ist auch dieser Unterschied aufgehoben.
a. Paul sucht einen Teller für den Kuchen.
b. Paul sucht einen Kuchenteller.
a. Paul und Tina besetzen (im Moment) ein Haus.
b. Paul und Tina sind (im Moment) Hausbesetzer.

\section{Charakteristika des Kopfs (N2)}

Scalise/Fábregas (2010:110) unterscheiden drei Arten von Köpfen: morphologische, kategorische und semantische. Der morphologische Kopf bestimmt die formalen Eigenschaften des Kompositums als lexikalisches Item, z.B. das Genus. Der kategorische bestimmt die

\footnotetext{
${ }^{10}$ Hierbei handelt es sich um ein Kriterium, was besonders für das Deutsche gilt, da die Zusammenschreibung (neben der Schreibung mit Bindestrich) die gängigste Form der Verschriftlichung ist. Im Englischen ist dies aufgrund orthografischer Unterschiede schwieriger. Neben Zusammen- und Bindestrichschreibung ist vor allem ein Leerzeichen zwischen N1 und N2 die Regel. Die Schreibweise kann somit für das Englische nicht als Kriterium herangezogen werden.
} 
lexikalische Kategorie und der semantische bestimmt die semantische Klasse des Gesamtworts (vgl. Scalise/Fábregas 2010:124). Die drei Arten von Köpfen fallen meist zusammen, jedoch nicht zwingend. Dies wird am Fall der Kopulativkomposita deutlich: Dort liegt der semantische Kopf nicht rechts, sondern ist eine Mischung aus den Bedeutungen der beiden Konstituenten. Da morphologischer und kategorischer Kopf jedoch zumeist zusammenfallen, werde ich diese beiden Köpfe unter der Bezeichnung ,morphosyntaktischer Kopf‘ zusammenfassen.

Die Funktion des morphosyntaktischen Kopfs fällt im Deutschen i.d.R. dem N2 zu, dessen Eigenschaften perkolieren zum Gesamtwort und legen somit dessen grammatische Merkmale fest. Damit geht einher, dass das N1 seine Merkmale nicht vererbt. Für Wunderlich (vgl. 1986:223) wird dies daran deutlich, dass das N1 seine Rektionseigenschaften und semantischen Argumente verliert (vgl. (25)), da diese Argumente existentiell gebunden und für syntaktische Realisierung blockiert sind.

??Trinkglas des Weins, ??Nachdenkpause über Anaphern, ??Esstisch der Nudeln

Eine Ausnahme räumt Wunderlich (vgl. 1986 224) im Falle von N1-Nominalisierungen wie in (26) ein. In diesem Fall sei eine Ausnahme pragmatisch lizenziert und es handele sich um ein periphäres Phänomen des Sprachgebrauchs. Ähnliches findet sich auch bei Härtl (vgl. 2015:170).

Abrißgenehmigung des schnellen Brüters, Elternverein behinderter Kinder, Parkgenehmigung im Hof, Ernennungsurkunde zum Professor

Neben der Eigenschaft des N2 morphosyntaktischer Kopf zu sein, fungiert es im Fall endozentrischer Determinativkomposita zumeist auch als semantischer Kopf. Dem N1 komme lediglich eine spezifizierende Funktion zu (vgl. Booij 2009a, Ortner/Ortner 1984). Dieser Vorstellung liegt eine Verallgemeinerung des oben erwähnten Prinzips ,ein NN ist ein N2‘ zugrunde (z.B.: ein Kuchenteller ist ein Teller, ein Computertisch ein Tisch). Allerdings gibt es auch Fälle wie in (27), in denen diese Abhängigkeit weniger eindeutig ist. Ein Hasenbraten ist sowohl Hase als auch Braten, gleiches gilt für den Heuhaufen. Ein Bronzelöwe ist sogar eher ein typischer Vertreter für Bronze als für Löwen.

$$
\text { Hasenbraten, Heuhaufen, Bronzelöwe }
$$

Noch deutlicher wird der Unterschied bei der Verwendung in Satzkontexten. Im Fall des N2 als semantischer Kopf wie in (28-a) kann bei gleichbleibender Bedeutung lediglich das N1 elidiert werden. In (28-b) kann sowohl das N1 als auch das N2 die Bedeutung des Gesamtkompositums tragen. Da das Gesamtkompositum sowohl Eigenschaften des N1 als auch Eigenschaften des N2 trägt, bezeichne ich diese Form als ,Schnittmengenbildung (mehr dazu in Kap. 3.3.3).

(28) a. Das ist aber ein schöner Kuchenteller. vs. Das ist aber ein schöner Teller/*Kuchen.

b. Bei uns gab es heute Hasenbraten. vs. Bei uns gab es heute Hase/Braten. 
Gegen eine Interpretation der Fälle unter (27) als Kopulativkomposita spricht, dass es sich bei den Konstituenten von Kopulativkomposita im Regelfall um semantisch gleichrangige Entitäten handelt, z.B. Berufe (Dichter-Sänger), Farben (schwarz-weiß), Länder (Österreich-Ungarn) oder Funktionen (Radio-Wecker). Das NN vereint die Eigenschaften beider Konstituenten ohne diese zu verändern. Die Konstituenten der Schnittmengenbildungen sind hingegen nicht gleichrangig, sondern modifizieren sich gegenseitig.

In diese Kategorie von Komposita fallen auch die sog. pleonastischen Zusammensetzungen nach Bloomer (vgl. 1996:70) in (29). Hierbei denotiert das NN dasselbe wie das N1, wobei das N2 allgemeiner denotiert und somit eine klassifizierende Funktion hat, die die Bedeutung des N1 verständlicher macht. Die Konstituenten-Denotate sind somit wie bei den Schnittmengenbildungen untrennbar verbunden.

Bilchmaus, Eichbaum, Farnkraut, Auerhahn, Bimsstein, Mastbaum, Guerillakrieg

Verwendet wird diese Form der Komposition vorrangig bei Art-Bezeichnungen, wenn das N1 weniger bekannt oder undeutlich ist, z.B. aufgrund seltenen Vorkommens oder Entlehnung. Dem N2 kommt hier quasi eine disambiguierende Funktion zu (vgl. Bloomer 1996: 73f). Dabei schließt die Bedeutung des N2 das N1 ein, der umgekehrte Fall gilt jedoch nicht. Bspw. ist jede Eiche ein Baum, jeder Baum ist jedoch keine Eiche.

\section{Fugenelemente}

Bei Fugenelementen handelt es sich um Elemente, die zwischen die Konstituenten eines Kompositums treten können. Eine verbreitete Auffassung ist, dass ihr Ursprung in der Flexionsmorphologie liegt, ihr grammatischer Wert jedoch aufgehoben ist (z.B. Erben 2000: 63f). Dies äußert sich zum einen darin, dass Fugenelemente auch nicht-paradigmatisch sein können, d.h. sogar Flexionsendungen entsprechen, die nicht dem Flexionsparadigma des N1 entstammen, wie in den Fällen unter (30-a). Zum anderen kann das Fugenelement auch einen Widerspruch zwischen der Semantik des N1 und der Oberflächenform erzeugen, wie in den Fällen unter (30-b), Brillengestell bezeichnet das Gestell einer einzelnen Brille und in einem Kinderwagen liegt i.d.R. nur ein Kind. Allerdings können Fugen wiederum dergestalt paradigmatisch sein, dass das N1 sogar umgelautet wird, wie im Fall von Gänsebraten.

Welches Fugenelement auftritt, scheint durch eine kontextsensitive Einsetzungsregel bestimmt zu werden; Laut-, Silben-, Wortbildungsstruktur und Flexionsklasse kommen hierbei als Einflussgrößen in Frage. Ein Indiz dafür sind bestimmte Regelmäßigkeiten, z.B. die regelhafte s-Fuge im Fall von Bildungen mit ung.11

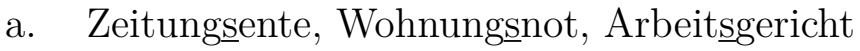
b. Brillengestell, Kinderwagen, Gänsebraten, Dankesrede

Die Funktion von Fugenelementen wird gemeinhin als Verbindung zwischen Konstituenten beschrieben (vgl. Coulmas 1988:325). Es ist jedoch anzunehmen, dass sie im Zuge der Wortverarbeitung geparst und somit zu einem gewissen Grad als morphosyntaktische Elemente interpretiert zu werden. Widersprechen sie der Semantik des N1, ist somit

\footnotetext{
${ }^{11}$ Da in nur ca. $30 \%$ der deutschen Komposita ein Fugenelement auftritt, sollen sie für diese Arbeit nur eine untergeordnete Rolle spielen (vgl. Dudengrammatik RN 1087 (Wermke et al. 2009:711)).
} 
anzunehmen, dass sie auch seiner Zugänglichkeit entgegen wirken (vgl. Coulmas 1988: 326). Stimmen sie überein, erleichtert dies die Zugänglichkeit. In Bezug auf [31-a) beobachtet Wunderlich (vgl. 1986:221), dass das Numerus des N1 für die NN-Interpretation semantisch unerheblich ist, ein N1-Bezug jedoch akzeptabler wird, wenn Pronomen und N1 wie in (31-b) im Numerus übereinstimmen. Erschwerend für die Akzeptabilität von (31-a) kommt hinzu, dass das NN selbst ebenfalls kongruent mit dem Pronomen ist und somit als Referent in Frage kommt, und aufgrund der Möglichkeit der Etablierung einer direkten Anapher vermutlich auch präferiert wird. Im Sinne eines Holzwegsatzes muss die Interpretation beim Lesen des weiteren Kontexts revidiert werden. Disambiguierung durch unterschiedliche Genera in N1 und N2 wie in (31-c) wirken dem entgegen.
a. *Was ein $\underline{H u n d}_{i}$ ehalter mit $\underline{\mathrm{ihm}}_{i}$ tun soll, wenn $\underline{\mathrm{er}}_{i}$ tollwütig wird.
b. ?Was Hunde $\underline{i}_{i}$ halter mit $\underline{\mathrm{ihnen}}_{i}$ tun sollen, wenn $\underline{\mathrm{sie}}_{i}$ tollwütig werden.
c. ??Was eine Hund ${ }_{i}$ ehalterin mit $\underline{\mathrm{ihm}}_{i}$ tun soll, wenn $\underline{\mathrm{er}}_{i}$ tollwütig wird.

Eine mögliche Begründung für den Einfluss von Fugenelementen ist, dass sie der Verschmelzung und nicht der syntaktischen oder semantischen Markierung dienen und deshalb der Konstituentenverfügbarkeit entgegenwirken (vgl. auch Kap. 2.2.2). Die nichtparadigmatischen Formen seien ein Argument dafür (vgl. Coulmas 1988:325f). Aus diesem Grund sei nach Coulmas (1988:324) der N1-Bezug in (32-a) möglich, die deutsche Entsprechung in (32-b) jedoch nicht (siehe dazu auch Dressler 2007:42).
a. The rocket launch had to be delayed because of some unexpected problems with its fuel tanks.
b. *Der Raketenstart musste wegen unerwarteter Probleme mit ihrem Treibstoff- tank verschoben werden.

Dieser Befund soll ungeachtet der theoretischen Auffassung von Fugenelementen als Anhaltspunkt dienen, Fugenelemente als Einflussfaktor für die N1-Zugänglichkeit in Betracht zu ziehen. Inwiefern N1 also tatsächlich generell unflektiert sind oder wahrgenommen werden, bleibt vorerst unklar.

\section{Der Sonderstatus des Nicht-Kopfs (N1)}

Der ersten Konstituente in nominalen Komposita werden zumeist spezielle, von frei vorkommenden Nomen abweichende Eigenschaften zugeschrieben. Zentral sei die Abwesenheit von Flexionsmerkmalen (vgl. Olsen 2000, Ortner/Ortner 1984).12 Für die Pluralbildung zeigen nun Clahsen et al. (2015:56), dass in deutschen Komposita zwar keine regelmäßigen (vgl. (33-a)), durchaus jedoch unregelmäßige Pluralformen im N1 auftreten können (vgl. (33-b)).
a. Autoliste vs. ${ }^{*}$ Autosliste
b. Wortliste vs. Wörterliste

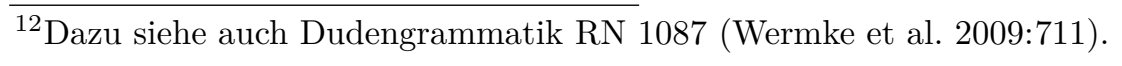


Wann es sich um einen echten Plural und wann um ein Fugenelement ohne grammatischen Wert handelt, kann an dieser Stelle nicht geklärt werden. Es ist jedoch festzustellen, dass im Fall von (33-b) die Fuge in der Form paradigmatisch ist, dass sich auch mit Umlautung einhergeht.

Eine weitere N1-Eigenschaft, die oben bereits erwähnt wurde, ist, dass es nicht separat modifiziert werden kann (vgl. (21-b)) . Eine Ausnahme bildet hier das Phänomen der sog. Klammerparadoxie, die Fälle wie (34) zulässt (vgl. Kap. 2.5.4).

$$
\text { eine gekochte Schinkenplatte }
$$

Darüber hinaus seien auch Appositionen (vgl. (35)] und Relativsätze (vgl. (36)] zu einem N1 unzulässig (vgl. Wunderlich 1986:219).

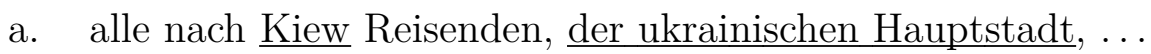

b. *alle Kiew-Reisenden, der ukrainischen Hauptstadt, ...

a. alle nach Kiew Reisenden, das besonders im Frühjahr attraktiv ist ...

b. *alle Kiew-Reisenden, das besonders im Frühjahr attraktiv ist ...

Die einzige Möglichkeit der N1-Modifikation ist die wortinterne Attribuierung, wie z.B. in Schnellkochtopf (vgl. Härtl 2015:163). Für Fälle mit verbaler Erstkonstituente führt Härtl (2015:169) die Beispiele in (37) an.

a. Nachtfahrbrille vs. ${ }^{*}$ Fahrbrille in der Nacht 13

b. Schnellsprechverbot vs ${ }^{*}$ Sprechverbot in schneller Weise

Die von Härtl aufgezeigte Form der wortinternen Attribuierung verbaler Elemente lässt sich jedoch (mit Einschränkungen) auch auf nominale Elemente übertragen, siehe (38). Diese Form der wortinternen N1-Attribuierung ist zwar nicht uneingeschränkt, aber dennoch systematisch produktiv. Demnach lässt sich die N1-Modifikation nicht generell ausschließen, sondern muss lediglich in Art und Form einschränkt werden. Dass dies nur wortintern möglich ist, lässt sich darauf zurückführen, dass ein vorangestelltes Attribut mit dem morphologischen Kopf, also dem N2, kongruent sein muss und ein Widerspruch zwischen semantischer und syntaktischer Form unzulässig ist, bzw. zu einer Klammerparadoxie führt.

Kochschinkenplatte, Schönwetterreise, ?Frischkuchenteller

Laut Härtl (vgl. 2015:169) ist der Grund für die Inakzeptabilität phrasaler Modifikation, dass die kanonische Wortfolge auf nicht lizenzierte Weise geändert sei. Ausnahmen stellen hier Fälle wie unter (39) dar. Härtl spricht hierbei von außergrammatischer Legitimation, welche nur im Falle obligatorischer Argumente möglich sei. Adjunktion wie in (40) sei hingegen nicht möglich (vgl. Härtl 2015:170).

a. Fahrgemeinschaft nach Italien

\footnotetext{
${ }^{13}$ Eine Paraphrasierung als Fahrbrille für die Nacht empfinde ich als akzeptabler.
} 


\section{b. Ausreiseverbot in die BRD}

*Spazierschuhe mit dem Hund

Wenn allerdings die Obligation der Argumentsättigung der Grund für die Akzeptabilität der Fälle unter (39) ist, stellt sich die Frage, weshalb Verwendungen ohne die obligatorischen Argumente dennoch akzeptabel sind, vgl. (41).
a. Ich habe mich einer Fahrgemeinschaft angeschlossen.
b. Das Ausreiseverbot hat viele DDR-Bürger massiv belastet.

Eine weitere Beschränkung des N1 liegt darin, dass es keinen Determinierer bei sich führen kann. Ausnahmen wie (42) werden u.a. von Meibauer (vgl. 2003:168) als Phrasenkomposita mit lexikalisiertem Erstglied beschrieben.

$$
\text { die Der-schöne-Rheingau-Laberei }
$$

Schließlich wird dem N1 Nicht-Referenzialität zugeschrieben (u.a. in Klos 2011, Ortner/Ortner 1984). Damit ginge einher, dass durch ein N1 keine individuellen, sondern typische Eigenschaften ausgedrückt werden:

$[\ldots]$ the first constituent does not refer to an entity distinct from the reference of the second constituent but rather serves as an indefinite or generic specification that further restricts the set of possible denotations of the second constituent, cf. computer industry: 'industry concerned with computers'. (Olsen 2000 898).

Auch diese Eigenschaft ist nicht allgemein gültig. In bestimmten Kontexten kann sich ein N1 auf etwas Spezifisches beziehen (vgl. u.a. Meineke 1991:85), bspw. können Eigennamen an der N1-Position auftreten, z.B. Dylan-Fan. Härtl (vgl. 2015:174) hebt Ad-hocBildungen hervor, z.B. sei der unangenehme Geruch einer Stinkedecke keine generelle Eigenschaft, sondern ein augenblicklicher Zustand.14

Darüber hinaus besteht, wie bereits erwähnt, die Annahme, dass ein N1 generell nicht referenziell sei. Allerdings gibt es auch frei vorkommende NPn, die nicht referenziell sind, z.B. NPn im Skopus von Quantoren, von Negationen, prädikative NPn und NPn in Adjunkten (vgl. Soehn 2005:156f). Treffender scheint mir zu sein, dass frei vorkommende NPn referenziell sind, jedoch - abhängig vom Kontext - nicht zwingend referieren. Ein N1 hingegen ist zumeist nicht referenziell, kann dies jedoch u.U. sein und in dem Fall auch referieren.

Bei Betrachtung der verschiedenen Wort-Kriterien und Charakteristika von Komposita entsteht insgesamt der Eindruck, dass es sich bei der Komposition an sich bereits um ein unscharfes Phänomen handelt. Die Wortkriterien genügen nicht, um eine strikte Trennung zwischen Morphologie und Syntax zu rechtfertigen (vgl. Haspelmath 2011). Darüber hinaus erscheinen die Kriterien nicht beobachtungsadäquat zu sein, da sich stets Ausnahmen finden lassen. Kriterien, die haltbar zu sein scheinen (z.B. die Zusammenschreibung),

\footnotetext{
${ }^{14}$ Allerdings lässt sich die Eigenschaft durch einen entsprechenden Kontext auch als generelle Eigenschaft konsolidieren, z.B. kann eine alte Hundedecke auch nach dem Waschen als Stinkedecke bezeichnet werden.
} 
sind allein nicht stark genug. Problematisch ist die Unterscheidung zwischen Kompositum und Phrase v.a. im Englischen aufgrund des Leerzeichens. Im Deutschen scheint das Problem vorrangig theoretischer Natur zu sein; in den meisten Fällen erweist sich eine Kombination der veschiedenen Kriterien als aussagekräftig. 15

Auch wenn für die Mehrzahl der Fälle kein Abgrenzungsproblem besteht, sind klare Kriterien wünschenswert und essentiell für die linguistische Theoriebildung. Als praktikable Lösung erscheint es mir, von einer Hierarchie von Charakteristika für Komposita auszugehen. Je mehr dieser Charakteristika zutreffen und je zentraler diese sind, umso wahrscheinlicher ist es, dass es sich um ein Kompositum handelt, bzw. umso typischer ist das Kompositum. Diese Vorstellung steht im Einklang mit Booijs Idee der relativen Kohäsion. Für die Zugänglichkeit eines N1 lässt sich daraus schlussfolgern: Je mehr der Kriterien zutreffen und je zentraler diese zutreffenden Kriterien sind, desto eher handelt es sich um ein typisches N1. Und je typischer ein N1 ist, desto eher ist von seiner Unzugänglichkeit auszugehen, da es sich dabei ebenfalls um eine typische N1-Eigenschaft handelt.

\subsubsection{Morphosyntax von Komposita}

\section{Theorien zur Strukturbildung}

Nach der Beschreibung der grammatischen Eigenschaften von Komposita und ihren Konstitutenten rückt als Nächstes ihr Aufbau ins Zentrum der Betrachtung. Dabei stellst sich die Frage nach den zugrundliegenden Bildungsprinzipien und dem grammatischen Status der Konstituenten.

Bei der Komposition handelt es sich um einen Konstruktionstyp zwischen Morphologie und Syntax. Diese Schnittstellenposition wird u.a. von Spencer (1991) thematisiert. Als typisch syntaktische Eigenschaften der Komposition nennt er die Rekursivität, die Konstituentenstruktur, das Kopf-Rechts-Prinzip, die Binärverzweigung und die Möglichkeit bei Rektionskomposita, das N1 als direktes Objekt des N2 zu interpretieren (vgl. Spencer 1991:310). Andererseits seien die Möglichkeit der Lexikalisierung, die Unflektierbarkeit des N1, und das postulierte Wirken lexikalischer Integrität typische Eigenschaften morphologischer Konstruktionen (vgl. Spencer 1991:312). Inwiefern diese Merkmale allgemeingültig sind, wurde bereits im vorhergehenden Abschnitt in Frage gestellt.

Durch die Positionierung der Komposition an der Morphologie-Syntax-Schnittstelle ergeben sich verschiedene Modellierungsmöglichkeiten, die wiederum verschiedene Konsequenzen für die Konstituenten sowie Ort und Art der Strukturbildung haben können. Dabei werden in der linguistischen Forschung verschiedene Traditionen und Beschreibungsansätze verfolgt.

Gemäß dem Ansatz der Transformationsgrammatik handelt es sich bei Komposita um reduzierte syntaktische Bildungen, z.B. Genitivphrasen oder Relativsatz-Tiefenstrukturen (vgl. Lees 1960). Ein Hauptkritikpunkt an der Transformationsgrammatik ist die Vermischung von Syntax und Semantik und die Ermangelung einer Erklärung für die Bildung von Mehrfachkomposita (vgl. Fanselow 1981:45).

Die beiden Hauptströmungen zur Wortbildung sind nun die lexikalistischen und die syntaktischen Ansätze. Lexikalistische Ansätze verorten die Wortbildung im Lexikon (vgl.

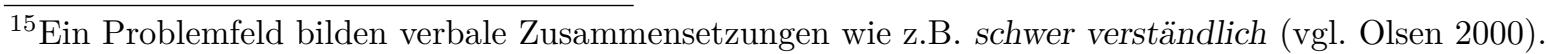


Di Sciullo/Williams 1987, Lieber 1982) oder in einem separaten morphologischen Modul (vgl. Ackema/Neeleman 2010). Bei der Komposition handelt es sich demzufolge um einen präsyntaktischen Prozess (vgl. Giegerich 2006, Härtl 2015). Alle nachfolgenden syntaktischen Prozesse greifen auf abgeschlossene Wortbildungsprodukte als Input zurück.

Syntaktische Ansätze beruhen auf der Vorstellung, dass gewisse Aspekte der X-BarTheorie auch in der Wortstruktur gelten (vgl. Selkirk 1982, Sproat 1985, Toman 1990). Weder im Instrumentarium noch bzgl. des Bildungsortes wird zwischen der Bildung von Wörtern und Phrasen getrennt (vgl. z.B. Harley 2009).

Daneben gibt es auch hybride Ansätze, die Annahmen der lexikalistischen und der syntaktischen Ansätze verbinden. Eine Diskussion der verschiedenen Ansätze sowie sich daraus ergebende Konsequenzen für den Konstituentenstatus erfolgt in Kapitel 4.

\section{Rektions- vs. Wurzelkomposita}

Dass es strukturell verschiedene Arten von Komposita gibt, wird bei nahezu allen Ansätzen vorausgesetzt. Die gebräuchlichste Unterscheidung ist die bereits in Kapitel 2.2.1 erwähnte Einteilung in Wurzelkomposita (root/primary compounds) und Rektionskomposita (synthetic/verbal/secondary compounds), so. u.a. bei Ackema/Neeleman (2004), Dressler (2007), Giegerich (2006), Meibauer (2007), Reinhard (2001), Roeper/Siegel (1978), Scalise/Bisetto (2009), Selkirk (1982), Spencer (1991).16

Die Tatsache, dass erstere in der englischsprachigen Literatur häufig als ,primary compounds' bezeichnet werden (z.B. bei Harley 2009), kennzeichnet diese Komposita-Art als Standardform und alle anderen Formen als Unterarten. In der deutschsprachigen Literatur ist häufig lediglich von ,Determinativkomposita' die Rede, was eine viel allgemeinere Beschreibungsebene repräsentiert. In Anlehnung an den im Englischen äquivalent verwendeten Ausdruck, root compounds' scheint mir die Bezeichnung,Wurzelkomposita' geeigneter, um sich auf Determinativkomposita wie unter (43) zu beziehen und von anderen Formen abzugrenzen. Die zentrale Eigenschaft von Wurzelkomposita ist die nicht-derivationale Natur der Lexeme (vgl. Scalise/Bisetto 2009:35). Aufgrund dieser Eigenschaft ist die Beziehung der beiden Kompositateile zueinander zwangsläufig unterspezifiziert und muss durch eine aus dem Weltwissen und dem sprachlichen Kontext abzuleitende Relation gefüllt werden (vgl. Kap. 2.2.4).

\section{Dachgarten, Tabakfabrik, Lederschuh, Kinderschokolade}

Bei Rektionskomposita handelt es sich um Verbindungen aus einer deverbalen Nominalisierung als Zweitglied und einem Erstglied, welches die interne Argumentstelle des dem Zweitglied zugrundeliegenden Verbs besetzt (z.B. Meibauer 2007, Scalise/Bisetto 2009, Spencer 1991). Die Relation der Kompositateile zueinander ist demnach nicht unterspezifiziert wie im Fall der Wurzelkomposita, sondern die Argumentstruktur des Verbs liefert den Interpretationsrahmen. Die Derivation des Verbs kann dabei auf verschiedenen Wegen erfolgen, in den Beispielen unter (44-a) zweimal durch Suffigierung und einmal durch Konversion.

\footnotetext{
${ }^{16}$ Es handelt sich hierbei um eine sprachspezifische Einteilung (vgl. Scalise/Bisetto 2009 35), die jedoch über die germanischen Sprachen hinweg als gültig betrachtet wird.
} 
(44) a. Autofahrer, Dachbegrünung, Möbelkauf

b. truck driver, truck driving, slum clearance (Spencer 1991;324)

Reinhard (2001:5f) nimmt Argumentstellensättigung als notwendiges Kriterium für Rektionskomposita an, entweder durch ein Objekt wie in (45-a) oder durch ein Subjekt an der N1-Position wie in (45-b).
a. Terminbesprechung
b. Mitarbeiterbesprechung

Andere Autor*inn*en gehen davon aus, dass das N1 ausschließlich die Position des internen Arguments einnehmen kann (vgl. Selkirk 1982, Spencer 1991), mehr dazu siehe Kapitel 4.2.2.

Neben der grundlegenden Unterscheidung zwischen Wurzel- und Rektionskomposita existieren weitere Mischformen. So benennt Reinhard (2001) die Kategorie der „deverbalen Determinativkomposita“, bei denen das Kompositum zwar ein deverbales Zweitglied beinhaltet, das N1 jedoch keine Argumentstelle sättigt, sondern als Modifikator im Sinne einer Adverbialbestimmung fungiert. Beispiele hierfür sind unter (46-a) aufgeführt. Weitere Formen sind u.a. Komposita mit relationalem Zweitglied wie (46-b)] (vgl. Fanselow 1981) oder präpositionalem Komplement wie (46-c) (vgl. Meibauer 2007).
a. Sonntagsfahrer, Radiowarnung, Kunstbegrünung, Notkauf
b. Bücherfan, Professorensohn
c. Prüfungsangst, Friedenssehnsucht

Diese Arbeit beschränkt sich auf die Unterscheidung zwischen Wurzel- und Rektionskomposita im Deutschen. Konkrete Merkmale dieser beiden Komposita-Arten und verarbeitungsrelevante Strukturunterschiede werden in Kapitel 4 thematisiert. Jedoch soll bereits an dieser Stelle eine Beobachtung vorangestellt sein: Bei Betrachtung der bereits aufgeführten und als akzeptabel eingeschätzten Fälle fällt auf, dass es sich überdurchschnittlich oft um Rektionskomposita zu handeln scheint, siehe (47). Ob es sich hierbei um einen systematischen Akzeptabilitätsunterschied handelt und wie sich dieser begründen ließe, gilt es zu ergründen.

(47) a. The rocket launch had to be delayed because of some unexpected problems with its fuel tanks. (Coulmas 1988:322)

b. Die Kinder sind auf Eiersuche. Wenn sie welche gefunden haben, kommen sie zurück. (Klos 2011:11)

c. 'Was Hundehalter mit $\underline{\text { ihnen }}$ tun sollen, wenn $\underline{\text { sie }}$ tollwütig werden. (Wunderlich 1986.221)

d. Ein Rosenhasser? Die tun doch niemandem was. (Tatort vom 05.05.2017) 


\subsubsection{Semantik von Komposita}

\section{Semantische Relationen}

Die Unterspezifiziertheit von Komposita wird bei der Interpretation von der durch Weltund Kontextwissen gestützten Wahl einer semantischen Relation aufgelöst, z.B. ob der Ausdruck Kuchenteller einen Teller mit einem oder für einen Kuchen denotiert.17Vielfach wird die Existenz eines begrenzten Sets an möglichen Relationen stipuliert, so z.B. bei Fanselow (1981), Levi (1978), Ortner/Ortner (1984).18

Das konkrete Set möglicher Relationen unterscheidet sich von Autor*in zu Autor*in. Ein Konsens liegt in der Annahme einer endlichen Zahl an Relationen, mit Hilfe derer sich ein Großteil der Komposita interpretieren lässt. Die wohl verbreitetste Klassifikation stammt von Judith Levi. Levi (1978:76f) unterscheidet neun Relationen: CAUSE, HAVE, MAKE, USE, BE, IN, FOR, FROM, ABOUT. In Tabelle 2.1 sind für diese Relationen Beispiele und ihre deutschen Entsprechungen aufgeführt.

\begin{tabular}{l|l|l}
\hline \hline semantische Relation & Bsp. Levi & Übertragung ins Deutsche \\
\hline CAUSE & tear gas & Tränengas \\
HAVE & picture book & Bilderbuch \\
MAKE & honeybee & Honigbiene \\
USE & manual labor & Handarbeit \\
BE & soldier ant & Soldatenameise \\
IN & field mouse & Feldmaus \\
FOR & horse doctor & Pferdearzt \\
FROM & olive oil & Olivenöl \\
ABOUT & linguistic lecture & Linguistikvorlesung \\
\hline \hline
\end{tabular}

Tabelle 2.1: Semantische Relationen nach Levi (1978:76f)

Eine weitere Relations-Klassifikation stammt von Downing (1977), speziell fürs Deutsche gibt es Klassifikationen u.a. von Ortner/Ortner (1984) und Fandrych/Thurmair (1994). Die verschiedenen Relations-Sets sind ähnlich und vergleichbar. Tabelle 2.2 bildet die drei genannten Relations-Klassifikationen auf die Levi'schen Relationen ab. Hierbei handelt es sich allerdings nicht um eine 1:1-Zuordnung, sondern um grobe Entsprechungen, die die Ähnlichkeiten zwischen den Modellen herausstellen sollen. Da es sich bei dem Levi'schen Modell um die in der Literatur verbreitetste Klassifikation handelt, werde ich mich im weiteren Verlauf der Arbeit vorrangig auf ihr Inventar beziehen.

Gemäß Booij (vgl. 2009a:205) basieren die Relationen auf paradigmatischen Verhältnissen zwischen Wörtern im Lexikon. Für die Wahl einer Relation wird nach gängiger Auffassung lexikalisches Wissen über die Konstituenten ebenso nutzbar gemacht wie Welt- und Kontext-Wissen (vgl. hierzu Meyer 1993:110). Dabei sind nicht alle Relationen gleichwertig, sondern es ist von Präferenzen und Wahrscheinlichkeiten auszugehen (vgl. Levi

${ }^{17}$ Dies vielfach verallgemeinert, gilt jedoch genaugenommen vorrangig für Wurzelkomposita.

${ }^{18}$ Levi (1978:6) spricht von ,Recoverably Deletable Predicates'. Die Annahme eines zugrundliegenden gelöschten oder nominalisierten Prädikats (vgl. Levi 1978 50) macht die Nähe von Levis Theorie zur Transformationsgrammatik deutlich. Auch wenn diese Form der Komposita-Analyse in der rezenten Grammatiktheorie nicht mehr verfolgt wird, gelten die von Levi aufgestellten Relationstypen nach wie vor als grundlegend (z.B. in Klos 2011). 


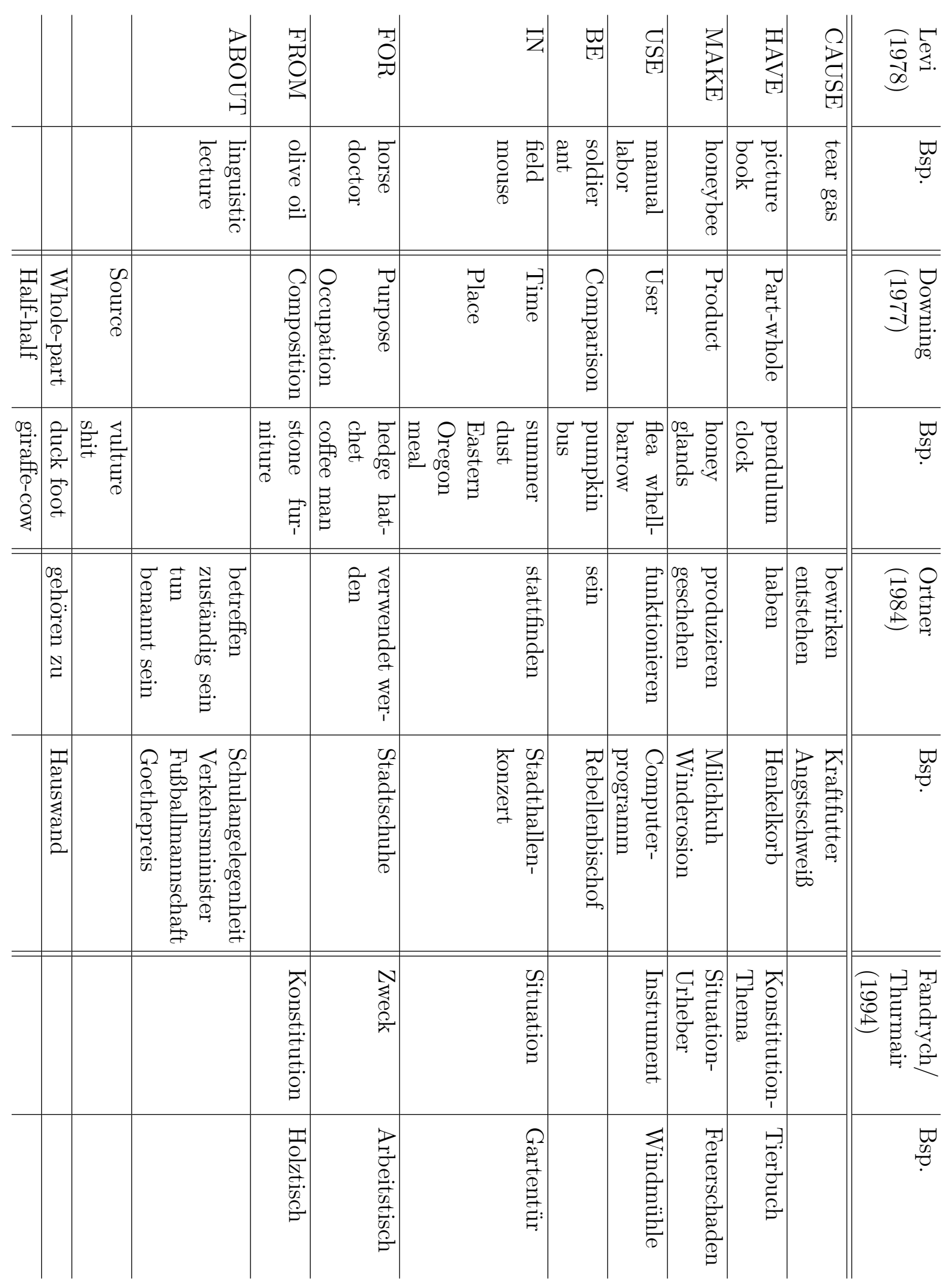

Tabelle 2.2: Vergleich der Klassifikation von Levi (1978) mit weiteren Relations-Klassifikationen 
1978 9). Durch Welt- und Konzeptwissen hat bspw. die Interpretation von Papierkorb als Korb für (FOR), statt aus (FROM) Papier die höhere Wahrscheinlichkeit, da das entsprechende Denotat eine größere lebensweltliche Häufigkeit hat und somit die präferierte Interpretation ist (vgl. hierzu Klos 2011:204ff) 19

Inwiefern die semantische Relation und ihre Auftretenswahrscheinlichkeit Einfluss auf die N1-Zugänglichkeit haben kann, gilt es zu prüfen.

\section{Kompositumstemplate}

Für die semantische Analyse von Komposita existieren ebenfalls verschiedene Ansätze. Ein Vorgehen liegt im Versuch, ein Template zur Genese kompositionaler Bedeutung zu erstellen.

So vertritt Booij (2009a:201ff) die Idee eines abstrakten Wortbildungsschemas. Dabei nimmt er ein Schema an, welches mit einer systematischen Korrelation zwischen Form und Bedeutung über bekannte komplexe Wörter generalisiert. Das Wissen über existierende Komposita dient somit als Voraussetzung zur Entwicklung eines analogen abstrakten Schemas.

Einen abstrakteren Ausgangspunkt wählt Olsen und definiert Komposita als:

[...] Strukturen, in denen zwei Prädikate $\mathrm{P}$ und Q in eine Konfiguration so eingeführt werden, dass ihre externen Argumente in einer impliziten Relation R zueinander stehen. Von einem semantischen Standpunkt aus betrachtet stellt ein Kompositum dadurch eine abstrakte Version des generellen Modifikationstemplates dar, welches im Allgemeinen für Modifikator-Kopf-Strukturen gilt [...] Die Annahme, dass die Interpretation eines Kompositums durch das Modifikationstemplate dargestellt werden kann, legt nahe, dass die lexikalisch-semantische Charakterisierung der Bedeutung eines Kompositums an dieser Stelle endet. Olsen 2012:135)

Olsens Template ist unter (48) wiedergegeben. Problematisch an dieser Darstellung ist, dass sie nicht für Rektionskomposita gültig ist, da diese nicht über eine unterspezifizierte Relation verfügen.

$$
\begin{aligned}
& \text { Kompositumstemplate nach Olsen }(2012: 135) \text { : } \\
& \lambda \mathrm{Q} \lambda \mathrm{P} \lambda \mathrm{v}[\mathrm{P}(\mathrm{v}) \& \mathrm{Q}(\mathrm{u}) \& \mathrm{R}(\mathrm{v}, \mathrm{u})]
\end{aligned}
$$

Olsen trennt dabei zwischen genuinen Formen des lexikalisch-semantischen Systems und den daraus abgeleiteten Konstituentenformen (vgl. Olsen 2012:163). Unter dieser Voraussetzung sei anzunehmen, dass die Bildung von Komposita auf Grundlage von lexikalischem Inventar und dem Kompositumstemplate möglich sei (vgl. Olsen 2012:166). Darüber hinaus geht Olsen davon aus, dass die Konstituenten(formen) sowohl ihr referenzielles Potenzial als auch ihr Thetaraster abgeben (vgl. Olsen 2012,156).

Die erste Konstituente eines Kompositums ist nicht-referentiell, da ihr externes Argument nicht für die Thetabindung durch einen Operator zur Verfügung steht.(Olsen 2012:156)

Bei Gültigkeit dieser Annahmen gilt es jedoch zu erklären, wie die bereits erwähnten Fälle von Argumentstellenzuweisung auf das N1 wie in (49-a) und durch das N1 (49-b) möglich sind.

\footnotetext{
${ }^{19}$ Vgl. hierzu das Beispiel Filtertüte aus Kapitel 2.2.2
} 
a. Autofahrer, Dachbegrünung

b. Fahrgemeinsaft nach Italien (Härtl 2015:163)

Eine besondere Herausforderung bei der semantischen Beschreibung von (Wurzel-)Komposita liegt in der Beschreibung der „impliziten Relation R“ (vgl. Olsen|2012:135). Die Konstituenten von Komposita können auf ganz unterschiedliche Weise zueinander in Relation stehen. Aufgrund der Implizitheit dieser Relation bleiben sie dennoch unterspezifiziert.

\section{Modellierung im Rahmen der DRT}

Eine andere Möglichkeit der semantischen Analyse von Komposita liegt in einer Abwandlung der DRT. Dieses Vorgehen ist besonders mit Hinblick auf Fragen zur anaphorischen Zugänglichkeit interessant. Für die Modellierung der Zugänglichkeit eines Diskursreferenten für Anaphern ist die Diskursrepräsentationstheorie (DRT) nach Kamp/Reyle (1993) eine verbreitete Theorie (siehe auch Averintseva-Klisch 2013, Lascarides/Asher 2007). Dabei handelt es sich um eine formalsemantische Theorie, die über Satzgrenzen hinausgeht und der eine repräsentationale Theorie der Interpretation zugrunde liegt. Ein Diskurs wird in Form einer Diskursrepräsentationsstruktur (DRS) dynamisch und prozessorientiert modelliert. Eine DRS wird in Form einer zweigeteilten Box dargestellt (vgl. z.B. $(50)$.

(50) Leon gießt die Rosen. Sie blühen.

\begin{tabular}{|c|}
\hline$x y$ \\
\hline Leon $(\mathrm{x})$ \\
rosen $(\mathrm{y})$ \\
$\mathrm{x}$ gießt $\mathrm{y}$ \\
$\mathrm{u}$ blüht \\
$\mathrm{u}=\mathrm{y}$ \\
\hline
\end{tabular}

Der obere Teil umfasst das Diskursuniversum (in (50) x y), in dem alle Diskursreferenten des Diskurses als Variablen aufgeführt werden. Der untere Teil beinhaltet die Bedingungen über diese Variablen. Diese Bedingungen ergeben sich aus der syntaktischen Struktur des Diskurses und den entsprechenden lexikalischen Einträgen. Eine anaphorische Beziehung wird in einer DRS nicht über Beziehungen zu Vorgänger-NPn dargestellt, sondern zu Diskursreferenten. Die Auflösung anaphorischer Bezüge erfolgt via Identifizierung von Variablen (in (50), $\mathrm{u}=\mathrm{y}$ ). Beschränkungen in der Zugänglichkeit werden i.d.R. über Blockierungen modelliert, z.B. durch Subordination des Wirkungsbereichs eines Operators. Die in untergeordneten Boxen enthaltenen Diskursreferenten sind für den nachfolgenden Diskurs nicht zugänglich (vgl. Kamp/Reyle 1993:105).

Um nun mit Hilfe dieses Instrumentariums Aussagen zur Zugänglichkeit von KompositaKonstituenten treffen zu können, entwirft Meyer (1993) (einem lexikalistischen Ansatz folgend) eine lexikalische DRS $\left(\mathrm{DRS}_{l e x}\right)$, die in die aktuell prozessierte DRS eines Diskurses insertiert wird, während dieser geparst wird (vgl. Meyer 1993:61). Meyers Modell erlaubt gleichzeitig die Integration von konzeptuellem und Diskurs-Wissen. Die $\mathrm{DRS}_{l e x}$ 
spiegelt die kontextinvariate Kernbedeutung wider und versteht sich als: „the contribution of the lexical entry to the utterance meaning of this lexical item" (Meyer 1993:81). Mit Hilfe der DRS $\mathrm{S}_{l e x}$ bildet Meyer Komposita mit relationalem N2 ab, z.B. Bücherfan in (51) (Meyer 1993:109).

Da das Kompositum die grammatischen Merkmale für Kategorie, Numerus und Genus vom N2 erhält, bettet Meyer die $\mathrm{DRS}_{l e x}$ des N1 in die des N2 ein (vgl. Meyer 1993:90). Für das N1 geht er generell von einer generischen Lesart aus, was durch den Generizitätsoperator (!) ausgedrückt wird (vgl. Meyer 1993:91). Zusätzlich beinhaltet die DRS $_{l e x}$ des N1 die verschiedenen Instantiierungen von Buch, die dieses im Kontext des Kompositums annehmen kann. Die gesamte $\mathrm{DRS}_{l e x}$ kann nun in eine übergeordnete DRS eingebettet werden.

Nach Meyers Darstellung eines Kompositums durch eine $\mathrm{DRS}_{l e x}$ ist ein N1 stets unzugänglich, da dem N1 (z) nur innerhalb seiner untergeordneten Box eine Variable zugeordnet werden kann und diese somit für Anaphern in späteren Diskursteilen, d.h. in übergeordneten Boxen nicht zugänglich ist.

Der große Vorteil dieser Modellierung ist, dass sie zum einen der Komplexität der kompositionalen Semantik gerecht zu werden versucht und ein Antezedens als Diskursreferent begriffen wird, d.h. die Beschreibung auf Diskursebene ohne syntaktische Grenzen möglich ist.

Bücherfan:

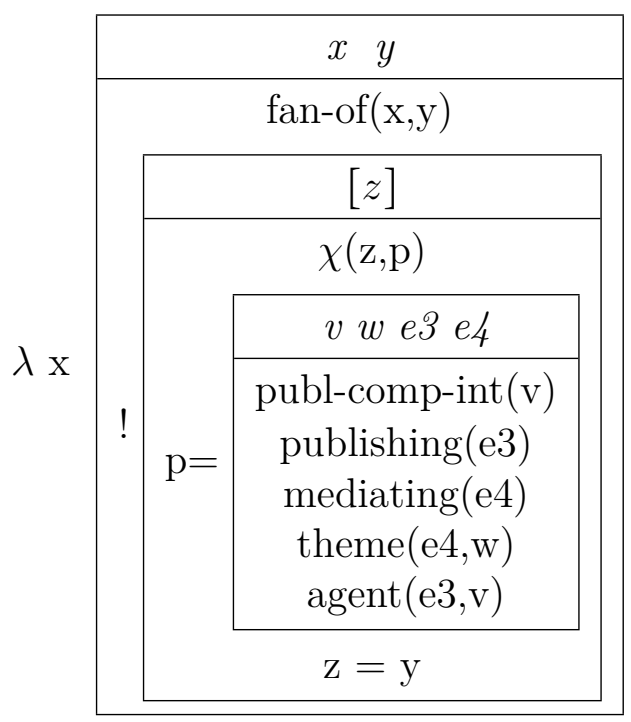

Nun wurde bereits thematisiert, dass ein N1 nicht prinzipiell generisch sein muss (vgl. $(16-b)$. Außerdem stellt sich die Frage, wie eine Integration einer $\mathrm{DRS}_{\text {lex }}$ in eine übergeordnete DRS aussehen kann und wie etwaige Einflussfaktoren der N1-Zugänglichkeit abgebildet werden können. Da die Einführung eines Diskursreferenten in das Diskursuniversum Voraussetzung für eine Wiederaufnahme ist, sei die Frage vorangestellt, ob ein N1 überhaupt einen Diskursrefereten etablieren kann. Dies illustriert Kadmon (2001) anhand der Murmelsätze von Barbara Partee: (52-a) und (52-b) haben die gleichen Wahrheitsbedingungen und beschreiben das Fehlen einer Murmel. Jedoch nur in (52-a) kann man sich mit einem Pronomen auf diese beziehen, weil nur dort ein linguistisches Antezedens für ein Pronomen vorliegt. 
(52) a. I dropped ten marbles and found all of them, except for one. It is probably under the sofa.

b. ?I dropped ten marbles and found only nine of them. It is probably under the sofa.

Der Bezug in (52-b) kann allerdings pragmatisch realisiert werden, z.B. durch eine zusätzliche Zeigegeste (vgl. Kadmon 2001:99). Im Gegensatz dazu ist (53) ohne Weiteres akzeptabel, da hier die Anapher in Form einer definiten NP realisiert wird und somit keine Inferenz notwendig ist. Eine definite Kennzeichnung kann im Vergleich zu einem Pronomen stets ohne linguistisches Antezedens verwendet werden (vgl. Kadmon 2001: 99).

I dropped ten marbles and found only nine of them. The missing marble is probably under the sofa.

Die Inakzeptabilität von (52-b) demonstriert, dass rein kontextuelle Faktoren nicht genügen, um einen Diskursreferent im Diskursuniversum zu etablieren. Dafür ist ein höheres Salienz-Niveau nötig. Unter einer salienten Information versteht Kadmon:

$[\ldots]$ information which the participants in the conversation have in fact inferred or become aware of. An entailment of the text will enter the DRS only if the language users have in fact inferred it. (Kadmon 2001 100).

Die Einführung eines Diskursreferenten allein genügt demnach nicht, damit dieser auch für ein Pronomen zugänglich ist:

$[\ldots]$ pronouns are constrained in use, and may pick as their antecedents only a subset of the discourse referents in the DRS. Here is where we could rely on some notion of salience. $[\ldots]$ Here, it seems that the relevant notion of 'salience' has to do with the language users' attention. (Kadmon 2001:100)

In diesem Sinne scheint der Diskursreferent in (52-a) salient genug, in (52-b) hingegen wird der Referent erst durch Entailment etabliert und ist dadurch nicht salient genug. Entscheidend sei der Grad an Aufmerksamkeit. Gemäß Kadmon (vgl. 2001:102) gehen Implikaturen in die DRS ein, Entailments nicht zwingend. Entailments erachtet sie im Gegensatz zu Implikaturen als Übermittlungsdetails, die an die Proposition gebunden sind und nach Übermittlung eines Statements verloren gehen können: „Syntactic, morphological and phonological information is already lost when utterances are mapped into DRSs." (Kadmon 2001:102)

Eventuell handelt es sich bei einem N1 ebenfalls um ein Übermittlungsdetail, welches verloren gehen kann, z.B. als Teil morphologischer Information. Es sei denn, es verfügt aufgrund von bestimmten Faktoren über einen hohen Grad an Aufmerksamkeit und damit Salienz. In Verbindung mit der Aufmerksamkeit wird wiederum der Kontext relevant: Lenkt der syntaktische Kontext die Aufmerksamkeit auf das N1, etabliert dieses einen Diskursreferenten, der auch im Diskursuniversum erscheint und somit durch ein Pronomen wiederaufgenommen werden kann. 


\subsection{Komposita in der Verarbeitungstheorie}

Die Frage nach der anaphorischen Zugänglichkeit von Komposita-Konstituenten ist nicht nur eine Frage nach grammatischen Beschränkungen, sondern auch Verarbeitungsaspekte spielen hierbei eine wichtige Rolle. Im Folgenden werden einige zentrale Aspekte der Komposita-Verarbeitung beleuchtet.

\subsubsection{Kenngrößen der Verarbeitungstheorie}

In den vorangegangen Abschnitten wurde bereits von den Begriffen ,Zugänglichkeit' und ,Akzeptabilität' Gebrauch gemacht. Die diesen Begriffen zugrundeliegenden verarbeitungstheoretischen Annahmen gilt es zunächst zu klären.

\section{Zugänglichkeit}

Mit Ward et al. (1991) verstehe ich die Interpretierbarkeit einer Anapher als eine Funktion der relativen Zugänglichkeit des Antezedens. Der Grad an Zugänglichkeit (accessibility) eines Antezedens, bzw. eines Referenten beschreibt seinen kognitiven Status und die Möglichkeiten seiner Wiederaufnahme durch eine Anapher. Diese Annahme basiert auf der ,Accessibility Theory' von Ariel (1988, 2001), deren Gegenstand es ist:

[...] how human language, specifically, the referential system, is responsive to facts about human memory, where memory nodes are not equally activated at any given time. [...] Accessibility theory offers a procedural analysis of referring expressions, as marking varying degrees of mental accessibility. (Ariel 200129)

Bei der Zugänglichkeit handelt es sich um eine graduelle Eigenschaft; die verschiedenen referenziellen Ausdrucksarten sind nach dem Grad ihrer Zugänglichkeit auf der Accessibility Marking Scale angeordnet. Grundlegend gilt: Über je weniger konzeptuellen Inhalt eine Anapher verfügt, desto höher muss der Grad an Zugänglichkeit des Referenten sein. Demzufolge handelt es sich bei Pronomen aufgrund ihres geringen semantischen Gehalts um Marker für ein Antezedens mit einem hohen Grad an Zugänglichkeit, z.B. im Gegensatz zu definiten Nominalphrasen (vgl. Ariel 2001:31). Einen wichtigen Einfluss auf den Zugänglichkeitsgrad eines Referenten schreibt Ariel den Faktoren Informativität, Disambiguität, phonologischer Umfang und v.a. Salienz zu (vgl. Ariel 2001:32). Insgesamt eröffnet sie eine breite Spanne an Faktoren, die auch linearen Abstand, semantische Bindung, syntaktische Faktoren etc. berücksichtigt. Der morphosyntaktische Status des Antezedens als N1 ist somit nur einer von vielen Zugänglichkeits-Faktoren (vgl. Ward et al. 1991). Außerdem nimmt Ariel an, dass frame-induzierte Entitäten einen höheren Grad an Zugänglichkeit haben als inferierbare Entitäten (vgl Ariel 2001:33).

In sum, I have argued that referring expressions are chosen according to the assessed degree of accessibility of the mental entities corresponding to them. Degree of accessibility depends on factors related to the inherent salience of the entity and on the unity between the antecedent and the anaphor. In addition, the conventional degree of accessibility coded by referring expressions is motivated by their relative informativity, rigidity and attenuation. (Ariel 2001:34) 
Die Accessibility Theory beschreibt allerdings ein komplexes psychologisches Konzept, welches nicht als ausschließliche Ursache für die Wahl des referenziellen Ausdrucks und dessen Interpretation gelten kann und soll (vgl. Ariel 2001:42).

Die Vorstellung, dass verschiedene Ausdrucksarten über verschiedene kognitive Status verfügen, basiert auf der Idee der Givenness Hierarchy nach Gundel et al. (1993) (vgl. auch Gundel 1996). Grundlegend für die Givenness Hierarchy ist die Annahme einer Korrelation zwischen dem Informationsgehalt eines Ausdrucks und seinem kognitiven Zustand. Demnach zeigen Sprecher*inn*en die Diskurszugänglichkeit eines Diskursreferenten durch die Verwendung eines bestimmten sprachlichen Ausdrucks an, d.h. die spezifische Anaphernform steht in Beziehung zum Grad der Zugänglichkeit des Antezedens.

The Givenness Hierarchy is an implicational scale, with statuses ordered from most restrictive (in focus) to least restrictive (type identifiable), with respect to the set of possible referents they include. (Gundel 1996 144f)

Der Ausgangspunkt ist dabei immer das Antezedens, welches durch seine konkrete Form festlegt, welche Arten von Anaphern für seine Wiederaufnahme akzeptabel sind. Dabei bildet die Skala unter (54) die verschiedenen Grade von Bekanntheit (givenness) ab und ordnet ihnen die möglichen anaphorischen Ausdrucksarten zu. Unbetonte und Null-Pronomen signalisieren konventionellerweise, dass der Referent fokussiert ist; demonstrative Determinierer signalisieren, dass der Referent zumindest bekannt (familiar) sein muss.

$$
\text { The Givenness Hierarchy (Gundel 1996:144f) }
$$

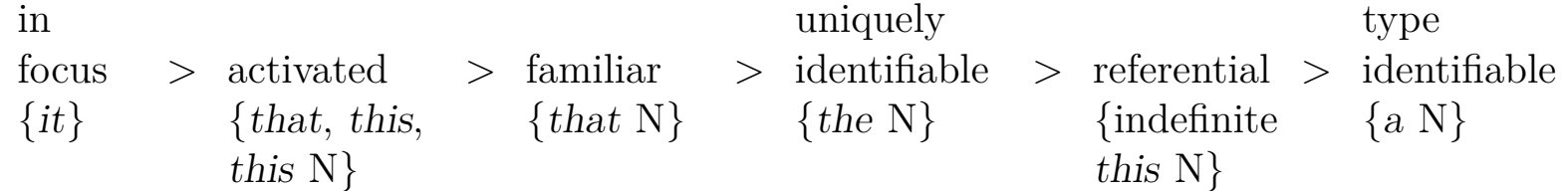

Schwarz (2000:43) unterscheidet darüber hinaus zwischen ,bekannt ${ }^{6}$ und ,erreichbar': Mit Bekanntheit bezieht sie sich auf die textuelle Ebene, Erreichbarkeit kann auch über Weltwissen gegeben sein.

Eine Folgerung aus der Giveness Hierarchy ist ebenfalls, dass indirekte Anaphern (inferrables) nur unter bestimmten Bedingungen durch Pronomen oder Demonstrativa hergestellt werden können:

\footnotetext{
[...] since pronouns and demonstratives determiners (unlike definite and indefinite articles and zero determiners) require the referent to be at least familiar (i.e. they require an existing representation of the referent), a condition which is generally not met by inferrables. On the other hand, there are instances when the interpretation of a phrase or sentence requires the addressee to automatically construct representations of entities that are inferred but not explicitly mentioned. In such cases, the inferrables could be coded with a pronoun or demonstrative determiner [...] (Gundel 1996 150f)
}

Fungiert ein Pronomen als indirekte Anapher, setzt dies in jedem Fall einen hohen Grad an Zugänglichkeit des Diskursreferenten voraus. Da es sich um einen nicht-expliziten Referenten handelt, bedeutet das, dass die Assoziation besonders stark sein muss, bzw., dass die zu ziehende Inferenz zur Erschließung des Referenten sehr plausibel sein muss. Diskurslinguistische Faktoren spielen dabei fraglos eine wichtige Rolle. Darüber hinaus bin ich der Auffassung, dass die Zugänglichkeit eines indirekten Referenten auch vom Ankerausdruck selbst abhängt, d.h. das ausdrucks-inhärente lexikalische und konzeptuelle 
Faktoren die Verfügbarkeit einer Inferenz und damit die Zugänglichkeit des Referenten beeinflussen, da sie Einfluss auf dessen Aktiviertheit haben.

Eine Übertragung der Giveness Hierarchy auf das Deutsche findet sich bei AverintsevaKlisch (vgl. 2013 75). Personalpronomen führt sie ebenfalls als mögliche Anaphern für fokussierte Referenten an, bei aktivierten Referenten hält sie auch das demonstrative der für möglich und dieser für referierende Ausdrücke. Diese Vorstellung sei an Beispiel (55) veranschaulicht. In (55-a) wird das N1 Gurke durch das Personalpronomen sie wiederaufgenommen und die Anapher scheint von eher geringer Akzeptabilität. Demzufolge scheint das N1 - wie zu erwarten - nicht fokussiert zu sein. In (55-b) ist die Anapher durch das Demonstrativpronomen der ersetzt und die Akzeptabilität nimmt deutlich zu. Die Ersetzung der Anapher durch das Demonstrativpronomen dieser in (55-c) steigert die Akzeptabilität meinem Eindruck nach nicht weiter. Sollte dieser Eindruck stimmen und allgemeingültig sein, würde dies auf Basis der Giveness Hierarchy nach AverintsevaKlisch (2013) bedeuten, dass Erstglieder nicht nur referieren, sondern sogar aktiviert sind. Dies lässt sich natürlich nicht mit einem einzelnen Beispiel als gesichert ansehen, zu vage ist hier die introspektive Einschätzung und zu vielfältig sind die Einflüsse durch das konkrete Satzmaterial. So scheint auch die Spezifität von Antezedens und Anapher die Akzeptabilität von (55-c) zu beeinflussen: Das Demonstrativpronomen dieser suggeriert einen konkreten Referenten, d.h. eine spezielle Gurke. Eine solche Gurke wird im vorausgehenden Kontextsatz allerdings nicht eingeführt, sondern nur die Gemüseart Gurken ganz allgemein.

(55) a. Der neue Gurkenschäler ist sehr praktisch. Sie lassen sich damit viel schneller bearbeiten.

b. Der neue Gurkenschäler ist sehr praktisch. Die lassen damit viel schneller bearbeiten.

c. Der neue Gurkenschäler ist sehr praktisch. Diese lassen damit viel schneller bearbeiten.

In diesem Sinne können mit Hilfe der Akzeptabilität von verschiedenen pronominalen Anaphern Aussagen zum kognitiven Status des N1 gemacht werden. Darüber hinaus bestätigt sich das Konzept einer kontinuierlichen Aktiviertheits-Skala. Je nach Position eines Referenten auf dieser Skala erscheint ein pronominaler Bezug als mehr oder weniger akzeptabel.

\section{Aktiviertheit}

Die Zugänglichkeit eines Ausdrucks wurde bisher als Funktion seines mentalen Erregungszustands beschrieben, d.h. seines Grads an Aktiviertheit. Bei der Aktiviertheit handelt es sich um eine Eigenschaft, die zunächst unabhängig vom anaphorischen Potenzial ist und von verschiedenen Faktoren beeinflusst wird. In der Giveness Hierarchy werden Zugänglichkeit und Aktiviertheit aufeinander abgebildet.

Unterschiede in Aktiviertheitsgraden spielen in verschiedenen Modellen der Sprachverarbeitung eine Rolle. Ein Modell ist das Mehrspeichermodell (multi store model) mit der Unterscheidung zwischen Kurz- und Langzeitgedächtnis (KZG und LZG), wie es bspw. Schwarz (2000) vertritt. Dabei werden verschiedene Aktiviertheitsgrade in den Speichern 
auf verschiedene Aufmerksamkeitsgrade abgebildet: Fokussierte und aktivierte Repräsentationen befinden sich im KZG, semi-aktivierte und de-aktivierte im LZG (vgl. Schwarz 2000:137ff). Je höher der Aktiviertheitsgrad einer Repräsentation ist, desto schneller ist sie verfügbar; fokussierte Repräsentationen sind somit am schnellsten verfügbar. Im LZG gibt es einen Schwellenwert für die Aktivierung einer Repräsentation, bspw. verfügen hoch-frequente Wörter über einen niedrigeren Schwellenwert. Eine Aktivierungsausbreitung führt zu einer Schwellenwertherabsetzung, d.h. die Aktivierung einer Repräsentation bewirkt eine Semi-Aktivierung verknüpfter Einträge. Beim Bau eines Textweltmodells bekommt jeder Textreferent einen Knoten im KZG (vgl. Schwarz 2000:46).

Direkte Anaphern beschreibt Schwarz als fokussiert, während das Antezedens noch aktiviert ist (vgl. Schwarz 2000:157). Bei der indirekten Anapher ist der Anker ebenfalls fokussiert und aktiviert eine kognitive Domäne, welche die Anapher explizit besetzt. Dabei bleibt der Anker im KZG aktiviert, die konzeptuelle Domäne ist semi-aktiviert und die Anaphern-NP verfügt über einen niedrigeren Schwellenwert. Alternativ sei der Anker im LZG aktiviert mit semi-aktiver Domäneninformation. Das setzt voraus, dass die relevante Domäne im Aufmerksamkeitsfokus liegt. Generell seien KZG-Repräsentationen schneller zu verarbeiten, weshalb indirekte Anaphern, die auf LZG-Zugriff basieren, längere Verarbeitungszeiten hätten (vgl. Schwarz 2000:137ff).

Ein anderes Modell der Sprachverarbeitung, welches mit seinen Vorhersagen dem Mehrspeichermodell jedoch nicht widerspricht, unterscheidet nach verschiedenen Verarbeitungstiefen. Beim Levels-of-Processing-Ansatz (vgl. Craik 2002, Craik/Lockhart 1972, Lockhart/Craik 1990) werden verschiedene Verarbeitungsebenen als Analysekontinuum zwischen ,early/shallow' und ,later/deep processing' betrachtet. Auf Ebene des ,shallow processings ${ }^{6}$ erfolgt phonemische und orthografische Verarbeitung, Verarbeitung von Oberflächenform, Farbe, Helligkeit etc., was in fragiler Erinnerung resultiert. Auf den sich anschließenden Ebenen des ,deep processings' erfolgt semantische Verarbeitung, Inferenz und Implikation, was in dauerhafter Erinnerung resultiert (vgl. Craik 2002 308).

Vertraute Stimuli mit reicher Semantik werden tiefer verarbeitet und können schneller abgerufen werden. Dabei ist die Abrufbarkeit zwar eine Funktion der Verarbeitungstiefe, aber auch andere Faktoren interagieren. So müssen nicht alle schnell abrufbaren Formen zwingend über eine hohe Verarbeitungstiefe verfügen (vgl. Craik/Lockhart 1972: 676). Gleichzeitig benötigt die Verarbeitung an sich mehr Zeit, je tiefer sie erfolgt, weshalb die Verarbeitungszeit allein nichts über Aufwand und Memorisierbarkeit aussagt (vgl. Craik/Lockhart 1972:681). Somit kann weder die Verarbeitungszeit noch die Zeit für den Abruf als eindeutiger Index für die Verarbeitungstiefe genutzt werden.

Nach Craik (vgl. 2002 311) führt weitere Verarbeitung auf oberflächlicher Ebene (z.B. einfaches Wiederholen) nicht zu einer besseren Memorisierbarkeit. Ein Effekt auf die Memorisierbakeit hat lediglich die Prozessierung auf einem tieferen Verarbeitungslevel. Um dorthin zu gelangen, muss der Ausdruck mit mehr Bedeutung angereichert werden.

Unbekannte, d.h. niedrig-frequente Wörter verhindern ein tiefes Verarbeitungslevel (vgl. Craik/Lockhart 1972:680). Betrachtet man ein relativ tiefes Verarbeitungslevel als Voraussetzung für gute Zugänglichkeit, würde dies in Bezug auf die Problematik des N1-Bezugs für hoch-frequente N1 eine höhere Zugänglichkeit prognostizieren.

Der Zusammenhang ist jedoch komplexer, denn bereits der Einfluss der semantischen Relation ist in beide Richtungen denkbar: Bspw. erfordert die Interpretation eines Kompositums mit einer seltenen und für das N1 unüblichen Relation, z.B. Dachschokolade, einen höheren Verarbeitungsaufwand und semantische Anreicherung. Dies müsste in einem 
tieferen Verarbeitungslevel und besserer N1-Zugänglichkeit resultieren. Gleichzeitig lässt sich argumentieren, dass ein semantisch transparentes und frequentes Kompositum wie z.B. Nussschokolade gerade durch seine Vertrautheit und Erreichbarkeit sowie seine vergleichsweise hohe Frequenz einen hohen Grad an Aktiviertheit und damit ein tieferes Verarbeitungslevel und bessere N1-Zugänglichkeit erreicht. Diese Überlegungen illustrieren das komplexe Zusammenspiel unterschiedlicher Faktoren, die Einfluss auf Aktiviertheit und Verarbeitungstiefe nehmen und jeweils im Einzelfall untersucht werden müssen 20

Verwandt mit der Annahme verschiedener Verarbeitungslevel ist der Good Enough Approach (vgl. Ferreira/Patson 2007, Ferreira et al. 2002). Nach diesem Ansatz ist die akkurate und detaillierte Repräsentation einer Äußerung nicht das oberste Ziel des Sprachverstehenssystems. Statt dessen wird eine Repräsentation angestrebt, die zur aktuellen Aufgabe passt, die durch den sprachlichen Input erfüllt werden soll, z.B. den Dialog aufrecht zu erhalten. Hierfür sind nicht unbedingt alle Details einer Äußerung notwendig, z.B. müssen nicht alle Ambiguitäten aufgelöst werden. Der Aufbau von Good-EnoughRepräsentationen erklärt auch das Zustandekommen vieler Missverständnisse.

Für die Problematik des N1-Bezugs kann dieser Ansatz zwei Anwendungen haben: Zum einen besteht die Gefahr, dass das N1 nur , good enough' bzw. ,shallow' prozessiert wird, da es nur als peripheres Detail wahrgenommen wird. Dies würde in einer schlechten anaphorischen Zugänglichkeit resultieren, bzw. diese erklären. Zum anderen ist denkbar, dass die Anapher nur ,shallow' prozessiert wird und für eine Good-Enough-Repräsentation der Äußerung gar nicht aufgelöst wird. Dies wiederum würde eine bessere Akzeptabilität der Anapher prognostizieren. Allerdings würden die Ergebnisse experimenteller Studien in diesem Fall verzerrt. Das Auflösen der Anapher muss daher für das Erfüllen der experimentellen Aufgabe notwendig sein und im experimentellen Design berücksichtigt werden.

Welchem Modell man auch folgen möchte, die Bedeutung für die Betrachtung der N1Zugänglichkeit ist ähnlich: Ein zugängliches N1 muss über ein relativ tiefes Verarbeitungslevel verfügen, bzw. über einen hohen Grad an Aktiviertheit. Nur so kann es durch eine Anapher abgerufen werden.

\section{Akzeptabilität und Grammatikalität}

Zugänglichkeit und Aktiviertheit eines Ausdrucks lassen sich - wie oben erwähnt - nicht unmittelbar ableiten, sondern nur über den Grad an Akzeptabilität einer anaphorischen Beziehung erschließen. Dabei stehen die Ausdrücke ,Akzeptabilität', ,Grammatikalität' und ,Gradienz' in einem engen Spannungsverhältnis zueinander.

Bei der Grammatikalität handelt es sich um eine kategoriale Eigenschaft, d.h. ein sprachlicher Ausdruck ist entweder grammatisch oder ungrammatisch. Bard et al. (vgl. 1996:33) definieren Grammatikalität als grammatikinhärentes Charakteristikum des linguistischen Stimulus. Die Akzeptabilität hingegen definieren sie als Charakteristikum des Stimulus, wie er durch die Sprecher*inn*en wahrgenommen wird. Die relative Akzeptabilität wiederum reflektiert eine Gradienz in der Bewertung. Die Akzeptabilitätsbewertung selbst

20 Marantz (2016) präsentiert Argumente für stärkere Aktiviertheit und bessere Zugänglichkeit sowohl für Fälle von ,deep' als auch von ,shallow processing،. 
ist die Antwort von Sprecher*inne*n auf eine linguistischen Nachfrage, z.B. die Angabe eines Werts auf einer Skala im Rahmen eines Experiments ${ }^{21}$

Bei Experimenten zeigt sich, dass Versuchspersonen ungrammatischen Sätzen verschiedene Grade an Akzeptabilität zuordnen, bspw. wird (56) als weniger akzeptabel bewertet als (57). In diesem Sinne sprechen Akzeptabilitätsgrade für Gradienz innerhalb der Ungrammatikalität. ${ }^{22}$ In experimentellen Settings ist den Versuchspersonen der Unterschied zwischen Grammatikalitäts- und Akzeptabilitäts-Bewertung zumeist unklar, sodass die beiden Ausdrücke synonym gebraucht werden können. Für die Formulierung der experimentellen Aufgabe ist der Unterschied jedoch nicht unerheblich, da der Ausdruck ,Grammatikalität' ein höheres Maß an Normativität konnotiert, was eine Beurteilung zugunsten des Normwissens und zu Lasten der Intuition mit sich bringen kann.

Mann der sah Katze eine, Gänse leben in glücklich.

Peter schläft das Buch.

Dieser Eindruck von Gradienz lässt sich durch Unterschiede in der Art der Regelverstöße erklären. So werden Sätze wie (56), in denen die Grundregeln von Phrasenstrukturen nicht beachtet werden, als weniger akzeptabel bewertet, als solche wie in (57), in denen (nur) Selektionsbeschränkungen verletzt werden. Neben der Art von Regelverstößen kann auch die Anzahl an Regelverstößen ein Rolle spielen. In (58) liegt eine Verletzung der koordinierten Struktur vor, in (59) zusätzlich eine Ob-Verletzung, was zu einer schlechteren Bewertung führt (vgl. Sprouse 2007).

(58) $\quad \mathrm{Wen}_{i}$ hat Peter [ $\mathrm{t}_{i}$ und Hans] eingeladen.

(59) $\quad$ Wen $_{i}$ fragst du dich ob [ Peter [ $t_{i}$ und Hans ] eingeladen hat ].

Bei Fällen mit akzeptablem N1-Bezug heißt es oft, die Beispiele seien „akzeptabler“ oder das N1 sei „zugänglicher“, bzw. „weniger unzugänglich“. Diese Formulierungen implizieren für das Phänomen ebenfalls graduelle Akzeptabilität. In diesem Sinne kann graduelle N1Unzugänglichkeit in Analogie zu den Akzeptabilitätsgraden für Ungrammatikalität gesetzt werden. So wie sich Akzeptabilitätsunterschiede bei der Beurteilung von Grammatikalität finden und an klaren Faktoren wie Art und Anzahl syntaktischer Regelverletzungen festmachen lassen, sehe ich diese Möglichkeit auch in Bezug auf die Akzeptabilitätsbewertung von N1-Bezügen und damit den Zugänglichkeitsgraden. Faktoren für die graduelle Akzeptabilität von N1-Bezügen können im Bereich der Verletzung von typischen N1Eigenschaften, bzw. im Vorhandensein typischer NP-Eigenschaften im N1 liegen. Je mehr prototypische Eigenschaften eines N1 es erfüllt und je weniger es sich wie ein selbstständiges Nomen, bzw. eine NP verhält, desto unzugänglicher ist es (konkrete Thesen dazu siehe Kap. 4). Diese Annahme ist plausibel, da es auch mehr oder weniger typische Nomen gibt. Crystal (1967) nennt hierzu die unter (60) wiedergegebenen hierarchisierten Nominalitätskriterien.

\footnotetext{
${ }^{21}$ Vgl. hierzu auch Featherston (2007) und der Begriff der Wohlgeformtheit (well-formedness).

${ }^{22}$ Vgl. Markiertheitsskala im Bereich der grammatischen Ausdrücke.
} 


\author{
Kriterien für Nominalität nach Crystal (1967): \\ - Möglichkeit als Subjekt zu agieren \\ - Flexion nach Numerus \\ - Gemeinsames Auftreten mit Artikel \\ - Morphologische Indikation wie nominale Suffigierung
}

Mit Hilfe dieser Eigenschaften entwirft Crystal (vgl. 1967:46) ein Venn-Diagramm mit sich überschneidenden Kreisen. Die Schnittmenge aller Kreise stellt die zentrale Klasse typischer Nomen dar. Je weniger Kreise sich schneiden, desto weniger typisch ist das Nomen. Auch Aarts (vgl. 2007:105) erachtet dies als eine sinnvolle Möglichkeit, Gradienz von Wortarten auszudrücken.

Die Menge an Verletzungen der Nominalitäts-Kriterien und die Menge an eingehaltenen N1-Kriterien wäre in diesem Sinne Ausdruck der Gradienz der Unzugänglichkeit. Diese Annahme wirft folgende Fragen für die vorliegende Arbeit auf: Was sind die für die N1Zugänglichkeit entscheidenden Eigenschaften dieser Konstituente, wie sind sie zu hierarchisieren und welche Arten von Interaktionen bestehen zwischen diesen Eigenschaften?

Auch ein gradueller Anaphern-Begriff wurde in der Literatur bereits thematisiert. Gemäß Reuland (vgl. 2006:57) ist es möglich, das Verhalten der anaphorischen Elemente mit Mechanismen der Grammatik zu verbinden. Die Variation sei durch Details in Merkmalsaufbau und Umgebung erklärbar und Interpretationsaspekte würden hauptsächlich durch das komputationale System und das Nutzungssystem bestimmt. Wie Fanselow et al. (2006) erklärt auch Reuland (2006:68) Gradualitäts-Phänomene durch die Befriedigung einiger, aber nicht aller Bedingungen.

Auch Verarbeitungsschwierigkeiten wirken sich auf die Akzeptabilitätsbewertung aus. Da Hörer*innen zunächst ihre präferierte Interpretation einer sprachlichen Äußerung verfolgen, kann bei Ambiguitäten in Syntax oder Semantik eine Reanalyse notwendig sein, z.B. im Fall von Homonymie oder Holzwegsätzen. Der zusätzliche Verarbeitungsaufwand durch die Reanalyse führt zu einer schlechteren Akzeptabilitäts- oder Grammatikalitätsbewertung (vgl. Crocker/Keller 2006:227). Fanselow/Frisch (2006) weisen nach, dass die Akzeptabilität bei steigender Verarbeitungsschwierigkeit sogar sinkt, wenn der Ausdruck eigentlich grammatisch ist. Weiterhin zeigen sie, dass die Anwesenheit einer lokalen Ambiguität die Gesamt-Akzeptabilität eines Satzes beeinflusst. Hierbei scheint es sich um einen Übertragungseffekt des Urteils der ursprünglicher Interpretation auf die Gesamtinterpretation zu handeln. Der Zusammenhang zwischen Akzeptabilität und Grammatikalität erscheint somit ebenfalls nicht strikt zu sein 23

Bei der anaphorischen Bezugnahme auf ein N1 handelt es sich insgesamt um ein Phänomen gradueller Akzeptabilität. Inwiefern dies Rückschlüsse auf die Grammatikalität zulässt, bleibt fraglich. Der Grad der Akzeptabilität einer Anapher liefert Rückschlüsse auf die Zugänglichkeit des N1 und ist abhängig von einer Vielzahl verschiedener grammatischer und außergrammatischer Faktoren, die Einfluss auf Aktiviertheit und Verarbeitungstiefe nehmen.

\footnotetext{
${ }^{23}$ Auch die Auftretenshäufigkeit kann Auswirkungen auf die Akzeptabilität haben und langfristig sogar
} auf die Grammatikalität, z.B. im Fall der Legitimierung der V2-Stellung in Nebensätzen. 


\subsubsection{Modelle des lexikalischen Zugriffs}

Eine grundlegende Frage zur Verarbeitung von Komposita ist die nach dem lexikalischen Zugriff, d.h. ob Komposita wie Monolexeme über einen eigenen Lexikoneintrag verfügen oder wie Sätze bei jedem Vorkommen neu gebildet werden. Dieser Überlegung entspricht die Unterscheidung zwischen ,full parsing ${ }^{6}$ und ,full listing'. Die Full-Parsing-Hypothesis geht ausschließlich von Einzelmorphemspeicherungen aus, was zu Folge hat, dass das Verstehen komplexer Wörter stets durch Analyse erfolgen muss. Der Vorteil liegt in einem schlanken Lexikon, der Nachteil in hohen Prozesskosten. Bei der Full-Listing-Hypthesis hingegen wird davon ausgegangen, dass Komposita als Ganzes abgespeichert werden, was niedrige Prozesskosten, aber eine hohe Speicherkapazität mit sich bringt (vgl. Dietrich 2007). Die beiden Modelle gelten lediglich als Archetypen; wahrscheinlicher ist eine hybride und ausdrucksabhängige Speicherform.

Einflussreiche Theorien auf diesem Gebiet der kongnitiven Linguistik stammen u.a. von Gary Libben. Ob ein komplexer Ausdruck dekomponiert oder als Ganzes abgerufen wird, hängt Libben (vgl. 1998:31) zufolge von einer Vielzahl von Faktoren ab, darunter Transparenz, Frequenz und Analyseaufwand, lexikalische Kategorie, morphologischer Typ und das semantische Verhältnis zwischen einzelnen Morphemen und dem komplexen Ausdruck.

Im Zusammenhang mit dem lexikalischen Zugang bei der Komposita-Interpretation sind drei Annahmen von Libben (1998) zu nennen: (i) der automatische Parser, (ii) die Theorie der drei Repräsentationsebenen und (iii) die Theorie der ,Maximization of Opportunity'. Die Annahme eines automatischen Parsers (i) beinhaltet die Vorstellung des Parsings als einen automatisch fortschreitenden Prozess mit lexikalischer Aktivierung, dem APPLE Parser (automatic progressive parsing and lexical excitation) (vgl. Libben 1998:35). Die Theorie der drei Repräsentationsebenen (ii) geht davon aus, dass die Wahrnehmung und Interpretation verschiedener Kompositaformen auf drei Ebenen (levels) erfolgt (vgl. Libben 1998:35ff). Auf der Stimulus-Ebene (stimulus level) erfolgt das Parsing und die Abfrage von lexikalischem und orthografischem Status, die lexikalische Ebene (lexical level) ist rein morphologisch und beinhaltet Wortformen und auf der konzeptuellen Ebene (conceptual level) spielen schließlich semantische Faktoren eine Rolle. Mit der Theorie der Maximization of Opportunity (iii) schließlich weist Libben die Ökonomie-Hypothese zurück, nach der es ein Abwägen zwischen Speicher- und Rechenaufwand gibt (vgl. Libben 2007). Stattdessen geht er davon aus, dass Morpheme sowohl als selbstständige Wörter als auch als Komposita-Konstituenten gespeichert werden. Das System ist also nicht maximal effizient, sondern verfügt über Redundanzen (vgl. Libben 2007:6f). Bei der Interpretation eines Tokens zerlegt der prälexikalische Parser den Ausdruck zunächst in alle möglichen Morpheme, welche bei der Bedeutungsfindung nützlich sein können.

Activating everything possible appears to be the easiest and most generally applicable mental architecture for the processing of multimorphemic words under the conditions of uncertainty that characterize word recognition. (Libben 2007:9f)

Kommt es zu Inkongruenz zwischen Einzelmorphem- und Ganzwortbedeutung, erfolgt eine Inhibierung nicht benötigter Bedeutungsteile, die mit Prozesskosten verbunden ist. Mit einer Reaktionszeitstudie liefern Libben et al. (2003) Evidenz dafür, dass auch komplexe Wörter über eine eigene Repräsentation verfügen, da signifikant längere Reaktionszeiten für getrennt geschriebenen Komposita im Gegensatz zu unmarkierter Zusammenschreibung gefunden wurden. 
Olsen unterstützt Libbens Theorie der Maximization of Opportunity und nimmt ebenfalls an, dass auch gebundene lexikalische Konstituenten über einen eigenen Lexikoneintrag verfügen (vgl. Olsen 2012:160). Ein einzelnes Morphem kann als gebundene Konstituente verschiedene Bedeutungen haben, bzw. im Gegensatz zum selbstständigen Wort kann eine spezielle Teilbedeutung stärker gewichtet sein, während andere Bededeutungsaspekte inhibiert werden. Daraus müsse wiederum eine Mehrfachspeicherung gebundener lexikalischer Konstituenten resultieren. Dies illustriert sie u.a. am Beispiel von Ecke in (61) Ecke müsse über vier Einträge im mentalen Lexikon verfügen: Einer für das Einzelwort und drei verschiedene für die verschiedenen Relationen, in denen das Morphem in einem Kompositum auftreten kann (vgl. Olsen 2012;156ff).
a. $\operatorname{Ecke}(\mathrm{v}, \mathrm{x})$
b. -ecke: Straßenecke (externes Argument des N1)
c. - -ecke1: Küchenecke (LOCATION)
d. - ecke2: Spielecke (PURPOSE)

Olsen nimmt an, dass „die Konstituenten etablierter Komposita eigene Einträge im mentalen Lexikon entwickeln und sich dadurch als Einheiten des mentalen Lexikons in einer kombinatorischen Bedeutung etablieren." (Olsen 2012:163).

Ob ein derart hoher Grad an Redundanz tatsächlich notwendig ist, bezweifle ich. Da die konkrete Relation zwischen zwei Komposita-Konstituenten nicht an der Oberfläche realisiert, sondern während der Wortverarbeitung erschlossen wird, erscheint mir das interpretative Erschließen einer Teilbedeutung eines mehrschichtigen, gebundenen Morphems nicht als weniger plausibel als die Auswahl eines Morphems mit spezifischerer Bedeutung. Zudem steht diese Trennung verschiedener Konstituentenmorpheme im Widerspruch zur Eigenschaft der semantischen Ambiguität, die ein zentrales Charakteristikum von Komposita ist.

Im Zusammenhang mit Libbens Modell wird auch oft von einer „dual route“ gesprochen, da sowohl der Weg des Einzelmorphem- als auch des Kompositum-Zugriffs gegangen wird. Psycholinguistische Evidenz für dieses Modell liefern u.a. Koester et al. (2004) mit Hilfe von EEG-Experimenten. Sie zeigen, dass während früher Verarbeitungsschritte Genus und semantische Informationen der Konstituenten unabhängig voneinander verarbeitet werden. So wurde ein Effekt für ein Mismatch des N1-Genus gefunden. Bei reinem Ganzwortzugriff dürfte die Genus-Information des N1 nicht zugänglich sein (vgl. Koester et al. 2004:1651). Gleichzeitig wurde kein Numerus-Effekt auf dem N1 gefunden, was wiederum gegen eine komplette morphosyntaktische Analyse und für ein hybrides Verarbeitungsmodell spricht (vgl. Koester et al. 2004:1656).

\subsubsection{Interpretationsmechanismen}

Das Merkmal der Unterspezifiziertheit wirft Fragen zur Bedeutungsinterpretation und zu den involvierten Interpretationsmechanismen beim Verstehen von Komposita auf. Sowohl bei den Wortbildungsschemata als auch bei den semantischen Relationen (vgl. Kap. 2.2.4) handelt es sich um linguistische Theorien und nicht um psychologische Modelle (vgl. Gagné/Shoben 1997:80). In diesem Sinne wäre es denkbar, dass das Auflösen der semantischen Relation kein Teil der Interpretation von Komposita ist. 
Ein $\mathrm{N}+\mathrm{N}$ zu verstehen heißt nicht, die Relation zwischen den unmittelbaren Konstituenten ermitteln zu können, sondern den Referenten identifizieren zu können. (Klos |2011.316)

Geht man davon aus, dass die semantische Relation zwischen den Konstituenten bei der Interpretation nicht aufgelöst wird, ergeben sich daraus zwei Konsequenzen: Zum einen sind die Relationen an sich nicht leicht oder schwer zu ermitteln, da sie gar nicht ermittelt werden. Zum anderen evozieren neugebildete Komposita ein geringeres Fremdheitsgefühl beim Hören als unbekannte Monolexeme. Durch die Bekanntheit der Konstituenten entsteht die Illusion von Durchsichtigkeit. In einem Benennungsexperiment von Klos (vgl. 2011:155) gaben Versuchspersonen Bedeutungen für Ad-hoc-Bildungen an. D.h. die Versuchspersonen fühlten sich in der Lage, Wörter zu erklären, die sie noch nie vorher gehört hatten. Dies belegt zudem, dass es sich bei der Komposition um einen uneingeschränkt produktiven Wortbildungstyp handelt. Als die generell involvierten Verarbeitungsaspekte identifiziert Klos die Konstituentensemantik, d.h. die lexikalische Bedeutung der Teile, und deren grammatische Bedeutung. Den grammatischen Merkmalen spricht sie nur geringen Einfluss zu. Als zugrundeliegende syntaktische Struktur erachtet sie lediglich das Determinationsschema als relevant (vgl. Klos 2011:266f).

Nach Meyer (vgl. 1993:103) ist die Interpretation weniger von spezifischen KompositionsRegeln, sondern mehr von einer Kombination von Merkmalen der Konstituentenkonzepte abhängig. Dabei erachtet er bei der kontextfreien Komposita-Interpretation drei Wissenstypen als relevant: die Argumentstruktur, lexikalisches Wissen und Konzeptwissen wie Skripts etc. Hierbei seien explizite gegenüber impliziten Informationen wie Hintergrundwissen präferiert, d.h. eine Interpretation auf der Basis von lexikalischem Wissen sei gegenüber einer konzeptbasierten Analyse präferiert (vgl. Meyer 1993:212).

Zur konzeptuellen Komposita-Interpretation existieren verschiedene Modelle. In den 80er und 90er Jahren des 20. Jahrhunderts waren besonders Schema-Modelle verbreitet. Dabei wird allgemein davon ausgegangen, dass das N1 und das N2 jeweils über ein Konzept verfügen und das Konzept des N1 einen Slot im Konzept des N2 füllt. Verschiedene Modelle unterscheiden nach Gewichtung von Faktoren, die darauf Einfluss haben, z.B. Kontext oder grammatische Merkmale. Ein ausführlicher Vergleich verschiedener Modelle findet sich in Meyer (1993:33).

Ein Beispiel für eine solche ,Slot Filling Theory" ist das ,interactive property attribution model' nach Estes/Glucksberg (2000). Dabei werden die relevanten Dimensionen des N2 mit den Eigenschaften des N1 abgeglichen, wie etwa in (62) (vgl. Estes/Glucksberg 2000: 29). Die Interpretation wird insgesamt von der Interaktion der Dimensionen und Merkmale geleitet.
a. Kompositum: shark lawyer
b. relevante Dimensionen für eine Attribuierung des N2: Temperament, Kom- petenz, Kosten
c. saliente Eigenschaften des N1: rücksichtslos, aggressiv und unmoralisch

Die Relationen können dabei abhängig von den entsprechenden Eigenschaften unterschiedlich gestaltet sein, was nach Estes/Glucksberg (vgl. 2000:32) keine Unterschiede in den involvierten kognitiven Prozessen mit sich bringen muss.

Ein anderes schema-basiertes Modell ist das der interaktiven Aktivation, demzufolge die relevanten Aspekte der semantischen Repräsentationen der Konstituenten aktiviert sein 
müssen (vgl. Coolen et al. 1993:235). Die gemeinsamen oder kompatiblen Bedeutungsaspekte verstärken sich gegenseitig. Die Theorie der interaktiven Aktivation wurde durch Coolen et al. (1993 236ff) in Priming-Experimenten verfolgt. Dabei zeigt sich, dass das N1 die Lesart des (ambigen) N2 festlegt.

Ein entscheidender Kritikpunkt an den schema-basierten Modellen ist, dass diese ausschließlich auf lexikalischer und konzeptueller Ebene arbeiten und somit Fälle von kontextabhängiger Bedeutung nicht erklären können; z.B. dass ein Marmormuseum je nach Kontext ein Museum aus oder über Marmor sein kann (vgl. Meyer 1993: 38).

Ein anderer Ansatz zur Erklärung der Komposita-Interpretation ist das CARIN-Modell von Gagné/Shoben (1997). Dabei handelt es sich im Gegensatz zu den oben erwähnten schema-basierten Modellen um einen relations-basierten Ansatz. Die entscheidende Rolle kommt hier der unterspezifizierten und konzeptuell nicht festgelegten Relation zu. Gagné/Shoben (1997) verbinden darin Wissen über Konzepte und Relationen, in denen die Konzepte genutzt werden. Das CARIN-Modell (Competition Among Relations in Nominals) geht davon aus, dass den Adressaten Bedeutung und Anwendung eines N1 bekannt sind und die verschiedenen Relationen für jedes N1-Konzept verschiedene Stärken im Sinne von Auftretenshäufigkeiten haben. Stehen mehrere „starke“ Rollen nebeneinander, verlangsamt sich die konzeptuelle Kombination.

[...] the CARIN model claims that the ease with which the appropriate relation can be found depends on both the strength of the to-be-selected relation and on the strength of the alternatives (Gagné/Shoben 1997 81)

Verschiedenen Relationen werden abhängig von der Häufigkeit eines Konzepts verschiedene Verfügbarkeiten zugeschrieben. Tritt ein Konzept häufig in Verbindung mit einem bestimmten N1 auf, verfügt dieses über einen Verarbeitungsvorteil. Die Schwierigkeit der Interpretation liegt somit nicht in den semantischen Relationen an sich, sondern an ihrer Häufigkeit in Verbindung mit einem N1. So neige z.B. mountain eher zu einer IN-Relation als zu einer FROM- oder ABOUT-Relation; deshalb sei mountain stream leichter zu verarbeiten als mountain magazine.

In summary, we suggest that people possess distributional knowledge about how often particular relations are used with modifiers and that this knowledge plays a large role in comprehending combined concepts. (Gagné/Shoben 1997.74).

Dabei stellt sich die Frage, warum die Relation an das N1 gebunden sein sollte. Gagné/Shoben (1997:83) betonen, dass das N1 linear an erster Stelle steht und deshalb die damit verbundenen Relationen zuerst aktiviert würden. Abgeleitet daraus halten sie auch eine semantische Privilegierung für möglich, die darüber entscheidet, wie die Repräsentation des N2 verändert werden soll. Mit dieser Vorstellung stehen sie den klassischen schema-basierten Modellen entgegen.

Zur Validierung ihres Modells berichtet Gagné (2001) einige Priming-Experimente. Hierbei erweist sich das N2 - wie auch in den anderen Studien - als effektiveres Prime. Allerdings wurde für N2-Primes kein Relations-Priming gefunden, sondern lediglich (morphologisches) Wiederholungs-Priming. Auch ein reines Relations-Priming konnte nicht nachgewiesen werden. Im Fall eines N1-Primes zeigte sich hingegen Relations-Priming. Dies betrachtet Gagné (2001:242ff) als Evidenz für die Gültigkeit des CARIN-Modells und die Assoziierung der Relation mit dem N1. Darüber hinaus zieht sie allerdings auch den Schluss, dass das N2 für anaphorische Referenz die wichtigere Konstituente sei, da auf 
das Denotat des NN auch durch ausschließliche Nennung des N2 referiert werden könne (vgl. Gagné 2001:251).

Allen Arten von Komposita (ad-hoc oder lexikalisiert) legen Gagné/Spalding (2007:168) den selben Verarbeitungsmechanismus zugrunde: Die Komposita werden dekomponiert und unter Verwendung einer Relation rekomponiert. Während gemäß Klos (2011) die Relation nicht eindeutig identifiziert werden muss, erachten Gagné/Spalding (2007:162f) die Etablierung der Relation als wichtigen Schritt.

Die bisher berichteten Theorien und Modelle zur Verarbeitung von Komposita lassen sich den lexikalistischen Wortbildungstheorien zuordnen. Deren entscheidendes Charakteristikum ist die Annahme, dass Wörter (z.T. mit Klang und Bedeutung) durch morphologische Prozesse im mentalen Lexikon konstruiert werden und anschließend als atomare Einheiten in die Syntax Eingang finden. Ein alternatives Modell stellt die Distribuierte Morphologie (DM) zur Verfügung. Wie in Kapitel 2.2.3 angesprochen, werden Wörter und Phrasen im Rahmen dieser Grammatiktheorie mit den gleichen Mitteln beschrieben. Statt der Vorstellung eines einzigen mentalen Lexikons herrscht in der DM die Annahme dreier distribuierter, nicht-komputationaler Listen: Das Lexikon, das Vokabular und die Enzyklopädie (vgl. Marantz 1997).

Ohne an dieser Stelle genauer auf die Annahmen der DM einzugehen, sei angemerkt, dass die Annahmen, die sich auf Grundlage der DM zur mentalen Repräsentation von Komposita machen lassen, nicht in Widerspruch zu den aus den lexikalistischen Theorien resultierenden Annahmen stehen. In Form von sukzessivem Einsatz der Listen ist auch im Rahmen der DM die Annahme mehrerer Verarbeitungsphasen plausibel, bspw. kommt die Enzyklopädie erst in der LF zum Einsatz. Kompositionalitätsgrade werden in der DM über Einsetzungsebenen modelliert. Dies hat den Vorteil, dass für Idiome und lexikalisierte Komposita eine einheitliche Modellierung möglich ist.

Während Libben (vgl. 1998:35) annimmt, dass alle Wortbildungsprodukte geparst werden, geht die DM einen Schritt weiter. Zur Dekomposition in der visuellen Worterkennung nimmt Marantz (2016) auf Basis bildgebender Verfahren an, dass morphologisches Parsing viel kleinteiliger vonstattengeht. Demnach finde Dekomposition statt, sobald sich Stamm und Suffix identifizieren lassen; unabhängig davon, ob die Aufteilung grammatisch ist. Mit Hilfe von Priming-Studien wurde z.B. gezeigt, dass excursion in excurs und ion dekomponiert wird, obwohl das nicht die zugrundeliegende Struktur ist. Die Idee eines automatischen und obligatorischen Parsings wird somit bestätigt und sogar verstärkt.

\subsubsection{Zentrale Verarbeitungsfaktoren}

Der Transparenz, der Lexikalisiertheit und der Frequenz von Komposita wird an vielen Stellen ein Einfluss auf Speicherung und Verarbeitung von Komposita zugesprochen, weshalb eine Diskussion der Begriffe erfolgen muss.

\section{Semantische Transparenz und Kompositionalität}

Die semantische Transparenz eines Kompositums wird von vielen Autor*inn*en als Kriterium für die Entscheidung zwischen Einzelmorphem- und Ganzwort-Speicherung angesehen (vgl. Klos 2011, Libben et al. 2003, Sandra 1990, Zwitserlood 1994). Dabei gilt es zwischen Transparenz der Konstituenten und Transparenz der gesamten Konstruktion zu 
unterscheiden. Letztere wird gemeinhin auch als Kompositionalität ${ }^{24}$ bezeichnet.

Die Unterscheidung zwischen , transparent, und ,opak ${ }^{6}$ beschreibt ein semantisches Charakteristikum eines Morphems, nämlich das Verhältnis zwischen seiner Bedeutung als Simplex und als Kompositums-Konstituente (vgl. Libben et al. 2003:61). Je ähnlicher sich die Bedeutung eines Morphems als Simplex und als Kompositums-Konstituente sind, desto transparenter ist die Konstituente. Demnach ist Butter in Butterbrot transparent und in Butterblume opak, da sich N1 und Simplex bei Butterblume nur wenige semantische Merkmale teilen, bei Butterbrot hingegen die meisten.

Der Grad an Kompositionalität eines komplexen Ausdrucks bezeichnet, inwiefern sich die Bedeutung des Ausdrucks mit Hilfe seiner Konstituentenbedeutungen erschließen lässt (vgl. Sandra 1990:550). Anders ausgedrückt: Die Kompositionalität beschreibt die semantische Bezogenheit zwischen der Bedeutung eines Kompositums und den Bedeutungen der Konstituenten (vgl. Schulte im Walde et al. 2016:150). Die Kompositionalitäts-Kriterien nach Klos (2011) sind, ob die Konstituenten austauschbar und die Bedeutung kontextunabhängig ist.

Dressler (vgl. 2007:40) gibt zu bedenken, dass die Bedeutung von Komposita nie voll transparent ist, da Komposita immer unterspezifiziert sind. Allerdings gelten sie als transparenter, wenn die Bedeutung des N2 ersichtlich ist und die Relation semantisch motiviert ist.

Sowohl Transparenz als auch Kompositionalität fasse ich mit Klos (vgl. 2011:159) als graduelle Merkmale auf. Bspw. handelt es sich bei den zentralen semantischen Merkmalen von Butter in Butterbrot um dieselben wie im Simplex, allerdings liegt die Butter im Fall der Konstituentenbedeutung nicht in ihrer prototypischen Blockform vor. Da die semantischen Merkmale von Simplex und Konstituente nicht identisch sind, kann der Ausdruck nicht als volltransparent gewertet werden. Der Grad an Kompositionalität wiederum ist größer, je eher sich die Semantik des Kompositums über die Teilbedeutungen erschließen lässt. Dies sei nach Klos (2011:273) umso eher der Fall, je mehr der folgenden Kriterien erfüllt sind:

- Konstituenten sind konkrete Nomina

- enger Bedeutungsumfang der Konstituenten

- Konstituenten sind monosem/quasi-terminologisiert

- Zweitglied ist relational oder rektional

- mind. 1 Konstituente hat stark kontextualisierende Semantik

- Konstituenten legen typische thematische Relation nahe

Kompositionalität und Transparenz sind zwei Eigenschaften eines Kompositums, die zwar in Zusammenhang stehen, jedoch nicht identisch sind und auch unabhängig voneinander existieren können (vgl. Libben 1998). So handelt es sich bei Handschuh um einen kompositionalen Ausdruck, dennoch ist das N2 nur in geringem Maße transparent.

Vielfach wurde die These aufgestellt, dass Transparenz und Kompositionalität eine kritische Rolle beim lexikalischen Zugriff spielen (vgl. Libben et al. 2003). Zur Überprüfung wurde eine Reihe von psycholinguistischen Experimenten durchgeführt.

Für niederländische Komposita untersuchte Sandra (1990), ob für transparente, opake und Pseudokomposita Unterschiede im Zugriff auf die Konstituenten bestehen. Hierfür

${ }^{24}$ Libben spricht zumeist auch hier von Transparenz, bzw. in Libben (1998 38) von „componentiality“. Was Libben als Transparenz des Gesamtworts beschreibt, bezeichne ich mit dem Terminus ,Kompositionalität: 
wurde ein semantisches Priming mit lexikalischer Entscheidungsaufgabe durchgeführt, bei denen das N1 oder das N2 als Prime dienten. Im Fall von opaken Komposita (z.B. Prime $=$ melkweg $(\mathrm{dt} .:$ Milchstraße), Probe $=$ koe $(\mathrm{dt} .:$ Kuh $))$ wurde genauso wie im Fall von Pseudokomposita (z.B. Prime = zon-de (dt.: Sünde), Probe = zomer (dt.: Sommer)) kein separates Konstituentenpriming nachgewiesen. Dies spricht gegen eine analytische Komposita-Interpretation und für separate Lexikoneinträge für die Konstituenten und das Kompositum. Im Fall von transparenten Komposita (z.B. Prime = melkfles (dt.: Milchflasche)) wurde für beide Konstituenten ein Priming-Effekt gefunden, was für eine Interpretation via Analyse und gegen eine Ganzwortspeicherung spricht (vgl. Sandra 1990:543ff). Diese Ergebnisse interpretiert Sandra als Indiz für unterschiedliche mentale Repräsentationen für transparente und opake Komposita und damit verbunden auch für Unterschiede in der Verarbeitung. Während transparente Komposita via Konstituentenrepräsentationen analysiert werden müssten, läge für opake Komposita eine separate Repräsentation als Einzelwort vor, welche als Ganzes abrufbar sei. Es erfolge somit keine automatische Dekomposition. Die Entscheidung darüber, ob eine kompositionale Bedeutung erzeugt oder eine Kompositums-Repräsentation abgerufen wird, erfolgt Sandra zufolge, indem der Parser registriert, ob das Wort nach dem ersten String weitergeht (vgl. Sandra 1990:554). Zu Sandras Befunden ist anzumerken, dass die von ihm als ,opak' kategorisierten Komposita jeweils nur eine opake Konstituente enthalten. Außerdem unterscheidet er in der Auswer-

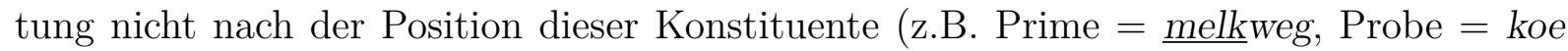
und Prime $=$ aardappel $(\mathrm{dt} .:$ Kartoffel $)$, Probe $=$ peer $(\mathrm{dt} .:$ Birne $))$. Abgesehen davon handelt es sich um semantisches Priming. Über den rein lexikalischen Zugriff sagen die Ergebnisse daher nichts aus. Als Konsequenz seiner Interpretation ergibt sich, dass opake Komposita schneller verarbeitet werden müssten, da für sie direkt ein Lexikoneintrag zur Verfügung steht.

Weitere Experimente in dieser Tradition wurden von Zwitserlood 1994:344) durchgeführt. Sie unterscheidet drei Stufen von Transparenz: ,voll transparent' (z.B. kerkorgel (dt.: Kirchenorgel)), ,teilweise transparent ${ }^{6}$ (z.B. drankorgel (dt.: Trunkenbold)) und ,opak (z.B. klokhuis (dt.: Kerngehäuse, wörtl.: Uhrenhaus)). Dabei versteht sie unter ,teilweise transparent', dass das Kompositum in seiner Bedeutung semantisch mit einer der Konstituenten relatiert ist. Ihre Experimente liefern Evidenz für morphologisches Priming für alle drei Arten von Komposita. Da der morphologische Aufbau vor der semantischen Verarbeitung verfügbar ist, spricht die Verfügbarkeit von morphologischen Informationen von Konstituenten opaker Komposita für separates Parsing aller Komponenten, d.h. alle Komposita werden als morphologisch komplex wahrgenommen (vgl. Zwitserlood 1994: 365), vgl. APPLE-Parser. Das semantische Priming hingegen förderte Unterschiede zutage. Der stärkste Effekt wurde für N2 in transparenten Komposita gefunden. Die Ergebnisse deuten darauf hin, dass echt opake Komposita über eine einzelne semantische Repräsentation verfügen und genau wie bei Pseudokomposita keine Verknüpfung zu den semantischen Repräsentationen der Teile besteht. Voll- und teiltransparente Komposita verfügen ebenfalls über eigene semantische Repräsentationen, allerdings ist diese mit den Teilrepräsentationen verknüpft (vgl. Zwitserlood 1994:365). In Einklang mit Libbens Theorie der Maximization of Opportunity schließt Zwitserlood daraus, dass die verschiedenen (lexikalischen) Repräsentationsformen aktiviert werden und somit kompetitieren. Nicht genutzte (semantische) Informationen werden inhibiert (vgl. Zwitserlood 1994:364).

Aufbauend auf den Untersuchungen von Zwitserlood fügen Libben et al. (2003) der Einteilung eine weitere Stufe hinzu und differenzieren somit die vier Arten von Komposita 
unter $(63) 25$

Transparenzklassifikation nach Libben et al. (2003):

- TT: transparent + transparent (z.B. car-wash)

- OT: opak + transparent (z.B. strawberry)

- TO: transparent + opak (z.B. jail bird)

- OO: opak + opak (z.B. hogwash)

Auch Libben et al. bedienen sich einer lexikalischen Entscheidungsaufgabe mit morphologischem Priming. Der größte Grad an Korrektheit bei der lexikalischen Entscheidung wurde bei den transparenten Komposita gefunden. Bei Betrachtung der Reaktionszeiten ergeben sich zwei Gruppen, zwischen denen ein signifikanter Unterschied gefunden wurde: $\mathrm{OO} / \mathrm{TO}$ und OT/TT. Dabei wurden für die Bedingung OO längere Reaktionszeiten als für die Bedingung TO gemessen, zwischen OT und TT wurde kein Unterschied gefunden. Da morphologisches Priming nachgewiesen wurde, nimmt Libben wie Zwitserlood an, dass für alle Arten von Komposita morphologische Dekomposition erfolgt. Dass für Komposita mit opakem Kopf längere Reaktionszeiten gemessen wurden, spricht gegen einen direkteren Abruf opaker Komposita, was Sandras Interpretation nahegelegt hatte. Auch der Einfluss der Konstitutenten-Transparenz konnte nachgewiesen werden, da für die Bedingungen OT und TO ein signifikanter Unterschied in den Reaktionszeiten gefunden wurde.

Diese Ergebnisse spiegeln Libbens Modell der drei Repräsentationsebenen wider: Entgegen Sandras Interpretation scheinen alle Komposita über identische Repräsentationen auf Stimulus- und lexikalischem Level zu verfügen, was sich in der Möglichkeit morphologischen Primings für alle Komposita zeigt. Auf konzeptuellem Level bestehen Unterschiede in Abhängigkeit vom Grad der Transparenz (vgl. Libben et al. 2003:36). Im Modell drückt Libben (1998) die verschiedenen Transparenzgrade über Verbindung zwischen lexikalischem und konzeptuellem Level aus (facilitatory links). Bei volltransparenten Komposita bestehen Verbindungen zur Ganzwortrepräsentation sowie zu den Einzelwortrepräsentationen auf dem konzeptuellen Level. Bei opaken Konstituenten besteht nur die Verbindung zur Ganzwortrepräsentation, weshalb kein semantisches Priming bei opaken Komposita festgestellt wurde, was wiederum auch Sandras Ergebnisse zeigen.

In seinem Aufsatz aus dem Jahr 2005 relativiert Libben den Unterschied zwischen opaken und transparenten Komposita. Sprachverarbeitung erfolge immer automatisch und obligatorisch, schließlich könne man sich auch nicht bewusst dagegen entscheiden, ein Wort zu verstehen. Der Unterschied zwischen opaken und transparenten Komposita liege also nicht im Stattfinden oder Nicht-Stattfinden eines Verarbeitungsschritts, sondern in der Menge an Verarbeitungsaufwand. Im Fall semantisch opaker Komposita erfolge ein zusätzlicher Verarbeitungsschritt, nämlich die Inhibition fälschlicherweise mitaktivierter Bedeutungsteile. Gleichzeitig schaffe die lexikalische Verarbeitung zwangsläufig große Datenmengen (vgl. Libben 2005:276).

Darüber hinaus reflektiert er die Einteilung in opak und transparent: Im Fall von bat seien trotz der Abweichung zwischen der Simplexbedeutung ,fliegendes Säugetier' und der Konstituentenbedeutung ,Art von fiktionalem Superheld' dennoch transparente Bildungen wie

${ }^{25}$ Darüber hinaus ließe sich als Zwischenstufe zwischen ,opak' und ,transparent' ,semitransparent' aufnehmen. Dazu könnten bspw. metaphorische motivierte Konstituenten zählen, z.B. Löwenzahn (vgl. Dressler 2007 41). 
Batman, Batmobile oder Batcape möglich (vgl. Libben 2005:277). Ich sehe hierin jedoch eher ein Indiz für die Unabhängigkeit von Transparenz und Kompositionalität sowie ein Beispiel für Analogiebildung.

Ein weiterer Punkt Libbens ist, dass Transparenz nicht als Eigenschaft an sich zu behandeln sei, sondern als psycholinguistischer Effekt von Bedeutungswechsel und Aktivierung (dazu siehe auch Gagné 2009 266). Methodenkritisch merkt er an, dass das jeweilige Paradigma einen entscheidenden Faktor darstellt; so sei keine unabhängige Variable wirklich unabhängig (vgl. Libben 2005:275ff).

Hierfür sprechen Unterschiede zwischen den Ergebnissen der Priming-Experimente mit anderen Paradigmen. Bei einer Eye-Tracking-Studie zur Komposita-Verarbeitung fanden Frisson et al. (2008:88) auf den Verarbeitungs-/Fixationszeiten keinen Effekt der Transparenz, weder bei frühen Werten, denen sie (wie Libben 1998) eine visuelle Verarbeitungsstufe mit automatischer Dekomposition zuordnen, noch bei späteren Werten, denen sie eine spätere Verarbeitungsstufe zuordnen, die sensitiv für Transparenz sein müsste. Im Unterschied zu den Priming-Experimenten zeigen diese Ergebnisse keinen Unterschied im Verarbeitungsaufwand zwischen transparenten und opaken Komposita und sprechen für Frisson et al. (2008:96) gegen eine „Berechnung“ von Bedeutung.

Zusammenfassend lässt sich festhalten, dass es sich bei Transparenz und Kompositionalität um graduelle Faktoren handelt, die Einfluss auf die Komposita-Verarbeitung haben können. Die Komposita-Verarbeitung an sich erfolgt vermutlich weder effizient noch zielgerichtet, sondern interaktiv und redundant.

\section{Lexikalisierung}

Eng verwandt mit dem Themenfeld um Transparenz und Kompositionalität ist der Begriff der Lexikalisiertheit. Im Allgemeinen gilt eine Lexikalisierung als eine Einheit im Lexikon, deren Bedeutung nicht kompositionell erschließbar, sondern „nennfest“ ist (vgl. Klos 2011:68). In diesem Sinne geht der Aufbau einer Lexikalisierung mit dem Abbau von Kompositionalität einher. Genauso wie bei der Kompositionalität, ist auch im Fall der Lexikalisierung von Komposita von einem graduellen Phänomen auszugehen (vgl. Klos 2011:60f). So ist z.B. Löwenzahn stärker lexikalisiert als Apfelbaum, was wiederum stärker lexikalisiert ist als Waldblume.

In Bezug auf den Einfluss des Lexikalisiertheitsgrads eines Kompositums auf dessen Verarbeitung geht Klos davon aus, dass mit steigender Nennfestigkeit weniger kompositionale Dekodierung und eher eine Verarbeitung als Simplex erfolgt (vgl. Klos 2011:166). Dabei sei nicht die Lexikalisiertheit an sich das entscheidende Verarbeitungskriterium, sondern der Wiedererkennungseffekt. Das bedeutet, dass ein Kompositum, wenn es hoch-frequent ist, über die Zeit an Kompositionalität verliert, da seine Bedeutung konsensuell festgelegt ist (vgl. Libben 1998).

Less lexicalization means more transparency, more lexicalization more opacity. More transparency implies more motivation of the compound via its members. (Dressler 2007:40)

Aus diesem Grund spricht sich Klos für eine holistische Verarbeitung von Lexikalisierungen aus (Klos 2011:253). Dagegen ist Jarema (2007) der Ansicht, dass lexikalisierte Komposita nicht anders verarbeitet werden als Neubildungen, da auch jede Lexikalisierung zunächst eine Neubildung war. 
Meiner Ansicht nach können lexikalisierte Ausdrücke durchaus transparent und kompositional sein. Der entscheidende Punkt, der eine Lexikalisierung ausmacht, ist die Bedeutungskonsolidierung, die sich in der starken Präferenz für eine Interpretation niederschlägt. Ein lexikalisiertes Kompositum ist in seiner Bedeutung weniger ambig als ein nicht-lexikalisiertes. So können die Beispiele Papierkorb und Filtertüte als Lexikalisierungen angesehen werden: Ihre Bedeutung ist konsensuell festgelegt und kann nur über einen sehr starken Kontextbezug ausgehebelt werden. Trotz alledem ist sie über ihre Konstituenten erschließbar. Es scheint mir plausibel, dass Lexikalisierungen tendenziell eher wie Simplizia und opake Komposita als Ganzes gespeichert werden, allerdings sehe ich darin keinen Anlass anzunehmen, dass Lexikalisierungen über einen geringen Grad an Kompositionalität verfügen müssen. Die Bedeutungskonsolidierung ist hier eher eine Frage der Frequenz.

\section{Frequenz}

Eine messbare Größe, mit der oft versucht wird Größen wie Kompositionalität und Lexikalisiertheit greifbar zu machen, ist die Frequenz. Dabei ist zunächst zwischen der Frequenz der Konstituenten als Simplizia und als Konstituenten (familiy size) und der Gesamtwortfrequenz eines Kompositums zu unterscheiden. Alle drei Frequenzarten haben möglicherweise einen Einfluss auf die Verarbeitung.

Im Unterschied zu Libben vertritt Olsen $(2012)$ in Bezug auf die NN-Frequenz die Ökonomie-Hypothese, die besagt, dass hoch-frequente Komposita schneller über die GanzwortRoute und niedrig-frequente Wörter effizienter via Dekomposition verarbeitet werden (vgl. auch Baayen et al. 1997).

Pollatsek et al. (2000) haben den Einfluss verschiedener Frequenzen auf die Verarbeitung mit Eye-Tracking-Experimenten im Finnischen untersucht. ${ }^{26}$ Für die Frequenz des N1 fanden sie einen Effekt auf der initialen Fixation, deren Dauer und die weiteren Fixationen. Erst folgende Fixationen zeigten einen Effekt für die N2-Frequenz und zeitgleich einen Effekt für die Ganzwort-Frequenz. Darüber hinaus beeinflusste die Ganzwort-Frequenz die Wahrscheinlichkeit einer dritten Fixation. Der gleichzeitige Einfluss von N2- und NN-Frequenz spricht für das Modell der dualen Route nach Libben, wonach gleichzeitig Konstituenten- und Ganzwortrepräsentation aktiviert werden. Darüber hinaus stellen sie fest, dass Komposita signifikant längere Gesamtfixationszeiten haben als gleichlange Simplizia, was gegen einen ausschließlichen Ganzwortzugriff spricht (vgl. Pollatsek et al. 2000:829).

Bei Rückschlüssen auf die Verarbeitung gilt es allerdings zu bedenken, dass es sich bei der Frequenz eines Ausdrucks nicht um eine mentale Größe handelt, sondern um eine statistische Kennzahl. Obwohl Frequenzen kein Teil der Grammatik sind, sind sie dennoch Teil der Erfahrung und können somit Einfluss auf Präferenzen haben.

Der Zusammenhang zwischen Transparenz, Kompositionalität, Lexikalisiertheit und Ganzwort-Frequenz ist in jedem Fall komplex (vgl. Schulte im Walde et al. 2016: 156).

In Libben et al. (2003) wurden vergleichbare Reaktionszeiten für OT- und für TT-Komposita im Gegensatz zu Komposita mit opakem Kopf gefunden. Die untersuchten TTKomposita verfügen über deutlich höhere Ganzwort-Frequenzen als die anderen drei Kom-

${ }^{26}$ Die Komposition im Finnischen ist mit der der germanischen Sprachen vergleichbar; Komposita verfügen hier ebenfalls über einen Initialakzent. 
posita-Gruppen. In diesem Fall handelt es sich somit nicht um einen Frequenzeffekt. Gleichzeitig ist es erstaunlich, dass die untersuchten volltransparenten Komposita insgesamt über höhere Frequenzen verfügen als die opaken. Sollte lediglich Verwendungshäufigkeit die Ursache oder zumindest Voraussetzung für Transparenz- und Kompositionalitätsabbau sein, wären höhere Frequenzen für opake Komposita zu erwarten. In einer Vorstudie zur Materialerstellung von Libben et al. (2003) hatten Annotator*inn*en die Aufgabe, Komposita hinsichtlich ihrer Transparenz zu bewerten. Es zeigte sich eine Tendenz dazu, frequente Komposita als voll transparent zu bewerten. Dies signalisiert, dass womöglich allein durch Vertrautheit mit dem Ausdruck der Eindruck von Transparenz entsteht.

Der Zusammenhang zwischen Frequenz, Transparenz und Lexikalisierung scheint geringer als zunächst angenommen. Lexikalisierte Ausdrücke können über einen niedrigen Grad an Transparenz und Frequenz verfügen (z.B. Apfelmännchen: DWDS-KK ${ }^{27} 2$ Treffer) oder auch über hohe Transparenz und hohe Frequenz verfügen (z.B. Haustür: DWDS-KK 1200 Treffer).

Mit Libben et al. (2003) nehme ich an, dass bei der Komposita-Verarbeitung in frühen Verarbeitungsschritten alle Teile aktiviert werden und erst in späteren Verarbeitungsschritten Unterschiede in der Verarbeitung verschiedener Komposita bestehen. Dabei gehe ich nicht davon aus, dass Komposita abhängig von ihren Eigenschaften schwerer oder leichter zu verarbeiten sind (vgl. Frisson et al. 2008), sondern lediglich unterschiedlich. Bspw. wäre es denkbar, dass bei transparenten Ad-hoc-Bildungen durch Kontextevaluierung zusätzlicher Verarbeitungsaufwand benötigt wird. Das gleiche Maß an zusätzlichem Verarbeitungsaufwand ist jedoch eventuell auch nötig, um bei opaken Komposita Teilbedeutungen der Konstituenten zu inhibieren. Außerdem muss zwischen Verstehen und Produktion unterschieden werden (vgl. Schreuder/Baayen 1995). Vor allem beim Erstellen von experimentellem Material gilt es, diese Faktoren und ihre möglichen Einflüsse in besonderem Maße zu berücksichtigen.

\subsubsection{Die Rolle des N1 bei der Komposita-Verarbeitung}

Nach der Betrachtung verschiedener Aspekte der Komposita-Verarbeitung soll nun zusammengetragen werden, welche Rolle dem N1 beigemessen wird und welche verarbeitungsrelevanten Eigenschaften ihm zugesprochen werden.

Das N1 muss einen entscheidenden Beitrag für die Ausdrucksbedeutung eines Kompositums leisten, sonst würde das N2 in der Form eines Simplex genügen (vgl. Gagné/Spalding 2007:160). Dass darin mehr als reine Modifikation liegt, wurde in den vorangegangenen Abschnitten bereits erläutert (vgl. auch Bücking 2010). Die Ergebnisse von Gagné (2009) und Libben et al. (2003) zeigen, dass das N1 zunächst auch morphologisch geparst wird. In späteren Verarbeitungsschritten gehen allerdings nicht zwingend alle morphologischen und morphosyntaktischen Eigenschaften des N1 verloren (vgl. Kap. 2.2.2). So zeigt Härtl (2015), dass ein verbales N1 u.U. seine Argumentstruktur behält, Clahsen et al. (2015) zeigen, dass das N1 Numerus-Eigenschaften tragen kann und Koester et al. (2004) zeigen einen Genus-Effekt für das N1.

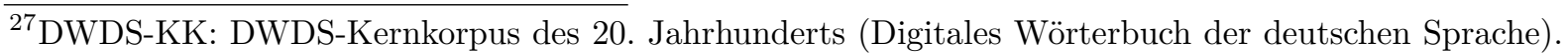
Berlin-Brandenburgische Akademie der Wissenschaften. http://www.dwds.de/r/ (23.10.2014). 
Eine Eigenschaft, die die Verarbeitung des N1 gegenüber dem N2 eventuell sogar erleichtert, ist seine lineare Voranstellung (position-in-the-string-effect). Dem gegenüber steht die Funktion des N2 als Kopf. Die Relevanz der N1-Position belegt Jarema (vgl. 2007:56) durch ein Priming-Experiment, bei dem sich ein größerer Priming-Effekt für das N1 bei rechtsköpfigen Komposita als für das N2 bei linksköpfigen Komposita zeigt.

To summarize, the significance of the first constituent in the left-to-right processing of compounds has been evidenced in typologically distinct languages (Finnish, German, Greek and Polish) and in both neurologically unimpaired and impaired populations. The variable first-position-in-the-string thus clearly contributes to the complexity of phenomena involved in compound recognition and production. (Jarema 2007:55)

Weitere Anhaltspunkte für die Art des Einflusses des N1 auf die Verarbeitung eines Kompositums liefern Pollatsek et al. (2000). In ihrem Eye-Tracking-Experiment zeigt sich ein Einfluss der N1-Frequenz auf die Dauer der ersten Fixation und der Gesamtverweildauer auf dem Kompositum. Die N2-Frequenz zeitigt erst bei späteren Kenngrößen einen Effekt (vgl. Pollatsek et al. 2000:820ff).

Darüber hinaus wird für N1 und N2 ein Unterschied in der Salienz innerhalb eines Kompositums angenommen. Libben et al. (2003) zeigen in einem Memory-Test für die Konstituenten, dass das N1 besser erinnert wird als das N2 und interpretieren dies als Indiz für eine höhere Salienz des N1. Gleichzeitig wurden OO-Komposita besser erinnert als TT-Komposita, was zusätzlich für einen Einfluss der Transparenz auf die Salienz spricht. In die gleiche Richtung argumentieren Libben et al. (2003:57) bei der Auswertung von Wiederholungseffekten: Die Reaktionszeiten bei TT- und OT-Komposita scheinen weniger durch Wiederholung beeinflusst zu werden als bei TO- und OO-Komposita. Dies spricht insgesamt für eine höhere Erstaktivierung beim Lesen der TO-/OO-Komposita. Dabei handelt es sich jedoch um Ergebnisse für das Gesamtkompositum. Inwiefern sich die Transparenz des N1 auf dessen Salienz auswirkt, bleibt unklar.

Insgesamt ist es plausibel, anzunehmen, dass ein hoher Grad an N1-Transparenz und Kompositionalität die N1-Zugänglichkeit fördert, da in dem Fall keine Bedeutungsteile des N1 unterdrückt werden und das N1 auch über frühe Verarbeitungsschritte hinaus wie das entsprechende Simplex verarbeitet wird. In Bezug auf die Frequenz ist denkbar, dass eine hohe NN-Frequenz bei gleichzeitig niedriger N1-Frequenz den N1-Bezug erschwert, da hier die Aktivierung des NN die Aktivierung des N1 überlagert. Andererseits ist eine hohe N1-Frequenz vermutlich förderlich, da dieses dadurch über einen höheren Grad an Aktiviertheit verfügt als das N2 und/oder das Gesamtkompositum (vgl. Kap. 2.3.1).

Auch ein Einfluss der semantischen Relation ist nicht auszuschließen. Bei der Einteilung der Relationen handelt sich zwar nicht um ein Verarbeitungsmodell, dennoch lassen sich anhand der Benennung von Relationen Aussagen über die bei der KompositumsInterpretation involvierten Konzepte treffen. Ebenso ist ein Einfluss der Häufigkeitsverteilung der Relationen für ein N1 denkbar, wie es das CARIN-Modell voraussagt.

Die berichteten Ergebnisse legen nahe, dass N1 und N2 nicht gleichrangig verarbeitet werden, das N1 jedoch nicht prinzipiell in allen Belangen dem N2 gegenüber benachteiligt ist. Vielmehr scheinen die beiden Konstituenten verschiedene Rollen inne zu haben und sich in der Interpretation gegenseitig zu beeinflussen. 


\subsection{Anaphorische Pronomen als Zugänglichkeitsmarker}

Der Grad an Zugänglichkeit eines linguistischen Ausdrucks ist keine Eigenschaft, die sich unmittelbar am Ausdruck ablesen lässt, sondern die erst über den Umweg der Akzeptabilität anaphorischer Bezüge bestimmt werden muss. In dieser Arbeit werden pronominale Diskursanaphern nutzbar gemacht, um den Grad an N1-Zugänglichkeit für ein Kompositum zu bestimmen. Aus diesem Grund gilt es das Phänomen des N1-Bezugs mit den zentralen Annahmen aus der Anaphorik in Verbindung zu bringen.

\subsubsection{Pronominale Diskursanaphern}

Mit einem sprachlichen Ausdruck referieren Sprachnutzer*innen auf ein Denotat in der außersprachlichen Welt. Für Pronomen ist die Identifizierung des Denotats allerdings nicht unmittelbar möglich. Pronomen verfügen nur über sehr geringen semantischen Gehalt und tragen, weil sie zu den Definita gehören, eine Existenz- und Unikalitätspräsupposition (vgl. Roberts 2002:1f). Darüber hinaus gelten klassischerweise die folgenden Annahmen:

\section{Presuppositions of Pronouns:}

Given a context $\mathrm{C}$, use of a pronoun $\mathrm{Pro}_{i}$ presupposes that it has as antecedent a discourse referent $x_{i}$ which is:

a) weakly familiar in $\mathrm{C}$,

b) salient in $\mathrm{C}$, and

c) unique in being the most salient discourse referent in $\mathrm{C}$ which is contextually entailed to satisfy the descriptive content suggested by the person, number and gender of $\operatorname{Pro}_{i}$. (Roberts 2005 14)

Ihr Defizit an semantischem Gehalt macht Pronomen zu relativ referierenden Ausdrücken. Für die Referenzfestlegung eines Pronomens, bzw. jeder Art von Anapher, ist somit ein Ausdruck als Antezedens nötig, auf den sich das Pronomen bezieht und mit dem ein*e Sprachnutzer*in auf ein Denotat in der Welt referiert (vgl. Pause 1991:548). Die Möglichkeit, sich mit einem anaphorischen Pronomen auf ein Antezedens zu beziehen, gibt wiederum Auskunft über die Zugänglichkeit des Antezedens (vgl. Kap. 2.3.1).

Besteht zwischen Antezedens und Anapher Referenzidentität, wird dies als Koreferenz bezeichnet (vgl. Pause 1991:549). Allerdings handelt es sich bei der Koreferenz nicht um ein notwendiges Kriterium für anaphorische Bezugnahme (vgl. Holler-Feldhaus 2004). So besteht z.B. im Fall der Identität von Eigenschaften keine Koreferenz (sloppy identity) (vgl. Schwarz 2000:55) und im Fall von indefiniten Antezedenten wie in (64) wird keine bestimmte Referenz festgelegt (vgl. ,donkey pronouns', z.B. bei Pause (vgl. 1991:549)).

(64) Wenn jemand ${ }_{1}$ einen $\underline{E s e l}_{2}$ besitzt, dann schlägt $\underline{\mathrm{er}}_{1} \underline{\mathrm{ihn}}_{2}$.

Während das Antezedens das Denotat festlegt, wird mit der pronominalen Anapher über den Bezug zum Antezedens relativ referiert. Dabei stellt sich die Frage, wie diese relative Referenzfestlegung erfolgt.

Traditionell werden Anaphern in der Generativen Grammatik ausschließlich satzorientiert behandelt. Die Anapher wird dabei als gebundene Variable interpretiert und der anaphorische Bezug über syntaktische Hierarchieverhältnisse festgelegt. In der Variante des Government and Binding geschieht dies über die Bindungsprinzipien nach Chomsky (1981) (vgl. dazu auch Reinhart (1991)). In vielen als unakzeptabel oder sogar als ungrammatisch 
bewerteten Fällen von anaphorischen N1-Bezügen wird nun Bindungsprinzip B verletzt. In Fällen wie (65) oder (66) wird das Pronomen innerhalb seiner Domäne gebunden und der Satz ist damit automatisch ungrammatisch. Die Verletzung eines Bindungsprinzips sagt allerdings nichts über die Zugänglichkeit einer Kompositums-Konstituente aus.

$$
\begin{aligned}
& { }^{*} \underline{\text { Divorce }}_{i} \text { lawyers often experience } \underline{\mathrm{it}}_{i} \text { themselves. (Levi 1978:72) } \\
& { }^{*} \underline{\text { Truck }}_{i} \text {-drivers fill them } \\
& i
\end{aligned}
$$

Anders verhält es sich, wenn keine syntaktischen Beschränkungen bei der anaphorischen Bezugnahme involviert sind. Um ohne syntaktisches Instrumentarium Beziehungen zwischen syntaktischen Einheiten beschreiben zu können, wurde die Ebene der Diskursrepräsentation eingeführt. Dabei handelt es sich um eine Zwischenebene zwischen sprachlichen Ausdrücken und Entitäten in der Welt, auf der Diskursreferenten etabliert werden (vgl. Karttunen 1976, Roberts 2002). Mithilfe dieses Modells können anaphorische Bezugnahmen unabhängig von Satzgrenzen betrachtet werden.

Bei einem Diskursreferenten handelt es sich um einen Redegegenstand in der Textwelt, auf den mit einer Anapher Bezug genommen werden kann. Referenzielle Terme, aber auch Quantorenphrasen und andere Arten von NPn können einen Diskursreferenten etablieren. Die Anapher muss lediglich in der ,Lebensspanne‘ des Diskursreferenten liegen, den das Antezedens bezeichnet (vgl. Pause 1991:550f). In der DRT wird die Lebensspanne von Diskursreferenten über Bedingungen der Zugänglichkeit zwischen Teilrepräsentationen eingeschränkt (vgl. Kamp/Reyle 1993). Syntaktische Strukturen werden somit nicht direkt gedeutet, sondern über die Zwischenebene der Diskursrepräsentation. Die für diese Arbeit entscheidende Frage ist somit, ob ein N1 einen Diskursreferenten etabliert (vgl. Kap. 2.2.4.

In den folgenden Untersuchungen werden ausschließlich Anaphern in Betracht gezogen, die über die Satzgrenze hinaus auf der Diskursebene wirken ${ }^{28}$ Die Auswirkungen dieses Unterschieds werden in (67-a) deutlich.29

$$
\begin{aligned}
& \text { a. } \quad \text { ?ruck }_{i} \text {-drivers often care for quality. That's why most of them fill them } \underline{\text { up }}_{i} \\
& \text { with Arco Diesel. }
\end{aligned}
$$

Die sich anschließende Frage ist, wie eine Anapher den entsprechenden Diskursreferenten als Antezedens identifiziert. Hierfür ist ein Such- und Vergleichsprozess notwendig (vgl. Schwarz 2000). Die Anapher verfügt über Genus- und Numerus-Merkmale, die mit den Eigenschaften der etablierten Diskursreferenten abgeglichen werden. Diese Merkmale allein genügen jedoch in vielen Fällen nicht. Mitunter kommen mehrere Diskursreferenten infrage; der Bezug ist in dem Fall ambig. In besonderen Fällen können entsprechende Merkmale auch missachtet werden (vgl. Pause 1991:549). Hierin liegt ein wichtiges Argument für die Akzeptabilität von anaphorischen N1-Bezügen.

\footnotetext{
${ }^{28}$ Von Seiten der psycholinguistischen Forschungstradition teilen auch Ward et al. (1991) und McKoon et al. (1993) die hier vertretene Auffassung von Anaphorik als Relation zwischen Sprache und Diskursentitäten.

${ }^{29}$ Erschwerend für die Zuordnung von Anapher und Antezedens wirkt hier, dass das Kompositum an sich ebenfalls im Plural steht und somit zumindest morphosyntaktisch als direktes Antezedens zur Verfügung steht.
} 


\subsubsection{Direkte vs. indirekte Anaphern}

Im Zusammenhang mit den Graden an Zugänglichkeit in Kapitel 2.3.1 wurden bereits verschiedene Arten von Anaphern erwähnt. Klassischerweise wird in der Anaphorik zwischen direkten, indirekten und Komplexanaphern unterschieden. Bei der direkten Anapher handelt es sich um die typische Form von Koreferenz zwischen Antezedens und Anapher 30 , bei der indirekten Anapher handelt es sich um eine interpretative Abhängigkeit zwischen Anker und Anapher und bei der Komplexanapher werden Sätze, Satzteile oder Propositionen als Antezedens verwendet (vgl. Schwarz 2000:129). Vor allem der Typ der indirekten Anapher ${ }^{31}$ spielt im Kontext dieser Arbeit eine entscheidende Rolle.

\section{Abgrenzung zwischen den Anapherarten}

Sowohl direkte als auch indirekte Anaphern wirken kohärenzstiftend und sind von vorangehenden Textstrukturen abhängig, jedoch unterscheiden sie sich in ihrer Art der Anbindung und der Referenzialisierung (vgl. u.a. Schwarz 2000).

Bei den indirekten Anaphern handelt es sich um definite Ausdrücke, die in interpretativer Abhängigkeit zu bestimmten Ausdrücken der vorausgehenden Textstruktur stehen und zwei textreferenzielle Funktionen haben: die Einführung neuer (bisher noch nicht erwähnter) Textreferenten und die Weiterführung des globalen referenziellen Bezugs. (Schwarz 2000 49)

Während direkte Anaphern sich durch Anwesenheit eines expliziten Antezedens auszeichnen, ist das Antezedens der indirekten Anapher ausschließlich implizit erwähnt und muss zunächst mithilfe eines „Anker-Ausdrucks“ (Schwarz 2000:50,75) erschlossen werden. Mit einer indirekten Anapher wird demnach nicht auf einen bereits etablierten Diskursreferenten referiert, sondern mit Hilfe eines Ankers im Text wird ein neuer Diskursreferent in der Textwelt etabliert. ${ }^{32}$ Indirekte Anapher und Anker sind über eine assoziative Relation miteinander verbunden. Der Referent der Anapher ist nur über zusätzliches Wissen (z.B. Weltwissen) zugänglich, welches durch den Anker-Ausdruck aktiviert wird.

Ein typisches Beispiel für eine direkte Anapher ist (68-a). Hierbei sind das Antezedens ein Auto und die NP-Anapher der Wagen koreferenziell. Bei der indirekten Anapher in (68-b) sind ein Auto und die Scheiben nicht koreferenziell, sondern der Ausdruck ein Auto dient als Anker für die Etablierung einer Teil-Ganzes-Relation, die die definite Verwendung der NP die Scheiben als indirekte Anapher ermöglicht.

a. Vor dem Haus parkt ein Auto. Der Wagen ist ganz schmutzig.

b. Vor dem Haus parkt ein Auto. Die Scheiben sind ganz schmutzig.

\footnotetext{
${ }^{30}$ Die oben angesprochenen Sonderfälle ohne Koreferenz seien hier außer Acht gelassen.

${ }^{31}$ Die Terminologie ist hierbei in der Literatur nicht einheitlich. Neben der Bezeichnung ,indirekte Anapher' finden sich auch die Bezeichnungen ,assoziative Anapher', ,Kontiguitätsanapher', ,inferrables u.a. Dabei wird in den meisten Fällen das gleiche Phänomen bezeichnet, lediglich die Forschungsperspektive unterschiedet sich mitunter (vgl. Schwarz 2000 48).

${ }^{32}$ Hierbei ist eine Abgrenzung zur Deixis vorzunehmen, mit der nicht nur außerhalb des Texts, sondern sogar außersprachlich referiert wird.
} 


\section{Anaphorische Pronomen}

Ein umstrittener Unterschied zwischen direkten und indirekten Anaphern besteht in der Möglichkeit, durch ein Pronomen realisierbar zu sein. Im Fall von direkten Anaphern gelten Pronomen als frequente und produktive Ausdrucksweise, da diese durch Unterspezifikation Bekanntheit signalisieren und somit die kognitive Verarbeitung erleichtern (vgl. Givenness Hierarchy in Kap. 2.3.1). In Bezug auf die indirekte Anaphorik wird die Möglichkeit des pronominalen Ausdrucks kontrovers behandelt. Während bei direkten Anaphern eine große Bandbreite an möglichen Ausdrucksklassen angenommen wird, ist die Standardform der indirekten Anapher eine definite NP. Auch im Fall direkter Anaphorik sind Pronomen durch ihren geringen semantischen Gehalt referenziell unterspezifiziert, im Fall von indirekten Anaphern liegt nun doppelte Unterspezifikation vor. Aus diesem Grund nehmen einige Autor*inn*en an, dass indirekte Anaphern per se nicht als Pronomen realisierbar sind, z.B. Erkü/Gundel (1987), Sanford et al. (1983), Schwarz (2000). Während bei der direkten Anapher in (69-a) die Verwendung des Pronomens es als Anapher unproblematisch ist, ist dies im Fall der indirekten Anapher in (69-b) nicht möglich, da die Anapher sie zu unspezifisch ist, um eine eindeutige Referenzzuordnung zu ermöglichen. Da das Pronomen nur Angaben zu Genus und Numerus beinhaltet und keinerlei Hinweise zur konkreten Relation, kann kein Bezug zu den Scheiben des Autos hergestellt werden.

a. Vor dem Haus parkt ein Auto. Es ist ganz schmutzig.

b. Vor dem Haus parkt ein Auto. ${ }^{*}$ Sie sind ganz schmutzig.

Es lassen sich allerdings auch relativierende Meinungen finden:

[...] pronouns do not in general require explicit antecedent expressions; and if they have antecedents, they need not be coreferential with them, but the relation may be arbitrarily more complicated. (Bosch et al. 2007.47)

In diesem Sinne formuliert auch Consten (vgl. 2004:84f), dass für die Verwendung von Pronomen als indirekte Anaphern Beschränkungen bestünden, die jedoch vage und schwer fassbar seien. Cornish et al. (2005) zeigen für das Englische und das Französische, dass die Wiederaufnahme eines impliziten Referenten durch (Nicht-Subjekt-)Pronomen möglich ist, wenn der Referent ,nuklear' ist. Diese Eigenschaft ist als konzeptuelle Zentralität und somit als nicht-sprachliche Eigenschaft aufzufassen. Dabei wird eine Skala beschrieben von ,nuklear' (zentrale Bestandteile des Diskursreferenten, z.B. Auto für parken) bis ,peripher (Instrumente oder Begleiterscheinungen, z.B. Rasierer für rasieren) 33

\section{Grade der Indirektheit}

Innerhalb der Klasse der indirekten Anaphern wird von verschiedenen Graden an Indirektheit ausgegangen (vgl. Consten 2004, Schwarz 2000). Dabei unterscheidet Schwarz (2000:118) nach Art des Wissens, das für die Anaphernresolution genutzt werden muss, drei Typen von indirekten Anaphern. Den geringsten Grad an Indirektheit misst sie den

\footnotetext{
${ }^{33}$ Weitere Ausführungen zum Einfluss konzeptueller Eigenschaften erfolgen in Kapitel 3 Zu den Möglichkeiten für pronominale indirekte Anaphern siehe auch die Auswertung der Kontroll-Items in Experiment 2 (Kap. 5 ).
} 
semantischen Typen bei, bei denen der Bezug lexikonbasiert hergestellt wird (vgl. Schwarz 2000:104). Ein Beispiel für eine indirekte Anapher auf Basis einer solchen semantischen Relation ist (70), Hier besteht zwischen dem Anker Wagen und der Anapher Lenkrad eine Teil-Ganzes-Relation.

$$
\text { Der Wagen ist hin. Das Lenkrad ist ja ganz verbogen. (Schwarz 2000:106) }
$$

Einen etwas höheren Grad an Indirektheit haben konzeptuelle Typen, die auf Weltwissen basieren. Dazu gehören u.a. schema-basierte Typen wie in (71). Hierbei dient das Restaurant-Skript als Interpretationsrahmen (vgl. Schwarz 2000:111).

Ein Kannibale sitzt im Restaurant eines Luxusdampfers. Der Kellner kommt und fragt, ob er die Speisekarte bringen soll. Sagt der Kannibale: „Nein, bringen sie mir bitte die Passagierliste." (Schwarz 2000:112)

Der höchste Grad an Indirektheit liegt bei den inferenzbasierten Typen vor. So ist für die Interpretation von (72) die Inferenz nötig, dass Briefe von Briefträgern übermittelt werden und wenn der Sprecher täglich einen Brief an seine Freundin geschrieben hat, diese täglich dem Briefträger begegnet ist, wodurch sich die beiden offensichtlich kennen und lieben lernten.

„Warum bist du so betrübt?“ „Jetzt habe ich meiner Freundin jeden Tag einen Eilbrief geschrieben, und nun heiratet sie den Briefträger!" (Schwarz 2000:115)

Natürlich ist die Einteilung in diese drei Typen nicht strikt, sondern mit fließenden Übergängen. Ebenso herrscht ein fließender Übergang zwischen direkter und indirekter Anapher, der durch eine Vielzahl an Grenzfällen markiert wird. Als einen solchen Mischtyp führt Schwarz (73) an. Hier kann der Ausdruck Auto als Antezedens betrachtet werden, welches durch die Anapher das zusammengepresste Eisenpaket wiederaufgenommen wird. Allerdings liegt das Denotat im Moment der Wiederaufnahme in einem stark veränderten Zustand vor, nämlich nicht mehr als Auto, sondern als Eisenpaket, und verfügt somit über neue Merkmale. Diese Merkmale können mithilfe des Ankers Schrottplatz inferiert werden. In diesem Sinne vereint es Eigenschaften einer direkten und einer indirekten Anapher. Insgesamt ist also von einem anaphorischen Kontinuum auszugehen.

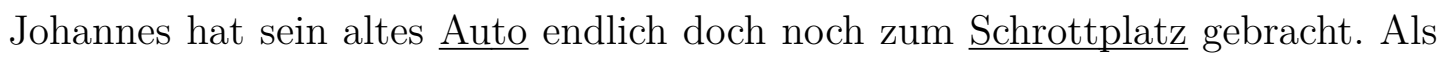
er dann aber das zusammengepresste Eisenpaket sah, wurde ihm weh um's Herz. (Schwarz 2000:148)

\section{Einordnung des N1-Bezugs}

An die Unterscheidung zwischen direkter und indirekter Anapher schließt sich die Frage nach der Verortung des N1-Bezugs an, die im Zusammenhang mit dem Begriff des Bridgings bereits angerissen wurde. Coulmas (1988) kategorisiert alle Bezüge auf semantisch Miterwähntes als ,indirekt‘. Hierzu zählt er das implizite Erwähnen der verlorenen Eltern im Ausdruck Waise ebenso wie das explizite Erwähnen von Obst im Kompositum Obstkuchen. Schwarz (2000:48) hingegen definiert indirekte Anaphern als Bezüge 
auf Entitäten außerhalb des geschriebenen Texts. Da das N1 Teil des geschriebenen Texts ist, spräche dies für eine Klassifizierung als direkte Anapher. Andererseits schreibt sie Komposita ebenfalls anaphorischen Inselstatus zu (vgl. Schwarz 2000:124f).

Ein grundlegendes Kriterium für indirekte Anaphern ist nach Schwarz (2000:49), dass ein neuer Diskursreferent aus der Anker-Domäne eingeführt wird. Hierfür müsse eine assoziative Relation zwischen Anker und Anapher etabliert werden. Da das N1 als avisierter Diskursreferent jedoch an der Oberfläche sichtbar ist, muss keine Relation assoziiert werden. Stattdessen muss das N1 extrahiert werden. Zudem herrscht die Annahme, dass Pronomen i.d.R. keinen neuen Diskursreferenten etablieren können, sondern sich lediglich auf einen solchen beziehen. Pronomem verfügen über einen so geringen semantischen Gehalt, dass die Assoziation einer semantischen Relation (z.B. Teil-Ganzes) nicht möglich ist. In diesem Sinne spricht einiges für eine Einordnung des N1-Bezugs als direkte Anapher. Plausibilität spielt nur dann eine Rolle, wenn der Satzkontext in den Resolutionsprozess einbezogen wird. Sollte dies notwendig sein, würde dies bedeuten, dass die Anapher erst im Laufe der Satzverarbeitung aufgelöst wird. Hierbei handelt es sich um eine Frage, die nur durch Experimente zur Online-Verarbeitung zu klären ist.

Sollte es sich beim N1-Bezug um indirekte Anaphern handeln, ist von einem geringen Grad an Indirektheit auszugehen. Ein hoher Grad an Indirektheit zeichnet sich durch das Stattfinden von Inferenzziehung aus. Im Fall eines N1-Bezugs sollte dies nicht nötig sein, da keine Informationen aus dem Weltwissen benötigt werden, sondern die Anapher auf Basis des Textwissens etabliert wird.

Härtl (2013) nimmt für Referenzetablierung wie in (74) Bridging an. Ob er den Diskursreferenten in diesem Fall jedoch als implizit oder explizit eingeführt erachtet, lässt er offen. Allerdings spricht er in vergleichbaren Fällen mit Erstglied-Bezug von „pragmatisch erzwungene[r] Rekonstruktion"(Härtl 2015:167).

(74) Ich habe auch Interesse an einem Tanzkurs, denn das möchte ich nun endlich mal lernen. (Härtl 2015:164)

Ebenfalls denkbar ist eine Interpretation des N1-Bezugs als Reanalyse-Prozess im Sinne von Semantic Coercion: Nach erfolglosem Abgleich der grammatischen Merkmale des Pronomens mit denen der vorerwähnten NPn setzt ein Re-Interpretationsprozess ein, für welchen das Pronomen als Trigger dient. Der Bezug auf das N1 ist nicht der präferierte Prozess, wird aber der aufwändigeren Etablierung eines neuen Diskursreferenten vorgezogen. In diesem Fall handelt es sich nicht um Bridging, da kein Weltwissen für die Referenzetablierung notwendig ist, sondern der Kotext weiter ausgewertet wird.

Ein weiteres Erklärungsmodell liefert der Good Enough Approach (vgl. Kap. 2.3.1).

Sollte sich pronominaler N1-Bezug unter bestimmten Bedingungen als systematisch akzeptabel erweisen, bedeutet dies zum einen, dass es sich entweder um direkte Anaphern handelt oder indirekte Anaphern doch pronominal realisiert werden können. Handelt es sich um eine Form der direkten Anapher, bleibt die Frage, warum die Mehrzahl der Fälle unakzeptabel ist.

Zum anderen können ggf. Verarbeitungsstudien Aufschluss über die Art der etablierten Anapher Aufschluss geben. Sollte es sich um eine Form der indirekten Anapher handeln, ist mit höheren Verarbeitungskosten aufgrund zusätzlicher Verarbeitungsschritte zu rechnen als im Fall von klassischen direkten Anaphern. Darüber hinaus ist vor allem der Zeitpunkt der Anaphernauflösung aussagekräftig: Geschieht dies erst zum Ende des 
Satzes, wäre dies ein Indiz dafür, dass die grammatischen Informationen des Pronomens nicht für eine Anaphernresolution ausreichen und weitere Wissensressourcen wie Kontextinformationen genutzt werden müssen. In dem Fall wäre die Annahme der Etablierung einer indirekten Anapher plausibel. Sollten jedoch Effekte auf der Anapher sichtbar werden, spräche dies für einen direkten Bezug durch morphosyntaktische Kongruenz mit dem N1.

Für die Bearbeitung dieser Fragestellungen soll folgende Terminologie gelten: Das NNKompositum, welches das N1 enthält, auf welches sich die Anapher bezieht, wird in Anlehnung an die Terminologie von Schwarz $(2000)$ als Anker, bzw. Anker-Ausdruck bezeichnet. Das N1 hingegen wird als Antezedens bezeichnet, da es sich hierbei um den avisierten Diskursreferenten handelt.

\subsubsection{Prozess der Anaphernresolution}

Wie in Kapitel 2.4.1 bereits angerissen, wird die Interpretation von Anaphern zumeist als Such- und Vergleichsprozess beschrieben (vgl. Schwarz 2000). Für direkte Anaphern bedeutet dies, dass nach der Wahrnehmung der Anapher ein Set möglicher Antezedenten ausgewählt und anschließend nach verschiedenen Kriterien mit der Anapher abgeglichen wird. Das ausschlaggebende Kriterium ist die Plausibilität. Die Interpretation indirekter Anaphern beschreibt Schwarz ähnlich (vgl. Schwarz 2000:50). Der Suchprozess besteht in dem Fall aus der Identifikation möglicher potenzieller Ankerkandidaten. Ist der Anker gefunden, kann eine kohärenzstiftende Relation zu einem Referenten in der kognitiven Domäne des Ankers etabliert werden. Dieser zusätzliche konstruktive Prozess der Relationsetablierung kann unterschiedlich gestaltet sein. Im einfachsten Fall wird eine Rolle in der kognitiven Domäne des Ankers gefunden, z.B. eine Teil-Ganzes-Relation wie in (68-b). Der kognitiv aufwändigste Fall ist die Inferenzziehung, bei der der Referent mit Hilfe von gegebenen Inhalten (Anker) und ergänzbaren Inhalten (Weltwissen) erschlossen, bzw. konstruiert wird (vgl. Schwarz 2000:140ff). Inferenz liegt demnach nicht in jedem Fall vor (vgl. Consten (2004:69f) und Schwarz (2000:36)). In der Frage nach dem Auftreten von Inferenzziehung spiegelt sich erneut die Gradienz der Indirektheit.

In der psycholinguistischen Literatur ist oft von ,Bridging' als einer speziellen Art der Inferenzziehung die Rede, bei der ein bestimmter Grad an Bezug zwischen neuer und bekannter Entität hergestellt wird (vgl. Burkhardt 2006). Als Voraussetzung für Bridging gibt Härtl 2015:165f) an, dass ein Referent direkt oder indirekt durch präsente Informationen in den Diskurs eingeführt worden sein muss. In (75-a) wird kein Referent eingeführt, in (75-b) ist er zwar semantisch vage, aber textuell eingeführt. In (75-c) wird ein indirekter Referent eingeführt, da Zigarette Teil des Lexikoneintrags von rauchen ist.
a. Mia hat den Fellkragen angenäht.
* Das war sicher ein grauer Strickmantel.
b. Mia hat den Fellkragen an etwas angenäht.

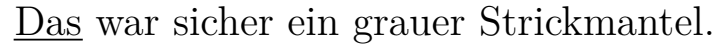
c. Mia hat heimlich geraucht.
Das war aber sicher nur eine Kräuterzigarette.

An dieser Stelle stellt sich die Frage, ob ein N1 ebenfalls als Teil des Lexikoneintrags eines Kompositums zu verstehen ist und somit ein N1-Bezug via Bridging vollzogen wird. Pro- 
blematisch wird diese Interpretation bei Ad-hoc-Bildungen: Besteht kein Lexikoneintrag für das Kompositum, kann das N1 auch nicht Teil dessen sein. Dennoch sind Bezüge wie in (76) (relativ) akzeptabel.

(76) Dort links ist das Zigaretten-Beet. Meisten werfen die Leute $\underline{\text { sie }}$ da einfach rein, bevor sie das Gebäude betreten.

Eine wichtige Annahme für den Vergleich von direkter und indirekter Anapher ist, dass es sich bei der Suche nach einem expliziten Referenten um die primäre Prozedur handelt, d.h. dass die direkte Anapher stets die präferierte Form ist.

Nur wenn der semantische Kernbestandteil der kognitiven Domäne des Ankers nicht die nötigen Informationen bereitstellt, wird der konzeptuelle Skopus aktiviert bzw. durch Inferenzziehung erweitert. (Schwarz 2000 99)

Aus diesem Grund müsste die Etablierung einer indirekten Anapher kognitiv aufwändiger sein (vgl. Sanford et al. 1983). Inferenzbasierte Anaphern müssten insgesamt die höchsten Verarbeitungskosten haben (vgl. Schwarz 2000:121f,157).

Wie leicht eine Anapher tatsächlich verarbeitet werden kann, hängt von einer Vielzahl von Faktoren ab. Schwarz (vgl. 2000:140f) nennt hier Distanz, Aufmerksamkeitsspanne, Art/Ort der Einführung des Antzedens/Ankers, und die Zahl möglicher Antezedenten/Anker. Pause (vgl. 1991:553) nennt darüber hinaus morphologische, syntaktische, semantische, textstrukturelle und pragmatische Beschränkungen.

Eine für die anaphorische Zugänglichkeit wichtige und in der psycholinguistischen Literatur viel diskutierte Eigenschaft ist die Salienz eines Ausdrucks, d.h. seine Sichtbarkeit in der mentalen Repräsentation. Der Einfluss der Salienz auf anaphorische Prozesse ist relativ gesichert; wodurch diese gesteuert wird, ist hingegen nur schwer einzugrenzen.

Als Faktoren, die die Salienz eines Ausdrucks beeinflussen, werden u.a. textstrukturelle Faktoren wie die syntagmatische Distanz genannt. So wird von einem Vorteil für letztgenannte Antezedenten ausgegangen (Recency Effect, vgl. Pause 1991:552). Im Fall von Diskursanaphern und besonders im Fall von N1-Bezügen kann Distanz die Zugänglichkeit allerdings erhöhen. Wie in (77) zu sehen, kann der Einfluss der Oberflächendistanz so weit gehen, dass sich damit sogar Postals berühmtes Beispiel für Unzugänglichkeit von Bedeutungbestandteilen ,reparieren' lässt.

(77) „I am an orphan." „What, that's terrible, Johnny, when did it happen? You never told me you were an orphan." Fitz was deeply concerned. „I am getting sort of used to it. They died when I was three." (Ward et al. 1991:467)

Eine andere Art von Salienzfaktoren sind die der informationsstrukturellen Fokussierung. Eine Möglichkeit der Salienzerhöhung durch Fokussierung bieten Spaltsätze, wie z.B. in $(78)$

(78) Nicht im Schweine-, sondern im Hühnerstall hat der kleine Karl sich versteckt. Dabei hat er sie alle aufgescheucht und wild gemacht. 
Darüber hinaus spielen auch in Bezug auf die Salienz syntaktische Faktoren eine Rolle, z.B. die grammatische Funktion von Antezedens und Anapher. Tendenziell besteht eine Präferenz für ein Antezedens in Subjektfunktion (Subjecthood Account) und in gleicher syntaktischer Funktion wie die Anapher (Parallelism Account, vgl. auch Smyth/Scholey 1994, Stevenson et al. 1994).

Die genannten Salienzfaktoren können einzeln wirken oder in einem gewichteten Zusammenspiel. Als oberstes Prinzip nehmen Pause (1991) und Schwarz (2000) die Plausibilität der anaphorischen Beziehung in Welt- und Kontextwissen an. In jedem Fall ist davon auszugehen, dass sie auch im Fall des N1 eine Rolle spielen und bei der Beschreibung von Zugänglichkeit in Betracht gezogen werden müssen.

\subsection{Verwandte Phänomene}

Neben der Einschränkung von N1-Bezügen existieren auch andere Formen von anaphorischen Bezügen, die aufgrund von grammatischen Beschränkungen ausgeschlossen werden, z.T. aber akzeptabel sind. Einige dieser Phänomene werden im Folgenden kurz umrissen. Ziel ist es, dabei Gemeinsamkeiten und Unterschiede zu den N1-Bezügen zu identifizieren und somit Näheres über die involvierten Mechanismen zu erfahren.

\subsubsection{Phrasenkomposita}

Meibauer (2003) bezeichnet Phrasenkomposita sie als einen okkasionellen, expressiven, schriftsprachlichen und peripheren Wortbildungstyp. Als Beispiele führt er die Fälle unter (79) an.

Abgerechnet-wird-am-Schluss-Taktik, Messer-und-Gabel-Handhabung (Meibauer 2003:153)

Strukturell handele es sich um Determinativkomposita der Form YP-X. Da sich in diesem Fall eine Phrase innerhalb eines $\mathrm{X}^{o}$ befindet, werden Phrasenkomposita oft als Argument für eine syntaktische Theorie der Wortbildung angeführt (vgl. Meibauer 2003:154f).

Auch für Phrasenkomposita gelte wie für reguläre NN-Komposita, dass es sich bei der ersten Konstituente nicht um eine DP handeln dürfe, vgl. (80). Eine mögliche Begründung hierfür sieht Meibauer in der Vermeidung von Referenzfestlegung. Die erste Konstituente eines Kompositums dürfe nicht referenziell sein, was sie durch einen Determinierer jedoch wäre. Ausnahmen wie (81) deklariert er als Ausnahme durch Lexikalisierung (vgl. Meibauer 2003:168).

$$
\begin{aligned}
& \text { *ein * die-graue-Schläfe-Effekt } \\
& \text { die Der-schöne-Rheingau-Laberei }
\end{aligned}
$$

Der Unterschied zwischen (80) und (81) scheint mir jedoch auf Denotatebene zu liegen: Das Erstglied die graue Schläfe wird in (80) unspezifisch verwendet, d.h. mit ihm wird nicht auf ein konkretes Denotat referiert. Der schöne Rheingau in (81) ist durch seine 
Eigenschaft als regionale Bezeichnung ein Eigenname, d.h. mit ihm wird direkt auf ein unikales Denotat referiert. Die Möglichkeit der Referenzfestlegung ist in diesem Fall somit keine allgemeine Eigenschaft der Konstituente, sondern resultiert aus Eigenschaften des Ausdrucks und seiner Verwendung. Meibauer (2003) sieht in der Nicht-Referenzialität eine allgemeine Eigenschaft der ersten Konstituente, weshalb auch (82-a) ungrammatisch sei (Meibauer 2003:169). Dem sei entgegengesetzt, dass Holz hier ebenfalls unspezifisch als Material verwendet wird und auch in einer phrasalen Verwendung artikellos auftritt (vgl. $(82-b)$.
a. *die $[$ das Holz + hütte]
b. Die Hütte ist aus (*das) Holz.

Ebenso könne ein Phrasenkompositum wie in (83) kein Genitivattribut oder Adjunkt bei sich tragen (vgl. Meibauer 2003:156). Dieses Beispiel erscheint mir jedoch vorrangig aufgrund seiner semantischen Ambiguität unakzeptabel: Entweder es handelt sich bei grauen Schläfen um einen allgemeinen Effekt oder um ein äußeres Merkmal von Peter. Für das Kompositum gibt es in dem Fall keine plausible Interpretation. In einem Kontext wie (84) ist jedoch eine Interpretation möglich und das Kompositum erscheint wesentlich akzeptabler.

\section{ein/der *Peters-graue-Schläfe-Effekt}

Seit Peter in die Jahre gekommen ist, gibt er sich gern weise und belehrend. Die Nachbarskinder lassen sich von seinen Vorträgen richtig einschüchtern. Wir nennen das den ,Peters-graue-Schläfe-Effekt؛

Als weitere Eigenschaften von Phrasenkomposita führt Meibauer an, dass ein enthaltenes Adjektiv unflektiert sein kann, dass das phrasale Betonungsmuster im Kompositum erhalten bleibt, und dass nur nominale Köpfe auftreten können, d.h. ein Phrasenkompositum kein Rektionskompositum sein kann (vgl. Meibauer 2003:156ff). Als Einwand für den letzten Punkt sei das Beispiel unter (85) genannt, das mir durchaus akzeptabel erscheint.

der Mamas-kalte-Füße-Beheizer

Zur Strukturanalyse von Phrasenkomposita gibt es verschiedene Ansätze, u.a. der syntaktische Ansatz nach Lieber (1992), die Konversionsanalyse nach Gallmann (1990) und die Zitatanalyse nach Wiese (1996) (mehr dazu siehe Kap. (73).

Nun stellt sich die Frage nach der anaphorischen Zugänglichkeit von nominalen Ausdrücken, die in der phrasalen Konstituente enthalten sind. Zu diesem Thema führt Meibauer (2003:167) die als akzeptabel bewerteten Fälle unter (86) an. Es scheint sich demnach bei Phrasenkomposita nicht kategorisch um anaphorische Inseln zu handeln.

(86) a. Damals wurde die $\left[\right.$ Gott $_{i}$-ist-tot $]$-Thematik in allen Zeitungen ausgebreitet, aber wir glaubten nicht daran, dass $\underline{\mathrm{er}}_{i}$ tot ist/aber wir hielten das Gegenteil [=Gott ist nicht tot $]$ für wahr. 
b. Die [ Rote-Augen $\left._{i}\right]$-Reduktion ist durchaus wünschenswert, weil die Fotografierten $\underline{\text { damit }}_{i}$ wie Zombies aussehen.

c. Gestern hat mich die $\left[\underline{\mathrm{Ohr}}_{i}\right.$-ab]-Bande überfallen, aber $\underline{\mathrm{es}}_{i}$ ist noch dran.

Dabei stellt Meibauer infrage, dass es sich wirklich um einen semantischen Bezug handelt. Ebenso hält er einen pragmatischen Schlussprozess für möglich, z.B. eine Implikatur (vgl. Meibauer 2003:167).

Wie auch bei den Fällen mit akzeptablem N1-Bezug erscheint mir dies zu kurz gegriffen. Analog zu den Ausnahmen von klassischen N1-Eigenschaften (vgl. Kap. 2.2.2p konnte auch für die phrasale Komponente von Phrasenkomposita gezeigt werden, dass die angeführten Eigenschaften keineswegs allgemeingültig sind. In beiden Fällen gehe ich von mehr oder weniger prototypischen Konstituenten aus. Wobei das Abweichen von der Prototypikalität die anaphorische Zugänglichkeit erhöht. Darüber hinaus dienen Phrasenkomposita als Indiz dafür, dass eine DP Teil eines Kompositums sein kann.

\subsubsection{Univerbierungen}

Bei Univerbierungen handelt es sich um Nomen-Verb-Gefüge, die wiederum hinsichtlich ihres Grads an Eigenständigkeit des Nomens unterschieden werden (vgl. Gallmann 1999). Beispiele mit abnehmender Eigenständigkeit sind in (87) aufgeführt.

Klavier spielen (Noun-Stripping) - teilnehmen (Partikelverb) - schlussfolgern (Univerbierung i.e.S.)

Für alle Arten von Univerbierungen gilt nach Gallmann (vgl. 1999:291f), dass die nominale Komponente nicht artikel- oder attributfähig und kein syntaktisches Wort sei, sondern Kopf-Adjunkt. Die Fügung neige zu Intransivierung und sei semantisch lexikalisiert, während die Teile syntaktisch selbstständig, d.h. trennbar seien.

Ähnlich wie bei NN-Komposita scheint es sich auch bei Univerbierungen um anaphorische Inseln zu handeln (vgl. (88)]: „Der nominale Bestandteil von (als Ganzes) intransitiven Nomen-Verb-Verbindungen ist gewöhnlich nicht referenzfähig." (Gallmann 1999:284)

Wir müssen diesem Umstand $\underline{\text { Rechnung tragen. }}$ * Sie ist sehr hoch.

Diese Eigenschaften der nominalen Konstituente erklärt Gallmann damit, dass das Nomen zwar seinen nominalen Status behält, jedoch keine Phrase projiziert, sondern ein Kopf-Adjunkt, also ein $\mathrm{N}^{o}$ sei.

Für den anaphorischen Bezug als vorrangig problematisch erachte ich die (durch Lexikalisierung entstandene) zumeist metaphorische Verwendung der nominalen Konstituente. Ist deren Bedeutung nicht metaphorisch zu interpretieren und hat die nominale Konstituente einen relativ hohen Grad an Eigenständigkeit, ist auch der anaphorische Bezug akzeptabler, vgl. (89).

(89) Ich werde erst wieder Klavier spielen, wenn es gestimmt wurde. 
Der Unterschied liegt darin, dass die Rechnung in Rechnung tragen keine Rechnung im Wortsinn denotiert, das Klavier in Klavier spielen hingegen schon. Hierin scheint auch ein entscheidender Faktor beim N1-Bezug zu liegen, denn auch metaphorisch verwendete N1 sind i.d.R. unzugänglich, siehe z.B. in (90),

(90) Ich habe mir eine Eselsbrücke gebaut. *Leider war er sehr störrisch.

\subsubsection{Idiome}

Bei Idiomen handelt es sich um lexikalisierte, unikale Wendungen, die morphologische Anomalien aufweisen und syntaktische Hinweise enthalten können, bspw. können Selektionsbeschränkungen verletzt werden (vgl. Soehn 2005:57ff). Ähnlich wie bei den lexikalisierten Univerbierungen heißt es für Idiome:

Auf die wendungsinternen Argumente kann man sich in der Regel nicht durch ein Pronomen beziehen, d.h. die Argumente sind nicht referenziell. (Soehn 2005 29)

Neben Beispielen wie (91-a) führt Soehn (2005:29) jedoch auch Ausnahmen wie (91-b) an.

(91) a. *Das $\underline{\mathrm{Ohr}}$, über $\underline{\text { das }}$ er mich gehauen hat, blutete.

b. Legendär ist bereits das Fettnäpfchen, in das die Bahn bei der Einführung ihres neuen Preissystems getreten ist [...]

Meines Erachtens liegt der Unterschied zwischen (91-a) und (91-b) darin, dass sich die anaphorische Bezugnahme in (91-b) innerhalb der Metapher bewegt, die durch das Idiom eingeführt wird, in (91-a) allerdings nicht. Wie bereits in Kapitel 2.5.2 konstatiert, gilt auch beim Bezug auf Teile von Idiomen, dass zwischen Antezedens und Anapher kein Widerspruch zwischen metaphorischer und wörtlicher Bedeutung bestehen darf. Dies ist ein Umstand, der sich nicht nur auf N1-Bezüge, sondern auf anaphorische Bezugnahmen im Allgemeinen ausweiten lässt 34

\subsubsection{Klammerparadoxie}

Erstglieder von Komposita gelten oft nicht nur als unzugänglich, eine weithin angenommene Eigenschaft ist auch, dass sie nicht durch Adjektive modifiziert werden können (vgl. Kap. 2.2.2). Ein Argument gegen die Gültigkeit dieser Annahme ist die sog. Klammerparadoxie.

Typische Fälle von Klammerparadoxie im Deutschen sind die Beispiele in (93), Aus Sicht der normativen Grammatik sollten diese ungrammatisch und unakzeptabel sein, dennoch lassen sich (nicht nur im Deutschen, vgl. (94) genug Fälle finden, um das Phänomen nicht als Kuriosum abtun zu können (vgl. Sternefeld 2003:2f).

\footnotetext{
${ }^{34}$ Ein Anzeichen für die Problematik des Wechsels zwischen metaphorischem und wörtlichem Sinn ist, dass ein solcher Wechsel oft als Mittel für Wortwitz eingesetzt wird, vgl. (92).

(92) A: Ich muss jetzt echt in die Falle.

B: Tu' dir nicht weh.
} 
(93) a. deutsche Sprachwissenschaft, gekochte Schinkenplatte

b. nukleare Kriegsgefahr, männliche Hormonproduktion, arabisches Wirtschaftswachstum (Wunderlich 1986:218)

(94) pure silk jacket, baroque flautist

Sternefeld (vgl. 2003:2) erklärt die Paradoxie als Widerspruch in der Strukturierung. Syntaktisch, morphologisch und orthografisch liegt die Struktur in (95) vor, semantisch jedoch die Struktur in (96). Die Paradoxie liegt nun darin, dass nach dem Fregeprinzip die Bedeutung des Ganzen eine Funktion ihres syntaktischen Aufbaus ist und sich somit morphosyntaktische und semantische Gliederung nicht grundsätzlich unterscheiden dürften.

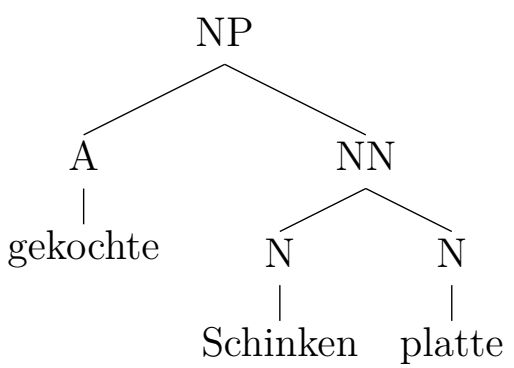

(96)

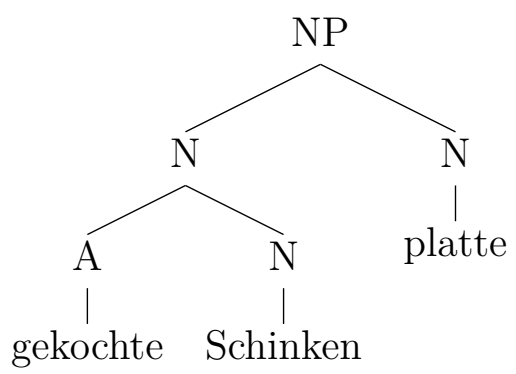

Für das Englische wird vorrangig innermorphologisch argumentiert. So wird dem Ausdruck unhappier die Bedeutung mehr unglücklich zugewiesen, was semantisch für Präfigierung vor Komparation spricht. Allerdings wird im Englischen der Komparativ von Dreisilbern mit more gebildet. Bei der morphosyntaktischen Strukturbildung muss demnach erst die Komparation und anschließend die Präfigierung erfolgen (vgl. Sternefeld 2003:3f). Insgesamt handelt es sich bei der Klammerparadoxie also um einen Widerspruch zwischen syntaktischer und semantischer Struktur.

Spencer erklärt das Zustandekommen der paradoxen Formen via Analogie-, bzw. Rückbildung (vgl. Spencer 1991:413ff). Wie in (97) dargestellt, entstehe die Klammerparadoxie als Ableitung aus einer bereits bestehenden Adjektiv-Nomen-Verbindung.

a. baroque flute $\rightarrow$ baroque flautist

b. modern languages $\rightarrow$ modern linguist

Ähnlich argumentieren auch Ackema/Neeleman (2004), die die Klammerparadoxie als Form der phrasalen Derivation beschreiben, d.h. als Phrasenkompositum mit einem Affix als Kopf. Die Annahme von Phrasalität belegen sie damit, dass das Adjektiv flektiert ist (vgl. Ackema/Neeleman 2004:167). Zumindest im Deutschen besteht in diesem Fall jedoch ein Widerspruch zur Orthografie, nach der im Fall eines Phrasenkompositums ein Durchkopplungsstrich statt eines Leerzeichens zwischen Adjektiv und N1 treten müsste.

Einen Erklärungsansatz für die Akzeptabilität der Konstruktion liefert Fabricius-Hansen (vgl. 1993 230f) mit der Beobachtung, dass bei den akzeptablen Fällen der adjektivischen N1-Modifikation ebenfalls eine zumindest hinreichende semantische Verträglichkeit zwischen Adjektiv und N2 besteht. So seien die Fälle unter (98-a) akzeptabler als die unter $(98-b)$. 
(98) a. atlantischer Störungsausläufer, deutsche Sprachwissenschaft

b. *siebenköpfiger Familienvater, *verregnete Feriengefahr

Übertragen auf die Problematik des anaphorischen N1-Bezugs würde dies bedeuten, dass die Anapher akzeptabler ist, wenn eine Interpretation als N2- oder NN-Bezugs zumindest semantisch verträglich ist.

Damit ließen sich Fälle wie (99) erklären, bei denen unklar ist, ob das Attribut aus der Übergabeposition zur nächsten Baugruppe das N1 oder das N2/NN von Leuchtdrucktaster näher beschreibt. Semantisch wäre beides plausibel und würde in der gleichen Funktionsinterpretation resultieren.

(99) Ist eine Palette zum Auslagern bereit, blinkt der Leuchtdrucktaster für den Transportweg aus der Übergabeposition zur nächsten Baugruppe.

(aus einer Betriebsanleitung)

Mit semantischer Kompatibilität lassen sich jedoch bei Weitem nicht alle Fälle von akzeptablen N1-Bezügen erklären. So ist ein N2/NN-Bezug in (100) semantisch keineswegs kompatibel.

(100) Wir mussten den Hundekorb zurückgeben. Er hat ein unfassbares Theater veranstaltet.

\subsubsection{Genitiv-Attribute}

Eine weitere Einschränkung für das N1 besteht in Bezug auf Genitiv-Attribute. Während sich diese sowohl auf das N2 (vgl. (101-a)) als auch auf das NN beziehen können (vgl. (101-b), ist ein Bezug auf das N1 nicht möglich (vgl. (101-c) (vgl. Fabricius-Hansen 1993:195,204).

(101) a. Personenüberwachung durch den Verfassungsschutz

b. Begleitumstände des Urnengangs

c. ?? ein Trinkglas des Weins, *ein Hauskauf aus Holz

Allerdings gibt es auch für diese Regel Ausnahmen, so z.B. die Fälle unter (102) (vgl. Fabricius-Hansen 1993).
a. Angriffswahrscheinlichkeit durch die Sowjetunion
b. eine Erfolgsbedingung der Republikaner
c. die Aufnahmebedingungen ihrer Studenten
d. Einreiseverweigerung in die DDR

Die Aussagekraft dieser Bildungsformen relativiert sie jedoch und erachtet deren Akzeptabilität als fraglich und am „unteren Ende der Normalitätsskala“; wobei die Akzeptabilität an den Grad der Lexikalisiertheit gekoppelt sei (vgl. Fabricius-Hansen 1993:213ff). Als Erklärung für die generelle Möglichkeit führt sie an, dass das N2 durch das N1 gesättigt sei, das N1 aber semantisch ergänzungsbedürftig bleibe. Die Interpretation richte sich 
schließlich nach pragmatischer Plausibilität (vgl. Fabricius-Hansen 1993:234ff). Härtl (vgl. 2015:169ff) spricht davon, dass derartige Fälle „außergrammatisch legitimiert“ seien und v.a. „funktional-kommunikative Faktoren“ eine entscheidende Rolle spielen. Auch bezieht er sich auf den bereits von Fabricius-Hansen angesprochenen Umstand, dass es sich bei vielen akzeptablen Fällen um obligatorische Argumente handelt.

Trotz allem bleibt der Befund, dass es sich auch in diesem Fall um Evidenz für die Akzeptabilität der Verletzung einer klassischen N1-Eigenschaft handelt.

Insgesamt konnte Kapitel 2.5 anschaulich machen, dass es sich bei der Akzeptabilität von anaphorischen Bezügen auf lexikalisch integrierte Einheiten zum einen um kein isoliertes Phänomen für NN-Komposita handelt (vgl. Phrasenkomposita, Univerbierungen und Idiome) und dass zum anderen auch für andere typische N1-Eigenschaften systematisch Gegenbeispiele zu finden sind (vgl. Klammerparadoxie und Genitiv-Attribute). In diesem Licht erscheint die Möglichkeit eines anaphorischen N1-Bezugs keineswegs als isoliertes Randphänomen, sondern lässt sich in eine systematische Reihe von verwandten Phänomenen einordnen.

\subsection{Stand der Forschung}

Die graduelle Akzeptabilität von anaphorischen N1-Bezügen an sich ist kein neuer Befund, sondern wurde bereits von einigen Autor*inn*en - vorrangig aus der Psycholinguistik thematisiert. Die wichtigsten bereits existierenden Ansätze und Untersuchungen sollen im Folgenden kurz zusammengetragen werden.

\subsubsection{Pragmatik}

Häufig werden akzeptable Fälle von anaphorischem N1-Bezug wie in (103) als „pragmatisch legitimiert" bezeichnet (vgl. Härtl 2015, Meibauer 2003, Wunderlich 1986).

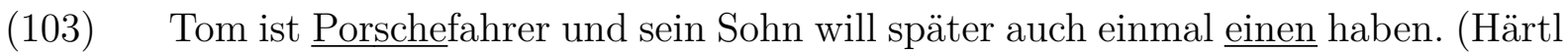
2015:9)

Gemäß der Giveness Hierarchy ist pronominaler Bezug möglich, sobald das Antezedens fokussiert oder zumindest aktiviert ist. Darüber hinaus ist ein Referent umso zugänglicher, je salienter er ist. Soll ein N1-Bezug nun durch Mittel der Pragmatik gewährleistet werden, müssen diese dafür sorgen, dass das N1 in den Fokus rückt und über hohe Salienz verfügt. Ward et al. (vgl. 1991:454) konstatieren, dass echte morphosyntaktische, also grammatische Verletzungen nicht durch pragmatische Faktoren repariert werden können und es sich somit bei einem pronominalen N1-Bezug nicht um eine echt morphosyntaktische Verletzung handeln könne. McKoon et al. (1993) betonen, dass die Zugänglichkeit eines Referenten von seiner Relevanz für den Rezipienten abhängt. In einer Reihe von Experimenten konnten sie Topikalität und Kontrast zu anderen Diskursentitäten als Faktoren identifizieren, die die Salienz eines Referenten erhöhen, der durch das N1 eingeführt wurde. In einer Studie zum Self-Paced-Reading mit anschließendem Memory-Test für das 
Antezedens fanden sie neben einem klaren zeitlichen Unterschied zwischen phrasalem Antezdens und Kompositum auch kürzere Lesezeiten für den Anaphernsatz bei Topikalität des N1 (Bsp.-Item siehe (104) aus McKoon et al. (1993:61)).

\section{a. High topicality}

Sam likes the outdoor life. Having grown up in rural Kentucky, he knows a lot about nature and is an expert at fishing and shooting. He goes on hunting trips as often as he can. He used to hunt just small game, like rabbit and

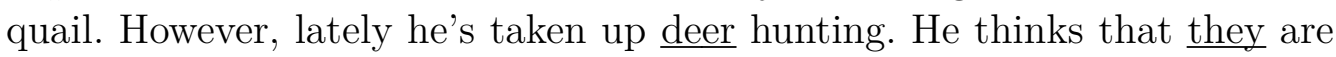
really exciting to track.

b. Low topicality

Sam has many interests in the outdoors, He's an avid skier, and each winter he takes about a month off from work to ski in Colorado. In the summertime, he visits his parents in Montana where he has a chance to do some mountain climbing. Lately, he's taken up deer hunting. He thinks that they are really exciting to track.

Sie kommen zu dem Schluss, dass die Zugänglichkeit eines N1 für Anaphern von den gleichen pragmatischen Variablen beeinflusst wird wie die Zugänglichkeit anderer Antezedenten, z.B. durch informationsstrukturelle Faktoren wie Topikalität (vgl. McKoon et al. 1993:72f). Diese Ergebnisse unterstreichen die Annahmen zur Salienz, die bereits in Kapitel 2.4.3 getroffen wurden.

\subsubsection{Transparenz und Kompositionalität}

Der Transparenz, der Kompositionalität und der Lexikalisiertheit (vgl. Kap. 2.3.4 von Komposita wird neben einem Einfluss auf die allgemeine Verarbeitung auch ein Einfluss auf die N1-Zugänglichkeit zugesprochen (vgl. Coulmas 1988, Härtl 2013, Schwarz 2000, Ward et al. 1991).

Im Zusammenhang mit anaphorischen Inseln heißt es bei Ward et al. (1991), dass ein transparentes Verhältnis zum Antezedens Zugänglichkeit ermöglicht, z.B. durch morphologische Präsenz im Kompositum. Aus diesem Grund seien die in orphan nicht morphologisch präsenten parents nicht zugänglich. Eine morphologische Verwandtschaft sei hingegen förderlich. Dabei betonen sie, dass die reine morphologische Präsenz alleine nicht genüge, sondern die Konstruktion transparent und regelhaft gebildet sein müsse. Daher sei der Bezug bspw. zu Länderbezeichnungen in regelhaften Ableitungen wie in (105) akzeptabel (vgl. Ward et al. 1991:465); cow im lexikalisierten Ausdruck cowboy hingegen nicht zugänglich (vgl. (106)) (siehe auch Lakoff/Ross 1972). Im Falle einer lexikalisierten Bedeutung werde das Kompositum nicht mehr auf der Basis seiner Teile interpretiert (vgl. Ward et al. 1991:454).

(105) Jean is a Frenchman, though he hasn't lived there for many years.

(106) Fritz is a cowboy. ${ }^{*}$ He says they can be difficult to look after.

Ähnliches formuliert auch Schwarz: 
Anaphorische Aufnahme der Konstituenten eines Kompositums ist möglich, wenn die Konstituenten ihre Autonomie behalten, also keine Idiomatisierung erfolgt ist, und wenn semantische und strukturelle Kompositionalität zusammenfallen. (Schwarz 2000:126)

Ebenso bei Coulmas:

Bei Wörtern, die in ihrer Form nicht kompositionell sind, ist der anaphorische Bezug auf Bestandteile ausgeschlossen. Umgekehrt [... ] wird ein solcher Bezug in dem Maße akzeptabler, wie Wörter kompositionell sind und die semantische Kompositionalität mit der formalen übereinstimmt. (Coulmas 1988.321)

Dabei geht Coulmas (1988) auch auf die verschiedenen Grade an Kompositionalität und Idiomatizität ein. Das Kompositum Fußgänger sei älter, lexikalisierter und weniger kompositionell als Atomwaffengegner. Demnach sei (107-a) direkt interpretierbar und weniger unzugänglich als (107-b) (vgl. Coulmas 1988:322).

(107) a. ??Atomwaffengegner haben gegen ihre Lagerung in Europa protestiert.

b. *Der Fußgänger hat sich in $\underline{\text { ihn }}$ geschossen.

Das Hauptkriterium für die N1-Zugänglichkeit ist nach Coulmas die Festigkeit der Bindung zwischen den Komposita-Konstituenten, da diese mit der Anwendbarkeit grammatischer Operationen korreliere. Ein Kriterium für diese Festigkeit sei die „Parallelität der formalen und semantischen Komposition" (Coulmas 1988:323). Wo die Interpretation der Bedeutung eines Kompositums mit Hilfe der Bedeutungen der Teile und ihrer Kasusrelation möglich sei, sei dies gegeben. Der Bezug Coulmas' auf kompositainterne Kasusrelationen lässt eine Nähe zur Transformationsgrammatik erahnen. Hierfür spricht auch, dass er die Umformbarkeit in eine Genitivkonstruktion als Kompositionalitätstest verwendet (z.B. river bank - river's bank (Coulmas 1988:323)). Dies erachte ich zum einen nicht als geeignetes Verfahren, da Genitivkonstruktionen vorrangig Possessoren beschreiben und es auch andere transparente Relationen gibt (z.B. Lederschuh $-{ }^{*}$ Schuh des Leders). Zum anderen nehme ich für das N1 typischerweise keine Kasusmarkierung an (vgl. Kap. 2.2.2), das Fugen-s interpretiere ich nicht als Genitivmarker und ich nehme keinen generellen Verarbeitungsunterschied zwischen den verschiedenen möglichen Relationen an (vgl. Kap. 2.3.3).

In Übereinstimmung mit Ward et al. (1991) und Schwarz (2000) spricht Coulmas allerdings auch von gradueller Transparenz und somit auch von gradueller Akzeptabilität.

Ist das N1 also im wörtlichen Sinne gebraucht, d.h. transparent, und ist die Bedeutung des Kompositums mit Hilfe der Bedeutungen seiner Teile erschließbar, also kompositional, ist das N1 weniger unzugänglich. Bzgl. der Grade an Transparenz will ich hier die Einteilung von Libben et al. (2003) bemühen und mit Tabelle 2.3 den Versuch einer Übertragung auf das Deutsche unternehmen.

\begin{tabular}{r|l|l}
\hline \hline & Libben (2003) & deutsche Bsp. \\
\hline transparent + transparent (TT) & car-wash & Autowäsche, Bücherregal \\
transparent + opak (TO) & jail bird & Trauerkloß, Kartoffelpuffer \\
opak + transparent (OT) & strawberry & Erdbeere, Eisbein \\
opak + opak (OO) & hogwash & Spießrute, Hasenfuß \\
\hline \hline
\end{tabular}

Tabelle 2.3: Kombinationen von transparenten und opaken Kompositagliedern 
Unter der Annahme, dass ein N1 transparent sein muss, um zugänglich zu sein, kann für OT- und OO-Komposita eine generelle Unzugänglichkeit des N1 angenommen werden. Es bleibt die Frage, ob ein Unterschied zwischen TT- und TO-Komposita besteht. In einer informellen Umfrage wurden die Beispiele für TT-Komposita unter (108) insgesamt als weniger akzeptabel bewertet als die Beispiele für TO-Komposita unter (109).

(108) a. Das Bücherregal nimmt eine komplette Wand im Wohnzimmer ein. Die meisten von ihnen hat Peter aber gar nicht gelesen.

b. Peter hat sich neue, teure Lederschuhe gekauft. Diesmal hat er sich vorgenommen, es regelmäßig zu pflegen.

(109) a. Paul ist heute ein richtiger Trauerkloß. Sie steht ihm seit dem verlorenen Spiel am Samstag förmlich ins Gesicht geschrieben.

b. Paul würde viel häufiger Kartoffelpuffer essen, wenn er sie dafür nicht selber reiben müsste.

Es handelt sich hier um unkontrollierte Ad-hoc-Beispiele und eine nicht-repräsentative Umfrage. Sollte sich der Eindruck jedoch bestätigen lassen, würde dies eine Interaktion zwischen den Konstituenten, ihrer Transparenz und ihren Konzepten nahelegen. Eine mögliche Interpretation des Ergebnisses ist, dass die Opakheit des N2 den Fokus bei der Interpretation auf das N1 lenkt und somit dessen Salienz erhöht wird.

\subsubsection{Weitere Ansätze}

Neben pragmatischen Faktoren und dem Einfluss von Transparenz gibt es noch weitere Ansätze; u.a. ist ein Einfluss der Form des anaphorischen Ausdrucks anzunehmen. Anhand von (110) identifiziert Schwarz (2000:126f) N1-Bezüge mit Demonstrativa als akzeptabler als mit Personalpronomen.

(110) Hans ist Flötist geworden, weil er glaubte, dass ? $\underline{\text { das }} /{ }^{*} \underline{\text { sie }}$ ein wundervolles Musikinstrument sei.

Dies lässt sich anhand der Givennes Hierarchy und der Accessibility Theory begründen: Je niedriger der Aktiviertheitsgrad eines Antzedens, desto markierter muss die Anapher sein. Ist ein N1 nicht fokussiert, sind Demonstrativa als Anaphern geeigneter als Personalpronomen.

Ein weiterer Ansatz ist die Betrachtung spezieller Ausdrucksklassen an der N1-Position. Hierzu zählt die Beobachtung Sadocks 1998:164, dass Eigennamen wie unter (111) zugängliche Erstglieder darstellen. Ähnliches formuliert auch Coulmas (1988). Er verbindet dies mit dem Faktor der Kompositionalität, indem er postuliert, die Verbindung der Konstituenten sei in diesem Fall „lockerer“. Deutsche Beispiele mit Eigennamen sind u.a. die Fälle unter (112).

(111) Volvo-owners tend to like them.

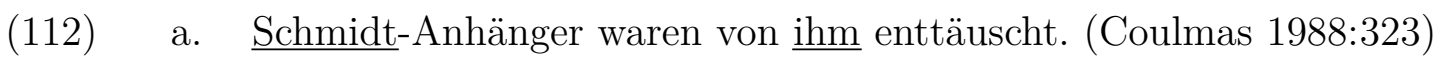


b. Viele Clinton-Anhänger haben hㅐ auch gewählt, weil er jünger als Dole ist. (Schwarz 2000: 126f)

Ein anderer Grund für die Zugänglichkeit von Eigennamen könnte in ihrer Referenzialität und Unikalitätspräsupposition liegen, mehr dazu in Kapitel 3.

\subsection{Zusammenfassung und Zielsetzung}

Komposita werden unter Annahme lexikalischer Integrität von vielen Autor*inn*en als anaphorische Inseln betrachtet. Gemäß der Lexical Integrity Hypothesis können syntaktische Prozesse nicht ausschließlich lexikalische Strukturen involvieren, und dies sei im Fall eines anaphorischen N1-Bezugs notwendig. Die erste Konstituente eines Kompositums könne nicht den Status einer NP haben und vor allem nicht referenziell sein.

Die allgemeine Gültigkeit der aus diesen Annahmen resultierenden Charakteristika von Erstgliedern wurde in den vorangehenden Abschnitten einer genaueren Betrachtung unterzogen (vgl. Kap. 2.2.2): Ein N1 könne nicht flektieren, Ausnahmen wurden für die unregelmäßige Deklination gefunden. Ein N1 sei nicht modifizierbar, Ausnahmen stellt die Klammerparadoxie dar. Ein N1 könne keinen Determinierer bei sich führen, jedoch ist dies innerhalb von Phrasenkomposita möglich. Ein N1 sei immer generisch und somit nicht-referenziell, allerdings können auch Eigennamen als N1 auftreten. Schnittmengenbildungen illustrieren, dass ein N1 auch die Funktion eines semantischen Kopfs übernehmen kann. Insgesamt erhärtet sich der Eindruck, dass die postulierten Eigenschaften auf einen Großteil der Komposita zutreffen, jedoch nicht allgemeingültig sind. Vielmehr scheint es mehr und weniger typische N1 zu geben, abhängig von Anzahl und Gewicht typischer N1-Eigenschaften.

Im Kontext von Annahmen auf Basis der LIH gilt es zu bedenken, dass diese nur innerhalb lexikalistischer Ansätze gültig ist. Im Rahmen von syntaktischen Ansätzen ist die strikte Trennung zwischen morphologischem und syntaktischem Modul aufgehoben und entsprechende Argumente verlangen nach einer alternativen theoretischen Basis.

Als Faktoren für die N1-Zugänglichkeit wurden bereits pragmatische Faktoren wie Kontext und Informationsstruktur attestiert. In Bezug auf die Komposita-Interpretation kommen vor allem der Transparenz und der Kompositionalität eine entscheidende Rolle zu. Je transparenter ein N1 ist, desto zugänglicher ist es. Ein möglicher Grund dafür könnte sein, dass es in dem Fall dem freien Vorkommen als Simplex ähnlicher ist und somit eher wie ein solches verarbeitet werden kann.

Zur Untersuchung weiterer Faktoren sollen pronominale Diskursanaphern nutzbar gemacht werden. Die Verwendung von Pronomen signalisiert durch deren geringen semantischen Gehalt Zugänglichkeit im Sinne eines hohen Aktiviertheitsgrads.

Eine besondere Herausforderung stellt die Positionierung des N1-Bezugs im Spannungsverhältnis von direkter und indirekter Anapher dar. Eine direkte Anapher kann i.d.R. durch ein Pronomen realisiert werden. Dies trifft auf N1-Bezüge nicht zu. Für die Etablierung einer indirekten Anaphern muss zusätzliches Wissen aktiviert werden, was im Falle eines morphologisch präsenten N1 wiederum nicht notwendig ist. Aussagekräftig wäre in Bezug auf diese Fragestellung Evidenz bzgl. des Zeitpunkts der Anaphernresolution. Findet die Auflösung erst im Zusammenhang mit der Verarbeitung des Kontexts statt, spräche dies für einen eher indirekten Anaphern-Typ. Findet der maßgebliche Resolutionsprozess 
jedoch schon auf der Anapher statt, spricht dies tendenziell für einen direkten AnaphernTyp, da die Genus- und Numerus-Informationen des Pronomens genutzt werden. Eine zentrale Frage ist auch, ob das N1 überhaupt einen Diskursreferenten etabliert.

In Hinblick auf experimentelles Arbeiten haben sich verschiedene Aspekte als beachtenswert herausgestellt: Liegt der Fokus auf dem Vergleich und der Analyse einzelner kontextunabhängiger Faktoren, gilt es Kontext (z.B. in Bezug auf die Informationsstruktur) und Syntax (z.B. in Hinblick auf Präferenzen für bestimmte syntaktische Funtkionen) in besonderem Maße zu kontrollieren. Weitere verarbeitungsrelevante Faktoren wie Transparenz und Frequenz sind ebenfalls zu reflektieren. Dabei gilt es eine Good-Enough-Lesart $\mathrm{zu}$ vermeiden und bspw. durch Verständnisfragen ,close reading' zu forcieren. Andernfalls können kurze Lesezeiten nicht zwangsläufig auf einen Verarbeitungsvorteil zurückgeführt werden.

Im Rahmen dieser Arbeit sollen aufbauend auf den bestehenden Annahmen ausdrucksinhärente grammatische und außergrammatische Faktoren der N1-Zugänglichkeit ermittelt, klassifiziert und hierarchisiert werden. Aufgrund des Umfangs an möglichen Einflussgrößen soll ein exhaustives Modell dabei nicht das oberste Ziel sein. Stattdessen soll der Fokus auf der empirischen Validierung und Objektivierung einer Auswahl von Faktoren liegen. In Zusammenhang damit steht die Gewinnung von Erkenntnissen zu Verarbeitungsprozessen bei der Komposita-Verarbeitung und der Anaphernresolution. 



\section{Konzeptuelle Faktoren}

In den bereits vorliegenden Arbeiten zur N1-Zugänglichkeit wurden vorrangig zwei Faktorarten analysiert: Die Pragmatik (vgl. Kap. 2.6.1) und die Transparenz (vgl. Kap. 2.6.2). Darüber hinaus deutet sich bereits an, dass es weitere Eigenschaften von Konstitutenten gibt, die die Zugänglichkeit beeinflussen können. Im folgenden Kapitel werden nun diskursunabhängige, ausdrucks-inhärente Eigenschaften untersucht, die entweder aus verschiedenen Arten von Wissen über das N1 als Einheit im mentalen Lexikon entstammen oder sich aus der Kombination der Konzepte im Kompositum ergeben. Dabei werden sowohl linguistische als auch nicht-linguistische Eigenschaften in Betracht gezogen.

\subsection{Allgemeine Vorüberlegungen}

Der Einfluss der Transparenz der Konstituenten und der Kompositionalität des Kompositums auf die N1-Zugänglichkeit kann als gesichert angesehen werden (vgl. Kap. 2.6.2). Je weiter die Bedeutung des N1 von der Bedeutung des Ausdrucks als Simplex abweicht, desto schlechter ist es zugänglich. Den Extremfall bildet hier der metaphorische Gebrauch. Die folgenden Analysen beschränken sich auf transparente Konstituenten. Dabei soll Transparenz und Kompositionalität zunächst via Paraphrasierbarkeit kontrolliert werden.

Bei der Genese von Beispielen zur Illustration einzelner Eigenschaften gilt es besonderes Augenmerk auf die Wahl der Anapher zu legen. Kongruenz von Pronomen und N1 in Genus und Numerus soll die Interpretation des Pronomens im Sinne einer direkten Anapher zumindest theoretisch ermöglichen. Dabei soll die Oberflächenform berücksichtigt werden, d.h. es wird die Möglichkeit der Interpretation von Fugenelementen als Flexionsmarker eingeräumt. Wie in Kapitel2.2.2 erläutert, ist ein N1-Bezug akzeptabler, wenn Pronomen und N1 im Numerus übereinstimmen, auch wenn dies nur Ausdruck der Oberflächenform ist und nicht semantisch relevant. Dies deckt sich mit dem Ansatz von Libben (1998), demzufolge Parsing obligatorisch und automatisch stattfindet (vgl. Kap. 2.3.2). Als Konsequenz daraus folgt, dass auch Fugenelemente Eingang in den Interpretationsprozess finden können. Demzufolge ist im Fall von nicht-paradigmatischen Fugen mit Verarbeitungsschwierigkeiten zu rechnen. Der tatsächliche Einfluss von Fugen auf die KompositaVerarbeitung ist unklar und nicht Gegenstand dieser Arbeit, kann jedoch Einfluss auf experimentelle Ergebnisse haben. Aufgrund dessen werden im Folgenden Fugenelemente bei der Genese von Beispielen soweit wie möglich vermieden oder zumindest wird auf das Flexionsparadigma des N1 Rücksicht genommen. 


\subsection{Konzeptuelles und grammatisches N1-Wissen}

Zunächst sollen Eigenschaften betrachtet werden, die Ausdruck verschiedener Arten von Wissen zum N1-Ausdruck sind und Einfluss auf die Aktiviertheit und damit auf die Zugänglichkeit haben könnten. Dabei wird sprachliches Wissen (z.B. die Eigenschaft ein Massenomen zu sein) genauso in Betracht gezogen wie Denotat- und Konzeptwissen (z.B. Konkretheit).

\subsubsection{Lebenswelteffekt}

In ihrer kognitionslinguistischen Dissertation „Vom Konkreten im Abstrakten“ vertritt Judith Schrauf (2011) die These, dass es theoretische Merkmale gibt, die psychologische Relevanz haben und dadurch die Sprachverarbeitung beeinflussen. In diesem Zusammenhang entwirft Schrauf den Begriff der „Lebenswelt“, der grundlegend auf das Kriterium der Subjektivität nach Frege (1892) zurückgeht (vgl. Schrauf 2011:160). Dabei handelt es sich um eine kontinuierliche Skala für Begriffe zwischen den Polen ,innere Lebenswelt‘ (z.B. Gewissen) und ,äußere Lebenswelt' (z.B. Auto). Darauf aufbauend entwirft Schrauf die Idee eines „Lebenswelteffekts“, demzufolge Ausdrücke mit einem starken Bezug zur inneren Lebenswelt des Menschen schneller verarbeitet werden als Ausdrücke mit einem schwachen Bezug zur inneren, bzw. einem starken Bezug zur äußeren Lebenswelt des Menschen (vgl. Schrauf 2011:198). Denkbar wäre nun, dass dieser Verarbeitungsvorteil mit einem Vorteil für die Zugänglichkeit einhergeht.

\section{Eigennamen}

Wie bereits in Kapitel 2.6.3 angerissen, werden Eigennamen (proper nouns) als Fälle von referenzfähigen Erstgliedern beschrieben (vgl. Coulmas 1988, Sadock 1998, Schwarz 2000, Wunderlich 1986), z.B. in den Fällen unter (1). In dem Sinne, dass bei Bezeichnungen für menschliche Referenten eine ontologische Nähe zwischen Denotat und Adressat*in besteht, könnte dies als Beispiel für den Lebenswelteffekt interpretiert werden.

(1) a. Was Picasso-Fans so alles veranstalten, wenn sie $\underline{\text { ihn }}$ verehren. (Wunderlich 1986:220)

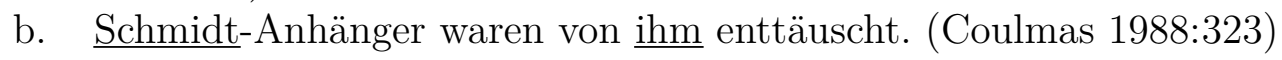

c. Viele Clinton-Anhänger haben ihn auch gewählt, weil er jünger als Dole ist. (Schwarz 2000:126f)

Neben Eigennamen in Form von Personenbezeichnungen sind auch Fälle mit Orts- (vgl. (2)) und Produktbezeichnungen (vgl. (3)] attestiert.

(2) a. Moskau-Reisende haben viel zu erzählen, wenn sie von dort zurückkommen. (Wunderlich 1986:220)

b. Jean is a Frenchman, though he hasn't lived there for many years. (Ward et al. 1991:465)

(3) a. Volvo-owners tend to like them. (Sadock 1998:164) 
b. Patty is a definite Kal Kan cat. Every day she waits for it. (Ward et al. 1991: 451)

Ein Grund für die gute Zugänglichkeit von Eigennamen an der N1-Position ist nach Coulmas (vgl. 1988:323), dass in dem Fall ein hoher Grad an Kompositionalität gegeben sei. Der Eigenname würde seine Eigenständigkeit teilweise behalten und die Verbindung mit dem N2 sei „lockerer“.

Entscheidender erscheint mir jedoch - unabhängig vom Lebenswelteffekt bei Personenbezeichnungen - der Einfluss der grammatischen Eigenschaften von Eigennamen zu sein. Der klassischen sprachphilosophischen Auffassung zufolge wird für Eigennamen generelle Referenzfähigkeit angenommen (vgl. Frege 1892, Kripke 1980), worin eine Voraussetzung für die pronominale Wiederaufnahme liegt. Eigennamen benötigen keinen Determinierer, um eine volle NP, bzw. DP zu bilden und sind somit im formalen Sinne auch an der N1-Position referenzfähig. Darüber hinaus präsupponieren Eigennamen Unikalität (vgl. Strawson 1950), was die Identifizierung und Referenzzuweisung erleichtert. Und schließlich denotiert ein Eigenname ein umgrenztes Individuum und zwar in allen möglichen Welten, was die Bezugnahme generell erleichtert.

\section{Animatheit}

Auch Fukumura und van Gompel gehen davon aus, dass referenten-inhärente Eigenschaften Einfluss auf die Zugänglichkeit des Referenten haben (vgl. Fukumura/van Gompel 2011:1472). Am Beispiel der Animatheit zeigen sie in drei Experimenten, wie die Zugänglichkeit und dadurch die Wahl der Anapher beeinflusst wird.

Beim ersten Experiment handelt es sich um eine Satzergänzungsaufgabe, bei der Versuchspersonen sich auf eine von zwei NPn beziehen müssen. Eine NP ist dabei stets animat, die andere inanimat; beide stehen im Plural (vgl. (4) aus Fukumura/van Gompel (2011. $1475)$ ).

a. The hikers carried the canoes a long way downstream. Sometimes, ...

b. The canoes carried the hikers a long way downstream. Sometimes, ...

Bei der Kodierung der Satzergänzungen wurde die Art des gewählten Anaphernausdrucks ausgewertet. Es zeigt sich, dass signifikant mehr Pronomen bei Bezug auf die erste NP und ebenfalls signifikant mehr Pronomen bei Bezug auf animate Referenten verwendet wurden (vgl. Fukumura/van Gompel 2011:1474ff). Das zweite und dritte Experiment bestätigt dieses Ergebnis unter anderen experimentellen Zielsetzungen. Insgesamt resümieren Fukumura/van Gompel (2011), dass die Animatheit eines Referenten dessen Grad an Aktiviertheit und somit dessen Zugänglichkeit beeinflusst.

Als Ursache für den erhöhten Grad an Zugänglichkeit führen Fukumura und van Gompel einen höheren Grad an Vorhersagbarkeit animater gegenüber inanimater Entitäten an und beziehen sich damit auf das elaboriertere Wissen und die stärkeren Assoziationsauslöser belebter Entitäten (vgl. Fukumura/van Gompel|2011:1493). Dem liegt die Annahme zugrunde, dass die ontologische Nähe zwischen Denotat und Sprachnutzer*in Einfluss auf die Sprachverarbeitung hat, was ich als eine weitere Ausprägung des Lebenswelteffekts betrachte, wie auch schon im Fall der Personenbezeichnungen. Außerdem sehen sie den 
Verarbeitungsvorteil für Ausdrücke für animate Denotate darin begründet, dass diese Aktionen initiieren können und deshalb häufig als Subjekt fungieren (vgl. Fukumura/van Gompel 2011:1472).

Aufbauend auf der Idee des Lebenswelteffekts soll der Einfluss der Animatheit auf die N1-Zugänglichkeit entlang der Animatheitsskala (5) nach Aissen (2003:437) angenommen werden.

$$
\text { Human }>\text { Animate }>\text { Inanimate }
$$

Dementsprechend müsste ein humanes N1, d.h. eine Personenbezeichnung wie in (6-a) am besten zugänglich sein und die Zugänglichkeit eines inanimanten N1, d.h. die Bezeichnung für einen unbelebten Gegenstand wie in (6-c) müsste in vergleichbarem Satzkontext deutlich schlechter zugänglich sein. Ein animates N1 in Form einer Bezeichnung für eine Tierart wie in (6-b) müsste in Bezug auf die Zugänglichkeit dazwischenliegen. Diese Einschätzung wurde durch informelle Umfragen unter Studierenden bestätigt.

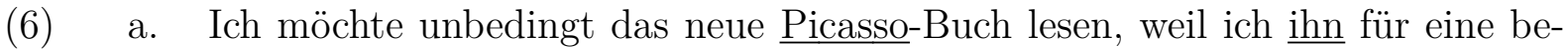
eindruckende Persönlichkeit halte.

b. Ich möchte unbedingt das neue Enten-Buch lesen, weil ich sie für beeindruckende Tiere halte.

c. Ich möchte unbedingt das neue Geigen-Buch lesen, weil ich sie für wundervolle Instrumente halte.

Zur Validierung dieser Annahmen ist allerdings eine systematische Untersuchung notwendig. Bei der Animatheit handelt es sich (im Gegensatz zu den Eigennamen) um eine Eigenschaft des Denotatwissen, d.h. um einen nicht-linguistischen Faktor.1

\section{Emotionale Valenz}

Schrauf spricht in Bezug auf psychologisch relevante Einflussgrößen für die Sprachverarbeitung vom Einfluss körperlicher Wahrnehmung, Emotionen und eigener Erfahrung (vgl. Schrauf 2011:207). Dabei läge ein primärer Verarbeitungseffekt für lebensweltlich relevante Entitäten vor, z.B. Ausdrücke für Emotionen und Vorstellungen im Gegensatz zu Ausdrücken aus dem Bereich der Wissenschaft und der Technik (vgl. Schrauf 2011:201). In diesem Sinne müsste (7-a) akzeptabler sein als (7-b), da der Ausdruck Angst über mehr emotionale Valenz verfügt als Wasser.

(7) a. Gunnar hat seit Tagen immer wiederkehrende Angstträume. Beim Aufwachen fühlt er sich komplett von ihr durchdrungen.

b. Gunnar hat seit Tagen immer wiederkehrende Wasserträume. Beim Aufwachen fühlt er sich komplett davon umgeben.

\footnotetext{
${ }^{1}$ Allerdings wurden bereits vereinzelt grammatische Reflexe gefunden. Bspw. beobachten Fanselow et al. (2011) einen Einfluss der Animatheit auf den Superioritäts-Effekt bei intervenierenden multiplen WFragen.
} 


\subsubsection{Wahrnehmungseffekt}

Neben dem Lebenswelteffekt spricht Schrauf auch von einem dem Lebenswelteffekt nachgeordneten „Wahrnehmungseffekt“. Dabei handelt es sich um einen Verarbeitungsvorteil für Ausdrücke, die sich auf sinnlich Wahrnehmbares beziehen, z.B. Bezeichnungen für Lebewesen und Dinge im Gegensatz zu Bezeichnungen für Maßeinheiten. Wie schon beim Lebenswelteffekt handelt es sich auch beim Wahrnehmungseffekt um einen Effekt des Weltwissens auf die Verarbeitung.

Ein Beispiel für Ausdrücke, die sinnlich Wahrnehmbares bezeichnen, sind Konkreta. Um fundierte Hypothesen zur Konkretheit aufstellen zu können, gilt es diese zunächst zu definieren. Schrauf (vgl. 2011;30) hält die dichotome Einteilung in ,konkret' vs. ,abstrakt' für zu eng und verwendet ,Konkretheit' daher als einen dynamischen Begriff für ein Kontinuum. Dabei impliziere Konkretheit eine Kombination aus sinnlicher Wahrnehmbarkeit und Abgrenzbarkeit durch den Bezug auf eine raum-zeitliche Dimension. ${ }^{2}$ Konkretheit sei mit Vorstellbarkeit verwandt, wobei letztere weiter gefasst sei und z.B. auch die Wahrnehmung von Zuständen umfasse (vgl. Schrauf 2011:125). Dies schränke sie in ihrer Verwendung ein und bewirke gleichzeitig eine höhere Kontextsensitivität abstrakter Entitäten (vgl. Schrauf 2011:83).

Darüber hinaus zieht Schrauf Parallelen zwischen Abstrakta und Metaphern. Beide zeigen in psycholinguistischen Experimenten ein ähnliches Verhalten. Für abstrakte Kategorien würden relativ mehr Metaphern verwendet, was die Konkretisierungsfunktion von Metaphern unterstreicht (vgl. Schrauf 2011:203). Trotz dieser Gemeinsamkeiten sei jedoch kein 1:1-Zusammenhang zwischen Abstrakta und Metaphern anzunehmen, da abstrakte Konzepte nicht zwingend metaphorisch repräsentiert sein müssen (vgl. Schrauf 2011:207). Im Zusammenhang mit der N1-Zugänglichkeit stärkt dies die Annahme besserer Zugänglichkeit für konkrete N1. Metaphorisch verwendete N1 wurden im Sinne opaker N1 als schlecht zugänglich bewertet. Sollten sich Abstrakta ähnlich den Metaphern verhalten, würde dies auch geringere Zugänglichkeit vermuten lassen. Ein genereller Verarbeitungsunterschied zwischen figurativer und wörtlicher Sprache kann durch Studien zu konventionellen Metaphern allerdings nicht nahegelegt werden (vgl. Schrauf 2011:100). Dies weist darauf hin, dass kein 1:1-Verhältnis zwischen allgemeinen Verarbeitungsvorteilen und der Zugänglichkeit als N1 bestehen muss, sondern von Fall zu Fall zu untersuchen ist.

In Bezug auf Verarbeitungsunterschiede zwischen Konkreta und Abstrakta zeichnen empirische Studien ein diffuses Bild (vgl. Schrauf 2011:80). Ein Reaktionszeitexperiment mit auditiv präsentierten Stimuli und lexikalischer Entscheidungsaufgabe zeigt einen leichten, jedoch nicht signifikanten Vorteil von Konkreta gegenüber Abstrakta (vgl. Schrauf 2011:152). Insgesamt geht Schrauf daher nicht von einem grundlegenden Verarbeitungsunterschied zwischen Konkreta und Abstrakta aus, sondern nimmt für beide die gleichen grundlegenden Verarbeitungsmechanismen an (vgl. Schrauf 2011:196). Ganz allgemein postuliert Schrauf auf Basis verschiedener Experimente jedoch die Existenz von Verarbeitungsunterschieden zwischen verschiedenen semantischen Kategorien, z.B. würden Bezeichnungen für Emotionen, menschliche Vorstellungen und Stoffe schneller verarbeitet als Bezeichnungen für Maße, Verhältnisse und Konzepte der Wissenschaft. Die Basis hierfür bilden Lebenswelt- und Wahrnehmungseffekt (vgl. Schrauf 2011:150).

\footnotetext{
${ }^{2}$ In der quantitativen Linguistik gibt es bereits Ansätze zur Objektivierung durch lexikologische Analysen auf Basis von Wörterbucheinträgen und entsprechenden Algorithmen (vgl. Schrauf 2011.42).
} 
Sollte ein Verarbeitungsunterschied zwischen Konkreta und Abstrakta bestehen, interagiert dieser vermutlich mit dem Lebenswelteffekt, wobei letzterer wahrscheinlich den stärkeren Einfluss hat. Lebensweltlich relevante Ausdrücke, z.B. Ausdrücke für Emotionen, haben einen klaren Verarbeitungsvorteil und können gleichzeitig Abstrakta sein. Vor diesem Hintergrund erscheint der Vergleich der Ausdrücke Angst und Wasser aus (7) ungeeignet. Die emotionale Valenz des Abstraktums Angst wiegt vermutlich schwerer als die Eigenschaft des Ausdrucks Wasser konkret zu sein. In diesem Sinne wäre ein Vergleich zwischen einem Konkretum und einem Abstraktum mit ähnlicher emotionaler Valenz geeigneter, z.B. Zaun und Schutz. In diesem Fall legt (8) einen Vorteil für das konkrete N1 nahe 3

a. Die Zaunaffäre hat der Politikerin das Genick gebrochen. Dabei war er als Maßnahme zum Wohle des Volkes gedacht.

b. Die Schutzaffäre hat der Politikerin das Genick gebrochen. Dabei war er als Maßnahme zum Wohle des Volkes gedacht.

Die Idee eines Wahrnehmungseffekts spiegelt sich auch im Begriff der „Weltimmanenz“ bei Hegarty et al. (2001) wider.

Another factor which seems to have an effect on whether or not a clausally introduced entity is brought into focus is the degree of world immanence of the entity and, correlatively, its manner and degree of individuation. (Hegarty et al. 2001 173)

Dabei beziehen sich Hegarty et al. (2001) auf das bereits von Asher (1993) entworfene Spektrum von Weltimmanenz. Einen niedrigen Grad an Weltimmanenz haben rein abstrakte Objekte wie Propositionen und Gedanken und einen hohen Grad konkrete Objekte sowie Ereignisse und Zustände mit kausalen, räumlichen und zeitlichen Eigenschaften.

Mit Hilfe dieser Annahmen lässt sich auch die Akzeptabilität von (9) erklären, die von Härtl (2015) mit pragmatischen Prozessen begründet wird: Beim N1 Porsche handelt es sich um einen Markennamen und damit um einen Eigennamen. Dieser referiert direkt und ist somit besser zugänglich. Gleichzeitig handelt es sich bei einem Auto um eine Entität, die konkret wahrnehmbar ist, einen hohen Grad an Weltimmanenz hat und als Individualnomen auch über einen hohen Individuationsgrad verfügt.

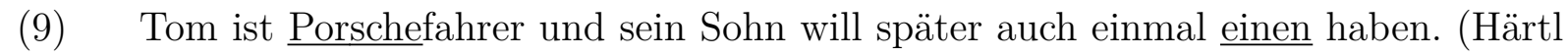
2015:167)

Während es sich bei der Konkretheit und ähnlichen Kategorien um nicht-linguistische Faktoren des Denotatwissens handelt, gibt es eine verwandte Eigenschaft, die über grammatische Reflexe verfügt: Die Zählbarkeit. Die Unterscheidung zwischen Massenomen (z.B. Brot, Rührei) und Individualnomen (z.B. Wurstbrot, Ei) bezieht sich vorrangig auf die Umgrenzbarkeit des Denotats (vgl. dazu Fanselow 1981:15f). Darüber hinaus verfügen Massenomen über die grammatische Eigenschaft, ohne Artikel referenzfähig zu sein (vgl. $(10)$.

${ }^{3}$ Ziel war es, zu Anschauungszwecken möglichst parallele Beispiele zu bilden, was wie bereits in (7) in eher unnatürlichen Sätzen resultiert. Allerdings liegen in beiden Fällen gleichsam Ad-hoc-Bildungen vor. 
Ich habe Wasser im Schuh. Siehst du, wie es an den Seiten heraustropft?

Aufgrund dessen liegt die Vermutung nahe, dass sie an der N1-Position über eine relativ gute Zugänglichkeit verfügen (vgl. Referenzfähigkeit bei Eigennamen). Andererseits ähneln Massenomen in Hinblick auf ihre unscharfen räumlichen Grenzen und der Vagheit des Denotats den Abstrakta, wohingegen Individualnomen den Konkreta näher stehen ${ }^{4}$ Individualnomen können leichter identifiziert und einheitlicher imaginiert werden als Massenomen, z.B. unterscheidet sich die Visualisierung von Wasser intersubjektiv stärker (z.B. als Fluss, Wasserfall, Wasser in einem Eimer, ...) als von ein Glas Wasser. Greift man das Konzept des Individuationsgrads von Hegarty et al. (vgl. 2001:173) auf, liegt die Vermutung nahe, dass die Umgrenzbarkeit eines Referenten dessen Zugänglichkeit erhöht. Dies wiederum spricht für eine bessere Zugänglichkeit von Individualnomen.

An dieser Stelle kommt es somit zu konträren Voraussagen für grammatische und konzeptuelle Eigenschaften innerhalb einer Ausdrucksklasse: Der Wahrnehmungseffekt spricht für eine bessere Zugänglichkeit von Individualnomen, der grammatische Einfluss entsprechend für eine bessere Zugänglichkeit von Massenomen. Die Frage, welche Eigenschaft den stärkeren Einfluss hat, lässt sich nur experimentell beantworten.

Ward et al. (1991:470ff) führen mit (11) sowohl Beispiele für akzeptable Bezüge zu Individualnomen an, als mit (12) auch für Massenomen. Dies lässt die Vermutung zu, dass keine der beiden Faktoreigenschaften in Bezug auf die Zugänglichkeit dominiert.
a. Officials in the Danish capital believe they've found a way to stop bicycle thefts - let people use them for free.
b. If you're a small business owner, or interested in starting one ...
c. I had a paper route once but my boss said I took too long deliverin' 'em.
a. At the same time as coffee beans were introduced, the Arabs made changes in coffee preparation that greatly improved its flavor.
b. I know, you probably get eight gazillion jokes from pragmatics students each

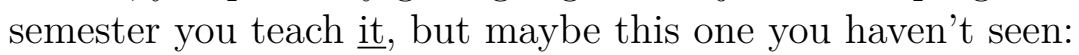
c. They're afraid it's the Gas and Electric man come to turn that off.

\subsubsection{Unikalität}

Der Wahrnehmungseffekt lässt sich dergestalt interpretieren, dass Ausdrücke für raumzeitlich umgrenzte, eindeutig identifizierbare und klar visualisierbare Denotate über eine relativ hohe Aktiviertheit verfügen und daher besser zugänglich sind als Ausdrücke, für die diese Eigenschaften nicht gelten. Diese Eigenschaft des Denotatwissens interpretiere ich als ihren Grad an Unikalität (vgl. Strawson 1950). Über den höchsten Grad an Unikalität verfügen Eigennamen: Mit einem Eigennamen bezeichnete Denotate (insbesondere Personennamen) verfügen nicht nur über klare raum-zeitliche Grenzen, sie sind auch eineindeutig identifizierbar, d.h. spezifisch und individuell. Fälle wie (13) demonstrieren, dass die Unikalität in der (außertextuellen) Welt zusammen mit dem Lebenswelt- und dem Wahrnehmungseffekt und der grammatischen Legitimation eine gute N1-Zugänglichkeit bewirkt.

\footnotetext{
${ }^{4}$ Die beiden Ausdrucksarten sind nicht identisch, denn Konkreta können ebenfalls Massenomen sein.
} 
(13) Der Papstbesuch wurde langfristig vorbereitet, um seine Sicherheit in jedem Fall gewährleisten zu können.

Insgesamt zeigt sich, dass spezielle Eigenschaften eines Ausdruckskonzepts dessen Verarbeitung und damit das Aktivationsniveau und die Zugänglichkeit beeinflussen können. Dabei ist davon auszugehen, dass Faktoren, die die Zugänglichkeit eines Diskursreferenten für anaphorische Bezugnahme allgemein beeinflussen, sich auch auf die N1-Zugänglichkeit auswirken, z.B. wie im Fall der Animatheit (vgl. Fukumura/van Gompel 2011).

Ein N1 wird allerdings niemals kontextfrei interpretiert, sondern stets im Zusammenhang mit einem N2. Daher gilt es im nächsten Schritt auch die spezifischen Eigenschaften des N2-Konzepts zu beleuchten. Die zentrale Frage ist dabei, inwiefern Eigenschaften des N2 die Zugänglichkeit des N1 beeinflussen können.

\subsection{Aspekte der Konzeptkombination}

\subsubsection{Vorüberlegungen}

Bei der Verbindung zweier Einzelausdrücke zu einem Kompositum werden auch die damit verbundenen Konzepte zu einem neuen NN-Konzept kombiniert (conceptual combination, vgl. Kap. 2.3.3). Diese Art der Verbindung wird oft mit adjektivischer Modifikation verglichen, was u.a. daran deutlich wird, dass das N1 in der englischprachigen Literatur meist als ,modifier' bezeichnet wird.

Bei der Modifikation eines Nomens durch ein Adjektiv entstehen v.a. Schnitt- und Teilmengenbildung. So bezeichnet z.B. kranker Freund die Schnittmenge aller kranken und aller befreundeten Individuen, analog bei begabter Violinist. Aber auch andere Modifikationsarten sind möglich, z.B. lässt sich fake gun nicht als Schnittmenge alles Künstlichen und aller Waffen beschreiben. Analog kann die Modifikation des N2 durch das N1 ganz unterschiedlicher Natur sein. Bei der Erstellung eines Gesamtkonzepts für das NN kommt es zu einer Interaktion der Ursprungskonzepte, die inferiert werden muss (vgl. Gagné/Spalding 2007).

[...] understanding a combined concept involves creating a new concept because a combined concept is more than a hybrid of its part. (Gagné/Spalding 2007:149)

Die entstandene Konzeptkombination kann sich in ihren Eigenschaften erheblich von den Eigenschaften der Ursprungskonzepte unterscheiden. Für adjektivische Modifikation führen Gagné/Spalding (2007:149) das Beispiel geschälter Apfel an: Während ein Apfel rot oder grün sein kann, ist er in geschältem Zustand hellgelb. Die Konzepteigenschaften ,Farbe' hat sich somit geändert. Auch der Beitrag von N1 und N2 zum Gesamtkonzept kann sich maßgeblich von den Konzepten der Simplizia unterscheiden, bspw. kann ein Bronzelöwe nicht brüllen und ein Hasenbraten keine Haken schlagen (vgl. dazu Partee 2003:1). Derartige Beispiele machen deutlich, dass es sich bei dem Wissen um die Konzepte und um die Beschaffenheit des Resultats der Interaktion um nicht-linguistisches Denotatwissen handelt.

Will man sich nun anaphorisch auf ein N1 beziehen, setzt dies zum einen voraus, dass das N1 unabhängig interpretiert werden kann und über eine eigenständige Repräsentation eines Diskursreferenten verfügt, auf den sich die Anapher beziehen kann. Zum anderen 
setzt es voraus, dass das N1-Konzept als Teil des NN-Konzepts nicht maßgeblich vom Konzept des entsprechenden Simplex abweicht, da der Bezug sonst wie im Fall des Wechsels zwischen metaphorischer und wörtlicher Ausdrucksverwendung unakzeptabel ist (vgl. Kap. 2.5.2). Daraus lässt sich die Annahme ableiten, dass, je mehr das Konzept des N1 vom Konzept des entsprechenden Simplex abweicht, die anaphorische Zugänglichkeit des N1 abnimmt und die Verarbeitungskosten durch zusätzliche Inferenz steigen.

Die konkrete Art der Kombination der Konzepte kann ganz unterschiedlich gestaltet sein, z.B. die Konstitution beschreiben oder eben auch eine Schnittmenge (vgl. Heuhaufen in Kap. 2.2.2). Eine Form der Systematisierung möglicher Konzeptkombinationen stellen die semantischen Relationen dar.

\subsubsection{Semantische Relation}

Dass die semantischen Relationen nicht Teil der Grammatik sind, wird u.a. daran deutlich, dass es sich dabei nicht um eine kategoriale Eigenschaft handelt, sondern verschiedene Einteilungen möglich sind (vgl. Kap. 2.2.4). Als Basis für die folgenden Untersuchungen dienen die neun Relationen von Levi (1978): CAUSE, HAVE, MAKE, USE, BE, IN, FOR, FROM, ABOUT 5

Betrachtet man nun akzeptable Fälle von N1-Bezügen wie (14-a) und unakzeptable Fälle von N1-Bezügen wie (14-b) in Hinblick auf die vorliegende semantische Relation, entsteht der Eindruck, dass die spezifische semantische Relation Einfluss auf die Art der Repräsentation des N1 und somit auch auf seine Zugänglichkeit hat. In diesem Fall liegt die Vermutung nahe, dass im Fall der BE-Relation eine geringere Zugänglichkeit besteht als im Fall der FROM-Relation.

(14) a. Heute sind Rindersteaks im Angebot. ?Da muss ich daran denken, was das für friedfertige Tiere sind.

b. Heute sind Schmetterlingssteaks im Angebot. *Da muss ich daran denken, was das für friedfertige Tiere sind.

Ähnliches zeigt sich beim Vergleich von Beispielen mit den Relationen HAVE und FOR. Insgesamt scheinen N1 in Komposita mit der HAVE-Relation wie unter (15) zugänglicher zu sein als N1 in Komposita mit der FOR-Relation wie unter (16).

(15) a. Oskar hat zum Geburtstag ein richtig dickes Bilderbuch bekommen. Nun sitzt er seit Tagen in seinem Zimmer und schaut sie sich mit Staunen an.

b. In seiner Freizeit kümmert sich Franz liebevoll um sein großes Rosenbeet. Er schneidet sie vorsichtig in Form und befreit sie regelmäßig von Unkraut.

(16) a. Aufgrund der vielen Bildschirmarbeit hat Philipp Augentropfen verschrieben bekommen. Allerdings tut er sich schwer, sie beim Anwenden offen zu halten.

b. Franz hat frischen Rosendünger gekauft. In diesem Jahr möchte er sie gern noch größer haben.

\footnotetext{
${ }^{5}$ Das Vorkommen weiterer semantischer Kategorien schließt auch Levi nicht aus, z.B. wohnen in, wachsen in oder räumliche und zeitliche Kategorien (vgl. Levi 1978 83).
} 
Dass metaphorisch verwendete N1 unzugänglich sind, lässt sich ebenfalls mit Hilfe der semantischen Relationen abbilden: Nach Levi lässt sich der metaphorische Gebrauch v.a. durch die BE-Relation ausdrücken (z.B. mother church, soldier ant, sister node).

Es konnte bisher allerdings nicht nachgewiesen werden, dass sich die verschiedenen semantischen Relationen in ihrer Komplexität unterscheiden (vgl. Shoben 1991). Auch ob die Relation bei der Kompositums-Interpretation aufgelöst wird, ist nicht final geklärt. Ob dies jedoch notwendig ist (vgl. Gagné/Spalding 2007:162) oder nicht (vgl. Klos 2011), spielt letztlich keine Rolle. Ob eine bestehende Systematik dem/der Sprachnutzer*in bewusst ist oder nicht, sagt nichts über den Einfluss der Systematik auf die mentale Verarbeitung aus.

Um systematische Aussagen zum Wesen der semantischen Relationen und ihrem tatsächlichen Einfluss auf die N1-Zugänglichkeit treffen zu können, seien zunächst zwei der neun Relationen für einen ausführlicheren Vergleich ausgewählt. Die Wahl fällt auf die Relationen HAVE und FOR, da hierfür beobachtungsgeleitet klare Tendenzen für die Zugänglichkeit bestehen. Außerdem handelt es sich um zwei Relationen, die, im Gegensatz zu z.B. HAVE und BE, relativ gut voneinander abgrenzbar sind. Schließlich ist die Unterscheidung zwischen beinhalten und benutzen eher objektivierbar als zwischen beinhalten und sein.

Die HAVE-Relation unterteilt Levi in $\mathrm{HAVE}_{1}$ und $\mathrm{HAVE}_{2} . \mathrm{HAVE}_{1}$ zählt sie zu den im Englischen weniger produktiven Relationen, $\mathrm{HAVE}_{2}$ und FOR zu den produktivsten (vgl. Levi 1978:86). HAVE 1 definiert Levi als: „function of identifying a possessed object or characteristic by naming both it and its ,possessor' (e.g., student power, horsepower, [...])“ (Levi 1978:89). Im Gegensatz dazu steht im Falle der HAVE 2 -Relation der Possesssor an der Kopfposition des Kompositums, z.B. in picture book. Im Folgenden beschränke ich mich auf die $\mathrm{HAVE}_{2}$-Relation. Die Eigenschaft des Possessors an der N2-Position betrachte ich als konkretes Beinhalten des N1 im wörtlichen Sinne, wie z.B. in Bilderbuch, vgl. (17). Beinhalten wiederum fasse ich als Umschließen des Schwerpunkts auf.

$$
\text { Bilderbuch } \rightarrow \text { Buch beinhaltet Bilder }
$$

Die FOR-Relation wird von Levi auch als „predicate of purpose“ beschrieben (vgl. Levi 1978:96). Dabei betont sie den Unterschied zwischen der semantischen Relation und dem lexikalischen Ausdruck for. Bei letzterem handelt es sich um einen weiter gefassten Begriff, während die semantische Relation viel konkreter umgrenzt ist. Die Semantik der FORKomposita definiert Levi dergestalt, dass das N2 für das N1 dienlich ist, wie z.B. in (18) (vgl. Levi 1978:96f).

$$
\text { Vogelkäfig } \rightarrow \text { Käfig für Vögel }
$$

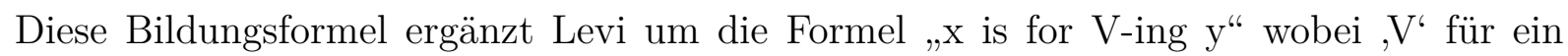
geeignetes Verb steht, z.B. beschützen im Fall von Vogelkäfig (vgl. Levi 1978:97). Manche dieser Verben seien mit Hilfe des N2 vorhersagbar, manche müssten individuell gelernt werden. Die Verbindung ist dann ein Element im mentalen Lexikon und kann nicht spontan interpretiert werden, z.B. das Abschwellen als Funktion von Nasentropfen (vgl. Levi 1978:97). Meiner Ansicht nach ist es hierfür jedoch nicht zwingend erforderlich, das Kompositum bereits zu kennen. Welt- und Kontextwissen können zur Bedeutungserschließung genügen, wie etwa in Beispiel (19). 
(19) a. Ich hab ganz trockene Augen, ich werde mir Augentropfen kaufen.

$\rightarrow$ Tropfen zum Befeuchten der Augen

b. Augenarzt zum Patienten: Um ihre Augen besser untersuchen zu können, bekommen sie diese Augentropfen.

$\rightarrow$ Tropfen zum Erweitern der Pupillen

In (19) liegt ein Kompositum in zwei verschiedenen FOR-Bedingungen vor; die ZweckBeziehung an sich kann demnach auch ambig sein (vgl. dazu Levi 1978:98). Eine konkretere Spezifizierung der FOR-Relation erachtet Levi jedoch nicht als zweckdienlich. Ziel sei es, eine möglichst breite Generalisierung zur Komposita-Produktion zu formulieren. Eine Spezifizierung bestimmter Verben könnte der vielseitigen Ambiguität, die ein Charakteristikum von kompositionalen Bildungen ist, nicht gerecht werden (vgl. Levi 1978: 97).

[T] he analysis provided here captures the essential generalization that all NPs of the form $\left[\mathrm{N}_{2}\right.$ for $\left.\mathrm{N}_{1}\right]$ (again, where for $>$ FOR) may be transformed into CNs of the form $\left[\mathrm{N}_{1}+\mathrm{N}_{2}\right]$ regardless of the possible verbs that could be supplied. In almost all cases, the predicate FOR indicates only this: that a relationship of intent or purpose exists (or is believed to exist) between the objects named by its two underlying arguments. (Levi 1978 97f)

Auch eine negative Art der Zweck-Beziehung kann somit abgedeckt werden, z.B. im Sinne von ,N2 ist gegen $\mathrm{N1}^{\text {‘ }}$ oder ,N2 dient der Beseitigung/Abschreckung von N1', vgl. (20) und (21) nach Levi (1978:99).

$$
\begin{aligned}
& \text { Rattengift } \rightarrow \text { Gift gegen/für die Beseitigung von Ratten } \\
& \text { a. fertility pills } \rightarrow \text { pills for fertility [to increase it] } \\
& \text { b. headache pills } \rightarrow \text { pills for headaches [to decrease it] }
\end{aligned}
$$

Beim Vergleich der Analysen der HAVE- und der FOR-Relation fällt auf, dass das N1 im Fall der HAVE-Relation eine zentrale Rolle für die Konstitution des Kompositums spielt. Das N1-Konzept ist, da es per definitionem im N2-Konzept ,enthalten' ist, ein zentraler Teil des NN-Konzepts. Bei der FOR-Relation hingegen ist das N1-Konzept eher eine Art Anwendungsgebiet, also nicht zwingend (zentraler) Teil des NN-Konzepts. Hierin könnte eine Ursache für Aktivierungsunterschiede liegen.

Insgesamt bleibt aber festzuhalten, dass keine systematischen Verallgemeinerungen gezogen werden können. Hierfür ist die Abgrenzung der Relationen untereinander zu unscharf und auch die semantische Heterogenität der Komposita innerhalb einer Relationsgruppe ist zu groß, so wird z.B. sowohl metaphorischer Gebrauch wie bei Schwesterknoten als auch die Beschreibung der Form wie bei Schmetterlingssteak von Levi unter der Relation BE zusammengefasst.

\subsubsection{Konzeptueller Fokus}

Im vorangegangenen Abschnitt wurde die Idee geschildert, dass die semantische Relation beeinflusst, wie zentral ein N1-Konzept für die Konstitution eines NN-Konzepts ist. Dies wiederum erweckt die Vorstellung von einer Art konzeptuellen Fokus bei Komposita. Unter dem konzeptuellen Fokus eines Kompositums verstehe ich das Teilkonzept des NN-Konzepts, das die zentralere Rolle im Gesamtkonzept spielt. 
Wie bereits in Kapitel 2.2.2 erläutert, hat ein klassisches NN seinen morphosyntaktischen und seinen semantischen Kopf im N2. Für die Festlegung des semantischen Kopfs wurden allerdings bereits Zweifelsfälle wie die Schnittmengenbildungen diskutiert. Handelt es sich also beim Denotat von Kuchenteller um einen Teller, denotiert Heuhaufen gleichzeitig Heu und einen Haufen sowie Hasenbraten sowohl einen Hasen als auch einen Braten. Das N1 und das N2 konstituieren sich in diesen Fällen gegenseitig. Unklar ist die Frage nach dem semantischen Kopf auch bei pleonastischen Zusammensetzungen wie Bilchmaus und Eichbaum (vgl. Bloomer 1996). Für derartige Fälle der Verlagerung des semantischen Kopfs nehme ich eine bessere N1-Zugänglichkeit an, da in diesen Fällen der konzeptuelle Fokus auf das N1 fällt oder zumindest weniger klar auf das N2 fällt, wie in Beispiel (22) demonstriert. Das N1 spielt hierbei eine zentralere Rolle als es bei klassischer Rechtsköpfigkeit der Fall ist. In diesem Sinne ist das NN weniger typisch.

(22) a. Die Kinder haben den ganzen Tag im Heuhaufen vor dem Haus gespielt. Abends haben sie es dann natürlich im ganzen Haus verteilt.

b. Am Sonntag gab es Hasenbraten. Die Kinder haben das Essen verweigert, weil sie ihn lieber weiter über den Hof gejagt hätten.

c. Helga hat im Ferienlager zum ersten Mal eine Bilchmaus gesehen. Wegen seiner großen Augen hat sie sich richtig erschreckt.6

d. Auf dem Dorfplatz steht ein massiver Eichenbaum. Paulas Opa schätzt, dass sie schon mindestens 100 Jahre alt ist.7

Die Verlagerung des konzeptuellen Kopfs lässt sich auch über semantische Relationen abbilden: Im Fall von Schnittmengenbildungen liegt die Relation FROM vor (der Braten besteht aus Hase, der Haufen aus Heu), bei den pleonastischen Zusammensetzungen BE (die Maus ist ein Bilch, der Baum ist eine Eiche), beim klar rechtsköpfigen Kuchenteller hingegen liegt die FOR-Relation vor.

Eine ähnliche Vorstellung von mehr oder weniger zentralen Bestandteilen komplexer Konzepte vertreten auch Cornish et al. (2005). Sie stellen die Zugänglichkeit impliziter Antezedenten durch (indirekte) pronominale Anaphern in einen Zusammenhang mit der $\mathrm{Nu}-$ klearität des Antezdens im Anker-Konzept (vgl. Kap. 2.4.2). Dabei ordnen sie den Stufen der Nuklearitäts-Skala unter (23) verschiedene Grade an Aktiviertheit entsprechend der Giveness Hierarchy zu (vgl. Cornish et al. 2005:374). Anhand von Lesezeitexperimenten im Englischen und Französischen belegen sie den Einfluss konzeptueller Nuklearität auf die Akzeptabilität pronominaler indirekter Anaphern. Eine Übertragung auf die Akzetabilität von N1-Bezügen erscheint mir plausibel.

Nuklearitätsskala nach Cornish et al. (2005)

nuklear $>$ peripher $>$ assoziativ

\footnotetext{
${ }^{6}$ Konfundierend wirkt in diesem Satz, dass der Ausdruck Bilchmaus veraltet ist, da es sich biologisch bei Bilchen um eine eigene Familie der Nagetiere handelt.

${ }^{7}$ In diesem Satz wird das Kompositum Eichenbaum im Gegensatz zu der von Bloomer verwendeten Form Eichbaum genutzt, um morphosytnatkische Reduktion des N1 als Störfaktor auszuschließen.
} 


\subsubsection{Raum-zeitliche Kontiguität (RZK)}

Sowohl bei den semantischen Relationen als auch beim Konzept des konzeptuellen Fokus handelt es sich nicht um Verarbeitungsmodelle. Darüber hinaus sind diese Eigenschaften vage und lassen keine eindeutige Kategorisierung von Komposita zu. Aus diesem Grund gilt es aus den Beobachtungen im Zusammenhang mit semantischen Relationen und dem konzeptuellen Fokus eine Eigenschaft abzuleiten, die trennschärfer und damit für eine experimentelle Überprüfung geeigneter ist.

Beim Vergleich von Fällen mit besserer N1-Zugänglichkeit (z.B. Bilderbuch, Heuhaufen, Rosenbeet) und mit schlechterer N1-Zugänglichkeit (z.B. Schmetterlingssteak, Rosendünger, Augentropfen) fällt auf, dass die Erstglieder in den gut zugänglichen Fällen bei der mentalen Visualisierung des NN zwingend präsent sind: Ein Denotat von Bilderbuch enthält Bilder und ein Denotat von Heuhaufen zwangsläufig Heu. Rosendünger hingegen kann unabhängig von der Anwesenheit von Rosen als Packung visualisiert werden, ebenso enthält die Repräsentation von Schmetterlingssteak keine Schmetterlinge. Man könnte also sagen, dass das NN-Konzept das Konzept des N1 im Sinne seines Konzepts als Simplex beinhaltet. Wenn ein NN-Denotat vorliegt, muss das entsprechende N1-Denotat ebenfalls raum-zeitlich vorhanden sein. Diese Eigenschaft des konzeptuellen Wissens über ein Kompositum und die Beziehung zwischen seinen Konstituenten bezeichne ich im Folgenden als ,raum-zeitliche Kontiguität‘ (RZK).

Mit der RZK ist nicht der allgemeine raum-zeitliche Bezug des N1 gemeint (vgl. Konkretheit), sondern seine raum-zeitliche Präsenz in Abhängigkeit vom N2. Dies bewirkt, dass ein und dasselbe N1 in Abhängigkeit vom jeweiligen N2 präsent oder absent sein kann. Ein Beet, das keine Rosen beinhaltet, würde i.d.R. nicht als Rosenbeet bezeichnet werden, für den Ausdruck Rosenbeet liegt somit raum-zeitliche Kontiguität der Konstituenten vor $(+\mathrm{RZK})$. Im Gegensatz dazu handelt es sich beim Denotat von Rosendünger um Granulat, welches zwar auf Rosenbeete gestreut wird, aber auch unabhängig von der Gegenwart von Rosen als Rosendünger bezeichnet werden würde (-RZK). Ein anderes Beispiel für raum-zeitliche Kontiguität ist Wasserfall, da Wasser ein zentraler Bestandteil des NN-Konzepts ist. Ein Bücherregal hingegen kann auch ohne die Anwesenheit von Büchern als Bücherregal bezeichnet werden, z.B. im Kontext einer Möbelausstellung. Es handelt sich also um ein Beispiel fehlender raum-zeitlicher Kontiguität. Weitere Beispiele sind in Tabelle 3.1 aufgeführt.

\begin{tabular}{l|l}
\hline \hline+ RZK & RZK \\
\hline Bilderbuch & Augentropfen \\
Rindersteaks & Schmetterlingssteak \\
Heuhaufen & Dosenöffner \\
Hasenbraten & Vogelkäfig \\
Bilchmaus & Feldmaus \\
Eich(en)baum & Kuchenteller \\
\hline \hline
\end{tabular}

Tabelle 3.1: Beispiele für eine Einteilung von Komposita nach raum-zeitlicher Kontiguität (RZK) der Konstituenten

In der individuellen Repräsentation, bzw. in der konkreten Ausgestaltung eines Denotats muss das N1 nicht zwingend präsent sein. Vielmehr geht es bei der RZK um Visulisierungstendenzen auf einer unspezifischen Ebene. 
Aus der Betrachtung der Komposita in Tabelle 3.1 und der entsprechenden Beispielsätze in (14) - (16) ergibt sich die Hypothese, dass im Fall von raum-zeitlicher Kontiguität der Kompositateile (+RZK) das N1 zentraler für das NN-Konzept ist als im Fall von fehlender raum-zeitlicher Kontiguität (-RZK). Bei einer Übertragung der semantischen Relationen auf die Beispiele in Tabelle 3.1 zeigt sich, dass sich in der Spalte ,+RZK häufiger HAVE-Relationen finden lassen als in der Spalte, - RZK' Dies wiederum deckt sich mit der Beobachtung bzgl. ihrer Zugänglichkeit und dem Eindruck, dass das N1 in Komposita mit einer HAVE-Relation zentraler für das Gesamtkonzept ist als in Fällen mit einer FOR-Relation.

Ein Grund für die Annahme, dass Komposita mit der Eigenschaft +RZK über eine höhere N1-Zugänglichkeit verfügen als Komposita mit der Eigenschaft -RZK liegt in der Annahme, dass je größer die Bedeutung des N1-Konzepts für das NN-Konzept ist, auch dessen Aktiviertheit größer ist, was sich auf die Zugänglichkeit auswirkt. Darüber hinaus besteht gemäß dem Wahrnehmungseffekt ein Verarbeitungsvorteil für raum-zeitlich gebundene Objekte (vgl. auch Langacker 1991).

Eine Möglichkeit der Objektivierung der Eigenschaft der RZK wäre ein Zeichenexperiment. Hierbei würden einer Reihe von Versuchspersonen nacheinander verschiedene Komposita präsentiert. Die Aufgabe der Versuchspersonen wäre es, diese Komposita zeichnerisch und in einer vorgegebenen Zeit skizzenhaft darzustellen. Anschließend würden die Zeichnungen dahingehend ausgewertet, wie groß und wie zentral das N1 in der Skizze dargestellt ist. Die Hypothese ist, dass die Visualisierungstendenz eines Ausdrucksteils etwas über dessen Bedeutung für das Konzept des Gesamtausdrucks aussagt. In diesem Sinne wäre im Fall von Wasserfall zu erwarten, dass zumindest Linien für fließendes Wasser gezeichnet werden. Im Fall von Bücherregal wäre eine reine Darstellung der Bretter denkbar. Gemittelt über eine statistisch aussagekräftige Anzahl an Versuchspersonen könnten sich somit Aussagen zu den Visualisierungstendenzen treffen lassen und die Eigenschaft der RZK objektivieren lassen.

\subsubsection{Interaktion der Teilkonzepte}

An die Frage nach der Bedeutung des N1-Konzepts für das NN-Konzept schließt sich die Frage an, wie sich Eigenschaften des N2 auf die Aktiviertheit des N1 auswirken. Meine Hypothese ist, dass ein N1 in einer NN-Repräsentation zentraler und damit zugänglicher ist, wenn das N2 weniger gut wahrnehmbar, z.B. abstrakt ist. Dies führt letztlich auch zu einer Verschiebung des konzeptuellen Fokus in Richtung N1 (vgl. Kap. 3.3.3). Verdeutlicht sei dies durch (24), Ein Einfluss der Relation ist an dieser Stelle auszuschließen, da in beiden Fällen die HAVE-Relation vorliegt. Dabei besteht die mentale Repräsentation des NN in (24-a) aus einem Baum, der Früchte trägt, in (24-b) hingegen besteht die Repräsentation des NN vorrangig aus Pflaumen, da das abstrakte N2 Traum kaum gegenständlich visualisierbar ist. Der N1-Bezug erscheint akzeptabler.

(24) a. Peter ist vom Pflaumenbaum gefallen. Sie purzelten minutenlang auf ihn herab.

b. Peter hatte einen Pflaumentraum. Sie purzelten minutenlang auf ihn herab. 8

${ }^{8}$ Bei Pflaumentraum handelt es sich um eine Ad-hoc-Bildung, die durch ihren Mangel an Konventionalisierung anfällig für die Verfügbarkeit unterschiedlicher Interpretationen ist. Die intendierte Inter- 
Ein weiterer Aspekt der Konzeptinteraktion, der sich in Zentralität, bzw. Nuklearität des N1 äußert, liegt in der Transparenz der Konstituenten (vgl. Kap. 2.6.2). Wie anhand der Beispiele in (25) zu sehen, scheint das transparente N1 in (25-a) zugänglicher, da das N2 opak ist. In diesem Fall liegt der Anknüpfungspunkt der NN-Repräsentation an die außersprachliche Welt sowie der konzeptuelle Fokus beim N1. Im Gegensatz dazu ist das N2 in (25-b) konzeptuell dominanter und bildet auch klar den semantischen Kopf. Das N1 ist hier kaum zugänglich.
a. Paul würde viel häufiger Kartoffelpuffer essen, wenn er $\underline{\text { sie }}$ dafür nicht selber reiben müsste.
b. Peter hat sich neue, teure Lederschuhe gekauft. Diesmal hat er sich vorge- nommen, es regelmäßig zu pflegen.

Diese Vorstellung lässt sich auch auf die Einteilung von Libben abbilden (63) in Kap. 2.3.4): Für TO-Komposita wird eine besser N1-Zugänglichkeit als für TT-Komposita prognostiziert. Für die OO- und OT-Komposita wird eine schlechte Zugänglichkeit prognostiziert, da die Interpretationen von N1 als Kompositums-Konstituente und als Simplex voneinander abweichen.

\subsubsection{Verarbeitungseffekte}

Gemäß dem CARIN-Modell (vgl. Gagné (2001), Gagné/Shoben (1997), Kap. 2.3.3) ist für die Verarbeitung von Komposita nicht die semantische Relation an sich relevant, sondern deren Auftretenshäufigkeit in Verbindung mit dem N1 und den konkurrierenden Alternativen (vgl. Gagné/Shoben 1997:81). Sollte nun nicht die Relation an sich die Verarbeitung beeinflussen, sondern entsprechend dem CARIN-Modell die Relations-Häufigkeit im Zusammenhang mit dem N1, stellt sich die Frage, ob und wie diese die N1-Zugänglichkeit beeinflusst.

Anhand des CARIN-Modells lässt sich die Annahme formulieren, dass Mechanismen, die die Gesamtinterpretation eines Kompositums erleichtern, die Verarbeitungstiefe und damit auch die Zugänglichkeit beeinflussen (vgl. Kap. 2.3.1). Darauf aufbauend lässt sich folgende Hypothese aufstellen: Je frequenter die Relation ist, mit der ein N1 auftritt, desto stärker wird das N1 aktiviert und desto zugänglicher ist es. Zur Veranschaulichung dieser Hypothese wurden exemplarisch die Komposita mit BERG- als N1 aus dem GermaNet (Henrich/Hinrichs 2011) betrachtet. Die Mehrheit dieser Komposita basiert auf der IN-Relation (ca. 75, z.B. Bergdorf), eine Minderzahl auf der ABOUT-Relation (ca. 5, z.B. Bergrecht). Gemäß der Annahme, dass eine höhere Relations-Häufigkeit die N1Zugänglichkeit erhöht, müsste (26-a) akzeptabler sein als (26-b). Dies scheint informellen Umfragen zufolge der Fall zu sein.

(26) a. Das Bergdorf feiert sein 750-jähriges Jubiläum. Zu diesem Anlass wird er mit viel Aufwand beleuchtet.

pretation besteht in einem Traum, in dem viele Pflaumen vorkommen. Ebenso plausibel wäre eine Interpretation als Traum, der eine sehr große Monster-Pflaume beinhaltet. Wird diese Interpretation präferiert, kommt es zwischen Anapher und Antezedens zu einem Numerus-Mismatch und das Beispiel ist weniger akzeptabel. 
b. Das Bergrecht feiert sein 750-jähriges Jubiläum. Zu diesem Anlass wird er mit viel Aufwand beleuchtet.

Andererseits ist ein Ausdruck Hofmeister (2011) folgend umso schwieriger zu verarbeiten, je komplexer er ist und je unerwarteter darin enthaltene Eigenschaften sind. Ein hoher Verarbeitungsaufwand resultiere wiederum in einer größeren Verarbeitungstiefe (deep processing), was wiederum in einem höheren Grad an Aktiviertheit und Zugänglichkeit resultiere. Demnach müssten die Beispiele unter (27) über zunehmende Verarbeitungsschwierigkeiten und dadurch auch über zunehmende Verarbeitungstiefe und Zugänglichkeit verfügen (vgl. Hofmeister 2011:391ff).
a. einfach: the dictator
b. komplex + typisch: the ruthless military dictator
c. komplex + untypisch: the loveable military dictator

Darauf aufbauend ließe sich argumentieren, dass bei Vorliegen von niedrig-frequenten Ausdrücken und untypischen Relationen Verarbeitungsschwierigkeiten entstehen, die eine größere Verarbeitungstiefe erfordern, was letztlich in besserer Zugänglichkeit resultiert.

In einem Lesezeitexperiment konnte Hofmeister nachweisen, dass die beiden komplexen Bedingungen (27-b) und (27-c) tatsächlich langsamer verarbeitet werden. Für die untypischen komplexen Ausdrücke wie (27-c) wurden die längsten Lesezeiten gemessen, d.h. wie erwartet verursachen diese die höchsten Verarbeitungskosten. Der Abruf erfolgte allerdings im Fall der typischen komplexen Ausdrücke wie (27-a) schneller. Daraus schlussfolgert er, dass sich eine höhere Aktiviertheit via ,deep processing' nicht belegen lässt. Eine Erleichterung des Abrufs lässt sich in Folge dessen nicht auf die Prozesskosten reduzieren (vgl. Hofmeister 2011:394). Darüber hinaus wurde bereits in Kapitel 2.3.1 thematisiert, dass von der reinen Verarbeitungsdauer nicht zwangsläufig auf den Grad der Verarbeitungstiefe geschlossen werden kann.

Die Ergebnisse von Hofmeister (2011) relativieren die Überlegungen zum Einfluss der Verarbeitungstiefe auf die Zugänglichkeit. Es lässt sich kein eindeutiger Zusammenhang zwischen Verarbeitungsvorteilen oder -schwierigkeiten, Verarbeitungstiefe und Aktiviertheit herstellen. Daraus leitet sich ab, dass Verarbeitungseffekte ohne empirische Belege in keinen direkten Zusammenhang mit der Zugänglichkeit gestellt werden können. Die Frequenz eines Ausdrucks oder einer Relation kann demnach nicht als Indiz für dessen Zugänglichkeit gewertet werden. Aus diesem Grund werden keine Hypothesen auf der Grundlage des CARIN-Modells verfolgt.

\subsubsection{Nuklearität}

In Kapitel 3.3 wurden verschiedene Aspekte der Kombination von Konzepten innerhalb von Komposita und deren Auswirkungen auf die N1-Zugänglichkeit beleuchtet. Dabei wurde festgestellt, dass je nach Art der Interaktion der konzeptuelle Fokus verschieden gelagert sein kann, d.h. dass das N1-Konzept über verschiedene Grade an Zentralität für das NN-Konzept verfügen kann. Dies deckt sich mit der Vorstellung von Cornish et al. (2005), dass Teile einer kognitiven Domäne über unterschiedliche Grade an Nuklearität verfügen können und dies wiederum Einfluss auf die Zugänglichkeit dieser Teile hat. In 
Analogie nehme ich an, dass ein N1 umso zugänglicher ist, je zentraler/nuklearer es für die NN-Repräsentation ist.

Der Grad an Zentralität/Nuklearität des N1 scheint z.B. von der semantischen Relation beeinflusst zu werden, die zwischen den Komposita-Konstituenten besteht. Exemplarisch wurden die Relationen HAVE und FOR näher betrachtet. Hierbei wurde ein stärkerer N1-Fokus im Fall von HAVE beobachtet.

Ausgehend von der Annahme, dass die spezifische Relation Einfluss auf die N1-Zugänglichkeit hat, die Einteilung von Komposita nach semantischen Relationen jedoch oft nur vage möglich ist, wurde ein Versuch der Abstraktion unternommen. Dabei kristallisierte sich die raum-zeitliche Kontiguität der Konstituenten (RZK) als geeignete Eigenschaft heraus.

Darüber hinaus wird ein Einfluss auf den konzeptuellen Fokus durch Eigenschaften wie Konkretheit oder Transparenz vermutet, die zwischen den Konstituenten verschieden ausgeprägt sein können. Ist das N1 konkret/transparent, während das N2 abstrakt/opak ist, spielt das N1 auch bei der Visualisierung eine größere Rolle und sollte somit zugänglicher sein. Dies lässt sich bspw. auf (28) anwenden: Beim N1 Croissant handelt es sich um einen konkreten Ausdruck, beim N2 Gerüche um einen relativ abstrakten. Der konzeptuelle Fokus liegt auf dem N1 und dieses ist somit relativ gut zugänglich.

[...] das Fenster ist offen und es ziehen die ganze Zeit Croissant-Gerüche von dem Bäcker unter uns zu mir hoch. Ich mag die ja nicht mal, aber [...] (vgl. 1)

Schließlich wurde herausgearbeitet, dass Eigenschaften, die Einfluss auf die Verarbeitung von Komposita haben, nicht unbedingt Einfluss auf die Zugänglichkeit des N1 haben müssen. Ein Zusammenhang zwischen Verarbeitungsschwierigkeit, Verarbeitungstiefe und Aktiviertheit lässt sich nicht sicherstellen.

Es lässt sich zusammenfassen, dass die involvierten Teilkonzepte nicht nur Einfluss auf das Konzept des Gesamtkompositums haben, sondern auch auf die Aktiviertheit des N1. Nachdem in Kapitel 3.2 die Unikalität der N1-Repräsentation als wichtigste Eigenschaft zur Steigerung der N1-Zugänglichkeit ausgemacht wurde, kommt nun der Grad an Nuklearität des N1-Konzepts hinzu.

\subsection{Experiment 1: Animatheit und Relation}

\subsubsection{Faktoren}

Einige Annahmen aus den vorhergehenden Abschnitten sollen nun in einem ersten Experiment überprüft werden. Ziel dieser Pilotstudie ist es in erster Linie, Erkenntnisse über die allgemeine Akzeptabilität und die Produktivität von N1-Bezügen sowie über verschiedene Resolutionsstrategien zu erlangen. Darüber hinaus gilt es ein geeignetes experimentelles Design für weitere Experimente zu entwickeln.

Des Weiteren sollen erste Faktoren identifiziert werden, die die Zugänglichkeit beeinflussen. Dabei wird je eine Eigenschaft aus dem Bereich des konzeptuellen N1-Wissens und aus dem Bereich der Konzeptkombination ausgewählt. 
Als ein Faktor aus dem Bereich des N1-Wissens (vgl. Kap. 3.2.1) wird die Animatheit herangezogen. Da es sich hierbei um einen Faktor handelt, dessen prinzipieller Einfluss auf die anaphorische Zugänglichkeit bereits nachgewiesen wurde (vgl. Fukumura/van Gompel 2011), ist nun die Frage, ob dies auch im Fall von Komposita-Erstgliedern gilt.

Der Faktor ANIMATHEIT ${ }^{9}$ ist in Experiment 1 als zweistufiger Faktor mit den Bedingungen ,+animat' und ,-animat' realisiert. Als animate Erstglieder wurden Bezeichnungen für Tierarten gewählt, da im Fall von Bezeichnungen für Menschen oder Menschen-Gruppen an der N1-Position bei der Interpretation des Kompositums ein größerer Grad an Vagheit besteht, z.B. eine Ambiguität zwischen individueller und institutioneller Lesart. Gemäß der Animatheitsskala nach Aissen (2003) liegen Bezeichnungen für Tiere in Bezug auf ihre Zugänglichkeit zwischen Menschen und unbelebten Objekten.

Aus dem Bereich des Wissens über die Konzeptkombination (vgl. Kap. 3.2.2) wird die semantische Relation als Faktor herangezogen. Da die semantischen Relationen als Ausgangspunkt für darauf aufbauende Konzepte wie dem konzeptuellen Fokus dienen, müssen sie in einem ersten Schritt in ihrem Einfluss bestätigt werden.

Der Faktor RELATION ist ebenfalls als zweistufiger Faktor realisiert. Die Komplexität des Experiments bei der Realisierung aller neun Relationen wäre enorm und das Set an Relationen stellt zudem kein Verarbeitungsmodell dar. Aus diesem Grund wurde der Faktor auf den Unterschied zwischen den beiden Relationen HAVE und FOR reduziert. Beobachtungsgeleitet wird hier ein Zugänglichkeitsunterschied durch einen Unterschied im konzeptuellen Fokus vermutet (vgl. Kap. 3.3.2).

\subsubsection{Methode}

Experiment 1 ist als explorative Pilotstudie zur Validierung grundlegender Tendenzen der N1-Zugänglichkeit angelegt. Es wurde eine Methode zur Analyse der Offline-Verarbeitung gewählt, da somit relativ einfach eine solide Datengrundlage geschaffen werden kann.

Eine mögliche Methode wäre eine Rating-Studie mit Akzeptabilitäts-Bewertung. Problematisch an dieser Methode ist, dass den Versuchspersonen (VPn) das Satzmaterial vorgegeben und von diesen qualitativ bewertet wird. Unabhängig von der Aufforderung, lediglich ihre Intuition als Korrektiv zu nutzen, agieren VPn im Modus der Bewertung stark normgeleitet (vgl. Schlesewsky et al. 2006:125f). Zudem spiegeln metalinguistische Urteile keine natürliche Sprachverarbeitung wider (vgl. Baayen 2014:117). Unter der Voraussetzung, dass N1-Bezüge generell selten sind und z.T. auch als Abweichung von der Norm betrachtet werden (vgl. Fabricius-Hansen 1993), bilden Ergebnisse eines Ratings somit nicht die tatsächliche Akzeptabilität ab. Ein weiteres Problem einer solchen Studie liegt darin, dass der Grund für eine konkrete Bewertung nicht auf eine konkrete Stelle im Satzmaterial zurückgeführt werden kann. Artefakte des Satzmaterials könnten dadurch einen starken Einfluss auf das Gesamtergebnis haben.

Eine direkte Resolutionsabfrage, bei der die VPn z.B. in Form einer Multiple-Choice-Task ein Antezedens auswählen, würde eindeutige Ergebnisse liefern. Allerdings hätte ein solche Aufgabe den Nachteil, dass die VPn auf den Untersuchungsgegenstand ,Pronomenresolution' aufmerksam gemacht würden.

Aufgrund dieser Probleme fällt die Wahl auf eine Satzergänzungsaufgabe mit einem Pronomen als Satzfragment. Im Zuge der Satzergänzung muss eine Resolution des Pronomens

\footnotetext{
${ }^{9}$ Zur Abgrenzung zwischen einer allgemeinen Eigenschaft und einem experimentellen Faktor sind Faktorbezeichnungen stets in Kapitälchen gesetzt.
} 
erfolgen, die anschließend interpretiert und ausgewertet werden kann. Als Methode zur Erforschung von Fragestellungen zur Pronomenresolution wird die Satzergänzungsaufgabe bereits seit den 1970er-Jahren eingesetzt (u.a. Garvey/Caramazza 1974) und findet auch in der aktuellen Anaphernforschung Anwendung (u.a. Ellert/Holler 2011). Die Satzergänzungsaufgabe ermöglicht die subtile Gewinnung von explorativen Daten zu verfügbaren Strategien der Anaphernresolution. Dahingehend sind die Daten natürlicher und weniger normativ. Letztlich lässt sich aus den Ergebnissen auch ableiten, ob die VPn die Zielstruktur des N1-Bezugs überhaupt bilden.

Bei der Satzergänzungsaufgabe handelt es sich um eine Kombination der Aufgaben ,Lesen“ und ,Vervollständigen' Prozesse der Sprach-Perzeption und -Produktion verbindet (vgl. Kaiser et al. 2011). Gleichzeitig erlaubt der produktive Teil Einsichten in die möglichen Formen der Bezugnahme.

Das Experiment wurde als Webfragebogen browserbasiert mit Sosci Survey 2.3 (Leiner 2013) erstellt. Die Satzergänzungen wurden von den VPn in ein Freitextfeld eingegeben. Anschließend erfolgte eine Annotation der Satzergänzungen in Hinblick auf die Anaphernresolution, d.h. es wurde im Nachhinein interpretiert, ob sich eine VP mit der Anapher im Kontext einer Satzergänzung auf das Antezedens bezieht oder nicht. Die Anzahl der N1-Bezüge wird als kategorialer Wert ausgewertet. Die Varianz in der Anzahl an Bezugnahmen zwischen den einzelnen Bedingungen spiegelt die Gradienz der N1-Zugänglichkeit wider und erlaubt Aussagen zur Einflussstärke der untersuchten Faktoren.

\subsubsection{Material}

Durch die Kombination der beiden zweistufigen Faktoren ergeben sich die vier Bedingungen -animat/HAVE, -animat/FOR, +animat/HAVE und +animat/FOR (vgl. Tab. 3.2). Für jede Bedingung wurden acht Komposita gebildet, woraus sich 32 experimentelle Items (Stimuli) ergeben.

\begin{tabular}{cccl}
\hline \hline Bed. & ANIMATHEIT & RELATION & Beispiel \\
\hline 1 & -animat & HAVE & Rosenbeet \\
2 & -animat & FOR & Dosenöffner \\
3 & + animat & HAVE & Hühnerstall \\
4 & +animat & FOR & Hundenapf \\
\hline \hline
\end{tabular}

Tabelle 3.2: Bedingungen und Beispiele für die in Experiment 1 verwendeten Komposita

Die FOR-Relation ist in der animaten Bedingung in drei Fällen eine , gegen'-Relation vor (Läusekamm, Insektenspray und Rattengift).

Das N1 liegt an der Oberfläche stets im Plural vor und das Kompositum ist in einen Kontextsatz eingebettet. An diesen Kontextsatz schließt sich ein zu vervollständigendes Satzfragment an, welches ausschließlich aus dem Plural-Pronomen Sie besteht. Das Satzfragment wurde bewusst auf die Anapher reduziert, um den VPn bei der Satzvervollständigung einen möglichst breiten Assoziationsspielraum einzuräumen. Durch das Fehlen eines Verbs ist der Numerus der Anapher nicht festgelegt, d.h. auch eine Interpretation des Pronomens als Singular feminin ist möglich. 
Ein Item besteht demnach jeweils aus einem Kontextsatz, welcher ein Kompositum enthält sowie einem sich anschließendem Satzfragment, welches aus dem Pronomen Sie besteht. Beispiel-Stimuli sind in Tabelle 3.3 wiedergegeben. Das vollständige Satzmaterial befindet sich in Anhang A,

Bei der Erstellung der Stimuli wurde darauf geachtet, dass das N1 der einzige Ausdruck ist, der mit der Anapher kongruent ist. Dementsprechend beinhalten die Kontextsätze keine (weiteren) femininen oder pluralischen Ausdrücke. Das N2 ist in $50 \%$ der Fälle maskulin und in $50 \%$ neutrum und stets inanimat.

\begin{tabular}{|c|c|c|c|}
\hline ANIMATHEIT & RELATION & $\begin{array}{l}\text { Ante- } \\
\text { zedens }\end{array}$ & Bsp.-Stimulus \\
\hline -animat & HAVE & N1 & $\begin{array}{l}\text { Leon hat in seinem Vorgarten das bunte Rosen- } \\
\text { beet angelegt. Sie ... }\end{array}$ \\
\hline -animat & HAVE & NP & $\begin{array}{l}\text { Leon hat in seinem Vorgarten das bunte Beet } \\
\text { mit den Rosen angelegt. Sie ... }\end{array}$ \\
\hline -animat & FOR & N1 & $\begin{array}{l}\text { Mirko hat zum Campen den praktischen Dosen- } \\
\text { öffner mitgenommen. Sie ... }\end{array}$ \\
\hline - animat & FOR & NP & $\begin{array}{l}\text { Mirko hat zum Campen den praktischen Öffner } \\
\text { für die Dosen mitgenommen. Sie ... }\end{array}$ \\
\hline +animat & HAVE & N1 & $\begin{array}{l}\text { Günther hat heute Morgen den alten Hühner- } \\
\text { stall ausgemistet. Sie ... }\end{array}$ \\
\hline +animat & HAVE & NP & $\begin{array}{l}\text { Günther hat heute Morgen den alten Stall mit } \\
\text { den Hühnern ausgemistet. Sie ... }\end{array}$ \\
\hline +animat & FOR & N1 & $\begin{array}{l}\text { Florian hat im Auslauf den großen Hundenapf } \\
\text { aufgefüllt. Sie ... }\end{array}$ \\
\hline +animat & FOR & NP & $\begin{array}{l}\text { Florian hat im Auslauf den großen Napf für die } \\
\text { Hunde aufgefüllt. Sie ... }\end{array}$ \\
\hline
\end{tabular}

Tabelle 3.3: Beispiel-Stimuli für alle Bedingungen in Experiment 1

Die Kontextsätze beginnen mit einem menschlichen Handlungsträger. Dessen Bezeichnung wurde dergestalt ausbalanciert, dass in $50 \%$ der Fälle ein Eigenname vorliegt und in $50 \%$ der Fälle eine Berufsbezeichnung. Die Handlungsträger sind stets maskulin und Singular. Die Zeitform in den Sätzen ist Perfekt. Die Komposita werden stets definit eingeführt und durch ein Adjektiv verlängert. Dies hat den Grund, dass komplexe Ausdrücke tendenziell länger fixiert werden als einfache (vgl. Sereno 1992,306). Die größere Verarbeitungsdauer soll einer Good-Enough-Repräsentation des Kompositums entgegenwirken.

Zur Überprüfung der Reliabilität des experimentellen Designs wurde zusätzlich der Kontrollfaktor FORM eingefügt: Neben der Verwendung in einem Kompositum kommt jedes N1 zusätzlich als Antezedens in einer phrasalen Struktur vor. Die Möglichkeit der Paraphrasieung dient gleichzeitig als Kontrolle der Transparenz des Kompositums.

Die Items wurden anhand der Kontrollbedingung gleichmäßig auf zwei Listen aufgeteilt. Ungeachtet der phrasalen Kontrollbedingung, handelt es sich um ein gemischtes Design, bei dem ,within subjects', aber ,between items' getestet wurde. Dies liegt daran, dass es sich bei der ANImATHeit um einen denotatsgebundenen Faktor handelt, d.h. ein Item kann nur animat oder inanimat sein. 
Zusätzlich zu den 32 experimentellen Items wurden 24 Filler-Items erstellt, um das experimentelle Design zu maskieren. Bei den Filler-Items handelt es sich um ein unabhängiges Experiment zur Abhängigkeit von Verb-Bias und Anapherart. Dabei wurden Verben mit NP1- und NP2-Bias mit Personal- und Demonstrativ-Pronomen kombiniert.

\subsubsection{Hypothesen}

Die Hypothesen für Experiment 1 sind:

(i) Ein N1-Bezug ist generell möglich und eine seltene, aber dennoch produktive Form des Sprachgebrauchs.

(ii) Die Etablierung einer direkten Anapher ist die präferierte Form der Anaphernresolution, dies sollte sich in der Auswertung des Kontrollfaktors FORM widerspiegeln. Daher wird eine signifikant höhere Anzahl an Bezügen auf das avisierte Antezedens in der phrasalen Bedingung erwartet. Ist dies der Fall, können die weiteren Daten als reliabel angesehen werden. Eine Auswirkung der Manipulation der Faktorstufen auf die phrasale Bedingung wird nicht vorhergesagt.

(iii) Für den Faktor ANIMATHEIT werden mehr N1-Bezüge in der Bedingung +animat als in der Bedingung -animat vorhergesagt. Damit wird die Übertragbarkeit des von Fukumura/van Gompel (2011) aufgezeigten Zugänglichkeitsfaktors von phrasalen Referenten auf Komposita-Erstglieder belegt. Zudem wäre dies ein Anzeichen für einen Lebenswelteffekt auf Basis der Animatheitsskala nach Aissen (2003).

(iv) Für den Faktor RELATION werden mehr N1-Bezüge in der Bedingung HAVE als in der Bedingung FOR vorhergesagt. Beobachtungsgeleitet wird hier ein Unterschied im konzeptuellen Fokus angenommen, der sich in einer stärkeren Fokussierung auf das N1 im Fall der HAVE-Relation mit einem damit einhergehenden Zugänglichkeitsvorteil ausdrückt.

(v) Eine Interaktion der Faktoren wird nicht vorhergesagt.

(vi) Für die Fälle, in denen kein Bezug auf das N1 erfolgt, wird eine explorative Auswertung der Resolutionsstrategien durchgeführt.

\subsubsection{Durchführung}

\section{Ablauf}

Nachdem eine VP die URL des Webfragebogens aufgerufen hat, erscheint ein Begrüßungsbildschirm, der Informationen und Instruktionen zur Durchführung des Experiments enthält. Anschließend folgt eine Seite mit Fragen zu demographischen Daten. Schließlich erscheinen die Items mit Freitextfeldern in randomisierter Reihenfolge. Die VPn wurden nun aufgefordert eine Satzergänzung in das Freitextfeld einzugeben (vgl. Abb. 3.1). Probeläufe zeigen, dass das Ausfüllen eines Fragebogens etwa 30 Minuten in Anspruch nimmt. Jeder VP wurde jedes Item entweder in einer Bedingung als Kompositum oder als Phrase präsentiert. Zusammen mit den Fillern hatte jede VP 56 Satzvervollständigungen einzugeben. 


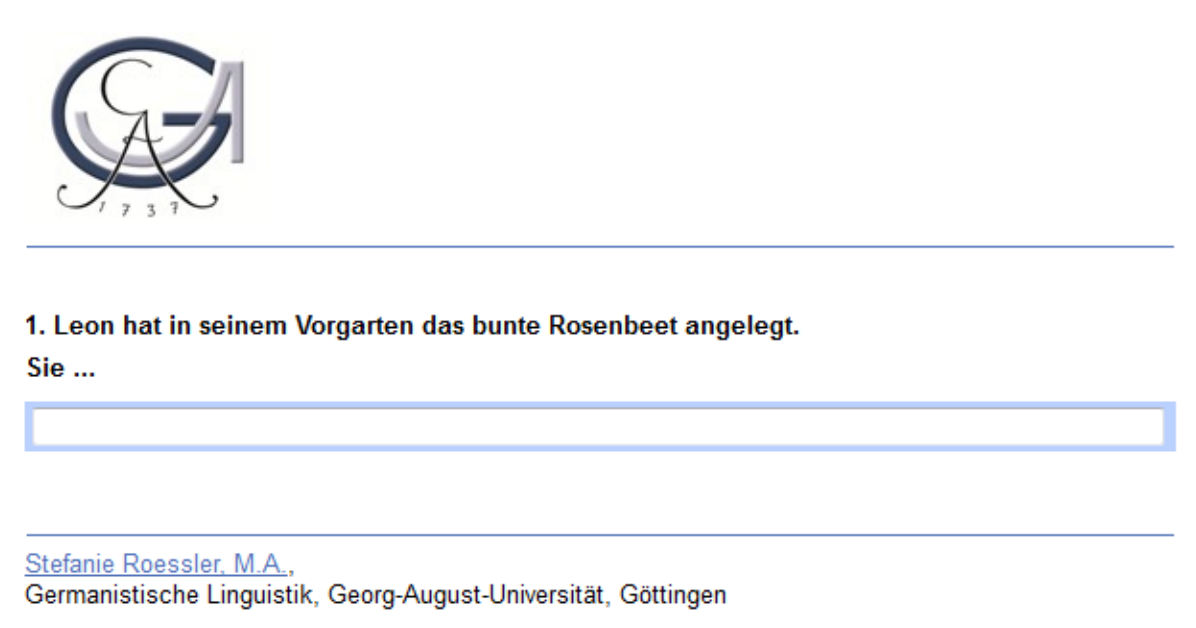

Abbildung 3.1: Screenshot aus Experiment 1 (Sosci Survey 2.3)

\section{Technik}

Das Experiment wurde mit Hilfe von SoSci Survey 2.3 (Leiner 2013) erstellt und browserbasiert als Webfragebogen von den VPn individuell auf ihren privaten Computern durchgeführt. Die URL für den Webfragebogen wurde als Link über verschiedene Portale der Universität Göttingen an Studierende verteilt.

\section{Versuchspersonen}

Insgesamt wurden 41 Datensätze erhoben. Im Zeitraum vom 25.07.2013 - 21.08.2013 wurden 39 VPn erhoben, von denen 3 Datensätze entfernt wurden, da die VPn nicht Deutsch als Muttersprache angaben. Bei der Sichtung der Antworten wurden darüber hinaus zwei unvollständige Datensätze entfernt. Zur Erhöhung der Stichprobengröße wurden am 26.08.2013 zwei VPn nacherhoben.

Für die Auswertung liegen somit letztlich Datensätze von 36 VPn vor.10 Davon waren 26 VPn weiblich und 10 männlich. Der Altersdurchschnitt lag bei 22,9 Jahren (Std. Abw. $=2.9)$.

\section{Datenaufbereitung}

Nach Abschluss der Erhebung wurden die Satzergänzungen hinsichtlich der Anaphernresolution interpretiert und kategorial kodiert. Dabei wurden N1-Bezüge mit ,1', sonstige Bezüge mit , $0^{`}$ und uninterpretierbare, ambige oder fehlende Satzergänzungen mit ,NA kodiert. Die Kodierung erfolgte durch zwei Annotatoren und einen Schlichter.

${ }^{10}$ Dies entspricht $17-20$ Datenpunkte für jedes Item in jeder Bedingung. Die Verteilung auf die beiden Listen ist 20:16. Die Ungleichverteilung ergibt sich aus der Tilgung einzelner Datensätze. Die Listenvergabe bei der Nacherhebung war nicht zu beeinflussen. 


\subsubsection{Auswertung}

Die Auswertung wurde mit Hilfe von R 3.2 (R Core Team 2013) durchgeführt. Dabei wurden die Plots mit Hilfe des Pakets „ggplot2“ (Wickham 2009) und die Inferenzstatistik mit dem Paket „lme4“ erstellt (Bates et al. 2013).

\section{Deskriptive Statistik}

Ein Vergleich zwischen phrasaler und Kompositabedingung (Faktor FORM) ermöglicht die Validierung des Designs und eine Einschätzung des generellen Verhaltens der VPn. Wie Tabelle $3.4 \mathrm{zu}$ entnehmen ist, wurde in der Kompositabedingung in $42 \%$ der Fälle ein N1-Bezug hergestellt. In der phrasalen Bedingung liegt der Bezug auf das entsprechende Antezedens bei $66 \%$ und damit deutlich höher.

\begin{tabular}{ccccc}
\hline \hline Faktor & Bed. & MW & Std. Abw. & $\mathrm{n}$ \\
\hline \multirow{2}{*}{ FORM } & Komp. & 0.42 & 0.49 & 561 \\
& Phrase & 0.66 & 0.47 & 568 \\
\hline \hline
\end{tabular}

Tabelle 3.4: Deskriptive Statistik der relativen Häufigkeit der N1-Bezüge für den Faktor FORM

Bei der Betrachtung der Kompositabedingung ergibt sich für den Faktor ANIMATHEIT ein höherer Mittelwert für die animaten N1 (vgl. Tab. 3.5) und für den Faktor RELATion ein höherer Mittelwert im Fall der HAVE-Relation (vgl. Tab. 3.6). Die Mittelwerte für die beiden Faktoren in Abhängigkeit voneinander sind in Tabelle 3.7] und Abbildung 3.2 wiedergegeben.

\begin{tabular}{ccccc}
\hline \hline Faktor & Bed. & MW & Std. Abw. & $\mathrm{n}$ \\
\hline \multirow{2}{*}{ ANIMATHEIT } & +animat & 0.58 & 0.49 & 278 \\
& -animat & 0.27 & 0.44 & 283 \\
\hline \hline
\end{tabular}

Tabelle 3.5: Deskriptive Statistik der relativen Häufigkeit der N1-Bezüge für den Faktor ANIMATHEIT für die Kompositabedingung

\begin{tabular}{ccccc}
\hline \hline Faktor & Bed. & MW & Std. Abw. & $\mathrm{n}$ \\
\hline \multirow{2}{*}{ RELATION } & FOR & 0.38 & 0.49 & 282 \\
& HAVE & 0.46 & 0.50 & 279 \\
\hline \hline
\end{tabular}

Tabelle 3.6: Deskriptive Statistik der relativen Häufigkeit der N1-Bezüge für den Faktor RELATION für die Kompositabedingung 


\begin{tabular}{ccccc}
\hline \hline ANIMATHEIT & RELATION & MW & Std. Abw. & $\mathrm{n}$ \\
\hline \multirow{2}{*}{ tanimat } & FOR & 0.56 & 0.50 & 140 \\
& HAVE & 0.60 & 0.49 & 138 \\
\hline \multirow{2}{*}{-animat } & FOR & 0.20 & 0.40 & 142 \\
& HAVE & 0.33 & 0.47 & 141 \\
\hline \hline
\end{tabular}

Tabelle 3.7: Deskriptive Statistik der relativen Häufigkeit der N1-Bezüge für die Faktoren ANIMATHEIT und RELATION für die Kompositabedingung

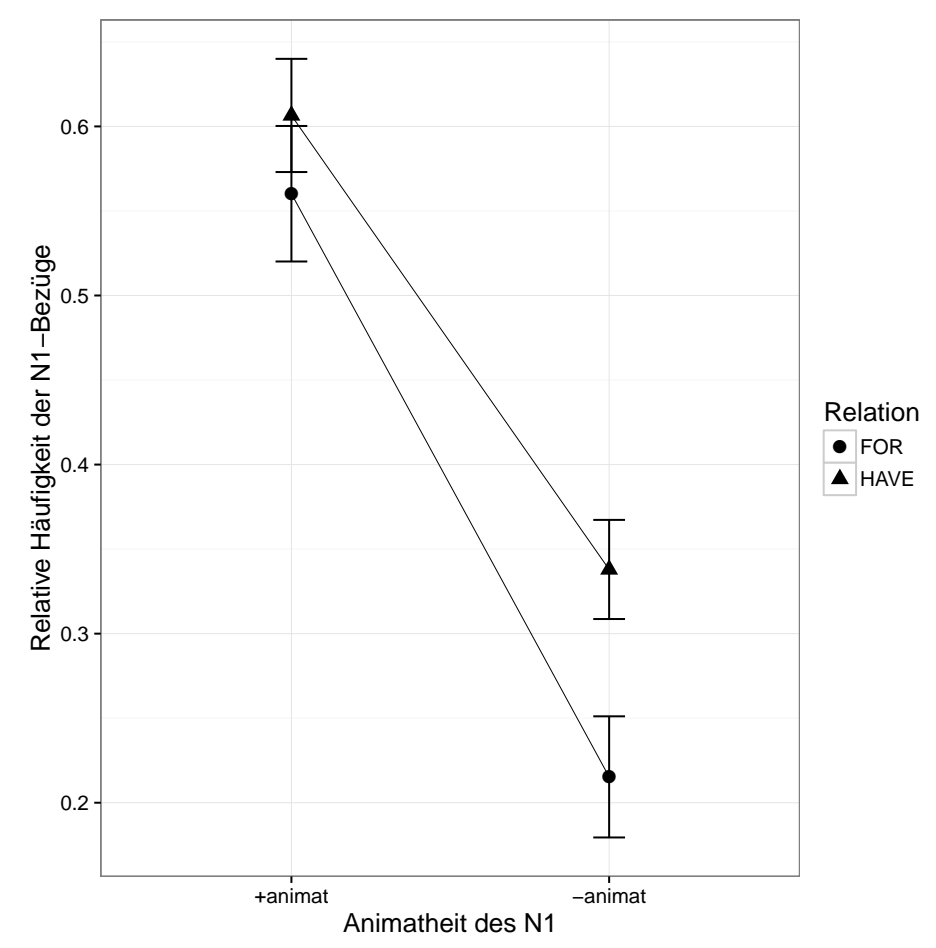

Abbildung 3.2: Plot der um die Zwischensubjektvarianz korrigierten Mittelwerte der N1-Bezüge ( $y$-Achse) in Abhängigkeit von den Faktoren ANIMATHEIT und RELATION für die Kompositabedingung; die Fehlerbalken repräsentieren +/- 1 Standardfehler des Mittelwerts

Entsprechende Berechnungen wurden für die phrasale Bedingung wiederholt (vgl. Tab. 3.8. 3.9 und 3.10). Die Unterschiede zwischen den Bedingungen scheinen geringer, aber in die gleiche Richtung ausgeprägt wie im Fall der Kompositabedingung.

\begin{tabular}{ccccc}
\hline \hline Faktor & Bed. & MW & Std. Abw. & $\mathrm{n}$ \\
\hline \multirow{2}{*}{ ANIMATHEIT } & +animat & 0.76 & 0.43 & 285 \\
& -animat & 0.56 & 0.50 & 283 \\
\hline \hline
\end{tabular}

Tabelle 3.8: Deskriptive Statistik der relativen Häufigkeit der N1-Bezüge für den Faktor ANIMATHEIT für die phrasale Bedingung 


\begin{tabular}{ccccc}
\hline \hline Faktor & Bed. & MW & Std. Abw. & $\mathrm{n}$ \\
\hline \multirow{2}{*}{ RELATION } & FOR & 0.62 & 0.49 & 284 \\
& HAVE & 0.70 & 0.46 & 284 \\
\hline \hline
\end{tabular}

Tabelle 3.9: Deskriptive Statistik der relativen Häufigkeit der N1-Bezüge für den Faktor RELATION für die phrasale Bedingung

\begin{tabular}{ccccc}
\hline \hline ANIMATHEIT & RELATION & MW & Std. Abw. & $\mathrm{n}$ \\
\hline \multirow{2}{*}{ tanimat } & FOR & 0.72 & 0.45 & 144 \\
& HAVE & 0.80 & 0.40 & 141 \\
\hline \multirow{2}{*}{-animat } & FOR & 0.51 & 0.50 & 140 \\
& HAVE & 0.61 & 0.49 & 143 \\
\hline \hline
\end{tabular}

Tabelle 3.10: Deskriptive Statistik der relativen Häufigkeit der N1-Bezüge für die Faktoren ANIMATHEIT und RELATION für die phrasale Bedingung

In Tabelle 3.11 und Abbildung 3.3 sind alle drei Faktoren in Beziehung zueinander gesetzt.

\begin{tabular}{cccccc}
\hline \hline FORM & ANIMATHEIT & RELATION & MW & Std. Abw. & $\mathrm{n}$ \\
\hline \multirow{4}{*}{ Komp. } & \multirow{2}{*}{+ animat } & FOR & 0.56 & 0.50 & 140 \\
& & HAVE & 0.60 & 0.49 & 138 \\
\cline { 2 - 6 } & \multirow{2}{*}{ animat } & FOR & 0.20 & 0.40 & 142 \\
& HAVE & 0.33 & 0.47 & 141 \\
\hline \multirow{3}{*}{ Phrase } & \multirow{2}{*}{+ animat } & FOR & 0.72 & 0.45 & 144 \\
\cline { 2 - 6 } & \multirow{2}{*}{-animat } & HAVE & 0.80 & 0.40 & 141 \\
\hline \hline
\end{tabular}

Tabelle 3.11: Deskriptive Statistik der relativen Häufigkeit der N1-Bezüge für die Faktoren FORM, ANIMATHEIT und RELATION

\section{Inferenzstatistik}

Für die inferenzstatistische Auswertung wurden Linear Mixed-Effects Models (LMEMs) mit logistischer Regression verwendet (vgl. Barr et al. 2013). Zunächst wurde der Unterschied zwischen Komposita- und phrasaler Bedingung betrachtet. Wie in Tabelle 3.12 zu sehen, erweist sich der Einfluss der FORM des Antezedens auf die Bezugszahlen als signifikant, d.h. es wurden signifikant mehr Bezugnahmen auf das dem N1 entsprechende phrasale Antezedens vorgenommen als auf das N1 im Kompositum 11

\footnotetext{
${ }^{11}$ Modellgleichung in $\mathrm{R}$ :

Generalized linear mixed model fit by maximum likelihood (Laplace Approximation) ['glmerMod']

Family: binomial ( logit)

Formula: resolution anker + (1+anker|subject $)+(1+a n k e r \mid$ item $)$

Data: d
} 


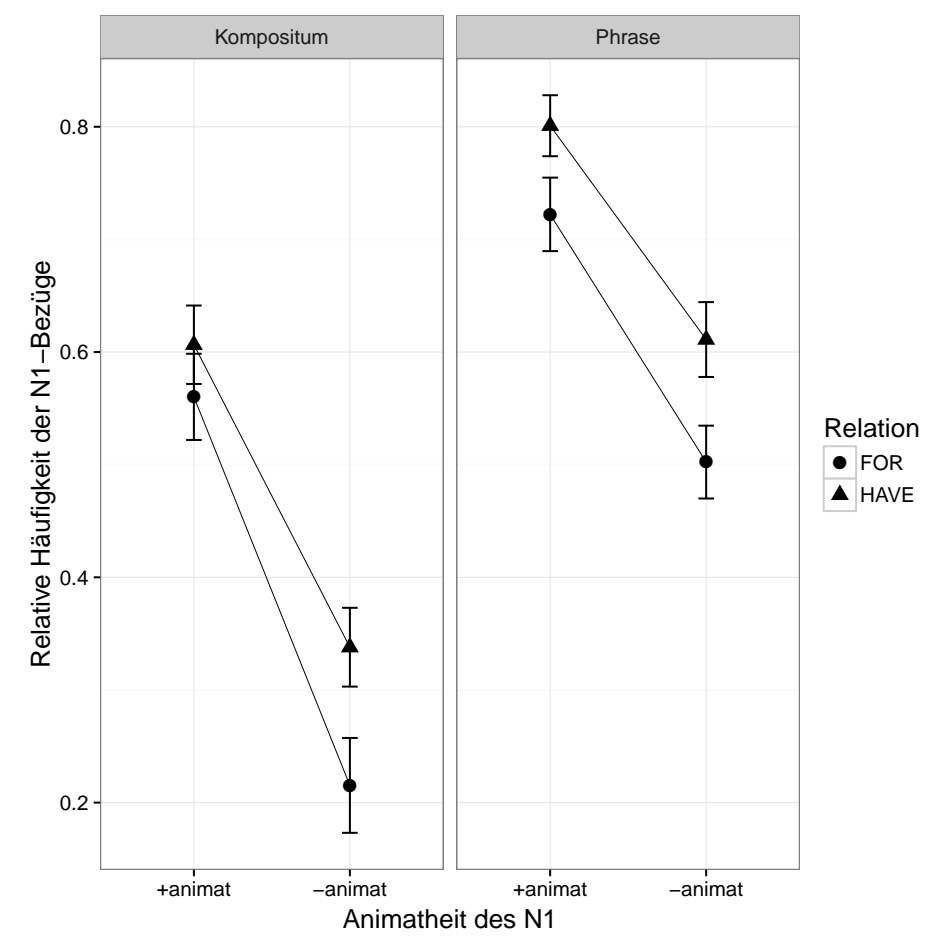

Abbildung 3.3: Plot der um die Zwischensubjektvarianz korrigierten Mittelwerte der N1Bezüge (y-Achse) in Abhängigkeit von den Faktoren FORM, ANIMATHEIT und RELATION

\begin{tabular}{c|cc}
\hline \hline Faktor & $|z|$ & $p$ \\
\hline FORM & 7.92 & $<0.001$ \\
\hline \hline
\end{tabular}

Tabelle 3.12: Inferenzstatistik (LMEM) für die N1-Bezüge für den Faktor FORM, komplettes Modell

Anschließend wurden die Daten für die beiden Formen von Antezedenten getrennt voneinander betrachtet. Da entsprechende Berechnungen somit nur auf dem halben Datensatz ausgeführt wurden, muss das $\alpha$-Level einer Bonferroni-Korrektur unterzogen werden, wodurch p-Werte in diesem Fall erst ab $p<0.025$ als signifikant betrachtet werden.

Im experimentellen Design sind die Faktoren zwischen den Items manipuliert, weshalb nur die Varianz zwischen den VPn als ,random slopes ${ }^{6}$ in die Berechnungen eingehen. ANIMATHEIT und RELATION bilden die ,fixed factors'. Der Faktor Animatheit zeitigt einen signifikanten Haupteffekt (vgl. Tab. 3.13).12 Für den Faktor RELATION zeigt sich kein signifikanter Effekt (vgl. Tab. 3.14) ${ }^{13}$ Die Interaktion ist ebenfalls nicht signifikant, wo-

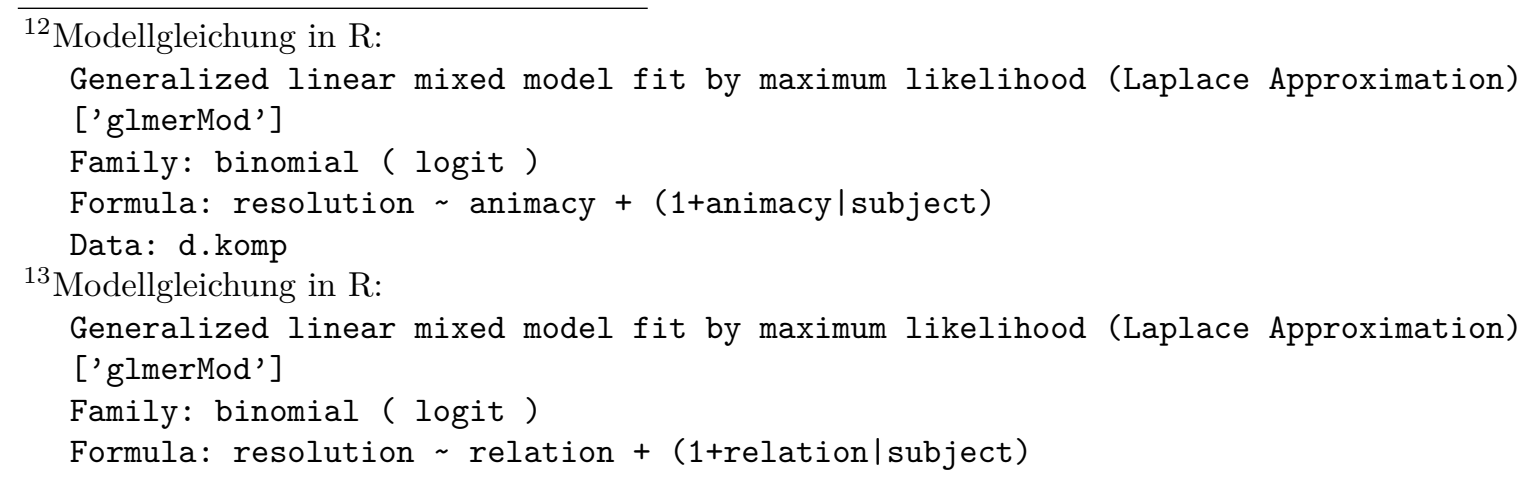


bei bei der Berechnung dieser aufgrund von Konvergenz-Problemen keine ,random slopes ${ }^{6}$ einbezogen werden konnten (vgl. Tab. 3.15).14

\begin{tabular}{c|cc}
\hline \hline Faktor & $|z|$ & $p$ \\
\hline ANIMATHEIT & 5.456 & $<0.001$ \\
\hline \hline
\end{tabular}

Tabelle 3.13: Inferenzstatistik (LMEM) für die N1-Bezüge für den Faktor ANIMATHEIT für die Kompositabedingung

\begin{tabular}{c|cc}
\hline \hline Faktor & $|z|$ & $p$ \\
\hline RELATION & 1.694 & 0.09 \\
\hline \hline
\end{tabular}

Tabelle 3.14: Inferenzstatistik (LMEM) für die N1-Bezüge für den Faktor RELATION für die Kompositabedingung

\begin{tabular}{c|cc}
\hline \hline Faktor & $|z|$ & $p$ \\
\hline ANIMATHEIT & 8.298 & $<0.001$ \\
RELATION & 2.590 & $<0.01$ \\
ANIMATHEIT $\times$ RELATION & 1.422 & 0.15 \\
\hline \hline
\end{tabular}

Tabelle 3.15: Inferenzstatistik (LMEM) für die N1-Bezüge für die Faktoren ANIMATHEIT und RELATION für die Kompositabedingung (ohne ,random slopes')

Im Modellvergleich $(\alpha$-Level $=0.0125)$ zeitigt der Faktor ANIMATHEIT sowohl innerhalb der Faktorstufe HAVE[ ${ }^{15}$ als auch innerhalb der Faktorstufe FOR ${ }^{16}$ ein signifikantes Ergebnis (vgl. Tab. 3.16.

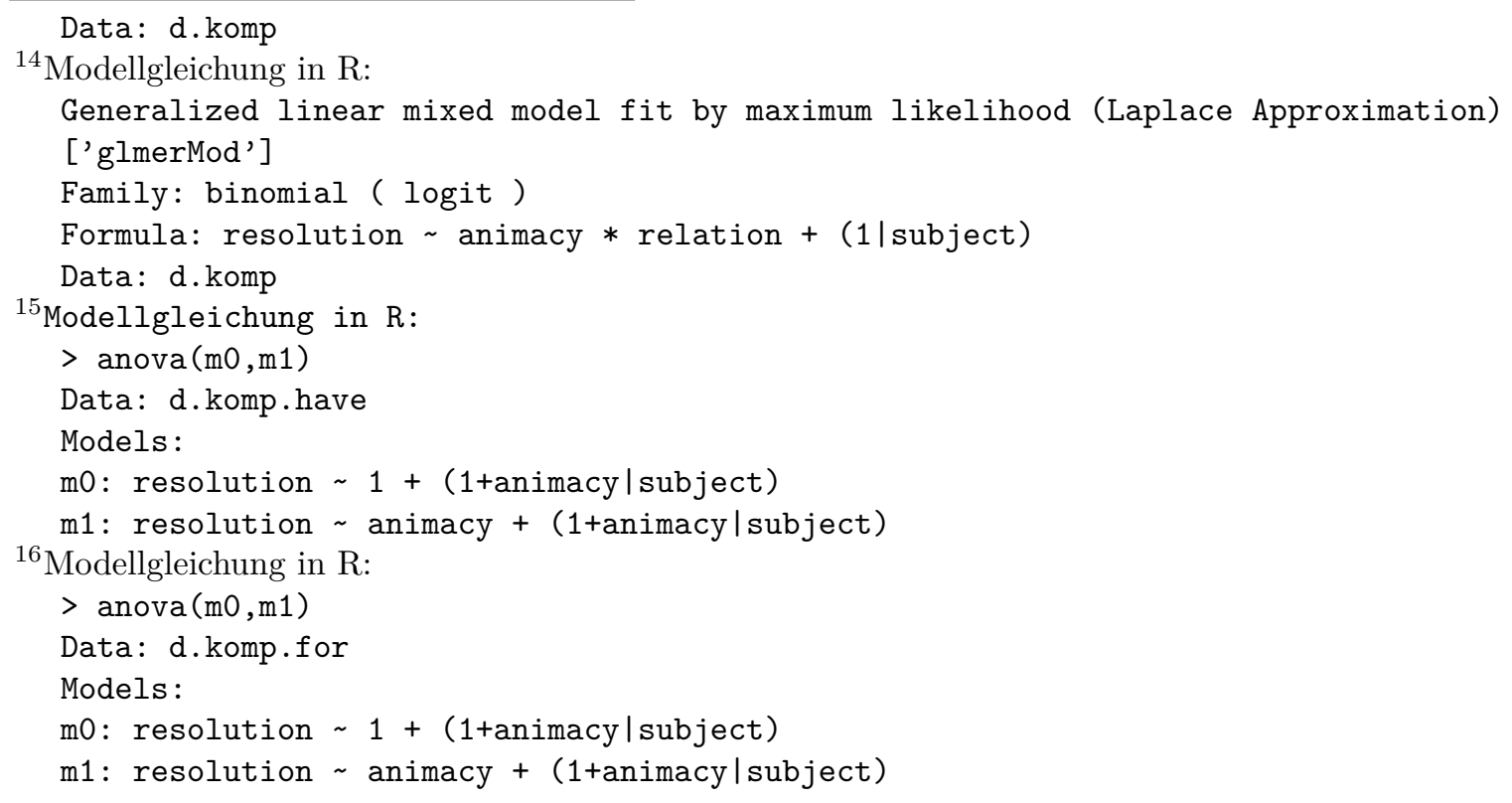




\begin{tabular}{cc|cc}
\hline \hline Faktor & RELATION & $L R \chi_{d f=1}^{2}$ & $p$ \\
\hline \multirow{2}{*}{ ANIMATHEIT } & HAVE & 17.052 & $<0.001$ \\
& FOR & 23.644 & $<0.001$ \\
\hline \hline
\end{tabular}

Tabelle 3.16: Inferenzstatistik (Modellvergleich) für den Faktor ANIMATHEIT in Abhängigkeit des Faktors RELATION für die Kompositabedingung

Der Faktor RELATION zeitigt innerhalb der Faktorstufe +animat 17 keinen signifikanten Effekt, innerhalb der Faktorstufe -animat ${ }^{18}$ hingegen schon (vgl. Tab. 3.17).

\begin{tabular}{cc|cc}
\hline \hline Faktor & ANIMATHEIT & $L R \chi_{d f=1}^{2}$ & $p$ \\
\hline \multirow{2}{*}{ RELATION } & +animat & 0.9232 & 0.3366 \\
& -animat & 8.5651 & $<0.0125$ \\
\hline \hline
\end{tabular}

Tabelle 3.17: Inferenzstatistik (Modellvergleich) für den Faktor RELATion in Abhängigkeit des Faktors ANIMATHEIT für die Kompositabedingung

In der phrasalen Bedingung weisen sowohl ANIMATHEIT als auch RELATION einen signifikanten Haupteffekt auf, jedoch ebenfalls keine Interaktion (vgl. Tab. 3.18).

\begin{tabular}{c|cc}
\hline \hline Faktor & $|z|$ & $p$ \\
\hline ANIMATHEIT & 5.877 & $<0.001$ \\
RELATION & 2.786 & $<0.01$ \\
ANIMATHEIT $\times$ RELATION & 0.284 & 0.78 \\
\hline \hline
\end{tabular}

Tabelle 3.18: Inferenzstatistik (LMEM) für die N1-Bezüge für die Faktoren ANIMATHEIT und RELATION für die phrasale Bedingung (Interaktion ohne ,random slopes')

\subsubsection{Interpretation und Diskussion}

In Bezug auf die Hypothesen in Kapitel 3.4.4 lassen sich die Ergebnisse wie folgt interpretieren:

(i) Es wurden von den VPn N1-Bezüge in ausreichend großer Zahl hergestellt, sodass diese Form der Anapher insgesamt als produktiv betrachtet werden kann.

(ii) Die FORM des Antezedens (Kompositum oder Phrase) zeitigt in den LMEMs einen

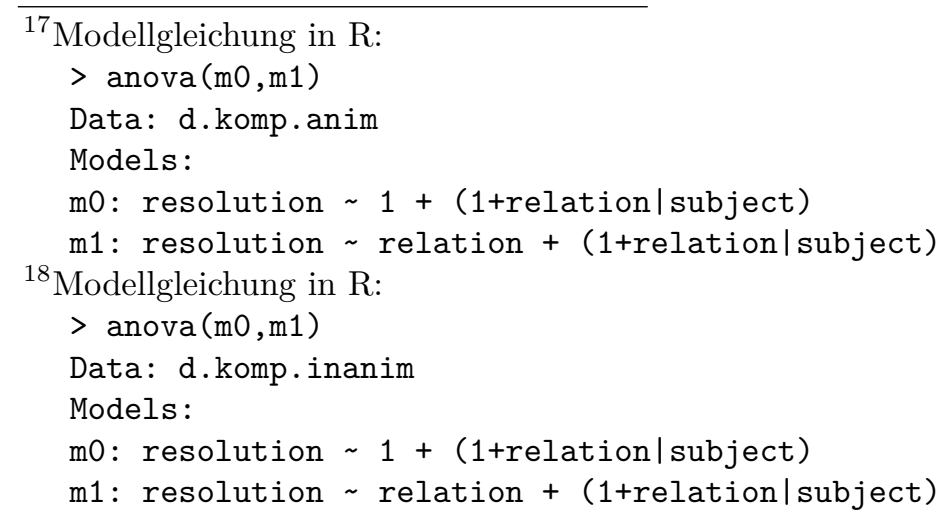


signifikanten Effekt. Dies bestätigt eine starke Präferenz für direkte Anaphern und weist die Ergebnisse der experimentellen Aufgabe als reliabel aus. Interessanterweise sind auch in der phrasalen Bedingung Effekte für die Faktoren ANIMATHEIT und RELATION zu verzeichnen. Es scheint sich demnach um Eigenschaften zu handeln, die die Aktiviertheit eines Referenten prinzipiell und unabhängig von seiner Form (N1 oder phrasale Konstituente) beeinflussen.

(iii) Für die Komposita konnte der Faktor AnIMATHEIT als signifikant bestätigt werden, d.h. in Übereinstimmung mit der Hypothese wurden im Fall animater N1 signifikant mehr N1-Bezüge hergestellt als im Fall inanimater. In Bezug auf diesen Faktor bestätigt sich damit der Einfluss von Denotatwissen und Lebenswelteffekt und die Übertragbarkeit von aktiviertheitsbeeinflussenden Faktoren von phrasalen Antezedenten auf KompositaErstglieder.

(iv) Für den Faktor RELATION konnte kein Effekt gefunden werden. Deskriptiv verhält sich der Faktor jedoch wie prognostiziert: Der Mittelwert der HAVE-Bedingung liegt über dem Mittelwert der FOR-Bedingung $(0.46>0.38)$. Die Tendenz geht somit in die prognostizierte Richtung.

(v) Eine Interaktion der beiden Faktoren konnte wie vermutet nicht gefunden werden. Im Modellvergleich zeigt sich jedoch, dass der Faktor RELATION innerhalb der inanimaten Items einen Einfluss hat und innerhalb der animaten nicht (vgl. Abb. 3.2). Die lässt sich dergestalt interpretieren, dass im Fall animater N1 diese Eigenschaft dominanter ist und andere Faktoren überlagern kann. Im Fall inanimater N1 können andere, weniger dominante Faktoren in Erscheinung treten. Es scheint sich demnach bei ANIMATHHEIT und RELATION um zwei Faktoren mit unterschiedlicher Einflussstärke zu handeln.

(vi) Neben N1-Bezügen wie in (29-a) wurden auch andere Resolutionsstrategien angewandt. Vorrangig wurde das Pronomen Sie als neues Agens im Plural, z.B. (29-b), oder als neues feminines Agens interpretiert, z.B. (29-c). Eine weitere Strategie beruht auf einem Fehler in der Materialerstellung: Bei 11 Items ist in der Adverbialbestimmung ein feminines Nomen enthalten. Diese Items verteilen sich jedoch relativ gleichmäßig auf die verschiedenen Bedingungen, weshalb nicht mit einer Beeinflussung des Gesamtergebnisses zu rechnen ist. Obwohl bei diesen Items eine direkte Anapher etabliert werden kann, geschah dies insgesamt nur in 15 Fällen (z.B. (29-d) ). Dies lässt darauf schließen, dass Kongruenz alleine keine anaphorische Beziehung garantiert, sondern auch andere Faktoren einen Einfluss haben, bspw. die syntaktische Funktion. Eine weitere Interpretation des Pronomens ist ein unspezifischer Bezug auf das Kompositum (29-e). Auch ambige Satzergänzungen wurden produziert, so z.B. in (29-f). Hierbei stellt sich die Frage, ob die VP die Anapher überhaupt auf ein konkretes Antezedens bezieht (vgl. Good-EnoughRepräsentation). Entsprechende Fälle gingen nicht in die Auswertung ein, sondern wurden mit ,NA' annotiert.

(29) a. Leon hat in seinem Vorgarten das bunte Beet mit den Rosen angelegt. Sie sind wunderschön.

b. Christoph hat im tiefen Winter den nahrhaften Meisenknödel aufgehängt. Sie lieben es, die Vögel zu beobachten.

c. Hannes hat seit der Lehre auf das rückfettende Kettenöl geschworen. Sie ist seiner Empfehlung gefolgt.

d. Der Schüler hat in den Sommerferien das gelbe Hamsterrad geputzt. Sie dauerten so lange, dass er deshalb Zeit für solche Dinge hatte.

e. Lukas hat zum Frühstück das warme Rosinenbrötchen gegessen. Sie schme- 
cken ihm immer sehr gut.

f. Der Tierpfleger hat mit großer Vorsicht den langen Tigerkäfig betreten. Sie beobachteten ihn dabei.

Mögliche Kritikpunkte am experimentellen Design könnten sein, dass die Auflösung nach dem N1 erzwungen wird, da kein anderes Nomen mit dem Pronomen in Numerus und Genus kongruent ist. Darüber hinaus wird durch einen definiten Artikel und ein Adjektiv das Kompositum in seiner Salienz erhöht. Dies ist jedoch in allen Items in allen Bedingung der Fall und hat somit keinen Einfluss auf die Ergebnisse der Faktorauswertung. Die insgesamt geringen Bezugszahlen und die verschiedenen beobachteten Ausweichstrategien belegen, dass ein N1-Bezug keinesfalls obligatorisch ist. Unterschiede zwischen den experimentellen Bedingungen lassen sich somit auf Unterschiede im Einfluss der Faktoren zurückführen. Gleiches gilt für die Konfundierung durch feminine Nomen in den Adverbialbestimmungen. Bei Betrachtung der einzelnen Satzergänzungen entsteht zudem nicht der Eindruck, dass die VPn eine Antwortstrategie entwickeln. Nur eine VP bezog das Pronomen durchgängig auf ein externes feminines Agens. Die übrigen VPn wechseln zwischen verschiedenen Arten von Bezugnahmen.

Abschließend gilt es einen kritischen Blick auf die Items zu werfen. Bei den inanimaten Fällen mit FOR fällt auf, dass das Kompositumsdenotat oftmals eine Veränderung am N1-Denotat bewirkt, z.B. ändert ein Dosenöffner den Zustand einer Dose von geschlossen zu offen. Im Zusammenhang damit fällt auf, dass in drei Fällen dieser Faktorkombination Rektionskomposita vorliegen, von denen zwei Verben der Zustandsänderung enthalten (Dosenöffner und Gurkenschäler). Dies geht mit Levis Beschreibung einher, dass die FORRelation durch ein zugrundeliegendes Verb ausgedrückt werden kann. Im Fall der beiden Rektionskomposita ist dieses nicht unterspezifiziert, sondern im N2 enthalten (öffnen und schälen).

Darüber hinaus lässt sich beobachten, dass die Relationen HAVE und FOR nicht so trennscharf sind, wie eingangs vermutet. Besonders bei den animaten N1 ist auffällig, dass das ,Beinhalten‘ meist zweckgebunden ist; z.B. beinhaltet ein Mäusezirkus Mäuse zu dem Zweck, dass sie darin Kunststücke vorführen und ein Schweinetransporter beinhaltet Schweine zu dem Zweck, sie von einem Ort zum anderen zu bringen. Innerhalb der inanimaten lässt sich die HAVE-Relation in den meisten Fällen mit einer Aufbewahrungsfunktion erschöpfend beschreiben. Funktionslos erscheint die HAVE-Relation lediglich im Falle der Schnittmengenbildungen. Im Experiment trifft dies nur auf Kräutertee zu. Dieser Zusammenhang spiegelt sich im Modellvergleich wider: Nur innerhalb der Gruppe der inanimaten N1 zeigt sich ein Einfluss der Relation.

Die Inferenzstatistik belegt, dass der Faktor ANIMATHEIT einen stärkeren Einfluss hat als der Faktor RELATION. Es bleibt die Frage, ob nun generell das Denotatwissen einen stärkeren Einfluss hat als Wissen über die Konzeptkombination. Dass für den Faktor RELATion kein Effekt gefunden wurde, kann zum einen bedeuten, dass die Komposita in den Items nicht sauber genug kontrolliert sind. Zum anderen kann es auch bedeuten, dass die Levi'schen Relationen zu vage voneinander abgrenzbar sind, um als experimenteller Faktor zu fungieren. Ein Einfluss der Konzeptkombination kann auf Basis dieser Ergebnisse nicht ausgeschlossen werden. Um sich der Idee des konzeptuellen Fokus experimentell anzunähern, eignet sich eventuell die in Kapitel 3.3.4 entwickelte Eigenschaft der raum-zeitlichen Kontiguität besser.

Die generellen Tendenzen für die Faktoren zeigen sich auf einem insgesamt höheren Niveau auch innerhalb der phrasalen Bedingung. Dies lässt die Vermutung zu, dass es sich 
zumindest bei der ANIMATHEIT um einen Faktor handelt, der einen generellen Einfluss auf die Wahl des Referenten ausübt. In diesem Sinne lassen sich Annahmen zur Beeinflussung von Aktiviertheitsgraden phrasaler Referenten, wie sie Fukumura/van Gompel (2011) machen, auch auf Komposita-Erstglieder übertragen.

Insgesamt konnte Experiment 1 zeigen, dass die Satzergänzungsaufgabe prinzipiell ein geeignetes Instrument für den Forschungsgegenstand ist, dass N1-Bezüge durchaus produziert werden und in jedem Fall auch, dass es graduelle Unterschiede in der Zugänglichkeit von Komposita-Erstgliedern gibt.

\subsection{Generizität}

Bei der Item-Erstellung stellte sich mehrfach die Frage, inwiefern generische Interpretationen von Antezedens und Anapher möglich sind und inwiefern dieser Interpretationsspielraum die Ergebnisse konfundieren kann. Aus diesem Grund widmet sich der letzte Abschnitt des Kapitels diesem Themenkomplex.

Bei der Beschreibung von charakteristischen Komposita-Eigenschaften ist in der Literatur häufig von der ,Generizität des N1' die Rede (vgl. z.B. Härtl 2013, Olsen 2012, Spencer 1991). Eine genauere Betrachtung dieses Begriffs im Zusammenhang mit Erstgliedern ist für weitere Überlegungen grundlegend. Klos (2011) und Ortner/Ortner (1984) zielen mit dem Begriff der Generizität darauf ab, dass das N1 keine individuellen, sondern typische Eigenschaften denotiert. Olsen (vgl. 2000:898) beschreibt damit die Art der N2-Spezifikation. Im folgenden Exkurs soll analysiert werden, inwiefern ein N1 wirklich generisch sein kann und wie sich diese Eigenschaft auf die Zugänglichkeit auswirkt.

Behrens (2005) versteht unter generischen Ausdrücken Ausdrücke, mit denen über eine Art generalisiert wird. Sie seien gegenüber episodischen Ausdrücken zeitstabil und prototypisch (vgl. Behrens 2005:275). Weiter heißt es, dass sich generische Ausdrücke auf eine allgemeine Qualität, anstatt auf eine individuelle Dimension beziehen. Im Allgemeinen beziehen sich generische Ausdrücke auf einen raum-zeitlich abstrakten Referent, d.h. auf etwas nicht sinnlich Wahrnehmbares (vgl. Behrens 2005:298f). Wie auch im Englischen, können generische Ausdrücke im Deutschen verschiedene Formen haben, z.B. definite oder indefinite NPn im Singular (vgl. (30-a) und (30-b) oder im Plural (im Englischen nur als ,bare plural', vgl. (30-c).
a. Die Boa (the boa) kann bis zu $5 \mathrm{~m}$ lang werden.
b. Eine Boa (a boa) kann bis zu 5 m lang werden.
c. Die Boas/Boas (boas) können bis zu $5 \mathrm{~m}$ lang werden.

Eine generische Interpretation entsteht aus einer Interaktion verschiedener Faktoren wie lexikalischer Semantik, pragmatischem Wissen, Diskurssituation und grammatischer Markierung. Gleichzeitig seien generische Ausdrücke in ihrer Interpretation oft ambig und synonym mit ihrer episodischen Form (vgl. Behrens 2005 276f). Krifka et al. (1995) explizieren, dass nicht ein Ausdruck an sich generisch sei, sondern dass er diese Eigenschaft vom Satz erbt. Generizität (als Gegenstück zu Episodizität) sei somit als Eigenschaft von Sätzen, nicht von Wörtern, zu betrachten. 
Eine definite Anapher - und dazu zählen auch Pronomen - kann nun gemäß Behrens (vgl. 2005:290) für anaphorische Bezüge auf generisch verwendete Ausdrücke auf die gleiche Art genutzt werden, wie auf episodisch verwendete. Demzufolge ist es prinzipiell möglich, sich mit einem Pronomen anaphorisch auf einen generisch verwendeten Ausdruck zu beziehen, da auch dieser einen Diskursreferenten etabliere. Ein Diskursreferent zu sein, präsupponiert nach Behrens (vgl. 2005 297) nicht, dass der Referent in Raum und Zeit lokalisiert oder individuell und distinkt ist. Ob ein linguistischer Ausdruck einen Diskursreferenten etabliert, sei dadurch prüfbar, ob er mit einer definiten Anapher koreferieren kann. Dies sei bei generischen Ausdrücken zur Bezeichnung von Arten gegeben. Bei einem Kompositums-Erstglied jedoch nicht (vgl. Behrens 2005:297). Dies läge jedoch nicht an der Generizität, sondern an der fehlenden Referenzialität.

Diese Begründung halte ich für zu kurz gegriffen. Zum einen ist fehlende Koreferenz kein Ausschlusskriterium für anaphorische Bezugnahme (vgl. Kap. 2.4.1), zum anderen ist die Frage der Referenzialität von Komposita-Erstgliedern nach wie vor ungeklärt.

Ein anderer Punkt in diesem Zusammmenhang ist, dass gerade die Generizität eines N1 dessen Referenzialität ermöglicht. Schließlich können generische Ausdrücke ohne Determinierer referieren (vgl. (31-a)), was auch für einen Ausdruck an der N1-Position gilt (vgl. $(31-b))$.

a. Rosen sind tückische Pflanzen. Man muss auf ihre Dornen Acht geben.

b. ?Rosenbeete sind tückische Bepflanzungen. Man muss auf ihre Dornen Acht geben.

An dieser Stelle stellt sich die Frage, ob der Begriff der Generizität im Zusammenhang mit Komposita-Erstgliedern angemessen ist. Betrachtet man den Ausdruck Rose in Rosenbeet als generisch verwendeten Ausdruck, würde dies bedeuten, dass dieser die spezielle Pflanzenart der Rosen in ihrer Gesamtheit denotiert. In diesem Sinne würde Rosenbeet ein Beet denotieren, welches die Gesamtheit aller Rosen beinhaltet. Dies ist jedoch nicht die präferierte Lesart. Vielmehr ist das präferierte Denotat von Rosen in Rosenbeet eine bestimmte Menge an unspezifischen, d.h. beliebigen Elementen der Art Rose. Aus diesem Grund halte ich in Bezug auf das N1 das Begriffspaar ,spezifisch/unspezifisch' für geeigneter, um die Unterscheidung zu beschreiben, die in der Literatur mit dem Begriff der ,Generizität‘ getroffen wird 19 Ein unspezifisches N1 verfügt demnach über keinen festgelegten Referenten, während ein spezifisches N1 über einen speziellen, festgelegten Referenten verfügt, bzw. ein episodisches N1 auf ein raum-zeitlich festgelegtes Ereignis referiert.

Wie die Beispiele in (32) belegen, sind nun N1 zwar in der Regel, jedoch nicht zwangsläufig unspezifisch (vgl. auch Meyer 1993:209). In (32-a) liegt die Spezifizität im Ausdruck des N1 selbst begründet, da es sich um einen per se spezifischen Eigennamen handelt. In (32-b) scheint die Spezifiztät des N1 durch den N2-Kontext bestimmt zu werden, da es sich beim Stinken um eine episodische Eigenschaft handelt. ${ }^{20} \operatorname{In}(33)$ wird deutlich, dass je nach Kontext das N1 entweder spezifisch oder unspezifisch interpretiert werden kann. Andererseits stellt sich in diesem Fall die Frage, ob das N1 wirklich spezifisch ist. Eventuell handelt es sich hier bei der Referentenfestlegung um einen nachträglichen Schlussprozess, der durch das Denotatwissen bedingt ist: Das NN wird spezifisch verwendet und ein Garten

\footnotetext{
${ }^{19}$ Eine ähnliche Diskussion ließe sich zu den Begriffen ,kind-denoting` und ,type-denoting‘ anführen.

${ }^{20}$ Kontextuell können natürlich auch andere Lesarten festgelegt werden.
} 
kann immer nur auf einem einzelnen Dach sein, also muss auch das Dach ein spezifisches Dach sein. Eine andere Interpretation der Bezugnahme wäre, dass sich eine spezifische Anapher auf ein unspezifisches Antezedens bezieht. Die Möglichkeit eines solchen Wechsels der Spezifizität zwischen Antezedens und Anapher gilt es näher zu betrachten, da hierbei von einem Einfluss auf die Akzeptabilität der Anapher auszugehen ist.
a. Chomsky-Verehrerin
b. Stinkedecke (Härtl 2015:174)

Ich glaube, ich hätte gerne einen $\underline{\text { Dach }}_{\mathrm{SPEZ} / \mathrm{UnSPEZ}}$ garten.

a. Die $_{\text {unspez }}$ sehen dann immer gleich viel schöner aus.

b. $\underline{\text { Meins }}_{\mathrm{SPEZ}}$ sähe dann auch gleich viel schöner aus.21

Bei einem unspezifischen N1 besteht laut Härtl (2015) die Möglichkeit, sich mit einem unspezifisch verwendeten anaphorischen Element darauf zu beziehen.22 Dies belegt er am Beispiel von raum-zeitlich nicht spezifizierten Ereignissen in (34) ${ }^{23}$

a. Ich habe auch Interesse an einem Tanzkurs, denn das möchte ich nun endlich mal lernen.(Härtl 2015:164)

b. Paul ist nun in einem Eislaufkurs. Das hatte er in der Schule nämlich nie gelernt. (Härtl 2015:171)

Der Bezug auf ein unspezifisches N1 mit Hilfe einer episodisch verwendeten Anapher wie in (35) ist Härtl (2015:171) zufolge nicht möglich.
a. Paul ist nun in einem Eislaufkurs. ${ }^{*}$ Das geschieht nächsten Mittwoch auf eigenen Gefahr.
b. Mia hat einen Audi-Fahrer kennengelernt. *Ihren eigenen hat sie nun ver- schrottet.

Grund für die Inakzeptabilität der Sätze unter (35) sei, dass eine episodisch referierende Anapher generell nicht auf ein unspezifisches Antezedens referieren könne (vgl. Härtl 2015. 173). Bei diesen konkreten Beispielen scheinen mir allerdings vorrangig andere Gründe vorzuliegen. In (35-a) besteht eine Präferenz für eine Komplexanapher und in (35-b) durch Kongruenz zwischen Pronomen und N2 eine Präferenz für eine direkte Anapher. Da diese Resolutionen nicht plausibel sind, ist der Bezug inakzeptabel.

Wie mit den Beispielen unter (32) angedeutet, sind auch spezifisch referierende N1 möglich, z.B. im Fall von Eigennamen, speziellen Konstituenten-Kombinationen und spezieller Satzsemantik. Für besagte spezielle Konstituenten-Kombinationen liegt ein Indiz für die Spezifizität des N1 im semantischen Numerus: Kann das N1 in einem definiten NN nur

\footnotetext{
${ }^{21}$ Die Frage der Akzeptabilität der Bezugnahmen sei an dieser Stelle außen vorgelassen. Das Beispiel dient lediglich der Unterscheidung zwischen spezifischer und unspezifischer Bezugnahme.

${ }^{22}$ Die Spezifizität einer Anapher wird immer über den Satzkontext festgelegt, da ein Pronomen einen zu geringen Informationsgehalt hat, um ein Interpretation seiner Spezifizität nur durch den Ausdruck selbst zu ermöglichen.

${ }^{23}$ Die Art der Herstellung von Koreferenz versteht Härtl $(2013,2015)$ als Bridging, also als pragmatisch verankert.
} 
im Singular interpretiert werden, liegt eine individuelle und damit spezifische Lesart vor, wie bspw. in (36-a) im Gegensatz zu (36-b). Bei diesem Beispiel ist allerdings die Frage, ob es sich wirklich um einen Unterschied in der Spezifizität handelt oder vielmehr um einen Unterschied in den Identitätsbedingungen. So sind die Identitätsbedingungen für das Denotat von Raketenstart wesentlich strikter als für das Denotat von Nagelbrett: Wird die Rakete ersetzt, denotiert Raktenstart ein anderes Ereignis. Werden einige Nägel ausgetauscht, kann Nagelbrett noch immer dasselbe Objekt denotieren.

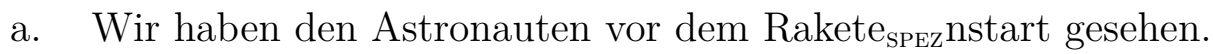

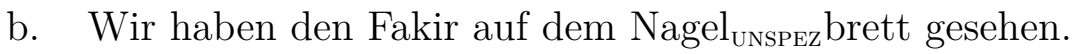

Für spezifische N1 führt Härtl (2015) an, dass die spezifische Verwendung einer Anapher z.B. im Fall von Eigennamen möglich ist (vgl. (37)).

Mia ist glühende Chomsky-Verehrerin.

Und er ist ja auch tatsächlich ein ziemliches Genie. (Härtl 2015:173)

Schließlich bleibt die Frage, ob der Bezug auf ein spezifisches N1 durch eine unspezifisch verwendete Anapher möglich ist. Für NPn wird diese Form der Bezugnahme als möglich erachtet (vgl. Gernsbacher 1991, Schwarz 2000) 24 Eine Übertragung auf Fälle mit spezifischem N1 im Sinne von (38) erscheint mir plausibel und akzeptabel.

a. Wir haben am Wochenende im Fernsehen den Raketenstart beobachtet. Ben will jetzt auch unbedingt in einer mitfliegen.

b. Wir haben am Wochenende im Museum das berühmte Löwenbild beobachtet. Ben will jetzt unbedingt einen streicheln.

Härtl (vgl. 2015:172) legt der Möglichkeit von anaphorischen N1-Bezügen das Parallelitätsprinzip zugrunde. Demzufolge kann sich eine unspezifisch verwendete Anapher auf ein unspezifisches Antzedens und eine spezifische Anapher auf ein spezifisches Antezedens beziehen. Gestützt wird diese Annahme von der Beobachtung, dass sich eine spezifisch verwendete Anapher nicht auf ein unspezifisches Antezedens beziehen kann (vgl. (35)]. Die Beispiele in (38) legen jedoch zusätzlich die Möglichkeit nahe, dass eine unspezifisch verwendete Anapher auf ein spezifisches N1 referieren kann, was dem Parallelitätsprinzip widerspricht.

Insgesamt konnte in diesem Exkurs herausgestellt werden, dass der Begriff der Generizität in Bezug auf das N1 ungeeignet ist und es sich bei der beobachteten N1-Eigenschaft eher um Spezifizität, bzw. Unspezifizität handelt. Diese Eigenschaft ist allerdings nur bei speziellen Ausdrucksklassen (z.B. Eigennamen) ausdrucks-inhärent, i.d.R. handelt es sich um ein komplexes Zusammenspiel verschiedener Faktoren, die die Spezifizität eines Ausdrucks festlegen. Eine endgültige Klärung der Frage, welche Auswirkungen der Satzkontext auf das N1 hat, kann hier nicht gegeben werden. Das liegt nicht zuletzt daran, dass die Frage

${ }^{24}$ Diese Form der Bezugnahme ordnet Schwarz (2000:124) als direkte Anapher ein. Es läge zwar kein Fall von Koreferenz vor, jedoch sei ein explizites Antezedens auf der Textoberfläche gegeben. Angesichts dieser Argumentation stellt sich die Frage, wieso ein textuell präsentes N1 nicht als explizites Antezedens gewertet wird. Erkü/Gundel (1987/544) sprechen bei episodischem Bezug auf ein generisches Antezedens nicht von Referenz-, jedoch von Form- und Sinnidentität. 
der Interpretation von nominalen Ausdrücken in generischen Sätzen Probleme aufwirft, die den Rahmen dieser Arbeit sprengen. Die Betrachtungen zur Spezifizität von Anapher und Antezedens sind nicht auf N1-Bezüge beschränkt, sondern unterliegen den gleichen Restriktionen wie NPn (vgl. dazu Gernsbacher 1991, Härtl 2015, Krifka et al. 1995, Schwarz 2000). In diesem Rahmen handelt es sich allerdings um einen Faktor, der die Akzeptabilität einer anaphorischen Bezugnahme beeinflusst. So kann die Art der anaphorischen Bezugnahme die konkrete Interpretation des N1 festlegen.

\subsection{Zwischenfazit}

Im Rahmen dieses Kapitels wurden zunächst Aspekte des konzeptuellen und grammatischen Wissens über das N1 betrachtet (Kap. 3.2). Dabei wurde ein Lebenswelteffekt beschrieben, gemäß dem z.B. Animatheit oder eine hohe emotionale Valenz die N1Zugänglichkeit erhöhen können. Darüber hinaus wurde ein Wahrnehmungseffekt thematisiert, nach dem ein Zugänglichkeitsvorteil für sinnlich Wahrnehmbares angenommen wird. Außerdem deutet sich eine Interaktion der beiden Effekte an, bei der der Lebenswelteffekt einen stärkeren Einfluss zu haben scheint.

Einige der untersuchten Ausdrucksarten verfügen zusätzlich über grammatische Reflexe. So sind Bezeichnungen für Eigennamen sowie Massenomen determiniererlos referenzfähig, was an der N1-Position einen Zugänglichkeitvorteil bewirken sollte. Für Eigennamen entspricht dies der Einflussrichtung des Denotatwissens, für Massenomen wirkt es dieser entgegen.

Des Weiteren wurden Einflüsse der Konzeptkombination innerhalb des Kompositums betrachtet (Kap. 3.3). Dabei wurde herausgearbeitet, dass der konzeptuelle Fokus eines NN verschieden gelagert sein kann, d.h. dass ein N1-Konzept über verschiedene Grade an Zentralität für das NN-Konzept verfügen kann. In der Regel liegt der konzeptuelle Fokus auf dem N2; je mehr sich der Fokus jedoch zum N1 verschiebt, desto zugänglicher wird es, so meine Hypothese. Als Faktoren für einen N1-Fokus wurden die semantische Relation und die raum-zeitliche Kontiguität der Konstituenten ermittelt. Darüber hinaus wurden spezifische Eigenschaften wie die Konkretheit des N2 in die Überlegungen einbezogen.

Insgesamt kristallisiert sich heraus, dass Eigenschaften, die generell die Zugänglichkeit eines Diskursreferenten erhöhen, auch im Speziellen die Zugänglichkeit eines N1 erhöhen können. Von einem generellen Verarbeitungsunterschied kann hingegen nicht per se auf einen Unterschied in der Zugänglichkeit geschlossen werden.

Im Anschluss an die theoretischen Untersuchungen wurde in Kapitel 3.4 eine Pilotstudie berichtet. In einer Satzergänzungsaufgabe wurde als Faktor des Denotatwissens die ANIMATHEIT und als Faktor der Konzeptkombination die RELATION bezüglich ihres Einflusses auf die N1-Zugänglichkeit überprüft. Dabei zeigte sich, dass N1-Bezüge von Muttersprachlern produziert werden, die Etablierung von direkten Anaphern jedoch präferiert wird. Darüber hinaus zeigte sich wie prognostiziert ein Haupteffekt für den Faktor ANIMATHEIT in Form einer höheren Anzahl an N1-Bezügen bei animaten N1 im Gegensatz zu inanimaten. Für die RELATION wurde kein Effekt gefunden, allerdings zeigt der Modellvergleich mehr N1-Bezüge in der Bedingung HAVE (stärkerer N1-Fokus) im Gegensatz zur Bedingung FOR (schwächerer N1-Fokus) im Fall inanimater N1. Dies deutet an, dass Aspekte des Denotatwissens über das N1 einen stärkeren Einfluss haben als Aspekte der Konzeptkombination. Eine nähere Betrachtung des experimentellen Materials zeigt 
außerdem, dass der Faktor RELATION ungeeignet ist, um Unterschiede in der Art der Konzeptkombination widerzuspiegeln.

Abschließend wurde in einem Exkurs ein möglicher Einfluss der Generizität von Anapher und Antezedens auf die Möglichkeiten der Referenzetablierung diskutiert. Dabei kristallisierte sich heraus, dass ein N1 i.d.R. nicht im eigentlichen Sinne generisch, sondern unspezifisch interpretiert wird. In der (Un-)Spezifizität liegt zwar ein Einflussfaktor für die Zugänglichkeit, in Bezug auf diesen verhalten sich N1 jedoch wie phrasale Antezedenten. Aus diesem Grund wird der Faktor nicht weiter untersucht, muss jedoch bei der Erstellung experimenteller Items berücksichtigt werden. 


\section{Strukturaspekte}

In Kapitel 3 wurden Unterschiede in der N1-Zugänglichkeit vor allem auf konzeptuelles Wissen über die Konstituenten und ihre Kombination zurückgeführt. Im Folgenden sollen nun grammatische Aspekte in den Vordergrund treten. Wie in Kapitel 2.2.3 angerissen, kann die morphosyntaktische Struktur von Komposita zu deren Klassifikation herangezogen werden. Zentral ist hier die Unterscheidung zwischen Rektions- und Wurzelkomposita. Zum einen stellt sich die Frage, wie sich der semantische Unterschied zwischen den beiden Komposita-Arten in der Struktur niederschlägt, nämlich Modifikation durch das N1 im Gegensatz zu Subkategorisierung des N1. Zum anderen bietet sich an dieser Stelle die Möglichkeit dem grammatischen Status der Konstituenten auf den Grund zu gehen.

Für beide Punkte ist es zentral, zunächst eine genaue Strukturbeschreibung zu unternehmen und etwaige Unterschiede auszumodellieren. Erst im Anschluss können Fragen zu Unterschieden in der Zugänglichkeit gestellt werden.

Im ersten Schritt gilt es nun ein geeignetes Framework zu finden, im Rahmen dessen eine Strukturbeschreibung erfolgen kann.

\subsection{Beschreibungsansätze}

Im Kontext dieser Arbeit sind v.a. zwei grundlegende Gruppen von Ansätzen von Interesse: Die lexikalistischen und die syntaktischen Ansätze.

\subsubsection{Lexikalistische Ansätze}

Lexikalistische Grammatikmodelle gehen davon aus, dass es sich bei Morphologie und Syntax um zwei getrennte Module der Grammatik handelt. Im morphologischen Modul werden Wörter, im syntaktischen Sätze gebildet. Zwischen den beiden Modulen herrscht eine sequenzielle Abfolge, wobei die Morphologie der Syntax vorausgeht. Das Resultat eines Wortbildungsprozesses ist ein Wort, welches eine abgeschlossene, atomare Einheit bildet, die den Input für die Syntax bildet. Eine Interaktion der beiden Module wird ausgeschlossen. Komposita werden als reine Wortbildungsprodukte betrachtet und somit ausschließlich dem morphologischen Modul zugeordnet. Innerhalb der Morphologie werden eigene Regeln angenommen; phonologische und semantische Phänomene innerhalb von Wörtern werden wortintern durch idiosynkratische Prinzipien erklärt.

Zentrale Vertreter*innen lexikalistischer Ansätze sind Aronoff (1994), Booij/van Haaften (1988), Di Sciullo/Williams (1987), Grimshaw (1992), Kiparsky (1982), Lapointe (1979), Levin/Rappaport Hovav (1995), Lieber (1982, 1992), Roeper/Siegel (1978), Selkirk (1982), Stump (2001), Williams (1981) u.a. 
Typisch für lexikalistische Modelle ist die These der Unzugänglichkeit von Wortteilen für syntaktische Prozesse entsprechend der LIH (vgl. Kap. 2.1). Wenn die Wortbildung abgeschlossen ist, können darauf folgende syntaktische Prozesse keine Teile des Wortbildungsprodukts einbeziehen, die kleiner sind als das Wortbildungsprodukt selbst. Das N1 kann somit auch nicht Ziel eines post-syntaktischen Prozesses wie diskurs-anaphorischer Bezugnahme sein. Komplexe Wörter werden als syntaktisch opak betrachtet (vgl. Chomsky 1970, Di Sciullo/Williams 1987, Lapointe 1979).

Der syntaktische Effekt der Rektion wird von Lexikalist*inn*en zumeist als Resultat morphologischer Prinzipien interpretiert (vgl. Spencer 1991). So postuliert Wunderlich, dass Wörter mit syntaktischer Rektion nicht als Zweitglied eines Kompositums auftreten, sondern in dem Fall ausschließlich semantische Rektion! vorliege. Als Unterart der Komposita werden somit auch Rektionskomposita als reine Wortbildungsprodukte aufgefasst. Selkirk (1982) legt Wurzel- und Rektionskomposita die gleiche Struktur zugrunde, da beide Arten durch das gleiche Regelset erzeugt würden. Gleichzeitig beschreibt sie jedoch, dass das N1 die Argumentstelle des N2 sättigt.

Die Tatsache, dass Argumentsättigung wie in (1) auch über eine Kategoriengrenze hinaus erfolgen kann, wird mit einer Lücke in der Strong Lexicalist Hypothesis² erklärt, die Argumente außerhalb der Wortdomäne zugänglich werden lasse. Vertreter*innen dieser Analyse sind u.a. Booij/van Haaften (1988), Di Sciullo/Williams (1987), Lieber (1983), Selkirk (1982). Bereits an dieser Stelle zeigt sich, dass einige lexikalistische Annahmen auch theorieintern nur unter Einräumung von Ausnahmen gültig sein können.

Eroberung Galliens, Besprechung der Arbeit, Fahrer des Autos

Eine weitere Ausnahme stellen Phrasenkomposita dar: Da Wortbildungsprodukte als atomare syntaktische Einheiten interpretiert werden, können lexikalistischen Annahmen zufolge in der $\mathrm{X}^{o}$-Kategorie keine phrasalen Einheiten auftreten. Dass dies dennoch möglich ist, zeigen die Beispiele unter (2) (siehe dazu auch Kapitel 2.5.1).
a. Die Wir-lassen-Sie-nicht-im-Regen-stehen-Wohngebäudeversicherung (aus einer Werbeanzeige eines Versicherungsunternehmens)
b. Sprich-mich-bloß-nicht-an-Miene, Nur-nicht-das-Sofa-verlassen-Wetter
c. Abgerechnet-wird-am-Schluss-Taktik, Messer-und-Gabel-Handhabung (Meibauer 2003)

Konsequenzen streng lexikalistischer Ansätze sind, dass kein syntaktisch relevanter Strukturunterschied zwischen verschiedenen Komposita-Arten besteht und Kompositateile per se unzugänglich sind. Die Etablierung einer Diskursanapher ist nur unter der Voraussetzung einer für spätere Textteile transparenten Wortstruktur möglich, was ausgeschlossen wird. Fälle von akzeptablen N1-Bezügen werden von Vertreter*inne*n lexikalistischer Ansätze vorrangig über pragmatische Legitimierung erklärt (z.B. Wunderlich 1986) und nicht

\footnotetext{
${ }^{1}$ Ich interpretiere „semantische Rektion“ in diesem Kontext als Theta-Rollen-Vergabe ohne Vorliegen einer Argumentstruktur.

${ }^{2}$ Nach der Strong Lexikalist Hypothesis (Selkirk 1984) werden Flexion und Derivation vom Lexikon abgedeckt; die Unterscheidung wird nicht durch ein grammatisches Framework bestimmt.
} 
als „echte“ N1-Zugänglichkeit. Allerdings werden mit der grenzüberschreitenden Subkategorisierung in (1) und den Phrasenkomposita in (2) bereits Grenzen lexikalistischer Ansätze sichtbar.

\subsubsection{Syntaktische Ansätze}

Einen alternativen Weg beschreiten die syntaktischen Ansätze. Das Wort wird hier nicht als opaker Baustein für folgende syntaktische Prozesse betrachtet, sondern alle in der Wortbildung beobachteten Phänomene werden gleichsam durch generelle syntaktische (und phonologische) Prinzipien erklärt. Der Bildung von Wörtern und phrasalen Strukturen wird eine gemeinsame Grammatikkomponente zugrunde gelegt sowie ein gemeinsames Set an Bildungsregeln. Da keine separate morphologische Komponente mit spezifischen Regeln angenommen wird, sind Phonologie und Syntax die relevanten interagierenden Ebenen der Grammatik. Die Fälle unter (1) und (2) stellen unter dieser Annahme keine Schwierigkeiten dar, da die Wortbildung mit syntaktischen Mitteln beschrieben wird.

Zentrale Vertreter*innen syntaktischer Ansätze sind Ackema (1995), Baker (1988), Bobaljik (2012), Borer (2013), Embick (2010, 2015), Embick/Noyer (2001), Fabb (1984), Halle/Marantz (1993), Harley (2009), Kuiper (1999), Lieber (1988), Marantz (1997, 2013b), Sproat (1985) u.a.

Innerhalb der syntaktischen Ansätze gibt es verschiedene Strömungen. So beschreiben z.B. Olsen (2000:904) und Toman (1990:423) eine Ähnlichkeit zwischen Wortstrukturen und syntaktischen Phrasen durch eine Rückführung beider Strukturbildungen auf die Prinzipien der X-Bar-Theorie. Aber auch im Rahmen des Minimalistischen Programms werden Aussagen zur Wortbildung getroffen (u.a. in Chomsky 1995, Epstein et al. 2016).

Ein zentrales Framework innerhalb der syntaktischen Ansätze ist die Distribuierte Morphologie (DM), deren Annahmen mit denen des Minimalistischen Programms vereinbar sind (u.a. Alexiadou 2001, Embick 2015, Embick/Noyer 2007, Halle/Marantz 1993, Harley 2014, Marantz 1997).

Die DM betrachtet die Syntax als das einzige generative System, dass der Grammatik zur Verfügung steht. Die Morphologie wird komplett auf Syntax und Phonologie distribuiert. Der internen Struktur von Wörtern wird eine syntaktische Architektur zugrunde gelegt, d.h. sie werden durch die gleichen syntaktischen Prinzipien beschrieben wie die interne Struktur von Phrasen und Sätzen. Es besteht lediglich eine Differenzierung zwischen wortinterner und wort-externer Syntax (vgl. Marantz 2013b:906f).

Auch die Bildung eines Kompositums - als Beispiel für eine komplexe Form - muss demnach mit syntaktischen Mitteln ausgeführt werden (vgl. Embick/Noyer 2007). Gemäß der DM arbeitet das komputationale System ausschließlich mit den Operationen ,Merge ${ }^{6}$ und ,Move', bzw. ,external' und ,internal Merge‘ nach Epstein et al. (2016). Wortbildung wird zumeist als (asymmetrisches) syntaktisches Merge beschrieben, z.B. bei Marantz (1996) und Embick/Noyer (2007).

Die terminalen Knoten bestehen in der DM aus kategorielosen Wurzeln und funktionalen Kategorien. Lexikalische Stämme werden durch Merge beider Knotenarten gebildet. Dabei werden funktionale Köpfe wie Derivations- und Flexionsaffixe als Morpheme betrachtet. Wortbildung wird als die Bildung komplexer syntaktischer Köpfe verstanden. Eine wichtige These der DM ist, dass das Wort keine Einheit mit Sonderstellung ist und 
somit keine absolute Grenze für die Anwendung spezifischer Regeln darstellt (vgl. Marantz (1997), Bobaljik (p.c.)). Wort-Syntax stellt sich somit als eine Frage des Skopus' für das Wirken einer Regel dar.

Die phonologischen Exponenten werden in der DM postsyntaktisch durch Late Insertion eingesetzt. Demnach wird eine syntaktische Struktur erst interpretiert und erhält seine phonologische Form anschließend durch Einsetzen eines Vocabulary Items nach dem Subset Principle an der PF-Komponente (vgl. Embick 2015, Halle/Marantz 1993).

There are three core properties which distinguish Distributed Morphology from other morphological theories: Late Insertion, Underspecification, and Syntactic Hierarchical Structure All the Way Down. (Harley/Noyer 1999.2)

Allgemeine Konsequenzen syntaktischer Ansätze sind (i) die prinzipielle Durchlässigkeit der Produkte aus Wortbildung und der Bildung phrasaler Strukturen sowie (ii) die Möglichkeit der Beschreibung der Struktur komplexer Wörter mit syntaktischen Prinzipien. Deren konkrete Ausgestaltung sowie sich daraus ergebende Implikationen sind vom jeweiligen Ansatz abhängig. Der syntaktische Ansatz trägt dem Fakt Rechnung, dass komplexe Wörter eine vorhersagbare Bedeutung haben können (vgl. Rektionskomposita). Lexikalisierte Bedeutungen können sowohl auf der Wort- als auch auf phrasaler Ebene eingesetzt werden. In der DM ist dies z.B. durch Einträge in der Enzyklopädie möglich (vgl. Marantz 1996:207).

\subsubsection{Hybride Ansätze}

Neben den lexikalistischen und den syntaktischen Ansätzen zur Wortbildung gibt es auch hybride Ansätze. Gemein haben diese zu großen Teilen, dass sie wie die lexikalistischen Ansätze sowohl von einem morphologischen als auch von einem syntaktischen Modul ausgehen; jedoch mit dem Unterschied, dass keine sukzessive Bildungsabfolge postuliert wird, sondern ein Wechselspiel zwischen den Modulen möglich ist.

Erste Ideen dazu stammen von Roeper/Siegel (1978), die der Bildung von Rektionskomposita das First Sister Principle zugrundelegen und bei den Komposita eine teils lexikalische, teils syntaktische Struktur annehmen. Es folgte die Idee, dass Wurzelkomposita im Lexikon und Rektionskomposita in der Syntax gebildet werden (vgl. Roeper 1988). Andere Ansätze nehmen ein paralleles Wirken der beiden Module an (u.a. Booij| 2009b).

Ein weiterer Ansatz stammt von Ackema/Neeleman (2004, 2010). Den Kern ihres Ansatzes bildet die Annahme zweier unabhängiger, aber kompetitierender generativer Submodule: Das der Wort-Syntax und das der phrasalen Syntax. Beide sind Teile eines syntaktischen Makromoduls. Wie die DM nehmen auch Ackema und Neeleman Merge als strukturbildende Operation an, jedoch unterscheiden sie zwischen syntaktischem und morphologischem Merge. Zwischen den beiden Submodulen werden zwei Arten von Interaktionen angenommen: ,Competition' und ,Insertion' (vgl. Ackema/Neeleman 2004:10). Unter Competition verstehen sie, dass syntaktisches Merge morphologischem Merge gegenüber stets präferiert wird. Letzteres muss durch lexikalische Informationen getriggert werden. Komplexe Ausdrücke (z.B. Komposita) können in Bezug auf ihren Bildungsort unterspezifiziert sein (vgl. Ackema/Neeleman 2004:88). Die Insertion beschreiben sie als: ,a relation of feature matching between two nodes in different representations" (Ackema/Neeleman 2004:90). Eine abgeschlossene Konstruktion der Wort-Syntax kann in eine phrasale Struktur eingesetzt werden. Teile davon sind dann wie in lexikalistischen Ansätzen unzugänglich. Da 
jedoch von einer Interaktionen zwischen phrasaler und Wort-Syntax ausgegangen wird, gäbe es sowohl syntaktisch aktive Wortbildungsprozesse als auch solche, die der Syntax gegenüber opak sind.

Bei dem Modell von Ackema und Neeleman handelt es sich um einen hybriden Ansatz, da Prinzipien lexikalistischer und syntaktischer Ansätze miteinander verbunden werden. So bestehen unterschiedliche Reihenfolgemöglichkeiten für die Bildung einer Einheit. Wird jedoch eine Einheit in eine andere insertiert, werden die Teile der insertierten Einheit unzugänglich, was im Ergebnis den Voraussagen der LIH entspricht. Ähnlich den syntaktischen Ansätzen gehen sie zwar von einem syntaktischen Makromodul aus, allerdings auch von morphologischem Merge. Ackema und Neeleman zufolge lässt sich die Wortbildung nicht mit rein syntaktischen Mitteln erklären, da Bindung und Kopf-Bewegung wortintern nicht möglich seien (vgl. Ackema/Neeleman 2010:23). Wenn Komposition allerdings via Merge von Köpfen erfolgt, wäre genau dies zu erwarten. Nach Ackema und Neeleman ist die Syntax bei der Komposition nicht direkt involviert, sondern hat via Competition einen indirekten Effekt auf die Komposition.

Auch Ackema und Neeleman gehen in ihrem hybriden Ansatz von lexikalischer Integrität aus. Elemente können in verschiedenen Reihenfolgen ineinander insertiert werden syntaktische in morphologische und diese wiederum in syntaktische, was die Bildung von Phrasenkomposita ermöglicht - aber jede Bildungseinheit wird als in sich abgeschlossen betrachtet (vgl. Ackema/Neeleman 2004:109f). Dies liege daran, dass Teile von komplexen Wörtern keine identifizierenden Funktionen einführen können, die durch die Syntax befriedigt werden müssten, d.h. Bewegung und Bindung aus Wörtern heraus wird ausgeschlossen (vgl. Ackema/Neeleman 2004:122). Wie sich dies bei Diskursanaphern verhält, bleibt unklar.

\subsubsection{Zusammenschau}

Eine Strukturanalyse verschiedener Komposita-Arten auf Basis lexikalistischer Ansätze stellt sich nicht als zielführend dar. Die Annahme einer separaten Wortbildungskomponente und der damit einhergehenden lexikalischen Integrität gibt keinen Anlass zur Annahme von Unterschieden in der Verarbeitung zwischen verschiedenen Arten von Komposita.

Bei den hybriden Ansätzen kommt es darauf an, welche Grundannahmen gelten. Solange diese die LIH nicht außer Kraft setzen, lassen sich auch mit diesen keine verarbeitungsrelevanten Unterschiede begründen.

Syntaktische Ansätze hingegen erlauben die Annahme verschiedener Strukturen und postulieren keine strikte Grenze zwischen Morphologie und Syntax. Dies eröffnet einen gewissen Spielraum für Annahmen zur Verarbeitung, die auf strukturellen Unterschieden basieren.

Eine für die Klärung der N1-Zugänglichkeit geeignete Theorie sollte liberal genug sein, Fälle von Durchlässigkeit zu erfassen, und gleichzeitig restriktiv genug, um Fälle von lexikalischer Integrität zu erklären. 


\subsection{Strukturaufbau der Komposita-Arten}

Die Unterscheidung zwischen Wurzel- und Rektionskomposita wurde in Kapitel 2.2 .3 bereits angesprochen und soll nun ausgeführt werden. Dabei werde ich die Bezeichnungen ,N1' und ,N2' als deskriptive Benennung der beiden an der Oberfläche sichtbaren Komposita-Konstituenten beibehalten, auch wenn sich zeigt, dass diese Bezeichnungen strukturell betrachtet irreführend sind.

\subsubsection{Wurzelkomposita}

Die kanonische Annahme zum Aufbau von Wurzelkomposita ist, dass (mind.) zwei nichtderivierte Einzellexeme aneinandergefügt werden (vgl. Scalise/Bisetto 2009:35), wobei das zweite Element die grammatischen Eigenschaften der Gesamtkonstruktion bestimmt. Darüber hinaus können Fugenelemente an das N1 herantreten; diese werden jedoch im Allgemeinen nicht als eigene Morpheme behandelt (vgl. Meibauer 2007:50f), siehe auch Kapitel 2.2.2. Daraus ergibt sich für Wurzelkomposita (ungeachtet der Fugenelemente) die Minimalstruktur unter (3) (z.B. bei Lieber 2009:367).

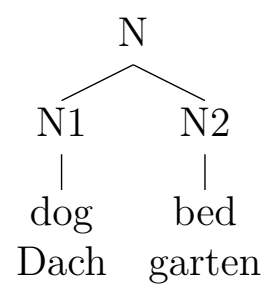

Die konkrete Relation der beiden Kompositateile zueinander ist unterspezifiziert und der Form nicht zu entnehmen. Zur Interpretation eines Kompositums muss diese Relation aus dem Welt- und Kontextwissen ergänzt werden (vgl. Kap. 2.2.4). Wurzelkomposita verfügen zumeist über eine aufgrund von Welt- oder Kontextwissen präferierte, aber dennoch ambige Lesart. So wird Dachgarten zumeist als ein Garten auf einem Dach interpretiert. Der Ausdruck lässt jedoch auch andere Interpretationen zu, bspw. als ein Garten mit verschiedenen Dächermodellen auf einer Baumesse.

Bei einer Paraphrasierung muss weiteres lexikalisches Material hinzugezogen werden, z.B. der Genitiv-Artikel (z.B. Hausbau für Bau des Hauses) oder eine Präposition (z.B. Dachgarten für Garten auf einem Dach).

\subsubsection{Rektionskomposita}

\section{Allgemeine Annahmen}

Der zentrale Unterschied zwischen Rektions- und Wurzelkomposita liegt darin, dass es sich beim N2 eines Rektionskompositums um eine deverbale Nominalisierung handelt. Das N1 hingegen ist das interne Argument des dem N2 zugrundeliegenden Verbs und 
wird durch dieses theta-markiert (für das Deutsche bspw. Klos 2011, Neef 2009).3 Das N1 hat die Funktion eines Arguments, wie es sonst nur bei Konstituenten in phrasalen Strukturen vorkommt (vgl. Reinhard 2001:5). Die an der Bildung eines Rektionskompositums beteiligten Elemente sind also ein nominales Element, ein verbales Element und ein nominalisierendes Derivationssuffix, entsprechend (4-a) und (4-b).

$$
\begin{array}{ll}
\text { a. } & \mathrm{N} 1+\mathrm{V}+\mathrm{N}^{a x} \\
\text { b. Dach-begrün-ung, Auto-fahr-er } \\
\text { c. Kopf-stütze, Haus-putz, Wasser-zufuhr, Öl-wechsel }
\end{array}
$$

Alternativ zu einem Derivationssuffix sind auch Konversionen wie in (4-c) möglich (dazu mehr in Kap. 4.3.5). Entscheidend ist, dass eine Nominalisierung stattfindet. Außerdem kann das Verb durch ein Präfix oder eine Partikel erweitert sein, bspw. bei be-grün im Gegensatz zu fahr. Ein Rektionskompositum kann weiter aus zusätzlichen nominalen oder adjektivischen Elementen bestehen und ebenfalls Fugenelemente enthalten. Entscheidend ist das verbale Element, welches ein nominales Element wortintern regiert, bzw. ihm eine Argumentstelle zuweist, während das nominale Element eine Theta-Rolle des verbalen Elements sättigt (vgl. Scalise/Bisetto 2009, Selkirk 1982). Adjunkte in N1-Position wie in (5) bilden im klassischen Verständnis keine Rektionskomposita (vgl. Selkirk 1982).

\section{Montagsbesprechung, Partydrink}

Zumeist fungiert das N1 als internes Argument (vgl. Reinhard 2001, Spencer 1991). Ob auch das externe Argument innerhalb eines Rektionskompositums realisiert werden kann, ist fraglich, bzw. sprachabhängig. So ist Mädchenschwimmen im Deutschen akzeptabel, girl-swimming im Englischen nicht (vgl. Selkirk 1982:34).4 Hier scheint es außerdem Unterschiede zwischen verschiedenen Komposita-Arten zu geben: Die Besetzung der externen Argumentstelle im Kompositum ist bei Konversionen produktiver (vgl. Lieber 2009), dazu ausführlicher in Kapitel 4.3.5. Die Frage der Besetzung der externen Argumentstelle eröffnet Fragen zu theoretischen Ableitungen: Das Subjekt wird mitunter nicht als regiert verstanden (z.B. Grewendorf 1988:122), bzw. nicht als Teil des Subkategorisierungsrahmens betrachtet (vgl. auch Marantz 1984). Um daraus resultierenden theoretischen Implikationen aus dem Weg zu gehen, liegt der Fokus in dieser Arbeit auf Rektionskomposita mit N1 als internem Argument und Derivationssuffix, vgl. (4-b).

Ein zentraler Bestandteil von Rektionskomposita ist die Nominalisierung. Dabei wird nach Ehrich (vgl. 1991:447f) eine Proposition nominalisiert. Die Nominalisierung ist dann keine Proposition mehr, sondern denotiert ein Ereignis oder dessen Resultatszustand. Ein sehr produktives Nominalisierungsaffix ist -ung. Ung-Nominalisierungen sind polysem, da sie Prozesse (z.B. Vorbereitung), Resultate (z.B. Heilung), entstandene Objekte (z.B. Versiegelung) oder auch Inhalte eines Verbalvorgangs (z.B. Erklärung) denotieren können (vgl. Reinhard 2001:59). Die entsprechende Lesart hängt vom jeweiligen Kontext ab. So

\footnotetext{
${ }^{3}$ In der Dudengrammatik (RN 1097) werden Rektionskomposita ausschließlich valenzgrammatisch durch Zuweisung der Rolle des Patiens und in seltenen Fällen des Agens erklärt (vgl. Wermke et al. 2009. $719)$.

${ }^{4}$ Lieber (2014 86) führt hierzu mit airline hiring ein Gegenbeispiel an. Darüber hinaus stellt sich bei Selkirks Beispiel die Frage, ob es sich im Englischen bei girl tatsächlich um ein externes Argument handelt, da auch die Paraphrasierung swimming for girls weniger akzeptabel als das deutsche Pendant Schwimmen für Mädchen ist.
} 
denotiert Ausgrabung in (6-a) ein Ereignis im Sinne eines Vorgangs, in (6-b) jedoch ein Resultat im Sinne eines gegenständlichen Objekts (vgl. Ehrich/Rapp 2000.246). Welche Interpretation vorliegt, geht also nicht aus der Form hervor, sondern muss auf der Ebene der konzeptuellen Struktur in Abhängigkeit vom Kontext ermittelt werden (vgl. Ehrich 1991:457).

(6) a. Bei der Ausgrabung sind viele Schichten des alten Troja zerstört worden.

b. Die Ausgrabung wird im Pergamon-Museum ausgestellt.

Andererseits teilen Nominalisierungen auch wichtige Eigenschaften mit ihrer verbalen Basis, bspw. argumentstrukturelle und selektionale Eigenschaften (vgl. Fu et al. 2001:550). In diesem Sinne vereinen Nominalisierungen verbale und nominale Merkmale: Wie Basisnomen haben sie eine kategoriale oder typenlogische Bedeutung und wie Basisverben deren lexikalische Bedeutung inkl. Argumentstruktur (vgl. Ehrich 1991:452). So können auch thematische Rollen durch Affixe besetzt werden, z.B. besetzt -er die Rolle des Agens oder -ung als Ereignisargument die Event-Rolle. Interessanterweise sind die obligatorischen Argumente eines Verbs bei der ung-Nominalisierung nur noch fakultativ (vgl. Reinhard 2001: 37f). So fordert herstellen eine $\mathrm{NP}_{\text {Nom }}$ und eine $\mathrm{NP}_{A k k}$ (vgl. (7-a) vs. (7-b)), im Fall der Nominalisierung in (7-c) ist der Satz jedoch auch ohne deren Realisierung grammatisch. Wird ein Argument realisiert, ist dies wie in (7-d) sowohl wortintern also auch -extern möglich (vgl. Reinhard 2001:38).
a. Die Firma stellt teure Stoffe her.
b. *Die Firma stellt her.
c. Die Herstellung nimmt viel Zeit in Anspruch.
d. Stoffherstellung, die Herstellung der Stoffe

Eine ung-Nominalisierung ist nur möglich, wenn das Basisverb einen Zustandswechsel beschreibt, d.h. nicht bei Zuständen und Vorgängen (vgl. Reinhard 2001:46). Dies erklärt, warum ung-Nominalisierungen häufig verbale Präfixe enthalten. Wie in (8) illustriert, können diese ein Vorgangsverb in ein Verb des Zustandswechsels umformen.
a. grünen $-{ }^{*}$ Grünung / begrünen - Begrünung
b. arbeiten $-*$ Arbeitung / verarbeiten - Verarbeitung
c. $\quad$ sorgen $-*$ Sorgung / entsorgen - Entsorgung

Eine semantische Besonderheit von Rektionskomposita gegenüber Wurzelkomposita ist ihre eindeutige Interpretierbarkeit. Im Gegensatz zu Wurzelkomposita sind Rektionskomposita nicht ambig, sondern ihre Interpretation ist stets strukturabhängig (vgl. Meyer 1993, Selkirk 1982). Indem das N1 eine Argumentstelle füllt, kann es nur in diesem Rahmen interpretiert werden (vgl. Meyer 1993:5). Bei transparenten Komposita entspricht die Interpretation der wortinternen Argumentsättigung der Interpretation der phrasalen, es ist also vom gleichen Mechanismus auszugehen (vgl. (9)).

(9) a. Fahrer des Autos

b. Autofahrer 
Wie oben erwähnt, hat auch das Affix eine spezifische Semantik und kann eine ThetaRolle sättigen. So denotiert ein Derivat auf -er den Verursacher einer Handlung, eines mit -ung hingegen bezieht sich auf den Prozess oder das Resultat eines Ereignisses. Alle für die Interpretation des Kompositums notwendigen Informationen sind somit in den Lexemsemantiken enthalten, sodass sich die Wortbedeutung durch eine Genitivphrase paraphrasieren lässt (vgl. z.B. Fanselow 1981:45). Bei der Bedeutung von Dachbegrünung handelt es sich demnach immer um den Prozess oder das Resultat des Begrünens eines Dachs, im Gegensatz zu den verschiedenen Lesarten von Dachgarten.

Worüber in der Literatur Uneinigkeit herrscht, ist die Reihenfolge der Konstituentenfügung (vgl. Lieber 2009). Während die Struktur von Wurzelkomposita aufgrund ihrer Zweigliedrigkeit auf den ersten Blick unkontrovers scheint, eröffnet die in (4-a) und (4-b) illustrierte Dreigliedrigkeit der Rektionskomposita eine Strukturdebatte. Unter der Prämisse der binären Verzweigung ergeben sich dabei zwei Möglichkeiten - die Linksverzweigung und die Rechtsverzweigung - deren Argumente im Folgenden einander gegenübergestellt werden.

\section{Rechts- vs. Linksverzweigung}

\section{a) Rechtsverzweigung: [N $\left.\left[\mathrm{V} \mathrm{N}^{A f}\right]_{N}\right]_{N}$}

Traditionell werden Rektionskomposita als N+N-Komposita analysiert, wobei das N2 eine deverbale Nominalisierung ist und als Kopf des Kompositums fungiert (vgl. Brandt et al. 1999:162). Diese Aussage impliziert eine rechtsverzweigende Struktur. Entsprechend der Analyse unter (10) wird ein verbales Element durch Anfügen eines nominalen Derivationssuffixes nominalisiert und die entstandene Nominalisierung bildet das Kopfnomen des Kompositums (N2).

Prinzipiell lassen sich alle Autor*inn*en, die von einem „deverbalen Kopf“ sprechen, zu den Vertreter*innen dieser Strukturbildungsvariante zählen. Dazu gehören u.a. Booij/van Haaften (1988), Klos (2011), Lieber (1992, 2004), Meyer (1993), Reinhard (2001), Scalise/Bisetto (2009), Selkirk (1982), Spencer (1991).

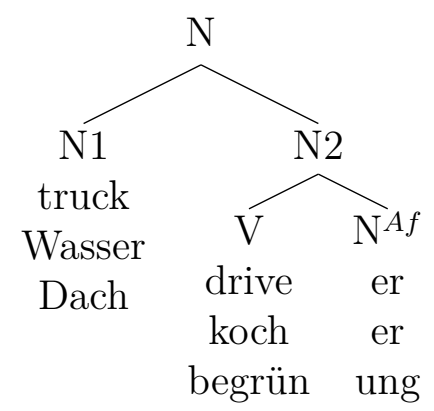

Da nun zwei unabhängig produktive Nomen vorliegen, können diese wie bei einem Wurzelkompositum zusammengefügt werden. Ein zentrales Argument für diese Art der Strukturbildung ist die Unproduktivität von NV-Konstruktionen wie (11), die als Zwischenschritt bei einer linksverzweigenden Struktur notwendig wären.

*truckdrive, ${ }^{*}$ wasserkoch, ${ }^{*}$ dachbegrün 
Geht man allerdings davon aus, dass das N1 eine Argumentstelle des Verbs sättigt, kommt es zu einem Verstoß gegen syntaktische Prinzipien, die Argumentstellensättigung nur zwischen Schwesterknoten zulassen und eine Vererbung der internen Argumentstruktur nicht über die erste Projektion hinaus erlauben. Dazu gehört das Theta-Kriterium der GBSyntax, das First Sister Principle nach Roeper/Siegel (1978), die First Order Projection Condition nach Selkirk (1982) $)^{5}$ und das Argument Linking Principle nach Lieber (1983). Kern dieser Prinzipien ist, dass Verbeigenschaften wie das Theta-Raster nur an SchwesterKnoten vererbt und nicht an den dominierenden Knoten einer anderen syntaktischen Kategorie übertragen werden können.

Eine rechtsverzweigende Analyse muss somit erklären, wie Rektion und Theta-RollenVergabe über eine Kategoriengrenze hinaus möglich sind. Will man für Rektionskomposita keine Argumentvergabe annehmen, gilt es zu klären, wie der semantische Unterschied zu Wurzelkomposita stattdessen erzeugt wird (siehe auch Brandt et al. (1999:162)).

Dass die Argumentstellen-/Theta-Rollen-Vergabe über Kategoriengrenzen hinaus allerdings prinzipiell möglich ist, wird an den Fällen unter (12) deutlich. Darüber hinaus zeigt (12-d), dass die entsprechende Theta-Rolle tatsächlich innerhalb des Kompositums vergeben wird und extern nicht (erneut) gesättigt werden kann. Wenn das N1 Auto nicht das interne Argument von fahr wäre, müsste (12-d) analog zu (12-c) grammatisch sein. Dies ist nicht der Fall.
a. driver of trucks (Di Sciullo/Williams 1987)
b. die Zerstörung der Stadt (Solstad 2010:219)
c. Fahrer des Autos, Beschluss des Erwerbs, Eroberung Galliens
d. *Autofahrer des Autos, *Autofahrer des Wagens, *Autofahrer des Porsches

Die Argumentstellenvergabe innerhalb einer rechtsverzweigenden Struktur bedarf somit einer Erklärung. Di Sciullo/Williams (1987) argumentieren, dass der Verbstamm gemeinsam mit dem Suffix eine zusammengesetzte Argument-Struktur bilde. Dabei begreifen sie das Affix als Funktor mit eigenem Theta-Raster, welches eine Funktion darstellt, die das Theta-Raster des Verbstamms als Wert aufnehmen kann. Das kompositionale ThetaRaster ist eine Eigenschaft des Gesamtworts entsprechend (13).

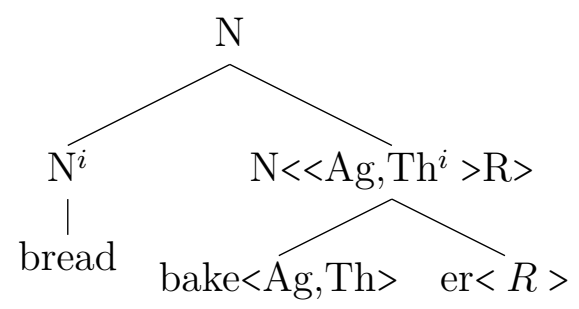

Demzufolge wird genau genommen durch das N1 nicht die Argumentstruktur des Verbs gesättigt, sondern die abgeleitete Argumentstruktur der Nominalisierung. Bei diesem Ansatz handelt es sich um eine lexikalistische Argumentation, da Di Sciullo/Williams (1987) von einer Verletzung des Theta-Kriteriums sprechen, was nur durch lexikalische Regeln

\footnotetext{
5 Selkirk (1982) formuliert die First Order Projection Condition als Alternative zum First Sister Principle. Demnach müssen alle Nicht-Subjekt-Argumente einer lexikalischen Kategorie $\mathrm{X}_{i}$ innerhalb der ersten Projektion von $\mathrm{X}_{i}$ gesättigt werden, d.h. innerhalb der direkt dominierenden Kategorie. Weitere Erläuterungen zur First Order Projection Condition finden sich auch bei Olsen (vgl. 2000:907).
} 
möglich sei.

Gemäß eines Ansatzes von Fanselow (1988) gilt das Theta-Kriterium nicht für Komposita. Die Stelle des internen Arguments wird stattdessen durch Funktionsapplikation gesättigt. Argumentvererbung könne dabei nur bei Derivationen mit Affixen ohne eigene Bedeutung stattfinden 6

Eine Erklärung auf Basis eines syntaktischen Ansatzes liefert Toman (1990), der den Aufbau von komplexen Wörtern mit Hilfe der X-Bar-Syntax beschreibt. Eine nominalisierte Verbform könne unter bestimmten Bedingungen die Argumentstruktur des Verbs bewahren. Dabei werde das Theta-Raster des verbalen Kopfs zunächst an den Mutterknoten vererbt und dann dem Nomen zugewiesen (vgl. Toman 1990:425f). Auch FabriciusHansen (vgl. 1993:195) nimmt an, dass die Argumentstruktur des Kopfs eines komplexen Worts zusammen mit relevanten syntaktischen Merkmalen entlang der Kopflinie an das Gesamtwort vererbt werden kann.

Ob man nun eine zusammengesetzte Argument-Struktur nach Di Sciullo/Williams (1987) annehmen möchte oder Weitergabe des verbalen Theta-Rasters nach Toman (1990) oder Fabricius-Hansen (1993), das Ergebnis ist letztlich das gleiche: Die Argumentvergabe über eine Kategoriengrenze hinweg ist möglich (siehe (12), , eine Rechtsverzweigung allein aufgrund dessen also nicht auszuschließen.

\section{b) Linksverzweigung: $\left[[\mathrm{N} \mathrm{V}]_{V} \mathrm{~N}^{A f}\right]_{N}$}

Unter der Annahme einer linksverzweigenden Struktur wie unter (14) wird zunächst das N1 mit einem Verb zusammengefügt. Syntaktische Ansätze gehen hierbei zumeist von Inkorporation aus. Das entstandene verbale Kompositum ist unproduktiv, wird jedoch in einem weiteren Schritt affigiert, dadurch nominalisiert und der Gesamtausdruck ist wieder eine produktive Konstruktion. Vertreter*innen dieser Form der Strukturbildung sind u.a. Ackema/Neeleman (2004), Booij (2009b), Fabb (1984), Lieber (1983), Sproat (1985).

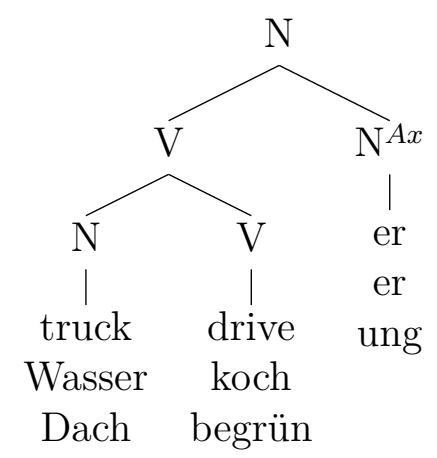

Das häufigste Argument für Linksverzweigung ist die Anordnung von Verb und Komplement in Schwesterposition. Das Festhalten an den Prinzipien der Argumentvererbung führt im Umkehrschluss dazu, dass die meisten Vertreter*innen der Linksverzweigung syntaktischen Ansätzen zuzuordnen sind. Wie bereits erwähnt, liegt hierin jedoch keine notwendige Voraussetzung für Argumentstellenvergabe.

${ }^{6}$ Ein Widerspruch dazu findet sich in Meyer (vgl. 1993 53). 
Eine der ersten linksverzweigenden Analysen stammt von Fabb (1984): Im Fall von (15-a) erfüllt truck das Theta-Kriterium von drive, während letzteres ersteres regiert. Demzufolge müssen beide Wurzeln auf syntaktischer Ebene Schwestern sein. Gleiches gilt für die Phrase unter (15-b), denn auch hier liegt innerhalb der NP eine Schwesterbeziehung vor. Dies gilt nicht für (15-c), weshalb die Konstruktion auch nicht grammatisch ist. Die Nominalisierung erfolge dabei durch syntaktische Affigierung. Denn gemäß der GB-Syntax handelt es sich bei Affixen genauso wie bei Wurzeln und Lexemen um lexikalische Elemente mit syntaktischen Eigenschaften, z.B. auf dem $\mathrm{X}^{o}$-Level zu stehen. Daraus resultiert nach Fabb die Struktur unter (16) 7
a. truck driver
b. driving of trucks
c. *Tom's driving was of trucks.

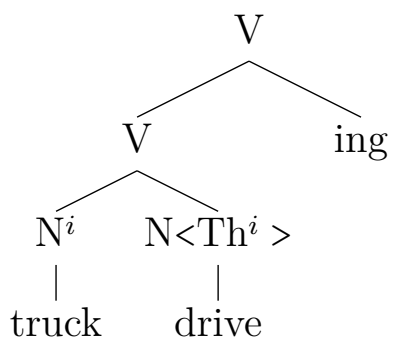

Auf die Theorie von Fabb baut Sproat (2005, 1985) auf. Auch Sproat ist Vertreter eines rein syntaktischen Ansatzes ohne Rückgriff auf eine lexikalische Komponente. Auch für die Komposition setzt er somit syntaktische Wohlgeformtheitsbedingungen an. Gleichzeitig erachtet er es als generell möglich, dass die Argumentstruktur in eine andere Kategorie perkolieren kann (vgl. Sproat 1985:223).

In Bezug auf Rektionskomposita folgt Sproat Fabb und nimmt an, dass das Verb Schwester des N1 ist und dass es seine Theta-Rolle an die Nominalisierung übergibt. Allerdings merkt er an, dass Theta-Rollen nur zur maximalen Projektion, also zur NP zugeschrieben werden können. In einem Kompositum stünden jedoch weder ein V' noch eine maximale Projektion zur Verfügung. Deshalb nimmt er nicht wie Fabb an, dass innerhalb des Rektionskompositums Theta-Markierung stattfindet, sondern Theta-Identifikation. Dabei werde das direkte Objekt zu einer Art Modifikator der Verbs (vgl. Sproat 1985:318).8 Die sich daraus ergebende Struktur ist unter (17) wiedergegeben, wobei ${ }^{* 6}$ und,$+^{6}$ die Theta-Identifikation kennzeichnen. Das Theta-Raster des Verbs perkoliert zum nächst höheren Knoten, wenn das Theta-Raster des (hier verbalen) Affixes und das Theta-Raster des Verbstamms durch einen regulären Prozess der Theta-Rollen-Erfüllung assoziiert werden. Das Theta-Raster des Affixes wird mit dem Theta-Raster des Verbs identifiziert, das Derivat erhält somit das Theta-Raster des Verbstamm. Es handelt sich um Vererbung durch syntaktische Affigierung.

\footnotetext{
${ }^{7}$ Beim hier mehrfach erwähnten Fall von truck driving gilt es zu bedenken, dass es sich um ein Gerundium handelt, welches nicht mit einer deutschen ung-Nominalisieurng identisch ist, die klar nominal ist.

${ }^{8}$ Wenn das N1 die Agens-Rolle erfüllt, handelt es sich Sproat zufolge nicht um ein Rektionskompositum, sondern um ein Wurzelkompositum, in dem Fall finde keine Theta-Rollen-Übertragung statt (vgl. Sproat 1985 276).
} 


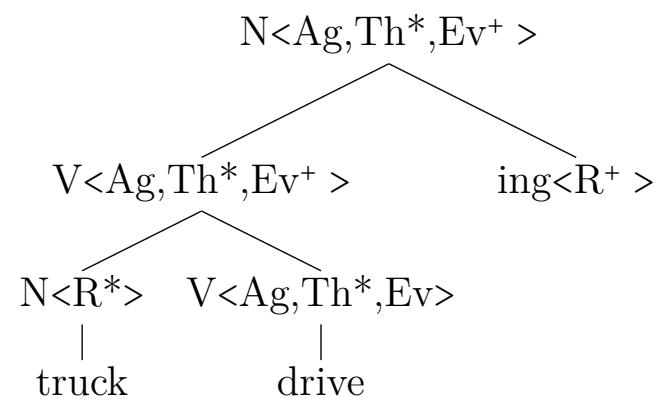

Dem Problem der affixlosen Nominalisierung bei Konversionen begegnet Sproat mit einem abstrakten Nominalisierungsmorphem (NOM). NOM hat eine syntaktische Funktion, kann aber verschiedene phonologische Formen annehmen, also auch Nullmorphem sein; ähnlich den Allomorphen im Flexionsparadigma. Weiterhin trägt es eine eigene ThetaRolle, die mittels Theta-Identifikation befriedigt werden kann (vgl. Sproat 1985:238ff). Da NOM den syntaktischen Kopf der Konstruktion bildet, legt es die spezielle Theta-Rolle des Verbs fest, welche das Nomen als Referenten auswählt (vgl. Sproat 1985:240). Die phonologische Form von NOM gilt als Funktion der lexikalischen Eigenschaften des individuellen Stamms. Angewendet auf die bestehende Struktur ergibt sich damit die Struktur unter (18)

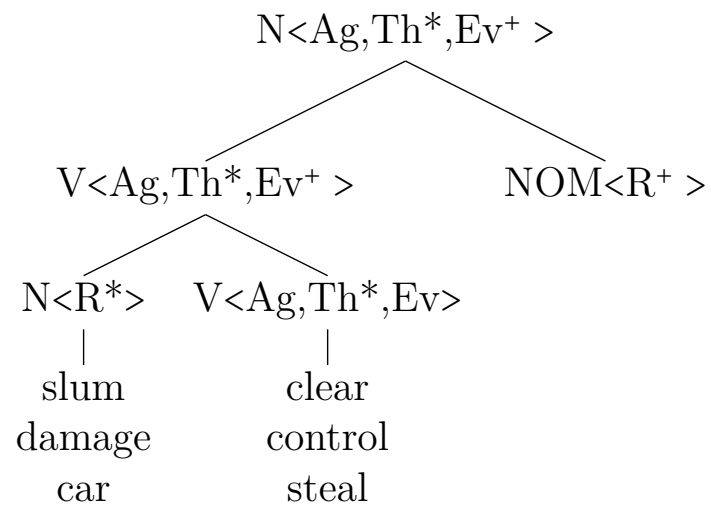

Zwar gewährleistet eine solche Struktur Argumentvergabe an Schwester-Knoten, dies allein genügt allerdings nach wie vor nicht als Argument gegen die Rechtsverzweigung. Stattdessen gibt es ein gravierendes Argument gegen die Linksverzweigung: Die Genese eines unproduktiven NV-Kompositums als Zwischenschritt der Kompositumsbildung. Diese Form ist weder im Deutschen noch im Englischen isoliert produktiv. Bei belegten NV-Komposita wie in (19) handelt es sich meist um lexikalisierte Formen, die als sog. Rückbildung erklärt werden. In diesem Sinne beobachtet Gaeta (vgl. 2010 231), dass ein NV-Konstrukt nur frei auftritt, wenn auch ein entsprechendes Kompositum existiert.

$$
\begin{aligned}
& \text { a. to baby-sit - baby-sitter } \\
& \text { b. staubsaugen - Staubsauger }
\end{aligned}
$$

Die meisten Vertreter*innen der Linksverzweigung sehen die Produktivität der Teilbildungen allerdings nicht als notwendige Voraussetzung an. Syntaktisch wird die NV-Komposition meist als Inkorporation beschrieben. Inkorporationen definiert Baker als „processes by which one semantically independent word comes to be 'inside' another" (Baker 1988:1). Die Inkorporation sei dabei ,the result of applying standard movement transformations 
to words rather than to full phrases“ (ebd.). Weiter heißt es, dass NV-Komposita nicht produktiv und deren nominale Elemente nicht-referenziell seien. Als Grund vermutet er, dass die inkorporierten Nomen oft generisch referieren (vgl. Baker 1988:78). Dass man hier differenzierter vorgehen muss, wurde bereits in Kapitel 3.5 thematisiert.

Auch Rivet (1999) hält Rektionskomposita für syntaktische Konstrukte, die eine Inkorporation enthalten. Komplexe Wörter betrachtet sie als Resultat einer syntaktischen $\mathrm{X}^{{ }_{-}}$ Bewegung, genauer zweifacher Kopfbewegung (vgl. Rivet 1999,320). Entsprechend der zwei möglichen Bildungsreihenfolgen nennt sie zwei „Bewegungsoptionen“: Entweder eine Bewegung des Kopfs der Komplement-NP an den verbalen Stamm und anschließend eine Inkoporation des komplexen Verbstamms in das nominale Affix; oder zuerst eine Inkorporation des Verbstamms in das Affix und anschließend eine Bewegung des Kopfs der Komplement-NP an das komplexe Nomen (vgl. Rivet 1999:320f). Für die Linksverzweigung argumentiert sie syntaktisch, indem sie sagt, dass sich ein Kopf nur an den nächsthöheren Kopf bewegen darf (strict cyclicity, vgl. Rivet 1999:323). Sollte Linksverzweigung tatsächlich die angemessene Strukturbildung sein und sollte dabei tatsächlich Inkorporation ein involvierter Mechanismus sein, ergibt sich ein neues Problem für die Zugänglichkeit des N1. Obwohl es sich bei der Inkorporation um ein syntaktisches Prinzip handelt, gelten inkorporierte Nomen im Deutschen i.d.R. als unzugänglich. In der deutschsprachigen Morphologie-Literatur wird Inkorporation zumeist wie Univerbierung als Oberbegriff für ein Kontinuum an Bindungsfestigkeit innerhalb von Nomen-Verb-Konstruktionen gebraucht, so u.a. bei Gallmann (vgl. 1999.272ff, siehe auch Kap. 2.5.2). Je nach Stärke der Verbindung verbleibt die N-Komponente als syntaktisches Wort (z.B. Klavier spielen) oder wird zum bloßen (nicht-syntaktischen) Wortteil (z.B. schlussfolgern). Generell beschreibt Gallmann nominale Bestandteile von intransitiven Nomen-Verb-Verbindungen als nicht referenzfähig (vgl. Gallmann 1999:284). Je lockerer jedoch die Verbindung, desto eher erscheint die N-Komponente zugänglich für anaphorische Bezüge (vgl. (20)]. Wie bereits in Kapitel 2.5.2 thematisiert, spielt hierbei auch die Differenzierung zwischen eigentlichem und uneigentlichem (metaphorischem) Gebrauch eine Rolle.

Ich werde erst wieder Klavier spielen, wenn es gestimmt wurde.

Ein weiteres Argument dafür, dass Inkorporation N1-Zugänglichkeit nicht prinzipiell ausschließt, liefern polysynthetische Sprachen. Inkorporationen sind dort wesentlich produktiver als im Deutschen und die N-Komponente ist dort zugänglich. Hierzu mehr in Kapitel 4.3 .4 .

Nicht als Inkorporation, sondern als eine Art Zusammenbildung beschreibt Erben (2000) die NV-Fügung. Dabei handele es sich um das morphologische Pendant zur syntaktischen Zusammenrückung. Gaeta (2010) deklariert die NV-Fügung als Wortgruppe (word group) und produktive Fälle als Rückbildungen.

Mit der Berufung auf das Betonungsmuster liefert Rivet (vgl. 1999:323) ein phonologisches Argument für die Linksverzweigung: Im Fall von dreigliedrigen Komposita wie unter (21) kann nur Linksverzweigung das korrekte Betonungsmuster hervorbringen.

PORschefahrfan vs. ${ }^{*}$ PorscheFAHRfan 
Diese Annahme manifestiert sich in der Compound Stress Rule:

Die Compound Stress Rule (CSR):

In einem Paar von Schwesternknoten $\left[{ }_{N}\right.$ A B $]$ ist B genau dann akzentuiert, wenn B verzweigt (d.h., A ist akzentuiert, wenn B nicht verzweigt). (Sternefeld 2008 10)

Dies illustriert Sternefeld anhand von (22), wo das Betonungsmuster die Lesart des ambigen Kompositums festlegt.

\section{MÄDchenhandelsschule vs. MädchenHANdelsschule}

Im Sinne der Compound Stress Rule würde die Verschiebung des Wortakzents auf das N1 im Fall von Rektionskomposita (vgl. (23-a) also für eine linksverzweigende Struktur sprechen. Allerdings stellt sich die Frage, ob dreigliedrige Komposita mit Rektionskomposita verglichen werden können, da das dritte Element in Rektionskomposita ein Affix ist und somit keinen Lexemstatus hat. Darüber hinaus beruht die Akzentverschiebung bei Sternefeld auf semantischen Kriterien. Bei Rektionskomposita handelt es sich um eine rein strukturelle Frage. Der Akzent dient in dem Fall nicht der Hervorhebung einer Lesart, da diese im Fall von Rektionskomposita nicht ambig ist. Dies zeigt sich auch bei dreigliedrigen Komposita. Liegt dort keine ambige Struktur vor, befindet sich sowohl bei (semantischer) Linksverzweigung wie in (23-b) als auch bei (semantischer) Rechtsverzweigung wie in (23-c) der Akzent auf der ersten Konsitutente. Es bleibt somit fraglich, ob das Betonungsmuster zur Klärung der Frage der Strukturbildungsreihenfolge beitragen kann.
a. BeGRÜNung vs. DACHbegrünung
b. HOLZschnitzkunst vs. ${ }^{*}$ HolzSCHNITZkunst
c. PaPIERschneidemaschine vs. ${ }^{*}$ PapierSCHNEIdemaschine

Ebenfalls für die Linksverzweigung, jedoch unabhängig von den genannten syntaktischen Ansätzen argumentieren Ackema/Neeleman (2004) in ihrem hybriden Ansatz. Gegen die Theorie der Rückbildung spricht für sie, dass dies nicht bei allen Komposita möglich ist (vgl. Ackema/Neeleman 2004:59); an der Inkorporations-Theorie problematisieren sie die Notwendigkeit von Bewegung. Bewegung und Bindung sind in ihrem Ansatz keine Operationen, die unterhalb der Wortgrenze stattfinden können. Stattdessen bezeichnen sie den Prozess der NV-Fügung als Insertion (Ackema/Neeleman 2010).

Ihr erstes Argument zielt darauf ab, dass auch das N2 im Falle von Rechtsverzweigung stets isoliert produktiv sein müsste, sollte die isolierte Produktivität eine notwendige Eigenschaft sein. Die Beispiele in (24) belegen, dass dies nicht der Fall ist und der Mangel an Produktivität somit kein ausreichendes Argument gegen die Linksverzweigung darstellt (vgl. Ackema/Neeleman 2004:56). Hierbei fällt allerdings auf, dass es sich in (24) um ein Funktionsverbgefüge und um ein Idiom handelt.9 Die alternativen Beispiele unter (25) stammen von Neef (2009:397).

(24) a. to make trouble/troublemaker vs. *maker of trouble

b. to break the ice/icebreaker vs. *breaker of the ice

${ }^{9}$ Für diese Beobachtung danke ich Andreas Blümel. 
a. Appetithemmer vs. ${ }^{*}$ Hemmer

b. Farbgebung vs. ${ }^{*}$ Gebung

Als weiteres Argument für die Linksverzweigung bemühen auch Ackema/Neeleman (2004) dreigliedrige Nominalkomposita. Bei ihren Beispielen aus dem Niederländischen unter (26-a) ist die Linksverzweigung klar semantisch begründbar. ${ }^{10}$ Wie in (26-b) und (26-c) ersichtlich, sind die beiden möglichen Teilbildungsschritte einzeln nicht grammatisch (vgl. Ackema/Neeleman 2004:56). Ähnliches beobachten sie auch bei Adjektiven: Unproduktive Teile wie langhaar werden erst durch Affigierung zum produktiven Adjektiv langhaarig (vgl. Ackema/Neeleman 2004:63). Semantisch sei es unplausibel zunächst die Bildung von haarig anzunehmen. Die Unproduktivität eine Teilbildung ist somit kein hinreichendes Argument gegen eine Linksverzweigung.
a. [[appel pluk]machine], [[hout snij]kunst]
b. *plukmachine
c. *De boerenknecht [appel plukt] de hele dag

Eine Erklärung für die Unproduktivität liefern Ackema/Neeleman (2004) mit dem Konzept der Competition, einem Wettbewerb zwischen Syntax und Morphologie:
[...] when projections of the same category merge, syntactic merger blocks morphological merger. Morphological merger is allowed when there is no syntactic competitor. As a result, affixation can have the effect of licensing morphological structures that do not occur in isolation. (Ackema/Neeleman 2004:63)

Konkret bedeutet das: (27-a) blockiert (27-b), weil die gleichen Kategorien gemergt werden und damit die syntaktische Variante die morphologische blockiert. (28-a) blockiert jedoch nicht (28-b), weil in dem Fall verschiedene Kategorien gemergt werden. Das Kompositum (28-b) kann also bestehen, die NV-Fügung (27-b) wird jedoch vom syntaktischen Äquivalent (27-a) blockiert (vgl. Ackema/Neeleman 2004:60ff).

$$
\begin{aligned}
& \text { a. } \text { drive a truck/drive trucks }=\mathrm{V}+\mathrm{N} \text { (syntaktisches Merge) } \\
& \text { b. } \quad \text { truck-drive }=\mathrm{V}+\mathrm{N} \text { (morphologisches Merge) } \\
& \text { a. driver of a truck }=\mathrm{N}+\mathrm{FP} \text { (syntaktisches Merge) } \\
& \text { b. } \quad \text { truck driver }=\mathrm{V}+\mathrm{N} \text { (morphologisches Merge) }
\end{aligned}
$$

Die Wettbewerbs-Theorie beantwortet die Frage nach dem Grund für die Unproduktivität der NV-Fügung und liefert gleichzeitig eine Begründung für die Linksverzweigung. Da verschiedene Kategorien gemergt werden, können (29-a) und (29-c) koexistieren. Allerdings können (29-b) und (29-c) nicht koexistieren, weil in dem Fall gleiche Projektionen germergt werden. Wenn also (29-b) die richtige Struktur wäre, dann würde sie durch die Existenz der syntaktischen Form [29-c) blockiert; da es die Form truck driver allerdings gibt, muss (29-a) die richtige Struktur sein, denn nur dann besteht kein Wettbewerb mit (29-c) (vgl. Ackema/Neeleman 2004:64).

(29) a. [[truck drive]er] $-\mathrm{V}+\mathrm{N}$ (morphologisches Merge)

\footnotetext{
${ }^{10}$ Ähnliche Beispiele zeigt Härtl (2015 177) für das Deutsche auf: [[Schuh-putz-]zeug], [[Schnell-koch-]
} topf], wobei mir diese Beispiele weniger eindeutig erscheinen. 
b. $\quad[\operatorname{truck}[$ drive er $]]-\mathrm{N}+\mathrm{N}$ (morphologisches Merge)

c. [[drive er] of trucks] $-\mathrm{N}+\mathrm{PP} / \mathrm{FP}$ (syntaktisches Merge)

Dass dennoch einzelne NV-Komposita frei vorkommen können, liege daran, dass diese idiomatisiert seien (z.B. to baby-sit) und das syntaktische Pendant die Bedeutung nicht tragen könne (z.B. *to sit a baby). In dem Fall besteht kein Wettbewerb und die morphologische Fügung wird nicht blockiert (vgl. Ackema/Neeleman 2004:60).

Eine andere Erklärung für die Unproduktivität der NV-Bildung stammt von Härtl (vgl. 2015:176). Anhand von (30) argumentiert er, dass interne Argumente von verbalen Köpfen nicht wortintern realisierbar sind. Nur im Fall von Adjunkten sei dies möglich (vgl. (30-b)). Sollte es sich hierbei tatsächlich um eine grammatische Beschränkung handeln, wäre dies ein starkes Argument für die Unproduktivität der NV-Bildungen.
a. *Max hat apfelgegessen.
b. Max hat hausgeschlachtet.

Schließlich sei noch ein typologisches Argument von Ackema/Neeleman (2010) angeführt: Für die Kreol-Sprache Saramaccan, deren Lexik hauptsächlich dem Englischen entstammt, ist $\mathrm{N}+\mathrm{N}-$ Komposition attestiert, jedoch keine $\mathrm{N}+\mathrm{V}$-Komposition (vgl. Ackema/Neeleman 2010.33). Die Ursprungssprachen, von denen Saramaccan abstammt, verfügen jedoch über $\mathrm{N}+\mathrm{V}$-Komposition. Wenn Rechtsverzweigung die adäquate Strukturbildung wäre, dann wäre der letzte Kompositionsschritt N+N. Dies und Affigierung ist im Saramaccan möglich. Rektionskomposita müssten also möglich sein. Ist jedoch Linksverzweigung die adäquate Strukturbildung, dürfte es keine Rektionskomposita geben, denn N+V ist keine mögliche Konstruktion. Da es im Saramaccan keine Rektionskomposita gibt, bleibt für Ackema/Neeleman (2010) nur der Schluss, dass Linksverzweigung die korrekte Analyse ist.

\section{c) Ternäre Bildung}

Um der Erzeugung unproduktiver Teilbildungen aus dem Weg zu gehen, wurden Ansätze zu ternärer Verzweigung formuliert, so z.B. von Štekauer (1998). Derartige Überlegungen sind vergleichbar mit der Idee der morphologischen Zusammenbildung im Sinne von (31) (vgl. Meibauer 2007:61ff).

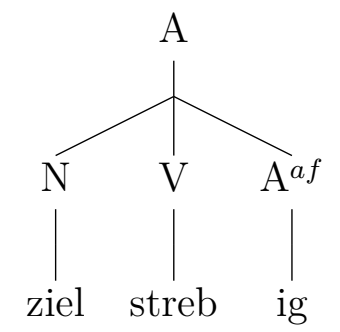

Auch Booij (2009a) und Gaeta (2010) postulieren die Gleichzeitigkeit von Komposition und Derivation durch eine sog. ,template conflation/unification؛ 
Through unification of the templates $[\mathrm{NV}]_{V}$ and $[\mathrm{V} \text { er }]_{N}$ we get the template $\left[[\mathrm{NV}]_{V} \text { er }\right]_{N}$. This latter, unified template can be qualified as productive, unlike the $\mathrm{N}+\mathrm{V}$ template in isolation. (Booij 2009a:214)

Als Argument führt Gaeta (2010:231f) an, dass es neben den unproduktiven NV-Komposita auch unproduktive Nominalisierungen gibt (z.B. * Sagung in Todsagung). Dies kann allerdings auch als Argument gegen die Notwendigkeit unabhängiger Produktivität der Teilbildungen geltend gemacht werden, wie bereits anhand von (25) thematisiert.

Ein Argument gegen ternäre Bildungsmuster ist, dass allgemeingültige Bildungsprinzipien erstrebenswert sind; die ternäre Struktur würde eine Ausnahme bilden.

\section{Zusammenschau}

Unter der Prämisse binärer Verzweigung treten zwei mögliche Strukturbildungsreihenfolgen für Rektionskomposita in Erscheinung: Die Rechts- und die Linksverzweigung. Bei der eher lexikalistisch orientierten Argumentation für Rechtsverzweigung kristallisiert sich die Bildung von Rektionskomposita als $\mathrm{N}+\mathrm{N}$ Adjunktion heraus, erweitert um ein Konzept der Argumentvererbung. Das deverbale Nomen erbt eine modifizierte Version der Argumentstruktur des zugrundeliegenden Verbs und schreibt der ersten Konstituente die Rolle des internen Arguments zu (vgl. Olsen 2014,42).

Bei der eher syntaktisch orientierte Argumentation für Linksverzweigung kristallisiert sich heraus, dass es sich bei Rektionskomposita genau genommen um derivierte NV-Komposita handelt.

Vertreter*innen der Rechtsverzweigung berufen sich darauf, dass Argumentvererbung über Kategoriengrenzen hinaus möglich ist und Vertreter*innen der Linksverzweigung entkräften das Argument der NV-Unproduktivität. Eine Möglichkeit der Analyse der NV-Konstruktion wäre im Sinne eines gebundenen Morphems, welches wie bspw. einige Verbstämme (z.B. ess-) oder Konfixe (z.B. bio-) ohne zumindest ein weiteres Affix nicht produktiv ist.

Ein weiteres Argument für die Linksverzweigung ist die Wettbewerbstheorie von Ackema/Neeleman (2004), welche jedoch nur im Rahmen ihres hybriden Ansatzes gültig ist, da sie die parallele Existenz von morphologischem und syntaktischem Merge voraussetzt.

Entscheidend an dieser Stelle ist die Frage nach einem Zusammenhang zwischen Bildungstyp und N1-Zugänglichkeit. Bei der Sichtung der Literatur stellt sich heraus, dass es auf beiden Seiten Autor*inn*en gibt, die von Strukturgleichheit zwischen Wurzel- und Rektionskomposita ausgehen (z.B. Borer 2013, Meyer 1993, Selkirk 1982), darunter auch Vertreter*innen syntaktischer Ansätze (z.B. Gaeta 2010, Harley 2009, Siebert 1999). Auf beiden Seiten gilt allerdings auch die Annahme von Argumentvererbung und Theta-RollenVergabe innerhalb der Rektionskomposita.

Eine Entscheidung für eine Strukturbildungsrichtung kann an dieser Stelle nicht getroffen werden, allerdings sollen zwei Annahmen als grundlegend für die weiteren Betrachtungen gelten: Innerhalb von Rektionskomposita findet Argument- und Theta-Rollen-Vergabe statt und die Strukturbildung erfolgt binär. 


\subsubsection{DM-Ansatz für Komposita}

Die vorgestellten lexikalistischen und hybriden Ansätze sind durch die strikte Trennung zwischen Syntax und Morphologie in ihren Möglichkeiten der Modellierung von Zugänglichkeit stark reglementiert. Deshalb wurde bereits in Kapitel 4.1.4 dafür argumentiert, die Modellierung des Strukturaufbaus verschiedener Komposita-Arten anhand eines syntaktischen Ansatzes zu verfolgen. Eine klare Entscheidung für eine Verzweigungsrichtung bei Rektionskomposita konnte nicht getroffen werden, allerdings tendieren die meisten syntaktischen Ansätze zu Linksverzweigung.

Im nächsten Schritt soll als Grundlage für strukturbasierte Hypothesen zur N1-Zugänglichkeit eine Detail-Analyse der beiden Komposita-Arten vorgestellt werden. Dabei stehen Fragen nach dem Ort und der Art der einzelnen Bildungsschritte und der Natur der einzelnen Konstitutenten im Fokus.

Als Grundlage der Analysen dient der syntaktische Ansatz der Distribuierten Morphologie (DM) nach Embick/Noyer (2007), Harley (2009), Marantz (1996, 1997). Das Framework der DM ermöglicht detaillierte Aussagen zu wortinterner Strukturbildung und liefert bereits Ansätze zur Analyse von Komposita. Zunächst werden die für diese Arbeit relevanten Grundannahmen der DM zusammengefasst und eine bereits bestehende KompositaAnalyse vorgestellt. Anschließend wird eine eigene Analyse fürs Deutsche skizziert.

\section{Grundannahmen}

Ein zentraler Punkt für die Analyse von Wortbildungsprodukten im Framework der DM ist, dass die Wortbildung auf syntaktischen Operationen beruht. Komposita werden somit genau wie Phrasen via Merge gebildet:

To imagine a theory in which the grammar constructs all words in the syntax by the same general mechanisms ("merge and move"; see Chomsky 1995) that construct phrases, it is useful to make the natural assumption that whether you get a "zero-level category" (wordlike unit) or a phrasal category by merging two constituents is a function of the (categories of the) constituents involved, not of the "merger" operation itself. That is, there is no reason not to build words in the syntax via "merger" (simple binary combination) as long as there are no special principles of composition that separate the combining of words into phrases from the combining of morphemes into words. (Marantz 1997 205)

Wie in Kapitel 4.1 .2 bereits angerissen, werden in der DM zwei Arten von terminalen Knoten unterschieden: Wurzeln (roots) und Merkmalsbündel (feature bundles, vgl. Harley 2009). Beide Arten von terminalen Knoten können frei und gebunden vorkommen und durch Merge verbunden werden. Wurzeln verfügen über keine Kategorie, sondern ausschließlich über einen semantischen Wert (z.B. $\sqrt{ } \mathrm{DACH})$. Ihre Interpretation variiert je nach syntaktischem Kontext, d.h. je nach dem, mit welchem kategorisierenden Merkmalsbündel (z.B. $\mathrm{n}^{o}, \mathrm{v}^{o}, \mathrm{a}^{o}$ ) sie mergen.

Ein Merkmalsbündel fungiert als Kategorisierer. Es kann kann null sein (z.B. bei Dach: $\left[[\sqrt{ } \mathrm{DACH}]_{\sqrt{ }} \mathrm{n}^{o}\right]_{n P}$ mit $\left.\mathrm{n}^{o}=\mathrm{NULL}\right)$ oder overt (z.B. bei gemütlich: $\left[[\sqrt{ } \text { GEMÜT }]_{\sqrt{ }} \mathrm{a}^{o}\right]_{a P}$ mit $\mathrm{a}^{o}=$ lich). Neben einer Kategorie tragen Merkmalsbündel verschiedene semantische Informationen. So verfügt das verbalisierende Bündel vo ${ }^{o}$.a. über Varianten mit der Bedeutung CAUSE (z.B. clarify), BECOME (z.B. grow), BE (z.B. fear); $\mathrm{n}^{o}$ wiederum hat als mögliche Ausprägungen ,the event or result of (z.B. mixing), , the agent or instrument of ' (z.B. mixer) oder ,the property of ' (z.B. elasticity). Merkmalsbündel können außerdem , gestapelt‘ werden: Eine Wurzel kann mit $n^{o}$ mergen; anschließend mergt das 
daraus resultierende Morphem mit $\mathrm{a}^{o}$, und das Produkt daraus wiederum mit $\mathrm{n}^{o}$ (z.B. pennilessness, vgl. Harley 2009:131f).

Wörter lassen sich somit als Merging-Produkte aus Wurzeln und Merkmalsbündel beschreiben. Mergt eine Wurzel mit einem $\mathrm{n}^{o}$ wird eine $\mathrm{nP}$ gebildet, mergt eine Wurzel mit einem $v^{o}$ eine vP. Der Unterschied zwischen Nomen und Verben liegt demnach ausschließlich in der funktionalen Strukturebene und ist keine lexikalische Eigenschaft.

In fact our usual syntactic categories of nouns, verbs, and adjectives aren't syntactic categories at all but morphological categories, categories that emerge during a derivation only in the context of certain functional projections. (Marantz 1996 17)

Ein Morphem ist ebenfalls ein kategorisiertes Merging-Produkt aus Wurzel und funktionalem Kopf, das im Gegensatz zum Wort noch nicht isoliert produktiv sein muss (vgl. Marantz 2013b). Das Mergen an sich wird z.T. mit einer Kopf-Bewegung verglichen, wobei terminale Knoten unter einer Kategorie eines Kopfs verbunden werden, jedoch als zwei unabhängige terminale Knoten unter diesem Kategorie-Knoten bestehen bleiben (vgl. Halle/Marantz 1993:116).

Eine Konsequenz aus den Annahmen der DM ist, dass es sich bei Derivation und Flexion nicht um zwei verschiedene Bildungsformen handelt; es sind lediglich verschiedene Arten von Merkmalsbündeln involviert (Harley 2009:131f). Die Redundanz zwischen lexikalischen und funktionalen Kategorien ist durch die Einteilung in Wurzeln und funktionale Merkmalsbündel aufgehoben. Auch auf Nominalisierungen eröffnet die DM einen neuen Blick: Diese sind nicht deverbal, da keiner der involvierten Knoten als Verb kategorisiert wird. So wird bspw. Zerstörung analysiert als $\left[[\sqrt{ } \text { ZERSTÖR }]_{\sqrt{ }} \mathrm{n}^{o}\right]_{n P}$. In dem Fall handelt es sich um ein Nomen, welches Argumente nimmt (vgl. Marantz 1996:22).

\section{Komposita in der DM (Harley 2009)}

Eine Analyse von englischen Komposita im Rahmen der DM schlägt Heidi Harley (2009) vor. Zusammengefasst beschreibt sie Komposition als zweifache Inkorporation: „the $\sqrt{ }$ containing constituents of a phrasal $\sqrt{ } \mathrm{P}$ incorporate[s] first within themselves and then in a category-creating head such as $\mathrm{n}^{o}$ or a $\mathrm{a}^{o "}$ (Harley 2009:136).

Dabei nimmt Harley an, dass die Selegierung interner Argumente eine Eigenschaft der Wurzel ist. Dies erkläre die Argumentsättigung der Fälle unter (32) ohne Annahme von Argumentweitergabe o. Ä. Die Wurzel mergt hier zuerst mit ihrem Argument ([[ $\sqrt{ }$ STUD $] \sqrt{ }$ $\mathrm{DP}($ chemistry $\left.)]_{\sqrt{ } P}\right)$, bevor diese wiederum mit ihrem kategorisierendem Merkmalsbündel mergt (vgl. Harley 2009:134).

a. student of chemistry

b. he studies chemistry

Hieraus lässt sich schlussfolgern, dass eine Wurzel Argumente selegieren kann, aber nur eine nominale Konstituente diese Argumentposition besetzen kann. Rektionskomposita wie truck driver legt Harley ein nominales Komplement zugrunde, welches über ein eigenes $\mathrm{n}^{o}$ verfügt (vgl. Harley 2009:135). Dieser n-Kopf wird mit der zweiten Wurzel ( $\sqrt{ }$ DRIVE) zusammengefügt, bevor die gesamte Wurzel-Phrase schließlich mit dem kategorisierenden $\mathrm{n}^{o}$ mergt (vgl. (33) aus Harley 2009:136). 


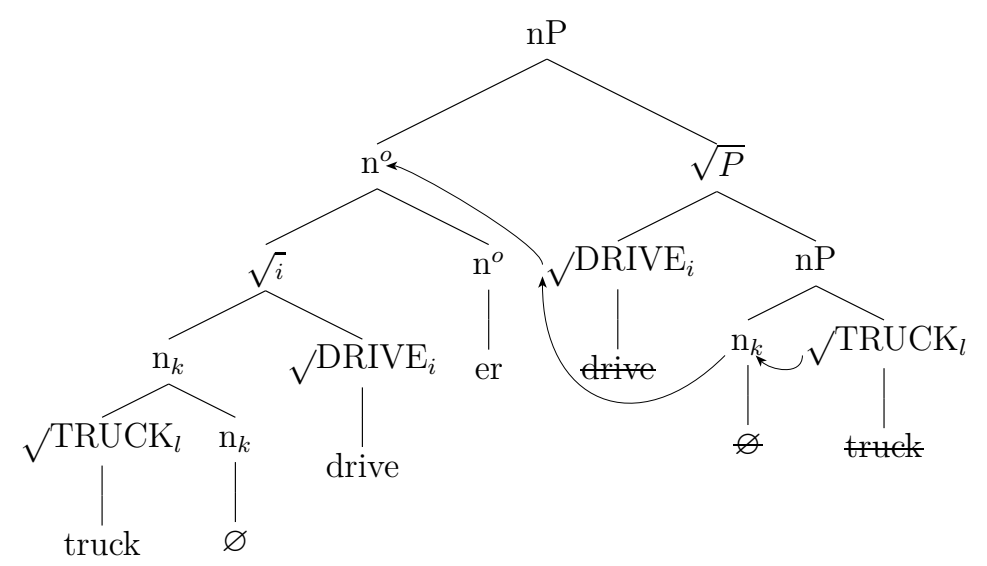

Die nP truck mergt also als Argument der Wurzel $\sqrt{ }$ DRIVE mit dieser und inkorporiert anschließend in sie. Die Inkorporation $(\sqrt{ } \mathrm{P})$ mergt mit dem agentiven $n^{o}$-er und vollzieht eine Kopf-Bewegung in dieses, wodurch der komplexe nP-Kopf (34) entsteht. Als letzter Schritt erfolgt die Vocabulary Insertion als truck driver (vgl. Harley 2009:136).

$$
\left[\left[\left[[\sqrt{ } \text { TRUCK }]_{\sqrt{ }} \mathrm{n}\right]_{n P} \sqrt{ } \mathrm{DRIVE}\right]_{\sqrt{ }} \mathrm{P} \mathrm{n}\right]_{n P}
$$

Nach dem gleichen Schema analysiert Harley auch Wurzelkomposita. In diesem Fall gibt es allerdings kein internes Argument, sondern eine semantische Relation zwischen den nominalen Wurzeln, die durch enzyklopädische und pragmatische Informationen in der interpretativen Komponente erschlossen werden muss. Das N1 wird auch hier als (bereits kategorisierte) Schwester der zweiten Wurzel eingeführt, bevor beides durch ein $n^{o}$ kategorisiert wird (vgl. Harley 2009:139). Hierfür nimmt sie die Struktur unter (35)] an (vgl. Harley 2009:140). In diesem Sinne legt Harley den beiden Komposita-Arten die gleiche Struktur zugrunde.

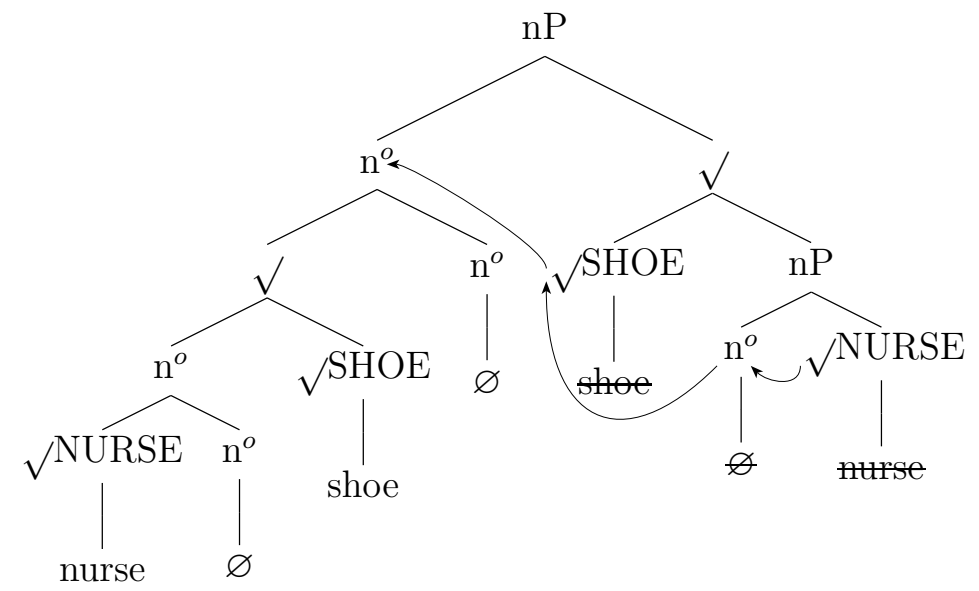

Gemäß Harley kann nur eine nP inkorporieren, keine DP. Wird die nP durch eine DP ersetzt, wird sie durch Stranding nach rechts verschoben und eine Phrase wird gebildet (z.B. 
driver of the truck, vgl. Harley 2009:136). Diese Beschränkung müsse auch für KompositaErstglieder gelten, da diese keine DP sein können, wobei sie sich auf Beispiele wie (36) bezieht.
a. *[trucks]-driver
b. *[the-truck]-driver

Eine Erklärung hierfür sei, dass nur in $\mathrm{n}^{o}$ und $\mathrm{a}^{o}$ inkorporiert werden kann. Wenn eine $\mathrm{nP}$ mit einem $\mathrm{D}^{o}$ mergt, müssen die kasus-relatierten $\mathrm{nP}$-features bereits DP-intern abgeglichen werden und sind somit nicht länger zugänglich für einen Abgleich via Inkorporation in eine Wurzel (vgl. Harley 2009:141).

Das gängigste Gegenargument gegen eine solche Sichtweise ist die Existenz von Phrasenkomposita. Bei Phrasenkomposita liegt das N1 in Gestalt einer DP vor, kann also eigentlich nicht inkorporieren. Harley betrachtet die wortinternen Phrasen (z.B. bikinigirls-in-trouble genre) als eingefrorene, kategorisierte Ausdrücke, die eine spezielle abstrakte Konzeptualisierung evozieren. Dafür spreche, dass sie auch affigiert werden können (z.B. the general bikini-girls-in-trouble-ness of it all). Die Phrase wird also durch NullDerivation zu einer nominalen Kategorie und kann anschließend Teil eines Derivats oder Kompositums $\left(\left[[\mathrm{XP}] \mathrm{n}^{o}\right]_{n P}\right)$ werden (vgl. auch Ackema/Neeleman 2004:Kap. 4).

Als Vertreterin einer linksverzweigenden Struktur bei Rektionskomposita äußert sich Harley auch zur Produktivität der NV-Inkorporation, bzw. zur Ungrammatikalität von $\mathrm{v}^{o_{-}}$ Inkorporationen wie *to truck-drive. Hier nimmt sie morphophonologische Beschränkungen an, bspw. dass das $\mathrm{v}^{o}$ im Englischen intern keine komplexen Köpfe mit mehr als einer Wurzel zulässt (vgl. Harley 2009:141).

Die Analyse von Komposita als Nomen-Inkorporation durch syntaktische Kopf-Bewegung und zweifaches Merge nimmt Harley als Gründe dafür an, dass sich komplexe Elemente in der phrasalen Syntax wie $\mathrm{X}^{\circ}$-Einheiten verhalten und bspw. als Diskursantezedens für pronominale Referenz unzugänglich seien (vgl. Harley 2009:133). An dieser Stelle muss eine tiefere Analyse ansetzen.

Harley geht darüber hinaus als Vertreterin eines syntaktischen Ansatzes von der Gültigkeit des Theta-Kriteriums aus. Eine Konsequenz daraus wäre, dass Nominalisierungen von klassisch transitiven Verben nicht ohne internes Argument auskommen dürften. Demnach sollten bspw. devourer oder carrier isoliert ungrammatisch sein 11 Derartige Probleme sind ein Argument für einen eher morphologisch orientierten Ansatz innerhalb der DM, welcher wiederum andere Fragen aufwirft, z.B. nach dem kategorialen Status eines Arguments.

\subsubsection{Modellierung fürs Deutsche}

Basierend auf den bisher ausgeführten Annahmen zur DM und der Analyse von Harley möchte ich im Folgenden eine Analyse für deutsche Komposita als komplexe Köpfe (ohne Inkorporation) vorschlagen.

Aufbauend auf den Annahmen in Kapitel 4.2.1 nehme ich für Wurzelkomposita die Arbeitsdefinition unter (37) an.

\footnotetext{
${ }^{11}$ Für diese Beobachtung danke ich Andreas Blümel.
} 

Arbeitsdefinition für Wurzelkomposita (WK):
Bei einem Wurzelkompositum handelt es sich um eine kompositionale Fügung mit nominalem Status, die mindestens zwei Wurzeln als Konstituenten enthält. Die grammatischen Eigenschaften der zweiten (bzw. letzten) Konstituente legen dabei die grammatischen Eigenschaften des Gesamtausdrucks fest. Zwischen die Konstituenten können Fugenelemente treten.

Für die konkrete Analyse von Wurzelkomposita wie Dachgarten halte ich zwei Strukturvarianten für möglich. Zunächst sehe ich im Unterschied zu Harley (2009) keinen Anlass für eine Kategorisierung der Wurzel $\sqrt{ }$ DACH. Stattdessen nehme ich an, dass die erste Konstituente im Status einer Wurzel verbleibt. Bei der Variante unter (38-a) mergen zunächst zwei Wurzeln und die entstandene $\sqrt{ } \mathrm{P}$ wird anschließend durch Merge mit einem $\mathrm{n}^{\circ}$-Kopf als Nomen kategorisiert. Damit beziehe ich mich auf die Idee des Root Merge nach Zhang (2007), gemäß dem Wurzeln über keinerlei syntaktische Merkmale verfügen, aber mergen können. Merge ist in diesem Sinne nicht merkmalsgetrieben. Root Merge nimmt Zhang für das Chinesische in den Fällen von Komposition an, in denen keine Subkategorisierung oder andere syntactico-semantischen Beziehungen vorliegen. Damit erklärt sie das Auftreten lexikalischer Integrität in den entsprechenden Fällen: Da Wurzeln über keinerlei Merkmale verfügen, kann ein Pronomen keine Kongruenz herstellen (vgl. Zhang 2007:177).12 Bei der Variante unter (38-b) mergt die zweite Wurzel $\sqrt{ }$ GARTEN zunächst mit einem $\mathrm{n}^{\circ}$-Kopf. Durch Merge der ersten Wurzel mit dem bereits kategorisierten N2-Kopfnomen entsteht ein nominales Kompositum.
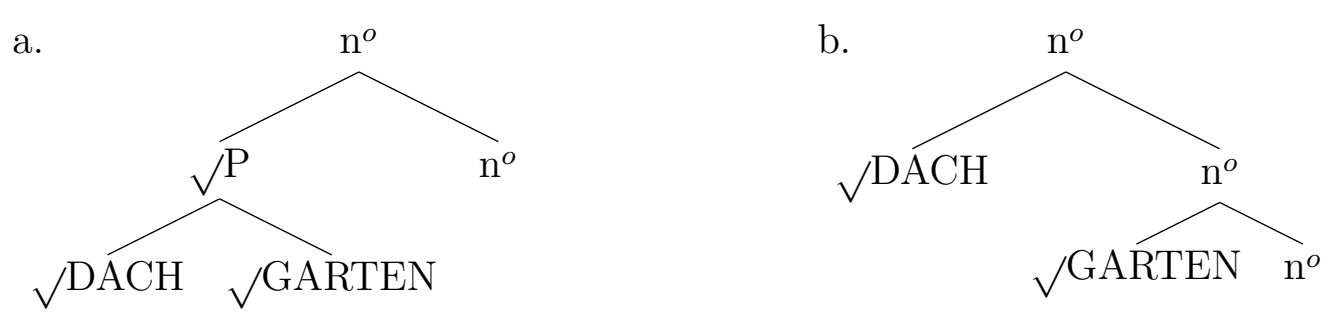

Diese beiden Strukturbildungsarten werfen einige Fragen auf. In (38-a) ist die Art der Verbindung zwischen den beiden Wurzeln unklar. Handelt es sich um gleichrangiges Merge (Set Merge), ist unklar, wie die zweite Konstituente den Status als grammatischer Kopf des Kompositums erlangt. Unter der Annahme von (Kopf-)Adjunktion ist unklar, ob und was $\sqrt{ }$ GARTEN projiziert. Im Fall von (38-b) ist unklar, ob es sich bei $\sqrt{ } \mathrm{DACH}$ um einen Spezifizierer oder um ein Adjunkt handelt. Für die Annahme von Adjunktion spricht in diesem Fall die Möglichkeit der rekursiven Verwendung der ersten Konstituente, z.B. [Dachwohnung]sgarten.13 Die Semantik der zweiten Konstituente wird durch Merge mit einem eigenen nominalen Merkmalsbündel erfasst und der Kopfstatus in der Konstruktion dadurch plausibilisiert. Das Problem an der Übertragung von Zhangs Prinzip des Root Merge ist, dass im Chinesischen exozentrische Komposita attestiert sind, bspw. können eine (scheinbar) adjektivische und eine (scheinbar) verbale Wurzel ein Nomen bilden. Diese Möglichkeit besteht für das Deutsche nicht. Im Deutschen besteht stets grammatische (endozentrische) Rechtsköpfigkeit, was für Pair Merge (statt des Set Merge im Chinesischen) spricht.

\footnotetext{
${ }^{12}$ Weiteres zu Besonderheiten von Komposita im Chinesischen siehe Kapitel 4.3.4

${ }^{13}$ Fugenelemente betrachte ich an dieser Stelle als postsyntaktisch insertierte phonologische Elemente. In diesem Sinne lässt ihre Anwesenheit keinen Rückschluss auf die Anwesenheit morphosyntaktischer Merkmale der angrenzenden Konstituenten zu.
} 
Dies alles spricht dafür, den Wurzelstatus nur für die erste Konstituente anzunehmen und der Struktur unter (38-b) Vorzug zu leisten. In beiden Varianten bleibt allerdings das N1 im Status einer Wurzel, womit sich wiederum einige typische Eigenschaften eines N1 erklären lassen: Wenn eine Konstituente kein Nomen ist, kann sie wie in (39-a) auch nicht nominal flektieren oder wie in (39-b) nicht modifiziert werden (vgl. Kap. 2.2.2).
a. *Dächergarten
b. *Grünesdachgarten, ${ }^{*}$ grünes Dachgarten

Nun stellt sich die Frage, inwiefern die Struktur von Rektionskomposita davon abweicht. Unter Berücksichtigung der Beobachtungen aus Kapitel 4.2.2 nehme ich die Arbeitsdefinition unter (40) an. Diese Definition steht im Einklang mit Selkirk (1982), Spencer (1991) und Scalise/Bisetto (2009), lässt jedoch mehr Freiräume für weitere Komponenten und den Strukturaufbau.

(40) Arbeitsdefinition für Rektionskomposita (RK):

Bei einem Rektionskompositum handelt es sich um eine kompositionale Fügung mit nominalem Status, die ein Element enthält, welches Argumente selegiert und einem weiteren Element wortintern den Status des (meist internen) Arguments zuweist.

Da in Kapitel 4.2 .2 keine Entscheidung über eine Präferenz von Links- oder Rechtsverzweigung getroffen werden konnte und die Fähigkeit der Argumentzuweisung in der DM kategorieunabhängig ist, wird am Beispiel des Rektionskompositums Dachbegrünung zunächst für beide Varianten eine Analyse vorgeschlagen.

Bei der linksverzweigenden Variante unter (41-a) wird die Wurzel $\sqrt{ }$ GRÜN durch Merge mit einem eigenen $v^{o}$ (realisiert durch das Präfix be) ${ }^{14}$ als Verb kategorisiert. Auch in diesem Fall gehe ich von der Wirksamkeit des Kopf-Rechts-Prinzips im Deutschen aus. Für die oberflächliche Inversion von Wurzel und Affix sind verschiedene Implementierungen denkbar, z.B. Local Dislocation in der PF (vgl. Embick/Noyer 2001). Der verbale Kopf selegiert nun Argumente und weist der ersten Konstituente den Status des internen Arguments zu. Geht man wie Harley (2009) davon aus, dass Nominalität eine Voraussetzung für Argumentstatus ist, muss nun die erste Konstituente via Merge mit einem eigenen $\mathrm{n}^{o}$ bereits nominalisiert worden sein, um als Argument selegiert werden zu können. Beide Konstituenten werden gemergt und anschließend durch Merge mit einem weiteren $\mathrm{n}^{o}$, welches durch -ung realisiert wird, nominalisiert.

Der Unterschied zur rechtsverzweigenden Variante unter (41-b) liegt darin, dass bei diesem der $\mathrm{v}^{o}$-Kopf zunächst mit einem $\mathrm{n}^{o}$ mergt. Der entstandene komplexe $\mathrm{n}^{o}$-Kopf verfügt über die entsprechende Argumentstruktur, die wie bereits bei der Linksverzweigung dem N1 Argumentstatus zuweist.

\footnotetext{
${ }^{14}$ Zur Verbalisierung durch bestimmte Affixe siehe auch Brandt et al. 1999 164).
} 
(41)

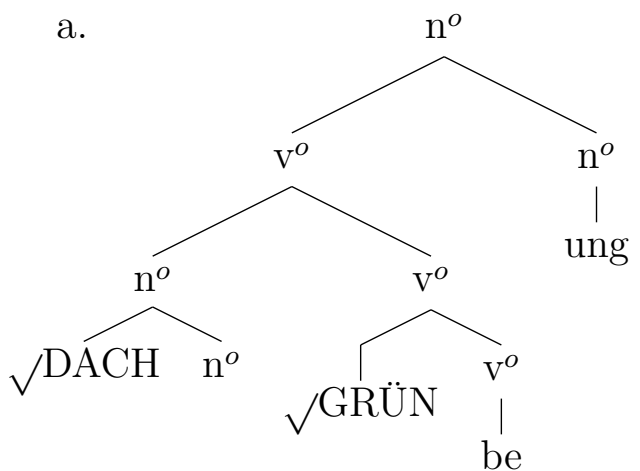

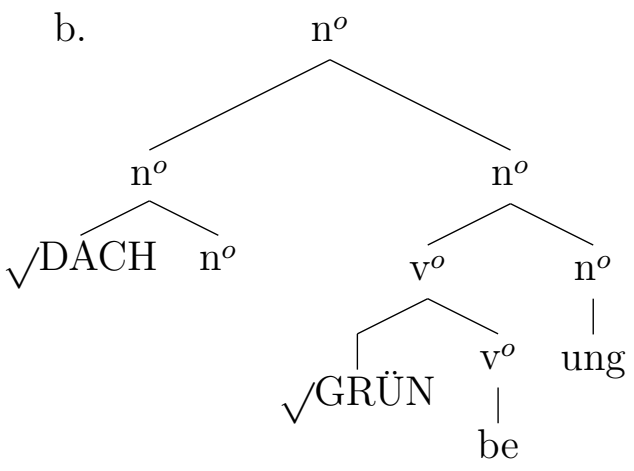

Harley und andere Vertreter*innen syntaktischer Ansätze argumentieren für eine linksverzweigende Struktur bei Rektionskomposita, weshalb ich diese Strukturbildungsrichtung im Folgenden vorrangig betrachte und nicht grundsätzlich auch eine rechtsverzweigende Alternative aufzeige, die jedoch meist ebenfalls möglich ist.

Die Analysen unter (41) gehen vom Zwischenschritt einer Verbalisierung aus, die durch ein overtes $\mathrm{v}^{o}$ induziert wird. Ebenso gibt es allerdings Rektionskomposita ohne ein Affix der Zustandsänderung o.Ä. (z.B. Autofahrer, Kopfstütze, ...). Bei solchen Fällen nehme ich Merge mit einem $\mathrm{v}^{o}$ an, welches nicht overt realisiert wird, sondern in Form eines NullAffixes vorliegt. Daraus resultiert die Struktur unter (42). Die Existenz von Null-Affixen wird im Rahmen der DM vor allem durch das Argument des Do-Supports im Englischen plausibilisiert (vgl. Halle/Marantz 1993:132ff). Damit liegt für beide Fälle eine uniforme Analyse im Stil einer Standard-VP vor. Die weitere Strukturbildung erfolgt wie in (41-a),

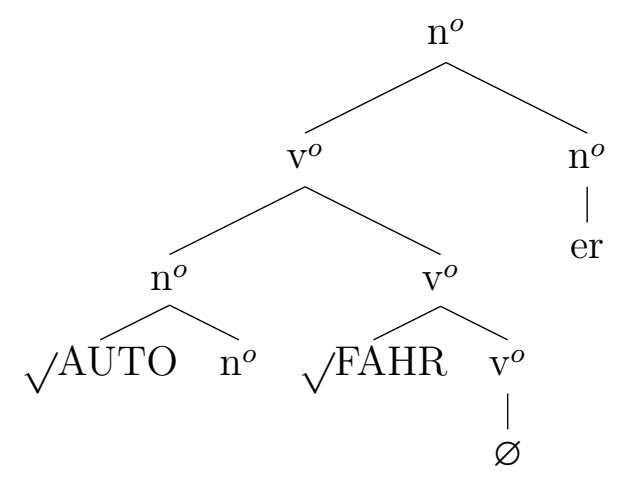

Alternativ wäre auch denkbar, dass die zweite Konstituente nicht mit einem $\mathrm{v}^{o}$ mergt, da dies für die Bereitstellung einer Argumentstruktur gemäß Harley (2009) nicht notwendig ist. Dies würde in der Struktur unter (43) resultieren. Aus Gründen der Konstruktionsuniformität präferiere ich allerdings die Struktur unter (42).

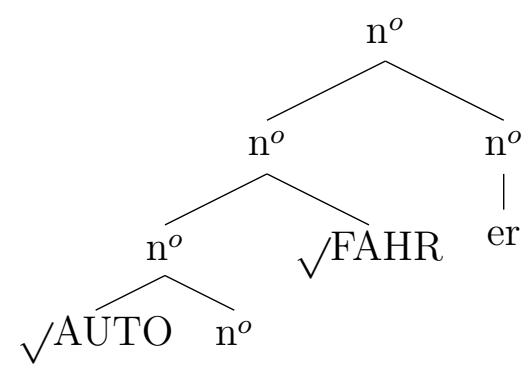




\subsection{Argumente für Unterschiede in der N1-Zugänglichkeit}

Bereits in Kapitel 2.2.3 wurde anhand von Beispielen wie (44) beobachtungsgeleitet für eine bessere N1-Zugänglichkeit von Rektions- gegenüber Wurzelkomposita argumentiert.

Ein Rosenhasser? Die tun doch niemandem was. (Tatort vom 05.05.2017)

Auf Basis der unter Kapitel 4.2.4 formulierten Annahmen soll im Folgenden nun theoriegestützt argumentiert werden. Die dafür entscheidende Frage ist die nach einem zentralen Unterschied in der Struktur der beiden Komposita-Arten, der sich auf die Zugänglichkeit der Konstituenten auswirken kann.

\subsubsection{Argumentstatus und Kategorisierung}

Im Zusammenhang mit verschiedenen Überlegungen zur N1-Zugänglichkeit stellte sich bereits mehrfach die Frage nach dem kategorialen Status des N1 und daraus resultierenden Konsequenzen für die Referenzialität (vgl. Kap. 2.1 und 2.4.1).

Ein zentraler Bestandteil der Definition von Rektionskomposita in Abgrenzung zu Wurzelkomposita ist die Eigenschaft des N1 den Status eines (meist internen) Arguments zu tragen und eine Theta-Rolle zugewiesen zu bekommen (vgl. z.B. Spencer 1991:309). Generell gilt die Annahme, dass ein Argument nominalen Status hat (vgl. Boase-Beier/Toman 1986:336). Da die erste Konstituente in Rektionskomposita Argumentstatus hat, muss sie über nominalen Status verfügen, den sie durch Merge mit einem no erhält (vgl. (41)]. Die Vorstellung, dass grammatische Erfordernisse eine Statusänderung bewirken können, findet sich z.B. auch bei Longobardi (1994) in Gestalt eines Raisings von N zu D.

Bei den Wurzelkomposita gibt es keine entsprechenden Erfordernisse, weshalb dafür argumentiert wurde, dass das Erstglied im Status einer kategorielosen Wurzel verbleibt (vgl. (38) ). Das Erstglied wird unkategorisiert als Wurzel mit der zweiten Konstituente gemergt. Von der zweiten Konstitutente erhält das Kompositum als finalen Schritt seine Kategorie (vgl. (38-b) . Dafür spricht, dass bei Wurzelkomposita an der N1-Position Ausdrücke (zumindest scheinbar) verschiedener Wortarten stehen können, z.B. ein Nomen in Dachgarten oder ein Verb in Hängegarten. Bei den Komposita unter (45) ist die Kategorie des N1 sogar ambig, da zier- und fried- nominal, adjektivisch oder verbal verwendet werden können.15 Hinzu kommt, dass es sich beim N1 in diesen Fällen um gebundene Morpheme handelt, die erst nach Anfügen einer Flexionsendung isoliert produktiv sind. Diese Beobachtung plausibilisiert den Wurzelstatus des Erstglieds in Wurzelkomposita. Für Rektionskomposita sind solche Fälle von gebundenen Morphemen an der N1-Position nicht attestiert.
a. Ziergarten
b. Friedhof

\footnotetext{
${ }^{15}$ Zur Aussage, dass das Erstglied nicht eindeutig kategorial festgelegt ist, siehe auch Erben $(200067)$.
} 
Wenn eine Wurzel nicht mit einem Merkmalsbündel mergt und somit nicht kategorisiert wird, kann sie keinen Wortstatus erlangen und auch nicht referenziell sein. Hierin scheint ein syntaktisches Argument für den Eindruck lexikalischer Integrität zu liegen. Anders verhält es sich im Fall von Rektionskomposita. Wenn das N1 über Argumentstatus verfügt, muss es nominal und damit auch refernziell sein. Ein Indiz gegen vollen nominalen Status ist die Unflektierbarkeit des N1 (vgl. Ackema/Neeleman 2004:63). Diese Eigenschaft muss für Rektionskomposita differenziert betrachtet werden.

Eine Aussage zur Kasusvergabe lässt sich im Fall des internen Arguments durch die Formidentität von Nominativ und Akkusativ im Deutschen schwer treffen. Allerdings konnten Clahsen et al. (2015) zeigen, dass Pluralmarkierungen am N1 u.U. möglich sind (siehe auch Kap. 2.2.2). Darüber hinaus lässt sich beobachten, dass sich der Numerus des N1 bei Rektionskomposita nach der Semantik richten kann, bei Wurzelkomposita jedoch nicht, siehe (46-a) vs. (46-b) ${ }^{16}$ Diese Beobachtung legt nahe, dass sich ein N1 in einem Rektionskompositum tendenziell mehr wie ein syntaktisches Wort verhält als in einem Wurzelkompositum.
a. Hausbau - Häuserbau
Dachbegrünung - Dächerbegrünung
b. Hausfarbe $-{ }^{*}$ Häuserfarbe/*Häuserfarben
Dachgarten - *Dächergarten/*Dächergärten

Ein weiteres Indiz für den Argumentstatus des N1 in Rektionskomposita ist die Sättigung einer Theta-Rolle. Dies wird u.a. dadurch deutlich, dass dieselbe Theta-Rolle nicht zusätzlich außerhalb gesättigt werden kann (siehe (12-d)). Bei Selkirk (1982) heißt es sogar, dass sich das Argument besser im Kompositum als außerhalb realisieren lässt. Tatsächlich ist die Interpretation des N1 in (47) als internes Argument die präferierte Lesart. Die Interpretation von tree als Ortsangabe mit nachgestelltem Argument ist nicht zugänglich.

*tree eater of pasta

Auch bei Grimshaw (1992) heißt es, dass Elemente im Kompositum vor wort-externen Elementen theta-markiert werden. Diese Beobachtung spricht darüber hinaus für eine linksverzweigende Struktur im Falle eines er-Suffixes, welches die Rolle des externen Arguments sättigt.

Andererseits gibt es auch Gegenbeispiele: Härtl (2013) führt mit (48-a) Fälle doppelter Argumentsättigung an. Ebenso führt Solstad (2010) mit (48-b) Fälle an, in denen sowohl das N1 als auch ein post-nominaler Genitiv die Thema-Rolle besetzten. Solstads Analyse dazu ist, dass das N1, z.B. Personen das N2, z.B. Beschreibung näher spezifiziert und mögliche weitere Thema-Argumente restringiert. Das N1 sei somit nicht als echtes Thema-Argument anzuerkennen, stattdessen würde das N1 selektionale und sortale Beschränkungen einführen.

a. Designanalyse des Geschirrs, Namensanalyse des Flusses, Stabilitätsprüfung der Dachkonstruktion, Belastbarkeitstest des Flugzeuges (Härtl 2013:166)

\footnotetext{
${ }^{16}$ Es handelt sich hierbei um eine Beobachtung, die es auf breiterer Basis zu überprüfen gilt. Einschränkend fällt bereits auf, dass Fugenelemente den semantischen Unterschied aufheben können und evtl. auch einige ungrammatische Fälle unter (46-b)] in entsprechendem Kontext plausibel gemacht werden können.
} 
b. die Personenbeschreibung der Täter, die Strukturbeschreibung des einfachen Arraymodells (Solstad 2010:241)

Allerdings identifizieren die Paraphrasen in (49) das (ursprüngliche) N1 als internes Argument und den postnominalen Genitiv als Attribut; denn Gegenstand der Analyse ist das Design und nicht das Geschirr.
a. das Design des Geschirrs wird analysiert
b. der Name des Flusses wird analysiert
c. die Stabilität der Dachkonstruktion wird geprüft
d. die Person des Täters wird beschrieben

Somit lässt sich letztlich strukturbasiert ein Argument für einen Unterschied in der Zugänglichkeit von Komposita-Erstgliedern bei Rektions- und Wurzelkomposita formulieren: Als Erstglied eines Wurzelkompositums wird eine Wurzel angenommen, die aufgrund des fehlenden kategorialen Status nicht referenziell und somit nicht zugänglich ist. Bei Rektionskomposita wird aufgrund des Argumentstatus für eine nominale Kategorisierung argumentiert. Da sich das Erstglied hier nominal, bzw. nominaler als im Fall von Wurzelkomposita darstellt, ist in diesem Fall auch eher mit Referenzialität und damit auch mit Zugänglichkeit zu rechnen. Gleichzeitig ist zu beachten, dass es sich hierbei um rein syntaktische Argumente handelt, die den Einfluss von Diskurs- und konzeptuellem Wissen auf die Zugänglichkeit unberücksichtigt lassen.

\subsubsection{VP in Rektionskomposita}

Die Annahme echter Argumentvergabe bei Rektionskomposita impliziert letztlich eine Analyse nach den Grundprinzipien einer VP sowie Linksverzweigung entsprechend (41-a). Die Möglichkeit des Vorliegens einer VP innerhalb eines Rektionskompositums ist allerdings umstritten. Harley (vgl. 2009:136) nimmt für truckdrive keine vP, sondern eine Wurzelphrase $(\sqrt{ } \mathrm{P})$ an und argumentiert für NV-Inkorporation und Kopfbewegung.17

Es gibt jedoch auch zahlreiche Argumente für das Vorliegen einer VP in Rektionskomposita. Grimshaw (1992) legt für Event-Nominalisierungen eine Argumentstruktur zugrunde, für Resultats-Nominalisierungen nicht. Manche Verben können nur die eine oder die andere Nominalisierungsart bilden, manche beide. In dem Fall ist die Lesart ambig und kontextabhängig. Für Borer (1993) resultiert das Auftreten einer Argumentstruktur bei Event-Nominalisierungen aus der Präsenz einer vollen VP. Dabei weise der Kopf der VP den Argumenten thematische Rollen zu, nicht das derivierte Nomen. Dies gelte allerdings nicht für Resultats-Nominalisierungen und „Simple Event Nominals“.

Auch in der DM gibt es entsprechende Annahmen. Alexiadou (2014) geht bei einer ObjektInterpretation von der Nominalisierung einer Wurzel aus, da kein Event involviert ist. Die Event-Lesart leitet sie von einer vollen Verbalstruktur ab. Als Indiz hierfür führt Alexiadou (2001:15f) Eigenschaften auf, die sonst nur mit VPn assoziiert werden, aber auch in Event-Nominalisierungen auftreten, z.B. Kasusvergabe.

17 Punske (2016) verwendet die postulierte fehlende N1-Referenzialität als Gegenargument für Harleys Annahme. 
López (2015) führt aus, dass Event-Denotation und Argumentstruktur gemeinsam erscheinen; vererbt von einer in der Struktur enthaltenen vP, die bei Resultats-Nominalisierungen nicht vorliegt. Entsprechend geht er bei Event-Nominalisierungen und Resultats-Nominalisierungen von der Struktur unter (50) aus (López 2015:679). Allerdings liegt seinem Ansatz zugrunde, dass Argumentstruktur und vP unabhängig voneinander auftreten. Bei ihm wird die Wurzel sowie ein internes Argument von einer EventP selegiert (vgl. López 2015:69).

$$
\begin{array}{ll}
\text { a. } & {\left[D P \text { the }\left[{ }_{n p} \text {-ation }\left[{ }_{v P} \mathrm{~V}\left[{ }_{\sqrt{ }} \mathrm{P} \text { examin- }\right]\right]\right]\right]} \\
\text { b. } & {\left[D P \text { the }\left[{ }_{n p} \text {-ation }\left[{ }_{\sqrt{ }} \mathrm{P} \sqrt{ } \text { examin- }\right]\right]\right]}
\end{array}
$$

Eine andere Analyse von Prozessnomen als VP findet sich bei Fu et al. (2001). Sie nehmen für englische Prozess-Nominalisierungen (im Gegensatz zu underivierten Versionen mit ähnlicher Semantik) eine zugrundeliegende VP-Struktur an. Als Argument hierfür dient ihnen die mögliche Präsenz von Adverbien bei Prozess-Nominalisierungen wie in (51) (Fu et al. 2001:555).18 Darüber hinaus nennen sie die Möglichkeit von ProzessNominalisierungen als Antezedens für do so zu fungieren (vgl. (52), Fu et al. 2001:571). In (51-a) und (52-a) liegen Prozess-Nominalisierungen vor, in den weniger akzeptablen Fällen (51-b) und (52-b) nicht derivierte Nomen. Ein wichtiger Punkt ist dabei, dass sie Prozess-Nominalisierungen lediglich eine VP, keine volle Satzstruktur (IP/TP oder CP) zugrundelegen (vgl. Fu et al. 2001:569).

(51) a. His transformation into a werewolf so rapidly was unnerving.

b. ??His metamorphosis into a werewolf so rapidly was unnerving.

(52) a. Sam's destruction of this documents this morning was preceded by Bill's doing so.

b. *Sam's version of the event and Bill's doing so were surprising.

Eine Besonderheit von Event-Nominalisierungen gegenüber anderen Nominalisierungsarten ist das Vorliegen einer Ereignisvariablen. Diese kann durch eine VP eingeführt werden. Ein Indiz für das Vorliegen einer Ereignisvariablen ist z.B. die Möglichkeit von iterativem Gebrauch wie in (53-a). Da die iterative Lesart möglich ist, scheint es sich um ein Ereignis zu handeln, d.h. eine VP liegt vor. Im Fall von (53-b) wird durch die er-Nominalisierung kein Ereignis denotiert und iterative Modifikation ist nicht möglich. Demnach ist nicht vom Vorhandensein einer VP oder einer sonstigen Projektion auszugehen, die eine Ereignisvariable zur Verfügung stellt.

a. Die tägliche Trinkwasserbereitung wird regelmäßig geprüft.

b. *Der tägliche Trinkwasserbereiter wird regelmäßig geprüft.

Alexiadou/Schäfer (2010) legen auch er-Nominalisierungen eine vP zugrunde. Als Teil des funktionalen Vokabulars besetzt die Derivationsendung -er bei Nominalisierungen eine externe Argumentstelle; entsprechend der Theta-Rolle des Verbs sind hier verschiedene

${ }^{18}$ López (2015:676f) zweifelt die Aussagekraft derartiger Fälle an und sagt, dass Beispiele von Nominalisierungen mit Adverben sehr sorgfältig konstruiert sein müssen: „Even a small change in the structure that should be irrelevant actually affects their acceptability substantially." 
Varianten möglich (z.B. Agens, Instrument, ... ). Von Nominalisierungen kann in dem Sinne gesprochen werden, in dem Alexiadou und Schäfer der Konstruktion eine vP zugrunde legen und sagen, dass alle er-Nominalisierungen, bei denen das -er das externe Argument bindet, die Struktur in (54) involvieren.

$$
[\mathrm{nP}-\mathrm{er}[\text { VoiceP[vP [RootP ]]]] }
$$

Bei Event- und er-Nominalisierungen scheint demnach die Annahme einer zugrundeliegenden vP theoretisch fundiert. Sollte es diesbezüglich zwischen den verschiedenen Arten von Rektionskomposita Unterschiede geben, müsste sich dies auch auf die N1-Zugänglichkeit auswirken. An dieser Stelle sei die Idee gradueller Nominalität aus Kapitel 2.2.2 aufgegriffen. Dort wurden typische und untypische N1 beschrieben. Hier zeigen sich nun typische und untypische Nomen, bzw. Nomen, bei denen mehr oder weniger der notwendigen Kriterien für Nominalität zutreffen. Je mehr dieser Kriterien zutreffen, desto typischer ist das Nomen, bzw. desto untypischer ist das N1 und desto zugänglicher müsste es sein.

In Konstruktionen, die eine vP beinhalten, müsste das N1 aufgrund seines Argumentstatus über einen höheren Grad an Nominalität verfügen und dadurch zugänglicher sein. Sollte im Fall von Event-Nominalisierungen sogar eine TP vorliegen, wäre dies ein Argument für einen wiederum höheren Grad an Nominalität und Zugänglichkeit.

Diese Vorstellung spiegelt sich in den Beispielen in (55) wider. In einer informellen Umfrage unter neun Studierenden wurde (55-a) mit Event-Nominalisierung durchschnittlich besser bewertet als (55-b) mit Resultats-Nominalisierung; (55-c) als Beispiel für eine erNominalisierung wurde am schlechtesten bewertet.19
a. Die Wasseraufbereitung wird Tag und Nacht am Laufen gehalten. Es muss ständig zur Verfügung stehen.
b. Die Wasseraufbereitung war ein voller Erfolg. Es steht nun allen zur Verfü- gung.
c. Der Wasseraufbereiter arbeitet Tag und Nacht. Es muss ständig zur Verfü- gung stehen.

\subsubsection{Referenzialität}

Anhand der detaillierteren Strukturbäume in Kapitel 4.2 .4 können Aussagen zum referenziellen Status des N1 auf Basis seiner grammatischen Eigenschaften getroffen werden. Unter der Annahme, dass Wurzelkomposita wie in (38) gebildet werden, hat ein N1 in einem Wurzelkompositum den Status einer kategorielosen Wurzel und ist somit nicht referenziell. Nimmt man für Rektionskomposita eine Struktur wie unter (41) an und geht man davon aus, dass das N1 bei Rektionskomposita im Falle einer Event-Nominalisierung Komplement in einer VP ist, stellt sich die Frage, welcher konkrete Status für ein solches N1 anzunehmen ist. Handelt es sich wie in (41-a) dargestellt um ein $n^{o}$, hätte das N1 nominalen Status. Dies wäre allerdings noch nicht ausreichend, um Argumentstellenfähigkeit und damit einhergehend Referenzialität zu gewährleisten. Eben jene ist jedoch

\footnotetext{
${ }^{19}$ Bei der Aufgabe handelte es sich um ein Akzeptabiltitäs-Ranking, bei dem die Studierenden den (in ihrer Reihenfolge pseudo-randomisierten) Sätzen Werte von 1 (am wenigsten akzeptabel) bis 3 (am akzeptabelsten) zuordnen sollten. Die aufsummierten Werte für die drei Bedingungen sind: a $(23)>$ $\mathrm{b}(18)>\mathrm{c}(13)$.
} 
Voraussetzung für anaphorische Zugänglichkeit.

Für weitere Analysen seien zwei Voraussetzungen aus dem Minimalistischen Programm angenommen. Zum einen ist D die für uneingeschränkte Referenzialität erforderliche Kategorie (vgl. Longobardi 1994). Zum anderen kann ein Knoten gleichzeitig minimal und maximal sein (vgl. Chomsky 1995:6).

Gegen die Annahme, dass es sich bei einem N1 um eine volle DP handelt, spricht die Inakzeptabilität von Determinierern, Präpositionen oder Adjektiven innerhalb des Kompositums (siehe (56) und Kapitel 2.2.2.
a. *das Dachbegrünung, *Das-Dach-Begrünung,
b. *Auf-dem-Dachbegrünung
c. *die Altesdachbegrünung

Es gibt allerdings auch Überlegungen, das N1 eines Rektionskompositums als maximale Projektion zu behandeln. Bspw. müsse ein N1 laut Rivet (1999) aufgrund seiner ThetaRolle über eine phrasale Kategorie verfügen (vgl. Rivet 1999:310ff). Allerdings geht sie statt von einer DP von einer NP aus. Sproat umgeht das Problem und spricht statt von Theta-Identifikation von Theta-Markierung, denn er schließt aus, dass das N1 eine maximale Projektion sein kann. Statt Argumentstatus und Referenzialität spricht er dem N1 anaphorischen Inselstatus zu (vgl. Sproat 1985:328ff, siehe auch Kap. 2.1).

Einen anderen Ansatz eröffnet Longobardi (1994) mit seiner Argumentation, dass auch ohne overten Determinierer eine (referenzielle) DP vorliegen kann. In den romanischen und germanischen Sprachen gibt es einige Arten von nominalen Ausdrücken, die in Argumentfunktion in der Oberflächenstruktur ohne overten Determinierer auftreten können: Eigennamen, Pronomen, Pluralformen und Massenomen. Determinierer versteht Longobardi semantisch als Operatoren, die eine Variable binden, wobei die D-Position die spezifische Referenz der DP bestimmt (vgl. Longobardi 1994:30f). Wenn nur DPn Argumentstatus aufweisen können, muss das Verhalten dieser Formen erklärt werden. Für das Englische nimmt Longobardi an, dass Pronomen in D basisgeneriert werden und Eigennamen keinen Operator benötigen (vgl. Longobardi 1994:36). Für generische Ausdrücke und Massenomen nimmt er eine D-N-Kette an (vgl. Longobardi 1994:50ff). Hierbei wird das Nomen an $\mathrm{N}$ basisgeneriert und in der LF zu D angehoben. Dabei entstehe die generische Lesart, wie sie für Komposita-Erstglieder häufig zu beobachten sei. Da nun kein Platz für einen weiteren Operator bleibe, seien die Ausdrücke kaum referenziell (vgl. Longobardi 1994: 48).

Für das Deutsche attestiert er einen Unterschied in Bezug auf die Verwendung expletiver Artikel, die wie in (57) z.T. möglich sind (vgl. Longobardi 1994:56f). Im Deutschen muss die D-Position für eine vollständige DP nicht besetzt sein, kann aber für den Fall, dass ein overter Determinierer auftritt, nicht durch das Nomen besetzt werden.
a. Biber bauen Dämme vs. Die Biber bauen Dämme
b. Milch ist weiß vs. Die Milch ist weiß
c. Hans ist angekommen vs. Der Hans ist angekommen

Nach Longobardis Ansatz können bestimmte Ausdrucksarten ohne overten Determinierer eine volle DP bilden und somit voll referenziell sein. Daraus lässt sich ableiten, dass diese an der N1-Position gut zugänglich sein müssten, da es kein Anzeichen dafür gibt, dass sich 
ihre kategorialen Eigenschaften für die Funktion als Kompositums-Konstituente ändern müssen. Für Eigennamen besteht diese Hypothese bereits (vgl. (58) nach Sadock (1998) und Kapitel 3.2.1), ebenso für Massenomen (vgl. Kap. 3.2.2). Interessanterweise handelt es sich bei Sadocks Beispielen unter (58) ebenfalls um Rektionskomposita.

a. Gingrich supporters think he is brilliant.

b. Volvo-owners tend to like them.

Trotz der Parallelen in Bezug auf die Eigennamen, lässt sich Longobardis Analyse - wie oben erwähnt - nicht unmittelbar auf das Deutsche übertragen. Besonders interessant ist dabei die Frage, warum in Phrasen Artikel und Adjektive fakultativ sein können (vgl. (57)), in NN-Komposita jedoch nicht zulässig sind (vgl. (56)].

Aufbauend auf Longobardis Ansatz schlage ich daher folgende Analyse vor 20 Innerhalb der Komposition findet zwischen Wurzel und entsprechendem Merkmalsbündel keine Kopf-Bewegung als Teil der Wortbildung statt, sondern ,external merge ${ }^{6}$, also Merge, das auf zwei separate Objekte angewandt wird (vgl. Marantz 2013a:99). Der Ansatz zu ,external pair merge' von Köpfen wird von Epstein et al. (2016) ausgeführt, dabei werden Parallelen zur Adjunktion gezogen. Da hierbei Merge auf zwei Köpfe appliziert, ordnen sie diese Operation als morphologisch ein (vgl. Epstein et al.|2016:98). Für die N1-Fügung kann in diesem Sinne eine basisgenerierte Adjunktionsstruktur angenommen werden, d.h. die Adjunktion findet hierbei nicht auf phrasaler Ebene statt, sondern als Kopf. Das N1 ist letztlich ein D. Die Besetzung des D-Kopfs durch das N1 erklärt die Ungrammatikalität von kompositainternen Determinierern und Adjektiven wie in (56). Eine Kopfbewegung zu D ist im Deutschen nur möglich, wenn dieses NULL ist (vgl. (59)), denn jede Bildung von $<$ D, N $>$ im Deutschen involviert auf der Oberflächenstruktur ein NULL-D. Durch die Besetzung der Komplement-Position durch NULL kann auch kein overter Determinierer oder ein Adjektiv hinzutreten. Da der Komplex ,D und $\mathrm{N}^{\prime}$ durch Basisgenerierung verkettet wird und keine NP vorliegt, wird prognostiziert, dass es auch keine APs gibt. Dass dies trotzdem in einem referenzfähigen Ausdruck resultiert, zeigen die Fälle von NPn im Deutschen, die ebenfalls keinen Determinierer bei sich tragen dürfen oder müssen: Eigennamen, Pluralformen, Massenomen und Pronomen (vgl. (57)).

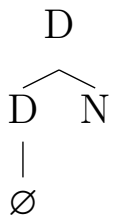

Unter der Voraussetzung, dass ein Element gleichzeitig minimal und maximal sein kann, ermöglicht der Status des N1 als D dessen Referenzialität. Dazu auch Alexiadou:

The basic insight is that $\mathrm{D}$ is the element that converts the nominal expression into a referential phrase that consequently can be used as an argument. (Alexiadou 2001.29)

Den Analysen von Epstein et al. (2016) und López (2015) folgend, gibt es keine zwingenden Gründe die Existenz von ,external pair merge' von Kopfadjunkten auszuschließen. Auch Halle/Marantz (1993) eröffnen diesen Handlungsspielraum:

\footnotetext{
${ }^{20}$ Für den Vorschlag dieser Analysemöglichkeit danke ich Andreas Blümel.
} 
[...] 'word formation' - the creation of complex syntactic heads - may take place at any level of grammar through such processes as head movement and adjunction and/or merger of structurally or linearly adjacent heads. (Halle/Marantz 1993 112)

Insgesamt ergibt sich für ein Rektionskompositum damit die Struktur unter (60).

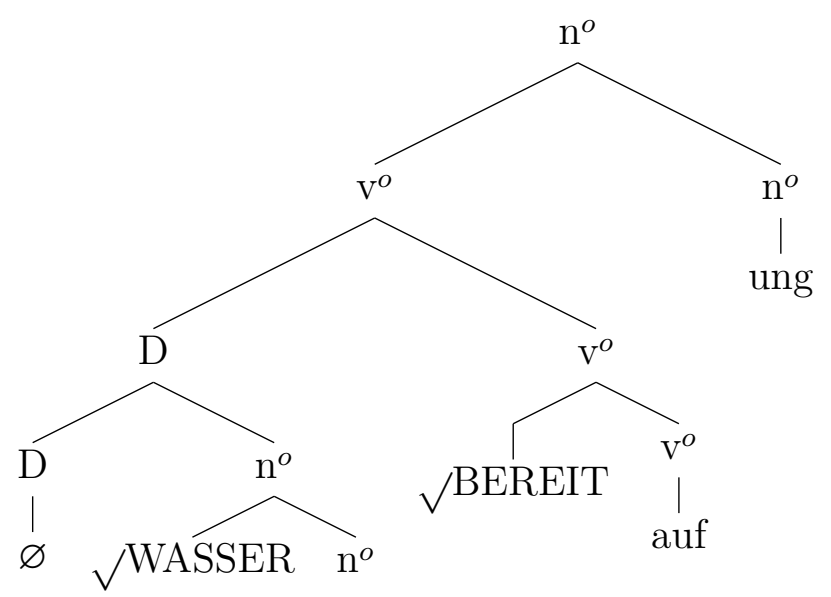

In der ursprünglichen Analyse wurde das N1 als no kategorisiert. Dadurch verfügt dieses über einen höheren Grad an Referenzialität als im Fall der Kategorisierung des N1 als $\sqrt{ } \mathrm{P}$ im Fall von Wurzelkomposita. Die Analyse des N1 als D (z.B. bei Massenomen) ermöglicht nun uneingeschränkte Referenzialität und Argumentstatus, erklärt die Ungrammatikalität von overten Artikeln und Adjektiven und verbleibt gleichzeitig unterhalb der phrasalen Ebene.

Die hier vorgestellten Analysen bedienen ausschließlich die typischen Fälle von Wurzelund Rektionskomposita. Selbstverständlich sind auch hier intermediäre Typen möglich, die entsprechend über eine Zwischenposition auf der Zugänglichkeitsskala verfügen. Dazu zählen z.B. Wurzelkomposita, bei denen die erste Konstituente durch Affigierung nominalen Status erhält, jedoch keine Argumentstelle besetzt (z.B. Raucherinse ${ }^{21}$ ).

\subsubsection{Weitere Evidenz}

\section{Typologische Argumente}

\section{Polysynthetische Sprachen}

Ein anschaulicher Fall für das Wirken syntaktischer Prinzipien innerhalb von Wörtern sind polysynthetische Sprachen. Für Inuit-Sprachen wurde gezeigt, dass polysynthetische Wörter syntaktisch auf dem XP-Level angesiedelt sind, sich prosodisch aber wie Wörter verhalten (vgl. Compton/Pittman 2010).

Darüber hinaus lässt sich mit Hilfe polysynthetischer Sprachen die Theorie der Linksverzweigung stützen, da im Gegensatz zu den germanischen Sprachen NV-Inkorporationen dort produktive Formen sind. Gleichzeitig ist das N1 in diesen Konstruktionen für pronominale Referenz zugänglich. Dies wurde bspw. für Kapampangan gefunden (vgl. Mithun

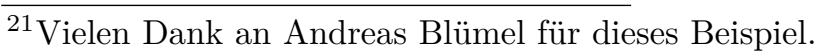


2010). Auch für Mohawk und Nahuatl existieren Belege von referierenden, inkorporierten Nomen:

An incorporated noun often refers to a generic or unspecific class, giving a reading rather similar to that of the English compound. However, it can also refer to a very specific object which is not focused in the discourse in languages like Mohawk and Nahuatl. (Baker 1988 78)

Um den Unterschied zu germanischen Sprachen wie dem Englischen zu erklären, geht Baker davon aus, dass Komposita im Englischen im Lexikon gebildet werden, weshalb das N1 unzugänglich sei. Bei den indianischen Sprachen liege hingegen ein syntaktischer Prozess vor. Die inkorporierte Form entstehe durch Bewegung aus einer Phrase, wobei die relevanten Strukturen erhalten bleiben. Dazu gehört, dass das N1 aus einer NP-Position kommt, somit referenziell ist und eine Spur hinterlässt. Analysiert man die Komposition jedoch im Sinne der DM als syntaktischen Prozess und nimmt man für Rektionskomposita eine zugrundeliegende NV-Inkorporation an, ist es nicht mehr plausibel, einen Referenzialitätsunterschied zu den Inkorporationen der polysynthetischen Sprachen anzunehmen. Ackema (1995) begründet einen Unterschied im referenziellen Potenzial von NV-Strukturen mit Unterschieden in der Grammatik der Sprachtypen und deren jeweiligem Verhältnis zwischen Syntax und Morphologie. So gäbe es in polysynthetischen Sprachen, in denen inkorporierte Nomen referenzfähig sind, kein Artikelsystem. Demnach seien dort NPn ohne Determinierer uneingeschränkt referenzfähig. Sprachen wie das Deutsche benötigen jedoch einen D-Kopf (vgl. Ackema 1995:115). Diesem Argument seien die in Kapitel 4.3.3 ausgeführten fakultativ determiniererlosen Ausdrucksklassen sowie die oben vorgestellte Analyse entgegengehalten.

Sollte sich die Analyse von Rektionskomposita als NV-Inkorporationen doch als adäquat herausstellen, kann das referenzielle Potenzial von NV-Strukturen polysynthetischer Sprachen als Argument für die N1-Zugänglichkeit sowie für die Linksverzweigung dienen. Allerdings gibt es auch ohne strikte Abgrenzung zwischen Syntax und Morphologie gravierende Unterschiede zwischen den Sprachtypen. So existieren in den Inuitsprachen keine Wurzelkomposita wie in den germanischen Sprachen, und ob bei Rektionskomposita wirklich Inkorporation vorliegt, bleibt strittig.

\section{Kontextsensitive Strukturen}

In einigen Sprachen lässt sich variables, kontextsensitives Verhalten von Komposita-Konstituenten beobachten. Im Chinesischen gibt es die sog. ,breakable compounds', die sich dadurch auszeichnen, dass sie je nach Kontext als Kompositum oder als Phrase interpretiert werden (vgl. Zhang 2007). Dies hat zur Folge, dass entsprechende Ausdrücke in manchen Kontexten Effekte lexikalischer Integrität aufweisen und in manchen nicht. Demnach kann ein ,breakable compound' auch durch zwei Strukturen realisiert werden: Merge von Wurzeln oder Bildung einer VP. Ein und dieselbe Konstituente wird also einmal syntaktisch als Teil einer VP interpretiert und einmal als Wurzel.

Für das Hebräische beschreibt Borer (2009) zum einen eine Form von opaken Komposita und zum anderen kompositionale syntaktische Konkatenationen, sog. ,Konstrukte6. Die beiden Formen sind z.T. nur schwer zu unterscheiden und teilen sich einige grundlegende strukturelle Eigenschaften. Allerdings werden die Konstrukte syntaktisch gebildet, 
die Komposita nicht (vgl. Borer 2009). Innerhalb der Klasse der Konstrukte unterscheidet Borer zwei Arten: M- und R-Konstrukte, wobei der Nicht-Kopf bei M-Konstrukten nicht-referenziell ist, bei den R-Konstrukten hingegen sogar referenziell sein muss, da er Argument des Kopfs ist (vgl. Borer 2009:498). Borers Beispiele für die einzelnen Ausdrucksarten sind unter (61) wiedergegeben. Zentral ist, dass zwischen den Formen auf der Oberfläche kein Unterschied besteht, jedoch sowohl ein Bedeutungsunterschied - die Komposita sind weniger transparent - als auch ein syntaktischer Unterschied (vgl. Borer 2009:498). Im Falle der R-Konstrukte nimmt Borer für den Nicht-Kopf eine DP an (vgl. Borer 2009:511).

$\begin{array}{lll}\text { Komposita } & \text { M-Konstrukte } & \text { R-Konstrukte } \\ \text { beyt (ha-)sefer } & \text { beyt (ha-)'ec } & \text { beyt (ha-)mora } \\ \text { Haus (das-)Buch } & \text { Haus (das-)Holz } & \text { Haus (der-)Lehrer } \\ \text { '(die) Schule' } & \text { '(das) hölzerne Haus' } & \text { '(das) Haus von (dem) Lehrer' }\end{array}$

Die Struktur der beschriebenen Formen des Chinesischen und des Hebräischen kann insofern als kontextsensitiv betrachtet werden. Je nach syntaktischer Umgebung handelt es sich bei einem ,breakable compound' im Chinesischen um ein Kompositum oder eine Phrase. Im Hebräischen entscheidet die jeweilige Semantik über das Vorliegen eines Kompositums oder eines Konstrukts. Ein ähnliches Verhalten nehme ich für das Deutsche an: Je nach Kontext, d.h. je nach Art von Kompositum, ist das N1 eine kategorielose Wurzel (Wurzelkompositum) oder es hat Wort-Status (Rektionskompositum).

\section{Transparenz der Bildung}

Ein weiteres Argument für Unterschiede in der N1-Zugänglichkeit zwischen Wurzel- und Rektionskomposita liegt in der Art und Weise, wie die beiden Komposita-Arten interpretiert werden. Dies wird bspw. anhand von (62) deutlich.
a. Papiermaschine
b. Papierherstellung

Bei einem Wurzelkompositum muss bei der Interpretation die Relation zwischen den beiden Komposita-Konstituenten aus dem Welt- und Kontextwissen antizipiert werden, weshalb Wurzelkomposita prinzipiell ambig sind (vgl. Kap. 2.2.4). Das Wurzelkompositum Papiermaschine denotiert eine Maschine, mit der Papier hergestellt wird (FOR-Relation). Das Denotat könnte in entsprechendem Kontext aber auch eine Modellmaschine sein, also eine Maschine aus Papier (FROM-Relation). Welche Interpretation die präferierte ist, richtet sich nach Weltwissen und Verwendungskontext.

Bei Rektionskomposita hingegen ist kein zusätzliches lexikalisches Material nötig, das zunächst erschlossen werden muss, um die Bedeutung des Kompositums zu paraphrasieren. Papierherstellung denotiert die Herstellung von Papier, bzw. den Prozess, bei dem Papier hergestellt wird. Auch für die zweite Paraphrasierung ist kein zusätzliches Wissen notwendig, da die Eventrolle des Verbs verbalisiert wird. Alle zur Interpretation notwendigen Bedeutungsteile liegen im Kompositum vor. 
The semantic relation between the first and the second constituent, however, is not constrained by general conceptual categories but by grammar proper. The only way to interpret the noun Appetithemmer is to give Appetit the role of the internal argument of the embedded verb. (Neef 2009 397)

Aufgrund der eindeutigen und kontextfreien Interpretierbarkeit von Rektionskomposita wird diesen oft ein höherer Grad an Kompositionalität zugesprochen (z.B. Klos 2011: 273). Wie in Kapitel 2.3.5 dargestellt, wird ein Zusammenhang zwischen der Transparenz und der Zugänglichkeit des N1 postuliert, in dem Sinne, in dem ein N1 umso zugänglicher ist, je mehr seine Bedeutung im Kompositum seiner Bedeutung als Simplex entspricht. Der höhere Grad an Transparenz bei Rektionskomposita gegenüber Wurzelkomposita spricht somit ebenfalls für eine bessere N1-Zugänglichkeit im Fall von Rektionskomposita.

Es existiert sogar die Ansicht, dass Wurzelkomposita generell nicht kompositional, sondern immer „gelistet" sind (vgl. Ackema/Neeleman 2004:83). Diese kategorische Einteilung in gelistete und geparste Komposita wurde im Rahmen dieser Arbeit bereits abgelehnt. Die Existenz von kompositionalen Wurzelkomposita zeigt sich in der Möglichkeit einer Ad-hocBildung wie unter (63), welche in entsprechendem Kontext verstanden werden würde. Die Interpretierbarkeit des Kompositums Stuhlglas resultiert vorrangig aus der Transparenz der Konstituenten und ihrer situativ-kompositionalen Fügung.

(63) In einer Küche stehen zwei Gläser. Ein Glas steht auf dem Tisch, ein Glas steht auf einem Stuhl.

A zu B: Kannst du mir mal das Stuhlglas geben?

Aufbauend auf dieser Beobachtung stellt sich die Frage, ob die Unterschiede in der Art der Interpretation von Rektions- und Wurzelkomposita tatsächlich etwas über deren Grad an Transparenz und Kompositionalität aussagen und tatsächlich Einfluss auf die N1Zugänglichkeit haben. Es fehlt Evidenz dafür, dass die Analyse anhand von morphosyntaktischen Merkmalen kognitiv weniger aufwändig ist als die Analyse durch Kontext- und Weltwissen.

Tendenziell scheinen Rektionskomposita über geringere Frequenzen als Wurzelkomposita zu verfügen ${ }^{22}$ Dies kann zum einen bedeuten, dass Wurzelkomposita tendenziell stärker lexikalisiert sind, zum anderen aber auch, dass Rektionskomposita ähnlich wie phrasale Strukturen produktiver sind und die verschiedenen Einzelausprägungen an sich seltener vorkommen. Die konkreten Auswirkungen der Frequenz bleiben unklar und eine direkte Abbildung auf die Transparenz ist nicht möglich (vgl. Kap. 2.3.4). Allerdings gibt es keine auf Transparenz, Kompositionalität oder Frequenz aufbauenden Annahmen, die den hier aufgestellten Hypothesen zum Unterschied zwischen Rektions- und Wurzelkomposita zuwiderlaufen.

\subsubsection{Weitere Formen von Komposita}

Abschließend sollen drei spezielle Formen von Komposita betrachtet und bzgl. ihrer Kompatibilität mit den hier getroffenen Annahmen überprüft werden.

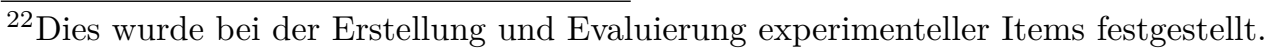




\section{Komposita mit Konversionen}

Rektionskomposita können durch Suffigierung, jedoch auch durch Konversion gebildet werden (vgl. Kap. 4.2.2). Bezüglich der Eigenschaften von Konversionen und deren Implikationen für die Komposita-Bildung gibt es verschiedene Ansichten. Di Sciullo/Williams (1987:39) vermuten mit Bezug auf (64-a), dass Konversionen generell keine Argumente nehmen können. Lieber (2014:86) führt mit (64-b) jedoch Korpusbelege an, die dies widerlegen.
a. *the hit of Bill, *the kick of Bill
b. Edmund Hillary's climb of Mount Everest, the stop of his car

Für Komposita mit Konversionen schlägt Lieber (2010) einen eigenen Kompositionstyp vor. Mit Bezug auf(65) nimmt sie an, dass das N1 bei Konversionen häufiger als externes Argument fungiert.

$$
\text { dog attack, bee sting, rainfall (Lieber 2010:128f) }
$$

Dies erklärt Lieber damit, dass die Koindizierung oben, also beim externen Argument beginnt. Bei Konversionen werde im Zuge der Nominalisierung keine Argumentstelle besetzt, weshalb für das Erstglied des Kompositums die externe Argumentstelle noch zur Verfügung steht. Liegt bspw. wie in burrito assembler das Affix -er vor, besetzt dieses die Position des externen Arguments und für das N1 bleibt die Position des internen Arguments (vgl. Lieber 2010:135ff). ${ }^{23}$ Problematisch an Liebers Erklärung ist, dass es neben -er auch andere Nominalisierungsaffixe gibt, die ihrerseits nicht die Position des externen Arguments besetzten und dennoch wird nur selten das externe Argument an die N1-Position vergeben. Ähnlich argumentiert auch Spencer (2011:495 FN 15) anhand des Beispiels truck driving. Hierbei besetzt das Suffix -ing die Event-Rolle. Wenn zunächst höhere Knoten besetzt würden, müsste girl driving akzeptabel und truck driving weniger akzeptabel sein. Dies ist nicht der Fall und somit ein Argument gegen eine im Baum abwärts gerichtete Besetzung der Argumentstellen.

Ebenso vertritt Grimshaw (1992:18) die Ansicht, dass das externe nicht vor dem internen Argument gesättigt werden kann. Mit (66) zeigt sie, dass das N1 in Komposita mit Konversionen kein echtes externes Argument ist und daher Komposita mit Konversionen als Wurzelkomposita zu analysieren seien (vgl. Grimshaw 1992:69).

$$
\text { bee sting - *bee stinging }
$$

Für das Deutsche zeigt Härtl (vgl. 2013:165), dass es sich um eine Frage der Verbart handelt. Die Beispiele in (67) illustrieren, dass im Fall von intransitiven Verben das externe Argument an der N1-Position stehen kann. Bei intransitiven Verben oder zumindest im Fall der Möglichkeit der intransitiven Verwendung der verbalen Basis wird die Rolle des internen Arguments gar nicht vergeben, die Rolle des externen Arguments kann also an das N1 vergeben werden.

$$
\text { Hundeheulen, Möwengeschrei }
$$

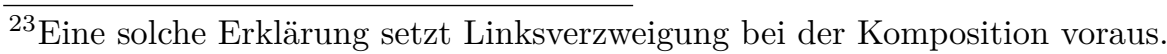


Dies scheint im Deutschen sowohl für unergative Verben wie in (68-a) als auch für unakkusative Verben wie in (68-b) möglich zu sein. Grimshaws Reihenfolge der Argumentvergabe bleibt damit gültig, da kein internes Argument vergeben wird und die Vergabe des externen Arguments als erstes erfolgen kann.
a. Hundebiss, Bienenstich
b. Wasserfall, Krokusblüte

Im Fall von Konversionen mit transitiven Verben kann das N1, wenn es Argumentstatus hat, nur als internes Argument fungieren. So zeigt sich in (69) und (70), dass die in den b-Fällen avisierte Lesart des N1 als externes Argument nicht zur Verfügung steht.
a. Die Kinderliebe der Eltern ist grenzenlos.
b. *Die Kinderliebe für ihre Eltern ist grenzenlos.

a. Der Fischfang von Klaas ist legendär.

b. *Der Fischfang des Balls ist legendär.

Auch Härtl bezeichnet die Lesart des N1 als externes Argument eines transitiven Verbs am Beispiel von Hundefressen als ungewöhnlich. Der Vorrang der wortinternen Vergabe der internen Argumentstelle bei transitiven Verben ist ein weiteres Argument für eine linksverzweigende Struktur von Rektionskomposita.

Zur Analyse von Konversionen schlägt Sproat (1985) in Analogie zur Bildung von Nominalisierungen mit Affigierung die Annahme eines Null-Morphems vor. Die Existenz von Null-Morphemen wird auch im Rahmen der DM angenommen (vgl. Halle/Marantz 1993: 132ff); das Hauptargument ist hier der Do-Support im Englischen. Für Rektionskomposita mit Konversionen nehme ich daher analog zu (41-a) an, dass als letzter Schritt der komplexe Kopf mit einem nominalen Merkmalsbündel mergt (siehe (71)), welches lediglich phonetisch nicht realisiert wird. Ansonsten entsprechen die Eigenschaften denen eines regulären Rektionskompositums. Ob dabei die zweite Wurzel mit einem verbalen Merkmalsbündel mergt (siehe (71-a) ) oder zunächst als Wurzel verbleibt (siehe (71-b)], ist unerheblich, da die Argumentstellenvergabe eine Eigenschaft der Wurzel ist.

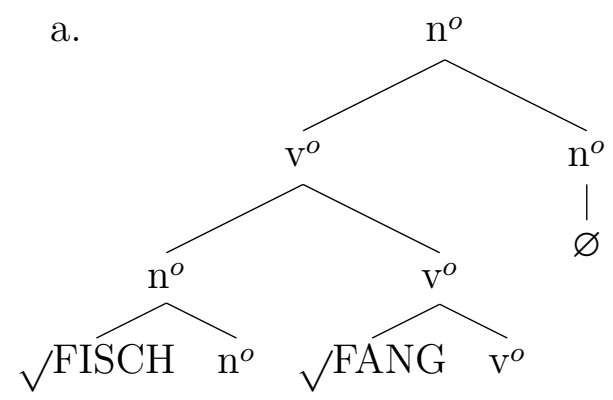

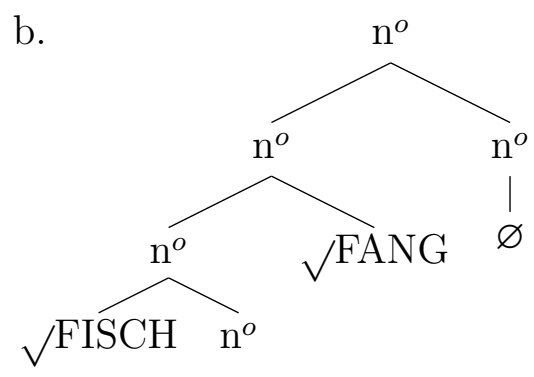

\section{Komposita mit relationalem Zweitglied}

Komposita mit relationalem Zweitglied (vgl. (72) scheinen auf den ersten Blick Wurzelkomposita zu sein. Allerdings wird die Relation, die nötig ist, um die Konstituenten in einen Zusammenhang zu bringen, nicht aus dem Welt- und Kontextwissen abgerufen, sondern ist Teil der Semantik des Kopfnomens. Im Gegensatz dazu ist bei nicht-relationalen 
Kopfnomen und damit ambigen Komposita Konzeptwissen zur Interpretation nötig (u.a. Fanselow 1981, Meyer 1993).

Ein relationales Nomen eröffnet eine Leerstelle. Wunderlich (vgl. 1986:221ff) beschreibt diese als "Argumentstelle", die jedoch semantisch als relational gekennzeichnet ist und nicht als syntaktisch subkategorisiert. Die Leerstelle kann wortintern oder phrasal realisiert werden. Geht man mit Higginbotham (1985) davon aus, dass alle Wortarten ein Theta-Raster haben, kann man hier ebenfalls von Theta-Rollen-Vergabe sprechen ${ }^{24}$ Diese erfordert eine spezifische Kategorie. Im vorliegenden Fall kann die entsprechende Rolle nur an ein Nomen vergeben werden. Wie bei den Rektionskomposita scheint also auch im Fall relationaler Zweitglieder durch die Anforderungen des Theta-Rasters eine Kategorisierung gefordert zu werden. Darüber hinaus geht der Einfluss des Zweitglieds in diesem Fall jedoch nicht, d.h. es gibt keinen Grund, für das N1 Argumentstatus anzunehmen und somit auch keinen Grund zur Annahme von Maximalität. Im Gegensatz zu Rektionskomposita kann in Komposita mit relationalem N2 keine VP vorliegen. Das N1 in Komposita mit relationalem Zweitglied liegt somit bzgl. seines Grads an Referenzialität zwischen den N1 von Wurzel- und Rektionskomposita. Diese Annahme wurde bei einer informellen Umfrage unter elf Studierenden getestet. Dabei wurde nach der Akzeptabilität der Sätze unter (73) gefragt. Es ergab sich der höchste Wert für die Bedingung (73-a) mit einem Rektionskompositum, gefolgt von Bedingung (73-b) mit einem Kompositum mit relationalem Zweitglied und der niedrigste Wert lag bei der Bedingung (73-c) mit einem Wurzelkompositum vor 25
a. Paul ist ein richtiger Büchersammler. Sie stapeln sich schon im ganzen Haus.
b. Paul ist ein richtiger Bücherfan. Sie stapeln sich schon im ganzen Haus.
c. Paul ist ein richtiger Bücherfreak. Sie stapeln sich schon im ganzen Haus.

\section{Phrasenkomposita}

Als dritte Sonderform seien die Phrasenkomposita erwähnt (siehe dazu auch Kapitel 2.5.1). Hierbei steht an der Position des N1 eine phrasale Konstituente, wie in den Beispielen unter (74)

Abgerechnet-wird-am-Schluss-Taktik, Messer-und-Gabel-Handhabung (Meibauer 2003:153)

Die Existenz von Phrasenkomposita wird oft als Argument für eine syntaktische Theorie der Wortbildung herangezogen, da hier konsensuell die Form YP $+\mathrm{X}$ zugrundegelegt wird (vgl. Meibauer 2003:155). Damit ist ihre Existenz an sich bereits ein Argument für die Möglichkeit von Maximalität unterhalb der Wortgrenze. Allerdings herrscht ebenfalls die Auffassung, dass auch in diesem Fall in der ersten Konstituente kein D-Kopf enthalten

\footnotetext{
${ }^{24}$ Zur Vergabe von Theta-Rollen durch relationale Nomen siehe auch Meyer (1993 104f).

${ }^{25}$ Bei der Aufgabe handelte es sich um ein Akzeptabiltitäs-Ranking, bei dem die Studierenden den (in ihrer Reihenfolge pseudo-randomisierten) Sätzen Werte von 1 (am wenigsten akzeptabel) bis 3 (am akzeptabelsten) zuordnen sollten. Die aufsummierten Werte sind: $\mathrm{a}(25)>\mathrm{b}(24)>\mathrm{c}(18)$.
} 
sein kann (siehe (75-a) , da ein Determinierer auf Referenzialität verweise, welche nicht gegeben sei (vgl. Härtl 2015:172f). Dennoch sind auch Fälle wie (75-b) attestiert. Meibauer (vgl. 2003:168f) nimmt an, dass es sich bei solchen Fällen um Lexikalisierungen handelt. Die erste Konstituente sei daher syntaktisch opak und als nicht-referenzielle DP zu beschreiben.26

$$
\begin{aligned}
& \text { a. *der Die-Grauen-Schläfen-Effekt (Härtl 2015:172) } \\
& \text { b. die Der-schöne-Rheingau-Laberei (Meibauer 2003:168) }
\end{aligned}
$$

Nach diesem Verständnis bewirkt die Einführung einer DP in ein Phrasenkompositum keine Referenzialität, was Meibauer anhand von Fällen wie (76) illustriert (vgl. Meibauer 2003:168f). Allerdings handelt es sich bei den Pronomen in diesen Beispielen nicht um Anaphern, sondern um Relativpronomen.

a. *die $\underline{\text { Holz }}+$ hütte, das man so günstig im Baumarkt bekommt

b. *die [das $\underline{\text { Holz }}+$ hütte], das man so günstig im Baumarkt bekommt

Auch Harley (2009) nimmt für Phrasenkomposita eine nicht-referenzielle, weil eingefrorene DP an. Im Fall von Diskursanaphern wie unter (77) schränkt Meibauer jedoch ein, dass eine in der phrasalen Komposita-Konstituente enthaltene DP als Antezedens fungieren kann (vgl. Meibauer 2003:167). Als Erklärung hierfür gibt er drei Möglichkeiten an: Die Sätze seien akzeptabel aufgrund eines semantischen Bezugs, aufgrund eines pragmatischen Schlussprozesses (Implikatur) oder bei Phrasenkomposita liege eventuell eine Ausnahme vom Inselstatus vor.
a. Damals wurde die [ $\underline{G o t t}_{i}$-ist-tot $]$-Thematik in allen Zeitungen ausgebreitet, aber wir glaubten nicht daran, dass $\underline{\mathrm{er}}_{i}$ tot ist.
b. Die $\left[\underline{\text { Rote-Augen }}_{i}\right]$-Reduktion ist durchaus wünschenswert, weil die Fotogra- fierten $\underline{\text { damit }}_{i}$ wie Zombies aussehen.
c. Gestern hat mich die $\left[\underline{\mathrm{Ohr}}_{i}\right.$-ab]-Bande überfallen, aber $\underline{\mathrm{es}}_{i}$ ist noch dran.

Die Analyse als Lexikalisierung liefert keine hinreichende Erklärung, da Phrasenkomposita auch ad hoc gebildet werden können. Angesichts von Fällen wie (78) spricht meiner Ansicht nach nichts gegen die Referenzialität einer in einer phrasalen Komposita-Konstituente enthaltenen DP. Ihr Grad an Zugänglichkeit hängt dann von der kontextuellen Einbettung ab. Neben Komposita mit einem D als erster Konstituente erscheinen Phrasenkomposita mit einer DP in der ersten Konstituente als weiteres Indiz für die Möglichkeit von Maximalität innerhalb von Komposita.

(78) Patrick ist heute in Wir-machen-die-Terrasse-schön-Laune. Sie soll endlich nicht mehr so trist aussehen.

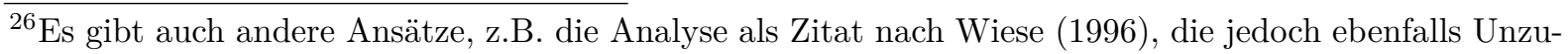
gänglichkeit postuliert und zu den lexikalistischen Ansätzen zu zählen ist.
} 


\subsection{Zwischenfazit}

Basierend auf Annahmen der DM wurden in diesem Kapitel zwei Arten von Komposita unterschieden: Zum einen Wurzelkomposita wie (79-a), die gemäß meiner Analyse unter (38) (mindestens) aus einer Wurzel und einem Wort bestehen. Dabei bleibt das Erstglied kategorielos; das Zweitglied wird durch den Kontext kategorisiert und legt die Kategorie des Gesamtworts fest ${ }^{27}$ Zum anderen Rektionskomposita wie unter (79-b), welche gemäß meiner Analyse unter (41) (mindestens) aus zwei kategorisierten Ausdrucksteilen und einem weiteren nominalen Merkmalsbündel bestehen, welches nicht overt realisiert sein muss. Die zweite Wurzel verfügt über eine Argumentstruktur, die eine Kategorisierung der ersten Konstituente auslöst, die dadurch eine Argumentstelle besetzen kann.
a. Dach-garten, Tabak-fabrik, Abend-veranstaltung
b. Dach-begrün-ung, Auto-fahr-er, Haus-putz- $\varnothing$

Mit Rektions- und Wurzelkomposita stehen sich syntaktisch-semantische Subkategorisierungsanforderungen und Modifikationsbeziehungen gegenüber; das N1 ist Argument oder Modifikator. Aus den herausgearbeiteten Unterschieden in der morphosyntaktischen Struktur von Wurzel- und Rektionskomposita resultieren Unterschiede im kategorialen Status der jeweiligen Erstglieder. Diese Unterschiede werden mit Unterschieden in der Referenzialität in Verbindung gebracht, die wiederum einen Reflex auf die Verarbeitung haben können. Meinen Annahmen zufolge wirkt sich dies letztlich auch auf den Grad an Zugänglichkeit der Erstglieder aus.

Als theoretische Basis dieser Annahme wurden vor allem drei Eigenschaften betrachtet: Zunächst wurde gezeigt, dass das N1 im Fall von Rektionskomposita über nominalen Status verfügt, welcher im Fall von Wurzelkomposita nicht plausibel ist. In der vorgestellten Analyse verfügt das N1 in Rektionskomposita über den Status eines $\mathrm{n}^{o}$. Im Gegensatz dazu wurde das N1 in Wurzelkomposita als $\sqrt{ } \mathrm{P}$ analysiert. Der Grad an Referenzialität und Zugänglichkeit des N1 ist somit bei Rektionskomposita höher. Als zweites wurde dafür argumentiert, dass das N1 in Rektionskomposita im Fall einer kompositumsinternen VP Argumentstatus und eine Theta-Rolle zugewiesen bekommt (z.B. bei ProzessNominalisierungen). Diese Eigenschaften erhöhen den Grad an Referenzialität über den eines lediglich nominalen Ausdrucks hinaus. Basierend auf Annahmen des Minimalistischen Programms wurde darüber hinaus eine Analyse des N1 in Rektionskomposita als D vorgeschlagen (z.B. bei N1 als Eigennamen oder Massenomen). In dem Fall verfügt das N1 über volle Referenzialität und liefert einen Erklärungsansatz für die Ungrammatikalität von kompositumsinternen Determinierern und Adjektiven.

Für diese generellen Überlegungen wurde weitere, unabhängige Evidenz gefunden. In polysynthetischen Sprachen sind NV-Inkorporationen eine produktive Form der Wortbildung und das N1 ist in diesen zugänglich. Die Analyse als Inkorporation stellt somit allein kein Argument gegen N1-Zugänglichkeit dar. Des Weiteren sind im Chinesischen und im Hebräischen Formen attestiert, die sich oberflächenstrukturell gleichen, aber über gravierende Unterschiede in der Strukturbildung verfügen. In Anbetracht dessen spricht nichts dagegen, auch verschiedenen Arten von Komposita verschiedene Strukturen zugrunde zu legen.

${ }^{27}$ Beim Zweitglied kann es sich auch um ein deverbales Nomen handeln, allerdings wird wortintern keine Argumentstelle gesättigt und somit keine Kategorisierung ausgelöst. 
Abschließend erfolgte eine Übertragung der Analyse auf andere Arten von Komposita. Es wurde gezeigt, dass sich Rektionskomposita mit Konversionen wie Rektionskomposita mit overtem Suffix analysieren lassen. Für das N1 in Komposita mit relationalem Zweitglied wurde ein Zugänglichkeitsgrad attestiert, der zwischen dem der Rektions- und Wurzelkomposita liegt. Dieser resultiert aus der Annahme, dass das N1 durch das Besetzen einer semantischen Leerstelle kategorisiert wird, jedoch Mangels VP keinen Argumentstatus zugewiesen bekommt. Schließlich wurde gezeigt, dass bei Phrasenkomposita eine volle DP in einer Kompositums-Konstituente enthalten sein kann und in diesem Fall auch ein hoher Grad an Zugänglichkeit besteht.

Wie bereits in den vorangegangenen Kapiteln thematisiert, handelt es sich bei der $\mathrm{Zu}$ gänglichkeit von Komposita-Erstgliedern um ein graduelles Phänomen. Neben den bereits attestierten verschiedenen konzeptuellen Einflüssen, scheint auch die morphosyntaktische Struktur zu diesem Eindruck von Gradienz beizutragen. Je nach konkretem Aufbau eines Kompositums, scheinen einem Erstglied verschiedene kategoriale Status zukommen zu können; von eher unzugänglichen, kategorielosen Wurzeln in Wurzelkomposita über nominale N1 in Rektionskomposita bis hin zu vollen DPn in Phrasenkomposita. Abbildung 4.1 veranschaulicht diese Skala wachsender Zugänglichkeit in Abhängigkeit vom kategorialen Status des Erstglieds.

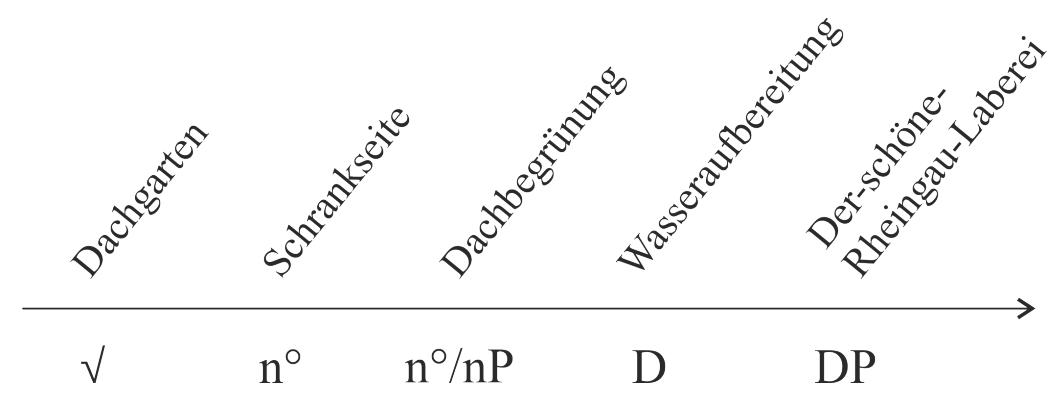

Abbildung 4.1: Verschiedene kategoriale Status von N1 angeordnet auf einer Skala mit steigender Zugänglichkeit

Die konkrete Strukturbildung der einzelnen Formen wurde in diesem Kapitel angerissen. Offene Fragen bestehen bzgl. Links- oder Rechtsverzweigung, dem Status des N2 und Möglichkeiten der Analyse weiterer Formen. Hier gilt es für eine breite theoretische Fundierung anzuknüpfen und in die Tiefe zu gehen. Diese Arbeit wechselt an dieser Stelle allerdings wieder zu einer psycholinguistischen Perspektive. Die nun zentrale Frage ist daher: Haben Unterschiede im Strukturaufbau und damit im kategorialen Status von Erstgliedern einen messbaren Reflex auf die Verarbeitung? Umgekehrt kann durch einen Nachweis von Unterschieden in der Verarbeitung auf das tatsächliche Bestehen von Unterschieden in der Struktur rückgeschlossen werden. Hierzu sollen im Folgenden experimentelle Arbeiten vorgestellt werden. 


\section{Experiment 2: RZK und Struktur}

\subsection{Faktoren}

Im Folgenden wird ein zweites Experiment berichtet, welches Hypothesen aus den Kapiteln 3 und 4 verfolgt und auf dem experimentellen Design der Pilotstudie aus Kapitel 3.4 aufbaut.

Als erster Faktor soll erneut ein Faktor der Konzeptkombination untersucht werden. In Kapitel 3.3 wurde die Annahme formuliert, dass es innerhalb von Komposita einen konzeptuellen Fokus gibt, der verschieden gelagert sein kann und der Auswirkungen auf die Aktiviertheit der Konstituenten hat. Je zentraler das N1 für das NN-Konzept ist, desto stärker ist es aktiviert und desto zugänglicher ist es. Als Kriterium für die Ausrichtung des konzeptuellen Fokus wurde in der Pilotstudie in Kapitel 3.4 die semantische Relation nutzbar gemacht. Hierfür wurde der Einfluss der Relationen HAVE und FOR überprüft. Dabei stellte sich heraus, dass die semantischen Relationen nicht eindeutig voneinander abzugrenzen sind und es sich somit um kein geeignetes Mittel handelt, um Aussagen zum konzeptuellen Fokus zu treffen. Die kategorische Eigenschaft der raum-zeitlichen Kontiguität (RZK) wurde als geeigneter für experimentelle Untersuchungen gewertet (vgl. Kap. 3.3.4 und Kap. 3.4.7), was im Folgenden überprüft werden soll.

Der Faktor RZK liegt in zwei Faktorstufen vor, siehe (1) In der Faktorstufe +RZK ist die raum-zeitliche Präsenz des N1-Konzepts bei der Visualisierung des NN-Konzepts obligatorisch. Die mentale Repräsentation von Autounfall muss auch die Repräsentation von Autos enthalten. Ohne die raum-zeitliche Anwesenheit von Autos kann kein Autounfall stattfinden. In der Faktorstufe -RZK ist die raum-zeitliche Präsenz des N1 für das NNKonzept fakultativ. Die mentale Repräsentation von Autoversicherung muss keine Autos enthalten, denn für die mentale Repräsentation einer Versicherungs-Police ist die Repräsentation des versicherten Objekts nicht zwingend erforderlich.

Die Untersuchung des Faktors RZK soll zum einen den Einfluss dieser Eigenschaft auf die N1-Zugänglichkeit belegen und zum anderen die Idee eines konzeptuellen Fokus bei der Komposita-Verarbeitung stützen.

Die Zuordnung der Komposita zu den Faktorstufen +/-RZK erfolgt anhand der Semantik der Komposita und nicht über den Satzkontext, da ausdrucks-inhärente und nicht kontextuelle Eigenschaften Gegenstand des Experiments sind.

$$
\begin{aligned}
& \text { a. +RZK: Autounfall } \\
& \text { b. -RZK: Autoversicherung }
\end{aligned}
$$

Als zweiter Faktor wird der in Kapitel 4 postulierte Unterschied in der Struktur von Wurzel- und Rektionskomposita untersucht. Der Faktor STRUKTUR ist ebenfalls zweistufig angelegt, siehe (2) Als Wurzelkomposita (WK) werden Komposita bezeichnet, deren Konstituenten durch eine unterspezifizierte semantische Relation verbunden sind, z.B. liegt 
bei Autounfall die Relation USE vor ${ }^{1}$ Als Rektionskomposita (RK) werden Komposita bezeichnet, bei denen das N1 als internes Argument des im Zweitglied enthaltenen Verbs interpretiert wird, z.B. ist bei einer Autoversicherung das Auto das versicherte Objekt. Wie in Kapitel 4 ausgeführt, werden aus den Unterschieden in der Struktur der beiden Komposita-Arten Unterschiede im referenziellen Status des jeweiligen N1 abgeleitet, womit letztlich Unterschiede in der N1-Zugänglichkeit motiviert werden. In der Bedingung WK wird für das N1 eine kategorielose Wurzel und damit eine schlechtere Zugänglichkeit angenommen als in der Bedingung RK, in der für das N1 eine nominale, weil argumentstellenfähige Konstituente angenommen wird. Die Untersuchung des Faktors STRUKTUR soll den Einfluss der Kompositums-Struktur auf die N1-Zugänglichkeit belegen.
a. WK: Autounfall
b. RK: Autoversicherung

Das im Folgenden berichtete Experiment hat zum einen das Ziel, Einsichten über den Einfluss der beiden Faktoren zu erlangen. Zum anderen sollen durch die Kreuzkombination eines konzeptuellen und eines grammatischen Faktors Einsichten in die Interaktion konzeptueller und grammatischer Faktoren gewonnen werden.

\subsection{Methode}

Als experimentelles Paradigma für Experiment 2 wurde analog zur Pilotstudie die Satzergänzungsaufgabe gewählt. Die Ergebnisse des ersten Experiments belegen, dass es sich dabei um eine für den Untersuchungsgegenstand geeignete Methode handelt. Ein Item besteht aus einem Kontextsatz, der ein Kompositum enthält, und einem darauf folgenden Satzfragment, welches ein Pronomen enthält, das grammatisch mit dem N1 im Kontextsatz kongruent ist. Die Aufgabe der VPn bestand darin, das Satzfragment intuitiv nach eigenem Ermessen zu vervollständigen. Im Anschluss wurden die Satzergänzungen in Hinblick auf die Pronomenresolution interpretiert und annotiert.

\subsection{Material}

\subsubsection{Komposita-Auswahl}

Als Basis für die experimentellen Items dienen Quadrupel von NN-Komposita, für welche in einem späteren Schritt Satzkontexte konstruiert wurden. Da zwei zweistufige Faktoren untersucht werden $(2 \times 2$-Design $)$, liegt jedes Item entsprechend Tabelle 5.1 in vier Bedingungen vor: $-\mathrm{RZK} / \mathrm{RK},+\mathrm{RZK} / \mathrm{WK},+\mathrm{RZK} / \mathrm{RK}$ und $-\mathrm{RZK} / \mathrm{WK}$. Um Vergleichbarkeit zu gewährleisten, setzen sich die Komposita innerhalb eines Items jeweils aus dem gleichen N1 und einem die jeweilige Bedingung erfüllenden N2 zusammen.

\footnotetext{
${ }^{1}$ Auch die Annahme einer zugrundliegenden HAVE-Relation ist möglich. Hier zeigt sich erneut die vage Abgrenzung der semantischen Relationen.
} 


\begin{tabular}{cccll}
\hline \hline Bed. & RZK & STRUKTUR & Beispiele & \\
\hline 1 & - RZK & RK & Geldtransporter & Müllvermeidung \\
2 & + RZK & WK & Geldmünze & Müllmenge \\
3 & + RZK & RK & Geldtransport & Müllverwertung \\
4 & - RZK & WK & Geldautomat & Müllauto \\
\hline \hline
\end{tabular}

Tabelle 5.1: Beispiele für die in Experiment 2 verwendeten Komposita in den vier Bedingungen mit den Faktoren RZK und STRUKTUR

Als Grundstock für Komposita diente die Komposita-Datenbank des GermaNet (Henrich/Hinrichs 2011). Die daraus infrage kommenden NN-Komposita wurden auf die vier Bedingungen aufgeteilt. Zur Komplettierung einzelner Items wurden z.T. in einzelnen Bedingungen weitere Komposita ergänzt. Die Zuordnung der Komposita zu den Faktorstufen des Faktors RZK, wurde anschließend durch zwei Hilfskräfte validiert. Hierfür wurden die Komposita gemischt und bzgl. ihrer RZK bewertet. Nur Komposita, bei denen ein Konsens in der Zuordnung bestand, wurden für das Experiment in Betracht gezogen.

Darüber hinaus wurden folgende Kriterien bei der Auswahl der Komposita angelegt: Da der Einfluss der Animatheit in Experiment 1 bereits gezeigt wurde, wurde dieser Faktor in Experiment 2 kontrolliert und nur inanimate N1 und N2 verwendet. Als N1 wurden konkrete Nomen verwendet; keine Eigennamen oder Singularetantums. Komposita mit Fugenelementen wurden ebenfalls ausgeschlossen; beide Konstituenten treten an der Oberfläche in der Singularform auf. Es wurde darauf geachtet, dass N1 und N2 stets über unterschiedliche Genera verfügen, um die Kongruenz mit dem anaphorischen Pronomen eindeutig zu machen. Da die Rektionskomposita aufgrund der Nominalisierungen oft Feminina und Maskulina sind, muss ein Großteil der N1 im Neutrum vorliegen. Das der Nominalisierung zugrundeliegende Verb ist stets transitiv. Die Art der Nominalisierung variiert und ist annotiert, um einen eventuellen Einfluss überprüfen zu können.

Ein weiterer zu kontrollierender Faktor ist die Transparenz (vgl. Coulmas (1988) und Kap. 2.6.2). Diese wurde durch einen Paraphrasierungs-Test so gut wie möglich sichergestellt. Darüber hinaus wurde die Frequenz kontrolliert. Auch wenn der Zusammenhang zwischen Transparenz und Frequenz nicht abschließend geklärt ist (vgl. Kap. 2.3.4), ermöglicht eine Kontrolle der Komposita-Frequenzen innerhalb der Items eine stärkere Homogenität und damit Vergleichbarkeit. In einem ersten Schritt wurde über die gerundeten Frequenzklassen aus dem Wortschatz der Universität Leipzig ${ }^{2}$ eine Vorauswahl getroffen. Für die verbleibenden Komposita wurden die Frequenzen im Kernkorpus 20 des DWDS3 ermittelt, sowie die Frequenzen aus dem DeReKo via COSMAS Il] Für die Werte aus dem DeReKo und dem Kernkorpus 20 wurden die z-Werte berechnet, um die Korpora vergleichbar zu machen. Anschließend wurden die Items ausgewählt, bei denen die Frequenzen der vier Komposita innerhalb eines Quadrupels nicht mehr als 0,5 $\sigma$ voneinander

\footnotetext{
${ }^{2}$ LWS: German newspaper corpus based on material crawled in 2011 (deu_newscrawl_2011). (C) 2014 Abteilung Automatische Sprachverarbeitung, Universität Leipzig. www.wortschatz.uni-leipzig.de/ (23.10.2014).

${ }^{3}$ DWDS-KK: DWDS-Kernkorpus des 20. Jahrhunderts (Digitales Wörterbuch der deutschen Sprache). Berlin-Brandenburgische Akademie der Wissenschaften. http://www.dwds.de/r/ (23.10.2014).

${ }^{4}$ Institut für Deutsche Sprache (2014b): Das Deutsche Refernzkorpus DeReKo/Archiv der Korpora geschriebener Gegenwartssprache 2014-II (Release vom 11.09.2014). Mannheim: Institut für Deutsche Sprache. www.ids-mannheim.de/DeReKo.

COSMAS II (Corpus Search, Management and Analysis System): (C 1991-2016 Institut für Deutsche Sprache, Mannheim. www.ids-mannheim.de/cosmas2/ (23.10.2014).
} 
abweichen.

Nach Abschluss der Frequenzanalyse blieben 27 Quadrupel übrig (vollständige Liste der Items siehe Anhang B. Da im Korpus besonders viele Komposita mit den Erstgliedern Geld, Öl und Wasser vorlagen, wurden diese für jeweils zwei Items genutzt.

Einige Ausdrucks-Eigenschaften konnten innerhalb der Komposita nicht ausreichend kontrolliert oder ausbalanciert werden. Dazu zählen Genus, Spezifizität und Zählbarkeit des N1 sowie Nominalisierungsart des N2. Um einen Einfluss dieser Eigenschaften im Nachhinein (zumindest deskriptiv) analysieren zu können, wurden diese Eigenschaften im Datenset annotiert.

\subsubsection{Item-Erstellung}

\section{Kontextsätze}

Nach der Auswahl der Komposita für die Items bestand der nächste Schritt in der Generierung von Kontextsätzen (Beispiel-Items siehe (3)] und (4)].

(3) 1 Armin hat in der Hafengegend einen verdächtigen Geldtransporter beobachtet. Dieses/Jenes hat er ...

2 Armin hat in der Hafengegend eine verdächtige Geldmünze gefunden. Dieses/Jenes hat er ...

3 Armin hat in der Hafengegend einen verdächtigen Geldtransport beobachtet. Dieses/Jenes hat er...

4 Armin hat in der Hafengegend einen verdächtigen Geldautomaten gesehen. Dieses/Jenes hat er ...

(4) 1 Silke hat beim Entsorgungswerk eine umweltschonende Müllvermeidung erlernt. Diesen/Jenen hat sie ...

2 Silke hat beim Entsorgungswerk eine umweltschonende Müllmenge produziert. Diesen/Jenen hat sie ...

3 Silke hat beim Entsorgungswerk eine umweltschonende Müllverwertung erlernt. Diesen/Jenen hat sie ...

4 Silke hat beim Entsorgungswerk ein umweltschonendes Müllauto bestellt. Diesen/Jenen hat sie ...

Die Kontextsätze sind parallel gebaut und innerhalb eines Items einheitlich. Zu Beginn eines Kontextsatzes steht jeweils ein Personenname in Subjektfunktion. Darauf folgt eine Adverbialbestimmung. Das Vollverb musste aufgrund semantischer Beschränkungen z.T. variiert werden. Das Konstanthalten des Verbs wäre mit Hilfe von Verba Dicendi und Sentiendi möglich, siehe (5). Hierbei wird das Gesamtkompositum jedoch in unspezifischer Lesart eingeführt, was eine Wiederaufnahme durch ein Pronomen erheblich erschwert und dadurch eine Verzerrung der Ergebnisse mit sich bringen würde. Aus diesem Grund wurde Varianz durch unterschiedliche Verben in Kauf genommen.

(5) Silke hat über ein Müllauto/eine Müllverwertung nachgedacht. 
Bei ambigen Komposita, z.B. Nominalisierungen mit -ung, die auf einen Prozess oder ein Ergebnis referieren können, wurde die für die Erfüllung der entsprechenden Bedingung des Faktors RZK benötigte Lesart durch das Verb forciert.

Den Komposita wurde neben einem indefiniten Artikel ein Adjektiv vorangestellt und die kritische NP somit verlängert. Durch eine solche Verlängerung der Phrase verlängert sich auch deren Lesezeit, was idealerweise mit erhöhter Aufmerksamkeit und leichterer Memorisierbarkeit einhergeht (vgl. Sereno 1992:306). Dabei kommt jedes Adjektiv nur in jeweils einem Item vor, dort jedoch in allen Bedingungen. Damit dies möglich ist, sind die Adjektive eher merkmalsarm gehalten, z.B. interessant.

Ziel war es, Sätze zu bilden, die vielfältige Anknüpfungspunkte für Assoziationen liefern und den VPn ermöglichen, kreative und gehaltvolle Satzergänzungen zu bilden, die wiederum eine möglichst eindeutige Annotation ermöglichen. Abschließend wurde kontrolliert, dass kein Element im Satz mit dem N1 genus-kongruent ist. Demzufolge hat die Anapher im Text lediglich das N1 als morphosyntaktisch kongruentes Antezendens. Die Positionierung des Pronomens vor einem Hilfsverb und einem weiteren Personalpronomen verhindert eine Interpretation als Determinierer.

\section{Wahl des anaphorischen Ausdrucks}

Eines der produktivsten Nominalisierungsmorpheme ist das Suffix -ung, welches Feminina bildet; ebenfalls produktiv ist das instrumentale Suffix -er, welches Maskulina bildet. Um die Anapher zu disambiguieren, muss das N1 stets ein anderes Genus tragen als das N2. Da nun in den meisten Items eines der beiden Suffixe auftritt, steht die Anapher in einem Großteil der Fälle im Neutrum (20 von 27 Items). Die entsprechende Anapher wäre demzufolge es. Das Pronomen es wird allerdings im Allgemeinen nur selten anaphorisch verwendet; meistens tritt es in expletiver Lesart, als Vorfeld-es, Korrelat oder - wenn anaphorisch - als Komplexanapher auf. Um zu überprüfen, ob die anaphorische Lesart überhaupt verfügbar ist, wurde als Vortest eine kleine Fragebogenstudie mit acht Versuchspersonen durchgeführt 5 In den kritischen Bedingungen wurde das es sowohl als Korrelat, z.B. in (6-a), als auch als Komplexanapher interpretiert, z.B. in (6-b). Eine anaphorische Verwendung mit N1-Bezug kam in keinem Fall vor. Darüber hinaus enthielt der Vortest Satzergänzung mit einem Vollverb, dass eine Handlung prädiziert, die für das jeweilige N1 typisch ist. In diesem Fall wurde wie in (6-c) stets ein N1-Bezug hergestellt.

(6) a. Tim hat im Herbst trotz schlechter Sicht einen schmierigen Ölfilter ausgewechselt. Er hat es ... nicht geschafft, auch noch die Reifen zu wechseln.

b. Tim hat im Herbst trotz schlechter Sicht einen schmierigen Ölfilm beseitigt. Er hat es ...gewissentlich ausgeführt - wie immer.

c. Tim hat im Herbst trotz schlechter Sicht einen schmierigen Ölwechsel vorgenommen. Er wischte es ... in ein dreckiges Handtuch.

Daraus schließe ich zum einen, dass das es theoretisch für einen N1-Bezug zur Verfügung steht, dies jedoch nicht die präferierte Resolution ist. Für Aussagen zur N1-Zugänglichkeit

\footnotetext{
${ }^{5}$ Für diesen Vortest wurden Fragebögen mit jeweils vier Sätzen erstellt. Ziel war es, verschiedene Varianten von Satzfragmenten bzgl. ihrer Eignung für das Experiment zu überprüfen. Dabei wurden 3 Items in verschiedenen Versionen sowie einige Filler getestet und an Freiwillige auf dem Campus ausgegeben.
} 
erscheint das Pronomen es somit ungeeignet. Die Verwendung von Vollverben ist jedoch ebenfalls keine Option, da die Satzfragmente für alle Items identisch sein sollen. Außerdem wäre die Verwendung eines Vollverbs aufgrund verschiedener Assoziationsstärken und zu starker inhaltlicher Einschränkung ein konfundierender Faktor.

Der Vortest deutet an, dass das Nicht-Auftreten eines N1-Bezugs nicht automatisch bedeutet, dass eine solche Möglichkeit ausgeschlossen ist, sondern lediglich, dass eine Präferenz für eine andere Interpretation vorliegt. Die Verwendung von es ist demnach für den Untersuchungsgegenstand ungeeignet.

Bei ihrer Übertragung der Givenness Hierarchy auf das Deutsche schließt AverintsevaKlisch (vgl. 2013 75) bei der Verwendung von Personalpronomen auf einen fokussierten Referenten, und bei der Verwendung von Demonstrativpronomen auf einen aktivierten Referenten. Diese Annahme lässt sich mit dem experimentellen Material dahingehend in Einklang bringen, dass es keinen Grund zur Annahme gibt, dass das N1 durch den Satzkontext fokussiert ist. Ein gewisser Grad an Aktiviertheit scheint mir jedoch plausibel, wenn ich davon ausgehe, dass das N1 einen Diskursreferenten etablieren kann. In diesem Sinne erscheinen Demonstrativpronomen als eine geeignete Form der Anapher.

$\mathrm{Zu}$ den Demonstrativpronomen zählen neben der, die und das auch Pronomen der Art dieser, jener und derselbe; wobei letztere von Bosch et al. (2007:146) als morphologisch komplex und selten eingestuft werden ${ }^{6}$ Insgesamt beschreiben sie Demonstrativpronomen als „lower ranked referring expressions“, die auf weniger saliente Antezedenten wie z.B. das grammatische Objekt referieren (vgl. Bosch et al. 2007:158f). Auf die Salienz des Antezedens haben neben der erwähnten grammatischen Rolle noch weitere Faktoren Einfluss, z.B. die syntagmatische Stellung der Satzglieder (vgl. Bosch et al. 2007:46) oder informationsstrukturelle Eigenschaften (vgl. Bosch et al. 2007.27). Bei Personalpronomen besteht eine Präferenz für einen Bezug auf das Diskurstopik. Im Kontrast referieren Demonstrativa i.d.R. gerade nicht auf das Diskurstopik (vgl. Bosch et al. 2007:50). Über allem steht die intrinsische Interpretation, d.h. das Weltwissen als dominierender Faktor der Pronomenresolution (vgl. Bosch et al. 2007:157). Auch Bosch et al. räumen ein, dass Pronomen nicht immer ein explizites Antezedens benötigen, mit dem sie koreferent sind, so z.B. im Fall von ,Donkey Pronouns', ,E-type Pronouns' und Pronomen mit inferriertem Antezedens:

[...] there are also occurences of both demonstratives and personal pronouns, that require no linguistic expression whatsoever that introduces a referent for the pronoun. (Bosch et al. 2007 47)

Das N1 ist in den Items nicht das Diskurstopik 7 und selbst unter der Voraussetzung, dass es als Antezedens zur Verfügung steht, verfügt es maximal über ein mittleres Salienzniveau. Ein Demonstrativpronomen erscheint somit als geeigneter anaphorischer Ausdruck für einen N1-Bezug. ${ }^{8}$

Die konkrete Wahl fiel auf die Demonstrativpronomen dieses/r und jenes/r. In ihrer Funktion als Demonstrativa referieren sie auf ein weniger salientes Antezedens und zeigen einen Topik-Wechsel an. Gleichzeitig sind Interpretationen als Komplexanapher, Vorfeld-es o.̈̈. grammatisch, jedoch weniger produktiv als beim Personalpronomen es. Sollte also ein

\footnotetext{
${ }^{6}$ Einschränkend heißt es: „Here it is not clear that their uses are similar to the simple demonstrative pronouns, however." (Bosch et al. 2007 146) Konkrete Unterschiede werden jedoch nicht benannt.

${ }^{7}$ Wobei dies z.B. durch die Verwendung von Spaltsätzen möglich wäre.

${ }^{8}$ Einflussfaktoren wie grammatische Funktion oder Informationsstruktur sind in den experimentellen Items kontrolliert.
} 
N1-Bezug hergestellt werden, bedeutet dies im Umkehrschluss, dass das entsprechende N1 tatsächlich zugänglich ist.

Für eine Entscheidung zwischen den Demonstrativa dieses und jenes liegen keine klaren Argumente vor. Jenes erscheint zum einen aufgrund seiner selteneren Verwendung stilistisch markiert, drückt zum anderen aber auch mehr Distanz aus, was für ein weniger salientes Antezedens als geeignet erscheint. Zur explorativen Untersuchung eines möglichen Unterschieds zwischen dieses und jenes fanden daher beide Demonstrativpronomen Eingang in das Experiment. Die Item-Zahl wurde hierfür verdoppelt, d.h. aus ursprünglich vier Listen wurden acht. Jedes Item tritt nun einmal mit dieses $/ r$ und einmal mit jenes $/ r$ als Anapher auf. Die Pronomenart wurde als weitere Bedingung in das Design aufgenommen. Jede VP sah in Abhängigkeit der ihm/ihr zufällig zugeteilten Liste jedes Item in einer der vier Bedingungen mit einem der beiden Demonstrativpronomen.

\subsubsection{Filler und Kontroll-Items}

Um den Untersuchungsgegenstand so gut wie möglich zu verbergen und um zu verhindern, dass die VPn eine Antwortstrategie entwickeln, mussten weitere, vom Aufbau abweichende Items in das Experiment integriert werden. Hierfür wurden zwei zusätzliche Item-Sets erstellt: Zum einen ein Set an Kontroll-Items und zum anderen ein reines Filler-Set.

\section{Kontroll-Items}

Mit Hilfe von 12 Kontroll-Items in zwei Bedingungen sollen Aussagen zur Validität des Designs und zu den Resolutionspräferenzen der VPn getroffen werden. Dabei soll geprüft werden, ob die VPn unter bestimmten Voraussetzungen im Rahmen des Designs direkte und indirekte Anaphern bilden. Dazu wurden Sätze konstruiert, die eine Tätigkeit beschreiben, bei der ein typisches Instrument involviert ist. Dieses Instrument ist mit der Anapher im nachfolgenden Satzfragment grammatisch kongruent. Die Wahl eines Instruments als Antezedens soll bewirken, dass der Bezug nicht zu offensichtlich und damit trivial ist. Dies basiert auf der Annahme von Cornish et al. (2005), dass Instrumente als periphere Bestandteile einer Aktion über eine schlechtere Zugänglichkeit verfügen als nukleare Bestandteil oder Elemente einer Teil-Ganzes-Beziehungen.

Die Kontroll-Items liegen in zwei Bedingungen vor: In Bedingung A wird das Instrument implizit erwähnt. Ein Bezug darauf entspricht somit einer indirekten Anapher. In Bedingung B wird es explizit erwähnt und ein Bezug darauf eintspricht somit einer direkten Anapher.

In der Bedingung A, z.B. (7-a) und (8-a), wird das Instrument implizit über einen Ankerausdruck erwähnt, z.B. Aufzug über in die vierte Etage fahren. Bezieht sich eine VP mit der Anapher ihn auf den Aufzug, kann daraus abgeleitet werden, dass die VP im Rahmen des Experiments in der Lage ist, indirekte Anaphern zu bilden.

In der Bedingung B, z.B. (7-b) und (8-b)] wird das Instrument explizit erwähnt, allerdings innerhalb einer Adverbialbestimmung, d.h. es handelt sich um einen Ausdruck mit niedriger Salienz. Bezieht sich eine VP mit der Anapher ihn hier auf den Aufzug, kann daraus abgeleitet werden, dass die VP im Rahmen des Experiments in der Lage ist, direkte Anaphern zu bilden. 
(7) a. Caro ist für das Vorstellungsgespräch in die vierte Etage gefahren.

Vorher hat sie ihn ...

b. Caro ist für das Vorstellungsgespräch in die vierte Etage mit dem Aufzug gefahren. Vorher hat sie ihn ...

(8) a. Anne hat beim Essen lustlos in der Suppe herumgerührt.

Dabei hat sie ihn ...

b. Anne hat beim Essen lustlos in der Suppe mit dem Löffel herumgerührt.

Dabei hat sie ihn ...

\section{Filler}

Als Filler-Set wurden 24 Items in zwei Bedingungen aus einer unabhängigen experimentellen Studie zu Negation und Verb-Bias integriert.

\subsection{Hypothesen}

Für Experiment 2 bestehen die folgenden Hypothesen:

(i) Für den konzeptuellen Faktor RZK werden für die Faktorstufe +RZK signifikant mehr N1-Bezüge als für die Faktorstufe -RZK erwartet, da angenommen wird, dass der konzeptuelle Fokus in der Bedingung +RZK stärker auf das N1 ausgerichtet ist als in der Bedingung -RZK und dies in entsprechenden Unterschieden in der N1-Zugänglichkeit resultiert (vgl. Kap. 3.3.4).

(ii) Für den grammatischen Faktor STRUKTUR werden für Rektionskomposita (RK) mehr N1-Bezüge als für Wurzelkomposita (WK) erwartet, da angenommen wird, dass das N1 in Rektionskomposita nominalen Status hat und in Wurzelkomposita lediglich in Form einer Wurzel vorliegt, was sich wiederum auf die Referenzialität der Erstglieder auswirken und in der Zugänglichkeit widerspiegeln sollte (vgl. Kap. 4).

(iii) Eine Interaktion wird dahingehend prognostiziert, dass der Faktor RzK einen stärkeren Einfluss als der Faktor STRUKTUR hat, da sich bereits in Experiment 1 andeutete, dass konzeptuelle Einflüsse einen stärkeren Einfluss auf die N1-Zugänglichkeit haben als grammatische.

(iv) Beim Faktor PRonomenarT handelt es sich um einen Pseudofaktor. Ein signifikanter Unterschied zwischen der Anzahl an N1-Bezügen zwischen den Pronomen dieses/ $r$ und jenes $/ r$ wird nicht erwartet.

(v) Zusätzlich werden weitere mögliche Einflussfaktoren annotiert und ausgewertet: Genus, Spezifizität und Zählbarkeit des N1 sowie die Nominalisierungsart. Dabei wird prognostiziert, dass weniger N1-Bezüge vorliegen, wenn das N1 im Neutrum vorliegt, da hier mehr Resolutionsalternativen vorliegen (vgl. Kap. 5.3.2). In Bezug auf die Spezifizität des N1 wird ein Vorteil für spezifische N1 vorhergesagt (vgl. Kap. 3.5). Für die Zählbarkeit und die Nominalisierungsart erfolgt eine explorative Analyse (vgl. Kap. 3.2.2). 


\subsection{Durchführung}

\subsubsection{Ablauf}

Das Experiment setzt sich aus 27 experimentellen Items (Stimuli) zusammen, die aufgrund der Kreuzkombination der zweistufigen Faktoren RZK und STRUKTUR in vier Bedingungen vorliegen. Die Items wurden verdoppelt und einmal mit dem Pronomen dieses $/ r$ und einmal mit dem Pronomen jenes $/ r$ verwendet, sodass das Stimulus-Set insgesamt 54 Items umfasst. Die Items wurden anhand der nun vorliegenden acht Bedingungen nach dem lateinischen Quadrat auf acht Listen aufgeteilt. Die 12 Kontroll-Items und die 24 Filler wurden entsprechend vervielfältigt und ebenfalls gleichmäßig auf die acht Listen aufgeteilt.

In der Durchführung setzte sich das Experiment aus zwei Blöcken zusammen, die von einer Pause separiert wurden. Da die Randomisierung der Items technisch nicht über die Pausengrenze hinweg realisiert werden konnte, wurden die Items im Vorfeld manuell auf die beiden Blöcke aufgeteilt. Hierbei wurde darauf geachtet, dass alphabetische und semantische Gruppierungen innerhalb der Blöcke vermieden werden. Die Items mit dieser/s und jener $/ \mathrm{s}$ wurden ebenfalls gleichmäßig auf die Blöcke verteilt 9 Das allgemeine methodische Vorgehen ist angelehnt an Ellert/Holler (2011) und baut auf der Pilotstudie auf.

In einem CIP-Pool mit stationären Rechnern wurden stets etwa zehn VPn zeitgleich getestet. Die VPn wurden dabei zunächst mündlich über den Ablauf des Experiments informiert. Die Arbeitsanweisung bestand darin, Sätze zu lesen, diese zügig und intuitiv zu ergänzen und sich dabei nicht von normativen Kriterien beeinflussen zu lassen. Das eigentliche Ziel des Experiments wurde den VPn zu keiner Zeit mitgeteilt. Zu Beginn des Experiments wurde eine Zusammenfassung der Instruktionen angezeigt und die Aufgabe anhand eines verfremdeten Beispiel-Items verdeutlicht. Nach Betätigung einer ,Weiter'Schaltfläche wurden zunächst einige demografische Daten abgefragt, um die Gültigkeit der Stichprobe sicherzustellen. Anschließend konnte das Experiment durch das erneute Betätigen einer ,Weiter'-Schaltfläche gestartet werden. Im Folgenden erschienen nacheinander die verschiedenen Items des ersten Blocks einer der acht Listen in randomisierter Reihenfolge. Unter dem Satzfragment erschien jeweils ein Freitextfeld. Die Satzeingabe war durch Betätigung einer ,Weiter'-Schaltfläche oder der ,Enter'-Taste zu bestätigen. Ein Beispiel für einen solchen Eingabe-Bildschirm ist unter Abbildung 5.1 wiedergegeben. Nach Bearbeitung des ersten Blocks, d.h. nach der Hälfte der Items, erschien ein Pausen-Bildschirm. Nach einer frei wählbaren Pausenzeit startet der zweite Block durch Betätigung der ,Weiter'-Schaltfläche. Nach dem letzten Item erschien einen Verabschiedungsbildschirm. Im Durchschnitt benötigte ein VP für einen Durchgang etwa 30 Minuten.

\subsubsection{Technik}

Anders als in der Pilotstudie wurden in Experiment 2 unsystematische Fehler wie äußere Ablenkungen besser kontrolliert, da das Experiment für alle Teilnehmer unter den glei-

\footnotetext{
${ }^{9}$ Dabei enthielten die Listen $1-4$ in den ungeraden Items dieses $/ r$ und die geraden jenes $/ r$ als Pronomen; in den Listen $5-8$ verhielt es sich umgekehrt.
} 


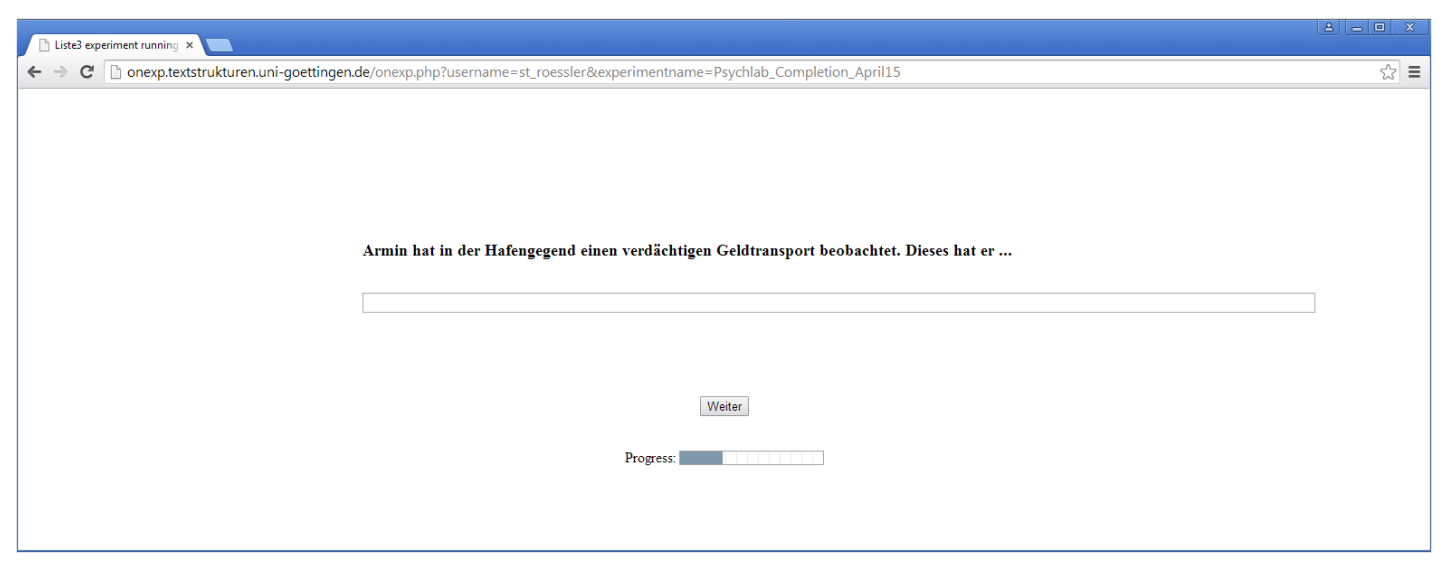

Abbildung 5.1: Screenshot aus Experiment 2 (OnExp 1.2)

chen Bedingungen an einem zentralen Ort durchgeführt wurde. Das Experiment wurde im Zeitraum vom 22.04. - 05.05.2015 im CIP-Pool der Philosophischen Fakultät der Universität Göttingen durchgeführt. Der CIP-Pool bot die Möglichkeit des zeitgleichen Nutzens mehrerer identisch konfigurierter Rechner. Als Software für die Erstellung des Experiments diente OnExp Version 1.2 (Onea 2012). Die Darstellung erfolgte unter Verwendung des Browsers ,Google Chrome'. Die Daten wurden anonymisiert unter Verwendung einer durchlaufenden VP-Nummer erhoben.

\subsubsection{Versuchspersonen}

Insgesamt wurden 60 VPn erhoben. Die Akquise der VPn erfolgte an den einzelnen Testtagen durch Flyer auf dem Göttinger Campus 10 In die Auswertung wurden insgesamt 56 VPn einbezogen. ${ }^{11}$ Dabei handelte es sich um Studierende der Universität Göttingen, die Deutsch als einzige Muttersprache angaben. 20 VPn waren weiblich und 36 männlich; der Altersdurchschnitt lag bei 22,71 Jahren (Std. Abw. = 2.67). Die Teilnahme erfolgte freiwillig und wurde vergütet.

\subsubsection{Datenaufbereitung}

Die unabhängigen Variablen des Experiments bilden die Faktoren RZK und STRUKTUR, sowie der Pseudofaktor PRONOMEnART. Im Zuge der Satzvervollständigung waren die VPn indirekt aufgefordert, das im Satzfragment enthaltene Pronomen aufzulösen. Um daraus Aussagen zur anaphorischen Zugänglichkeit des entsprechenden N1 ableiten zu können, wurden die Satzergänzungen daraufhin interpretiert und annotiert. Hierbei wurde kodiert, ob eine VP in einer Satzergänzung einen N1-Bezug herstellt (1) oder nicht (0). Inkohärente oder fehlende Satzergänzungen wurden als Interpretationsschwierigkeit durch mangelnde Zugänglichkeit eines Antezedens interpretiert und ebenfalls mit ,0` kodiert.

\footnotetext{
${ }^{10}$ Die Flyer informierten darüber, dass es sich um ein Experiment zur Untersuchung von Sprachintuition handelt, bei welchem an einem Computerbildschirm Sätze zu vervollständigen sind. Darüber hinaus war eine geschätzte Dauer von 30 Minuten und die Vergütung angegeben. Die Versuchspersonen konnten ohne Voranmeldung zu angegebenen Uhrzeiten im CIP-Pool erscheinen.

${ }^{11}$ Drei VPn mussten aufgrund von linguistischem Hintergrundwissen und eine aufgrund der Teilnahme an Vorstudien zu den Items des Filler-Sets von der Auswertung ausgeschlossen werden.
} 
Die Kodierung wurde durch zwei Annotatoren und einen Schlichter angefertigt. Bei der abhängigen Variablen handelt es sich um die relative Häufigkeit der N1-Bezüge in der jeweiligen Bedingung.

\subsection{Auswertung}

Alle statistischen Berechnungen und Plots wurden mit R 3.2 (R Core Team 2015) unter Windows 7 vorgenommen. Für die Plots wurde das R-Paket „ggplot2“ (Wickham 2009), für die Linear Mixed-Effects Models das Paket „lme4“ (Bates et al. 2015) verwendet.

\subsubsection{Deskriptive Statistik}

Die deskriptive Auswertung der Hauptfaktoren RZK und STRUKTUR erfolgt auf Basis der mittleren Anzahl an N1-Bezügen in den jeweiligen Bedingungen.

Für den Faktor RZK zeichnet sich deskriptiv ein höherer Mittelwert für die Faktorstufe +RZK im Gegensatz zu -RZK ab, vgl. Tabelle 5.2 .

\begin{tabular}{ccccc}
\hline \hline Faktor & Bed. & MW & Std. Abw. & n \\
\hline \multirow{2}{*}{ RZK } & + RZK & 0.18 & 0.38 & 749 \\
& -RZK & 0.10 & 0.30 & 763 \\
\hline \hline
\end{tabular}

Tabelle 5.2: Deskriptive Statistik der relativen Häufigkeit der N1-Bezüge für den Faktor RZK

Für den Faktor STRUKTUR zeichnet sich deskriptiv ein höherer Mittelwert für die Faktorstufe WK im Gegensatz zu RK ab, vgl. Tabelle 5.3 .

\begin{tabular}{ccccc}
\hline \hline Faktor & Bed. & MW & Std. Abw. & n \\
\hline \multirow{2}{*}{ STRUKTUR } & RK & 0.12 & 0.33 & 756 \\
& WK & 0.15 & 0.36 & 756 \\
\hline \hline
\end{tabular}

Tabelle 5.3: Deskriptive Statistik der relativen Häufigkeit der N1-Bezüge für den Faktor STRUKTUR

Bei der gemeinsamen Betrachtung beider Faktoren deutet sich an, dass die Struktur innerhalb der Faktorstufe +RZK einen größeren Einfluss auf die N1-Zugänglichkeit hat als innerhalb der Faktorstufe -RZK, vgl. Tabelle 5.4 und Abbildung 5.2 .

\begin{tabular}{ccccc}
\hline \hline RZK & STRUKTUR & MW & Std. Abw. & $\mathrm{n}$ \\
\hline \multirow{2}{*}{+ RZK } & RK & 0.15 & 0.35 & 371 \\
& WK & 0.21 & 0.41 & 378 \\
\hline \multirow{2}{*}{ RZK } & RK & 0.10 & 0.30 & 385 \\
& WK & 0.10 & 0.30 & 378 \\
\hline \hline
\end{tabular}

Tabelle 5.4: Deskriptive Statistik der relativen Häufigkeit der N1-Bezüge für die Faktoren RZK und STRUKTUR 


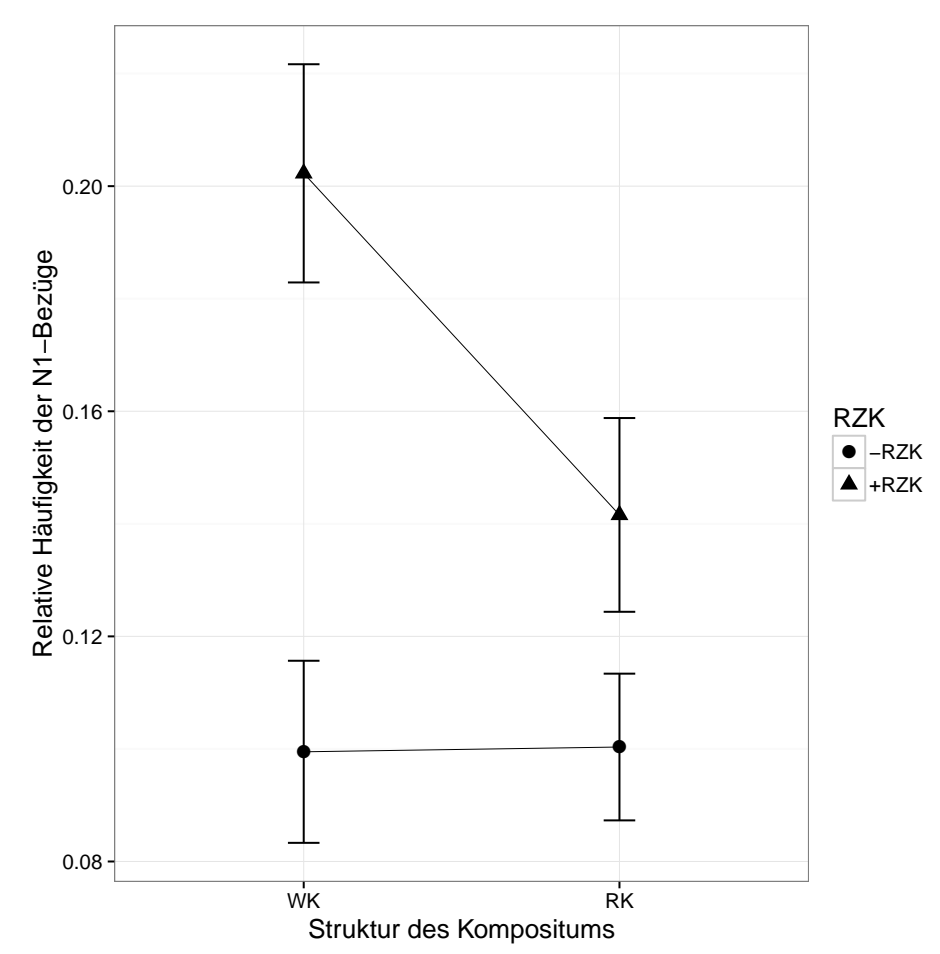

Abbildung 5.2: Plot der um die Zwischensubjektvarianz korrigierten Mittelwerte der N1Bezüge (y-Achse) in Abhängigkeit von den Faktoren RZK und STRUKTUR; die Fehlerbalken repräsentieren +/- 1 Standardfehler des Mittelwerts

Das Ergebnis der Analyse der Pronomenart ist Tabelle $5.5 \mathrm{zu}$ entnehmen. Es zeigt sich, dass die Zahl der N1-Bezüge im Fall von dieses/r etwas höher ist als im Fall von jenes/r.

\begin{tabular}{ccccc}
\hline \hline Faktor & Bed. & MW & Std. Abw. & $\mathrm{n}$ \\
\hline \multirow{2}{*}{ PRONOMEN } & dieses $/ \mathrm{r}$ & 0.15 & 0.35 & 756 \\
& jenes $/ \mathrm{r}$ & 0.13 & 0.34 & 756 \\
\hline \hline
\end{tabular}

Tabelle 5.5: Deskriptive Statistik der relativen Häufigkeit der N1-Bezüge für den Faktor PRONOMENART

In Tabelle 5.6 und Abbildung 5.3 sind die Werte für alle drei Faktoren in Abhängigkeit voneinander dargestellt. 


\begin{tabular}{cccccc}
\hline \hline PRONOMEN & RZK & STRUKTUR & MW & Std. Abw. & $\mathrm{n}$ \\
\hline \multirow{3}{*}{ dieses $/ \mathrm{r}$} & \multirow{2}{*}{+ RZK } & RK & 0.16 & 0.37 & 182 \\
\cline { 2 - 6 } & \multirow{2}{*}{-RZK } & WK & 0.23 & 0.42 & 189 \\
\hline \multirow{3}{*}{ jenes/r } & \multirow{2}{*}{+ RK } & 0.10 & 0.30 & 196 \\
& \multirow{2}{*}{+ RK } & RK & 0.09 & 0.29 & 189 \\
\cline { 2 - 6 } & \multirow{2}{*}{- RZK } & WK & 0.13 & 0.33 & 189 \\
& & RK & 0.11 & 0.39 & 189 \\
\hline \hline
\end{tabular}

Tabelle 5.6: Deskriptive Statistik der relativen Häufigkeit der N1-Bezüge für die Faktoren RZK, STRUKTUR und PRONOMENART

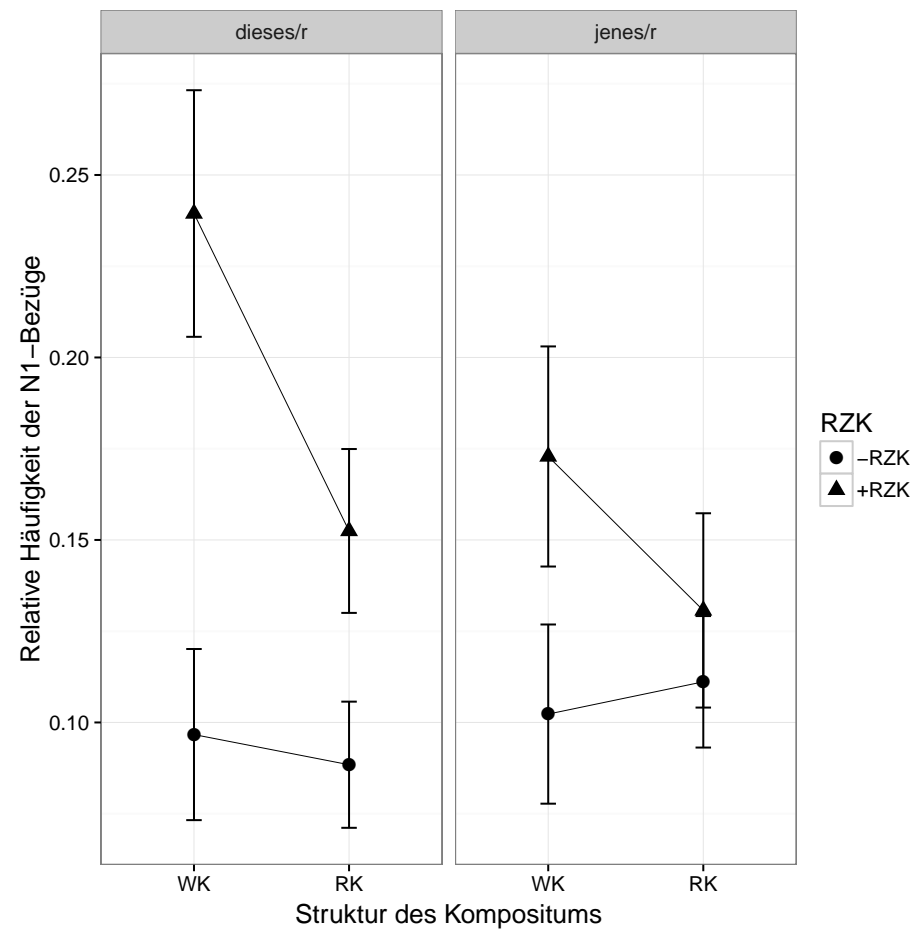

Abbildung 5.3: Plot der um die Zwischensubjektvarianz korrigierten Mittelwerte der N1Bezüge (y-Achse) in Abhängigkeit von den Faktoren RZK, STRUKTUR und PrONOMENART

\subsubsection{Inferenzstatistik}

Die Inferenzstatistik wurde mit Hilfe von Linear Mixed-Effects Models (LMEMs) mit logistischer Regression durchgeführt (vgl. Barr et al. 2013). Als ,random factors ${ }^{6}$ wurden Items und VPn in das Modell aufgenommen (within items/within subjects design), die ,fixed factors' bilden die Faktoren RZK und strukTur. Da das komplette Modell nicht konvergierte, wurden die Items mit $\mathrm{MW}=0$ aus dem Modell entfernt (Items 7, 11 und 12), sodass die Auswertung auf der Grundlage von nur 24 Items erfolgt.

Der Faktor RZK zeitigt darin einen signifikanten Haupteffekt (vgl. Tab. 5.7). 12

${ }^{12}$ Modellgleichung in $\mathrm{R}$ :

Generalized linear mixed model fit by maximum likelihood (Laplace Approximation) 


\begin{tabular}{c|cc}
\hline \hline Faktor & $|z|$ & $p$ \\
\hline RZK & 2.777 & $<0.01$ \\
\hline \hline
\end{tabular}

Tabelle 5.7: Inferenzstatistik (LMEM) für die N1-Bezüge für den Faktor RzK mit 24 Items, $56 \mathrm{VPn}$ und Item und VP als, random factors"

Für den Faktor STRUKTUR lässt sich ebenfalls ein signifikanter Haupteffekt verzeichnen (vgl. Tab. 5.8).13

\begin{tabular}{c|cc}
\hline \hline Faktor & $|z|$ & $p$ \\
\hline STRUKTUR & 2.136 & $<0.05$ \\
\hline \hline
\end{tabular}

Tabelle 5.8: Inferenzstatistik (LMEM) für die N1-Bezüge für den Faktor STRUKTUR mit 24 Items, 56 VPn und Item und VP als ,random factors"

Die Interaktion der beiden Faktoren stellt sich als marginal signifikant heraus (vgl. Tab. 5.9) und wird im Folgenden mit Hilfe von Modellvergleichen aufgelöst. Dazu wurden die Daten der VPn mit MW = 0 (VP 6 und 16) sowie die ,random slopes ${ }^{6}$ aus dem Modell entfernt, da das komplette Modell andernfalls nicht konvergiert.14

\begin{tabular}{c|cc}
\hline \hline Faktor & $|z|$ & $p$ \\
\hline RZK & 4.785 & $<0.001$ \\
STRUKTUR & 2.147 & $<0.05$ \\
RZK $\times$ STRUKTUR & 1.718 & 0.0859 \\
\hline \hline
\end{tabular}

Tabelle 5.9: Inferenzstatistik (LMEM) für die N1-Bezüge für die Faktoren RzK und STRUKTUR mit 24 Items und 54 VPn, ohne, random slopes'

Der Faktor RZK zeitigt innerhalb der Faktorstufe RK unter Berücksichtigung des auf 0.025 gesenkten $\alpha$-Levels (Bonferroni-Korrektur) einen marginal signifikanten Effekt ohne Berücksichtigung der ,random slopes‘. Für die Faktorstufe WK konvergiert das Modell nicht

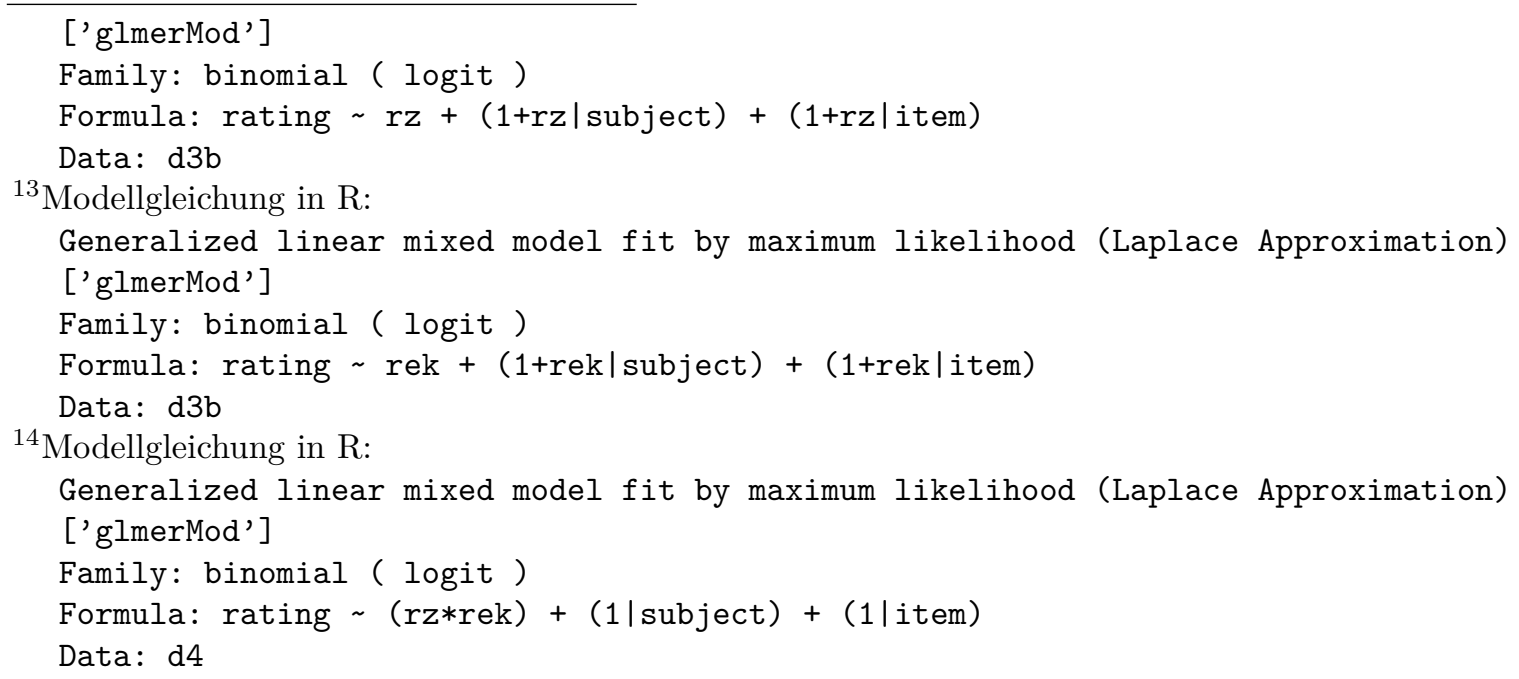


(vgl. Tab. 5.10) 15

\begin{tabular}{cc|cc}
\hline \hline Faktor & STRUKTUR & $L R \chi_{d f=1}^{2}$ & $p$ \\
\hline \multirow{2}{*}{ RZK } & RK & 4.8315 & 0.028 \\
& WK & - & - \\
\hline \hline
\end{tabular}

Tabelle 5.10: Inferenzstatistik (Modellvergleich) für den Faktor RZK in Abhängigkeit des Faktors STRUKTUR mit 24 Items und 54 VPn ohne ,random slopes ${ }^{6}$

Für den Faktor STRUKTUR innerhalb der Faktorstufe +RZK konvergiert das Modell ebenfalls nicht. Innerhalb der Faktorstufe -RZK zeitigt der Faktor sTRUKTUR keinen Effekt (vgl. Tab. 5.11) ${ }^{16}$

\begin{tabular}{cc|cc}
\hline \hline Faktor & RZK & $L R \chi_{d f=1}^{2}$ & $p$ \\
\hline \multirow{2}{*}{ STRUKTUR } & + RZK & - & - \\
& - RZK & 0.0334 & 0.8549 \\
\hline \hline
\end{tabular}

Tabelle 5.11: Inferenzstatistik (Modellvergleich) für den Faktor sTRUKTUR in Abhängigkeit des Faktors RZK mit 24 Items, 54 VPn und Item und VP als ,random factors ${ }^{6}$

Demnach kann in Bezug auf die Interaktion lediglich ausgesagt werden, dass der Faktor RZK Einfluss auf die N1-Bezüge im Fall von Rektionskomposita hat und die STRUKTUR keinen Einfluss auf Komposita ohne RZK hat.

Abschließend wurde auch der Einfluss des (Pseudo-)Faktors PRONOMENART untersucht. Für diesen zeichnet sich kein signifikanter Einfluss ab (vgl. Tab. 5.12). ${ }^{17}$ Aufgrund fehlender Hypothesen wurden keine Berechnungen zu Interaktionen der Pronomenart mit den Hauptfaktoren durchgeführt.

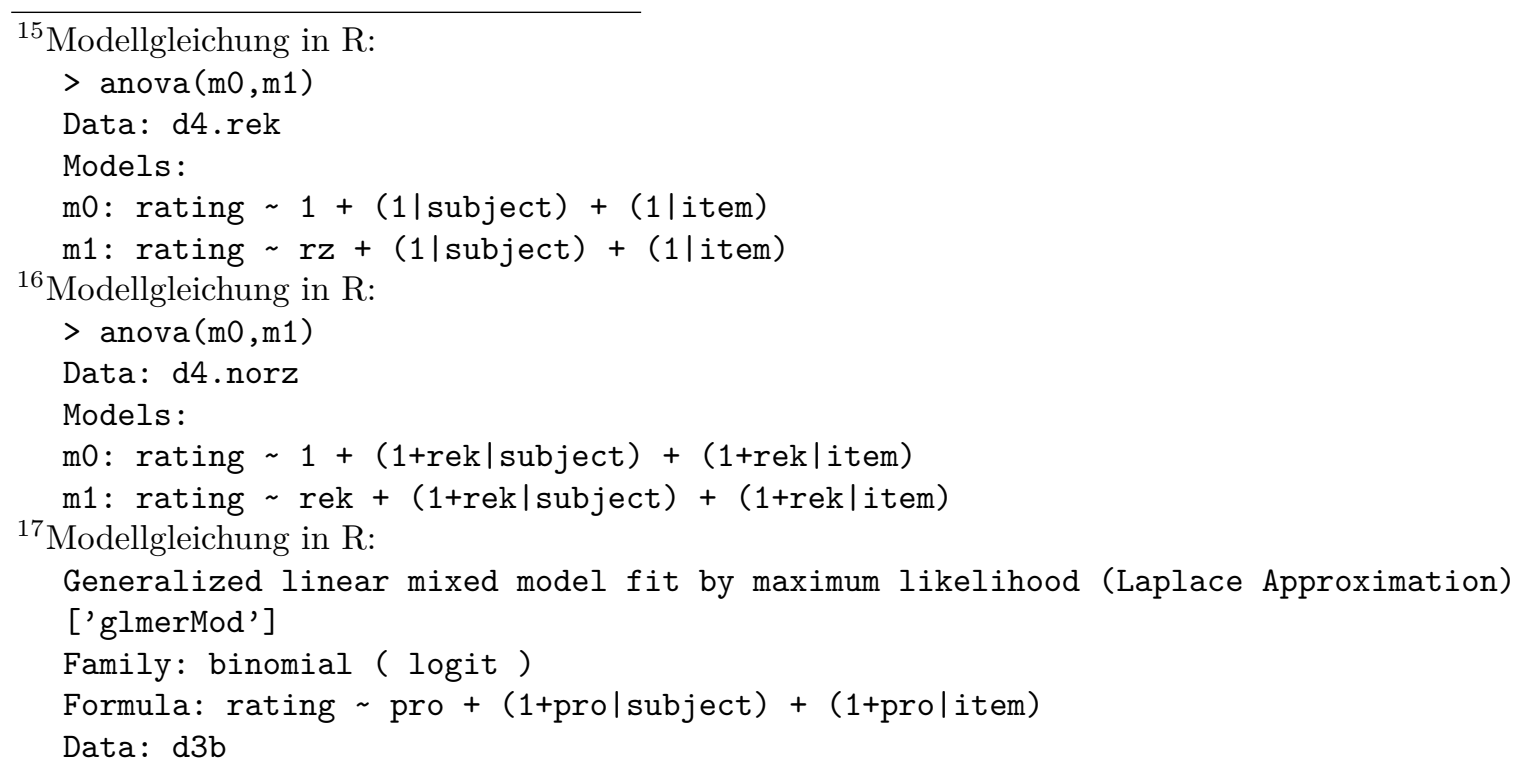




\begin{tabular}{c|cc}
\hline \hline Faktor & $|z|$ & $p$ \\
\hline PRONOMEN & 0.797 & 0.426 \\
\hline \hline
\end{tabular}

Tabelle 5.12: Inferenzstatistik (LMEM) für die N1-Bezüge für den Faktor PRONOMENART mit 24 Items, 56 VPn und Item und VP als ,random factors ${ }^{6}$

\subsubsection{Interpretation}

Die Ergebnisse lassen sich in Bezug auf die unter Kapitel 5.4 formulierten Hypothesen wie folgt interpretieren:

(i) Für den Faktor RZK wurde ein signifikanter Haupteffekt gefunden $(p<0.01)$, der anhand der deskriptiven Daten dergestalt zu interpretieren ist, dass in der Faktorstufe +RZK eine signifikant höhere Zahl an N1-Bezügen vorliegt als in der Faktorstufe -RZK. Dies ist konform mit der Hypothese zum Einfluss der RZK, d.h. die RZK der Konstiutenten eines Kompositums beeinflusst die Zugänglichkeit des N1.

(ii) Für den Faktor STRUKTUR wurde ebenfalls ein signifikanter Haupteffekt gefunden $(p<0.05)$. Dieser ist anhand der deskriptiven Daten dergestalt zu interpretieren, dass im Fall von Rektionskomposita die Zahl der N1-Bezüge geringer ist als im Fall von Wurzelkomposita. Das bedeutet, der kategoriale Status scheint nicht wie prognostiziert die Zugänglichkeit des N1 zu beeinflussen oder die Annahmen aus Kapitel 4 sind nicht zutreffend. Eine nähere Betrachtung des Ergebnisses ist Gegenstand der nachfolgenden Detailanalysen.

(iii) Es deutet sich eine Interaktion der Faktoren RZK und STRUKTUR an $(p<0.1)$. Allerdings lässt sich anhand der statistischen Berechnungen lediglich aussagen, dass der Faktor STRUKTUR keinen Einfluss auf das Subset der Faktorstufe -RZK hat, der Faktor RZK sich jedoch auf das Subset der Rektionskomposita auswirkt. Darüber hinaus zeigt sich entgegen der Hypothese in den deskriptiven Daten, dass innerhalb der Faktorstufe +RZK die Wurzelkomposita über einen höheren Mittelwert $(\mathrm{MW}=0.21)$ verfügen als die Rektionskomposita (MW = 0.15). Für diesen Unterschied konvergiert das Modell jedoch nicht, weshalb eine inferenzstatistische Validierung des Zusammenhangs nicht möglich ist. Da der Einfluss des Faktors STRUKTUR innerhalb der Faktorstufe -RZK nicht signifikant ist, entsteht der Eindruck, dass der Effekt der Kompositums-Struktur von den Komposita der Faktorstufe +RZK getragen wird. Wie oben bereits erwähnt, gilt es an dieser Stelle detailliertere Analysen vorzunehmen.

(iv) Für den (Pseudo-)Faktor PRONOMEnART wurde wie erwartet kein Effekt gefunden. Deskriptiv ist eine höhere Zahl an N1-Bezügen im Fall des frequenteren Demonstrativpronomens dieses/r im Gegensatz zu jenes/r zu verzeichnen.

(v) Die Analyse der weiteren möglichen Einflussfaktoren wird im Folgenden vorgestellt.

\subsection{Weitere Analysen}

Neben den Faktoren RZK und STRUKTUR wurden im Vorfeld weitere mögliche Einflussgrößen annotiert. Auf diese soll nun ein Blick geworfen werden. Darüber hinaus soll das Ergebnis für den Faktor STRUKTUR näher analysiert werden sowie die Validität der Stichprobe auf Item- und VP-Ebene überprüft werden. 


\subsubsection{Post-hoc-Analyse des Struktur-Effekts}

Entgegen Hypothese (ii) zeitigt der Faktor STRUKTUR in der Inferenzstatistik einen signifikanten Effekt für eine bessere N1-Zugänglichkeit bei Wurzelkomposita.

Bei kritischer Betrachtung des Materials fällt auf, dass v.a. in der Bedingung +RZK/ WK (der Bedingung mit dem höchsten Mittelwert (vgl. Tab. 5.4)) einige Schnittmengenbildungen vorliegen. Wie in Kapitel 2.2.2 erläutert, verstehe ich unter Schnittmengenbildungen Komposita, bei denen das NN sowohl die Merkmale des N1 als auch des N2 trägt, z.B. Heuhaufen. In Kapitel 3.3.2 wurde für Schnittmengenbildungen aufgrund der Verschiebung des konzeptuellen Fokus' in Richtung des N1 und aufgrund des geringen Bedeutungsbeitrags des N2 eine hohe N1-Zugänglichkeit angenommen. Da einige Schnittmengenbildungen in den Items auftreten, z.B. Gaswolke, und dabei ungleichmäßig im Material verteilt sind, ist hier ein Einfluss auf das Gesamtergebnis denkbar. Aus diesem Grund wurde SCHNiTTMENGENBILDUNG als Faktor nachträglich annotiert und ausgewertet.

In der deskriptiven Statistik zeigt sich ein deutlicher Vorteil für Schnittmengenbildungen bei großen Unterschieden in den Fallzahlen (vgl. Tab. 5.13). Die Inferenzstatistik weist diesen Vorteil als signifikant aus (vgl. Tab. 5.14).

\begin{tabular}{ccccc}
\hline \hline Faktor & Bed. & MW & Std. Abw. & n \\
\hline \multirow{2}{*}{ SCHNITTMENGENBILDUNG } & ja & 0.24 & 0.43 & 294 \\
& nein & 0.11 & 0.32 & 1218 \\
\hline \hline
\end{tabular}

Tabelle 5.13: Deskriptive Statistik der relativen Häufigkeit der N1-Bezüge für den Faktor SCHNITTMENGENBILDUNG

\begin{tabular}{c|cc}
\hline \hline Faktor & $|z|$ & $p$ \\
\hline SCHNITTMENGENBILDUNG & 4.324 & $<0.001$ \\
\hline \hline
\end{tabular}

Tabelle 5.14: Inferenzstatistik (LMEM) für die N1-Bezüge für den Faktor sCHNITTMENGENBILDUNG mit 27 Items, $56 \mathrm{VPn}$ und Item und VP als ,random factors"

Dieses Ergebnis spricht für einen Effekt von Schnittmengenbildung auf die N1-Zugänglichkeit. Basierend auf dieser Beobachtung soll nun ihre die Verteilung im und ihr Einfluss auf das Material betrachtet werden.

\begin{tabular}{cccccc}
\hline \hline SCHNITTMENGENBILDUNG & RZK & STRUKTUR & MW & Std. Abw. & $\mathrm{n}$ \\
\hline \multirow{3}{*}{ ja } & \multirow{2}{*}{+ RZK } & RK & 0.46 & 0.51 & 28 \\
& & WK & 0.22 & 0.42 & 266 \\
\cline { 2 - 6 } & \multirow{2}{*}{- RZK } & RK & - & - & - \\
& & WK & - & - & - \\
\hline \multirow{3}{*}{ nein } & \multirow{2}{*}{+ RZK } & RK & 0.12 & 0.32 & 343 \\
& & WK & 0.17 & 0.38 & 112 \\
\cline { 2 - 6 } & \multirow{2}{*}{- RZK } & RK & 0.10 & 0.30 & 385 \\
& & WK & 0.10 & 0.30 & 378 \\
\hline \hline
\end{tabular}

Tabelle 5.15: Deskriptive Statistik der relativen Häufigkeit der N1-Bezüge für die Faktoren RZK, STRUKTUR und SCHNITTMENGENBILDUNG 
Wie Tabelle 5.15 zu entnehmen, treten Schnittmengenbildungen in Bezug auf den Faktor RZK nur in der Faktorstufe +RZK auf. Dies ist plausibel, da es sich bei RZK und Schnittmengenbildung um verwandte Konzepte handelt, die sich teilweise auch gegenseitig bedingen. Darüber hinaus fällt auf, dass im Fall von Wurzelkomposita zahlenmäßig wesentlich mehr Schnittmengenbildungen vorliegen (z.B. Fleischsorte, Haarstrang, Papiertüte, Geldmünze) als im Fall von Rektionskomposita (z.B. Filmbearbeitung, Theatervorführung). Auch dies ist plausibel, da das N1 in Schnittmengenbildungen oft das Material des NN beschreibt und das N2 in Rektionskomposita z.T. nicht gegenständlich ist, also über keine Materialeigenschaften verfügt. Die hohe Zahl an Schnittmengenbildungen und der starke Einfluss dieser liefern einen Erklärungsansatz für den relativ hohen Mittelwert der Bedingung +RZK/WK. Innerhalb der Gruppe der Schnittmengenbildungen weisen die Rektionskomposita einen höheren Mittelwert als die Wurzelkomposita auf $(0.46>0.22)$. In den Fällen ohne Schnittmengenbildungen ist dies zwar nicht der Fall, allerdings ist hier die Differenz der Mittelwerte deutlich geringer $(0.12<0.17)$. Die Fallzahlen sind insgesamt so unausgeglichen, dass keine inferenzstatistischen Berechnungen durchgeführt werden konnten.

In Bezug auf den Einfluss der Schnittmengenbildungen lässt sich zusammenfassen, dass das Material in Hinblick auf diesen Faktor unausgeglichen ist und dieser das Gesamtergebnis wahrscheinlich konfundiert. Dies wird als Erklärungsansatz für das Ergebnis des Faktors STRUKTUR betrachtet. Das wiederum bedeutet, dass der tatsächliche Einfluss der morphosyntaktischen Struktur des Kompositum auf dessen N1-Zugänglichkeit ungeklärt bleibt, da er nicht sauber durch das experimentelle Material repräsentiert wird. Unter Einbeziehung der Ergebnisse des Faktors SCHnitTmengenbiLdung zeigt sich, dass Wurzelkomposita entgegen des Ergebnisses der Einzelauswertung des Faktors STRUKTUR nicht per se über eine bessere N1-Zugänglichkeit verfügen (siehe Tab. 5.3). Der hohe Mittelwert für Wurzelkomposita ergibt sich vielmehr in Interaktion mit konzeptuellen Faktoren wie RZK und schnitTmengenbildung. Für belastbare Aussagen zum Struktureinfluss ist anderes Material und ein anderes experimentelles Design notwendig.

Darüber hinaus kann diese Post-hoc-Auswertung als erstes Indiz dafür gewertet werden, dass konzeptuelle Faktoren (hier: SCHNITTMENGENBILDUNG) einen stärkeren Einfluss haben als grammatische (hier: STRUKTUR).

Die Analyse der Schnittmengenbildungen ist nur eine von mehreren möglichen Post-hocAnalyse. Für weitere Überlegungen hierzu befindet sich das komplette Item-Material in Anhang $B$.

\subsubsection{Genus}

Die N1 und somit auch die Pronomen liegen in den experimentellen Items im Maskulinum (dieser/jener) und im Neutrum (dieses/jenes) vor. Ein Vortest bestätigte bereits, dass im Fall von Pronomen im Neutrum eine starke Präferenz für die Verwendung als Expletivum, Komplexanapher o. Ä. vorliegt und somit eine alternative und vermutlich sogar präferierte Resolutionsmöglichkeit anstelle eines N1-Bezugs besteht (vgl. Kap. 5.3.2). Aus diesem Grund wurden im Satzfragment Demonstrativpronomen verwendet. Nicht-anaphorische Interpretationen sind auch damit möglich, jedoch weniger typisch als im Fall von es. Für das Maskulinum-Pronomen liegt keine vergleichbare Resolutionsalternative vor, weshalb in entsprechenden Fällen mit mehr N1-Bezügen zu rechnen ist. 
In der Tat zeichnet sich in der deskriptiven Statistik ein deutlicher Vorteil der MaskulinumPronomen ab (vgl. Tab. 5.16), der sich in der Inferenzstatistik als signifikant bestätigt (vgl. Tab. 5.17).

\begin{tabular}{lcccc}
\hline \hline Faktor & Bed. & MW & Std. Abw. & $\mathrm{n}$ \\
\hline \multirow{2}{*}{ GENUS } & mask. & 0.38 & 0.48 & 392 \\
& neutr. & 0.05 & 0.23 & 1120 \\
\hline \hline
\end{tabular}

Tabelle 5.16: Deskriptive Statistik der relativen Häufigkeit der N1-Bezüge für den Faktor N1-GENUS

\begin{tabular}{c|cc}
\hline \hline Faktor & $|z|$ & $p$ \\
\hline GENUS & 6.568 & $<0.001$ \\
\hline \hline
\end{tabular}

Tabelle 5.17: Inferenzstatistik (LMEM) für die N1-Bezüge für den Faktor N1-GENUS mit 24 Items, $56 \mathrm{VPn}$ und Item und VP als ,random factors ${ }^{6}$

Der Genus-Effekt lässt sich somit belegen, auch wenn die Fallzahlen ungleich auf die beiden Faktorstufen verteilt sind 18

Dieses Ergebnis bestätigt zum einen die klare Präferenz für die Resolutionsalternative im Fall von Neutrum-Pronomen. Zum anderen zeigt der Mittelwert von 0.05 für NeutrumPronomen auch, dass in Einzelfällen ein N1-Bezug vorgenommen wird, obwohl eine zugängliche Resolutionsalternative zur Verfügung steht. Dies stärkt die Annahme von gradueller N1-Zugänglichkeit und von der Produktivität von N1-Bezügen.

\subsubsection{Spezifizität}

In Kapitel 3.5 wurde ein Einfluss der Spezifizität eines N1 auf seine Zugänglichkeit postuliert. Die in diesem Experiment verwendeten N1 weisen diesbezüglich Unterschiede auf. Die Eigenschaft eines N1, spezifisch oder unspezifisch zu sein, liegt hierbei primär im Kompositum selbst. So denotiert Filmrecht ein Recht, welches für viele Filme im unspezifischen Sinne gilt, Filmbewertung kann sich jedoch auf einen ganz speziellen Film beziehen. Darüber hinaus kann die Eigenschaft der Spezifizität auch vom Kontext des Kompositums mitbestimmt werden. Da der Bezug auf das N1 durch ein Demonstrativpronomen erfolgt, ist die Anapher stets spezifisch. Für unspezifische N1 sollten somit deutlich weniger N1-Bezüge vorliegen, da ein Wechsel zwischen unspezifischer und spezifischer Lesart eine Reanalyse oder zumindest höhere Verarbeitungskosten verursacht. Die deskriptiven Daten bestätigen diese Annahme (vgl. Tab. 5.18).

\begin{tabular}{ccccc}
\hline \hline Faktor & Bed. & MW & Std. Abw. & $\mathrm{n}$ \\
\hline \multirow{2}{*}{ SPEZIFIZITÄT } & unspezifisch & 0.09 & 0.29 & 896 \\
& spezifisch & 0.20 & 0.40 & 616 \\
\hline \hline
\end{tabular}

Tabelle 5.18: Deskriptive Statistik der relativen Häufigkeit der N1-Bezüge für den Faktor N1-SPEZIFIZITÄT

\footnotetext{
${ }^{18} \mathrm{Da}$ das N1-Genus innerhalb der Items identisch ist, ist kein Einfluss auf das Ergebnis der Hauptfaktoren anzunehmen.
} 
Die Inferenzstatistik weist den Effekt der Spezifizität als signifikant aus (vgl. Tab. 5.19). Bei dieser Analyse ist jedoch anzumerken, dass die Annotation subjektiv ist und die spezifischen und unspezifischen N1 ungleichmäßig über die und innerhalb der Items verteilt sind. In Anbetracht dessen sind die Ergebnisse lediglich als Tendenz anzusehen.

\begin{tabular}{c|cc}
\hline \hline Faktor & $|z|$ & $p$ \\
\hline SPEZIFIZITÄT & 3.310 & $<0.001$ \\
\hline \hline
\end{tabular}

Tabelle 5.19: Inferenzstatistik (LMEM) für die N1-Bezüge für den Faktor N1SPEZIFIZITÄT mit 27 Items, $56 \mathrm{VPn}$ und Item und VP als ,random factors ${ }^{6}$

\subsubsection{Zählbarkeit}

Das experimentelle Material wurde in Bezug auf konzeptuelle Eigenschaften wie Konkretheit und Animatheit des N1 kontrolliert. Eine Eigenschaft, die nicht kontrolliert, sondern nur grob ausbalanciert werden konnte, ist die Zählbarkeit ${ }^{19}$ Wie in Kapitel 3.2.2 diskutiert, handelt es sich dabei um eine grammatisch und konzeptuell relevante Eigenschaft. Grammatisch ist mit besserer N1-Zugänglichkeit bei Massenomen an der N1-Position zu rechnen, da diese auch ohne Determinierer uneingeschränkt referenziell sind. Konzeptuell ist mit einem Vorteil für die Zugänglichkeit von Individualnomen an der N1-Position zu rechnen, da diese durch ihre raum-zeitliche Abgrenzung einen Vorteil im Sinne von Unikalität und Visualisierbarkeit mit sich bringen.

Im Zuge der inferenzstatistischen Auswertung der Hauptfaktoren fiel bereits auf, dass einige Items den Mittelwert 0 aufweisen, d.h. das entsprechende N1 von allen VPn als unzugänglich empfunden wurde. Bei diesen N1 handelt es sich um die Massenomen Wasser, Metall und Gas. In der deskriptiven Auswertung des Faktors N1-ZÄHLBARKEIT bestätigt sich der Eindruck, dass für Massenomen an der N1-Position weniger Bezugnahmen bestehen als im Fall von Individualnomen (vgl. Tab. 5.20). In diesem Sinne deutet sich für den Faktor N1-ZÄHLBARKEIT ein stärkerer Einfluss der konzeptuellen als der grammatischen Eigenschaften an. Inferenzstatistisch lässt sich dieser Einfluss jedoch nicht belegen (vgl. Tab. 5.21). Gleichzeitig handelt es sich auch nicht um einen sauber kontrollierten Faktor, d.h. auch hier sind die Ergebnisse lediglich als Tendenz zu werten.

\begin{tabular}{ccccc}
\hline \hline Faktor & Bed. & MW & Std. Abw. & $\mathrm{n}$ \\
\hline \multirow{2}{*}{ N1-ZÄHLBARKEIT } & Massenom. & 0.13 & 0.34 & 840 \\
& Individualnom. & 0.15 & 0.35 & 672 \\
\hline \hline
\end{tabular}

Tabelle 5.20: Deskriptive Statistik der relativen Häufigkeit der N1-Bezüge für den Faktor N1-ZÄHLBARKEIT

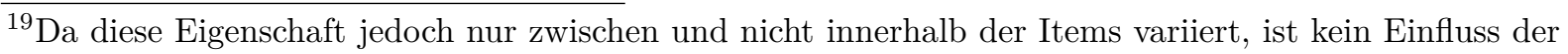
Zählbarkeit auf das Ergebnis der Hauptfaktoren anzunehmen.
} 


\begin{tabular}{c|cc}
\hline \hline Faktor & $|z|$ & $p$ \\
\hline N1-ZÄHLBARKEIT & 0.064 & 0.949 \\
\hline \hline
\end{tabular}

Tabelle 5.21: Inferenzstatistik (LMEM) für die N1-Bezüge für den Faktor N1ZÄHLBARKEIT mit 24 Items und 56 VPn ohne, random slopes

\subsubsection{Nominalisierungsart}

Um über genügend Rektionskomposita zu verfügen, die den Item-Kriterien entsprechen, wurden verschiedene Nominalisierungsarten einbezogen. Diese bringen z.T. unterschiedliche morphosyntaktische und semantische Merkmale mit sich, weshalb ein Einfluss der Nominalisierungsart auf das Gesamtergebnis nicht mit Sicherheit ausgeschlossen werden kann. Ein Überblick über die verschiedenen Nominalisierungsarten und ihren Einfluss auf die Bezugszahlen ist Tabelle $5.22 \mathrm{zu}$ entnehmen.

\begin{tabular}{llccc}
\hline \hline Nominalisierungsart & Bsp. & MW & Std. Abw. & $\mathrm{n}$ \\
\hline -ung & Filmbewertung & 0.20 & 0.40 & 406 \\
Konversion & Geldtransport & 0.04 & 0.19 & 168 \\
-er & Wasserkocher & 0.03 & 0.16 & 112 \\
Ablaut-Konversion & Fleischgenuss & 0.02 & 0.15 & 42 \\
-erei & Metallgießerei & 0 & 0 & 14 \\
-tor & Videoprojektor & 0 & 0 & 14 \\
\hline \hline
\end{tabular}

Tabelle 5.22: Deskriptive Statistik der relativen Häufigkeiten der N1-Bezüge für den Faktor NOMINALISIERUNGSART für Rektionskomposita

Bei der Betrachtung der deskriptiven Werte fällt auf, dass Nominalisierungen auf -ung die zahlenmäßig größte Gruppe bilden. Auch der höchste Mittelwert fällt dieser Gruppe zu. Eine mögliche Erklärung hierfür ist, dass ung-Komposita eine Prozesslesart zur Verfügung stellen, was entsprechend der Hypothese zum Einfluss abstrakter N2 die N1Zugänglichkeit fördert (siehe auch Kap. 3.3.5). In diesem Fall müssten allerdings auch andere Rektionskomposita mit abstraktem N2, wie z.B. Fleischgenuss, über eine höhere N1-Zugänglichkeit verfügen. Wie Tabelle 5.22 zu entnehmen, ist dies nicht der Fall. Allein auf den Faktor KONKRETHEIT lässt sich das Ergebnis somit nicht zurückführen. Insgesamt ist diese Analyse primär explorativ. Aufgrund der unregelmäßigen Fallzahlen lassen sich keine inferenzstatistischen Berechnungen ausführen.

\subsubsection{Validierung des Designs}

\section{Bewertung des Faktors RZK}

Einzelne Auswertungsschritte ergaben, dass das Material trotz vielfältiger Kontrollen bei der Erstellung einige Störfaktoren enthält, so z.B. die Schnittmengenbildungen. Bei der Betrachtung der Ergebnisse auf Item-Ebene zeigt sich jedoch, dass das Gesamtergebnis einen guten Schnitt durch die Items darstellt und nicht durch einzelne Items getragen wird (siehe Anhang Abb. B.3. 
Ein Ziel des Experiments ist die Abbildung des Einflusses der Interaktion der Konstituenten-Konzepte und des konzeptuellen Fokus auf die N1-Zugänglichkeit. Dies wurde versucht am Beispiel des Faktors RZK greifbar zu machen und soll an dieser Stelle diskutiert werden. Anlass für die Entscheidung für diesen Faktor war, dass sich die in der Literatur verbreiteten semantischen Relationen in Experiment 1 (vgl. Kap. 3.4) als ungeeignet für die experimentelle Überprüfung erwiesen. Bei der Analyse der Items zeigte sich, dass die Relationsklassen nach Levi (1978) zwar theoretisch plausibel sind, für die konkrete Einteilung von Komposita jedoch zu vage Kriterien liefern. Dies zeigte sich erneut bei der Materialerstellung für Experiment 2. Die Einteilung der Komposita nach +/-RZK ist hingegen relativ trennscharf. Aus heuristischem Interesse wurden die Komposita auch nach den Levi-Klassen (erweitert um die Whole-Part-Beziehung) annotiert. Dabei erwiesen sich viele Fälle als uneindeutig. Eine Aufteilung der Relationen auf +RZK und -RZK war ebenfalls nicht möglich, allerdings sind Tendenzen zu erkennen. Eine stärkere Tendenz zu +RZK ist bei Komposita mit den Relationen HAVE, BE, FROM und Whole-Part zu verzeichnen. Dagegen sind Komposita mit den Relationen FOR und MAKE vorrangig -RZK. Die USE-Relation ist in etwa gleichmäßig auf beide Gruppen verteilt. Bei ähnlichem theoretischen Hintergrund erscheint die RZK damit insgesamt als der experimentell besser operationalisierbare Faktor.

Bei der Betrachtung der Mittelwerte der einzelnen Relationen zeigt sich, dass die Relationen, die sich relativ klar -RZK oder +RZK zuordnen lassen, auch entsprechend klare Ergebnisse haben. So verfügen Whole-Part und FROM als typische Vertreter für + RZK jeweils über einen relativ hohen Mittelwert. FOR und MAKE als typische Vertreter für -RZK haben hingegen niedrigere Mittelwerte (vgl. Tab. 5.23) ${ }^{20}$ Die explorative Auswertung der Relationen unterstützt somit in ihren Tendenzen die Ergebnisse des Faktors RZK.

\begin{tabular}{cccc}
\hline \hline semant. Relat. & MW & Std. Abw. & $\mathrm{n}$ \\
\hline Whole-Part & 0.29 & 0.46 & 56 \\
FROM & 0.24 & 0.43 & 210 \\
ABOUT & 0.24 & 0.43 & 98 \\
BE & 0.21 & 0.41 & 112 \\
FOR & 0.11 & 0.31 & 756 \\
HAVE & 0.11 & 0.31 & 28 \\
USE & 0.05 & 0.21 & 126 \\
MAKE & 0.04 & 0.2 & 70 \\
IN & 0.02 & 0.13 & 56 \\
\hline \hline
\end{tabular}

Tabelle 5.23: Deskriptive Statistik der relativen Häufigkeit der N1-Bezüge für die verschiedenen semantischen Relationen

\section{Analyse von VPn und Kontroll-Items}

Neben der Auswertung der Varianz zwischen den Items zur Hypothesenüberprüfung soll auch einen Blick auf die Varianz zwischen den VPn geworfen werden. Hierbei entsteht nicht der Eindruck, dass das Gesamtergebnis von einzelnen VPn getragen wird, sondern das Mittel repräsentiert (siehe Anhang Abb. B.3).

${ }^{20}$ Detailliertere Analysen wurden nicht durchgeführt, da die Einteilung subjektiv ist und die Fallzahlen der Relationen stark voneinander abweichen. 
Dass dennoch Unterschiede im Verhalten der VPn zu finden sind, lässt vermuten, dass unterschiedliche Präferenzen bei der Pronomenresolution vorliegen. Dafür spricht, dass z.B. zwei VPn (6 und 16) gar keinen N1-Bezug herstellen, andere dies dafür in allen Bedingungen tun (z.B. 17 und 37).

Zur Überprüfung des generellen Umgangs der VPn mit verschiedenen Anapherarten wurden 12 Kontroll-Items in das Experiment integriert (vgl. Kap. 5.3.3). Die Items lagen in zwei Bedingungen vor: Bedingung A enthält eine indirekte Anapher, bei der sich das Pronomen im Satzfragment auf ein implizites Instrument aus dem Vorsatz bezieht. In Bedingung B ist das entsprechende Instrument explizit erwähnt, wodurch die Etablierung einer direkten Anapher möglich ist.

Alle VPn bezeugten in ihren Satzergänzungen die Fähigkeit zur Anaphernresolution und der Bildung kohärenter Satzergänzungen. Dies spricht zum einen für ein zielführendes experimentelles Design und zum anderen musste keine VP aufgrund dessen von der Auswertung ausgeschlossen werden.

Bei genauerer Betrachtung der Satzergänzungen für Bedingung A zeigt sich, dass sich 26 der 56 VPn auf das implizite Antezedens beziehen, z.B. (9-a). Alle VPn waren zudem in der Lage, kohärente Resolutionsalternativen zu bilden, z.B. eine weitere Person einzuführen, z.B. (9-b), Bei der Analyse von Bedingung B zeigt sich, dass die VPn in den meisten Fällen das morphosyntaktisch kongruente Antezedens identifizieren konnten und sich mit der Anapher darauf bezogen, z.B. (9-c). In einigen Fällen wurde jedoch trotz der Möglichkeit der Bildung einer direkten Anapher ein indirektes Antezedens genutzt, was die Produktivität dieser Art der Anapher unterstreicht, z.B. (9-d). Dies kann zusätzlich auch als Indiz für die Präferenz animater Antezedenten gewertet werden. Nur 6 VPn bildeten weniger als 4 direkte Anaphern. ${ }^{21}$ Dabei ist anzumerken, dass 3 der 12 Instrumente im Neutrum stehen, d.h. als Anapher ein es im Satzfragment auftritt und somit eine starke Präferenz für die Verwendung als Komplexanapher o.Ä. besteht. Die Intensität dieser Präferenz zeigt sich darin, dass diese Art der Interpretation z.T. sogar der Bildung einer direkten Anapher vorgezogen wird, z.B. in (9-e). Gleichzeitig bestärkt dieses Beispiel die These von Cornish et al. (2005), dass es sich bei Instrumenten um periphere Antezedenten handelt.

(9) a. Caro ist für das Vorstellungsgespräch in die vierte Etage gefahren. Vorher hat sie ihn ... per Knopfdruck bestellt.

b. Caro ist für das Vorstellungsgespräch in die vierte Etage gefahren. Vorher hat sie ihn ... verbal angegriffen

c. Caro ist für das Vorstellungsgespräch in die vierte Etage mit dem Aufzug gefahren. Vorher hat sie ihn ... mit einem Knopfdruck angefordert

d. Caro ist für das Vorstellungsgespräch in die vierte Etage mit dem Aufzug gefahren. Vorher hat sie ihn ... zum letzten Mal gesehen.

e. Alexander ist am Freitag zur Konferenz mit dem Auto gefahren. Dafür hat er es ... nicht geschafft noch im Seminar vorbei zu kommen.

Die statistische Auswertung der Kontroll-Items unterstützt die explorativ gewonnenen Erkenntnisse: Die deskriptive Statistik in Tabelle 5.24 zeigt einen deutlich höheren Mittelwert für die Anzahl an Bezügen auf das explizite Antezedens - im Gegensatz zum im-

${ }^{21}$ Die 6 VPn verteilen sich relativ gleichmäßig auf verschiedene Listen, sodass dies nicht von konkreten Items abzuhängen scheint. 
pliziten. Die Inferenzstatistik weist diesen Unterschied als signifikant aus (vgl. Tab. 5.25).

\begin{tabular}{ccccc}
\hline \hline Faktor & Bed. & MW & Std. Abw. & $\mathrm{n}$ \\
\hline \multirow{2}{*}{ ANTEZEDENS } & implizit & 0.12 & 0.33 & 336 \\
& explizit & 0.80 & 0.40 & 336 \\
\hline \hline
\end{tabular}

Tabelle 5.24: Deskriptive Statistik der relativen Häufigkeit der anaphorischen Bezüge auf das implizit oder explizit erwähnte Antezedens in den Kontroll-Items

\begin{tabular}{c|cc}
\hline \hline Faktor & $|z|$ & $p$ \\
\hline ANTEZEDENS & 7.770 & $<0.001$ \\
\hline \hline
\end{tabular}

Tabelle 5.25: Inferenzstatistik (LMEM) für die Bezüge auf das Instrument-Antezedens in den Kontroll-Items mit 12 Items, 56 VPn und Item und VP als ,random factors'

Der Mittelwert des experimentellen Item-Sets für die N1-Bezüge über alle 4 Bedingungen hinweg liegt bei 0.14 (Std. Ab. $=0.34, \mathrm{n}=1512$ ) und damit zwischen den Mittelwerten der direkten und indirekten Anaphern in den Kontroll-Items. Dies lässt sich dergestalt interpretieren, dass es sich bei einem N1-Bezug um eine Form der Anapher handelt, die in Bezug auf den Grad an Zugänglichkeit des Referenten zwischen direkten und indirekten Anaphern liegt, jedoch den indirekten Anaphern näher steht. Allerdings ist diese Interpretation unter Vorbehalt zu betrachten, da die Fallzahlen sehr stark voneinander abweichen (1512 vs. 336) und die Items mit den N1-Bezügen aufgrund der experimentellen Manipulation insgesamt sehr heterogen sind. Darüber hinaus weichen die experimentellen Items und die Kontroll-Items in ihrem Aufbau voneinander ab.

Nach der Durchführung des Experiments wurden die VPn befragt, was sie für das Ziel des Experiments halten und ob ihnen etwas aufgefallen sei. Verschiedene Ideen wurden geäußert, z.B. die Untersuchung von Assoziationen zu bestimmten Vornamen. Die häufigste Antwortet war, dass Ergänzungen zu dieses im Gegensatz du dieser „einfacher zu finden" seien, da in dem Fall eine „neutrale Antwort" möglich sei. Diese Einschätzung stützt den Eindruck der Stärke der Präferenz für die Resolutionsalternativen bei Neutrum-Pronomen. Darüber hinaus wurde angegeben, dass es sich bei jenes $/ r$ um ein eher ungebräuchliches Pronomen handelt und dass bei vielen Sätzen der Eindruck bestehe, dass der Vorgängersatz nicht „zum Satzende passt“ oder kein Bezug möglich sei. Keine VP gab an, dass Komposita oder Komposita-Konstitueten Ziel der Untersuchung sein könnten. Ein systematischer Zusammenhang zwischen Pronomen und N1 wurde nicht erwähnt.

\section{Resolutionsalternativen}

Abschließend soll ein explorativer Blick auf die durch die VPn gegebenen Satzergänzungen geworfen werden. Bei der Annotation dieser Satzergänzungen zeigt sich, dass bestimmte Formen von Bezugnahmen häufiger verwendet werden als andere. Neben N1-Bezügen wie (11-a) wurden besonders häufig Komplexanaphern gebildet, die sich auf den gesamten im Kontextsatz beschrieben Vorgang beziehen, z.B. (11-b) ${ }^{22}$ Darüber hinaus fiel auf,

\footnotetext{
${ }^{22}$ Fälle mit N1-Bezügen in späteren Satzteilen wurden nicht als N1-Bezug gewertet, wie z.B. (10)
} 
dass viele VPn eine klare Präferenz für ein Antezedens hatten und diese sogar entgegen der grammatischen Kongruenz verfolgten. Plausibilität wurde hier über Grammatikalität gestellt, z.B. (11-c) ${ }^{23}$ Im Umkehrschluss lässt ein realisierter N1-Bezug darauf schließen, dass das N1 in dem Fall tatsächlich relativ salient ist und der Bruch mit der lexikalischen Integrität zu Gunsten der Plausibilität in Kauf genommen wird.

(11) a. Paula hat neulich im Seminar eine umstrittene Filmbewertung gelesen. Diesen hat sie ... vorher gesehen

b. Daniel hat schon vor Wintereinbruch eine teure Autopanne verursacht. Jenes hat er ... bereut

c. Sophie hat im Anatomiepraktikum eine ideale Kopfhaltung vorgeführt. Jenen hat sie ... zuvor vor dem Spiegel geübt.

d. Marie hat in Kolumbien eine interessante Tabakverarbeitung kennengelernt. Jenen hat sie ... erzählt, was sie dort gesehen hat.

e. Guido hat mit der Feuerwehr eine bedenkliche Wasserverschmutzung entdeckt. Jenes hat er ... davon in Kenntnis gesetzt.

f. Mattis hat auf der Klassenfahrt eine typische Papiermaschine besichtigt. Dieses hat er ... noch nie gesehen.

g. Silke hat beim Entsorgungswerk eine umweltschonende Müllmenge produziert. Diesen hat sie ... nicht gesehen.

h. Marie hat in Kolumbien eine interessante Tabakverarbeitung kennengelernt. Diesen hat sie ... zum ersten Mal in ihrem Leben gesehen, diesen speziellen Teil des Landes.

Eine weitere häufig angewandte Strategie ist der Bezug auf extratextuelle humane Referenten im Singular oder Plural, z.B. (11-d), Einige Fälle erwecken den Eindruck, dass die Pronomen jenes und jener als Eigennamen interpretiert wurden, z.B. (11-e), Einige Satzergänzungen waren wiederum sehr allgemein und damit ambig formuliert, z.B. (11-g). In solchen Fällen, wie auch schon in ähnlichen Fällen in Experiment 1, bleibt unklar, ob die VP die Anapher wirklich aufgelöst hat oder lediglich einen grammatischen Satz gebildet hat. Sollte dies der Fall sein, wäre dies ein Beispiel für eine Good-Enough-Resolution an der Schnittstelle zwischen Sprachrezeption und -produktion.

Weitere auftretende Strategien sind die Verwendung von Kataphern, z.B. (11-h), die Verwendung weiterer indirekter Referenten oder Teilkomplexbildungen oder der Gebrauch der Pronomen als Deiktika, was eine imaginierte situative Einbettung nahelegt.

Neben diesen verschiedenen Strategien gibt es auch Fälle von inkohärenten Satzergänzungen. In einigen Fällen wird deutlich, dass der VP kein Ausdruck als Antezedens plausibel scheint, z.B. in (12). Dies wiederum ist ein sicheres Indiz für die Unzugänglichkeit eines N1.

(12) Silke hat beim Entsorgungswerk ein umweltschonendes Müllauto bestellt. Diesen hat sie ... hier fällt mir zu ,Diesen' überhaupt nichts ein.

(10) Thomas hat wegen der nervigen Insekten einen alten Fensterspalt abgedichtet. Dieses hat er ... mit so viel Wut in der Brust getan, dass das Fenster wohl nie wieder richtig aufgehen wird

${ }^{23} \mathrm{Zu}$ einem ähnlichen Ergebnis kommt auch Klos (2011:163) in einem ihrer Experimente, bei dem VPn die Bedeutung von Zufallskomposita angeben sollten. Gab es keine naheliegende Interpretation, drehten manche VPn das Determinationsverhältnis um, um eine plausiblere Interpretation zu finden. 
Einige Komposita wurden z.T. anders interpretiert als durch die Zuweisung zu einer Bedingung intendiert, was der allgemeinen Unterspezifiziertheit von Komposita geschuldet ist. So wurde das Kompositum Kabelverbindung mal als Verbindung von und mal als Verbindung aus Kabeln interpretiert. Erschwerend kommt hinzu, dass sich die Interpretation der einzelnen VPn nicht unbedingt an der Satzergänzung erkennen lässt.

Darüber hinaus ließen sich anhand der Satzergänzungen einige Schnittmengenbildungen identifizieren. In diesen Fällen war die Entscheidung zwischen N1- und NN-Bezug nicht zweifelsfrei möglich, z.B. in (13). Grammatikalisch ist eine Lesart als N1-Bezug plausibel, rein inhaltlich ist aber auch ein NN-Bezug möglich. Da auch an anderen Stellen Ungrammatikalität in Kauf genommen wird, bleibt der Bezug hier unklar.

a. Sarah hat für die Polarexpedition vorsichtshalber eine einfache Ölreserve angelegt. Jenes hat sie ... dann samt Rucksack mitgenommen.

b. Sarah hat für die Polarexpedition vorsichtshalber eine einfache Ölreserve angelegt. Dieses hat sie ... bis heute noch nicht aufgebraucht.

Schließlich geben die Satzergänzungen auch Aufschluss über die RZK an sich. Bei der Einteilung der Bedingungen wurden nur die Komposita als +RZK klassifiziert, bei denen die Anwesenheit des N1 zwingend für die Konstitution des NN ist. Trotz alledem ist in Fällen von -RZK die Repräsentation des N1 möglich. Dies führte dazu, dass ein N1 in manchen Fällen als absent betrachtet wurde (z.B. in (14-a) und in manchen als präsent (z.B. in (14-b) . Hierin zeigt sich die Kontextabhängigkeit des Faktors, die auch zu einem gewissen Grad die Varianz zwischen Items und VPn erklärt.

\author{
Marie hat in Kolumbien eine interessante Tabakdose aufgestöbert. \\ Diesen hat sie ... \\ a. dort hinein getan \\ b. aufgeraucht und musste in ärztliche Behandlung.
}

\title{
5.8. Interpretation und Diskussion
}

Die Ergebnisse von Experiment 2 bestätigen den Einfluss des Faktors RZK. Für die Faktorstufe +RZK liegen signifikant mehr N1-Bezüge als für -RZK vor $(p<0.01)$. Beim Vergleich der Ergebnisse für den Faktor RZK mit den Ergebnissen für die semantischen Relationen aus Experiment 1 stellt sich die RZK als der geeignetere Faktor heraus. Die Aufteilung von Komposita auf die Faktorstufen +/-RZK erweist sich als deutlich trennschärfer. Die Relationen, die relativ sicher einer der beiden Faktorstufen zugeordnet werden konnten, bestätigen die Ergebnisse der Auswertung der RZK. Diese Ergebnisse sprechen auch für die in Kapitel 3.3 entwickelte Idee eines konzeptuellen Fokus und seinen Einfluss auf die N1-Zugänglichkeit.

Der Faktor STRUKTUR zeitigt ebenfalls ein signifikantes Ergebnis $(\mathrm{p}<0.05)$, allerdings entgegen der Hypothese mit mehr N1-Bezügen im Fall von Wurzelkomposita. In der Einzelauswertung der Bedingungen zeigt sich, dass der Effekt maßgeblich von der Faktorstufe +RZK getragen wird. In einer Post-hoc-Analyse wurde in dieser Faktorstufe eine höhere Zahl an Schnittmengenbildungen gefunden. Der Einfluss dieses unkontrollierten konzeptuellen Faktors scheint das Ergebnis für den Faktor STRUKTUR zu konfundieren. Zudem 
sind die Pronomenresolutionen in den entsprechenden Fällen oft ambig, da sie sowohl als N1- als auch als NN-Bezug gelesen werden können (vgl. (13)). Dies belegt zum einen die These, dass konzeptuelle Faktoren strukturelle Faktoren überlagern. Zum anderen zeigt sich, dass dieses Experiment keine fundierten Aussagen zum Einfluss der KompositumsStruktur erlaubt.

Die Interaktion RZK $\times$ STRUKTUR ist marginal signifikant $(\mathrm{p}<0.1)$. Aufgrund von Konvergenzproblemen kann allerdings nur ausgesagt werden, dass für die Faktorstufe -RZK kein Einfluss des Faktors STRUKTUR besteht und für Rektionskomposita ein Einfluss des Faktors RZK besteht. Es zeigt sich, dass der Effekt des Faktors STRUKTUR durch die Faktorstufe +RZK getragen wird, wie bereits oben thematisiert. Für die Art des Demonstrativpronomens konnte kein Einfluss nachgewiesen werden. Deskriptiv liegen mehr N1-Bezüge im Fall von dieses/ $r$ als von jenes/ $r$ vor.

Der Einfluss des Genus konnte bestätigt werden. Bei der Auswertung zeigte sich außerdem, dass selbst bei Vorliegen einer präferierten Strategie zur Anaphernresolution (z.B. als Komplexanapher), vereinzelt N1-Bezüge auftreten. Dies spricht für die Produktivität dieser Form des anaphorischen Bezugs. Auch der Einfluss der Spezifizität des N1 ist in prognostizierter Richtung signifikant. Bei der Auswertung der N1-Zählbarkeit zeigte sich deskriptiv eine leicht höhere Zahl an N1-Bezügen für Individualnomen und damit eine Tendenz zum stärkeren Einfluss konzeptueller Eigenschaften.

Insgesamt zeichnet sich ab, dass konzeptuelle Faktoren einen stärkeren Einfluss auf die N1-Zugänglichkeit haben als strukturelle. So ergab die Post-hoc-Analyse der Interaktion von RZK $\times$ STRUKTUR, dass die unbalancierte Verteilung von Schnittmengenbildungen im Material maßgeblich für die gute N1-Zugänglichkeit einiger Wurzelkomposita verantwortlich ist. Argumentativ in die gleiche Richtung geht die Beobachtung, dass deskriptiv ein Vorteil für die N1-Zugänglichkeit im Fall von Individualnomen gegenüber Massenomen besteht. Schließlich belegen Satzergänzungen wie (11-c), dass zu Gunsten der Plausibilität sogar grammatische Restriktionen wie Kongruenz missachtet werden. Insgesamt schließe ich daraus, dass das Material trotz vielfältiger Kontrollbemühungen zu unausgeglichen ist, um verlässliche Aussagen zum Struktureinfluss ableiten zu können. Die Kombination mit einem konzeptuellen Faktor, wie es in diesem Experiment versucht wurde, scheint ungeeignet.

Die Auswertung der Kontroll-Items mit direkten und indirekten Anaphern legt eine Interpretation des N1-Bezugs als Hybrid zwischen den beiden Anapherarten nahe, allerdings mit größerer Nähe zur indirekten Anapher.

Die Betrachtung der Satzergänzungen insgesamt zeigt, dass die VPn mehrheitlich verschiedene Resolutionsstrategien anwenden und das experimentelle Design daher den N1-Bezug keineswegs forciert. Dies sprich einmal mehr dafür, dass in den Fällen mit N1-Bezug das N1 tatsächlich relativ salient ist.

Einzelne Satzergänzungen zeugen jedoch auch davon, dass der Kontext der Komposita nicht eindeutig genug ist, um eine Interpretation des N1 entsprechend der jeweiligen Bedingung klar festzulegen. Ebenso zeigt sich ein starker Einfluss von Weltwissen und individuellen Präferenzen.

Der Einfluss der RZK auf die N1-Zugänglichkeit kann durch die Ergebnisse von Experiment 2 bestätigt werden. Der Einfluss der morphosyntaktischen Struktur der Komposita bleibt unklar. Um valide Aussagen dazu treffen zu können, muss das experimentelle 
Material stärker kontrolliert werden. Komposita und Kontexte müssen eindeutig zu interpretieren und die Interpretation muss eindeutig messbar sein. Die Annotation der Satzergänzungen ist hier zu ungenau. In diesem Sinne haben sich die Satzergänzungsaufgabe und die Kombination verschiedenartiger Faktoren nicht als optimal erwiesen.

Ferner erwies sich die inferenzstatistische Auswertung als problematisch, da die LMEMs in einigen Fällen nicht oder nur bei Vereinfachung des Modells durch Ausschluss einzelner Items, VPn oder der ,random slopes' konvergierten. Dies hängt aller Wahrscheinlichkeit nach mit dem Datentyp zusammen. Da es sich bei der AV um eine binär kodierte Variable handelt, liegen kategoriale Daten mit relativ geringer Messwiederholung vor 7 Datenpunkte pro Zelle). Hierzu heißt es bei Barr et al. (2013):

$[\ldots]$ it is our impression that fitting maximal LMEMs is less often successful for categorical data than for continuous data. (Barr et al. 2013 276)

Als erste Annäherung an das Phänomen ist die Satzergänzungsaufgabe ein probates Mittel. Für detailliertere Erkenntnisse ist jedoch eine Studie zur Online-Verarbeitung notwendig. Ein geeignetes Paradigma scheint hier ein Leseexperiment mit Eye-Tracking zu sein. Blickverhaltensdaten sind kontinuierliche Daten, die unmittelbar ohne Zwischenschritt der Interpretations-Annotation ausgewertet werden können. Komposita und Pronomen können dabei in festen Diskursen auftreten, die via Kontext nur eine spezifische Interpretation zulassen. 


\section{Experiment 3: Struktur}

\subsection{Vorüberlegungen}

\subsubsection{Faktor}

Entgegen der Annahmen aus Kapitel 4 legt Experiment 2 eine bessere N1-Zugänglichkeit bei Wurzel- als bei Rektionskomposita nahe (siehe Kap. 5). Der Effekt wird allerdings maßgeblich von der Bedingung +RZK/WK getragen. In einer Post-hoc-Analyse des experimentellen Materials zeigte sich, dass bei den Komposita in dieser Bedingung überdurchschnittlich viele Schnittmengenbildungen vorliegen, die aufgrund ihrer spezifischen Art der Konzeptkombination über eine besonders gute N1-Zugänglichkeit verfügen. Dies wiederum bedeutet, dass der gemessene Vorteil für die N1-Zugänglichkeit bei Wurzelkomposita in Experiment 2 nicht auf dem Unterschied in der morphosyntaktischen Struktur beruht, sondern ebenfalls konzeptuell begründet ist.

Durch das mehrfaktorielle Design in Experiment 2 konnte herausgearbeitet werden, dass der Einfluss von konzeptuellen Faktoren wie der RZK stärker ist als der Einfluss von grammatischen Faktoren wie der STRUKTUR. Dieser Einfluss scheint sogar so stark zu sein, dass der grammatische Faktor überlagert wird.

Um nun unabhängige Aussagen zum Einfluss des strukturellen Aufbaus von Komposita auf die N1-Zugänglichkeit treffen zu können, gilt es Wurzel- und Rektionskomposita direkt miteinander zu vergleichen. Dabei müssen zum einen alle möglichen konzeptuellen Einflüsse bestmöglich kontrolliert werden und zum anderen auch die Komposita eine vergleichbare Semantik haben. Dies war in Experiment 2 aufgrund des mehrfaktoriellen Designs nicht möglich.

In Experiment 2 treten darüber hinaus deutlich die Grenzen der Satzergänzungsaufgabe als experimentelles Paradigma in Erscheinung. Die kategorialen Daten des Ratings führten bei der inferenzstatistischen Analyse mit LMEMs vermehrt zu Konvergenzproblemen (vgl. Kap. 5.6.2). Außerdem handelt es sich um ein Verfahren, welches zwar Sprachperzeption und -produktion verbindet, mit dem sich jedoch ausschließlich Aussagen zur OfflineVerarbeitung treffen lassen. Frühe Unterschiede in der Verarbeitung und ihr räumlicher Ursprung können mit diesem Verfahren nicht sichtbar gemacht werden.

Um den Einfluss der Kompositums-Struktur auf die N1-Zugänglichkeit genauer zu beleuchten und feinkörnigere Informationen zum Prozess der Anaphernresolution zu erhalten, soll im Folgenden das Eye-Tracking-Paradigma genutzt werden. Beim Eye-Tracking können Blickbewegungen und Lesezeiten über einen längeren Leseprozess hinweg aufgezeichnet werden. In diesem Fall werden den VPn Antezedens und Anapher im Kontext von kurzen Texten präsentiert. Die Interpretation der Anaphern wird dabei über den Kontext festgelegt. Für die Auswertung stehen verschiedene kontinuierliche, intervallskalierte Daten als abhängige Variable zur Verfügung. Das Eye-Tracking-Paradigma bietet die Möglichkeit, den gesamten Text zeitgleich darzustellen, was ein natürliches Leseverhalten mit 
Blickbewegungen zu vorausgehenden Textstellen ermöglicht. Somit werden Daten über Ort und Dauer von Fixationen, und Sakkaden mit genauer räumlicher Auflösung gewonnen. Durch Lesezeiten lassen sich Verarbeitungsschwierigkeiten offenlegen und lokalisieren und somit detaillierte Erkenntnisse zum Anaphernresolutions-Prozess gewinnen.

Experiment 3 besteht aus drei Teilexerimenten, die auf der Basis eines gemeinsamen Sets an Komposita durchgeführt werden. Experiment 3a bedient sich zunächst erneut einer Satzergänzungsaufgabe, um einen Vergleich der Ergebnisse mit den vorhergehenden Experimenten zu ermöglichen. Gleichzeitig ermöglicht die Überprüfung von Items in zwei verschiedenen Paradigmen einen direkten Paradigma-Vergleich, also einen Vergleich der Ergebnisse aus Satzergänzungsaufgabe und Eye-Tracking. Experiment 3b ist ein Kohärenzrating, in welchem die Texte der Items hinsichtlich ihrer Reliabilität für das EyeTracking überprüft werden. Experiment 3c ist schließlich das Eye-Tracking-Experiment.

\subsubsection{Komposita-Auswahl}

Die drei Teilexperimente von Experiment 3 basieren auf einem gemeinsamen Set an Komposita, die 24 Items bilden. Jedes der 24 Items beinhaltet zwei Komposita mit demselben N1, aber verschiedenen N2 (siehe Anhang C.1). Dadurch tritt jedes N1 einmal in einem Rektions- (RK) und einmal in einem Wurzelkompositum (WK) auf. In einer dritten Bedingung (Mono) wird das N1 als Monolexem, d.h. als Simplex verwendet. Das N1 fungiert hier als Antezedens für eine direkte Anapher. Bei dieser Bedingung handelt es sich um eine Kontrollbedingung, da in diesem Fall die Anaphernresolution immer direkt und damit auch schneller als in den beiden Kompositabedingungen etabliert werden sollte. Beispiele für die drei Bedingungen finden sich in Tabelle 6.1 1

\begin{tabular}{ccll}
\hline \hline Bed. & \multicolumn{1}{c}{ STRUKTUR } & Beispiele & \\
\hline 1 & Rektionskompositum (RK) & Dachbegrünung & Tabakverarbeitung \\
2 & Wurzelkompositum (WK) & Dachgarten & Tabakfabrik \\
3 & N1 als Monolexem (Mono) & Dach & Tabak \\
\hline \hline
\end{tabular}

Tabelle 6.1: Beispiele für die in Experiment 3 verwendeten Komposita in den drei Bedingungen des Faktors STRUKTUR

Bei der Auswahl geeigneter Komposita wurden zunächst die Komposita aus Experiment 2 als Grundlage genommen. Einige wurden ausgetauscht, sodass u.a. Genus und Zählbarkeit des N1 zwischen den Items besser ausbalanciert sind und kein N1 doppelt vorkommt. Bei der Auswahl der Items für Experiment 2 wurden die Frequenzen der Komposita streng kontrolliert; nach genauerer Betrachtung der Ergebnisse erscheint es jedoch sinnvoll, dies zugunsten einer strengeren Kontrolle der semantischen und v.a. konzeptuellen Faktoren nachrangig zu betrachten. Spezifische Eigenschaften des N1 sind dahingehend kontrolliert, dass innerhalb eines Items immer das gleiche N1 steht. Darüber hinaus wurden weitere mögliche Einflussfaktoren bestmöglich ausbalanciert und annotiert, darunter neben Zählbarkeit und Genus des N1 auch die Konkretheit des N2 sowie die RZK (siehe Tab. C.2 in Anhang C.1).

\footnotetext{
${ }^{1}$ In der Beschreibung des experimentellen Designs bezeichne ich das N1 als ,Antezedens' und das Kompositum als ,Anker', in der Bedingung Mono fällt beides zusammen.
} 
Zur Vereinheitlichung wurden als Rektionskomposita ausschließlich Nominalisierungen mit dem Derivationssuffix -ung verwendet. Dabei handelt es sich um eine der frequentesten Nominalisierungsarten des Deutschen (vgl. Kap. 5.7.5), was das Auffinden geeigneter Komposita erleichtert. Da ung-Nominalisierungen sowohl über eine Prozess- als auch über eine Resultatslesart verfügen (vgl. Kap. 4.2.2) und dies die Verarbeitung der Anapher beeinflussen kann, wurde versucht die Lesart über den Kontext zu disambiguieren und gleichzeitig die beiden Lesarten auszubalancieren. Bei den zugrundeliegenden Verben handelt es sich in 8 Fällen um (underivierte) Wurzelverben², in 4 Fällen um Partikelverben und in 12 Fällen um Präfixverben.

Es wurde darauf geachtet, die Semantik zwischen den Komposita in RK und WK ähnlich und damit vergleichbar zu gestalten, um mögliche Unterschiede in den Daten möglichst nur auf die Struktur zurückführen zu können (z.B. Dachbegrünung vs. Dachgarten).

\section{Transparenztests}

Wie in Kapitel 2.3.4 dargelegt, ist von einem Einfluss von Transparenz, Kompositionalität und Lexikalisiertheit auf die N1-Zugänglichkeit auszugehen. Einer Konfundierung aufgrund des Einflusses dieser Faktoren sollte vorgebeugt werden. Da eine quantitative Messung nicht möglich ist, wurden zwei verschiedene Tests angewandt. Diese sollen sicherstellen, dass alle Items über einen niedrigen Grad an Lexikalisiertheit sowie über einen hohen Grad an Transparenz und Kompositionalität verfügen. Die Tests sind im Folgenden jeweils am Beispiel des Kompositums Dachgarten erläutert.

\section{Test 1: Substitution}

Zunächst wurde geprüft, ob die Teile des Kompositums durch andere Lexeme austauschbar sind und ob das neue Kompositum auf die gleiche Weise interpretiert wird. Die Leitfrage dazu lautet: Ist es möglich, die Kompositums-Konstituenten auszutauschen, sodass die gleiche Relation durch andere Konstituenten hergestellt wird? Ist der Test erfolgreich, lässt sich schlussfolgern, dass die Relation der Kompositaglieder und ihre Fügung transparent sind.

Bspw. denotiert Dachgarten einen Garten auf einem Dach; es gibt aber auch Hinterhofgärten, also Gärten in Hinterhöfen. Die Konzeptkombination im zweiten Kompositum ist die gleiche wie im ersten: In beiden Fällen beschreibt das N1 den Ort des N2 und ist in dieser Funktion durch einen anderen Ort austauschbar. Formalisieren lässt sich dieser Test mit der Formel xy : x wie zy : z, also Dachgarten : Dach wie Hinterhofgarten : Hinterhof.

\section{Test 2: Definition}

Bei transparenten Wurzelkomposita stellt das N2 den Oberbegriff dar und das N1 charakterisiert diesen näher, z.B. denotiert Dachgarten einen Garten, der sich auf einem Dach befindet. Bei Rektionskomposita bezeichnet das Denotat einen Prozess oder das Resultat des Prozesses, welcher durch das dem N2 zugrundeliegenden Verb prädiziert wird. Dabei bildet das N1 das direkte Objekt des Verbs. So denotiert Dachbegrünung das Resultat

\footnotetext{
${ }^{2}$ Analog zum Begriff, Wurzelkompositum' benutze ich den Ausdruck, Wurzelverben' um mich auf Verben mit underivierter Wurzel, d.h. ohne Präfixe oder Partikel zu beziehen.
} 
des Prozesses, bei welchem ein Dach begrünt wird. Wenn ein Kompositum kompositional ist, müsste es sich anhand dieses Schemas definieren lassen. Zur Überprüfung wurden die Komposita anhand ihrer Bedingungen auf Listen aufgeteilt und randomisiert vier Hilfskräften vorgelegt. Diese wurden gebeten, die ihnen präsentierten Begriffe zu definieren. Die Definitionen wurden dahingehend geprüft, ob sie den Ausdruck anhand des entsprechenden Bildungsmusters (auch unter Verwendung von Synonymen) erklären.

Es fanden nur Komposita Eingang in das endgültige Stimulus-Material, die beide Tests bestanden. Die endgültigen Items sind im Anhang C.1 aufgelistet.

\subsection{Experiment 3a: Satzergänzungsaufgabe}

Experiment 3a besteht wie bereits Experiment 1 (vgl. Kap 3.4) und Experiment 2 (vgl. Kap. 5) aus einer Satzergänzungsaufgabe. Dadurch soll ein direkter Vergleich der Ergebnisse mit den bisherigen Experimenten ermöglicht werden. Darüber hinaus wird durch die Verwendung der gleichen Komposita in einer Satzergänzungsaufgabe und in einem EyeTracking-Experiment ein Paradigmen-Vergleich innerhalb von Experiment 3 möglich.

\subsubsection{Methode}

Das methodische Vorgehen in Experiment 3a entspricht der Methode aus Experiment 1 und 2. Die Items in Experiment 3a beginnen jeweils mit einem Einleitungssatz. Darauf folgt ein Satz, der mit dem kritischen Kompositum (bzw. dem Simplex in der Bedingung Mono) beginnt. Schließlich folgt ein Satzfragment, welches ein Pronomen enthält, das grammatisch ausschließlich mit dem N1 kongruent ist. Die VPn haben die Aufgabe, möglichst schnell und intuitiv eine geeignete Satzergänzung zu finden und per Tastatur in ein Freitextfeld einzugeben. Die Satzergänzungen wurden anschließend hinsichtlich der Pronomenresolution interpretiert und annotiert.

\subsubsection{Material}

Um die in Kapitel 6.1.2 beschriebenen Komposita wurden 24 Items konstruiert. Jedes Item besteht aus einem Einleitungssatz, einem Satz mit dem kritischen Kompositum und einem Satzfragment, welches nur aus einem Pronomen und einem Modalverb besteht (siehe Beispiel-Item (1)]. Die Kriterien, die zur Erstellung der Sätze nach diesem Prinzip geführt haben, richten sich nach Erfordernissen des Eye-Trackings und werden an späterer Stelle erläutert.

(1) a. Karl ist ein richtiger Heimwerker geworden. Die Dachbegrünung ist wirklich gut gelungen. Es kann ...

b. Karl ist ein richtiger Heimwerker geworden. Der Dachgarten ist wirklich gut gelungen. Es kann ...

c. Karl ist ein richtiger Heimwerker geworden. Das Dach ist wirklich gut gestaltet. Es kann ... 
Zusätzlich sind im Experiment zwei Filler-Sets enthalten. Bei Filler-Set 1 handelt es sich um ein weiteres, unabhängiges experimentelles Set, welches den IC-Bias bei Verben zum Gegenstand hat. Dieses beinhaltet 48 Items in je zwei Bedingungen.

Filler-Set 2 ist ein Filler-Set, welches in seinem Design verschiedene Aspekte der beiden anderen Sets verbindet und somit die Systematik dieser maskieren soll. Das Filler-Set besteht aus 16 Items.

Alle drei Sets wurden nach dem lateinischen Quadrat auf sechs Listen aufgeteilt. Das hier besprochene experimentelle Set wurde dafür zunächst auf drei Listen aufgeteilt, welche anschließend verdoppelt wurden. Die Items wurden innerhalb jeder Liste für jeden experimentellen Durchlauf automatisch randomisiert.

\subsubsection{Hypothesen}

Die Experiment 3a zugrundeliegende Hypothese ist, dass abhängig von der KompositumsStruktur die N1-Zugänglichkeit variiert und sich dies auf die Zahl der etablierten N1Bezüge bei der Pronomenresolution niederschlägt. Die unabhängige Variable ist dabei die experimentelle Bedingung STRUKTUR, die abhängige Variable ist die relative Häufigkeit von N1-Bezügen in den drei Bedingungen. Die Art der Pronomenresolution in den Satzergänzungen wurde von drei Kodierern und einer Schlichterin interpretiert und annotiert. Dabei wurde annotiert, ob eine VP ein Satzfragment so fortgesetzt hat, dass sich das Pronomen auf das N1 bezieht oder ob eine andere Art der Satzergänzung gewählt wurde. Bei der Annotation wurde ein N1-Bezug mit , $1^{\text {‘ }}$ und ein anderweitiger Bezug mit ,0` kodiert. Je höher der Mittelwert für eine Bedingung, desto mehr N1-Bezüge (bzw. Bezüge auf das Simplex in der Bedingung Mono) wurden hergestellt.

Die konkrete Hypothese lautet, dass in der Bedingung Mono die meisten Bezüge zum avisierten Antezedens hergestellt werden, da hier eine direkte Anapher etabliert werden kann. In den Kompositabedingungen (Bed. RK und WK) ist das Antezedens als N1 im NN-Ankerausdruck enthalten und somit in jedem Fall schlechter zugänglich. In diesem Sinne dient Mono als Kontrollbedingung zur Überprüfung des experimentellen Designs. Für die Überprüfung der zentralen Hypothese aus Kapitel 4 werden die N1-Bezugszahlen der Bedingungen RK und WK verglichen. Aufgrund des postulierten Unterschieds im kategorialen Status der N1 wird im Fall von Rektionskomposita eine bessere N1-Zugänglichkeit und damit mehr N1-Bezüge erwartet als im Fall von Wurzelkomposita. Daher wird eine höhere relative Häufigkeit an N1-Bezügen für die Bedingung RK im Vergleich zu WK prognostiziert. Daraus ergibt sich Gesamthypothese: MW(Mono) > MW(RK) > MW(WK).

\subsubsection{Durchführung}

Das Experiment wurde in zwei Erhebungen durchgeführt: Die erste Erhebung erfolgte vom 04.05. - 12.05.2016 mit 36 VPn. Im ersten Auswertungsschritt zeigte sich jedoch ein Fehler in der Programmierung der Iteration. Aus diesem Grund wurde vom 30.05. 01.06.2016 Daten von weiteren 6 VPn erhoben. 


\section{Ablauf}

Die Durchführung der beiden Erhebungen erfolgte an den Rechnern des psycholinguistischen Labors des Courant Forschungszentrums, Textstrukturen' (CRC) in Göttingen. Jeder Durchgang dauerte ca. 30 - 45 Minuten. Die Versuchsleitung instruierte die VPn über den Ablauf des Experiments und über die Aufgabe, möglichst schnell und intuitiv Sätze zu vervollständigen. Anschließend wurde der Ablauf des Experiments jeder VP noch einmal schriftlich am Bildschirm vorgestellt. Daraufhin konnten sich die VPn selbstständig durch das Experiment arbeiten. Ein Fortschrittsbalken informierte über den Fortschritt im Experiment. Am Ende des Experiments erfolgte in einem Feedbackbildschirm die Aufforderung, eine Vermutung zum Gegenstand des Experiments anzustellen.

\section{Technik}

Das Experiment wurde mit OnExp 1.3.1 (Onea 2012) programmiert und an den Rechnern des CRC-Labors browserbasiert durchgeführt.

\section{Versuchspersonen}

Im ersten Erhebungszeitraum wurden 36 VPn getestet, bei der Nacherhebung weitere 6, sodass insgesamt 42 Datensätze vorliegen. Ein Datensatz musste aus Gründen der demografischen Homogenität entfernt werden, sodass die Auswertung auf Basis von 41 Datensätzen erfolgt. Dabei handelt es sich um Studierende der Universität Göttingen mit Deutsch als einziger Muttersprache. Von den VPn waren 27 weiblich und 14 männlich, das mittlere Alter lag bei 23,05 Jahren (Std. Abw. = 2.95) ${ }^{3}$ Die Teilnahme am Experiment erfolgte freiwillig und wurde vergütet.

\section{Datenaufbereitung}

Nach Abschluss der Erhebung wurden die Daten zusammengeführt und die Satzergänzungen bzgl. der Auflösung des Pronomens in einem Rating ausgewertet. Dabei gaben drei Kodierer unabhängig voneinander ihre Einschätzung ab. Für die Fälle, in denen Uneinigkeit bei der Kodierung herrschte, wurde im Nachhinein eine Schlichterin hinzugezogen. Beim Rating wurde unterschieden, ob das Pronomen sich durch die Satzergänzung auf das N1 bezieht (1) oder ob die VP eine Resolutionsalternative gewählt hat und sich das Pronomen auf etwas anderes bezieht (0). Fälle, bei denen die Bezugnahme von allen Kodierern als uneindeutig bewertet wurde, wurden mit ,NA' gekennzeichnet.

Aufgrund des Programmierfehlers in der ersten Erhebung liegen für die einzelnen Fälle unterschiedlich viele Satzergänzungen und somit unterschiedlich viele Datenpunkte vor, was bei einigen inferenzstatistischen Berechnungen zu Problemen führt. Aus diesem Grund wurde der Datensatz gekürzt und vereinheitlicht. Dabei wurden alle Fälle auf jeweils 10 Beobachtungen beschnitten. In 7 Fällen lagen weniger als 10 Beobachtungen vor. Um

\footnotetext{
${ }^{3}$ Das experimentelle Set ist auf 3 verschiedene Listen aufgeteilt: auf Liste 1 wurden 15 VPn getestet, auf Liste $214 \mathrm{VPn}$ und auf Liste $312 \mathrm{VPn}$.
} 
eine gleichmäßige Verteilung der Beobachtungen auf die drei Bedingungen zu gewährleisten, wurde eine weitere Beobachtung entfernt. Im finalen Datensatz liegen für jede der drei Bedingungen 236 Beobachtungen vor.

Keine der VPn gab im Feedback-Feld eine Vermutung zum Hintergrund des Experiments an, die präzise genug war, als dass die VP von der Auswertung hätte ausgeschlossen werden müssen.

Während des Ratings fiel auf, dass bei drei Items ein Nomen im Kontextsatz auftritt, welches das Genus des Pronomens trägt und somit als potenzielles Antezedens die Wahl der Bezugnahme beeinflusst. Da dies allerdings in den entsprechenden Items in allen drei Bedingungen vorkommt, bewirkt dies keine Konfundierung des Ergebnisses, sondern vermindert lediglich insgesamt die Wahrscheinlichkeit eines N1-Bezugs in den entsprechenden Items. Aus diesem Grund wurden die entsprechenden Items im Datensatz belassen.

\subsubsection{Auswertung}

Alle statistischen Berechnungen und Plots wurden mit R 3.2 (R Core Team 2015) vorgenommen. Für die Plots wurde das R-Paket „ggplot2“ (Wickham 2009) verwendet, für die Linear Mixed-Effects Models das Paket „lme4“ (Bates et al. 2015).

\section{Deskriptive Auswertung des kompletten Datensatzes}

Der komplette, aber unausgeglichene Datensatz (vor der Aufbereitung durch Kürzung) wird zumindest für eine deskriptive Auswertung herangezogen, da dieser eine größere Stichprobe abbildet.

\begin{tabular}{ccccc}
\hline \hline Faktor & Bed. & MW & Std. Abw. & n \\
\hline \multirow{4}{*}{ STRUKTUR } & RK & 0.26 & 0.44 & 275 \\
& WK & 0.22 & 0.42 & 265 \\
& Mono & 0.84 & 0.37 & 281 \\
\hline \hline
\end{tabular}

Tabelle 6.2: Deskriptive Statistik der relativen Häufigkeit der N1-Bezüge auf dem gesamten Datensatz für den Faktor sTRUKTUR

Wie in Tabelle 6.2 und Abbildung 6.1 zu sehen, zeigt sich eine Verteilung der mittleren Werte des Ratings gemäß der Hypothese (MW(Mono) $>\mathrm{MW}(\mathrm{RK})>\mathrm{MW}(\mathrm{WK}))$. Besonders deutlich zeigt sich hier der Abstand zwischen direkter (Bed. Mono) und indirekter Anapher (Bed. RK und WK).

\section{Deskriptive Auswertung des gekürzten Datensatzes}

Im nächsten Schritt erfolgt eine deskriptive Auswertung des aufbereiteten, d.h. gekürzten Datensatzes. 


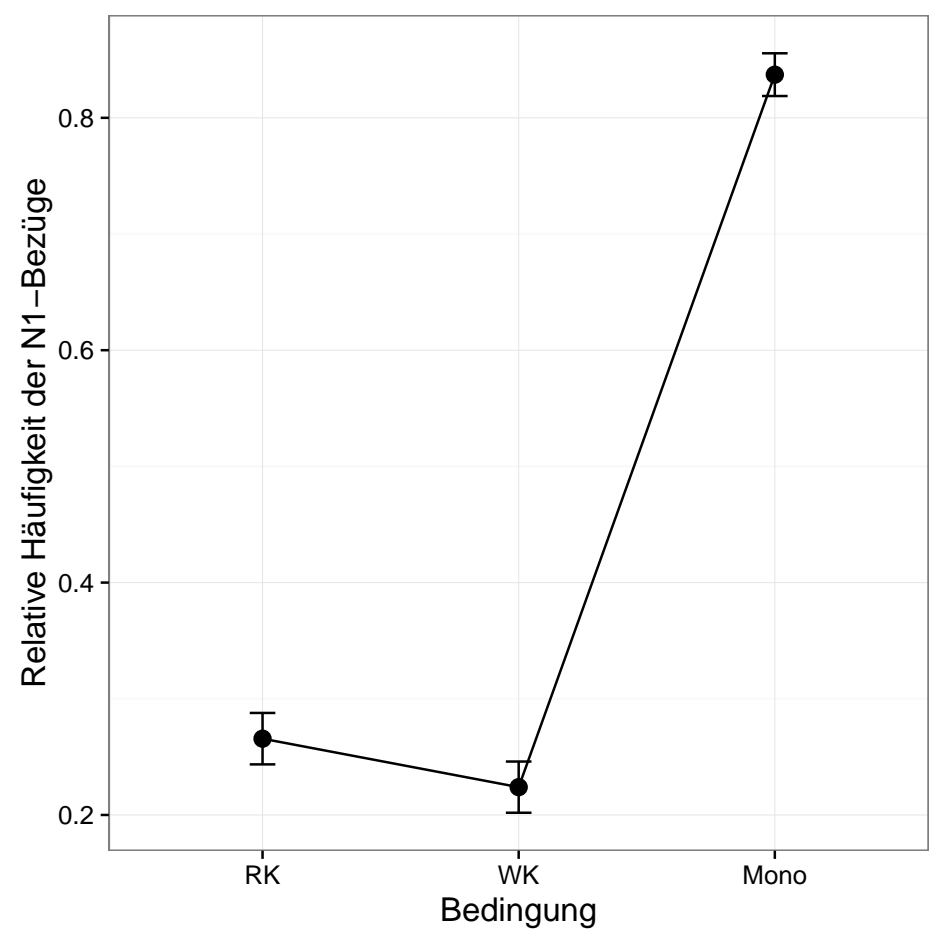

Abbildung 6.1: Plot der um die Zwischensubjektvarianz korrigierten Mittelwerte der N1Bezüge ( $\mathrm{y}$-Achse) in Abhängigkeit vom Faktor sTRUKTUR (x-Achse) auf dem gesamten Datensatz; die Fehlerbalken repräsentieren +/-1 Standardfehler des Mittelwerts

\begin{tabular}{ccccc}
\hline \hline Faktor & Bed. & MW & Std. Abw. & n \\
\hline \multirow{4}{*}{ STRUKTUR } & RK & 0.24 & 0.43 & 236 \\
& WK & 0.23 & 0.42 & 236 \\
& Mono & 0.84 & 0.37 & 236 \\
\hline \hline
\end{tabular}

Tabelle 6.3: Deskriptive Statistik der relativen Häufigkeit der N1-Bezüge auf dem gekürzten Datensatz für den Faktor STRUKTUR

Der Unterschied zwischen Bedingung RK und WK ist hierbei geringer als beim kompletten Datensatz, jedoch ebenfalls in prognostizierter Richtung ausgeprägt (siehe Tab. 6.3). Auch hier ist der Abstand zwischen Bedingung Mono und den Kompositabedingungen deutlich sichtbar.

\section{Inferenzstatistik für den gekürzten Datensatz}

Die Berechnungen für die Inferenzstatistik erfolgten auf dem gekürzten Datensatz unter Verwendung von LMEMs (vgl. Barr et al. 2013) mit Items und VPn als ,random factors ${ }^{6}$ und der experimentellen Bedingung als ,fixed factor'. Da die komplette RandomEffects-Struktur nicht konvergiert und auch eine höhere Iteration sowie die Entfernung der Korrelation kein Konvergieren ermöglicht, erfolgte die Berechnung letztlich ohne Einbeziehung der ,random slopes 4 Der experimentelle Faktor STRUKTUR zeitigt darin ein

${ }^{4}$ Modellgleichung in $\mathrm{R}$ :

Generalized linear mixed model fit by maximum likelihood (Laplace Approximation) 


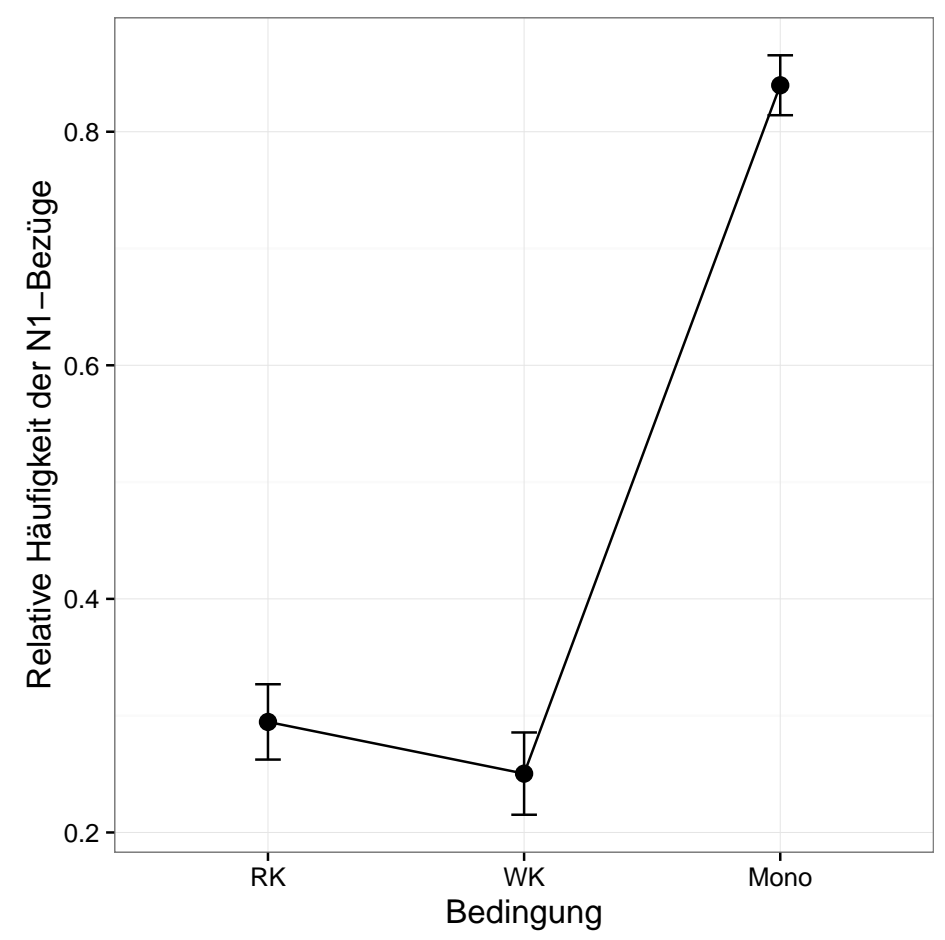

Abbildung 6.2: Plot der Mittelwerte der relativen Häufigkeit der N1-Bezüge auf dem gekürzten Datensatz für den Faktor sTRUKTuR

signifikantes Ergebnis (siehe Tab. 6.4).5 Der Modellvergleich ist in Tabelle 6.5 wiedergegeben. ${ }^{6}$

\begin{tabular}{c|cc|cc}
\hline \hline Faktor & $|z|$ & $p$ & $|z|$ & $p$ \\
\hline STRUKTUR & 7.713 & $<0.001$ & 8.023 & $<0.001$ \\
\hline \hline
\end{tabular}

Tabelle 6.4: Inferenzstatistik (LMEM) für die N1-Bezüge auf dem gekürzten Datensatz für den Faktor strukTur, Kontraste (RK)/(Mono) und (WK)/(Mono)

\begin{tabular}{c|cc}
\hline \hline Faktor & $L R \chi_{d f=2}^{2}$ & $p$ \\
\hline STRUKTUR & 317.88 & $<0.001$ \\
\hline \hline
\end{tabular}

Tabelle 6.5: Inferenzstatistik (Modellvergleich) auf dem gekürzten Datensatz für den Faktor STRUKTUR

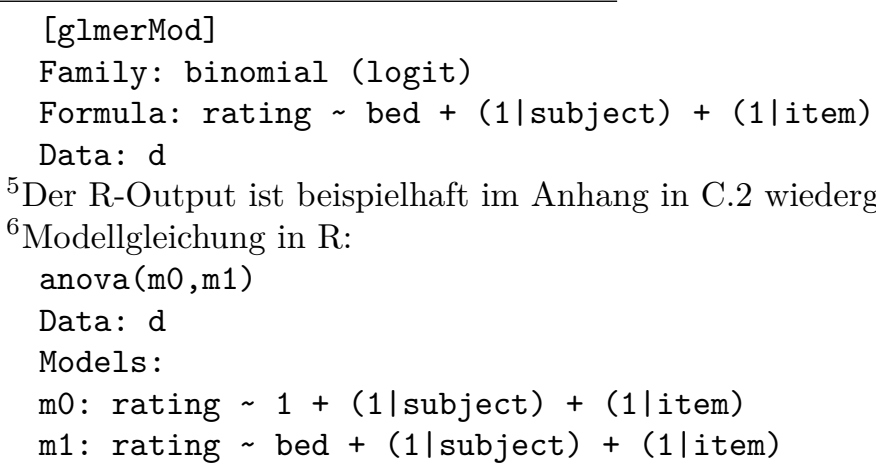


Die Berechnungen wurden auf den geplanten Kontrasten (RK)/(Mono) und (WK)/(Mono) durchgeführt und zusätzlich auf den geplanten Kontrasten (WK)/(RK) und (Mono)/(RK) wiederholt. Die Ergebnisse hierfür bewegen sich auf dem gleichen Signifikanzniveau.

Anschließend wurde die Kontrollbedingung Mono aus den Daten entfernt und nur die kritischen Bedingungen RK und WK in das Modell sowie in den Modellverlgeich einbezogen. Der Unterschied zwischen den beiden Bedingungen ist nicht signifikant (vgl. Tab. 6.6 und 6.7).

\begin{tabular}{c|cc}
\hline \hline Faktor & $|z|$ & $p$ \\
\hline STRUKTUR & 0.263 & 0.792 \\
\hline \hline
\end{tabular}

Tabelle 6.6: Inferenzstatistik (LMEM) für die N1-Bezüge auf dem gekürzten Datensatz für den Faktor strukTur ohne Bed. Mono

\begin{tabular}{c|cc}
\hline \hline Faktor & $L R \chi_{d f=1}^{2}$ & $p$ \\
\hline STRUKTUR & 0.0734 & 0.7865 \\
\hline \hline
\end{tabular}

Tabelle 6.7: Inferenzstatistik (Modellvergleich) für die N1-Bezüge auf dem gekürzten Datensatz für den Faktor STRUKTUR ohne Bed. Mono

\section{Interpretation}

Die Daten der deskriptiven Auswertung entsprechen der Gesamthypothese MW(Mono) > $\mathrm{MW}(\mathrm{RK})>\mathrm{MW}(\mathrm{WK})$. Der Vorteil für die Bedingung Mono ist dabei signifikant. Da sich die Kontrollbedingung wie erwartet verhält, kann das experimentelle Design als reliabel interpretiert werden.

Der Unterschied zwischen RK und WK ist nicht signifikant, verhält sich jedoch deskriptiv wie prognostiziert, d.h. in der Bedingung RK liegen mehr N1-Bezüge vor als in der Bedingung WK. Dies steht im Gegensatz zu den Ergebnissen aus Experiment 2.

Demnach hat die Überarbeitung der Items, die Fokussierung auf den grammatischen Faktor und die Kontrolle möglichst vieler konzeptueller Einflussgrößen das erwartete Ergebnis in der Satzergänzungsaufgabe deskriptiv zutage gefördert. Dies spricht für die Interpretation der Ergebnisse aus Experiment 2 als Überlagerung von Faktoren.

Allerdings zeigen sich bei den Berechnungen für die Inferenzstatistik erneut die Nachteile kategorialer Daten und die Notwendigkeit der Überprüfung durch ein anderes Paradigma.

\subsubsection{Weitere Analysen}

Weitere mögliche Faktoren wurden zwischen den Items bestmöglich ausbalanciert und annotiert. Die so erfassten Eigenschaften können nun ebenfalls für eine Analyse herangezogen werden. Dabei gilt es zu bedenken, dass diese Faktoren nicht im Fokus des Designs stehen und daher nicht ideal verteilt sind. Die aus den Analysen gewonnenen Ergebnisse sind daher lediglich als Indizien zu betrachten.

Die folgenden Analysen wurden auf dem gekürzten Datensatz durchgeführt, da in diesem die Verteilung der Eigenschaften gleichmäßiger ist. 


\section{Raum-zeitliche Kontiguität (RZK)}

\section{Hypothese}

In Experiment 2 wurde neben dem Faktor STRUKTUR die RZK der Komposita-Konstituenten untersucht. In Experiment 2 handelte es sich dabei um einen Faktor ,within items ${ }^{6}$, in Experiment 3 wurde er zwischen den Items bestmöglich ausbalanciert, wobei der Einfluss des Satzkontexts bei der Annotierung einbezogen wurde. Die Hypothese zur RZK ist nun - wie auch in Experiment 2 - ein Zugänglichkeitsvorteil für das N1 im Falle der notwendigen konzeptuellen Präsenz des N1. Demnach werden für die Bedingung + RZK mehr N1-Bezüge erwartet als für die Bedingung -RZK. Die Hypothese lautet somit: $\mathrm{MW}(+\mathrm{RZK})>\mathrm{MW}(-\mathrm{RZK})$.

Die Auswertung erfolgt ausschließlich unter Berücksichtigung der Bedingungen RK und WK, da der Faktor nur für Komposita gültig ist.

\section{Ergebnisse}

Die Ergebnisse der deskriptiven Auswertung der RZK sind in Tabelle 6.8 und Abbildung 6.3 dargestellt. Es zeigt sich wie erwartet ein höherer Mittelwert für die Bedingung + RZK.

\begin{tabular}{ccccc}
\hline \hline Faktor & Bed. & MW & Std. Abw. & $\mathrm{n}$ \\
\hline \multirow{2}{*}{ RZK } & - RZK & 0.21 & 0.41 & 206 \\
& +RZK & 0.26 & 0.44 & 266 \\
\hline \hline
\end{tabular}

Tabelle 6.8: Deskriptive Statistik der relativen Häufigkeit der N1-Bezüge auf dem gekürzten Datensatz für den Faktor RZK

\begin{tabular}{c|cc}
\hline \hline Faktor & $|z|$ & $p$ \\
\hline RZK & 0.662 & 0.508 \\
\hline \hline
\end{tabular}

Tabelle 6.9: Inferenzstatistik (LMEM) für die N1-Bezüge auf dem gekürzten Datensatz für den Faktor RZK

Die Inferenzstatsitk mittels LMEMs mit kompletter Random-Effects-Struktur fördert jedoch kein signifikantes Ergebnis zutage (vgl. Tab. 6.9).

\section{Interpretation}

Die Verteilung der Anzahl an N1-Bezügen auf die Bedingungen +/-RZK entspricht dem prognostizierten Muster und bestätigt damit die Ergebnisse aus Experiment 2. Die fehlende Signifikanz dieses Ergebnisses könnte darauf gründen, dass die Items nicht auf die Analyse dieses Faktors ausgelegt sind. Der Faktor ist hier ,between items ${ }^{6}$ kontrolliert und im Vergleich zu Experiment 2 weniger deutlich ausgeprägt. Bei der Erstellung der Items für Experiment 3 lag der Fokus auf der semantischen Ähnlichkeit zwischen den strukturell verschiedenen Komposita und der Plausibilität der Satzeinbettung. 


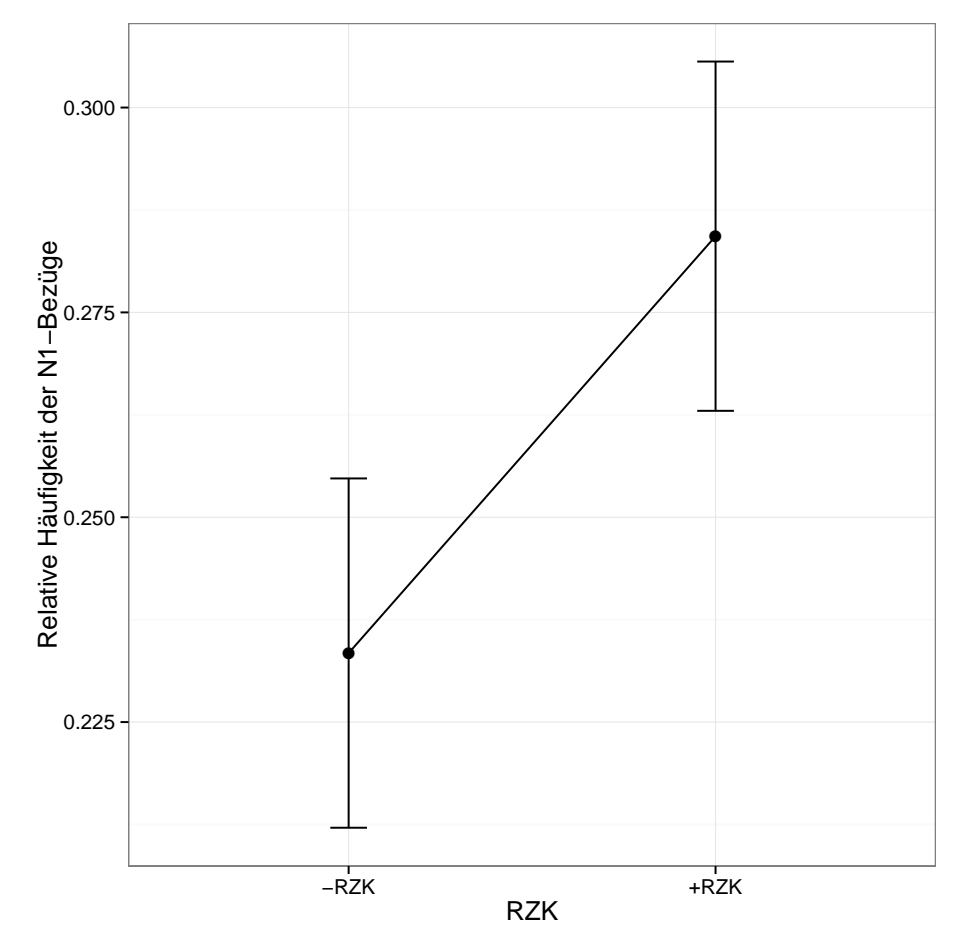

Abbildung 6.3: Plot der Mittelwerte der relativen Häufigkeit der N1-Bezüge auf dem gekürzten Datensatz für den Faktor RZK

\section{N2-Konkretheit}

\section{Hypothese}

Ein weiterer konzeptueller Faktor ist die N2-KOnkRETheIT. Das N1 ist in Experiment 3 immer konkret. Tritt nun ein abstraktes N2 zusammen mit einem konkreten N1 auf, verschiebt sich - so die Annahme aus Kapitel 3.3.5 - der konzeptuelle Fokus des Kompositums in Richtung N1 und dieses ist besser zugänglich. Daraus resultiert folgende Hypothese für die den Faktor N2-KONKRETHEIT: MW(-konkret) > MW(+konkret).

Die Berechnungen erfolgen wieder nur auf den Bedingungen RK und WK, da in Bedingung Mono nur eine Konstituente vorliegt.

\section{Ergebnisse}

Die Ergebnisse der deskriptiven Auswertung der N2-KONKRETHEIT spiegeln die Annahmen der Hypothese wider (siehe Tab. 6.10 und Abb. 6.4): Im Fall abstrakter N2 liegen mehr N1-Bezüge vor.

\begin{tabular}{ccccc}
\hline \hline Faktor & Bed. & MW & Std. Abw. & $\mathrm{n}$ \\
\hline \multirow{2}{*}{ N2-KONKRETHEIT } & -konkret & 0.29 & 0.46 & 237 \\
& +konkret & 0.18 & 0.39 & 235 \\
\hline \hline
\end{tabular}

Tabelle 6.10: Deskriptive Statistik der relativen Häufigkeit der N1-Bezüge auf dem gekürzten Datensatz für den Faktor N2-KONKRETHEIT 


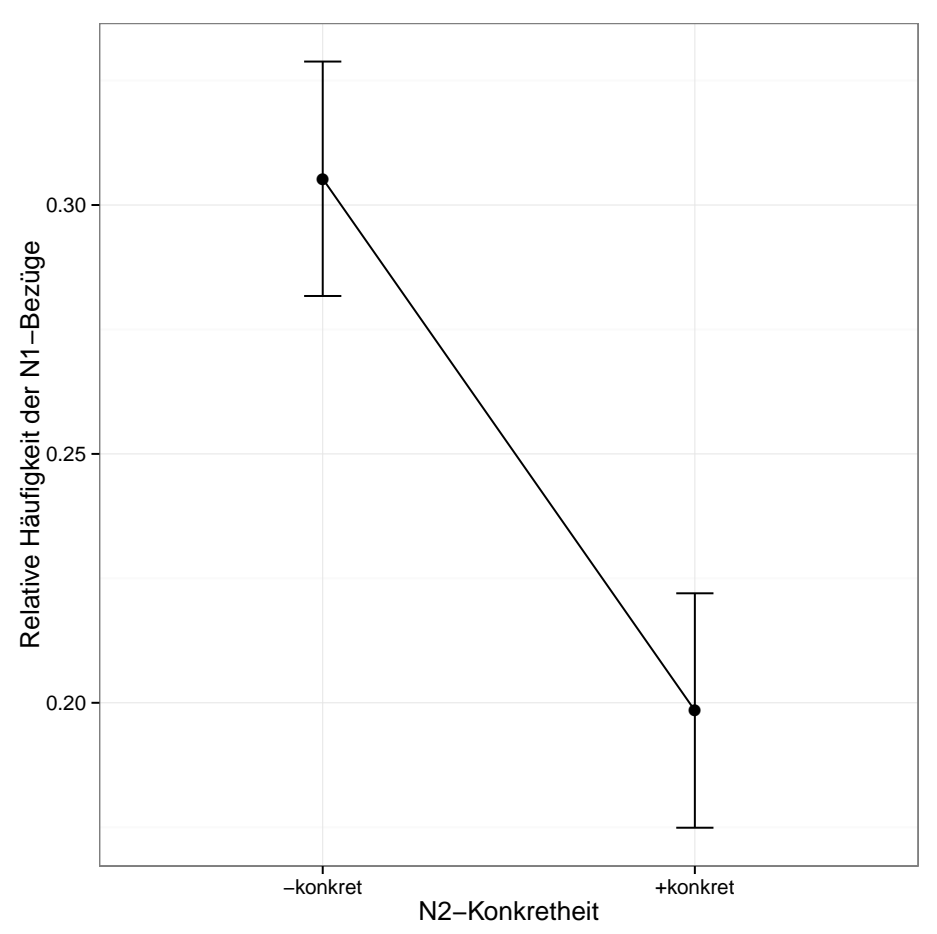

Abbildung 6.4: Plot der Mittelwerte der relativen Häufigkeit der N1-Bezüge auf dem gekürzten Datensatz für den Faktor N2-KONKRETHEIT

Bei der inferenzstatistischen Auswertung ergaben sich bei den LMEMs mit kompletter Random-Effects-Struktur Konvergenzprobleme. Die Ergebnisse der Berechnung ohne ,random slopes ${ }^{6}$ sind in Tabelle 6.11 wiedergegeben.

\begin{tabular}{c|cc}
\hline \hline Faktor & $|z|$ & $p$ \\
\hline N2-KONKRETHEIT & 2.591 & $<0.01$ \\
\hline \hline
\end{tabular}

Tabelle 6.11: Inferenzstatistik für die N1-Bezüge auf dem gekürzten Datensatz für den Faktor N2-KONKRETHEIT

\section{Interpretation}

Der Einfluss des Faktors N2-KONKRETHEIT kann in prognostizierter Richtung bestätigt werden. Dadurch werden auch die damit in Zusammenhang stehenden Annahmen zum Einfluss des konzeptuellen Fokus gestützt.

\section{Nominalisierungslesart}

\section{Hypothese}

Der Faktor N2-KONKRETHEIT lässt sich auch im Subset der Rektionskomposita betrachten. Diese liegen in Experiment 3 ausschließlich in Form von ung-Nominalisierungen vor, die sich wiederum aufgrund von Wortsemantik und Kontext in Prozess- und ResultatsNominalisierungen unterscheiden lassen. In Hinblick auf den Einfluss der Konkretheit 
können Prozesse wie abstrakte Nomen und Resultate wie konkrete aufgefasst werden. Gemäß der Hypothese zur N2-KONKRETHEIT besteht nun die Annahme, dass im Fall von Prozess-Nominalisierungen mehr N1-Bezüge vorliegen: MW(Prozess) > MW(Resultat).

\section{Ergebnisse}

Die Ergebnisse der deskriptiven Auswertung (siehe Tab. 6.12 bestätigen die Hypothese: Im Fall einer Prozesslesart liegen mehr N1-Bezüge vor.

\begin{tabular}{ccccc}
\hline \hline Faktor & Bed. & MW & Std. Abw. & $\mathrm{n}$ \\
\hline \multirow{2}{*}{ NOM.-LESART } & Resultat & 0.22 & 0.41 & 106 \\
& Prozess & 0.26 & 0.44 & 130 \\
\hline \hline
\end{tabular}

Tabelle 6.12: Deskriptive Statistik der relativen Häufigkeit der N1-Bezüge auf dem gekürzten Datensatz für den Faktor NOMINALISIERUnGSLESART

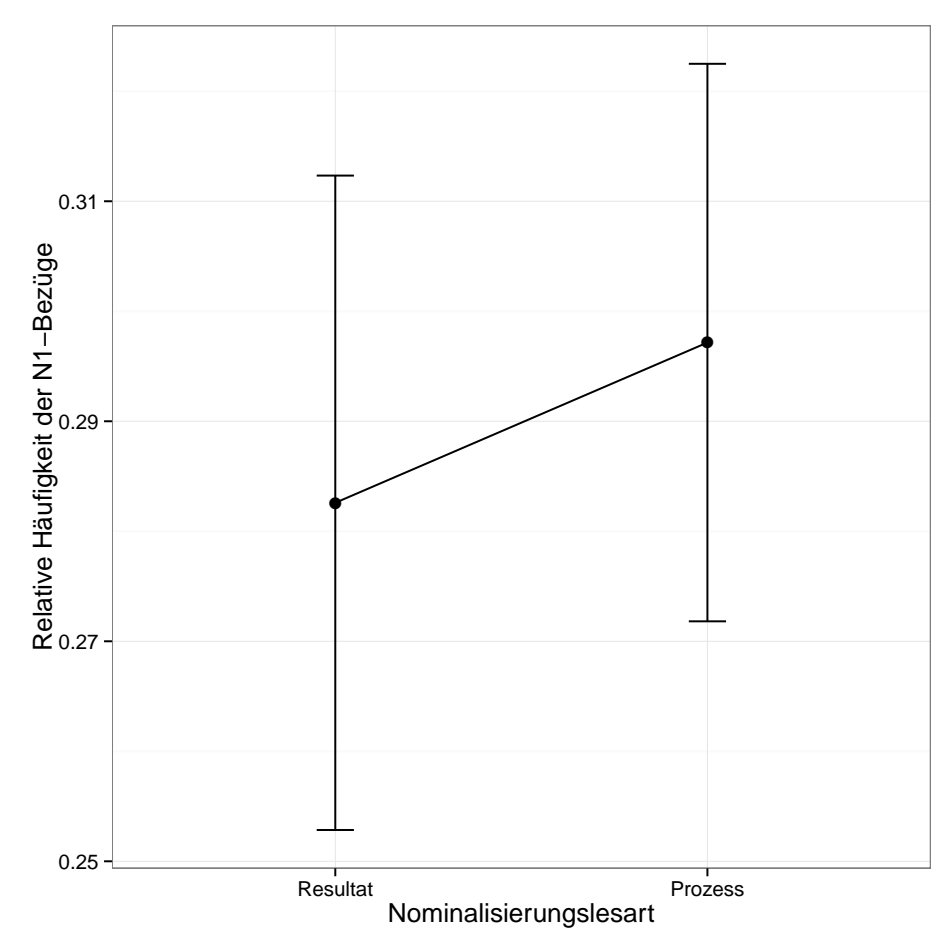

Abbildung 6.5: Plot der Mittelwerte der relativen Häufigkeit der N1-Bezüge auf dem gekürzten Datensatz für den Faktor NOMINALISIERUNGSLESART

Bei der inferenzstatistischen Auswertung treten erneut Konvergenzprobleme auf. Da in diese Auswertung lediglich die Items in der Bedingung RK einbezogen wurden, kann die dadurch verminderte Itemzahl eine Ursache sein. Das Ergebnis der Auswertung ohne ,random slopes' ist nicht signifikant (siehe Tab. 6.13). 


\begin{tabular}{c|cc}
\hline \hline Faktor & $|z|$ & $p$ \\
\hline NOM.-LESART & 0.404 & 0.686 \\
\hline \hline
\end{tabular}

Tabelle 6.13: Inferenzstatistik für die N1-Bezüge auf dem gekürzten Datensatz für den Faktor NOMINALISIERUNGSLESART

\section{Interpretation}

Deskriptiv stützt die Auswertung der NOMINALISIERUnGSLESART die Ergebnisse der Auswertung der N2-KONKRETHEIT. Wie erwartet liegen mehr N1-Bezüge im Fall von ProzessNominalisierungen vor. Die inferenzstatistische Auswertung ist nicht signifikant. Dies kann zum einen am subtilen Unterschied liegen, der für das Satzverständnis nicht notwendigerweise aufgelöst werden muss. Zum anderen kann es auch ein Problem des Datenmaterials sein: Da der Faktor nur in einer Bedingung vorliegt, konnte nur ein Drittel der Daten zur Auswertung herangezogen werden. Die geringe Datenmenge führt wiederum zu Konvergenzproblemen.

\section{N1-Zählbarkeit}

\section{Hypothese}

Wie in Kapitel 3.2.2 dargestellt, vereint der Faktor N1-ZÄHLBARKEIT zwei gegenläufige Hypothesen in sich. In Bezug auf den konzeptuellen Einfluss des Faktors ist aufgrund eines höheren Grads an Unikalität mit einem Vorteil für die N1-Zugänglichkeit im Fall von Individualnomen an der N1-Position zu rechnen, vergleichbar mit konkreten N1. Bezogen auf den grammatischen Einfluss ist aufgrund determiniererloser Referenzfähigkeit mit einem Vorteil für Massenomen zu rechnen. In Experiment 2 wurde deskriptiv ein Vorteil für Individualnomen gefunden (vgl. Kap. 5.7.4), welcher als Indiz für einen stärkeren Einfluss konzeptueller Faktoren interpretiert wurde. Auch in diesem Experiment soll der Einfluss der N1-ZÄHLBARKEIT auf die beiden Kompositabedingungen betrachtet werden.

\section{Ergebnisse}

In der deskriptiven Auswertung (siehe Tab. 6.14) kann kein Unterschied zwischen den Bedingungen festgestellt werden.

\begin{tabular}{ccccc}
\hline \hline Faktor & Bed. & MW & Std. Abw. & $\mathrm{n}$ \\
\hline \multirow{2}{*}{ N1-ZÄHLBARKEIT } & Individualnom. & 0.24 & 0.43 & 274 \\
& Massenom. & 0.24 & 0.43 & 198 \\
\hline \hline
\end{tabular}

Tabelle 6.14: Deskriptive Statistik der relativen Häufigkeit der N1-Bezüge auf dem gekürzten Datensatz für den Faktor N1-ZÄHLBARKEIT 


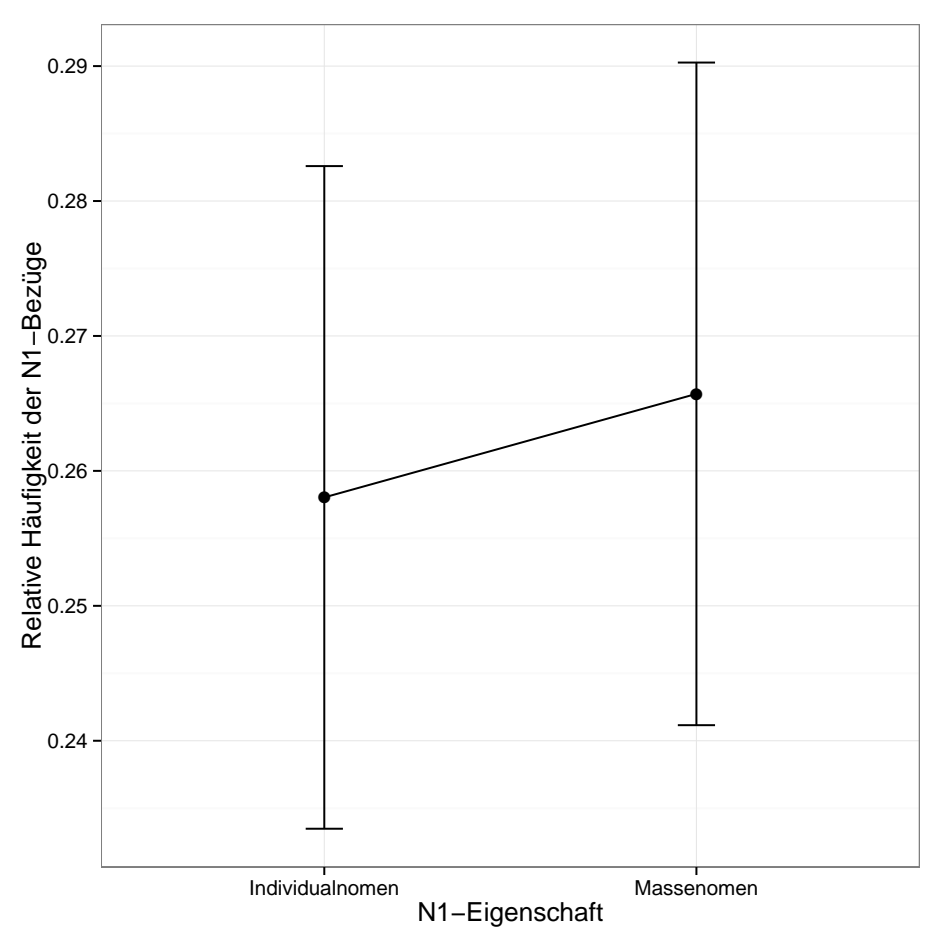

Abbildung 6.6: Plot der Mittelwerte der relativen Häufigkeit der N1-Bezüge auf dem gekürzten Datensatz für den Faktor N1-ZÄHLBARKEIT

Für die LMEMs ist ein Konvergieren nur unter Ausschluss der ,random slopes ${ }^{6}$ möglich; das Ergebnis wird dabei als nicht signifikant ausgewiesen (vgl. Tab. 6.15).

\begin{tabular}{c|cc}
\hline \hline Faktor & $|z|$ & $p$ \\
\hline N1-ZÄHLBARKEIT & 0.41 & 0.682 \\
\hline \hline
\end{tabular}

Tabelle 6.15: Inferenzstatistik für die N1-Bezüge auf dem gekürzten Datensatz für den Faktor N1-ZÄHLBARKEIT

\section{Interpretation}

Für den Faktor N1-ZÄHLBARKEIT konnte kein Unterschied zwischen den beiden Bedingungen festgestellt werden. Dieses Ergebnis legt nahe, dass die Eigenschaft der Zählbarkeit des N1 bei den vorliegenden Komposita keinen Einfluss auf die N1-Zugänglichkeit ausübt.

\section{Genus}

\section{Hypothese}

Wie bereits in Experiment 2 deutlich wurde, sind Satzergänzungsaufgaben sensibel für Genus-Unterschiede zwischen Pronomen. So wurde in Experiment 2 eine Präferenz für die Resolution des Neutrum-Pronomens als Vorfeld-es und Komplexanapher festgestellt. Diese frequente Form der Resolution des Neutrum-Pronomens und die Abwesenheit einer derartigen Strategie für das Maskulinum-Pronomen führte zu einem Mehr an N1-Bezügen 
im Falle der Maskulina. Ein entsprechendes Ergebnis wird auch für das vorliegende Experiment erwartet: MW(mask/er) > MW(neutr/es).

Auch bei den Berechnungen zum Faktor Genus wurde die Bedingung Mono ausgeschlossen.

\section{Ergebnisse}

Die Ergebnisse der deskriptiven Auswertung stützen die Hypothese (siehe Tab. 6.16). Für das maskuline Pronomen liegt ein deutlich höherer Mittelwert vor.

\begin{tabular}{ccccc}
\hline \hline Faktor & Bed. & MW & Std. Abw. & $\mathrm{n}$ \\
\hline \multirow{2}{*}{ GENUS } & $\mathrm{m}$ & 0.31 & 0.46 & 214 \\
& $\mathrm{n}$ & 0.17 & 0.38 & 258 \\
\hline \hline
\end{tabular}

Tabelle 6.16: Deskriptive Statistik der relativen Häufigkeit der N1-Bezüge auf dem gekürzten Datensatz für den Faktor GENus

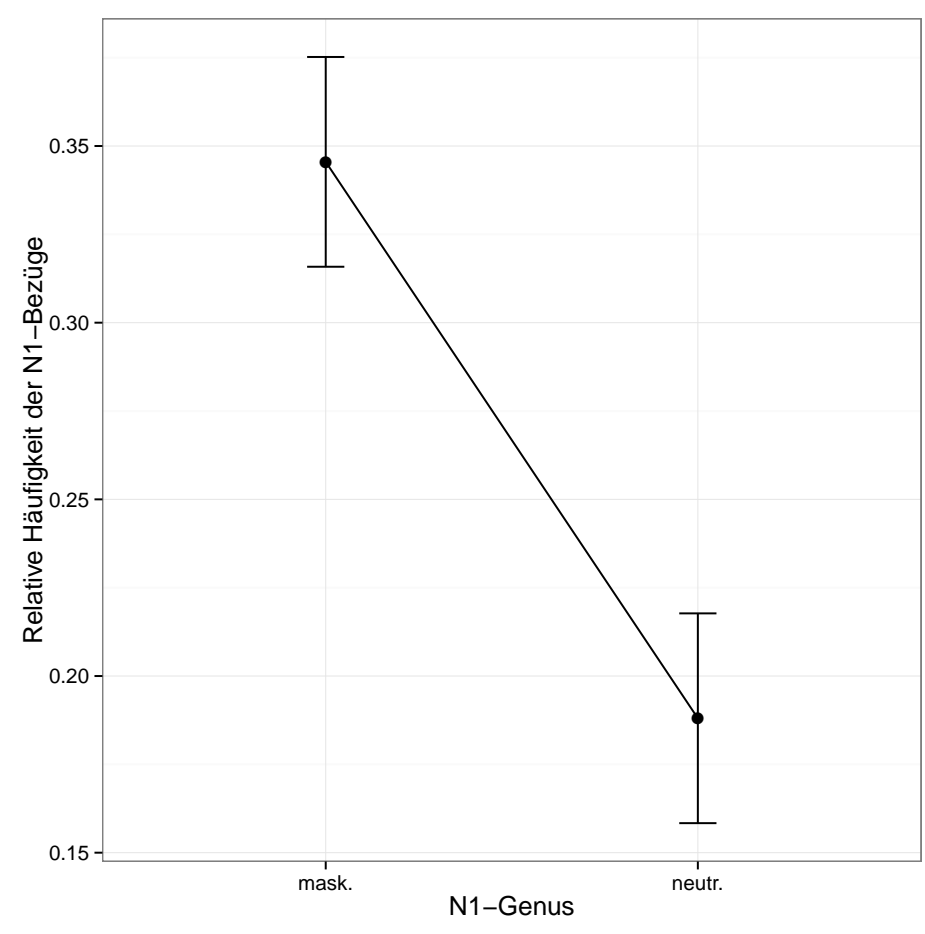

Abbildung 6.7: Plot der Mittelwerte der relativen Häufigkeit der N1-Bezüge auf dem gekürzten Datensatz für den Faktor GENus

In der Inferenzstatistik zeigt sich ein klarer Effekt (siehe Tab. 6.80, Berechnungen ohne ,random slopes $\left.{ }^{6}\right)$.

\begin{tabular}{c|cc}
\hline \hline Faktor & $|z|$ & $p$ \\
\hline GENUS & 584.0 & $<0.001$ \\
\hline \hline
\end{tabular}

Tabelle 6.17: Inferenzstatistik für die N1-Bezüge auf dem gekürzten Datensatz für den Faktor GENUS 


\section{Interpretation}

Die Ergebnisse der Auswertung des GENUs stützen die Ergebnisse aus Experiment 2: Das Neutrum-Pronomen wird präferiert als Vorfeld-es interpretiert, was die Zahl der N1Bezüge mindert. Die Betrachtung der Satzergänzungen stützt diese Interpretation. Für das Maskulinum-Pronomen gibt es keine entsprechende Resolutionsalternative. Wenn in dem Fall der N1-Bezug umgangen wird, wird zumeist eine weitere Person in den Diskurs eingeführt. Das Zur-Verfügung-Stehen einer frequenten Resolutionsalternative beeinflusst somit die Anzahl der N1-Bezüge.

\section{Frequenz}

Abschließend soll ein Blick auf den Einfluss verschiedener Frequenzen auf die N1-Zugänglichkeit geworfen werden, da diese im Gegensatz zu Experiment 2 hier weniger stark kontrolliert wurden. Dabei wird zum einen die N1-FREQUENZ und zum anderen die NNFREQUENZ ausgewertet. Die Daten basieren auf den Häufigkeitsklassen des Leipziger Wortschatzes (LWS)7 Die Berechnungen erfolgen auf Basis der beiden Kompositabedingungen.

\section{N1-Frequenz}

\section{Hypothese}

Zur Analyse der N1-FREQUENZ wurden die Items in drei Gruppen aufgeteilt. Die erste Gruppe umfasst die N1 mit den höchsten Frequenzen (Häufigkeitsklassen 6- 8, 6 Items), die zweite Gruppe umfasst die mittleren Frequenzen (Häufigkeitsklassen 9 - 10, 12 Items) und die dritte Gruppe umfasst die Ausdrücke mit den niedrigsten Frequenzen (Häufigkeitsklassen 11 - 13, 6 Items).

Eine mögliche Hypothese zum Einfluss der N1-Frequenz ist, dass ein N1 mit hoher Frequenz stärker aktiviert und somit besser zugänglich ist (zu diesem Zusammenhang siehe auch Kap. 2.3.4 und Baayen/Schreuder (1999)). Dies steht der Hypothese zum Aktiviertheitsvorteil durch , deep processing' entgegen. Die hier als niedrig frequent klassifizierten Ausdrücke sind jedoch keine seltenen oder markierten Ausdrücke, sondern lediglich im Vergleich zu den anderen weniger frequent, weshalb ich nicht von einem Einfluss der Verarbeitungstiefe ausgehe. Die Hypothese zur N1-FREQUENZ lautet somit: MW(hoch) > $\mathrm{MW}($ mittel $)>\mathrm{MW}$ (niedrig).

\section{Ergebnisse}

Die deskriptive Auswertung der N1-FREQUEnZ ergibt das Muster MW(niedrig) > MW(mittel) > MW(hoch), was gegenläufig zur Hypothese ist (siehe Tab. 6.18).

\footnotetext{
${ }^{7}$ LWS: German newspaper corpus based on material crawled in 2011 (deu_newscrawl_2011). (C) 2016 Abteilung Automatische Sprachverarbeitung, Universität Leipzig. www.wortschatz.uni-leipzig.de/ (23.08.2016).
} 


\begin{tabular}{ccccc}
\hline \hline Faktor & Bed. & MW & Std. Abw. & n \\
\hline \multirow{3}{*}{ N1-FREQUENZ } & hoch & 0.19 & 0.39 & 117 \\
& mittel & 0.25 & 0.43 & 238 \\
& niedrig & 0.26 & 0.44 & 117 \\
\hline \hline
\end{tabular}

Tabelle 6.18: Deskriptive Statistik der relativen Häufigkeit der N1-Bezüge auf dem gekürzten Datensatz für den Faktor N1-FREQUENZ

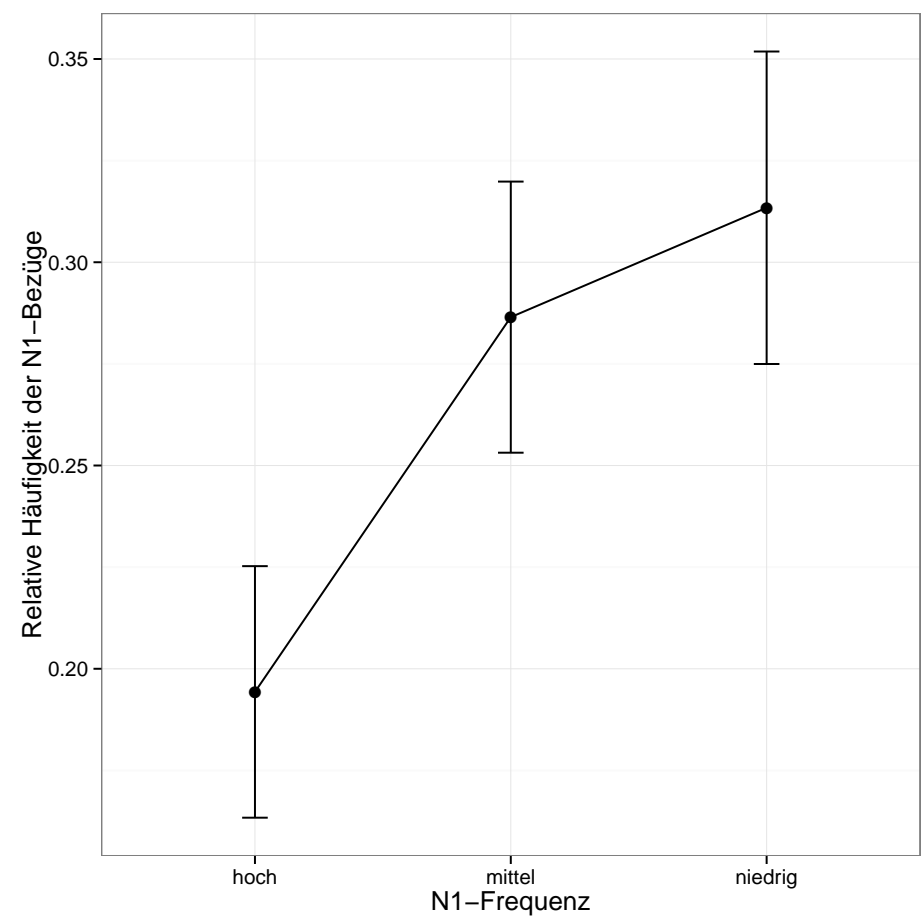

Abbildung 6.8: Plot der relativen Häufigkeit der N1-Bezüge auf dem gekürzten Datensatz für den Faktor N1-FREQUENZ

Die Inferenzstatistik (ohne,random slopes ${ }^{6}$ ) weist dieses Ergebnis nicht als signifikant aus (vgl. Tab. 6.19).

\begin{tabular}{c|cc|cc}
\hline \hline Faktor & $|z|$ & $p$ & $|z|$ & $p$ \\
\hline N1-FREQUENZ & 0.618 & 0.536 & 0.899 & 0.368 \\
\hline \hline
\end{tabular}

Tabelle 6.19: Inferenzstatistik für die N1-Bezüge auf dem gekürzten Datensatz für den Faktor N1-FREQUENZ

\section{Interpretation}

Die der Hypothese widersprechende Verteilung der Mittelwerte ist entweder dem Zufall zuzuschreiben (siehe Inferenzstatistik und stark schwankende Fallzahlen) oder niedrigfrequente N1 sind tendenziell zugänglicher. Sollte dies der Fall sein, würde dies eine Interpretation gemäß der Idee des , deep processings ${ }^{6}$ nahelegen. Ein derartiger Einflss kann nur 
durch ein speziell darauf ausgerichtetes Experiment belegt werden. Ich tendiere eher zur Annahme einer zufälligen Verteilung, da die Einzelfrequenzen nicht so stark aufgefächert sind, als das überhaupt eine Varianz in der Verarbeitungstiefe möglich wäre.

\section{NN-Frequenz}

\section{Hypothese}

Ein möglicher Einfluss der NN-Frequenz könnte darin liegen, dass eine hohe NN-Frequenz eine Konsensualisierung und dadurch Bedeutungskonsolidierung auslöst, was letztlich eine Voraussetzung für Lexikalisierung ist. Das N1 sollte in solchen Fällen schlechter zugänglich sein. Allerdings gibt es auch Komposita mit hoher NN-Frequenz, die über eine kompositionale Bedeutung verfügen (vgl. Diskussion Kap. 2.3.4). Da die Items in diesem Experiment hinsichtlich Kompositionalität und damit Lexikalisiertheit kontrolliert wurden, prognostiziere ich keinen Einfluss der NN-Frequenz auf die Zugänglichkeit des N1.

Die Frequenzen aller Ankerausdrücke wurden analog zur N1-Frequenz aus dem LWS ermittelt und in drei Gruppen aufgeteilt. Die Gruppe ,hoch` besteht dabei ausschließlich aus Monolexemen. Für die Fragestellung zur NN-FREQUENZ sind nur die beiden Kompositabedingungen relevant. Aus diesem Grund befinden sich in der Auswertung nur die Gruppen ,mittel' und ,niedrig6.

\section{Ergebnisse}

Die deskriptive Auswertung legt einen Zugänglichkeitsvorteil für die Gruppe ,niedrig' nahe (siehe Tab. 6.20). In der Inferenzstatistik (komplette Random-Effects-Struktur) zeitigt der Unterschied jedoch kein signifikantes Ergebnis.

\begin{tabular}{ccccc}
\hline \hline Faktor & Bed. & MW & Std. Abw. & n \\
\hline \multirow{2}{*}{ NN-FREQUENZ } & mittel & 0.21 & 0.41 & 248 \\
& niedrig & 0.27 & 0.44 & 224 \\
\hline \hline
\end{tabular}

Tabelle 6.20: Deskriptive Statistik der relativen Häufigkeit der N1-Bezüge auf dem gekürzten Datensatz für den Faktor NN-FREQUENZ

\begin{tabular}{c|cc}
\hline \hline Faktor & $|z|$ & $p$ \\
\hline NN-FREQUENZ & 0.978 & 0.328 \\
\hline \hline
\end{tabular}

Tabelle 6.21: Inferenzstatistik für die N1-Bezüge auf dem gekürzten Datensatz für den Faktor NN-FREQUENZ

\section{Interpretation}

Ein Einfluss der NN-FREQUENZ kann anhand der Inferenzstatistik nicht gefunden werden. Die deskriptiven Daten sprechen allerdings für einen Vorteil im Fall von niedrig-frequenten NN-Komposita. Dies könnte bedeuten, dass entsprechende Komposita tatsächlich weniger 


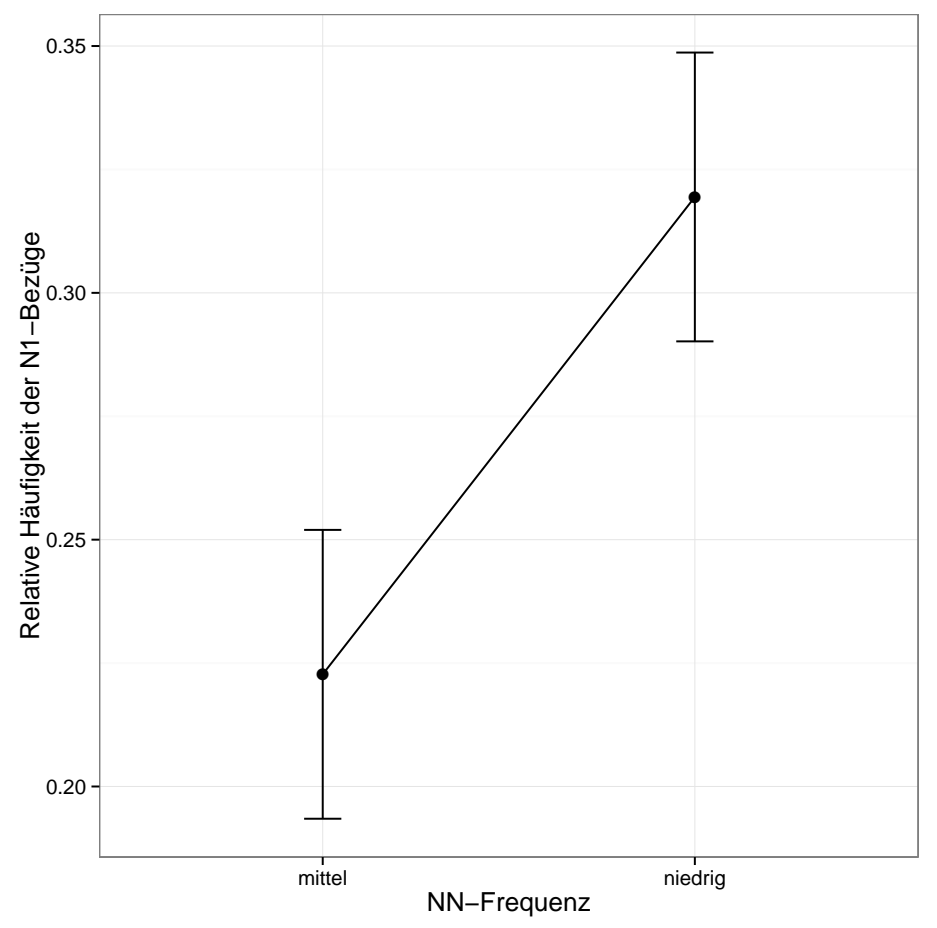

Abbildung 6.9: Plot der Mittelwerte der relativen Häufigkeit der N1-Bezüge auf dem gekürzten Datensatz für den Faktor NN-FREQUENZ

stark lexikalisiert sind und eher dekomponiert werden. Insgesamt ist allerdings die Interpretation des Ergebnisses entsprechend der Hypothese naheliegender, und zwar dass die NN-Frequenz keinen Einfluss auf die N1-Zugänglichkeit ausübt.

\subsubsection{Interpretation und Diskussion}

In der Kontrollbedingung Mono liegen signifikant mehr Bezüge auf das Antezedens vor als in den beiden Kompositabedingungen. Der Unterschied zwischen direkter Anapher und N1-Bezug ist damit klar ersichtlich und das experimentelle Design kann als reliabel betrachtet werden.

Ein Einfluss der struKTuR auf die N1-Zugänglichkeit deutet sich in diesem Satzergänzungsexperiment deskriptiv an. Der Unterschied zwischen den Bedingungen RK und WK ist nicht signifikant, entspricht aber im Gegensatz zu Experiment 2 deskriptiv der Hypothese für mehr N1-Bezüge bei Rektionskomposita. Dieses Ergebnis bestätigt die Annahme, dass das Ergebnis für den Faktor STRUKTuR in Experiment 2 durch konzeptuelle Faktoren beeinflusst wurde. Allerdings führen auch hier die kategorialen Daten mit geringer Messwiederholung zu Konvergenzproblemen.

In der Auswertung der weiteren Einflussgrößen konnte der Einfluss des Faktors RzK nicht bestätigt werden, die deskriptiven Daten sind jedoch in prognostizierter Richtung als Vorteil für die Bedingung +RZK ausgeprägt. Der Einfluss des Faktors N2-KONKRETHEIT kann in prognostizierter Richtung bestätigt werden. Es scheint einen Vorteil für die N1Zugänglichkeit im Fall von abstrakten N2 zu geben. In die gleiche Richtung weisen die deskriptiven Daten zur NOMINALISIERUNGSLESART, die einen Vorteil für die Prozesslesart andeuten, jedoch nicht signifikant sind. Die Ergebnisse für die Faktoren N2-KONKRETHEIT 
und NOMinAlisierungslesart stützen die Annahmen zum Einfluss des konzeptuellen Fokus' eines Kompositums auf die N1-Zugänglichkeit. Für den Faktor N1-ZÄHLBARKEIT konnte keinerlei Einfluss festgestellt werden. In Bezug auf den Faktor GENus konnten wie erwartet signifikant mehr Bezüge bei maskulinen Pronomen gefunden werden. Bei der Betrachtung der Frequenzen von N1 und N2 konnten keine signifikanten Ergebnisse zutage gefördert werden.

Die Ergebnisse des Faktors STRUKTuR zeigen, dass die Überarbeitung der Daten und die gezielte Ausrichtung des Experiments auf diesen Faktor den subtilen grammatischen Unterschied sogar im Offline-Paradigma der Satzergänzungsaufgabe zutage fördern kann. Eine detaillierte Analyse der während der Anaphernresolution ablaufenden Prozesse und eine aussagekräftigere Inferenzstatistik soll das Eye-Tracking ermöglichen.

\subsection{Experiment 3b: Kohärenzrating}

Für das Eye-Tracking-Experiment wurden mehrzeilige Diskurse erstellt, die die bereits in Experiment 3a verwendeten Komposita enthalten. In Experiment 3b werden diese Diskurse zunächst in einem Kohärenzrating auf ihre Eignung als experimentelle Items geprüft. Gleichzeitig ermöglicht das Kohärenzrating einen Einblick in die Auswirkungen der experimentellen Manipulation auf den Kohärenzeindruck der Diskurse.

\subsubsection{Methode}

Beim Kohärenzrating wurden die Diskurse VPn mit der Aufgabe präsentiert, für jeden Diskurs anzugeben, für wie kohärent sie diesen empfinden. Dafür stand ihnen eine 7 Punkte-Skala zur Verfügung $(1=$ sehr inkohärent und $7=$ völlig kohärent). Da unklar ist, was genau eine VP unter ,Kohärenz' versteht, wurde die Aufgabe mündlich paraphrasiert und es wurde ein kohärenter und ein inkohärenter Beispieltext zur Orientierung vorgegeben. Die konkrete Aufgabenstellung ist im Anhang C.3.2 wiedergegeben. Das Experiment wurde als Pen-and-Paper-Studie durchgeführt und die Antworten wurden im Nachhinein von Hand digitalisiert.

\subsubsection{Material}

Die bereits in Experiment 3a verwendeten Komposita wurden in Diskurse, d.h. in sechszeilige Textblöcke eingebettet. Die Verwendung in einem mehrzeiligen Kontext ermöglicht zum einen einen natürlicheren Lesefluss und zum anderen wird das Untersuchungsziel stärker maskiert. Ankerausdruck (Kompositum) und Anapher (Pronomen) befinden sich etwa mittig im Textblock. Dabei steht das Kompositum zu Beginn eines Satzes und das anaphorische Pronomen in einem Folgesatz, der die Anapher disambiguiert. Die Frage ist nun, ob die avisierten Interpretationen in den verschiedenen Bedingungen unterschiedlich gut zugänglich sind.

Da das Kohärenzrating als Vortest für das Eye-Tracking dient, werden bei der Erläuterung des konkreten Materialaufbaus bereits hier Kriterien genannt, die erst für das Eye-Tracking relevant sind. 
Ein Item besteht jeweils aus einem sechs-zeiligen Text in drei Bedingungen, der aus Gründen der Vergleichbarkeit stets wie folgt aufgebaut ist: In der ersten Zeile wird in einem Satz eine Person und eine Situation eingeführt. Zeile 2 und 3 bestehen aus einem Satz, der die Situation weiter ausführt. Die ersten drei Zeilen enthalten keine Ausdrücke, die das N1 primen oder ein plausibles Antezedens für die Anapher darstellen könnten.

Zeile 4 beginnt mit einem definiten Artikel, gefolgt vom kritischen Kompositum in der entsprechenden Bedingung. Die das Kompositum einbettenden Sätze entsprechen denen der Satzergänzungsaufgabe. Am Ende der vierten Zeile beginnt ein neuer Satz mit einem Pronomen (er oder es), welches sich anaphorisch auf das N1 der Vorsatzes bezieht. Darauf folgt ein Modalverb. Der Satz wird in der fünften Zeile beendet. Der das Kompositum enthaltende Satz variiert zwischen den Bedingungen, um das Kompositum möglichst plausibel einzubetten. Der Anaphern-Satz wiederum ist über alle drei Bedingungen hinweg identisch. Somit kann dessen Lesezeit als AV ausgewertet werden. Inhaltlich stellen die Zeilen 4 und 5 eine allgemeine Aussage dar, die bspw. einen Gedankengang enthält.

Zeile 6 schließt den Text mit einem weiteren Satz ab, der zumeist den Gefühlszustand der Person oder ein Fazit aus den Ereignissen beinhaltet. Es wurde darauf geachtet, dass das Geschlecht der Person ungleich dem Genus des N1 ist. Ein Beispiel-Item in allen drei Bedingungen ist in (2) wiedergegeben 8

a. 1 Karl ist ein richtiger Heimwerker geworden.

2 Seit die Kinder ausgezogen sind,

3 ist er nur noch am Bauen und Buddeln.

4 Die Dachbegrünung ist wirklich gut gelungen. Es kann

5 jetzt bei gutem Wetter wunderbar als Rückzugsort genutzt werden.

6 Als nächstes will sich Karl den Keller vornehmen.

b. 1 Karl ist ein richtiger Heimwerker geworden.

2 Seit die Kinder ausgezogen sind,

3 ist er nur noch am Bauen und Buddeln.

4 Der Dachgarten ist wirklich gut gelungen. Es kann

5 jetzt bei gutem Wetter wunderbar als Rückzugsort genutzt werden.

6 Als nächstes will sich Karl den Keller vornehmen.

c. 1 Karl ist ein richtiger Heimwerker geworden.

2 Seit die Kinder ausgezogen sind,

3 ist er nur noch am Bauen und Buddeln.

4 Das Dach ist wirklich gut gestaltet. Es kann

5 jetzt bei gutem Wetter wunderbar als Rückzugsort genutzt werden.

6 Als nächstes will sich Karl den Keller vornehmen.

Die beiden kritischen Regionen für Antezedens und Anapher befinden sich stets in der vierten Zeile. Bei der Konstruktion dieser Zeile wurde besonderes Augenmerk auf die Anforderungen des Eye-Trackings gelegt. Die Zeile beginnt mit einem definiten Artikel, gefolgt vom kritischen Kompositum, bzw. Monolexem als Subjekt. Die Verwendung des definiten Artikels dient der Hervorhebung und Betonung des Nomens. Da die Konstruktion in allen Items und allen Bedingungen identisch ist, ist dadurch keine Beeinflussung der

\footnotetext{
${ }^{8}$ Die Hervorhebungen dienen der Sichtbarmachung von Ankerausdruck und Anapher und sind im Experiment nicht zu sehen.
} 
Ergebnisse zu erwarten. Nach dem Punkt beginnt noch in derselben Zeile ein Satz mit der pronominalen Anapher im Subjekt und einem sich anschließenden Modalverb. Beendet wird der Satz in der Folgezeile. Somit stehen die beiden kritischen Ausdrücke - Anapher und Antezedens - jeweils ein Wort vom Zeilenrand entfernt. Dieser maximale lineare Abstand soll bewirken, dass die Blickbewegungen so eindeutig wie möglich zugeordnet werden können. Bei einer Positionierung der Anapher in der Folgezeile wäre eine Zuordnung von Fixationen zwischen den Zeilen uneindeutig. Regressionen zwischen Anapher und Antezedens können so auf einer Zeile erfolgen, haben aber gleichzeitig genug Abstand, um nicht mit reinen Korrekturfixationen verwechselt zu werden. Der Mindestabstand von Beginn des Antezedens bis zum Beginn der Anapher liegt bei 28 Zeichen. Bei Regressionen mit einer Spanne von mehr als 10 Zeichen kann man von einem Verständnisproblem ausgehen (vgl. Rayner 1998:375). Direkt vor der Anapher befindet sich die durch einen Punkt markierte Grenze des Vorgängersatzes. Die Markierung einer Satzgrenze vor der Anapher dient der Minderung der parafovealen Verarbeitung der Anapher auf dem links angrenzenden Wort. Der Punkt signalisiert die Grenze zwischen zwei Sinneinheiten. Ziel ist es, die Verarbeitung der Anapher möglichst erst mit der Anapher und damit innerhalb der entsprechenden Interest Area (IA) beginnen zu lassen. Gleichzeitig ist eine Verminderung der parafovealen Verarbeitung des Pronomens wünschenswert, da letztere ein Überspringen des Pronomens begünstigen würde (vgl. Rayner 1998:381). Die Gefahr, dass das Pronomen übersprungen, also nicht separat fixiert wird, besteht bereits durch seine Länge: Wörter mit nur zwei bis drei Buchstaben werden nur zu $25 \%$ fixiert (vgl. Rayner 1998: 375). Darüber hinaus wird die Fixationswahrscheinlichkeit für das Pronomen durch seine Frequenz vermindert (vgl. Rayner 1998:387). Dem soll durch eine Verlängerung der IA mit einem Modalverb entgegengewirkt werden. Die Verwendung ein und desselben Verbs in allen Items könnte den VPn auffallen und ihre Aufmerksamkeit darauf lenken. Die geschlossene Klasse der Modalverben bietet hier eine gute Möglichkeit der kontrollierten Variation und gleichzeitiger Vergleichbarkeit durch eine verwandte Semantik. Idealerweise lässt sich durch das Modalverb zusätzlich der Spill-Over-Effekt des Pronomens auffangen (vgl. Rayner 1998:377), demzufolge die Pronomenverarbeitung erst auf der Folgefixation, also in dem Fall auf dem Modalverb stattfindet. Als rechter Kontext des Pronomens kann das Verb dessen Verarbeitung auch inhaltlich beeinflussen (vgl. Rayner 1998:382), was wiederum für die geschlossene Klasse der Modalverben spricht. Schließlich vergrößert die Zusammenfassung von Pronomen und Modalverb in einer IA die Wahrscheinlichkeit einer Fixation vor dem Zeilenumbruch. Die letzte Fixation in einer Zeile erfolgt meist 5 7 Buchstaben vor Zeilenende (vgl. Rayner 1998:375). Die Modalverben haben mindesten 4 Zeichen, sodass anzunehmen ist, dass in der kritischen IA nach Ende des Kompositumssatzes zumindest eine Fixation stattfindet, deren Dauer ausgewertet werden kann. Durch den auf das Modalverb folgenden Zeilenumbruch wird die Verarbeitung des Pronomens gefördert, da die Prozessierung während der Dauer der erzwungenen Sakkade in die nächste Zeile fortschreitet (vgl. Rayner 1998:373), unterdessen jedoch keine neuen Informationen hinzukommen.

Die Prozessierung des Pronomens ist am Zeilenende allerdings noch nicht beendet, weshalb auch der Rest des Satzes zur Auswertung herangezogen werden soll. Aus dem Grund ist dieser innerhalb eines Items über alle drei Bedingungen hinweg identisch.

Die beiden Sätze in den Zeilen 4 und 5 beginnen beide mit dem jeweils kritischen Ausdruck im Subjekt. Somit werden sowohl Subjecthood als auch Parallelism Account berücksichtigt (vgl. Smyth/Scholey 1994, Stevenson et al. 1994), siehe Kapitel 2.4.3.

Das Kohärenzrating wurde mit 24 Stimuli durchgeführt, wobei es sich um Textblöcke 
handelt, die wie oben beschrieben konstruiert wurden und die in den drei Bedingungen RK, WK und Mono vorliegen. Die für das Eye-Tracking gezielt gesetzten Zeilenumbrüche wurden für das Kohärenzrating entfernt, damit der Text DIN-A4-Breite einnimmt. Andernfalls besteht die Gefahr, dass die VPn auf die parallele Struktur der Items aufmerksam werden und diese zu ergründen versuchen. Zusätzlich wurden 6 Filler-Items in zwei Bedingungen hinzugefügt. Bei den Fillern handelt es sich um ähnlich konstruierte kurze Texte in zwei Bedingungen. In Bedingung A sind die Filler-Texte kohärent und ohne Auffälligkeiten. In Bedingung B sind die Filler-Texte inkohärent, plausible Handlungsreihenfolgen sind vertauscht oder zusammenhangslos und es werden Ausdrücke verwendet, die nicht in den Kontext passen. Mit Hilfe dieser Filler soll ein Ausnutzen des gesamten Spektrums der Kohärenz-Skala möglich sein. Ein Beispiel für ein kohärentes und ein inkohärentes Filler-Item ist in (3) wiedergegeben.

a. Hendrik hat im Sommer den Angelschein bestanden. Die nächsten Wochen hat er viel Zeit am See verbracht. Es ist für ihn pure Entspannung, die Ruhe zu genießen, während die Angelschnur im Wasser hängt. Er hat sogar schon einen Hecht gefangen.

b. Hendrik hat im Sommer den Angelschein bestanden. Die nächsten Wochen hat er viel Zeit am See verbracht. Es ist für ihn purer Wein, die Ruhe zu genießen, damit der Teilchenbeschleuniger im Wasser hängt. Er hat sogar schon einen Hecht gefangen.

Zusammengenommen ergeben Stimuli und Filler 30 Items. Die Items wurden entsprechend ihren Bedingungen nach dem lateinischen Quadrat auf drei Listen aufgeteilt. Für jede Liste wurden vier verschiedene Item-Reihenfolgen erstellt, sodass 12 Versionen vorlagen.

\subsubsection{Hypothesen}

Das Kohärenzrating verfolgt zwei Ziele: Zum einen soll ermittelt werden, ob sich die Items untereinander homogen verhalten und sich kein Text aufgrund spezieller, unkontrollierter Eigenschaften hervorhebt. Zum anderen soll anhand des Ratings der VPn analysiert werden, ob die experimentelle Manipulation Einfluss auf die generelle Wahrnehmung der Texte hat und sich auf die Offline-Verarbeitung auswirkt.

Die abhängige Variable des Ratings ist die numerische Bewertung der Texte durch die VPn auf einer ordinalen 7-Punkte-Skala. Je höher der durchschnittliche Rating-Wert eines Texts ist, als desto besser und damit kohärenter wurde er bewertet.

Die erste Hypothese ist, dass die Items untereinander ähnliche mittlere Ratingwerte aufweisen und sich in ihrem Kohärenzgrad ähneln. Dazu soll das Material bzgl. seiner Varianz zwischen den Items geprüft werden.

Der Hypothese zur Auswirkung der strukturellen Manipulation auf die Textkohärenz sind einige Vorüberlegungen voranzustellen: Welche Faktoren für eine VP bei der Kohärenzbewertung ausschlaggebend sind, lässt sich nicht explizit abfragen, da dies die Aufmerksamkeit auf spezielle Textteile lenken würde. Die Bewertung durch die VPn soll intuitiv und ohne explizite Textanalyse erfolgen. Bei der Bewertung soll der Gesamteindruck im Vordergrund stehen. Sollten nun spezifische Texteigenschaften maßgeblichen Anteil daran haben, z.B. einzelne Formulierungen in einzelnen Items, sollte dies an der Varianz zwischen 
den Items sichtbar werden. Innerhalb eines Items, also zwischen den drei Bedingungen, unterscheiden sich die Texte lediglich in Zeile 4 um einige Wörter.

Sollte nun das Ergebnis des Ratings Unterschiede zwischen den Bedingungen zutage fördern, kann dies zwei Ursachen haben: Zum einen kann die Anapher und damit der Grad an N1-Zugänglichkeit den Kohärenzeindruck beeinflussen. In dem Fall wird ein Ergebnis entsprechend der generellen Hypothese für Experiment 3 erwartet, d.h. der höchste Ratingwert sollte bei Bedingung Mono liegen, da hier ein direkte Anapher etabliert wird. Den zweithöchsten Wert erwarte ich für die Bedingung RK und den niedrigsten Wert für die Bedingung WK $(\mathrm{MW}(\mathrm{WK})<\mathrm{MW}(\mathrm{RK})<\mathrm{MW}($ Mono $))$.

Eine andere Ursache kann in der Art der Einführung des Kompositums liegen. Das Kompositum erscheint als Definitum relativ unvermittelt im Text, da Priming-Effekte durch inhaltliche Hinführung bestmöglich ausgeschlossen werden sollten. Für die Bedingung RK erwarte ich in dem Fall den höchsten Kohärenzwert, da durch die Argumentstellenvergabe im Kompositum eine Einbettung des N1 in einen Handlungskontext erfolgt. An zweiter Stelle erwarte ich Bedingung Mono, da es sich hier um ein unvermittelt auftretendes Definitum handelt. Das schlechteste Ratingergebnis erwarte ich für die Bedingung WK, da es sich hierbei wie schon bei der Bedingung Mono um ein Definitum handelt, welches, durch seine Eigenschaft ein Kompositum zu sein, zudem noch merkmalsreicher ist und dadurch noch unvermittelter erscheint $(\mathrm{MW}(\mathrm{WK})<\mathrm{MW}($ Mono $)<\mathrm{MW}(\mathrm{RK}))$.

\subsubsection{Durchführung}

\section{Ablauf}

Das Rating wurde im Januar 2016 als Pen-and-Paper-Studie mit Studienanfänger*inne*n in zwei Basisseminaren der Germanistischen Linguistik an der Georg-August-Universität Göttingen durchgeführt.

Die Aufgabe der VPn bestand darin, intuitiv zu entscheiden, wie kohärent ihnen ein Text erscheint und dies auf einer 7-Punkte-Skala anzugeben. Dabei bedeutet ,1' sehr inkohärent und, $7^{`}$ völlig kohärent. Die konkrete Aufgabenstellung befindet sich in Anhang C.3.2.

\section{Versuchspersonen}

In die Auswertung gingen Datensätze von 24 deutschen Muttersprachler*inne*n ein, davon waren 16 weiblich und 8 männlich. Der Altersdurchschnitt lag bei 21,54 Jahren (Std. $\mathrm{Abw} .=2.17) 9$

\section{Datenaufbereitung}

Die Ratingwerte der VPn wurden händisch in eine Datenmatrix übertragen.

\footnotetext{
${ }^{9}$ Auf jeder Version wurden zwei VPn getestet, außer auf Variante 7 nur eine und auf Variante 2 drei VPn.
} 


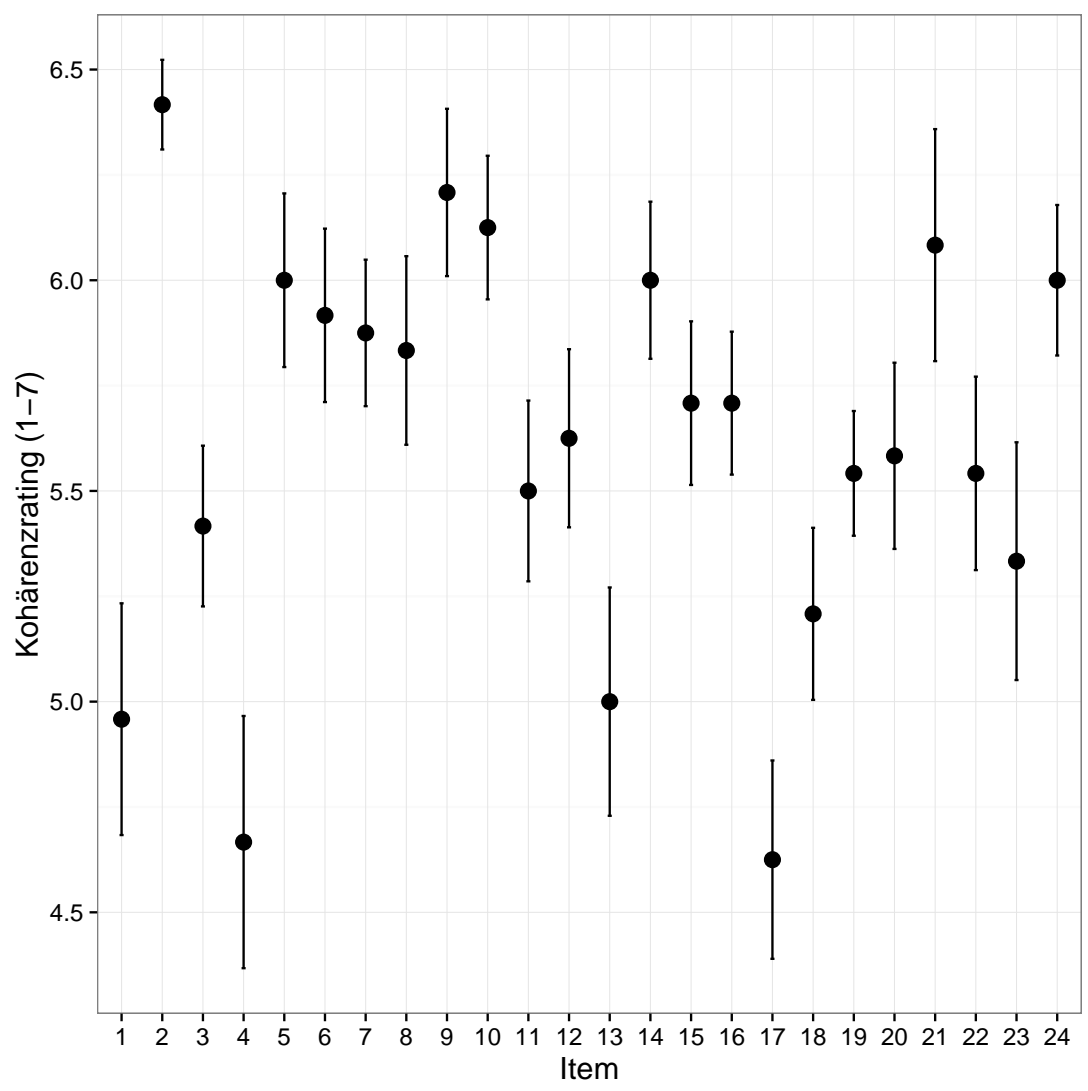

Abbildung 6.10: Plot der Mittelwerte des Kohärenzratings für die 24 Items

\subsubsection{Auswertung}

\section{Auswertung nach Items}

Zunächst wurde deskriptiv ausgewertet, wie stark sich die Kohärenzbewertungen zwischen den Items unterscheiden. In diesem Auswertungsschritt wird der Frage nachgegangen, ob die Items im Mittel untereinander als ähnlich kohärent wahrgenommen werden.

Die Mittelwerte der experimentellen Items liegen über alle drei Bedingungen hinweg zwischen 4.62 und 6.42 und damit insgesamt in der kohärenten Hälfte der Skala. Der Wert 4 wird als neutrale Mitte betrachtet. Da sich für kein Item ein Mittelwert $<4$ ergibt, wird kein Item aufgrund von abweichender Kohärenz vom Experiment ausgeschlossen. Die mittleren Bewertungen der einzelnen Items sind in Abbildung 6.10 dargestellt ${ }^{10}$

\section{Auswertung nach Versuchspersonen}

Als nächstes wurde ausgewertet, ob alle VPn die experimentelle Aufgabe korrekt gelöst haben, d.h. wirklich die wahrgenommene Textkohärenz der Bewertung zugrunde liegt. Zu diesem Zweck wurden die Ergebnisse der Filler-Texte betrachtet. Drei der sechs FillerTexte waren voll kohärent und drei sehr inkohärent. Wenn die Aufgabe entsprechend der Aufgabenstellung gelöst wurde, sollte die Bewertung der Texte dies widerspiegeln. Nur in

\footnotetext{
${ }^{10}$ Eine Auflistung der Einzelwerte findet sich im Anhang in Tabelle C.5 und Abbildung C.4
} 
dem Fall sind auch die Bewertungen der Stimuli aussagekräftig.

Die Mittelwerte der einzelnen VPn in den beiden Filler-Bedingungen sind in Abbildung 6.11 dargestellt. Innerhalb der kohärenten Filler liegen die Mittelwerte aller VPn zwischen 4.67 und 7, d.h. stets in der kohärenten Hälfte $(>4)$. Für die inkohärenten Filler liegen die Mittelwerte zwischen 1.0 und 3.67, d.h. stets im inkohärenten Bereich $(<4)$. Da das Rating das intendierte Ergebnis zeigt, kann das Design als geglückt erachtet werden und keine VP muss von der Auswertung ausgeschlossen werden.

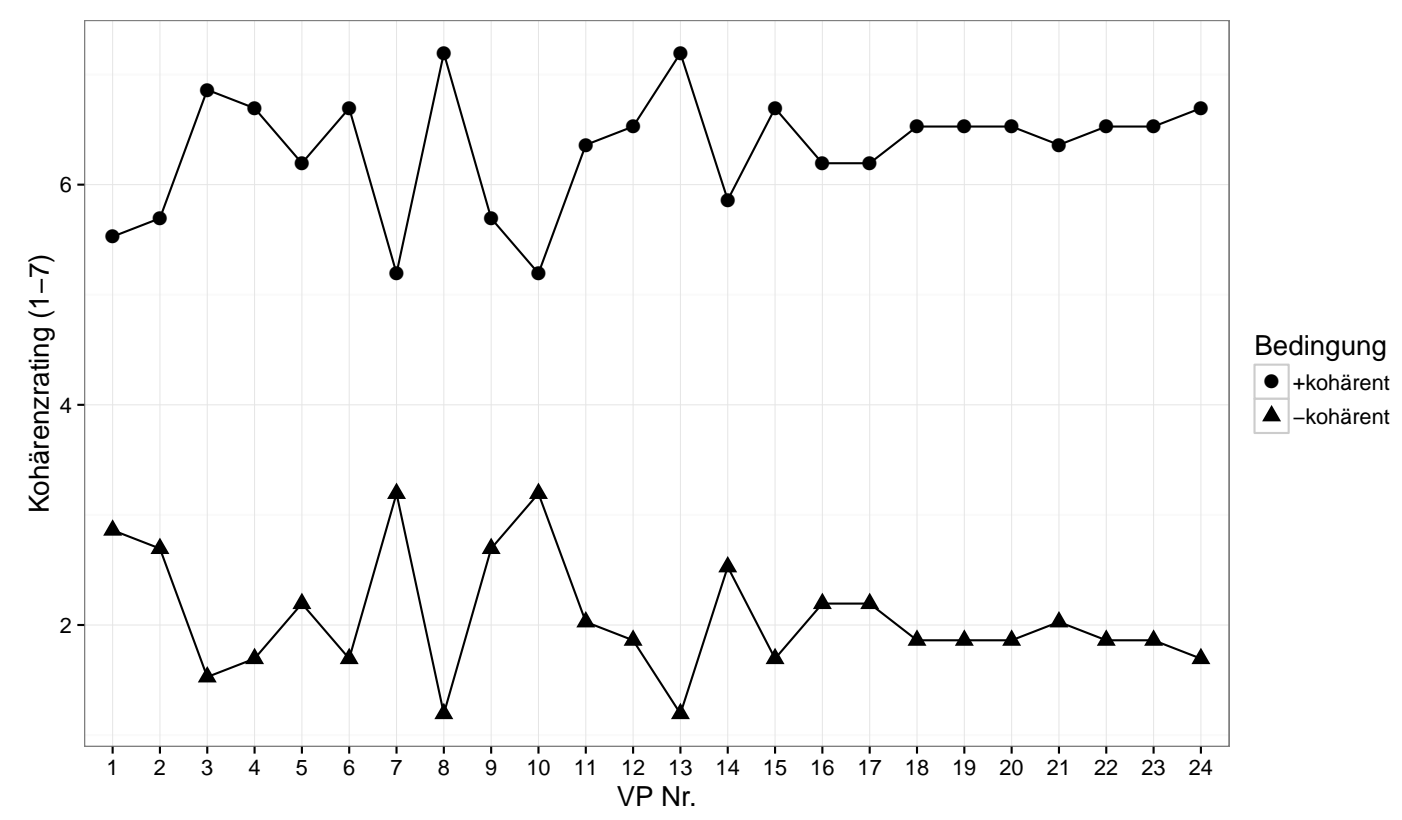

Abbildung 6.11: Plot der Mittelwerte des Kohärenzratings (y-Achse) der 24 Filler-Items für die $24 \operatorname{VPn}$ (x-Achse)

\section{Auswertung nach Bedingung}

Der nächste Schritt ist die Auswertung nach der experimentellen Manipulation für den Faktor struktur. Dem liegt die Frage zugrunde, ob die experimentelle Manipulation Auswirkungen auf den Kohärenzeindruck hat. Die Ergebnisse der deskriptiven Auswertung sind in Tabelle 6.22 und Abbildung 6.12 dargestellt.

\begin{tabular}{ccccc}
\hline \hline Faktor & Bed. & MW & Std. Abw. & n \\
\hline \multirow{4}{*}{ STRUKTUR } & RK & 5.74 & 1.25 & 192 \\
& WK & 5.44 & 1.28 & 192 \\
& Mono & 5.68 & 1.27 & 192 \\
\hline \hline
\end{tabular}

Tabelle 6.22: Deskriptive Statistik des Kohärenzratings für den Faktor sTRUKTuR 


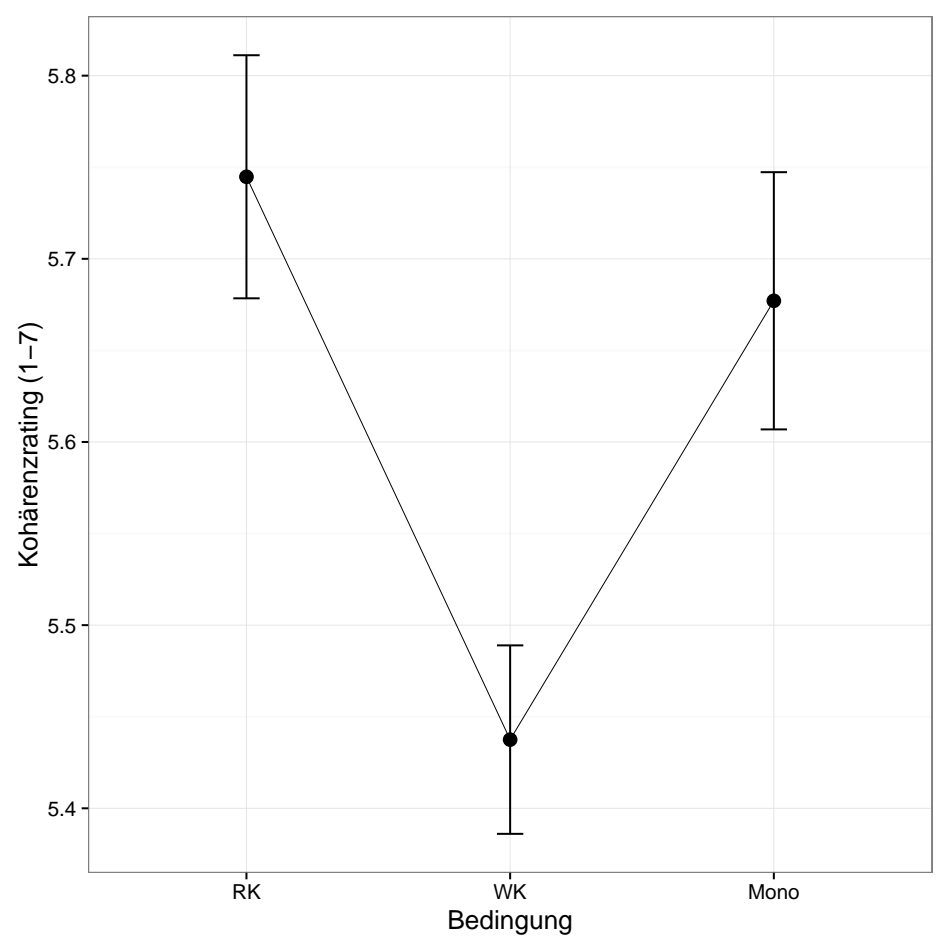

Abbildung 6.12: Plot der um die Zwischensubjektvarianz korrigierten Mittelwerte des Kohärenzratings (y-Achse) in Abhängigkeit vom Faktor sTrukTur (x-Achse); die Fehlerbalken repräsentieren +/- 1 Standardfehler des Mittelwerts

Die deskriptive Statistik weist für die Rektionskomposita den höchsten Mittelwert aus, gefolgt von den Monolexemen. Mit etwas Abstand folgen die Wurzelkomposita mit dem niedrigsten Mittelwert. Zur Validierung dieses Ergebnisses erfolgt die inferenzstatistische Überprüfung mit Hilfe einer ANOVA, da beim Kohärenzrating ordinal skalierte Daten vorliegen. Die Manipulation zeitigt in den ANOVAs einen Effekt (siehe Tab. 6.23) 11 Zusätzlich wurde als konservativster Schätzer minF' nutzbar gemacht (vgl. Barr et al. 2013). Dieser ist nicht signifikant 12

\begin{tabular}{c|cc|cc|cc}
\hline \hline & $F_{1}(2,46)$ & $p$ & $F_{2}(2,46)$ & $p$ & $\operatorname{minF}$ & $p$ \\
\hline Rating & 4.352 & $<0.05$ & 4.144 & $<0.05$ & 2.12 & $>0.05$ \\
\hline \hline
\end{tabular}

Tabelle 6.23: Inferenzstatistik (ANOVA omnibus-by-items, ANOVA omnibus-bysubjects, minF') des Kohärenzratings für den Faktor sTRUKTUR

Dieses Ergebnis legt eine Interpretation in Richtung der zweiten Ursache zum Einfluss der experimentellen Manipulation auf den Kohärenzeindruck nahe. Das bedeutet, dass die Einbettung des Kompositums in den Text einen stärkeren Einfluss auf den Kohärenzeindruck des Gesamttexts hat als die Art der anaphorischen Bezugnahme.

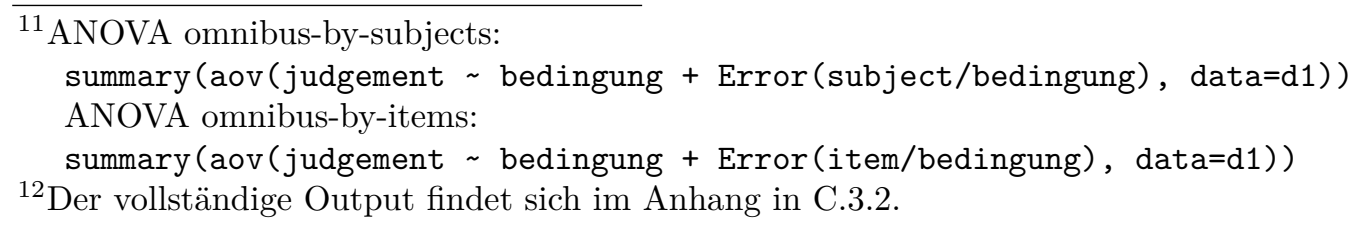




\subsubsection{Interpretation und Diskussion}

Die Auswertung nach Items ergibt, dass alle Items als kohärent eingestuft wurden. Somit können diese für das Eye-Tracking-Experiment genutzt werden.

Die Auswertung für die drei Bedingungen des Faktors STRUKTUR ergibt für den Kohärenzgrad die Abfolge MW(RK) > MW(Mono) > MW(WK). Die ANOVAs bestätigen dieses Muster als signifikant. Dies legt die Interpretation nahe, dass die Art der textuellen Einbettung des Ankerausdrucks einen maßgeblichen Einfluss auf den Kohärenzeindruck des gesamten Texts hat. Der Unterschied zwischen den Bedingungen wird in diesem Paradigma demnach nicht von der Interpretierbarkeit der Anapher, also von der N1-Zugänglichkeit beeinflusst. Dass die Texte der Bedingung Mono nicht generell als kohärenter eingestuft wurden, signalisiert darüber hinaus, dass die experimentelle Manipulation nicht vordergründig ersichtlich ist.

\subsection{Experiment 3c: Eye-Tracking}

\subsubsection{Methode}

Das dritte Teilexperiment zur Untersuchung des Einflusses der Kompositums-Struktur auf die N1-Zugänglichkeit ist ein Eye-Tracking-Experiment, d.h. eine Studie zu Blickbewegungen beim Lesen. Beim Eye-Tracking handelt es sich um ein Paradigma zur OnlineVerarbeitung, da mit hoher zeitlicher Auflösung Blickbewegungen und Fixationen beim Lesen, und damit während der Verarbeitung aufgezeichnet werden.

VPn bekommen Texte mit N1-Bezug präsentiert und nach jedem Text eine Verständnisfrage gestellt. Während des Lesens werden die Blicke aufgezeichnet. Dadurch ergeben sich zwei Arten von AVn mit räumlicher und zeitlicher Auflösung: Blicke - in Form von Fixationen - und Blickbewegungen - in Form von Sakkaden.

\subsubsection{Material}

Experiment 3c besteht wie die anderen beiden Teilexperimente 3a und 3b aus 24 Items in je drei Bedingungen (vgl. Tab. 6.1). Die Items entsprechen den Stimuli aus Experiment 3b, z.B. (2), Auf jedes Item folgte eine Entscheidungsfrage zum Inhalt des entsprechenden Texts. Dabei hatten $50 \%$ der Fragen ja als korrekte Antwort. Die Fragen bezogen sich in unterschiedlichem Detailgrad auf verschiedene Textstellen und sollten genaues Lesen (close reading) und eine anhaltende Konzentration der VPn gewährleisten sowie zur Überprüfung dieser herangezogen werden. Darüber hinaus wurde bei einem Viertel der Items in der Verständnisfrage nach dem Ziel der Anapher gefragt. Dies ermöglicht später eine Auswertung bzgl. der Unterschiede in der Memorisierung der Antezedenten zwischen den Bedingungen.

Um eine effiziente Auswertung zu ermöglichen, wurden die Texte jeweils in acht Interest Areas (IAs) eingeteilt. Dies erfolgte nach folgendem Prinzip: 


\begin{tabular}{|c|c|c|c|}
\hline Zeile 1: & \multicolumn{3}{|c|}{ IA 1} \\
\hline Zeile 2: & \multicolumn{3}{|c|}{ IA 2} \\
\hline Zeile 3: & \multicolumn{3}{|c|}{ IA 3} \\
\hline Zeile 4: & IA 4 (Antezedens-Region) & IA 5 (Spillover-Region) & IA 6 (Anaphern-Region) \\
\hline Zeile 5: & \multicolumn{3}{|c|}{ IA 7 (Satzergänzung) } \\
\hline Zeile 6: & \multicolumn{3}{|c|}{ IA 8} \\
\hline
\end{tabular}

Bezogen auf ein Item ergibt sich daraus beispielhaft folgende Aufteilung: ${ }^{13}$

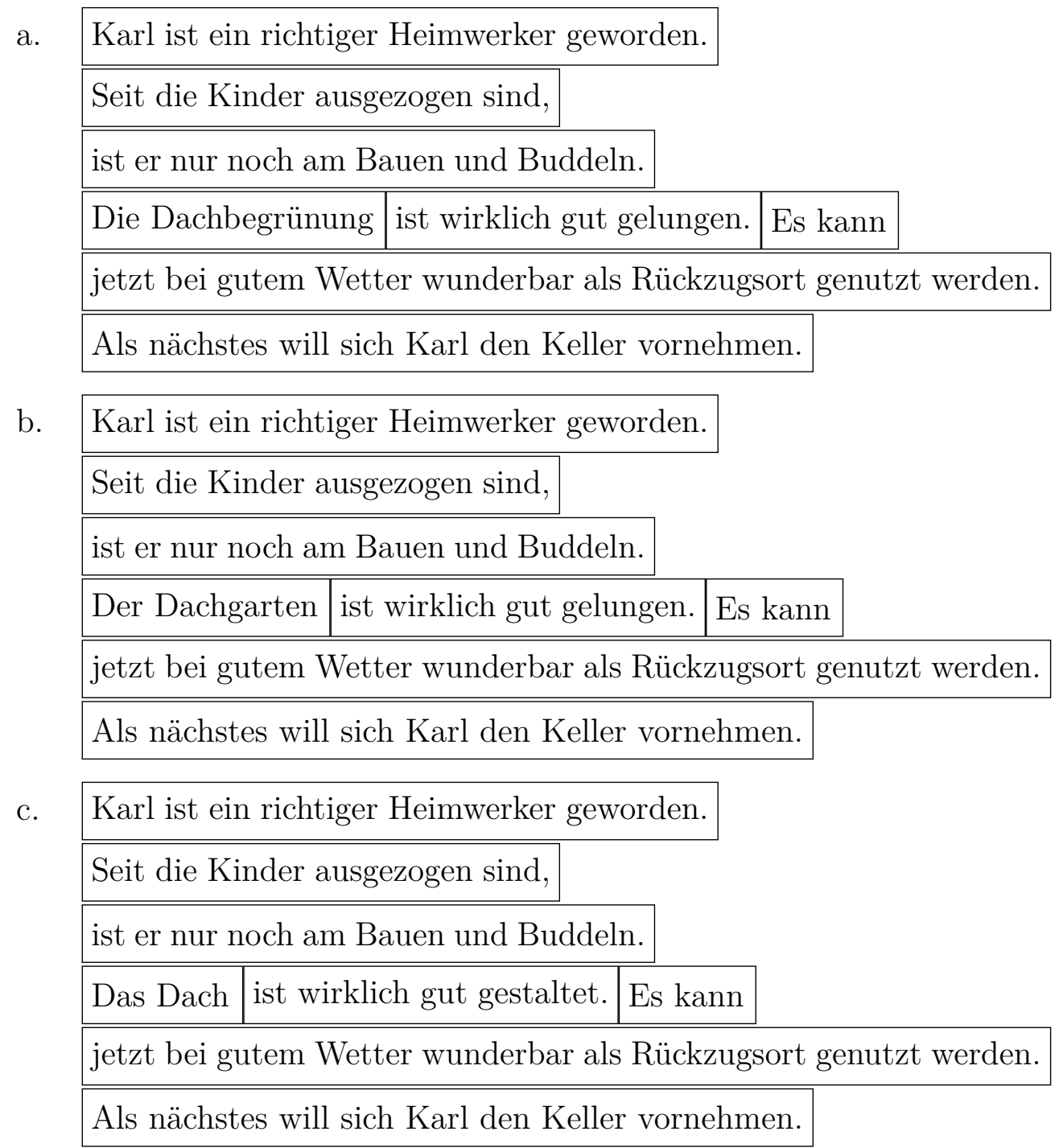

Auch in diesem Experiment wurden mögliche Einflussgrößen, die bei der Materialerstellung berücksichtigt wurden, im Datensatz annotiert. Dazu gehören Zählbarkeit und Genus des N1, die jedoch nur zwischen und nicht innerhalb der Items variieren. Schwieriger ist

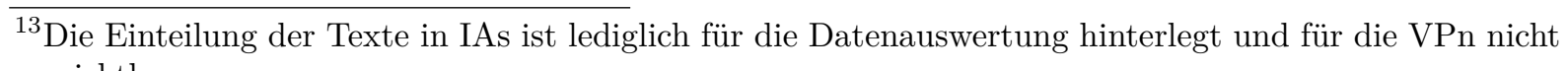
sichtbar. 
die Kontrolle von Eigenschaften, die sowohl innerhalb als auch zwischen den Items variieren: die RZK, die N2-Konkretheit, die Nominalisierungslesart der Rektionskomposita sowie die Art des nominalisierten Verbs. Die genaue Verteilung dieser Eigenschaften innerhalb der Items ist im Anhang in Tabelle C.2 wiedergegeben. Darüber hinaus wurden Frequenz ${ }^{14}$ und Länge der Ankerausdrücke annotiert; außerdem der Abstand zwischen Antezedens und Anapher, bestimmt durch die Zeichenzahl vom Beginn des Kompositums bis zum Beginn der Anapher.

Als Filler wurden zusätzlich zwei Sets aus unabhängigen Experimenten integriert. Dabei handelt es sich zum einen um ein Experiment zum Right Frontier Constraint mit 18 Items in drei Bedingungen und zum anderen um ein Experiment zu Markern von erlebter Rede mit 12 Items in zwei Bedingungen. Insgesamt wurden alle Items nach dem lateinischen Quadrat auf drei Listen aufgeteilt. Da beim zweiten Filler-Set nur zwei statt drei Bedingungen vorliegen, wurden die Items zwischen den Listen aufgeteilt. Jeder VP wurden im Verlauf des Experiments insgesamt 50 Texte präsentiert. Die Items wurden automatisch randomisiert. Jede VP bekam jedes Item in genau einer Bedingung präsentiert, und über alle Items hinweg sah jede VP jede Bedingungen gleich oft (within items/within subjects design).

\subsubsection{Hypothesen}

Bei der Auswertung von Blickbewegungen spielen zwei Arten von AVn eine Rolle: Die Fixationen in eine IA, die nach ihrer Anzahl und Dauer ausgewertet werden können, und die Sakkaden zwischen zwei Fixationen, die nach ihrer Anzahl, dem Ausgangspunkt und dem Ziel ihrer Bewegung ausgewertet werden können. Die Varianz in den Messungen spiegelt dabei die Varianz in der Verarbeitung wider (vgl. Rayner 1998:376). Der zentrale Parameter ist die mittlere Fixationsdauer in einer IA, da diese mit Verarbeitungsschwierigkeiten korreliert (vgl. Radach et al. 2002). Die für dieses Experiment zur Auswertung herangezogenen AVn sind in Tabelle 6.24 aufgeführt und erläutert.

Zur Veranschaulichung der AVn ist in Abbildung 6.13 ein hypothetisches Blickmuster beim Lesen der kritischen IAs abgebildet. Der Eintritt in die Antezedens-Region (IA 4) erfolgt mit der Fixation 1 (FFD für IA 4). Da es sich um eine längere IA handelt, ist von mehreren Fixationen (1 und 2) innerhalb der FPFD auszugehen. Anschließend erfolgt der Übertritt in die nächste IA (IA 5/Spillover-Region). Der Satz, der mit dem Ankerausdruck beginnt, endet in der IA 5; somit ist in der Fixation 6 mit einem ,sentence wrap-up' zu rechnen. Fixation 7 ist die erste Fixation in der Anaphern-Region (IA 6). Da es sich bei der Anaphernresolution um einen Prozess der Textintegration und somit um einen eher späten Verarbeitungsschritt handelt, werden während der ersten Fixationen in der IA 6 noch keine Unterschiede zwischen den Bedingungen erwartet. Regressive Sakkaden in vorhergehende Regionen werden erst nach einer Progression in die nächste IA (IA 7/Satzergänzung) erwartet. Spätestens während der Verarbeitung der IA 7 werden jedoch Unterschiede zwischen den Bedingungen erwartet, die sich in der GesamtVerweildauer (TRT) der IA 7 (Fixationen 9, 10, 12) widerspiegeln. Für die IA 6 werden Unterschiede ebenfalls vorrangig für die TRT erwartet, da diese auch Refixationen nach dem Lesen der Satzergänzung beinhaltet. Auch Regressionen in die IA 4 (Fixation 11)

\footnotetext{
${ }^{14}$ Als Frequenzen wurden der absoluter Wert aus dem Kernkorpus 20 des DWDS und die Häufigkeitsklasse aus dem Leipziger Wortschatz aufgenommen.
} 


\begin{tabular}{|l|l|}
\hline \multicolumn{2}{|l|}{ AVn zu Fixationen } \\
\hline $\begin{array}{l}\text { FFD } \\
\text { First Fixation Duration }\end{array}$ & Dauer der initialen Fixation in einer bestimmten IA \\
\hline $\begin{array}{l}\text { FPFD } \\
\text { First Pass Fixation Duration } \\
\text { (First Run Dwell Time) }\end{array}$ & $\begin{array}{l}\text { Summierte Fixationsdauern in einer IA, bevor diese } \\
\text { das erste Mal verlassen wird }\end{array}$ \\
\hline $\begin{array}{l}\text { SPFD } \\
=\text { Second Pass Fixation Dura- } \\
\text { tion (Second Run Dwell Time) }\end{array}$ & $\begin{array}{l}\text { Summierte Fixationsdauern in einer IA im zweiten Le- } \\
\text { sedurchlauf, d.h. nach dem zweiten Eintritt bis zum } \\
\text { nächsten Austritt aus der IA }\end{array}$ \\
\hline $\begin{array}{l}\text { RPD } \\
=\text { Regression Path Duration } \\
\text { Trmmierte Fixationsdauern, bis eine IA erstmals } \\
\text { durch eine Sakkade in eine folgende IA verlassen wird } \\
\text { (d.h. inkl. Refixationen in vorhergehende IAs) }\end{array}$ \\
\hline $\begin{array}{l}\text { Total Reading Time } \\
\text { Dwell Time) }\end{array}$ & $\begin{array}{l}\text { Summierte Fixationsdauer aller Fixationen innerhalb } \\
\text { einer IA, ohne Refixationen einer früheren IA }\end{array}$ \\
\hline \hline $\begin{array}{l}\text { AVn zu Sakkaden } \\
\text { RIC } \\
=\text { Regression In Count }\end{array}$ & $\begin{array}{l}\text { Anzahl der Regressionen in eine IA aus einer folgen- } \\
\text { den IA }\end{array}$ \\
\hline $\begin{array}{l}\text { ROC } \\
=\text { Regression Out Count }\end{array}$ & $\begin{array}{l}\text { Anzahl der Regressionen aus einer IA in eine vorher- } \\
\text { gehende IA, bevor die IA das erste Mal durch eine } \\
\text { Sakkade in eine folgende IA verlassen wird }\end{array}$ \\
\hline
\end{tabular}

Tabelle 6.24: Liste verwendeter AVn (vgl. SR Research Ltd. 2002-2007; 64ff)

sind während der Anaphernresolution zu erwarten. Im Beispiel würde sich die Fixation 11 auf die ROCs der IA 7 und die RICs der IA 4 sowie auf die RPD der IA 7 (Fixationen 9, 10, 11, 12) auswirken.

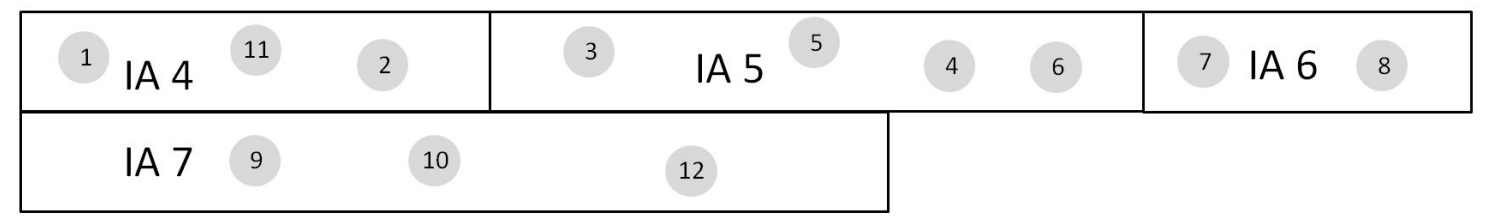

Abbildung 6.13: Beispiel für mögliches Blickverhalten

Die generelle Arbeitshypothese für das Experiment ist, dass sich die verschiedenen Kompositums-Strukturen im Verarbeitungsaufwand unterscheiden und sich dies auf das Blickverhalten auswirkt. In der Bedingung Mono sollte die Verarbeitung am leichtesten fallen, da hier eine direkte Anapher etabliert wird, was stets die präferierte Prozedur ist. Deutlich mehr Verarbeitungsaufwand sollte in den Bedingung RK und WK nötig sein, da hier das Antezedens in Form des N1 zunächst aus dem Ankerausdrucks extrahiert werden muss. Dabei wird für die Bedingung RK ein Vorteil gegenüber der Bedingung WK vorhergesagt, da für das N1 in einem Rektionskompositum aufgrund seines nominalen Status eine bessere Zugänglichkeit prognostiziert wird, als für das N1 eines Wurzelkompositums, für 
das nur Wurzelstatus angenommen wird (vgl. Kap. 4). Die Arbeitshypothese ist in (5) dargestellt.

$$
\begin{aligned}
& \text { Hypothese in Bezug auf den Verarbeitungsaufwand: } \\
& \mathrm{H}_{1} \text { : Mono }<\mathrm{RK}<\mathrm{WK}
\end{aligned}
$$

Daraus werden in Bezug auf die Fixationsdauern folgende konkrete Hypothesen abgeleitet: Für die IA 4 werden keine systematischen Unterschiede bei den AVn erwartet, bis auf Varianzen, die sich aus den Unterschieden der Ausdrücke in Länge und Frequenz ergeben. Gleiches gilt für die IA 5. Für die IA 6 sollten sich abhängig von der experimentellen Bedingung Unterschiede in den mittleren Fixationsdauern zeigen. Da die FFD und die FPFD vorrangig Auskunft über den lexikalischen Zugang liefern, werden die Unterschiede v.a. in späteren Fixationsdauern (RPD, TRT) erwartet. Diese bilden auch den Textintegrationsprozess ab, wozu die Anaphernresolution zählt (vgl. Rayner 1998:377). Am aussagekräftigsten ist die TRT, weil in dieser alle Verzögerungen enthalten sind, die durch Sakkaden verursacht werden. Für die mittlere TRT der IA 6 besteht demnach die Hypothese TRT(Mono) $<$ TRT(RK) $<$ TRT(WK).

Womöglich handelt es sich bei einem Effekt der Anaphernresolution in diesem Experiment um einen globalen Effekt, d.h. der Effekt besteht über den gesamten Satz fort. Die durch das Pronomen induzierten Verarbeitungsschwierigkeiten wären dann nicht primär am Pronomen selbst messbar, sondern die Lesezeit für den ganzen Satz würde sich verlängern (vgl. Ehrlich 1983:262). Um diesem Phänomen Rechnung zu tragen, wird auch die summierte Fixationsdauer für die IA $7 \mathrm{zu}$ Rate gezogen. Da es sich um eine große Region handelt, bei der nicht genau vorhergesagt werden kann, an welcher Stelle die Anaphernresolution zu Verzögerungen führt, gilt die Hypothese hierfür ausschließlich für die TRT (TRT(Mono) $<$ TRT $(\mathrm{RK})<\mathrm{TRT}(\mathrm{WK}))$. Bei der Verarbeitung der Anapher werden Regressionen auf das Antezedens prognostiziert, diese sollten sich auf Verweildauern der IA 4 niederschlagen, die Regressionen nach dem Anaphernlesen beeinhalten, d.h. auch für die TRT der IA 4 gilt TRT(Mono) $<$ TRT(RK) $<$ TRT(WK). Generell sind die TRT und die RPD die wichtigsten AVn, da die Anaphernresolution tendenziell zu den späten Prozessen gezählt wird (siehe auch Garnham et al. 2006).

In Bezug auf Sakkaden gelten folgende Annahmen: Nach dem Auftreten eines Pronomens ist man bestrebt, dieses möglichst schnell aufzulösen. Die Resolution ist dabei nicht zwingend an eine Regression zum Antezedens gebunden, denn die Funktion der Regression ist nicht der Zugang zu Informationen, sondern die Informationsüberprüfung, wenn eine unlösbare Inkonsistenz besteht, z.B. ein Genus-Mismatch (vgl. Ehrlich 1983:264f). Aufgrund dieser Annahme ist davon auszugehen, dass abhängig von der experimentellen Bedingung die Anzahl an Regressionen zum Antezedens in Bezug auf Verarbeitungsschwierigkeiten der Anapher aussagekräftig ist. Daraus folgt, dass die Anzahl der Regressionen in die IA 4, also zum Antezedens, Verabeitungsschwierigkeiten widerspiegelt, was zur Hypothese $\mathrm{RIC}($ Mono $)<\mathrm{RIC}(\mathrm{RK})<\mathrm{RIC}(\mathrm{WK})$ führt. Außerdem sollte dieser Überprüfungsprozess in IA 6 und 7 ausgelöst werden und sich in deren ROCs niederschlagen (ROC(Mono) < $\mathrm{ROC}(\mathrm{RK})<\mathrm{ROC}(\mathrm{WK}))$. Für die Bedingung Mono wird stets auch die geringste Zahl an Refixationen vorausgesagt, da ein Ausdruck weniger Refixationen evoziert, je zugänglicher er ist.

Vor allem bei der Auswertung der IA4 müssen Länge und Frequenz der verschiedenen Ankerausdrücke berücksichtigt werden. Eine Konfundierung kann durch die Möglichkeit 
einer parafovealen Verarbeitung der Anapher während einer Fixation in der IA 5 erfolgen (vgl. Radach et al. 2002:71). Das Einfügen einer Satzgrenze direkt vor der Anapher soll einen ,sentence wrap-up' bewirken und damit die parafoveale Verarbeitung des Pronomens vermindern. Sollte diese dennoch maßgeblich stattfinden, müsste sie sich in systematischen Unterschieden in der TRT der IA 5 niederschlagen, welches es zu überprüfen gilt. Schließlich gilt es zu bedenken, dass es sich bei der Anaphernresolution um einen eher unscharf zu lokalisierenden Prozess handelt. Die Wortverarbeitung kann sich generell über mehrere Fixationen verteilen (vgl. Radach et al. 2002:72) und besonders Pronomen und Artikel werden aufgrund ihrer geringen Länge und hohen Frequenz häufig übersprungen (vgl. Rayner 1998,387). Aus diesem Grund ist gerade bei Pronomen von einem Spillover-Effekt auszugehen, d.h. die Verarbeitung des Pronomens dauert an und beeinflusst die Fixationszeiten für das folgende Wort (vgl. Radach et al. 2002:71). Aus diesem Grund wird die Anaphern-Region durch ein Modalverb verlängert und auch nur mit diesem gemeinsam ausgewertet. Die Hypothesen für das Blickverhalten sind in Tabelle 6.25 zusammengefasst.

\begin{tabular}{l|l|l}
\hline \hline IA & AV & Hypothese \\
\hline 6 & FFD & - \\
6 & FPFD & - \\
6 & TRT & Mono $<$ RK $<$ WK \\
6 & ROC & Mono $<$ RK $<$ WK \\
7 & TRT & Mono $<$ RK $<$ WK \\
7 & ROC & Mono $<$ RK $<$ WK \\
4 & TRT & Mono $<$ RK $<$ WK \\
4 & RIC & Mono $<$ RK $<\mathrm{WK}$ \\
5 & TRT & - \\
\hline \hline
\end{tabular}

Tabelle 6.25: Zusammenfassung der Hypothesen für die verschiedenen Interest Areas $(I A s)$ und abhängigen Variablen $(A V n)$

\subsubsection{Durchführung}

\section{Ablauf}

Das Experiment wurde in Einzelsitzungen im Eye-Tracking-Labor des Seminars für Deutsche Philologie in Göttingen durchgeführt. Den VPn wurden an einem Computerbildschirm die Items randomisiert präsentiert. Nach jedem Item musste eine Verständnisfrage dazu per Tastendruck mit, ja، oder ,nein' beantwortet werden. Der Ablauf des Experiments wurde zunächst mündlich von der Versuchsleitung mit den VPn besprochen. Vor dem Start des Experiments wurden einige demografische Daten erhoben.

Anschließend wurden die VPn gebeten, ihren Kopf in der Kopfstütze zu positionieren und die Hände auf die Tastatur zu legen. Der Sichtkontakt zwischen VP und Versuchsleitung wurde durch einen Sichtschirm verhindert, um eine etwaige Ablenkung der VP zu vermeiden. Danach wurde ein Übungs-Item präsentiert und eine 9-Punkte-Kalibrierung durchgeführt. Die VPn wurden instruiert, die Items in normaler Lesegeschwindigkeit zu lesen und anschließend die Leertaste zu betätigen. Nach jedem Item wurde ihnen eine Verständnisfrage präsentiert, welche sie mit ,ja (Taste, $1^{6}$ ) oder ,nein ${ }^{6}$ (Taste , $0^{`}$ ) beantworten sollten. $\mathrm{Ob}$ die Antwort korrekt ist, wurde auf einem sich anschließenden Feedback-Bildschirm 
angezeigt. Das nächste Item erschien jeweils nach erneuter Leertasten-Betätigung. Nach 10 Items erfolgte jeweils eine Pause, deren Länge die VPn selbst bestimmen konnten und an die sich eine erneute Kalibrierung anschloss.

\section{Technik}

Das Experiment wurde mit Experiment Builder 1.10.165 (SR Research Ltd.) erstellt. Die Items wurden auf einem Computer-Bildschirm von DELL mit schwarzer Schrift auf weißem Grund dargestellt (Courier New 12, Zeilenabstand 2,5). Das Eye-Tracking erfolgte mit einem Eye Link 1000 (SR Research Ltd.) mit einer Abtastfrequenz von $1000 \mathrm{~Hz}$ und einer Beleuchtungsstärke von 75 \% . Gemessen wurde die Relation Pupille-Kornealreflex. Da diese nur bei Unbeweglichkeit des Kopfs aussagekräftig ist (vgl. Richardson/Spivey 2004), wurde die Kopfstütze des Eye Link verwendet. Der Abstand zwischen Kopf und Bildschirm betrug $58 \mathrm{~cm}$. Als Eingabegerät für die VPn diente ein CMStorm Gaming Keyboard 15 Die Beantwortung der Entscheidungsfragen erfolgte über die farblich gekennzeichneten Tasten , $1^{`}$ (=ja) und ,0` (=nein) des Buchstabenblocks.

\section{Versuchspersonen}

Als VPn wurden 30 Studierende der Universität Göttingen getestet, die alle Deutsch als einzige Muttersprache angaben. Das Experiment wurde im April 2016 durchgeführt. Die Dauer eines Durchgangs betrug im Durchschnitt ca. 45 Minuten pro VP. In die Auswertung gingen 27 VPn ein, davon waren 20 weiblich und 7 männlich. Alle waren Rechtshänder. Der Altersdurchschnitt lag bei 25,6 Jahren (Std. Abw. = 3.61). Beim Eye-Tracking wurde nur das dominante Auge aufgezeichnet; bei fünf VPn war dies das linke, bei 22 VPn das rechte Auge. Die Teilnahme erfolgte freiwillig und wurde vergütet. Der eigentliche Untersuchungsgegenstand war den VPn unbekannt und konnte in einer anschließenden Befragung auch von keiner VP korrekt benannt werden.

\section{Datenaufbereitung}

Nach Erhebung der Daten mussten drei VPn von der Auswertung ausgeschlossen werden: VP 1 (Liste 1) und VP 15 (Liste 3) aufgrund von technischen Problemen während der Aufzeichnung und VP 20 (Liste 2), um die Verteilung der Daten auf die drei Listen auszugleichen.

Als Kriterium zur Überprüfung der Aufmerksamkeit der VPn beim Lesen wurden die Antworten zu den Verständnisfragen ausgewertet. Die Grenze wurde dabei bei max. 1/4 falscher Antworten angelegt. Demnach wäre eine VP bei mehr als 12 falschen Antworten von der Auswertung ausgeschlossen worden, da in dem Fall davon auszugehen ist, dass die VP nicht konzentriert gelesen hat und somit die Blickbewegungen in den Items auch keinen konzentrierten Leseprozess abbilden. Dies war jedoch nicht der Fall, d.h. keine VP musste aufgrund vieler falsch beantworteter Verständnisfragen ausgeschlossen werden $(\mathrm{MW}=0.93$, Std. Abw. $=0.25, \operatorname{Min}=0.82, \operatorname{Max}=0.98)$.

\footnotetext{
${ }^{15}$ Das Keyboard (Cooler Master Model SGK-4030-GKCL1-DE) war über PS/2 angeschlossen und auf doppelte Wiederholungsrate eingestellt.
} 


\subsubsection{Auswertung}

Die Auswertung erfolgt für die einzelnen IAs getrennt nach Fixationen und Regressionen. Dabei wird zuerst die Auswertung der IAs 6 und 7 berichtet, da diese Regionen für die Anaphernresolution am aufschlussreichsten sind. Anschließend folgt die Auswertung der IAs 4 und 5 .

Im Gegensatz zu den bisher durchgeführten Satzergänzungsaufgaben, für die aufgrund der kategorialen Daten LMEMs genutzt wurden, wird für die intervallskalierten Daten des Eye-Trackings (wie auch bereits beim Kohärenzrating) die ANOVA und als konservativster Schätzer minF' nutzbar gemacht (vgl. Barr et al. 2013). Die Berechnungen erfolgten - wenn nicht anders angegeben - auf den Rohdaten. Diese verhalten sich annähernd normalverteilt. Die Auswertung wurde wieder mit R 3.2 durchgeführt.

\section{Auswertung der Anaphern-Region (IA 6)}

\section{Fixationen}

\section{Hypothesen}

Bei der Anaphern-Region handelt es sich um die IA am Ende der vierten Zeile, die aus der pronominalen Anapher (er oder es) und einem Modalverb (kann, soll oder muss) besteht, welches der Verlängerung der IA dient. Unterschiede in der Gesamtverweildauer in dieser Region können als Indiz für einen Unterschied in der Zugänglichkeit der forcierten Interpretation der Anapher gewertet werden. Für die FFD und die FPFD werden noch keine signifikanten Ergebnisse erwartet, da diese frühen Verarbeitungsschritte vorrangig den lexikalischen Zugang und die syntaktische Strukturzuweisung abbilden. Ein Unterschied zwischen Kontroll- und Kompsitabedingungen ist allerdings bereits wahrscheinlich. Ein Einfluss der Anaphernresolution wird erst bei späteren Prozessen, also in der RPD und vor allem in der TRT erwartet. Die Hypothese für die TRT lautet (stellvertretend für die analysierten AVn): TRT(Mono) $<$ TRT(RK) $<$ TRT(WK).

\section{Ergebnisse}

Die deskriptiven Daten für die erste Fixation in der IA 6 in Tabelle 6.26 und Abbildung 6.14 zeigen einen deutlichen Unterschied zwischen der Bedingung Mono und den beiden Kompositabedingungen. Diese Muster ist jedoch nicht signifikant (vgl. Tab. 6.27) ${ }^{16}$

\footnotetext{
${ }^{16}$ In $\mathrm{R}$ verwendete Formeln für die ANOVAs: summary (aov(IA_FIRST_FIXATION_DURATION bed + Error(vp/bed), d3.ia.ana)) 


\begin{tabular}{cccccc}
\hline \hline Faktor & AV & Bed. & MW & Std. Abw. & n \\
\hline \multirow{4}{*}{ STRUKTUR } & FFD & RK & 208.06 & 79.77 & 214 \\
& & WK & 208.92 & 98.7 & 211 \\
& & Mono & 196.01 & 87.17 & 214 \\
\hline \hline
\end{tabular}

Tabelle 6.26: Deskriptive Statistik für die FFD der Anaphern-Region (IA 6) für den Faktor STRUKTUR

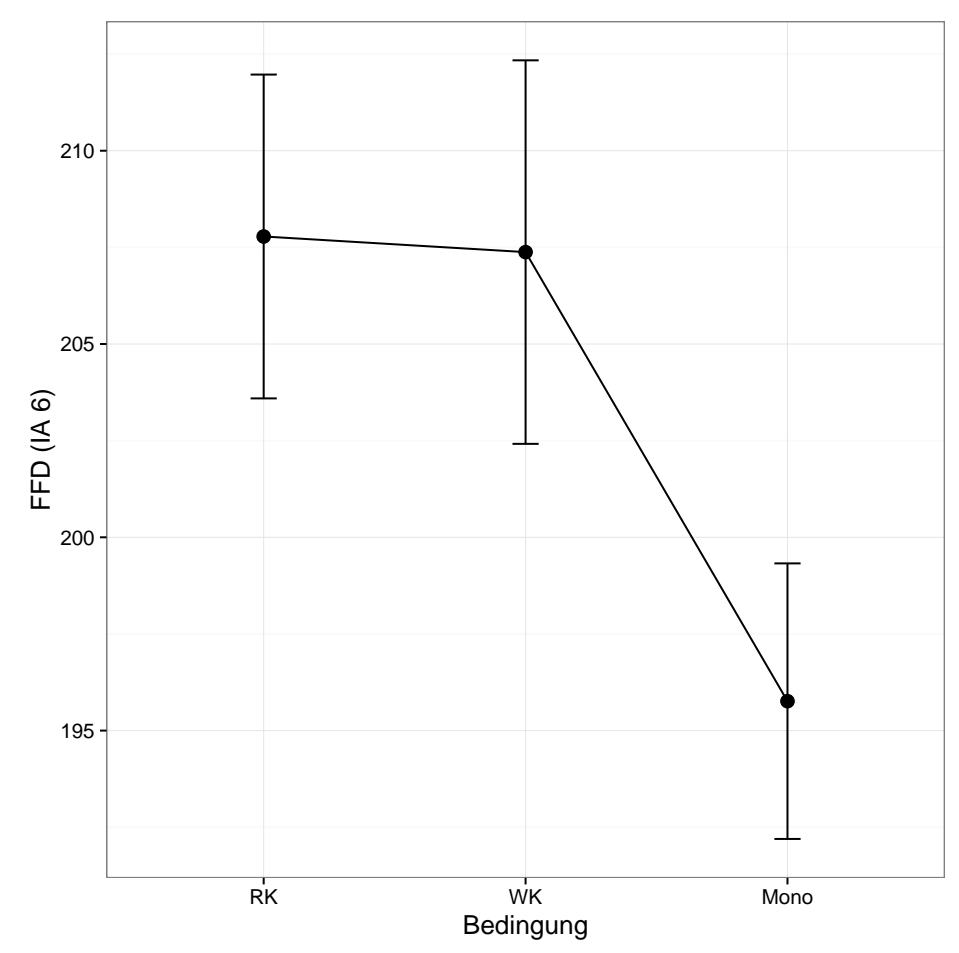

Abbildung 6.14: Plot der um die Zwischensubjektvarianz korrigierten Mittelwerte der FFD der Anaphern-Region (IA 6) (y-Achse) in Abhängigkeit vom Faktor STRUKTUR (xAchse); die Fehlerbalken repräsentieren +/- 1 Standardfehler des Mittelwerts

\begin{tabular}{c|cc|cc|cc}
\hline \hline AV & $F_{1}(2,52)$ & $p$ & $F_{2}(2,46)$ & $p$ & $\operatorname{minF}^{\prime}(2,95)$ & $p$ \\
\hline FFD & 1.77 & 0.18 & 1.365 & 0.266 & 0.77 & $>0.05$ \\
\hline \hline
\end{tabular}

Tabelle 6.27: Inferenzstatistik (ANOVA omnibus-by-subjects, ANOVA omnibus-byitems, minF') für die FFD der Anaphern-Region (IA 6) für den Faktor sTRUKTUR

Die FPFD inkludiert alle Fixationen des ersten Lesedurchlaufs der IA, z.B. auch Korrekturbewegungen. Hierbei zeigt sich in den deskriptiven Daten (vgl. Tab. 6.28 und Abb. 6.15) das Muster FPFD(Mono) $<\operatorname{FPFD}(\mathrm{RK})<\operatorname{FPFD}(\mathrm{WK})$, welches erst für spätere Fixationszeiten prognostiziert wurde. Die Inferenzstatistik bestätigt dieses als signifikant (siehe Tab. 6.29). Es liegt somit bereits auf der FPFD ein Effekt vor. Bemerkenswert daran ist, dass die FPFD ausschließlich Fixationen vor dem Lesen der disambiguierenden Satzergänzung beinhaltet. Aus diesem Grund können hier nur morphosyntaktische Eigenschaften für Verarbeitungsunterschiede verantwortlich sein und nicht der weitere Kontext. Generell wurde für die eher kurze IA nicht mit mehreren Fixationen gerechnet. Diese scheinen 
allerdings stattgefunden zu haben, da ein deutlicher Unterschied zu den Daten der FFD besteht.

\begin{tabular}{cccccc}
\hline \hline Faktor & AV & Bed. & MW & Std. Abw. & n \\
\hline \multirow{4}{*}{ STRUKTUR } & \multirow{2}{*}{ FPFD } & RK & 254.47 & 130.27 & 214 \\
& & WK & 286.85 & 183.91 & 211 \\
& & Mono & 235.16 & 129.64 & 214 \\
\hline \hline
\end{tabular}

Tabelle 6.28: Deskriptive Statistik für die FPFD der Anaphern-Region (IA 6)

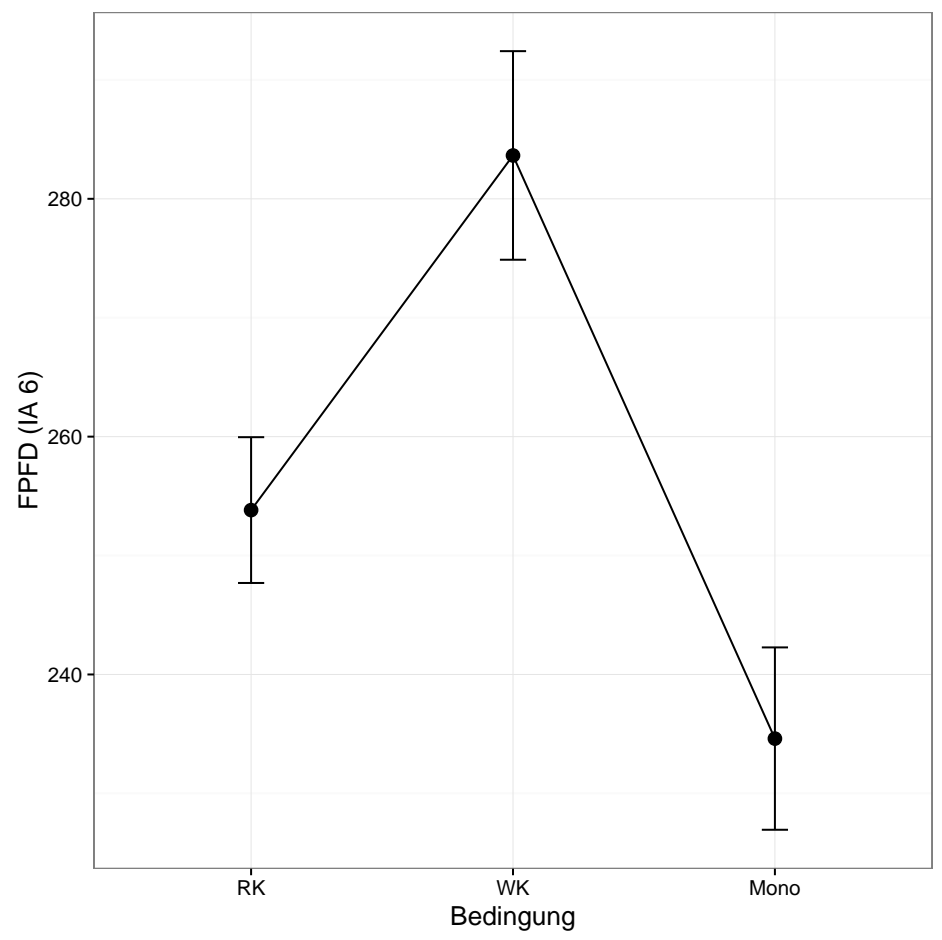

Abbildung 6.15: Plot der Mittelwerte der FPFD der Anaphern-Region (IA 6) für den Faktor STRUKTUR

\begin{tabular}{c|cc|cc|cc}
\hline \hline $\mathrm{AV}$ & $F_{1}(2,52)$ & $p$ & $F_{2}(2,46)$ & $p$ & $\operatorname{minF}^{\prime}(2,98)$ & $p$ \\
\hline FPFD & 7.393 & $<0.01$ & 8.369 & $<0.001$ & 3.93 & $<0.05$ \\
\hline \hline
\end{tabular}

Tabelle 6.29: Inferenzstatistik für die FPFD der Anaphern-Region (IA 6)

Der Unterschied zwischen den Bedingungen RK und WK ohne der Kontrollbedingung Mono wird zumindest von den ANOVAs ebenfalls als signifikant ausgewiesen (vgl. Tab. 6.30).

\begin{tabular}{c|cc|cc|cc}
\hline \hline $\mathrm{AV}$ & $F_{1}(1,26)$ & $p$ & $F_{2}(1,23)$ & $p$ & $\min F^{\prime}(1,49)$ & $p$ \\
\hline FPFD & 5.548 & $<0.05$ & 6.22 & $<0.05$ & 2.93 & $>0.05$ \\
\hline \hline
\end{tabular}

Tabelle 6.30: Inferenzstatistik für die FPFD der Anaphern-Region (IA 6) ohne Bed. Mono 
Das Muster der FPFD zeigt sich auch in der SPFD, also im zweiten Lesedurchlauf. Da es sich bei Pronomen und Modalverben um hochfrequente Ausdrücke handelt, ist ein zweiter Lesedurchgang nicht unbedingt zu erwarten, weshalb für diese AV v.a. die Unterschiede in den Fallzahlen ein Indiz für Verarbeitungsschwierigkeiten liefern. Wie Tabelle 6.31 $\mathrm{zu}$ entnehmen ist, spiegeln die Fallzahlen das prognostizierte Muster wider (n(Mono) < $\mathrm{n}(\mathrm{RK})<\mathrm{n}(\mathrm{WK}))$. Eine Interpretation ist schwierig, da bei der SPFD unklar ist, ob die VPn zwischen dem ersten und zweiten Lesedurchgang eine Sakkade in frühere oder spätere IA getätigt haben. Zur Aufklärung des Blickverhaltens sind die FPFD, die RPD und die TRT aussagekräftiger.

\begin{tabular}{cccccc}
\hline \hline Faktor & AV & Bed. & MW & Std. Abw. & $\mathrm{n}$ \\
\hline \multirow{4}{*}{ STRUKTUR } & \multirow{2}{*}{ SPFD } & RK & 246.74 & 148.79 & 54 \\
& & WK & 253.03 & 136.98 & 71 \\
& & Mono & 207.31 & 97.33 & 35 \\
\hline \hline
\end{tabular}

Tabelle 6.31: Deskriptive Statistik für die SPFD der Anaphern-Region (IA 6)

Die RPD umfasst alle Fixationen vor der ersten Sakkade in eine folgende IA. In den Mittelwerten zeigt sich das prognostizierte Muster (vgl. Tab. 6.32). Auffällig ist vor allem der hohe Mittelwert für die Bedingung WK. Das Muster ist jedoch nicht signifikant (vgl. Tab. 6.33).

\begin{tabular}{cccccc}
\hline \hline Faktor & AV & Bed. & MW & Std. Abw. & n \\
\hline \multirow{4}{*}{ STRUKTUR } & RPD & RK & 293.76 & 237.86 & 214 \\
& & WK & 332.02 & 279.31 & 211 \\
& Mono & 291.6 & 238 & 214 \\
\hline \hline
\end{tabular}

Tabelle 6.32: Deskriptive Statistik für die RPD der Anaphern-Region (IA 6)

\begin{tabular}{c|cc|cc|cc}
\hline \hline $\mathrm{AV}$ & $F_{1}(2,52)$ & $p$ & $F_{2}(2,46)$ & $p$ & $\min F^{\prime}(2,98)$ & $p$ \\
\hline $\mathrm{RPD}$ & 1.779 & 0.179 & 2.204 & 0.144 & 0.98 & $>0.05$ \\
\hline \hline
\end{tabular}

Tabelle 6.33: Inferenzstatistik für die RPD der Anaphern-Region (IA 6)

Die letzte und wichtigste Fixationszeit der Anaphern-Region ist die TRT, die als Summe aller Fixationen in der IA Auskunft über die gesamte Verarbeitungsdauer der Region gibt. In der TRT sind neben den frühen, eher lexikalischen Verarbeitungsprozessen, auch späte, einbettende und interpretierende Prozesse enthalten, d.h. auch Refixationen nach Verarbeitung folgender Textteile. Da die Interpretation einer Anapher kontextabhängig ist, ist dies die aussagekräftigste AV der Region. Wie Tabelle 6.34 und Abbildung 6.17 zu entnehmen ist, zeigt die TRT der Anaphern-Region deutlich das prognostizierte Muster. 


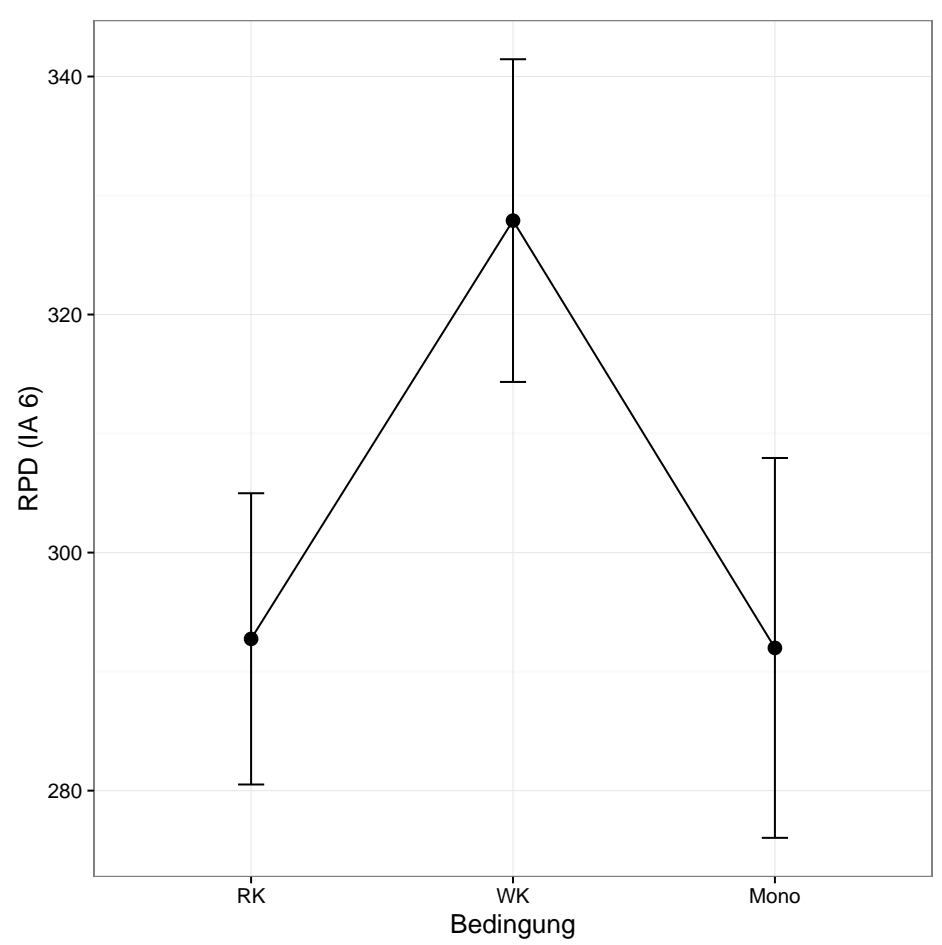

Abbildung 6.16: Plot der Mittelwerte der RPD der Anaphern-Region (IA 6)

\begin{tabular}{cccccc}
\hline \hline Faktor & AV & Bed. & MW & Std. Abw. & n \\
\hline \multirow{4}{*}{ STRUKTUR } & & RK & 343.25 & 261.03 & 216 \\
& & WK & 399.53 & 294.97 & 216 \\
& & Mono & 271.07 & 163.26 & 216 \\
\hline \hline
\end{tabular}

Tabelle 6.34: Deskriptive Statistik für die TRT der Anaphern-Region (IA 6)

Die Inferenzstatistik über alle Bedingungen liefert ein signifikantes Ergebnis (vgl. Tab. 6.35). Bei der Auswertung des Unterschieds zwischen den beiden Kompositabedingungen ist lediglich die ANOVA-by-subjects signifikant (vgl. Tab. 6.36).

\begin{tabular}{c|cc|cc|cc}
\hline \hline AV & $F_{1}(2,52)$ & $p$ & $F_{2}(2,46)$ & $p$ & $\operatorname{minF}^{\prime}(2,87)$ & $p$ \\
\hline TRT & 15.54 & $<0.001$ & 8.564 & $<0.001$ & 5.52 & $<0.01$ \\
\hline \hline
\end{tabular}

Tabelle 6.35: Inferenzstatistik für die TRT der Anaphern-Region (IA 6)

\begin{tabular}{c|cc|cc|cc}
\hline \hline AV & $F_{1}(1,26)$ & $p$ & $F_{2}(1,23)$ & $p$ & $\operatorname{minF}^{\prime}(1,36)$ & $p$ \\
\hline TRT & 7.451 & $<0.05$ & 2.164 & 0.155 & 1.36 & $>0.05$ \\
\hline \hline
\end{tabular}

Tabelle 6.36: Inferenzstatistik für die TRT der Anaphern-Region (IA 6) ohne Bed. Mono

\section{Interpretation}

Die Auswertung der Fixationszeiten für die Anaphern-Region (IA 6) bestätigt die generelle Arbeitshypothese. Über alle untersuchten Fixationszeiten hinweg liegen für die 


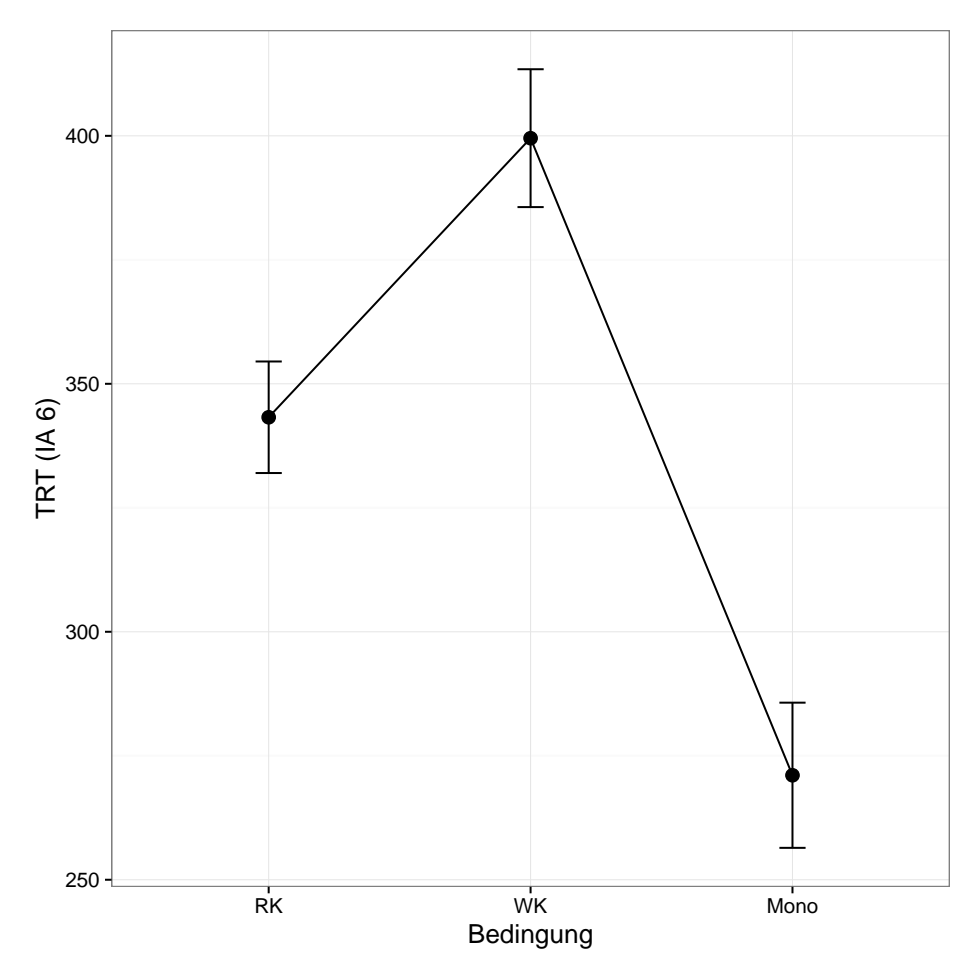

Abbildung 6.17: Plot der Mittelwerte der TRT der Anaphern-Region (IA 6)

Kontrollbedingung Mono die kürzesten Fixationszeiten vor. In Bezug auf die Kompositabedingungen liegen für die Bedingung RK tendenziell kürzere Fixationszeiten vor als für die Bedingung WK.

In der FFD ist bereits ein Unterschied zwischen den Kompositabedingungen und der Kontrollbedingung zu verzeichnen. Der Unterschied zwischen direkter Anapher und N1-Bezug scheint somit bereits auf frühe Verarbeitungsprozesse Einfluss zu nehmen. Für die erste Fixation wurde aufgrund ihrer Funktion für den rein lexikalischen Zugang kein Unterschied zwischen den beiden Kompositabedingungen erwartet. Entgegen der ursprünglich formulierten Prognose ist der Verarbeitungsvorteil für die Bedingung RK gegenüber der Bedingung WK jedoch bereits auf der FPFD zu sehen und sogar signifikant. Der Einfluss der experimentellen Manipulation auf den ersten Lesedurchlauf der Anaphern-Region ist ein Indiz für einen unverzüglich einsetzenden Anaphernresolutions-Prozess. Da zu diesem Zeitpunkt des Lesefortschritts ausschließlich die morphosyntaktischen Informationen der möglichen Antezdenten und des Pronomens zur Verfügung stehen und noch kein Kontext für die Resolution bekannt ist, spricht dieses Ergebnis für einen Unterschied in der Nutzbarkeit der morphosyntaktischen Informationen. Ohne Kenntnis des disambiguierenden Kontexts kann zu diesem Zeitpunkt noch keine Inferenzziehung stattgefunden haben. Der Unterschied zwischen den mittleren FPFDs der Bedingung RK und WK auf der Anaphern-Region spricht daher deutlich für einen Einfluss der Kompositums-Struktur auf die N1-Zugänglichkeit.

Die SPFD spiegelt das prognostizierte Muster in den Fallzahlen wider, d.h. im Auftreten eines zweiten Lesedurchgangs. Darüber hinaus ist das Muster in der RPD zu sehen, dort jedoch nicht signifikant. Auffällig ist hier die hohe mittlere Fixationszeit der Bedingung WK. Für die TRT ist das prognostizierte Muster signifikant. Dieser Effekt scheint maßgeblich durch den Effekt der FPFD getragen zu werden. Dass bei der inferenzstatistischen Analyse des Unterschieds zwischen den Bedingungen RK und WK für die TRT 
nur die ANOVA-by-subjects signifikant ist, spricht für eine Verunreinigung des Effekts durch spätere Maße, z.B. durch Regressionen. Die Schlussfolgerung, dass es sich bei den Unterschieden in den Fixationszeiten zwischen den drei Bedingungen um Auswirkungen des anaphorischen Bezugs handeln muss, resultiert daraus, dass das lexikalische Material (z.B. Er muss) in allen drei Bedingungen identisch ist.

\section{Regressionen}

\section{Hypothesen}

Für die ROCs der IA 6 wird kein Effekt erwartet, da der Suchprozess erst nach der Einbettung der Anapher beginnt. Die deskriptiven Werte der RPD lassen jedoch höhere Werte für die Bedingung WK erwarten. Für die RICs wird ein Effekt erwartet. Nach dem Lesen der Satzeinbettung sollten im Falle von Unsicherheiten bei der Anaphernresolution Eigenschaften des Pronomens, z.B. sein Genus, überprüft werden.

\section{Ergebnisse}

In den deskriptiven Daten der ROCs (Tab. 6.37) hat die Bedingung WK den höchsten Wert. Allerdings hat die Kontrollbedingung nicht den niedrigsten Wert, sodass das Muster nicht interpretierbar ist.

\begin{tabular}{cccccc}
\hline \hline Faktor & AV & Bed. & MW & Std. Abw. & $\mathrm{n}$ \\
\hline \multirow{4}{*}{ STRUKTUR } & ROC & RK & 0.07 & 0.25 & 214 \\
& & WK & 0.09 & 0.30 & 211 \\
& & Mono & 0.08 & 0.28 & 214 \\
\hline \hline
\end{tabular}

Tabelle 6.37: Deskriptive Statistik für die ROCs der Anaphern-Region (IA 6)

\begin{tabular}{c|cc|cc|cc}
\hline \hline $\mathrm{AV}$ & $F_{1}(2,52)$ & $p$ & $F_{2}(2,46)$ & $p$ & $\operatorname{minF}^{\prime}(2,87)$ & $p$ \\
\hline $\mathrm{ROC}$ & 0.673 & 0.514 & 0.372 & 0.691 & 0.24 & $>0.05$ \\
\hline \hline
\end{tabular}

Tabelle 6.38: Inferenzstatistik für die ROCs der Anaphern-Region (IA 6)

Bei Betrachtung der deskriptiven Daten der RICs (siehe Tab. 6.39) zeigt sich deutlich ein hypothesenkonformes Muster, welches als signifikant bestätigt werden kann (vgl. Tab. 6.40). Demnach wird die Anaphern-Region je nach Bedingung unterschiedlich oft refixiert.

\begin{tabular}{cccccc}
\hline \hline Faktor & AV & Bed. & MW & Std. Abw. & n \\
\hline \multirow{3}{*}{ STRUKTUR } & \multirow{2}{*}{ RIC } & RK & 0.15 & 0.41 & 214 \\
& & WK & 0.18 & 0.46 & 211 \\
& & Mono & 0.03 & 0.19 & 214 \\
\hline \hline
\end{tabular}

Tabelle 6.39: Deskriptive Statistik für die RICs der Anaphern-Region (IA 6) 


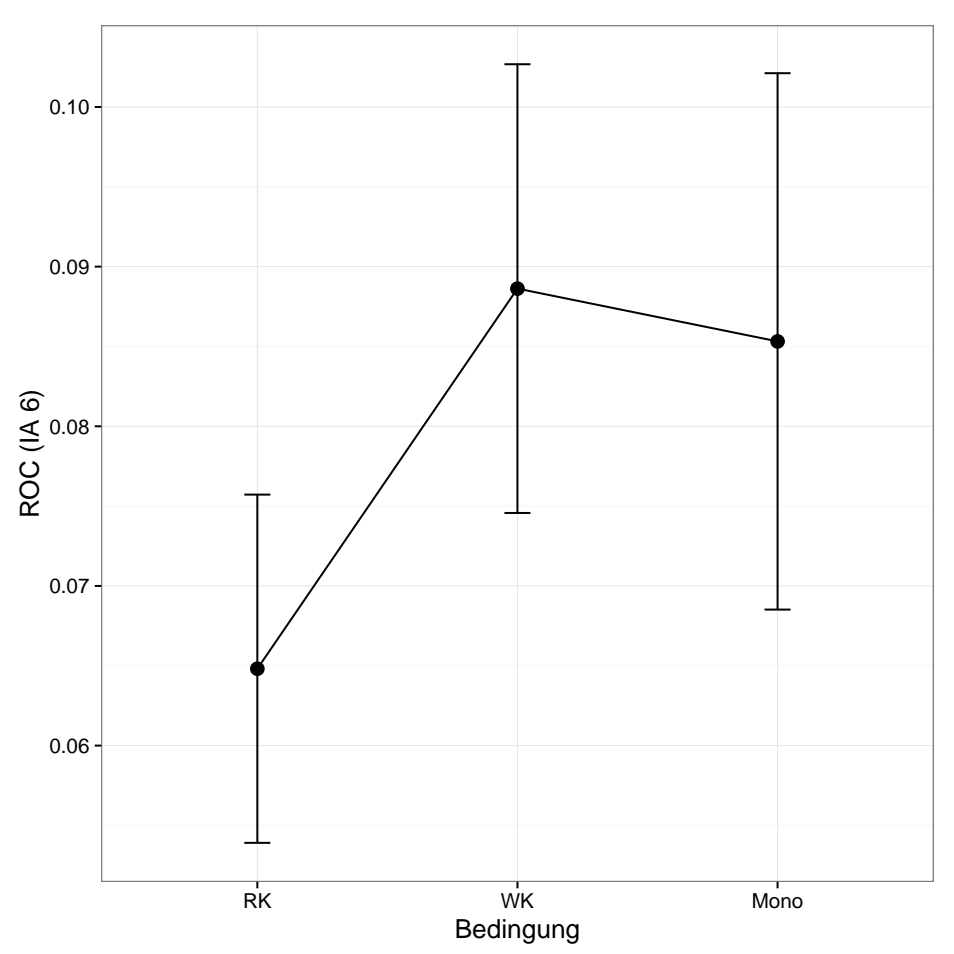

Abbildung 6.18: Plot der Mittelwerte der ROCs der Anaphern-Region (IA 6)

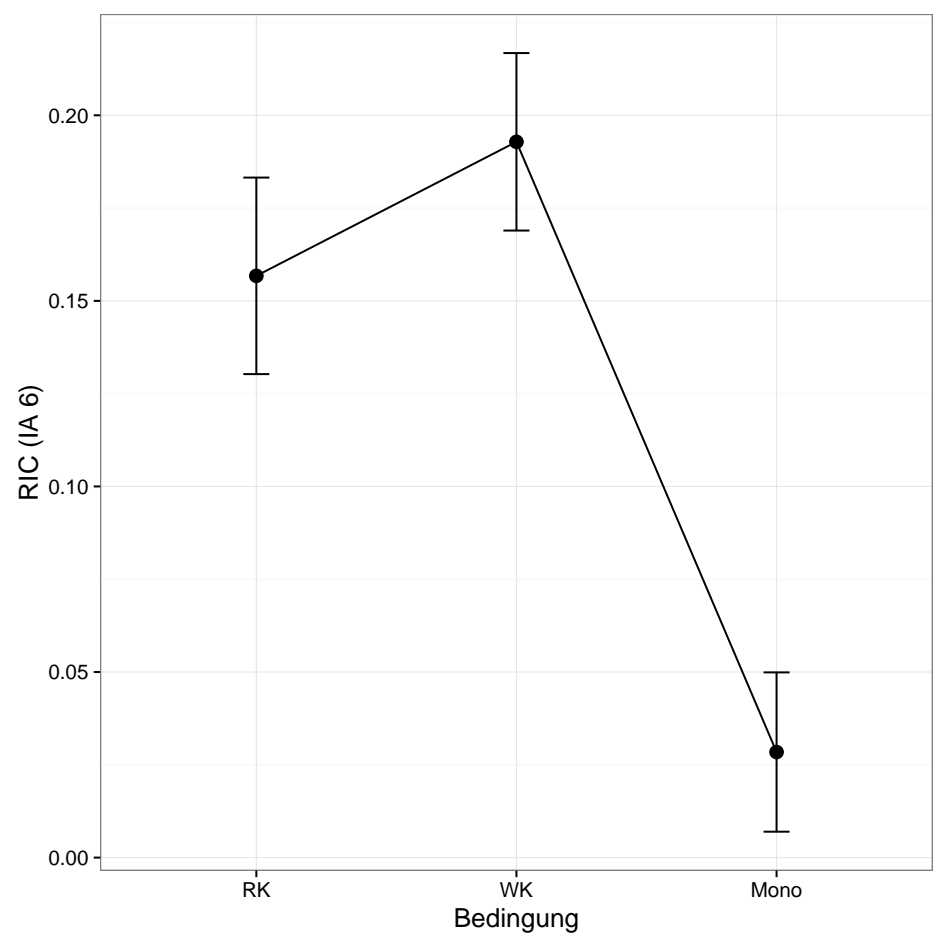

Abbildung 6.19: Plot der Mittelwerte der RICs der Anaphern-Region (IA 6)

\begin{tabular}{c|cc|cc|cc}
\hline \hline $\mathrm{AV}$ & $F_{1}(2,52)$ & $p$ & $F_{2}(2,46)$ & $p$ & $\operatorname{minF}^{\prime}(2,94)$ & $p$ \\
\hline $\mathrm{RIC}$ & 8.466 & $<0.001$ & 6.424 & $<0.01$ & 3.65 & $<0.05$ \\
\hline \hline
\end{tabular}

Tabelle 6.40: Inferenzstatistik für die RICs der Anaphern-Region (IA 6) 
Der Unterschied zwischen den Kompositabedingungen kann nicht als signifikant bestätigt werden (vgl. Tab. 6.41).

\begin{tabular}{c|cc|cc|cc}
\hline \hline $\mathrm{AV}$ & $F_{1}(1,26)$ & $p$ & $F_{2}(1,23)$ & $p$ & $\operatorname{minF}^{\prime}(1,46)$ & $p$ \\
\hline $\mathrm{RIC}$ & 0.468 & 0.5 & 0.312 & 0.582 & 0.19 & $>0.05$ \\
\hline \hline
\end{tabular}

Tabelle 6.41: Inferenzstatistik für die RICs der Anaphern-Region (IA 6) ohne Bed. Mono

\section{Interpretation}

Für die Regressionen aus der Anaphern-Region in frühere Regionen (ROCs) lässt sich wie erwartet kein Effekt finden. Dies lässt sich darauf zurückführen, dass es sich bei der Anapher um einen kurzen und frequenten Ausdruck handelt, d.h. der Suchprozess muss nicht zwangsläufig von der Anapher ausgehen, sondern kann auch später im Satz seinen Ausgangspunkt haben, da die Verarbeitungsschwierigkeit nicht im Ausdruck selbst liegt, sondern in seinem referenziellen Bezug. Diese Annahme erklärt auch das Ausbleiben eines Effekts der RPD auf der Anaphern-Region. Gleichzeitig schließt dies nicht aus, dass anapherninduzierte Suchprozesse stattfinden; diese können ihren Ausgangspunkt in einer späteren IA nehmen.

Der höchste Wert für die Zahl an Regressionen in die Anaphern-Region (RICs) liegt in der Bedingung WK vor, wie es bereits die Auswertung der RPD nahelegt. Aus dem hypothesenkonformen Effekt für die RICs lässt sich ableiten, dass die Anapher abhängig von der Bedingung in unterschiedlichem Maße für Unsicherheit in der Satzinterpretation sorgt. Je größer die Unsicherheit bzgl. des Bezugs ist, desto größer ist die Wahrscheinlichkeit einer Refixation der Anaphern-Region zur Überprüfung des Pronomens, z.B. seines Genus. Diese Unsicherheit wird dadurch erhöht, dass sich die beiden verwendeten Pronomen er und es nur in einem Buchstaben unterscheiden. Darüber hinaus stützen die Ergebnisse der Auswertung der RICs die These zur Zusammensetzung der TRT aus FPFD und späteren Refixationen. Der Unterschied zwischen RK und WK ist in den RICs jedoch nicht signifikant.

Wenn die Interpretation korrekt ist, dass die Refixationshäufigkeiten der Anaphern-Region aus einer Unsicherheit bzgl. des anaphorischen Ausdrucks nach Lesen der Satzergänzung resultiert, sollten die Regressionen aus den Folgeregionen heraus ebenfalls das Hypothesenmuster zeigen. Dies soll als nächstes geprüft werden.

\section{Auswertung der Satzergänzung (IA 7)}

Die IA 7 beinhaltet die Satzergänzung der Anapher, d.h. es handelt sich um den die Anapher disambiguierenden Satzteil in der Folgezeile. Die Satzergänzung ist über alle drei Bedingungen hinweg identisch und kann somit als eine IA ausgewertet werden. Da die Interpretation der Anapher einen maßgeblichen Beitrag zur Satzbedeutung liefert, müssten sich Verarbeitungsunterschiede bei der Anaphernresolution auch in den Gesamtlesezeiten der Satzergänzung niederschlagen. 


\section{Fixationen}

\section{Hypothesen}

Da der Anaphernresolutions-Prozess erst bei der Textintegration oder sogar erst im ,sentence wrap up' abgeschlossen wird, wird ein Effekt auf der TRT der IA 7 erwartet $(\mathrm{TRT}($ Mono $)<\mathrm{TRT}(\mathrm{RK})<\mathrm{TRT}(\mathrm{WK}))$. Da die Region sich zwischen den Bedingungen nicht unterscheidet, werden für frühere Fixationszeiten - die vorrangig lexikalische Verarbeitung widerspiegeln - keine Effekte erwartet.

\section{Ergebnisse}

Da es sich bei der IA 7 um eine große IA handelt, die eine komplette Zeile einnimmt, ist die FFD keine relevante AV. Das Ergebnis der FPFD ist nicht interpretierbar, da hier die Kontrollbedingung die längste mittlere Fixationszeit aufweist (Tab. 6.42). Das Muster ist nicht signifikant (Tab. 6.43).

\begin{tabular}{cccccc}
\hline \hline Faktor & AV & Bed. & MW & Std. Abw. & n \\
\hline \multirow{4}{*}{ STRUKTUR } & \multirow{2}{*}{ FPFD } & RK & 1203.38 & 632.67 & 216 \\
& & WK & 1136.52 & 572.17 & 216 \\
& & Mono & 1203.4 & 549.77 & 215 \\
\hline \hline
\end{tabular}

Tabelle 6.42: Deskriptive Statistik für die FPFD der Satzergänzung (IA 7)

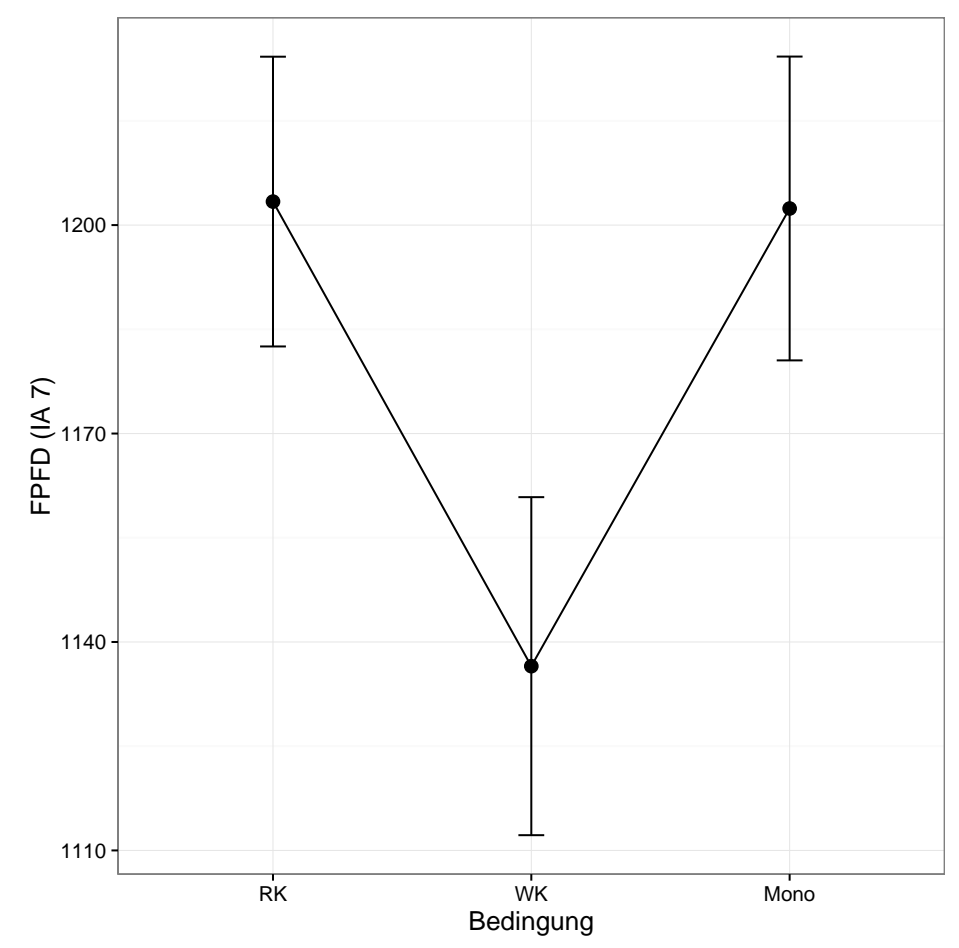

Abbildung 6.20: Plot der Mittelwerte der FPFD der Satzergänzung (IA 7) 


\begin{tabular}{c|cc|cc|cc}
\hline \hline $\mathrm{AV}$ & $F_{1}(2,52)$ & $p$ & $F_{2}(2,46)$ & $p$ & $\operatorname{minF}^{\prime}(2,95)$ & $p$ \\
\hline FPFD & 1.968 & 0.15 & 1.577 & 0.217 & 0.88 & $>0.05$ \\
\hline \hline
\end{tabular}

Tabelle 6.43: Inferenzstatistik für die FPFD der Satzergänzung (IA 7)

In den Daten der SPFD zeigt sich ein deutlicher Unterschied zwischen den Kompositabedingungen und der Kontrollbedingung (Tab. 6.44). Auch bei dieser IA spiegeln die Fallzahlen des zweiten Lesedurchlaufs das Muster der Hypothese wider. Gleichzeitig ist eine weitere Auswertung aufgrund der unterschiedlichen Fallzahlen nicht sinnvoll.

\begin{tabular}{cccccc}
\hline \hline Faktor & AV & Bed. & MW & Std. Abw. & n \\
\hline \multirow{4}{*}{ STRUKTUR } & \multirow{2}{*}{ SPFD } & RK & 712.42 & 526.01 & 73 \\
& & WK & 718.93 & 582.23 & 89 \\
& & Mono & 551.43 & 526.19 & 49 \\
\hline \hline
\end{tabular}

Tabelle 6.44: Deskriptive Statistik für die SPFD der Satzergänzung (IA 7)

Wie bereits in der SPFD zeigt sich auch in der RPD v.a. ein Unterschied zwischen den Komposita und der Kontrollbedingung. Das Ergebnis der RPD unter Berücksichtigung aller Bedingungen ist signifikant (vgl. Tab. 6.46). Der Unterschied zwischen den Bedingungen RK und WK entspricht nicht der Hypothese und ist in der Einzelauswertung nicht signifikant (vgl. Tab. 6.47).

\begin{tabular}{cccccc}
\hline \hline Faktor & AV & Bed. & MW & Std. Abw. & n \\
\hline \multirow{4}{*}{ STRUKTUR } & RPD & RK & 1677.06 & 1102.58 & 216 \\
& & WK & 1650.69 & 907.35 & 216 \\
& Mono & 1285.57 & 595.7 & 215 \\
\hline \hline
\end{tabular}

Tabelle 6.45: Deskriptive Statistik für die RPD der Satzergänzung (IA 7)

\begin{tabular}{c|cc|cc|cc}
\hline \hline $\mathrm{AV}$ & $F_{1}(2,52)$ & $p$ & $F_{2}(2,46)$ & $p$ & $\min F^{\prime}(2,96)$ & $p$ \\
\hline $\mathrm{RPD}$ & 13.51 & $<0.001$ & 11.73 & $<0.001$ & 6.28 & $<0.01$ \\
\hline \hline
\end{tabular}

Tabelle 6.46: Inferenzstatistik für die RPD der Satzergänzung (IA 7)

\begin{tabular}{c|cc|cc|cc}
\hline \hline $\mathrm{AV}$ & $F_{1}(1,26)$ & $p$ & $F_{2}(1,23)$ & $p$ & $\operatorname{minF}^{\prime}(1,46)$ & $p$ \\
\hline $\mathrm{RPD}$ & 0.095 & 0.761 & 0.062 & 0.805 & 0.04 & $>0.05$ \\
\hline \hline
\end{tabular}

Tabelle 6.47: Inferenzstatistik für die RPD der Satzergänzung (IA 7) ohne Bed. Mono

Die Ergebnisse für die TRT der Satzergänzung entsprechen schließlich der Hypothese und die ANOVAs weisen für die Manipulation ein signifikantes Ergebnis auf (vgl. Tab. 6.49). Die Auswertung des Einzelvergleichs der Bedingung RK und WK ist allerdings nicht signifikant (vgl. Tab. 6.50). 


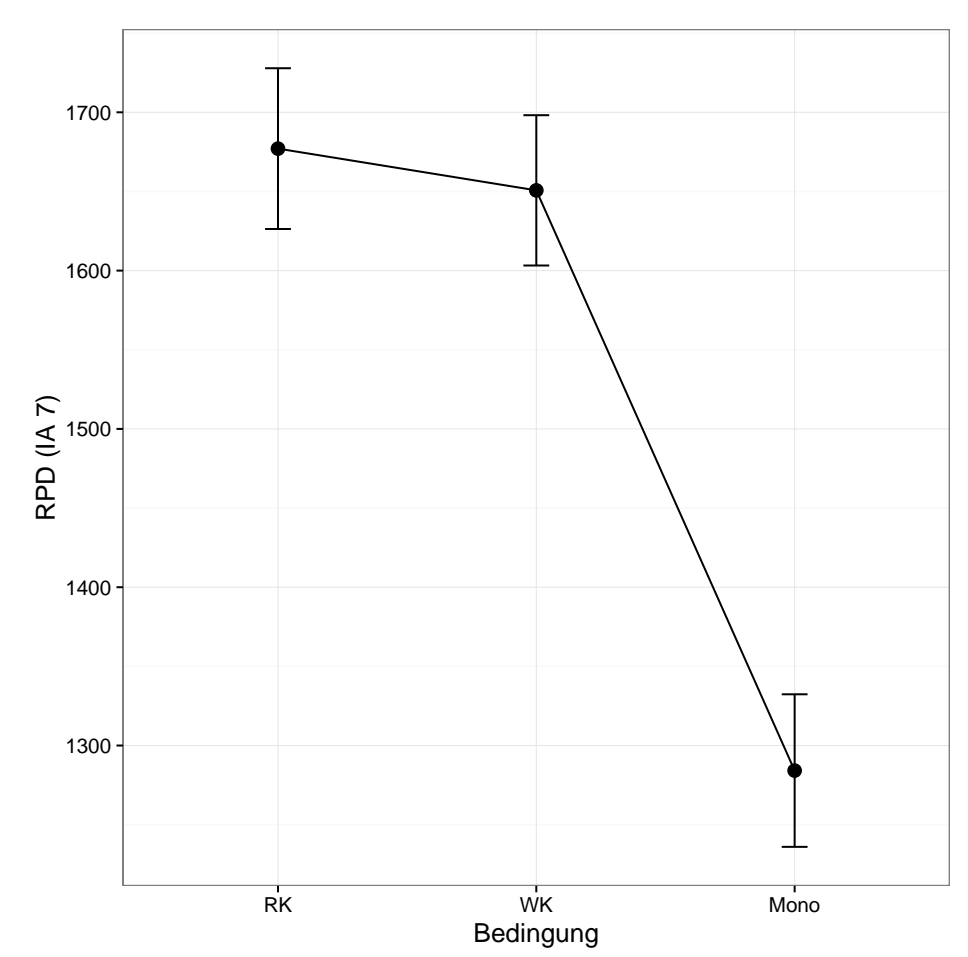

Abbildung 6.21: Plot der Mittelwerte der RPD der Satzergänzung (IA 7)

\begin{tabular}{cccccc}
\hline \hline Faktor & AV & Bed. & MW & Std. Abw. & $\mathrm{n}$ \\
\hline \multirow{4}{*}{ STRUKTUR } & \multirow{2}{*}{ TRT } & RK & 1556.29 & 753.87 & 216 \\
& & WK & 1568.93 & 790.4 & 216 \\
& & Mono & 1345.75 & 650.09 & 216 \\
\hline \hline
\end{tabular}

Tabelle 6.48: Deskriptive Statistik für die TRT der Satzergänzung (IA 7)

\begin{tabular}{c|cc|cc|cc}
\hline \hline AV & $F_{1}(2,52)$ & $p$ & $F_{2}(2,46)$ & $p$ & $\operatorname{minF}^{\prime}(2,85)$ & $p$ \\
\hline TRT & 10.32 & $<0.001$ & 5.256 & $<0.01$ & 3.48 & $<0.05$ \\
\hline \hline
\end{tabular}

Tabelle 6.49: Inferenzstatistik für die TRT der Satzergänzung (IA 7)

\begin{tabular}{c|cc|cc|cc}
\hline \hline AV & $F_{1}(1,26)$ & $p$ & $F_{2}(1,23)$ & $p$ & $\operatorname{minF}^{\prime}(1,43)$ & $p$ \\
\hline TRT & 0.043 & 0.837 & 0.023 & 0.88 & 0.01 & $>0.05$ \\
\hline \hline
\end{tabular}

Tabelle 6.50: Inferenzstatistik für die TRT der Satzergänzung (IA 7) ohne Bed. Mono

\section{Interpretation}

Die TRT der Satzergänzung weist einen reliablen Effekt in die erwartete Richtung auf. Für die weiteren AVn wurde kein Effekt vorhergesagt. Es zeigt sich jedoch in der SPFD und der RPD ein deutlicher Unterschied zwischen Komposita und Kontrollbedingung. Das Ergebnis der FPFD ist maßgeblich durch das konkrete lexikalische Material geprägt und daher nicht interpretierbar. Die SPFD und die RPD hingegen können durch Regressionen in frühere Regionen beeinflusst sein. Da diese beiden AVn als über die rein lexikalische 


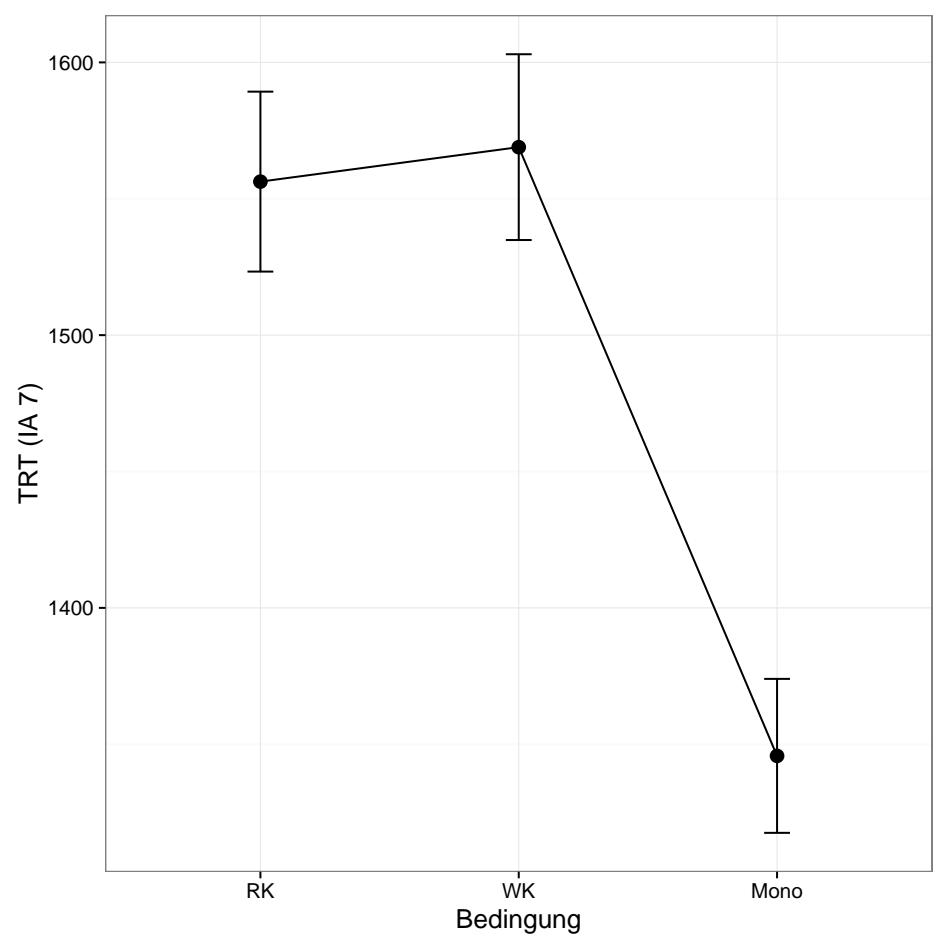

Abbildung 6.22: Plot der Mittelwerte der TRT für die Satzergänzung (IA 7)

Verarbeitung hinausgehende AVn bereits weitere Verarbeitungsschritte abbilden, kann der grundlegende Unterschied zwischen Kontroll- und Kompositabedingungen bereits hier in Erscheinung treten. Der Unterschied zwischen RK und WK ist nicht signifikant, jedoch deskriptiv in der TRT sichtbar.

Als Ursache für den der Hypothese zuwiderlaufenden Unterschied zwischen den beiden Kompositabedingungen in der RPD nehme ich die Größe der IA an: Die gesamte Zeile wurde als eine IA definiert, da das in ihr enthaltene Material sich zwischen den Items in Lexik und Syntax unterscheidet und somit eine weitere Untergliederung nicht systematisch möglich ist. Aufgrund dessen lässt sich nicht bestimmen, an welcher Stelle längere und kürzere Fixationen zu verorten sind und wodurch diese ausgelöst werden. Die durch die lexikalische und strukturelle Varianz erzeugten Unterschiede verdecken somit einen möglichen Effekt. Auf eine einheitlichere Gestaltung der Satzergänzung wurde zugunsten von Plausibilität und Natürlichkeit der Items verzichtet. Nach Aufsummieren aller Verarbeitungsphasen und Fixationen zeigt sich in der TRT ein hypothesenkonformer Effekt. Diesem Ergebnis zufolge wirkt sich die Manipulation des Ankerausdrucks auf spätere Verarbeitungsprozesse innerhalb der Verarbeitung der Satzergänzung aus, z.B. im ,sentence wrap-up' Der Verarbeitungsunterschied für die IA 7 lässt sich innerhalb der IA nicht genau lokalisieren, sollte sich aber in den ROCs der Region wiederfinden, also in den Suchprozessen, die in der Region ihren Anfang und vorhergehende Regionen als Ziel haben, z.B. die Antezedens- oder die Anaphern-Region. 


\section{Regressionen}

\section{Hypothesen}

Es wird eine Abbildung des durch die IA 7 ausgelösten Such- und Rückversicherungsprozesses in Form von Regressionen aus der Region heraus erwartet: ROC(Mono) < $\operatorname{ROC}(\mathrm{RK})<\operatorname{ROC}(\mathrm{WK})$.

\section{Ergebnisse}

Die ROCs der Satzergänzung zeigen einen hypothesenkonformen Effekt (siehe Tab. 6.51 und 6.52.

\begin{tabular}{cccccc}
\hline \hline Faktor & AV & Bed. & MW & Std. Abw. & n \\
\hline \multirow{4}{*}{ STRUKTUR } & \multirow{2}{*}{ ROC } & RK & 0.36 & 0.69 & 216 \\
& & WK & 0.39 & 0.64 & 216 \\
& & Mono & 0.07 & 0.27 & 215 \\
\hline \hline
\end{tabular}

Tabelle 6.51: Deskriptive Statistik für die ROCs der Satzergänzung (IA 7)

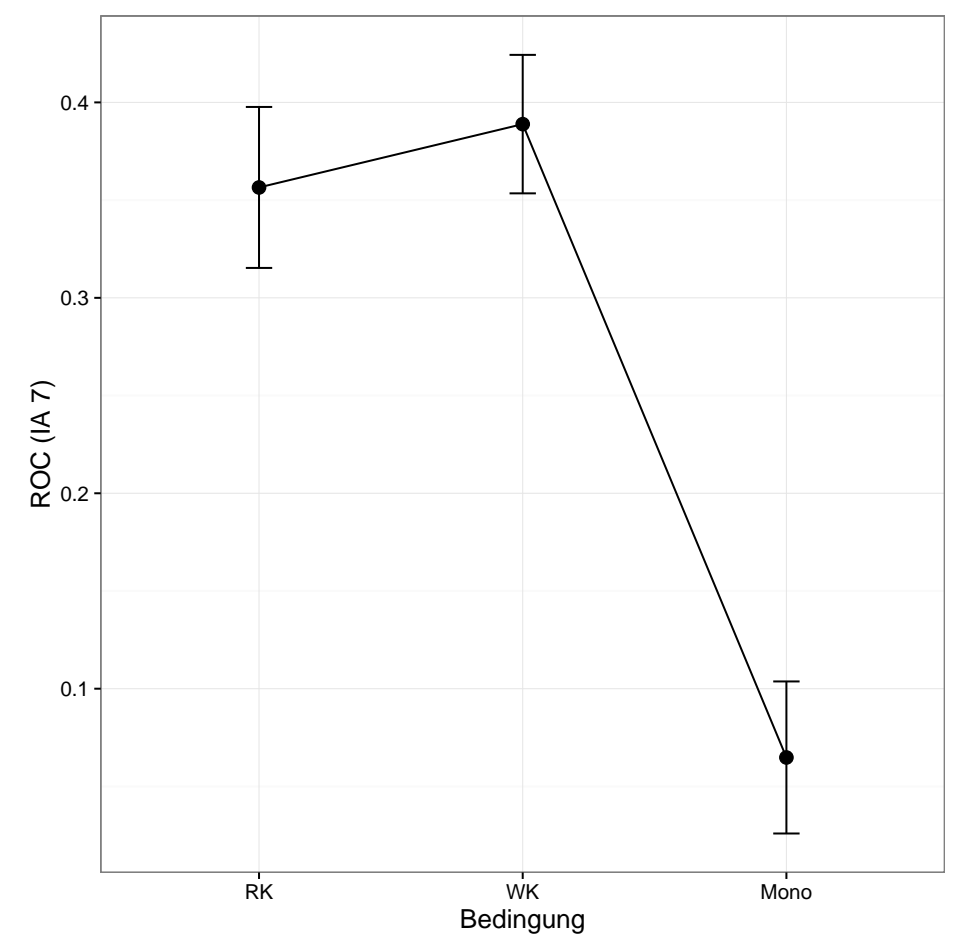

Abbildung 6.23: Plot der Mittelwerte der ROCs der Satzergänzung (IA 7)

\begin{tabular}{c|cc|cc|cc}
\hline \hline $\mathrm{AV}$ & $F_{1}(2,52)$ & $p$ & $F_{2}(2,46)$ & $p$ & $\operatorname{minF}^{\prime}(2,97)$ & $p$ \\
\hline ROC & 14.34 & $<0.001$ & 12.68 & $<0.001$ & 6.73 & $<0.01$ \\
\hline \hline
\end{tabular}

Tabelle 6.52: Inferenzstatistik für die ROCs der Satzergänzung (IA 7) 
Der Unterschied zwischen den beiden Kompositabedingungen verhält sich deskriptiv hypothesenkonform, ist allerdings nicht signifikant (vgl. Tab. 6.53).

\begin{tabular}{c|cc|cc|cc}
\hline \hline $\mathrm{AV}$ & $F_{1}(1,26)$ & $p$ & $F_{2}(1,23)$ & $p$ & $\operatorname{minF} F^{\prime}(1,46)$ & $p$ \\
\hline $\mathrm{ROC}$ & 0.239 & 0.629 & 0.164 & 0.69 & 0.1 & $>0.05$ \\
\hline \hline
\end{tabular}

Tabelle 6.53: Inferenzstatistik für die ROCs der Satzergänzung (IA 7) ohne Bed. Mono

\section{Interpretation}

Gemäß der Auswertung der ROCs löst das Lesen der Satzergänzung in hypothesenkonformer Weise regressive Sakkaden aus. Während der sententiellen Einbettung der Anapher wird ein Such- und Rückversicherungsprozess ausgelöst. Dies stützt die Interpretation der Fixationszeiten der IA 7.

\section{Auswertung der Antezedens-Region (IA 4)}

\section{Fixationen}

\section{Hypothesen}

Vor dem Lesen der Anapher werden für die Antezedens-Region (IA 4) keine systematischen Unterschiede erwartet, d.h. es werden keine Effekte für FFD, FPFD und RPD prognostiziert. Die FFD ist bei längeren Wörtern wenig aussagekräftig und FPFD und RPD spiegeln ausschließlich die Verarbeitung der Ankerausdrücke wider. Für diese werden unabhängig vom anaphorischen Bezug keine generellen Verarbeitungsunterschiede vorhergesagt. Mit dem Lesen der Anapher (IA 6) setzt der Anaphernresolutions-Prozess ein, der im Fall von Verarbeitungsschwierigkeiten mit Refixationen der Antezedens-Region verbunden sein sollte, weshalb systematische Unterschiede in der TRT der IA 4 (d.h. inkl. Refixationen) erwartet werden: TRT(Mono) $<\mathrm{TRT}(\mathrm{RK})<\mathrm{TRT}(\mathrm{WK})$.

Da sich die Ankerausdrücke hinsichtlich ihrer Wortlänge und Frequenz unterscheiden, müssen diese beiden Größen als Einflussfaktoren berücksichtigt werden (vgl. Rayner 1998. 387). Dabei liegt in der Bedingung Mono stets der kürzeste Ausdruck vor, das Kompositum in der Bedingung WK ist etwas länger und in der Bedingung RK liegen aufgrund der Derivationsaffixe die meisten Zeichen vor. Da auf einem Ausdruck mehr Fixationen stattfinden können, je länger er ist, wirkt sich dies auch auf die Gesamt-Fixationszeit eines Ausdrucks aus. Daher wird für alle Fixationszeiten der IA 4 ein Längeneffekt nach dem Muster Fix.(Mono) < Fix.(WK) < Fix.(RK) erwartet.

Bezüglich der Frequenzen verfügt Bedingung Mono stets über die höchsten Werte ${ }^{17}$ In den meisten Fällen ist das Wurzelkompositum frequenter als das Rektionskompositum. Je frequenter ein Ausdruck ist, desto größer ist sein Verarbeitungsvorteil und desto kürzer sollte seine Fixationszeit sein. Somit wird für die IA 4 ein Frequenzeffekt nach dem Muster Fix.(Mono) < Fix.(WK) < Fix.(RK) erwartet. Länge und Frequenz sollten sich demnach

\footnotetext{
${ }^{17}$ Die Frequenzwerte basieren auf den absoluten Werten der entsprechenden Ausdrücke aus dem Kernkorpus 20 des DWDS. Da der Einfluss der Frequenz auf die Lesezeit exponentiell ist, wurden die Werte einem natürlichen Logarithmus unterzogen. Um den Wert 0 auszuschließen, wurde zu allen Werten 0.5 addiert.
} 
in gleicher Weise auf die Fixationszeiten auswirken.

\section{Ergebnisse}

Wie Tabelle 6.54 und Abbildung 6.24 zu entnehmen ist, zeigt die FPFD der Rohdaten deutlich das Muster FPFD(Mono) $<$ FPFD(WK) $<$ FPFD(RK), welches auch signifikant ist (siehe Tab. 6.55). Gemäß der Hypothese lässt sich dies als Längen- und/oder Frequenzeffekt deuten. Bei einer Wiederholung der Berechnungen mit den Residuen der FPFD, nachdem der Effekt der Länge herausfaktorisiert wurde (resFPFD.L), besteht dieser Effekt nicht länger. Bei der Berechnung auf den Residuen der Frequenz (resFPFD.F) kommt der Längeneffekt erneut zum Tragen. Der Einfluss der Länge scheint somit stärker als der Einfluss der Frequenz. Bei Zusammenfassung von Längen- und Frequenzresiduen (resFPFD.LF) zeigt sich wiederum kein interpretierbares Muster und kein Effekt.

\begin{tabular}{|c|c|c|c|c|c|}
\hline Faktor & AV & Bed. & MW & Std. Abw. & $\mathrm{n}$ \\
\hline \multirow{12}{*}{ STRUKTUR } & \multirow{3}{*}{ FPFD } & RK & 588.69 & 265 & 216 \\
\hline & & WK & 491.21 & 268.41 & 216 \\
\hline & & Mono & 292.83 & 134.35 & 215 \\
\hline & \multirow{3}{*}{ resFPFD.L } & RK & 3.96 & 264.1 & 216 \\
\hline & & WK & 13.62 & 266.46 & 216 \\
\hline & & Mono & -17.66 & 133.63 & 215 \\
\hline & \multirow{3}{*}{ resFPFD.F } & RK & 35.68 & 268.7 & 216 \\
\hline & & WK & -19.13 & 267.14 & 216 \\
\hline & & Mono & -16.63 & 139.68 & 215 \\
\hline & \multirow{3}{*}{ resFPFD.LF } & RK & -8.27 & 263.91 & 216 \\
\hline & & WK & 6.87 & 266.08 & 216 \\
\hline & & Mono & 1.41 & 133.73 & 215 \\
\hline
\end{tabular}

Tabelle 6.54: Deskriptive Statistik für die FPFD der Antezedens-Region (IA 4) mit Berücksichtigung der Längen- und Frequenzresiduen

\begin{tabular}{c|cc|cc|cc}
\hline \hline $\mathrm{AV}$ & $F_{1}(2,52)$ & $p$ & $F_{2}(2,46)$ & $p$ & $\operatorname{minF}$ & $p$ \\
\hline FPFD & 83.14 & $<0.001$ & 96.55 & $<0.001$ & $(2,98) 44.67$ & $<0.001$ \\
resFPFD.L & 0.923 & 0.401 & 1.095 & 0.343 & $(2,98) 0.5$ & $>0.05$ \\
resFPFD.F & 3.657 & $<0.05$ & 3.489 & $<0.05$ & $(2,97) 1.79$ & $>0.05$ \\
resFPFD.LF & 0.205 & 0.815 & 0.256 & 0.775 & $(2,98) 0.11$ & $>0.05$ \\
\hline \hline
\end{tabular}

Tabelle 6.55: Inferenzstatistik für die FPFD der Antezedens-Region (IA 4) mit Berücksichtigung der Längen- und Frequenzresiduen

Eine Auswertung der SPFD wird für die Antezedens-Region nicht für sinnvoll erachtet, da bei dieser Größe unklar ist, ob zwischen erstem und zweiten Lesedurchgang eine Fixation nach rechts, z.B. zur Anapher stattgefunden hat. Da sich dies auch von VP zu VP unterscheiden kann, ist eine einheitliche Interpretation der SPFD nicht möglich. Die RPD beinhaltet lediglich Sakkaden in vorhergehende IAs, ein Einfluss der Anapher ist daher auszuschließen. Für die RPD wurden entsprechend der Analysen für die FPFD Berechnungen auf den Rohdaten und den verschiedenen Residuen durchgeführt (vgl. Tab. 6.56 und 


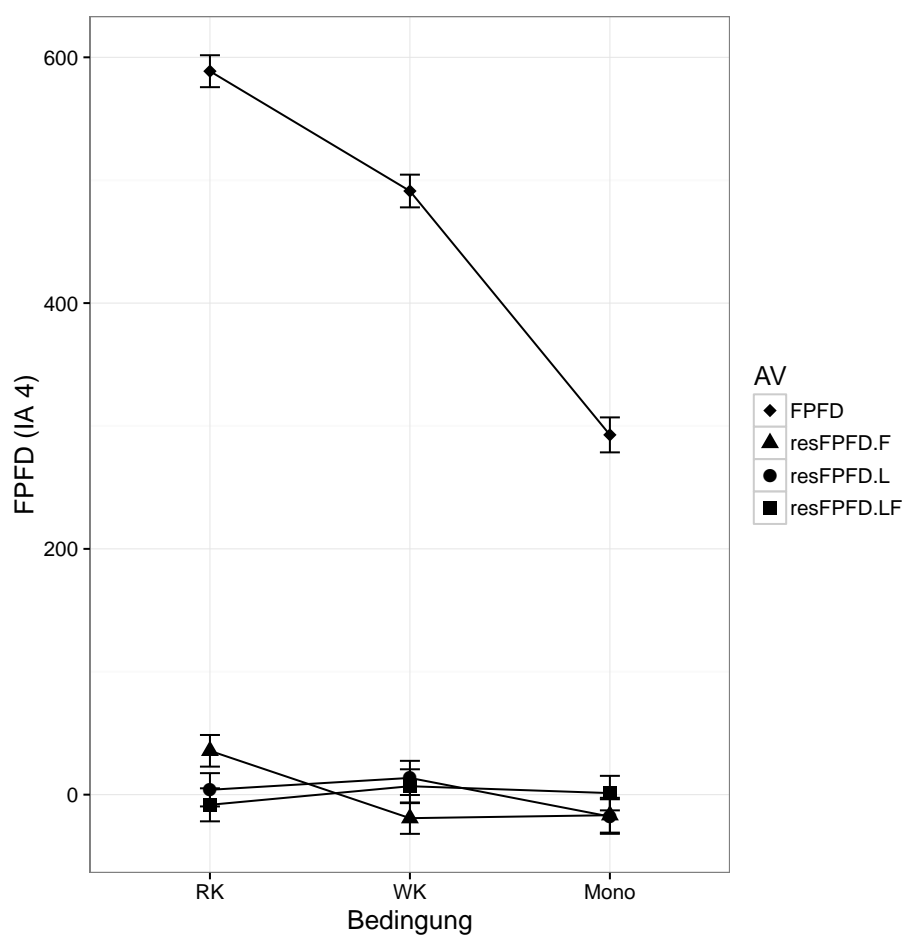

Abbildung 6.24: Plot der Mittelwerte der FPFD der Antezedens-Region (IA 4) mit Berücksichtigung der Längen- und Frequenzresiduen

6.57). Es zeigt sich wie bereits bei der FPFD ein deutlicher Längen- und Frequenzeffekt. Bei den Berechnungen auf den verschiedenen Residuen wird kein Effekt ersichtlich. Ein Unterschied in der Stärke des Einflusses von Länge oder Frequenz ist hier nicht ersichtlich.

\begin{tabular}{cccccc}
\hline \hline Faktor & AV & Bed. & MW & Std. Abw. & n \\
\hline \multirow{4}{*}{ RPD } & RK & 631.06 & 356.53 & 216 \\
& & WK & 548.34 & 325.44 & 216 \\
& & Mono & 309.83 & 197.19 & 215 \\
\cline { 2 - 6 } & \multirow{3}{*}{ resRPD.L } & RK & -2.14 & 353.97 & 216 \\
& & WK & 30.39 & 327.91 & 216 \\
& & Mono & -28.38 & 197.5 & 215 \\
\cline { 2 - 6 } STRUKTUR & RK & 29.1 & 360.32 & 216 \\
& \multirow{3}{*}{ resRPD.F } & WK & -6.42 & 323.52 & 216 \\
& & Mono & -22.78 & 204.35 & 215 \\
\cline { 2 - 6 } & \multirow{3}{*}{ resRPD.LF } & RK & -18.19 & 353.85 & 216 \\
& & WK & 21.54 & 327.36 & 216 \\
& & Mono & -3.38 & 198.02 & 215 \\
\hline \hline
\end{tabular}

Tabelle 6.56: Deskriptive Statistik für die RPD der Antezedens-Region (IA 4) mit Berücksichtigung der Längen- und Frequenzresiduen 


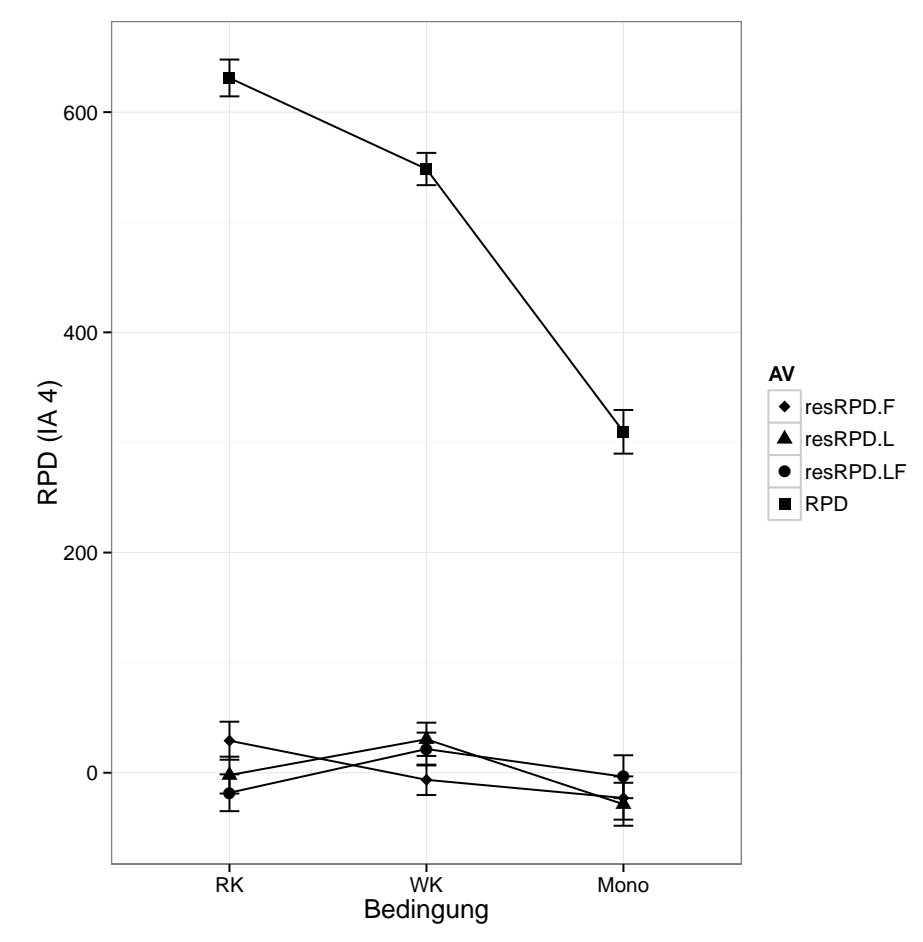

Abbildung 6.25: Plot der Mittelwerte der RPD der Antezedens-Region (IA 4) mit Berücksichtigung der Längen- und Frequenzresiduen

\begin{tabular}{c|cc|cc|cc}
\hline \hline $\mathrm{AV}$ & $F_{1}(2,52)$ & $p$ & $F_{2}(2,46)$ & $p$ & $\operatorname{minF}(2,98)$ & $p$ \\
\hline $\mathrm{RPD}$ & 62.94 & $<0.001$ & 73.31 & $<0.001$ & 33.87 & $<0.001$ \\
resRPD.L & 1.994 & 0.146 & 2.11 & 0.133 & 1.03 & $>0.05$ \\
resRPD.F & 1.647 & 0.203 & 1.669 & 0.2 & 0.83 & $>0.05$ \\
resRPD.LF & 0.923 & 0.404 & 0.996 & 0.377 & 0.48 & $>0.05$ \\
\hline \hline
\end{tabular}

Tabelle 6.57: Inferenzstatistik für die RPD der Antezedens-Region (IA 4) mit Berücksichtigung der Längen- und Frequenzresiduen

Die TRT der IA 4 beinhaltet alle Fixationen in der Region, d.h. auch Refixation nach dem Lesen späterer Textteile. Beim Verarbeiten der Anapher ist anzunehmen, dass bei Resolutionsschwierigkeiten die möglichen Antezedenten häufiger und/oder länger refixiert werden. In diesem Sinne kann die TRT der IA 4 Auskunft über den Einfluss der Anapher geben. Wie in Tabelle 6.58 ersichtlich, zeigt die TRT der Antezedens-Region mit dem Muster TRT(Mono) < TRT(WK) < TRT(RK) einen deutlichen Längeneffekt, welcher durch die Inferenzstatistik als reliabel bestätigt wird (siehe Tab. 6.59). Bei den Berechnungen auf den Längenresiduen (resTRT.L) zeigt sich das Muster TRT(Mono) $<$ TRT(RK) < TRT(WK), welches durch die ANOVAs als signifikant bestätigt wird. Auf den Frequenzresiduen (resTRT.F) zeichnet sich erneut der Längeneffekt ab. Bei den Berechnungen auf den Längen- und Frequenzresiduen (resTRT.LF) zeigt sich kein interpretierbares Muster und auch kein Effekt. 


\begin{tabular}{cccccc}
\hline \hline Faktor & AV & Bed. & MW & Std. Abw. & n \\
\hline \multirow{4}{*}{ TRT } & RK & 822.81 & 487.93 & 216 \\
& & WK & 712.46 & 406.34 & 216 \\
& & Mono & 348.5 & 175.42 & 216 \\
\cline { 2 - 6 } & \multirow{3}{*}{ resTRT.L } & RK & 7.4 & 496.69 & 216 \\
& & WK & 54.94 & 413.06 & 216 \\
& & Mono & -62.33 & 177.37 & 216 \\
\cline { 2 - 6 } STRUKTUR & RK & 37.07 & 485.02 & 216 \\
& \multirow{3}{*}{ resTRT.F } & WK & -2.65 & 414.75 & 216 \\
& & Mono & -34.42 & 189.17 & 216 \\
\cline { 2 - 6 } & \multirow{3}{*}{ resTRT.LF } & RK & -27.74 & 494.54 & 216 \\
& & WK & 35.52 & 414.32 & 216 \\
& & Mono & -7.78 & 178.55 & 216 \\
\hline \hline
\end{tabular}

Tabelle 6.58: Deskriptive Statistik für die TRT der Antezedens-Region (IA 4) mit Berücksichtigung der Längen- und Frequenzresiduen

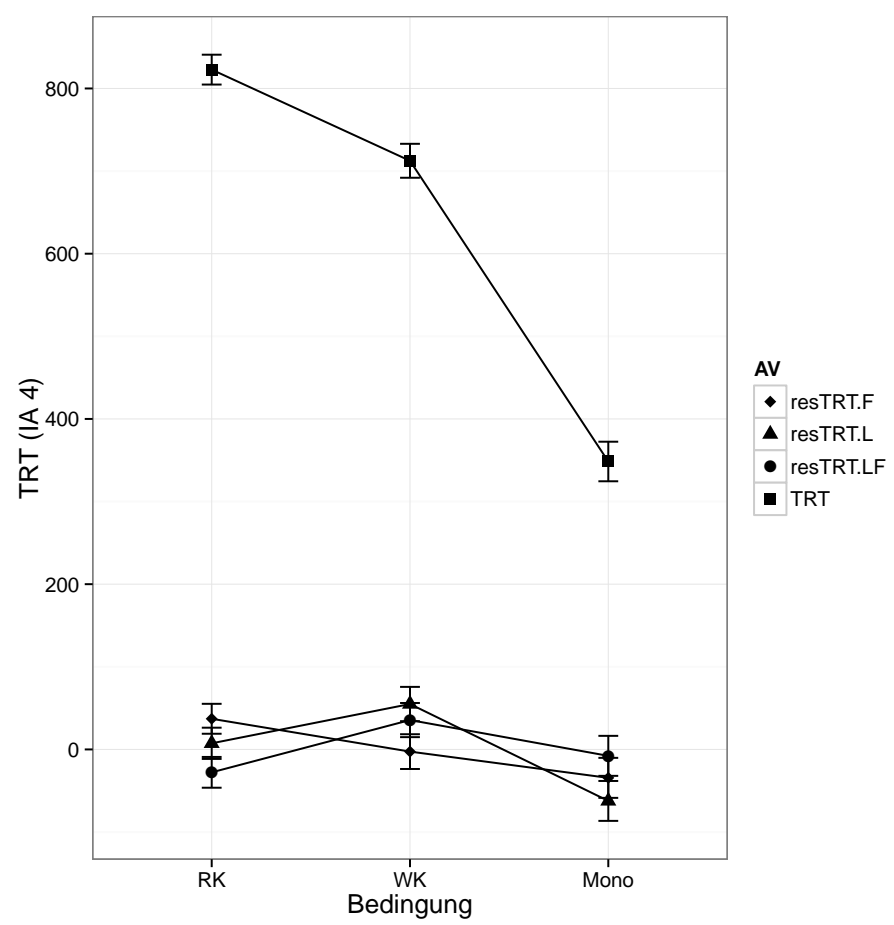

Abbildung 6.26: Plot der Mittelwerte der TRT der Antezedens-Region (IA 4) mit Berücksichtigung der Längen- und Frequenzresiduen 


\begin{tabular}{c|cc|cc|cc}
\hline \hline AV & $F_{1}(2,52)$ & $p$ & $F_{2}(2,46)$ & $p$ & $\operatorname{minF}^{\prime}$ & $p$ \\
\hline TRT & 93.17 & $<0.001$ & 65.29 & $<0.001$ & $(2,93) 38.39$ & $<0.001$ \\
resTRT.L & 5.077 & $<0.01$ & 3.305 & $<0.05$ & $(2,91) 2$ & $>0.05$ \\
resTRT.F & 1.891 & 0.161 & 1.107 & 0.339 & $(2,89) 0.7$ & $>0.05$ \\
resTRT.LF & 1.539 & 0.224 & 0.98 & 0.383 & $(2,91) 0.6$ & $>0.05$ \\
\hline \hline
\end{tabular}

Tabelle 6.59: Inferenzstatistik für die TRT der Antezedens-Region (IA 4) mit Berücksichtigung der Längen- und Frequenzresiduen

\section{Interpretation}

Die Auswertung der Rohdaten der FPFD der Antezedens-Region (IA 4) zeigt einen signifikanten Einfluss der Länge der Ankerausdrücke. Auf den Residuen von Länge und Frequenz ist kein Effekt zu verzeichnen. Ein ähnliches Ergebnis zeigt sich für die RPD. Auf den Rohdaten ist ein signifikanter Einfluss von Länge und Frequenz zu verzeichnen. Sobald hier einer der beiden Einflussgrößen herausfaktorisiert ist, ist kein Effekt mehr zu sehen. Die Ergebnisse für FPFD und RPD legen nahe, dass es - wie prognostiziert - keinen (längen- und frequenzunabhängigen) Unterschied in der Verarbeitung der AntezedensRegion zwischen den drei Bedingungen gibt. Dies wiederum bedeutet, dass Wurzel- und Rektionskomposita sich nicht im Verarbeitungsaufwand an sich unterscheiden. Ein Einfluss der Anapher (z.B. durch parafoveale Wahrnehmung) ist zu diesem Zeitpunkt ausgeschlossen, da auf den Ankerausdruck zunächst die Spillover-Region (IA 5) folgt. Effekte für spätere Regionen lassen sich somit auf den Einfluss der Anapher zurückführen. Bei der Auswertung der TRT, d.h. unter Einbeziehung möglicher Refixationen, zeigt sich auf den Rohdaten zunächst ebenfalls ein deutlicher Einfluss von Länge und Frequenz. Auf den um den um Frequenz- und Längeneffekt bereinigten Daten ist kein interpretierbares Muster erkennbar. Sollten demnach Refixationen in die Antezedens-Region erfolgt sein, sind diese zahlenmäßig zu gering oder zu kurz, um die TRT maßgeblich zu beeinflussen. Daraus lässt sich schlussfolgern, dass die Regressionen aus der IA 7 nicht den Ankerausdruck zum Ziel hatten. Unterstützend sollen hierfür abschließend die Regressionen in die IA 4 ausgewertet werden.

\section{Regressionen}

\section{Hypothesen}

Für die Regressionen aus der Anaphern-Region (IA 6) konnte kein Effekt gefunden werden, jedoch für die Regressionen aus der Satzergänzung (IA 7). Somit liegt die Vermutung nahe, dass der Such- und Rückversicherungsprozess nicht direkt von der Anapher ausgeht, sondern als seinen Ausgangspunkt andere Stellen im Satz hat. Da die zahlenmäßige Verteilung der Regressionen aus der Satzergänzung heraus hypothesenkonform ist, lässt sich vermuten, dass die IA 4 Ziel des Suchprozesses ist (neben der bereits als Ziel bestätigten IA 6). Sollte diese Vermutung - entgegen der Indizien der Auswertung der TRT korrekt sein, sollten entsprechende Unterschiede durch Regressionen in die AntezedensRegion sichtbar sein. Die Hypothese für die RICs der Antezedens-Region lautet somit: $\mathrm{RIC}($ Mono $)<\mathrm{RIC}(\mathrm{RK})<\mathrm{RIC}(\mathrm{WK})$. 


\section{Ergebnisse}

Bei der Auswertung der Regressionen in die Antezedens-Region (siehe Tab. 6.60 zeigt sich bei den um Einflüsse von Länge und Frequenz bereinigten Daten deskriptiv das Muster $\mathrm{RIC}(\mathrm{RK})<\mathrm{RIC}(\mathrm{Mono})<\mathrm{RIC}(\mathrm{WK})$. Dieses ist jedoch nicht signifikant. Bei den unbereinigten Daten ist weder ein Längen- noch ein Frequenzeffekt sichtbar, in der Bedingung WK liegen stets höhere Werte vor als in der Bedingung RK.

\begin{tabular}{cccccc}
\hline \hline Faktor & AV & Bed. & MW & Std. Abw. & n \\
\hline \multirow{5}{*}{ RIC } & RK & 0.52 & 0.76 & 216 \\
& & WK & 0.57 & 0.78 & 216 \\
& & Mono & 0.38 & 0.59 & 215 \\
\cline { 2 - 6 } & \multirow{3}{*}{ resRIC.L } & RK & 0 & 0.76 & 216 \\
& & WK & 0.07 & 0.78 & 216 \\
& & Mono & -0.07 & 0.59 & 215 \\
\cline { 2 - 6 } STRUKTUR & RK & -0.02 & 0.76 & 216 \\
& \multirow{3}{*}{ resRIC.F } & WK & 0.05 & 0.78 & 216 \\
& & Mono & -0.02 & 0.59 & 215 \\
\cline { 2 - 6 } & \multirow{3}{*}{ resRIC.LF } & RK & -0.03 & 0.76 & 216 \\
& & WK & 0.05 & 0.78 & 216 \\
& & Mono & -0.02 & 0.59 & 215 \\
\hline \hline
\end{tabular}

Tabelle 6.60: Deskriptive Statistik für die RICs der Antezedens-Region (IA 4) mit Berücksichtigung der Längen- und Frequenzresiduen

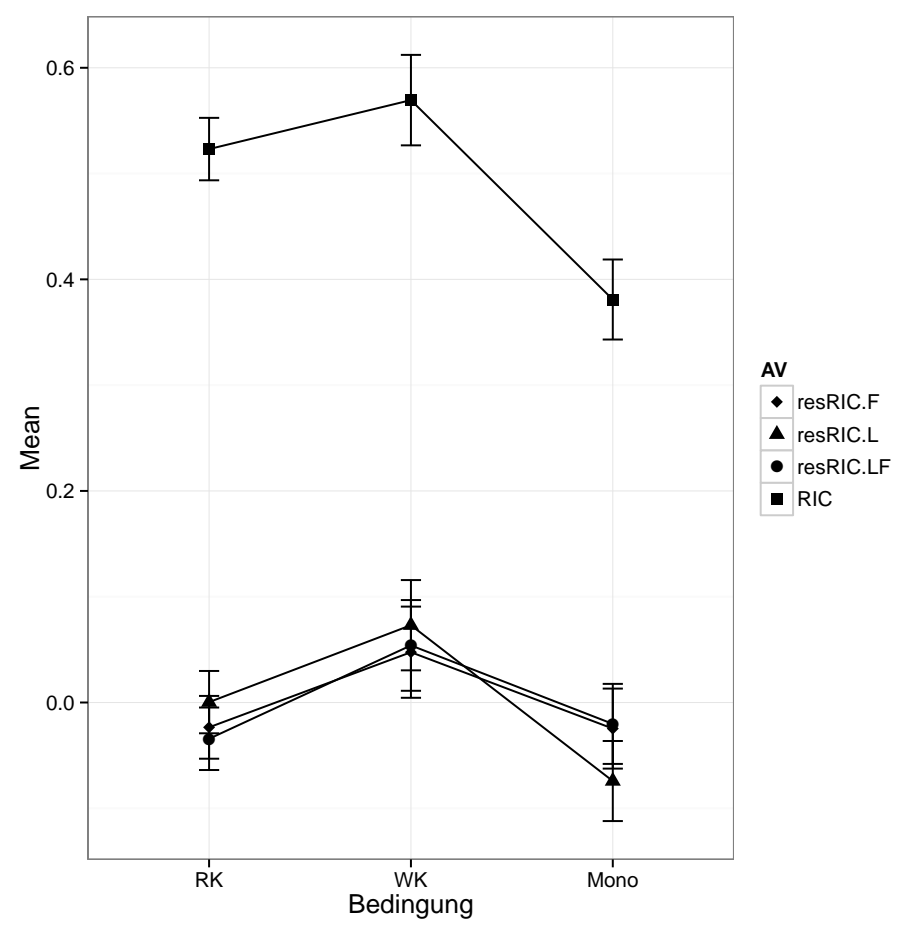

Abbildung 6.27: Plot der Mittelwerte der RICs der Antezedens-Region (IA 4) mit Berücksichtigung der Längen- und Frequenzresiduen 


\begin{tabular}{c|cc|cc|cc}
\hline \hline $\mathrm{AV}$ & $F_{1}(2,52)$ & $p$ & $F_{2}(2,46)$ & $p$ & $\min F^{\prime}(2,82)$ & $p$ \\
\hline $\mathrm{RIC}$ & 4.71 & $<0.05$ & 2.158 & 0.127 & 1.48 & $>0.05$ \\
resRIC.L & 2.66 & $<0.1$ & 1.20 & 0.31 & 0.83 & $>0.05$ \\
resRIC.F & 0.831 & 0.441 & 0.373 & 0.691 & 0.26 & $>0.05$ \\
resRIC.LF & 1.095 & 0.342 & 0.496 & 0.612 & 0.34 & $>0.05$ \\
\hline \hline
\end{tabular}

Tabelle 6.61: Inferenzstatistik für die RICs der Antezedens-Region (IA 4) mit Berücksichtigung der Längen- und Frequenzresiduen

\section{Interpretation}

Länge und Frequenz der Ausdrücke scheinen auf die RICs keinen maßgeblichen Einfluss auszuüben, da ein entsprechendes Muster in keiner der AVn gefunden wurde. Auf den um Längen- und Frequenzeinfluss bereinigten RICs konnte weder ein interpretierbares Muster noch ein Effekt gefunden werden. Dies bestätigt die Annahmen zur TRT: Der vorrangig in der IA 7 ausgelöste Suchprozess scheint nicht gezielt auf die IA 4, also nicht auf den Ankerausdruck gerichtet zu sein.

\section{Auswertung der Spillover-Region (IA 5)}

\section{Hypothesen}

Für die Anaphern-Region konnte für einige Fixationszeiten das prognostizierte Muster Fix. (Mono) $<$ Fix. $($ RK) $<$ Fix. (WK) gefunden werden. Ein Argument gegen den Einfluss des Faktors STRUKTUR wäre nun, dass dieses Verhalten durch die Spillover-Region (IA 5) verursacht wird und nicht erst durch die Anapher am Zeilenende. Um dies zu überprüfen, soll die Spillover-Region ausgewertet werden, die sich zwischen der Antezedens- und der Anaphern-Region befindet. Diese Region unterscheidet sich zwischen den Bedingungen, da sich verschiedene Komposita nicht immer gleich gut in denselben Kontext einbetten lassen. Diese kontextuelle Einbettung sollte jedoch keine Verarbeitungsunterschiede zwischen den Bedingungen mit sich bringen, da sie nicht systematisch mit den Bedingungen variiert. Es ist jedoch möglich, dass die verschiedenen Kontexte und die daraus resultierenden Unterschiede sich auf die Ergebnisse auswirken. Aufgrund parafovealer Verarbeitung können Effekte in angrenzende Regionen übertreten und damit nicht eindeutig zugeordnet werden (vgl. Radach et al. 2002 71). Besteht nun für die Spillover-Region ein nachweisbarer Unterschied zwischen den Bedingungen, wäre unklar, ob die Effekte der angrenzenden IAs ausschließlich von diesen evoziert werden, oder ob es sich um Spillover-Effekte handelt. Zeigt die Region jedoch keinen Effekt oder sogar ein von der Arbeitshypothese abweichendes Muster, können die Effekte auf der IA 6 sicher der experimentellen Manipulation zugeschrieben werden. Zudem sollte das Einfügen einer Satzgrenze unmittelbar vor der Anapher deren parafoveale Verarbeitung vermindern.

Für die Spillover-Region werden demnach gezielt keine Effekte erwartet. Lediglich ein Einfluss der Länge auf die Verarbeitungszeit wird prognostiziert, da diese zwischen den Bedingungen variiert. Dies geschieht jedoch nicht systematisch, sodass diesbezüglich keine konkrete Hypothese besteht, sondern der Längeneffekt lediglich herausfaktorisiert wird. 


\section{Ergebnisse}

Die Berechnungen wurden auf den Rohdaten und auf den Längenresiduen durchgeführt, da das Material zwischen den Bedingungen in der Zeichenzahl variiert. Für die FPFD zeigt sich sowohl auf den Rohdaten als auch auf den Längenresiduen das Muster $\operatorname{FPFD}($ Mono $)<\operatorname{FPFD}(\mathrm{WK})<\operatorname{FPFD}(\mathrm{RK})$ (siehe Tab. 6.62).

\begin{tabular}{cccccc}
\hline \hline Faktor & AV & Bed. & MW & Std. Abw. & n \\
\hline \multirow{3}{*}{ FPFD } & RK & 745.14 & 428.96 & 216 \\
& WK & 718.35 & 350.33 & 216 \\
& & Mono & 711.36 & 419.65 & 215 \\
\cline { 2 - 6 } STRUKTUR & RK & 35.53 & 415 & 216 \\
& resFPFD.L & WK & 19.49 & 342.08 & 216 \\
& & Mono & -55.28 & 408.23 & 215 \\
\hline \hline
\end{tabular}

Tabelle 6.62: Deskriptive Statistik für die FPFD der Spillover-Region (IA 5) mit Berücksichtigung der Längenresiduen

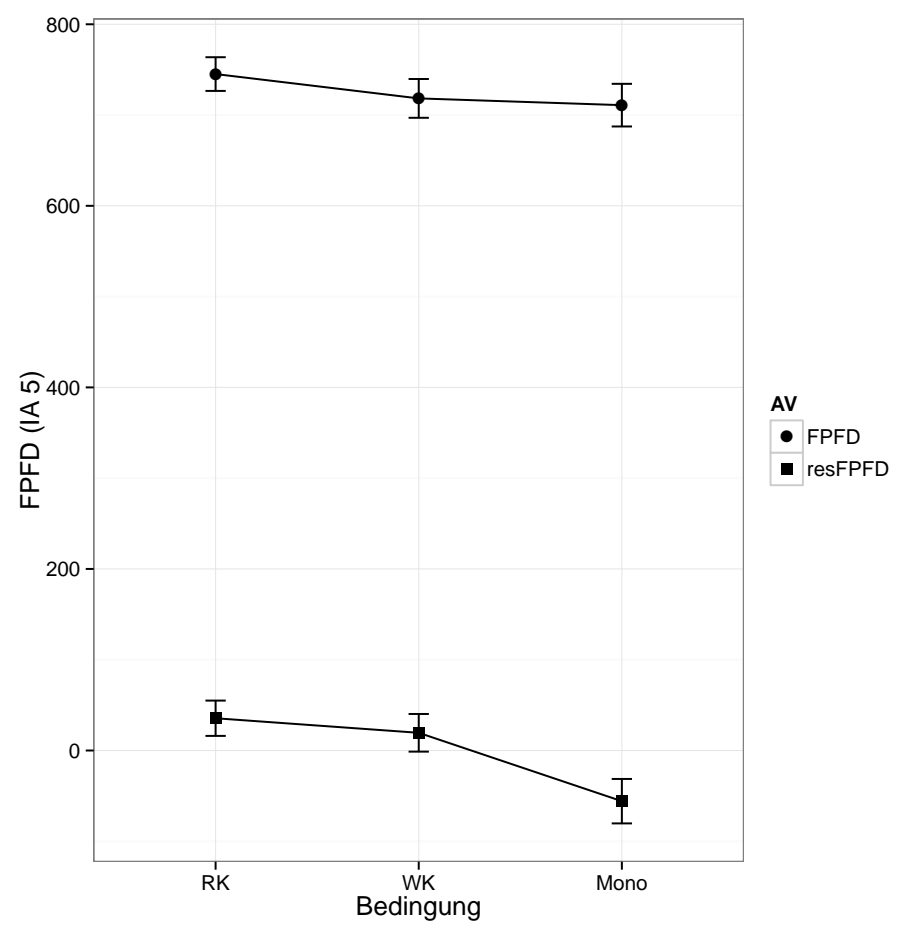

Abbildung 6.28: Plot der Mittelwerte der FPFD der Spillover-Region (IA 5) mit Berücksichtigung der Längenresiduen

\begin{tabular}{|c|c|c|c|c|c|c|}
\hline $\mathrm{AV}$ & $F_{1}(2,52)$ & $p$ & $\bar{F} F_{2}(2,46)$ & $p$ & $\min F^{\prime}$ & $p$ \\
\hline FPFD & 0.473 & 0.626 & 0.499 & 0.61 & $(2,98) 0.24$ & $>0.05$ \\
\hline resFPFD.L & 3.345 & $<0.05$ & 4.746 & $<0.05$ & $(2,97) 1.96$ & $>0.05$ \\
\hline
\end{tabular}

Tabelle 6.63: Inferenzstatistik für die FPFD der Spillover-Region (IA 5) mit Berücksichtigung der Längenresiduen 
Für die RPD ergibt sich auf den Rohdaten das Muster $\operatorname{RPD}(\mathrm{WK})<\operatorname{RPD}($ Mono $)<$ $\mathrm{RPD}(\mathrm{RK})$ und auf den Längenresiduen das Muster $\mathrm{RPD}(\mathrm{Mono})<\mathrm{RPD}(\mathrm{WK})<\mathrm{FPFD}(\mathrm{RK})$ (siehe Tab.6.64).

\begin{tabular}{cccccc}
\hline \hline Faktor & AV & Bed. & MW & Std. Abw. & n \\
\hline \multirow{4}{*}{ RPD } & RK & 1124.45 & 1149.54 & 216 \\
& & WK & 985.96 & 554.74 & 216 \\
& & Mono & 1118.28 & 847.27 & 215 \\
\cline { 2 - 6 } STRUKTUR & RK & 82.76 & 1109.39 & 216 \\
& \multirow{3}{*}{ resRPD.L } & WK & -31.59 & 558.06 & 216 \\
& & Mono & -51.41 & 831.51 & 215 \\
\hline \hline
\end{tabular}

Tabelle 6.64: Deskriptive Statistik für die RPD der Spillover-Region (IA 5) mit Berücksichtigung der Längenresiduen

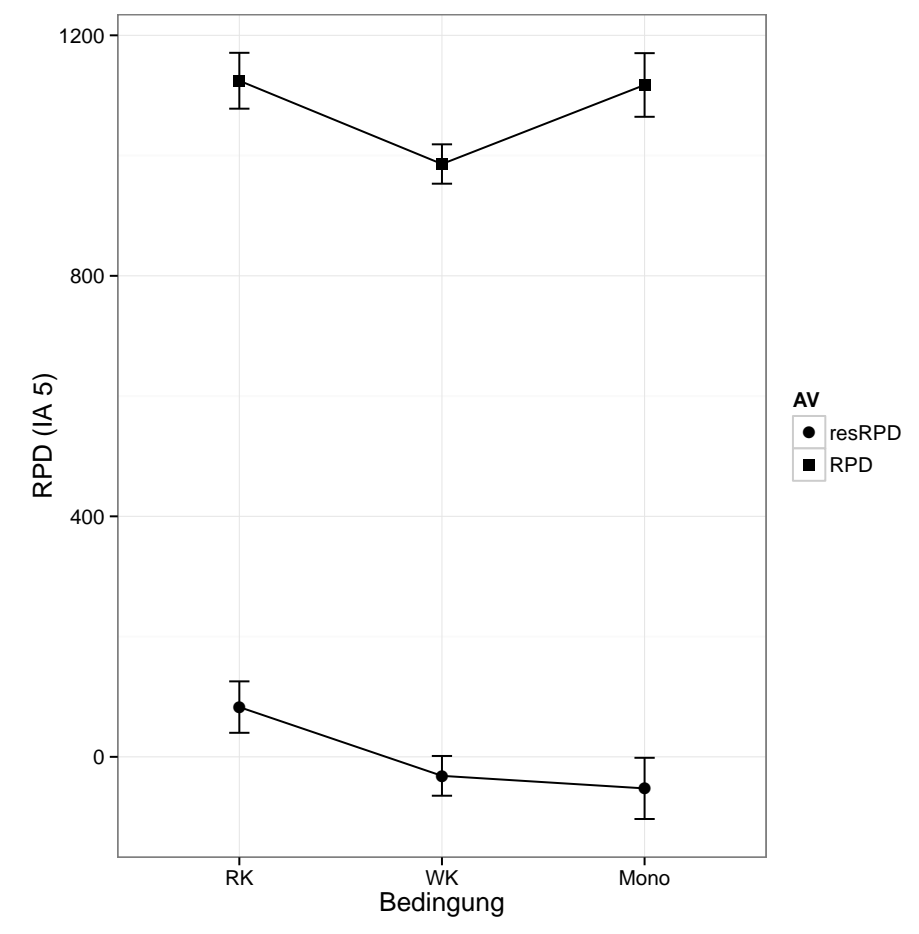

Abbildung 6.29: Plot der Mittelwerte der RPD der Spillover-Region (IA 5) mit Berücksichtigung der Längenresiduen

\begin{tabular}{c|cc|cc|cc}
\hline \hline $\mathrm{AV}$ & $F_{1}(2,52)$ & $p$ & $F_{2}(2,46)$ & $p$ & $\min F^{\prime}(2,96)$ & $p$ \\
\hline $\mathrm{RPD}$ & 2.015 & 0.144 & 1.712 & 0.192 & 0.93 & $>0.05$ \\
resRPD.L & 1.92 & 0.157 & 1.677 & 0.198 & 0.9 & $>0.05$ \\
\hline \hline
\end{tabular}

Tabelle 6.65: Inferenzstatistik für die RPD der Spillover-Region (IA 5) mit Berücksichtigung der Längenresiduen

Bei Betrachtung der TRT der Spillover-Region zeigt sich auf den Rohdaten das Muster TRT(WK) $<$ TRT(Mono) $<$ TRT(RK) und auf den Längenresiduen TRT(Mono) < 


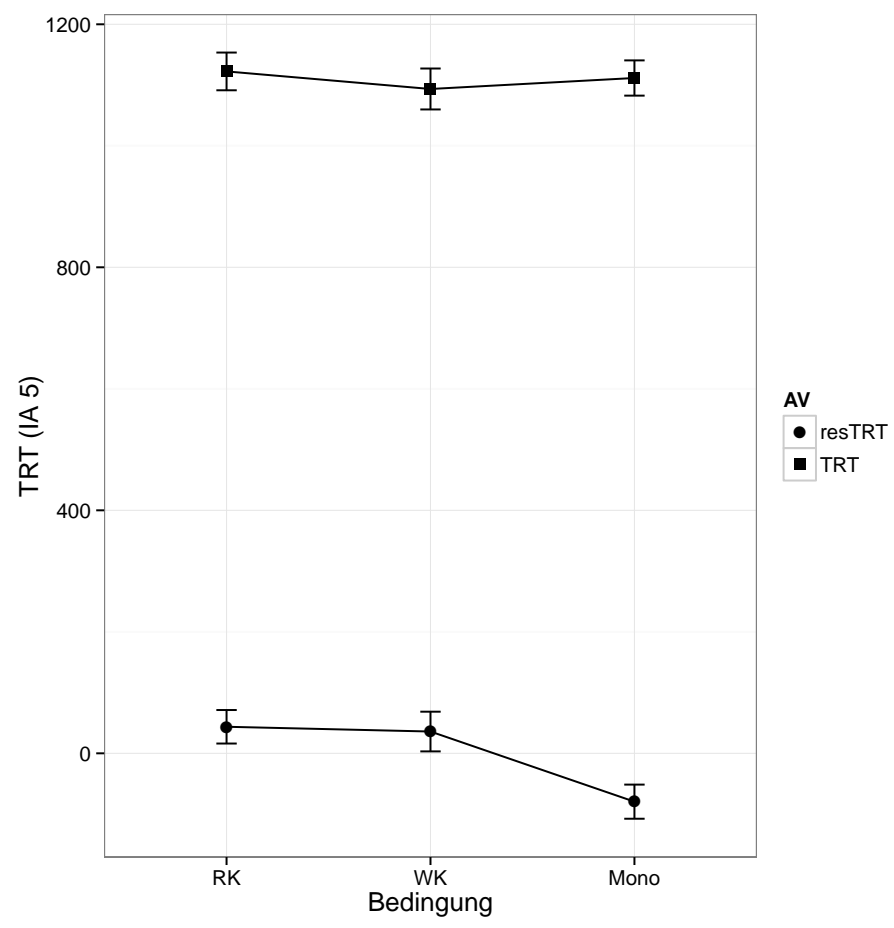

Abbildung 6.30: Plot der Mittelwerte der TRT der Spillover-Region (IA 5) mit Berücksichtigung der Längenresiduen

TRT $(\mathrm{WK})<\operatorname{TRT}(\mathrm{RK})$ (siehe Tab. 6.66).

\begin{tabular}{cccccc}
\hline \hline Faktor & AV & Bed. & MW & Std. Abw. & n \\
\hline \multirow{4}{*}{ TRT } & RK & 1122.43 & 632.24 & 216 \\
& WK & 1093.52 & 566.72 & 216 \\
STRUKTUR & Mono & 1111.58 & 594.08 & 216 \\
\cline { 2 - 6 } & \multirow{3}{*}{ resTRT.L } & RK & 43.7 & 604.03 & 216 \\
& & WK & 35.87 & 548.79 & 216 \\
& Mono & -79.57 & 557.4 & 216 \\
\hline \hline
\end{tabular}

Tabelle 6.66: Deskriptive Statistik für die TRT der Spillover-Region (IA 5) mit Berücksichtigung der Längenresiduen

\begin{tabular}{c|cc|cc|cc}
\hline \hline $\mathrm{AV}$ & $F_{1}(2,52)$ & $p$ & $F_{2}(2,46)$ & $p$ & $\operatorname{minF}{ }^{\prime}$ & $p$ \\
\hline TRT & 0.145 & 0.865 & 0.107 & 0.899 & $(2,94) 0.06$ & $>0.05$ \\
resTRT.L & 3.641 & $<0.05$ & 4.501 & $<0.05$ & $(2,98) 2.01$ & $>0.05$ \\
\hline \hline
\end{tabular}

Tabelle 6.67: Inferenzstatistik für die TRT der Spillover-Region (IA 5) mit Berücksichtigung der Längenresiduen

\section{Interpretation}

Bei Betrachtung der Analyse von IA 5 ist kein interpretierbarer Effekt zu verzeichnen. Auf den verschiedenen Längenresiduen findet sich stets das Muster Fix.(Mono) $<$ Fix. (WK) $<$ 
Fix.(RK), für die FPFD und die TRT sogar signifikant. Dies entspricht nicht dem Muster der Arbeitshypothese, wie es in der angrenzenden Region IA 6 gefunden wurde. Wo das Muster der Arbeitshypothese gefunden wurde, kann es nicht auf das Material der SpilloverRegion zurückgeführt werden. Die Gegenthese eines Spillover-Effekts konnte widerlegt werden. Eine parafoveale Verarbeitung der Anapher scheint nicht in relevantem Maße stattgefunden zu haben.

\subsubsection{Interpretation und Diskussion}

Die Arbeitshypothese zum Einfluss des Faktors STRUKTUR auf die N1-Zugänglichkeit besagt, dass die Verarbeitung der Anapher in der IA 6 am leichtesten in der Bedingung Mono fällt, da hier eine direkte Anapher etabliert wird. Bei den beiden Kompositabedingungen sollte die Verarbeitung in der Bedingung RK leichter fallen als in der Bedingung WK, da dem N1 in der Bedingung RK ein höherer Grad an Referenzialität zugeschrieben wird als in der Bedingung WK. Dies sollte sich dergestalt in allen Zeitmaßen und Refixationszahlen niederschlagen, dass die niedrigsten Werte in der Kontrollbedingung Mono vorliegen und für die Bedingung RK stets niedrigere Werte als für die Bedingung WK vorliegen.

Bei der Auswertung der Anaphern-Region (IA 6) zeigt sich das prognostizierte Muster in der FPFD, den Fallzahlen der SPFD, in der RPD sowie in der TRT. Demnach zeigt sich ein Einfluss der experimentellen Manipulation des Ankerausdrucks sowohl in frühen als auch in späten Verarbeitungsphasen der Anapher. Die Manipulation zeitigt in der FPFD und in der TRT einen hypothesenkonformen Effekt. Der Unterschied zwischen den Bedingungen RK und WK wird durch die Inferenzstatistik der FPFD sowie durch die ANOVA-by-subjects der TRT als signifikant ausgewiesen. Die Anzahl der Regressionen aus der Anaphern-Region stellt kein interpretierbares Muster dar, demnach löst die Anapher an sich keinen Suchprozess aus. Allerdings weisen die Regressionen in die Anaphern-Region einen hypothesenkonformer Effekt aus. Die Anapher stellt somit das Ziel eines Suchprozesses dar.

Bei der Auswertung der Satzergänzung der Anapher (IA 7) wurden für die FPFD, die SPFD und die RPD unterschiedliche Muster gefunden. Das Muster der FPFD ist nicht interpretierbar; SPFD und RPD illustrieren einen Unterschied zwischen den Kompositabedingungen und der Kontrollbedingung. Insgesamt handelt es sich bei der IA 7 um eine große Region, für die keine Detailauswertung möglich ist. Vor allem für frühe, lexikalische Prozesse können daher auf Grundlage der Auswertung dieser Region keine Aussagen zum Einfluss der Anaphernresolution getroffen werden. Für die TRT, d.h. für die Gesamtlesezeit, die auch alle Verarbeitungsschritte mit Refixationen beinhaltet, konnte ein hypothesenkonformer Effekt gefunden werden. Dieser wird zusätzlich durch einen hypothesenkonformen Effekt für die Anzahl der Regressionen aus der Region heraus gestützt. Die Region erscheint somit als Ursprung eines durch die experimentelle Manipulation beeinflussten Informations-Suchprozesses.

Bei der Auswertung der Antezedens-Region (IA 4) konnte zunächst für alle Fixationszeiten ein deutlicher Einfluss der Länge und der Frequenz des Ankerausdrucks bestätigt werden. Nachdem diese Einflussgrößen herausfaktorisiert wurden, konnte für keine der betrachteten $\mathrm{AVn}$ ein interpretierbares Ergebnis gefunden werden, dass auf einen Einfluss des Faktors STRUKTUR bei der Verarbeitung schließen lässt.

Die Auswertung der Spillover-Region (IA 5), also der Satzeinbettung des Ankerausdrucks, wurde durchgeführt, um die Unabhängigkeit der Ergebnisse der angrenzenden Regionen 
zu belegen. Dabei konnte, wie erwartet, für keine AV ein interpretierbares Muster gefunden werden. Vor allem weichen die Muster der IA 5 vom Muster der IA 6 sogar dahingehend ab, dass für die Bedingung WK in dieser Region nicht die jeweils höchsten Werte vorliegen. Eine Interpretation der Effekte der Anaphern-Region als Spillover-Effekte kann durch diese Analyse ausgeschlossen werden.

Insgesamt deutet die Auswertung des Blickverhaltens darauf hin, dass die experimentelle Manipulation der Ankerausdrücke in hypothesenkonformer Weise einen Einfluss auf die Verarbeitung der Anapher und ihrer Satzeinbettung hat. Nicht alle relevanten AVn verzeichnen signifikante Effekte. Allerdings liegen auch für keine AV Ergebnisse vor, die dezidiert für eine Falsifizierung der Arbeitshypothese sprechen. Stattdessen zeigt sich, dass der Prozess der Anaphernresolution z.T. anders verläuft, als dies ursprünglich vermutet wurde.

Die detaillierte Analyse der Eye-Tracking-Daten hat v.a. für zwei Verarbeitungsschritte neue Einsichten zutage gefördert: (i) Für den Zeitpunkt der Anaphernresolution und (ii) für den Ablauf des Such- und Rückversicherungsprozesses während der Anaphernresolution.

Für den Zeitpunkt der Anaphernresolution (i) wurde ein später Effekt, d.h. ein Effekt auf der TRT der Anapher und der Satzeinbettung erwartet, da hier mit einer Reanalyse zu rechnen ist. Sollte es sich beim N1-Bezug tatsächlich um eine indirekte Anapher handeln, wäre Inferenzziehung nötig, was nur unter Einbeziehung des Satzkontexts (IA 7) möglich ist. Tatsächlich wurde für den Unterschied zwischen den Kompositabedingungen ein später Effekt auf der IA 6 gefunden (signifikante ANOVA-by-subjects für die TRT) und deskriptiv durch die TRT der IA 7 bestätigt. Allerdings wurde auf der IA 6 zusätzlich ein Effekt auf der FPFD gefunden: Der Verarbeitungsvorteil für die Bedingung RK gegenüber der Bedingung WK ist für diese AV signifikant. Die FPFD enthält ausschließlich die Fixationen vor dem Fortfahren des Lesevorgangs, d.h. ein Einfluss des Kontexts der IA 7 ist für diese Fixationszeit ausgeschlossen. Die einzigen während dieses Verarbeitungsschritts zur Verfügung stehenden Informationen sind die Informationen aus dem vorangehenden Kontext, d.h. die möglichen Antezedenten, und die morphosyntaktischen Informationen des Pronomens. Dass in der FPFD der Anaphern-Region Unterschiede zwischen den Kompositabedingungen vorliegen, kann nur bedeuten, dass die morphosyntaktischen Informationen des N1 nutzbar gemacht wurden. Daraus lässt sich zum einen schließen, dass das N1 abhängig von der Art des Kompositums unterschiedlich verarbeitet wird und zum anderen, dass der Anaphernresolutions-Prozess bereits zu diesem frühen Zeitpunkt zu einem wesentlichen Teil erfolgt. Diese beiden Erkenntnisse sprechen gegen das Verständis des N1-Bezugs als klassische indirekte Anapher.

Für den Such- und Rückversicherungsprozess (ii) im weiteren Verlauf des Anaphernresolutions-Prozesses zeigt sich, dass dieser nicht bei der Anapher seinen Ausgang hat (kein interpretierbares Ergebnis für die ROCs der IA 6), sondern während der Verarbeitung der Satzergänzung einsetzt (Effekt für die ROCs der IA 7). Als Ziel der Informationssuche konnte nicht dezidiert das Kompositum ausgemacht werden (kein Effekt für die RICs der IA 4). Dies spricht für ein ungerichtetes Absuchen des Texts. Gleichzeitig zeigt sich, dass das Pronomen Unsicherheit auszulösen scheint und selbst ein Ziel im Rückversicherungsprozess ist (Effekt für die RICs der IA 6).

Eine Gegenüberstellung der Hypothesen aus Kapitel 6.4.3/Tabelle 6.25 und den entsprechenden Ergebnissen ist in Tabelle 6.68 dargestellt. 


\begin{tabular}{l|l|l|l}
\hline \hline IA & AV & Hypothese & Ergebnis (deskriptiv) \\
\hline 6 & FFD & - & Mono $<\mathrm{RK}=\mathrm{WK}$ \\
6 & FPFD & - & Mono $<\mathrm{RK}<\mathrm{WK}$ \\
6 & TRT & Mono $<\mathrm{RK}<\mathrm{WK}$ & Mono $<\mathrm{RK}<\mathrm{WK}$ \\
6 & ROC & Mono $<\mathrm{RK}<\mathrm{WK}$ & $\mathrm{RK}<\mathrm{Mono}<\mathrm{WK}$ \\
7 & TRT & Mono $<\mathrm{RK}<\mathrm{WK}$ & $\mathrm{Mono}<\mathrm{RK}<\mathrm{WK}$ \\
7 & ROC & Mono $<\mathrm{RK}<\mathrm{WK}$ & Mono $<\mathrm{RK}<\mathrm{WK}$ \\
4 & TRT & Mono $<\mathrm{RK}<\mathrm{WK}$ & $\mathrm{RK}<\mathrm{Mono}<\mathrm{WK}$ \\
4 & RIC & Mono $<\mathrm{RK}<\mathrm{WK}$ & $\mathrm{RK}<\mathrm{Mono}<\mathrm{WK}$ \\
5 & TRT & - & Mono $<\mathrm{WK}<\mathrm{RK}$ \\
\hline \hline
\end{tabular}

Tabelle 6.68: Zusammenfassung der Hypothesen und deskriptiven Ergebnisse für die verschiedenen Interest Areas (IAs) und abhängigen Variablen (AVn)

\subsubsection{Weitere Analysen}

Abschließend werden einige Analysen zu weiteren möglichen Einflussgrößen vorgestellt, die kontrolliert, annotiert und bereits in Experiment 3a zur Analyse herangezogen wurden. Wo nicht anders angegeben, wurden die Auswertungen auf der TRT der Anaphern-Region (IA 6) durchgeführt, da diese Region aufgrund ihrer geringen Größe und einheitlichen Gestaltung am sichersten zu interpretieren ist. Da die Faktoren nicht ideal ausbalanciert werden konnten, bzw. z.T. ,between-items' bestehen, wurde hier die ANOVA-by-items ohne, random slopes' ausgeführt.

\section{Raum-zeitliche Kontiguität (RZK)}

Als Faktor der Konzeptkombination und Indiz für einen konzeptuellen Fokus ist die RZK von besonderem Interesse. Bereits in der Satzergänzungsaufgabe in Experiment 2 (vgl. Kap. 5) konnte sie in ihrem Einfluss bestätigt werden. In der Satzergänzungsaufgabe in Experiment 3a (vgl. Kap. 6.2.6) konnte der Einfluss nur deskriptiv bestätigt werden.

\section{Hypothesen}

Die Annotation erfolgte unter Berücksichtigung des Kontextsatzes, der mitunter Einfluss auf die Interpretation des Kompositums und damit auch auf den Faktor RZK hat. Entsprechend der Ergebnisse aus Experiment 2 erwarte ich, dass in der Faktorausprägung +RZK die N1-Zugänglichkeit erhöht ist, also die TRT der Anaphern-Region entsprechend kürzer ist: $\mathrm{TRT}(+\mathrm{RZK})<\mathrm{TRT}(-\mathrm{RZK})$.

Darüber hinaus ist für einen Vergleich mit Experiment 2 eine Betrachtung der Interaktion der Faktoren RZK und STRUKTUR interessant. Hier wird entgegen der Ergebnisse aus Experiment 2 keine Interaktion erwartet, da die Items stärker kontrolliert wurden.

\section{Ergebnisse}

Da es sich bei der RZK um einen Faktor handelt, der nur bei Komposita auftritt ist, wurden die Berechnungen ausschließlich auf Basis der beiden Kompositabedingungen durchgeführt. In den deskriptiven Daten (vgl. Tab. 6.69) zeigt sich ein Verarbeitungsvorteil für 
die Faktorstufe +RZK. Die ANOVA-by-subjects weist diesen Unterschied als signifikant aus (vgl. Tab. 6.70) 18

\begin{tabular}{cccccc}
\hline \hline Faktor & AV & Bed. & MW & Std. Abw. & n \\
\hline \multirow{2}{*}{ RZK } & \multirow{2}{*}{ TRT } & -RZK & 391.87 & 296.71 & 189 \\
& & +RZK & 355.46 & 265.09 & 243 \\
\hline \hline
\end{tabular}

Tabelle 6.69: Deskriptive Statistik für die TRT der Anaphern-Region (IA 6) für den Faktor RzK (ohne Bed. Mono)

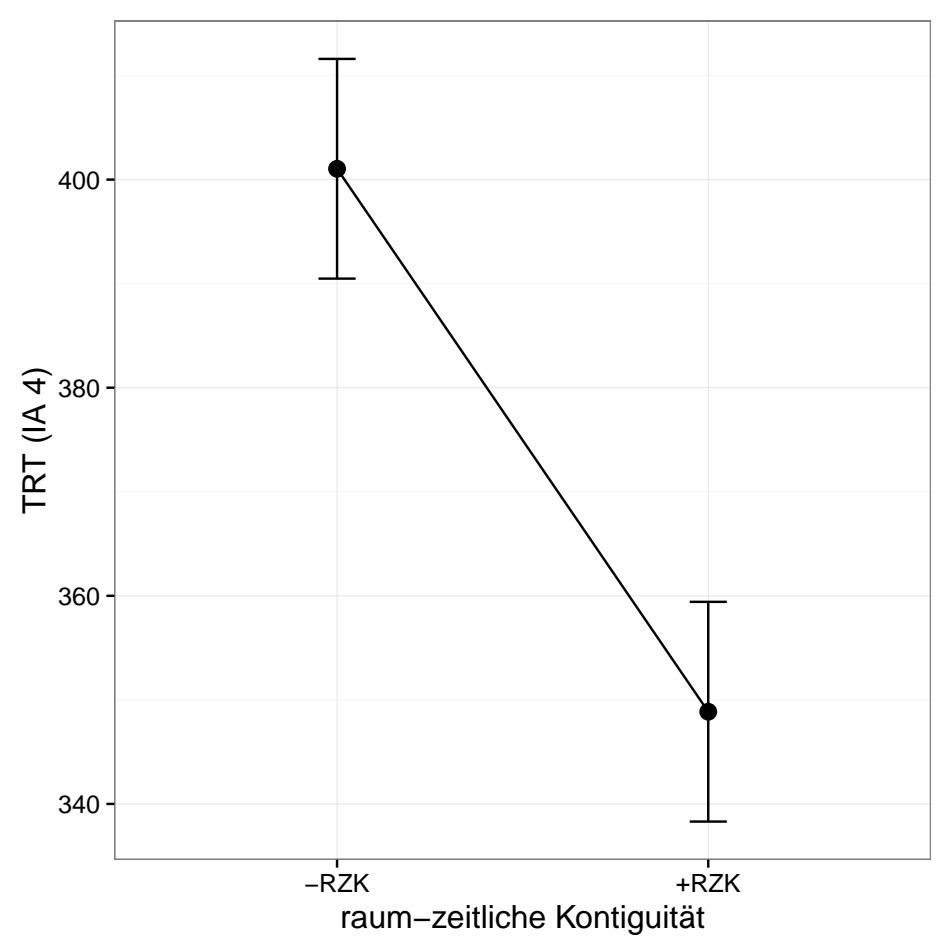

Abbildung 6.31: Plot der Mittelwerte der TRT der Anaphern-Region (IA 6) für den Faktor RZK (ohne Bed. Mono)

\begin{tabular}{c|cc|cc}
\hline \hline Faktor & $F_{1}(1,26)$ & $p$ & $F_{2}(1,22)$ & $p$ \\
\hline RZK & 6.032 & $<0.05$ & 0.394 & 0.531 \\
\hline \hline
\end{tabular}

Tabelle 6.70: Inferenzstatistik für die TRT der Anaphern-Region (IA 6) für den Faktor RZK (ohne Bed. Mono)

Die Ergebnisse der deskriptiven Auswertung der RZK in Kombination mit dem Faktor STRUKTUR sind in Tabelle 6.71 dargestellt. Es zeigt sich, dass sowohl innerhalb der Faktorstufe RK als auch innerhalb der Faktorstufe WK die kürzere TRT für die Faktorstufe + RZK vorliegt. Die Differenz ist jedoch innerhalb der Wurzelkomposita größer und auch

\footnotetext{
${ }^{18}$ In $\mathrm{R}$ verwendete Formeln für die ANOVAs:

summary (aov(IA_DWELL_TIME rz_mk + Error (vp/rz_mk), d3.ia.ana.komp))

summary(aov(IA_DWELL_TIME rz_mk + Error(item), d3.ia.ana.komp))
} 
nur dort und nur für die ANOVA-by-subjects signifikant (vgl. Tab. 6.72).

\begin{tabular}{cccccc}
\hline \hline AV & STRUKTUR & RZK & MW & Std. Abw. & n \\
\hline \multirow{4}{*}{ TRT } & \multirow{2}{*}{ RK } & - RZK & 359.47 & 289.94 & 90 \\
& \multirow{2}{*}{ WK } & +RZK & 331.67 & 238.78 & 126 \\
\cline { 3 - 6 } & & -RZK & 421.33 & 301.17 & 99 \\
& + RZK & 381.08 & 289.63 & 117 \\
\hline \hline
\end{tabular}

Tabelle 6.71: Deskriptive Statistik für die TRT der Anaphern-Region (IA 6) für den Faktor RZK in Abhängigkeit vom Faktor strukTur (ohne Bed. Mono)

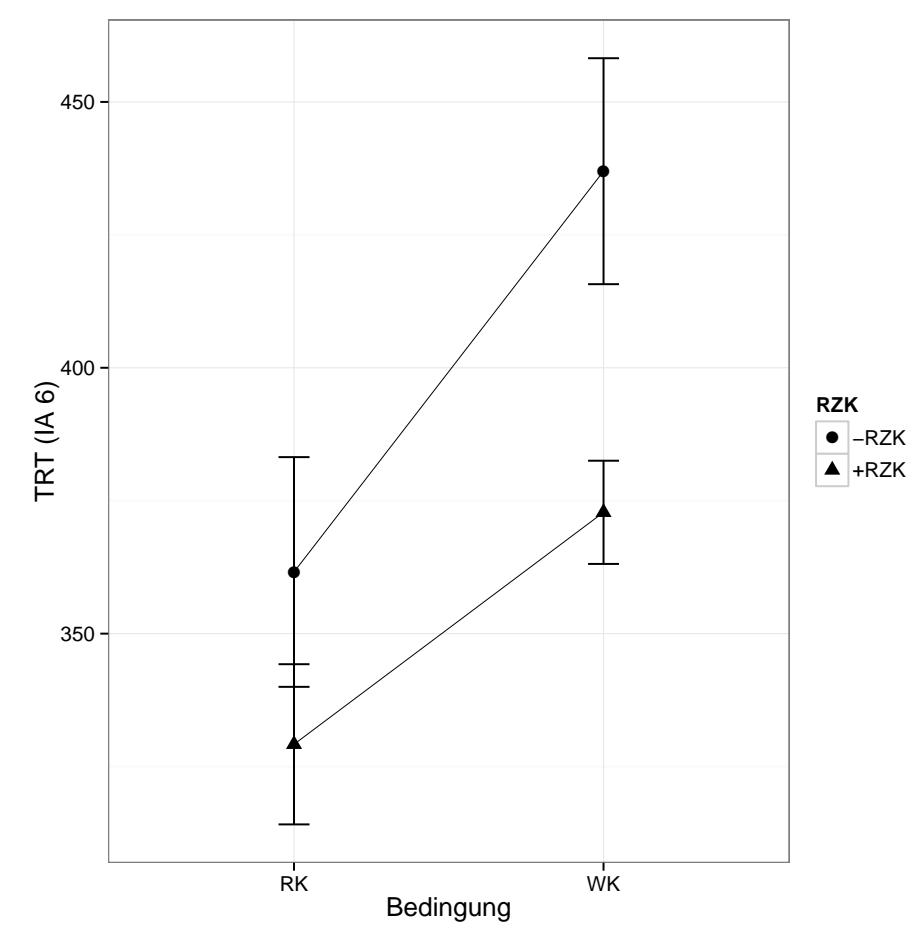

Abbildung 6.32: Plot der Mittelwerte der TRT der Anaphern-Region (IA 6) für den Faktor RZK in Abhängigkeit vom Faktor sTRUKTUR (ohne Bed. Mono)

\begin{tabular}{c|cc|cc}
\hline \hline Faktor & $F_{1}(1,26)$ & $p$ & $F_{2}(1,22)$ & $p$ \\
\hline RK & 1.178 & 0.288 & 0.432 & 0.518 \\
WK & 6.667 & $<0.05$ & 0.572 & 0.458 \\
\hline \hline
\end{tabular}

Tabelle 6.72: Inferenzstatistik für die TRT der Anaphern-Region (IA 6) für den Faktor RZK in Abhängigkeit vom Faktor STRUkTUR (ohne Bed. Mono)

\section{Interpretation}

Der Einfluss der RZK konnte in prognostizierter Richtung durch die ANOVA-by-subjects bestätigt werden. Bei einer Analyse der Interaktion der RZK mit der Bedingung STRUKTUR ergab sich wie erwartet ein von den Ergebnissen von Experiment 2 abweichendes Resultat. Die Faktoren wirken unabhängig voneinander in die jeweils prognostizierte Richtung. 
Entgegen des Ergebnisses aus Experiment 2 zeigt sich in Experiment 3c ein unabhängiger Verarbeitungsvorteil für die Faktorstufen +RZK und RK. Der stärkere Einfluss der RZK auf die N1-Zugänglichkeit von Wurzelkomposita lässt vermuten, dass ein konzeptueller Faktor wie die RZK stärker zum Tragen kommt, wenn die Beziehung der Konstituenten zueinander unterspezifiziert ist, wie es im Fall von Wurzelkomposita gegenüber Rektionskomposita der Fall ist.

Die Unterschiede zwischen den Ergebnissen des Eye-Tracking-Experiments 3c und des Satzergänzungsexperiments 2 lassen sich dem experimentellen Paradigma zuschreiben: Mithilfe der Aufzeichnung von Blickbewegungen lassen sich durch die Messung von Daten der Online-Verarbeitung subtilere Unterschiede finden, als es bei einer Satzergänzungsaufgabe möglich ist. Dafür spricht auch, dass die Ergebnisse im Satzergänzungsexperiment 3a nicht signifikant sind. Zum anderen handelt es sich bei den Stimuli im Eye-TrackingExperiment um vollständige Texte, die somit weniger Interpretationsspielraum lassen. Dies ermöglicht auch eine klarere Einschätzung der RZK für die einzelnen Komposita. In Experiment 2 ergab sich während der Auswertung der Satzvervollständigungen, dass die Einschätzung der RZK von VPn und Annotatoren z.T. voneinander abwich.

Insgesamt bestätigen die Ergebnisse von Experiment 2 und 3 die RZK item- und methodenunabhängig als Einflussfaktor für die N1-Zugänglichkeit.

\section{N2-Konkretheit}

Der nächste untersuchte Faktor basiert ebenfalls auf der Annahme eines konzeptuellen Fokus': Die Konkretheit des N2.

\section{Hypothese}

Die Items beinhalten als N1 ausschließlich konkrete Nomen. Das N2 - und damit auch das Gesamtkompositum - ist mitunter konkret und mitunter abstrakt. Die Hypothese dazu lautet, dass bei abstraktem N2 und konkretem N1 der konzeptuelle Fokus auf das N1 verschoben ist und dadurch die N1-Zugänglichkeit erhöht wird (vgl. Kap. 3.3.5). Im Fall eines konkreten N2 konkurrieren zwei Konkreta um die konzeptuelle Dominanz. Bei der Auswertung des Faktors N2-KOnkRETheIT wurde erneut die Bedingung Mono außen vor gelassen, da in dem Fall kein N2 vorliegt. Die Hypothese lautet: TRT(-konkret) < TRT(+konkret).

\section{Ergebnisse}

In den deskriptiven Daten (Tab. 6.73) zeigt sich eine kürzere mittlere TRT für die Bezüge zu Ankerausdrücken mit abstraktem N2 als zu Ankerausdrücken mit konkretem N2. In der Inferenzstatistik zeitigt der Faktor N2-KONKRETHEIT kein signifikantes Ergebnis (Tab. 6.74). 


\begin{tabular}{cccccc}
\hline \hline Faktor & AV & Bed. & MW & Std. Abw. & $\mathrm{n}$ \\
\hline \multirow{2}{*}{ N2-KONKRETHEIT } & \multirow{2}{*}{ TRT } & -konkret & 342.00 & 247.13 & 216 \\
& & +konkret & 400.78 & 306.48 & 216 \\
\hline \hline
\end{tabular}

Tabelle 6.73: Deskriptive Statistik für die TRT der Anaphern-Region (IA 6) für den Faktor N2-Konkretheit (ohne Bed. Mono)

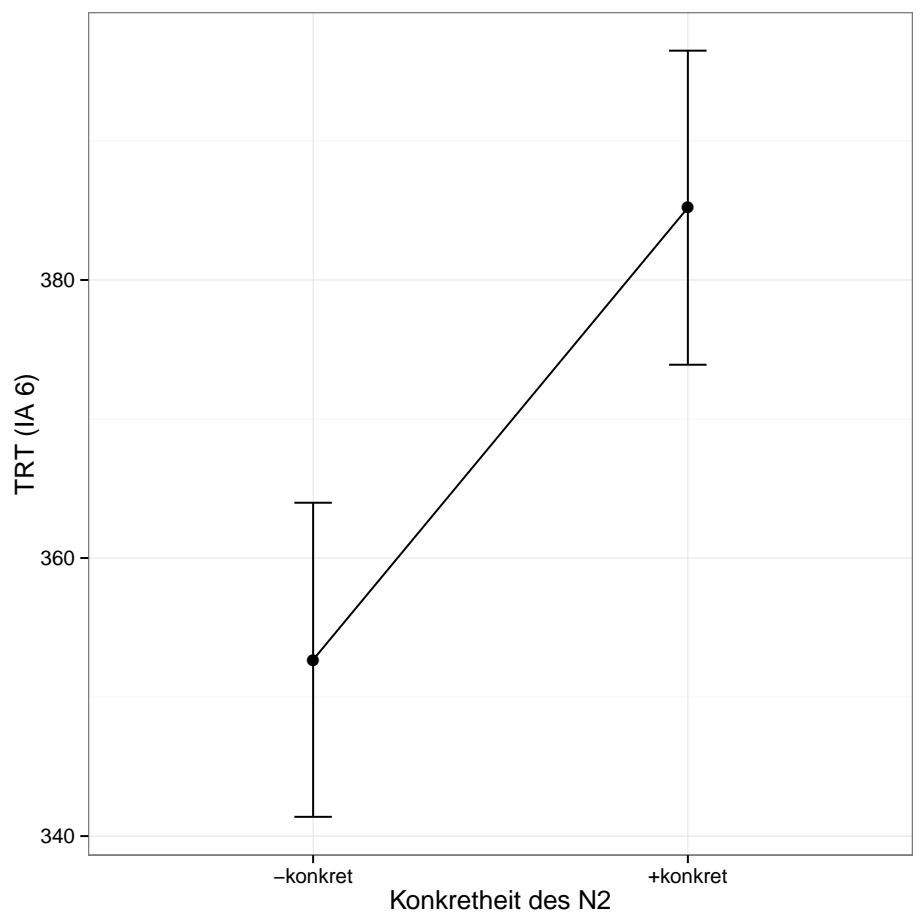

Abbildung 6.33: Plot der Mittelwerte der TRT der Anaphern-Region (IA 6) für den Faktor N2-Konkretheit (ohne Bed. Mono)

\begin{tabular}{c|cc|cc}
\hline \hline Faktor & $F_{1}(1,26)$ & $p$ & $F_{2}(1,22)$ & $p$ \\
\hline N2-KONKRETHEIT & 2.024 & 0.167 & 1.415 & 0.247 \\
\hline \hline
\end{tabular}

Tabelle 6.74: Inferenzstatistik für die TRT der Anaphern-Region (IA 6) für den Faktor N2-KOnKRETHEIT (ohne Bed. Mono)

\section{Interpretation}

Gemäß den Ergebnissen scheint wie prognostiziert für ein N1 eine bessere Zugänglichkeit zu bestehen, wenn das N2 abstrakt ist. Inferenzstatistisch lässt sich dies jedoch nicht bestätigen. Entsprechende Ergebnisse wurden bereits in der Satzergänzungsaufgabe (Exp. 3a) gefunden. Unter Berücksichtigung beider Teilexperimente kann somit unabhängig vom experimentellen Paradigma von Indizien für eine Abhängigkeit der N1-Zugänglichkeit von der N2-KONKRETHEIT gesprochen werden. Dabei gilt es sich zu vergegenwärtigen, dass für diese Fragestellung die TRT der Anapher ausgewertet wurde, d.h. nicht die Verarbeitung des Kompositums wird widergespiegelt, sondern die Verarbeitungsunterschiede bei der Anaphernresolution. Es werden keine Aussagen zu einem generellen Einfluss der Konkretheit auf die Verarbeitung getroffen. 


\section{Nominalisierungslesart}

\section{Hypothesen}

Verwandt mit der Frage der Konkretheit ist die Unterscheidung zwischen Prozess- und Resultatsnominalisierungen innerhalb der Rektionskomposita. Da Prozessnominalisierungen als Abstrakta aufgefasst werden können, und Resultatsnominalisierungen konkreter sind, müsste sich das Ergebnis der Analyse der N2-KONKRETHEIT auch in dieser Unterscheidung widerspiegeln: TRT(Prozess) $<$ TRT(Resultat).

Die Einteilung der Items in Prozess- und Resultatsnominalisierungen erfolgte unter Berücksichtigung der Satzkontexte.

\section{Ergebnisse}

Die Berechnungen wurden auf den TRTs in der Bedingung RK durchgeführt. In den deskriptiven Daten findet sich der prognostizierte Vorteil für den N1-Bezug bei Prozessnominalisierungen (siehe Tab.66.75). In den ANOVAs schlägt sich der Faktor als marginal signifikant nieder (siehe Tab. 6.76).

\begin{tabular}{cccccc}
\hline \hline Faktor & AV & Bed. & MW & Std. Abw. & $\mathrm{n}$ \\
\hline \multirow{2}{*}{ NOM.-LESART } & \multirow{2}{*}{ TRT } & Resultat & 385.27 & 309.97 & 99 \\
& & Prozess & 307.70 & 205.75 & 117 \\
\hline \hline
\end{tabular}

Tabelle 6.75: Deskriptive Statistik für die TRT der Anaphern-Region (IA 6) für den Faktor nOMINALISIERUNGSLESART (nur Bed. RK)

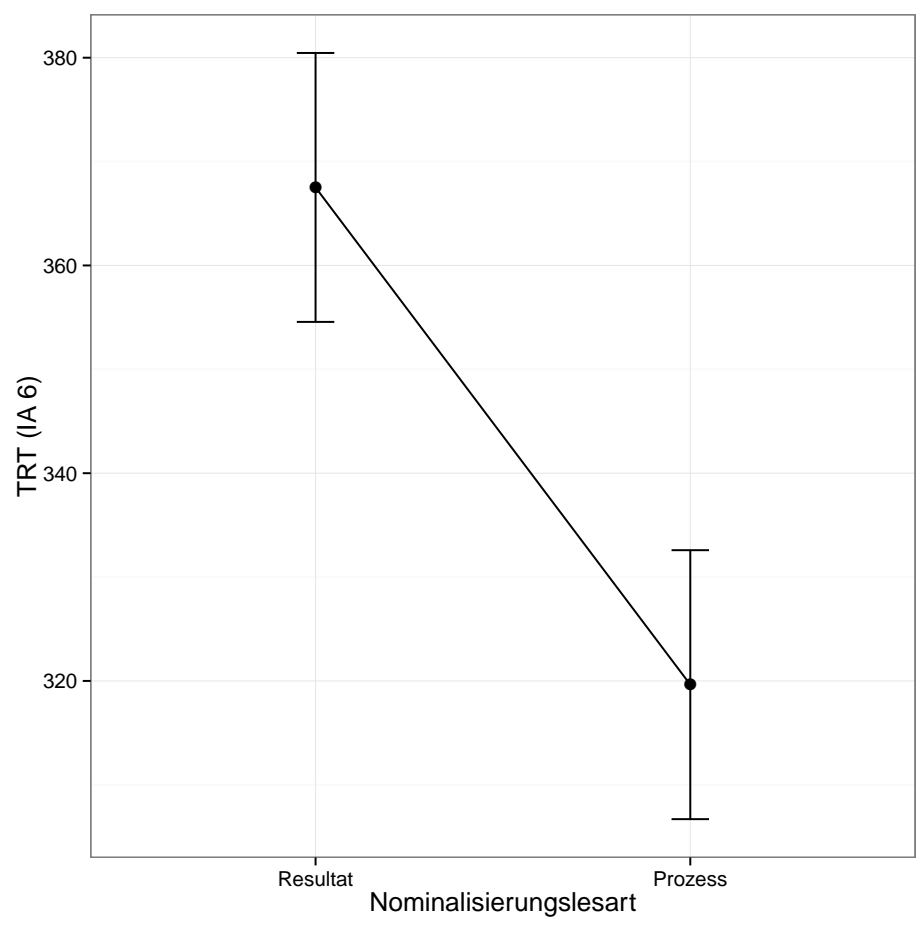

Abbildung 6.34: Plot der Mittelwerte der TRT der Anaphern-Region (IA 6) für den Faktor NOMINALisierungsLESART (nur Bed. RK) 


\begin{tabular}{c|cc|cc}
\hline \hline Faktor & $F_{1}(1,26)$ & $p$ & $F_{2}(1,22)$ & $p$ \\
\hline NOM.-LESART & 3.418 & $<0.1$ & 3.984 & $<0.1$ \\
\hline \hline
\end{tabular}

Tabelle 6.76: Inferenzstatistik für die TRT der Anaphern-Region (IA 6) für den Faktor NOMINALISIERUNGSLESART (nur Bed. RK)

\section{Interpretation}

Die Ergebnisse der Analyse der NOMINALISIERUnGSLESART stützen die Hypothese zum Einfluss des Faktors N2-KONKRETHEIT. Da für die Auswertung der NOMINALISIERUnGSLESART jedoch nur die Rektionskomposita nutzbar gemacht werden konnten, basiert diese nur auf einer geringen Datenmenge. Außerdem handelt es sich bei der Einteilung um eine subjektive Einschätzung. Andererseits werden die Ergebnisse auch durch die Ergebnisse der Satzergänzungsaufgabe in Experiment 3a gestützt.

\section{N1-Zählbarkeit}

\section{Hypothese}

Ein weiterer konzeptueller Faktor ist die N1-ZÄHLBARKEIT, d.h. ob es sich bei einem N1 um ein Massenomen oder um ein Individualnomen handelt. In Bezug auf den konzeptuellen Einfluss des Faktors wird mit einem Vorteil für die Zugänglichkeit von Individualnomen an der N1-Position gerechnet; in Bezug auf den grammatischen Einfluss mit einem Vorteil für Massenomen aufgrund determiniererloser Referenzfähigkeit. Vor diesem Hintergrund erfolgt eine explorative Auswertung. Da der Faktor unabhängig vom N2 ist, erfolgte die Auswertung in diesem Fall inklusive der Bedingung Mono.

\section{Ergebnisse}

Die deskriptiven Ergebnisse für die N1-ZÄHLBARKEIT sind in Tabelle 6.77 dargestellt. Die Anaphern-Region hat im Fall des Bezugs auf ein Massenomen eine kürzere Gesamtlesezeit. Die ANOVA-by-subjects weist diesen Vorteil als marginal signifikant aus (vgl. Tab.6.78).

\begin{tabular}{cccccc}
\hline \hline Faktor & AV & Bed. & MW & Std. Abw. & $\mathrm{n}$ \\
\hline \multirow{2}{*}{ N1-ZÄHLBARKEIT } & \multirow{2}{*}{ TRT } & Massenom. & 320.13 & 239.23 & 270 \\
& & Individualnom. & 350.68 & 259.24 & 378 \\
\hline \hline
\end{tabular}

Tabelle 6.77: Deskriptive Statistik für die TRT der Anaphern-Region (IA 6) für den Faktor N1-ZÄHLBARKEIT

\begin{tabular}{c|cc|cc}
\hline \hline Faktor & $F_{1}(1,26)$ & $p$ & $F_{2}(1,22)$ & $p$ \\
\hline N1-ZÄHLBARKEIT & 3.581 & $<0.1$ & 2.906 & 0.102 \\
\hline \hline
\end{tabular}

Tabelle 6.78: Inferenzstatistik für die TRT der Anaphern-Region (IA 6) für den Faktor N1-ZÄHLBARKEIT 


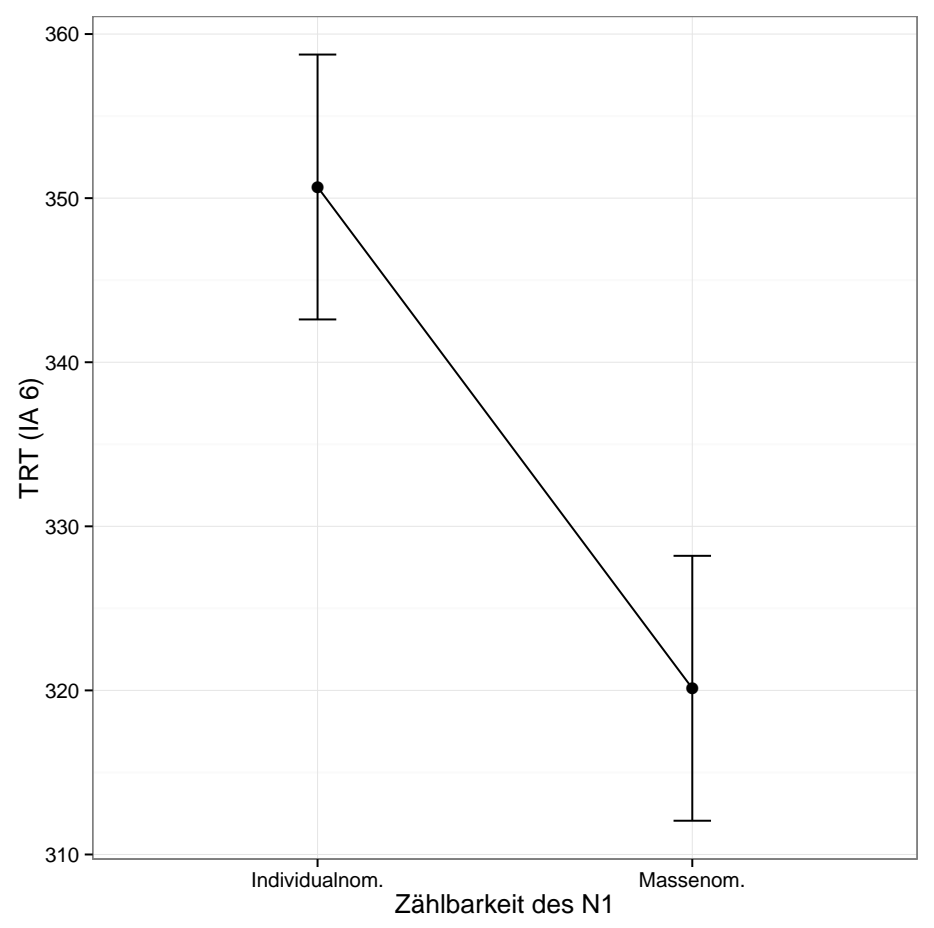

Abbildung 6.35: Plot der Mittelwerte der TRT der Anaphern-Region (IA 6) für den Faktor N1-ZÄHLBARKEIT

\section{Interpretation}

Während in Experiment 2 ein nicht signifikanter Vorteil für Individualnomen gefunden wurde (vgl. Kap. 5.7.4) und in Experiment 3a kein Unterschied zwischen den beiden Faktorstufen gefunden wurde (vgl. Kap. 6.2.6), zeigt sich im Eye-Tracking ein umgekehrtes Bild. Entgegen der Hypothese zum Vorteil für Individualnomen aufgrund stärkerer konzeptueller Einflüsse zeigt sich hier ein marginaler Vorteil für Massenomen. Eine mögliche Erklärung ist, dass das Material in diesem Experiment strenger kontrolliert ist als in Experiment 2 und durch das Eye-Tracking-Paradigma auch subtile grammatische Effekte zutage gefördert werden können. Andererseits hat sich bei der Kodierung gezeigt, dass die Einteilung in Masse- und Individualnomen keinesfalls immer eindeutig möglich ist. So gibt es stets mehr oder weniger eindeutige Fälle, z.B. Fleisch. Darüber hinaus ist die Zählbarkeit kontextsensitiv. Insgesamt lassen die Ergebnisse den Schluss zu, dass das Wirken einer Einflussgröße immer auch abhängig von den konkreten Items und dem Paradigma ist. Insgesamt stellt sich die N1-ZÄHLBARKEIT somit im Gegensatz zur N2-KONKRETHEIT nicht als relevanter Einflussfaktor dar.

\section{Genus}

Die Satzergänzungsaufgaben in Experiment 2 und 3a haben für die pronominalen Anaphern einen starken Genus-Effekt gezeigt. Ob dieser auch im Eye-Tracking besteht oder ein reiner Paradigma-Effekt ist, wird als nächstes geprüft. 


\section{Hypothesen}

Bei 13 Items ist das N1 und entsprechend auch die pronominale Anapher maskulin (er) und in 11 Fällen steht es im Neutrum (es). Aufgrund des Befunds aus Experiment 2, dass eine der häufigsten Satzergänzungen für ein Neutrum-Pronomen eine Interpretation als Vorfeld-es ist, besteht die Vermutung, dass auch in einer Lese-Aufgabe das NeutrumPronomen zunächst nicht-anaphorisch interpretiert wird. In diesem Fall würde - im Gegensatz zum maskulinen Pronomen - kein Resolutionsprozess angestoßen. Nach dem oder beim Lesen der disambiguierenden Satzergänzung müsste dann eine Revision dieser Interpretation erfolgen. Ist diese Annahme korrekt, müssten frühe Fixationszeiten der Anaphern-Region wie FFD und FPFD für das Neutrum-Pronomen kürzer als für das maskuline sein $(\mathrm{FFD} / \operatorname{FPFD}(\mathrm{n})<\mathrm{FFD} / \operatorname{FPFD}(\mathrm{m}))$. Während des Lesens der Satzergänzung muss die Interpretation dann revidiert werden. Aus diesem Grund müsste für die TRT ein umgekehrtes Verhältnis vorliegen, da diese Refixationen nach dem Lesen der Satzergänzung beinhaltet und demnach einen Revisionsprozess abbilden sollte (TRT $(m)<\operatorname{TRT}(n))$.

\section{Ergebnisse}

\begin{tabular}{cccccc}
\hline \hline Faktor & AV & Bed. & MW & Std. Abw. & n \\
\hline \multirow{6}{*}{ FFD } & $\mathrm{m}$ & 206.85 & 97.05 & 293 \\
& & $\mathrm{n}$ & 202.15 & 77.51 & 346 \\
\cline { 2 - 6 } GENUS & \multirow{2}{*}{ FPFD } & $\mathrm{m}$ & 258.31 & 158.57 & 293 \\
\cline { 2 - 6 } & \multirow{2}{*}{ TRT } & $\mathrm{n}$ & 259.02 & 144.91 & 346 \\
\hline \hline
\end{tabular}

Tabelle 6.79: Deskriptive Statistik für die Anaphern-Region (IA 6) für den Faktor GEnus

\begin{tabular}{c|cc|cc}
\hline \hline AV & $F_{1}(1,26)$ & $p$ & $F_{2}(1,22)$ & $p$ \\
\hline FFD & 0.408 & 0.528 & 0.418 & 0.525 \\
FPFD & 0.007 & 0.933 & 0.004 & 0.952 \\
TRT & 1.698 & 0.204 & 2.114 & 0.16 \\
\hline \hline
\end{tabular}

Tabelle 6.80: Inferenzstatistik für die Anaphern-Region (IA 6) für den Faktor GENUs

Wie in Tabelle 6.79 zu sehen, liegen für die FFD in der Neutrum-Bedingung kürzere Fixationszeiten vor. Für die FPFD sind sie in der Maskulinum-Bedingung minimal kürzer. Bei der TRT hat wiederum die Neutrum-Bedingung kürzere Fixationszeiten. Demnach scheint die Verarbeitung des Neutrum-Pronomens tendenziell schneller zu verlaufen. Allerdings lässt sich keine der AVn als signifikant bestätigen (Tab. 6.80).

\section{Interpretation}

Sollte der auf der FFD sichtbare deskriptive Verarbeitungsvorteil für das Neutrum-Pronomen aus einer Interpretation als Vorfeld-es resultieren, müsste sich das Verhältnis spätestens in der TRT umkehren. Das ist nicht der Fall. Dies wiederum könnte bedeuten, dass das Neutrum-Pronomen generell kürzere Verarbeitungszeiten hat. Dass der Unterschied 
nicht signifikant ist, könnte daran liegen, dass die IA nicht ausschließlich aus dem Pronomen besteht, sondern zusätzlich ein Modalverb beinhaltet. Die Unterschiede zwischen den Einflüssen der beiden Genera auf die Sprachverarbeitung sind beim Lesen womöglich zu gering, um sich signifikant auf die Fixationszeiten der gesamten IA auszuwirken.

Aus dem sich hier zeigenden Vorteil für die Neutrum-Bedingung in der TRT lässt sich jedoch nicht mit Sicherheit schließen, dass keine Uminterpretation stattfindet. Es kann lediglich ausgesagt werden, dass sich auf der TRT der Anaphern-Region keine Verzögerungen finden lassen, die für einen solchen Uminterpretationsprozess sprechen. Möglicherweise findet dieser jedoch statt, ohne Refixationen des Pronomens auszulösen. Beim Lesen der disambiguierenden Satzergänzung (IA 7) findet entsprechende Uminterpretation eventuell statt, ohne dass das Pronomen erneut fixiert wird, da es sich bei diesem um einen sehr kurzen und frequenten Ausdruck handelt (vgl. Ehrlich 1983 264f). Sollte diese Post-hocHypothese korrekt sein, müsste ein Genuseffekt auf der Satzergänzung (IA 7) zu finden sein, welcher sich in einer längeren TRT für die Neutrum-Bedingung auswirken müsste $(\operatorname{TRT}(\mathrm{m})<\operatorname{TRT}(\mathrm{n}))$.

\begin{tabular}{cccccc}
\hline \hline Faktor & AV & Bed. & MW & Std. Abw. & $\mathrm{n}$ \\
\hline \multirow{2}{*}{ GENUS } & \multirow{2}{*}{ TRT } & $\mathrm{m}$ & 1405.72 & 667.15 & 297 \\
& & $\mathrm{n}$ & 1561.9 & 790.09 & 351 \\
\hline \hline
\end{tabular}

Tabelle 6.81: Deskriptive Statistik für die TRT der Satzergänzung (IA 7) für den Faktor GENUS

\begin{tabular}{c|cc|cc}
\hline \hline Faktor & $F_{1}(1,26)$ & $p$ & $F_{2}(1,22)$ & $p$ \\
\hline GENUS & 11.18 & $<0.01$ & 2.599 & 0.121 \\
\hline \hline
\end{tabular}

Tabelle 6.82: Inferenzstatistik für die TRT der Satzergänzung (IA 7) für den Faktor GENUS

Wie post-hoc prognostiziert, ist die TRT der Satzergänzung in der Neutrum-Bedingung größer als in der Maskulinum-Bedingung (vgl. Tab. 6.81). Die ANOVA-by-subjects weist diesen Unterschied als signifikant aus (vgl. Tab. 6.82).

Insgesamt lässt sich also auf der Anaphern-Region ein leichter, jedoch nicht signifikanter Verarbeitungsvorteil für das Neutrum-Pronomen finden. Auf der Satzergänzung findet sich ein Effekt in umgekehrter Richtung. Dieses Ergebnis lässt die Interpretation zu, dass das Neutrum-Pronomen zunächst als Vorfeld-es interpretiert wird, keinen AnaphernresolutionsProzess auslöst und deshalb eine kürzere Lesezeit hat. Alternativ wäre auch eine Interpretation als Komplexanapher denkbar, was schlussendlich die gleichen Auswirkungen für die Verarbeitung mit sich bringen würde. Das Maskulinum-Pronomen kann nur als Anapher interpretiert werden und evoziert bei schwer zugänglichem Antezedens bereits beim Lesen des Pronomens Verarbeitungsschwierigkeiten und damit längere Fixationszeiten. Während der Disambiguierung beim Lesen der Satzergänzung findet eine Uminterpretation des Neutrum-Pronomens hin zu einer Anapher statt, die Interpretation des MaskulinumPronomens hat Bestand. Dies spiegelt sich in der längeren Gesamtfixationszeit der Satzergänzung für die Neutrum-Bedingung wider. Allerdings ist für die Uminterpretation keine Regression zur Anapher notwendig, da kein Mismatch zwischen grammatischen Informationen besteht. Daher ist es wahrscheinlich, dass die Uminterpretation zu einer leichten 
Verzögerung führt, jedoch keine Irritation auslöst und somit keine Regressionen zum Pronomen nötig macht, da es sich um einen regulären Verarbeitungsprozess handelt. Diese These passt auch zu den Ergebnissen von Experiment 2, bei dem die VPn beide Verwendungsmöglichkeiten des Pronomens nutzten.

Die Auswirkungen des Faktors Genus auf die Fixationen der Anaphern-Region sind nur gering; auf der IA 6 gar nicht signifikant und auf der IA 7 nur für die ANOVA-by-subjects. Im Gegensatz dazu zeitigt das GENUs in den Satzergänzungsaufgaben in Experiment 2 und 3a ein signifikantes Ergebnis. In diesem Sinne kann der Genus-Effekt als paradigmaabhängig gewertet werden; die Auswirkungen des Genus des Pronomens unterscheiden sich zwischen Sprachproduktion und -perzeption. Während in der Satzergänzungsaufgabe bei schlechter N1-Zugänglichkeit und „erzwungener“ Resolution das Vorfeld-es (oder eine Komplexanapher) die präferierte Wahl ist, muss beim Lesen die Resolution nicht direkt nach dem Pronomen erfolgen, sondern kann zu einem späteren Zeitpunkt abgeschlossen werden.

\section{Frequenz}

Wie bereits in Experiment 3a in Kapitel 6.2.6, soll auch in Experiment 3c der Einfluss verschiedener Frequenzen betrachtet werden.

\section{N1-Frequenz}

\section{Hypothesen}

Eine Hypothese, für die eine Auswertung von Frequenzen dienlich sein kann, besteht darin, dass ein Ausdruck umso zugänglicher ist, je leichter er zu verarbeiten ist. Ein Gegenentwurf dazu wäre das ,deep processing, bei dem davon ausgegangen wird, dass erhöhter Verarbeitungsaufwand zu höherer Aktiviertheit und damit zu besserer Zugänglichkeit führt (vgl. Diskussion in Kap. 2.3.1). Rayner (vgl. 1998;376) postuliert einen direkten Einfluss der Frequenz auf die Fixationsdauer. Wenn nun ein allgemeiner Verarbeitungsvorteil wie hohe Frequenz die Zugänglichkeit eines Ausdrucks erhöht, so müssten auch N1 mit hoher Frequenz zugänglicher sein als N1 mit niedriger Frequenz.

Im Folgenden soll in einem ersten Schritt geprüft werden, ob ganz allgemein eine höhere Frequenz einen Verarbeitungsvorteil bewirkt. In einem zweiten Schritt gilt es zu überprüfen, ob eine höhere N1-Frequenz auch eine bessere Zugänglichkeit bewirkt. Sollten sich beide Annahmen bestätigen lassen, können Spekulationen zum Zusammenhang zwischen Verarbeitungsvorteilen und N1-Zugänglichkeit unternommen werden.

\section{Ergebnisse}

Schritt 1: Besteht für Items mit einer höheren Frequenz ein Verarbeitungsvorteil?

Sollte es sich bei hoher Frequenz um eine Eigenschaft handeln, die einen generellen Verarbeitungsvorteil bewirkt, so sollte dies bei steigender Frequenz an kürzeren Verarbeitungszeiten sichtbar werden. Um dies zu überprüfen, sollen die FPFDs der Antezedens-Region verschieden frequenter Ankerausdrücke miteinander verglichen werden. Die FPFD wurde als AV gewählt, da während dieser ersten größeren Verarbeitungsphase der Großteil der 
Verarbeitung des Ankerausdrucks abläuft und ein Einfluss der Anapher auszuschließen ist. Als Wert für die Frequenz der Ankerausdrücke wurden die Häufigkeitsklassen aus dem Leipziger Wortschatz verwendet.

Die Ausdrücke wurden nach ihren Häufigkeitsklassen in drei Gruppen eingeteilt: Die erste Gruppe umfasst die Ausdrücke mit den höchsten Frequenzen (Häufigkeitsklassen 6 - 12 (23 Fälle, vorrangig Monomorpheme)), die zweite Gruppe umfasst die mittleren Frequenzen (Häufigkeitsklassen 13 - 18 (26 Fälle)) und die letzte Gruppe umfasst die Ausdrücke mit den niedrigsten Frequenz (ab Häufigkeitsklasse 19 (23 Fälle, vorrangig Rektionskomposita)). Die Hypothese zur Verteilung der FPFD lautet FPFD(hoch) $<$ FPFD(mittel) < FPFD(niedrig). Die Berechnungen erfolgten auf den Längenresiduen der FPFD, da auch der Ausdruckslänge ein Einfluss auf die Verarbeitung zugesprochen wird und diese zwischen den Ausdrücken z.T. stark variiert (z.B. Öl vs. Ölverteilung).

Wie in Tabelle 6.83 zu sehen, zeigt sich eine Verteilung der mittleren, um einen Einfluss der Länge korrigierten FPFDs gemäß dem prognostizierten Muster FPFD(hoch) < $\operatorname{FPFD}($ mittel $)<\mathrm{FPFD}$ (niedrig)

Je höher also die Frequenz eines Ausdrucks ist, desto kürzer ist seine FPFD. Daraus lässt sich ableiten, dass eine höhere Frequenz die Verarbeitung erleichtert. Die Ergebnisse sind allerdings nicht signifikant (Tab. 6.84).

\begin{tabular}{cccccc}
\hline \hline Faktor & AV & Bed. & MW & Std. Abw. & $\mathrm{n}$ \\
\hline \multirow{3}{*}{ NN-FREQUENZ } & & resFPFD.L & -17.97 & 134.06 & 206 \\
& & mittel & -2.03 & 249.82 & 234 \\
& & niedrig & 20.18 & 277.09 & 207 \\
\hline \hline
\end{tabular}

Tabelle 6.83: Deskriptive Statistik für die Längenresiduen der FPFD der AntezedensRegion (IA 4) für den Faktor NN-FREQUENZ

\begin{tabular}{c|cc|cc}
\hline \hline $\mathrm{AV}$ & $F_{1}(2,52)$ & $p$ & $F_{2}(2,21)$ & $p$ \\
\hline resFPFD.L & 1.383 & 0.26 & 0.92 & 0.414 \\
\hline \hline
\end{tabular}

Tabelle 6.84: Inferenzstatistik für die Längenresiduen der FPFD der Antezedens-Region (IA 4) für den Faktor NN-FREQUENZ

Schritt 2: Bewirkt ein Verarbeitungsvorteil eine bessere Zugänglichkeit?

Im ersten Schritt konnte anhand der NN-Frequenzen der Antezedenten gezeigt werden, dass es eine Tendenz für höhere Frequenzen gibt, einen Verarbeitungsvorteil mit sich zu bringen. Nun soll ausgehend von dieser Erkenntnis geprüft werden, ob ein Verarbeitungsvorteil eine bessere Zugänglichkeit mit sich bringt. Besteht dieser Zusammenhang, sollte eine höhere N1-Frequenz eine bessere N1-Zugänglichkeit zur Folge haben. Dies wiederum sollte sich auf die TRT der Anapher auswirken.

Als Frequenzen wurden die Häufigkeitsklassen des Leipziger Wortschatzes für die N1, d.h. für die Monolexeme genutzt. Der Frequenz-Wert des Monolexems (=N1) wurde allen drei Bedingungen eines Items zugewiesen. Anhand der Frequenzen wurden drei Gruppen von Items gebildet: Die erste Gruppe umfasst die N1 mit den höchsten Frequenzen (Häufigkeitsklassen $6-8$ (6 Items)), die zweite Gruppe umfasst die mittleren Frequenzen (Häufigkeitsklassen 9 und 10 (12 Items)) und die dritte Gruppe umfasst die N1 mit den 


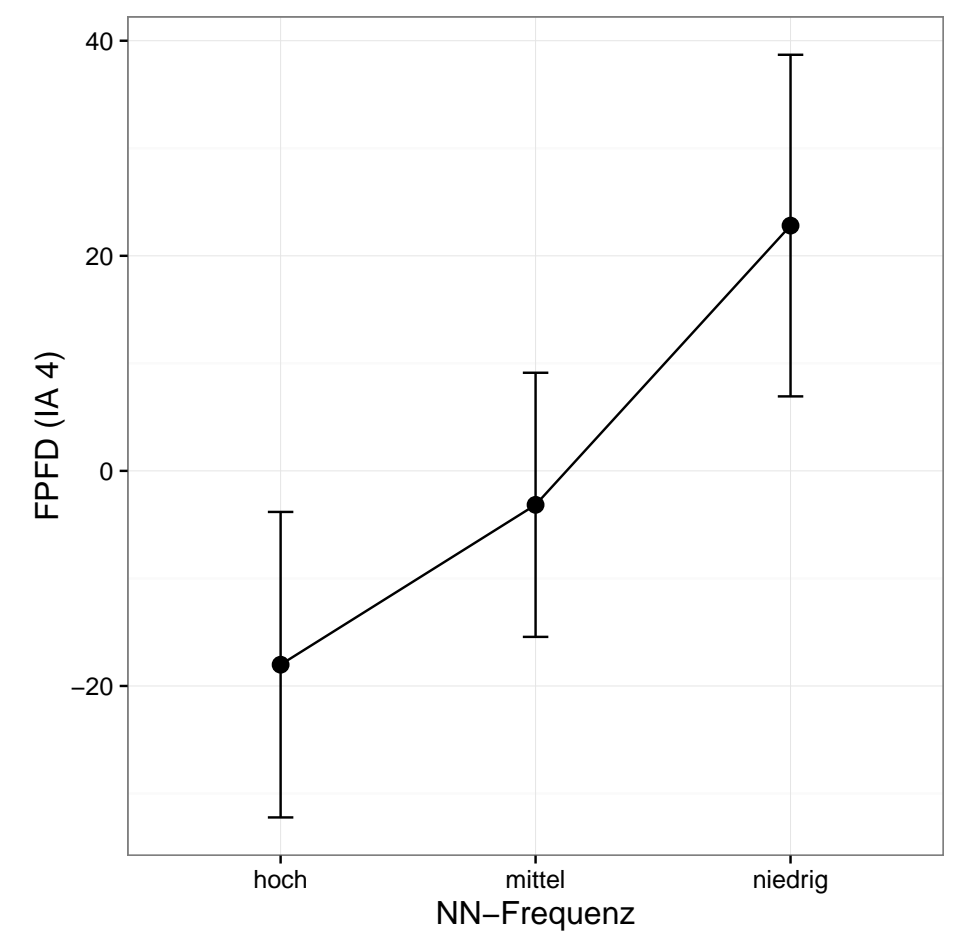

Abbildung 6.36: Plot der Mittelwerte der Längenresiduen der FPFD der AntezedensRegion (IA 4) für den Faktor NN-FREQUENZ

niedrigsten Frequenzen (Häufigkeitsklassen 11 - 13 (6 Items)).

Die Hypothese lautet, dass mit steigender Frequenz auch die Zugänglichkeit steigt, was in einem erleichterten Resolutionsprozess, d.h. kürzeren TRTs der Anapher sichtbar werden sollte. Das prognositizierte Ergebnis in Bezug auf die TRTs ist demnach TRT(hoch) < TRT(mittel) $<$ TRT(niedrig).

Wie in Tabelle 6.85 zu sehen, kann dieses Muster gefunden und in Tabelle 6.86 als signifikant bestätigt werden.

\begin{tabular}{|c|c|c|c|c|c|}
\hline Faktor & AV & Bed. & MW & Stabw. & $\mathrm{n}$ \\
\hline \multirow{3}{*}{ N1-FREQUENZ } & \multirow{3}{*}{ TRT } & hoch & 319.91 & 195.91 & 162 \\
\hline & & mittel & 329.8 & 254.56 & 324 \\
\hline & & niedrig & 372.29 & 289.59 & 162 \\
\hline
\end{tabular}

Tabelle 6.85: Deskriptive Statistik für die TRT der Anaphern-Region (IA 6) für den Faktor N1-FREQUENZ

\begin{tabular}{c|cc|cc}
\hline \hline AV & $F_{1}(2,52)$ & $p$ & $F_{2}(2,21)$ & $p$ \\
\hline TRT & 3.549 & $<0.05$ & 2.801 & $<0.1$ \\
\hline \hline
\end{tabular}

Tabelle 6.86: Inferenzstatistik für die TRT der Anaphern-Region (IA 6) für den Faktor N1-FREQUENZ 


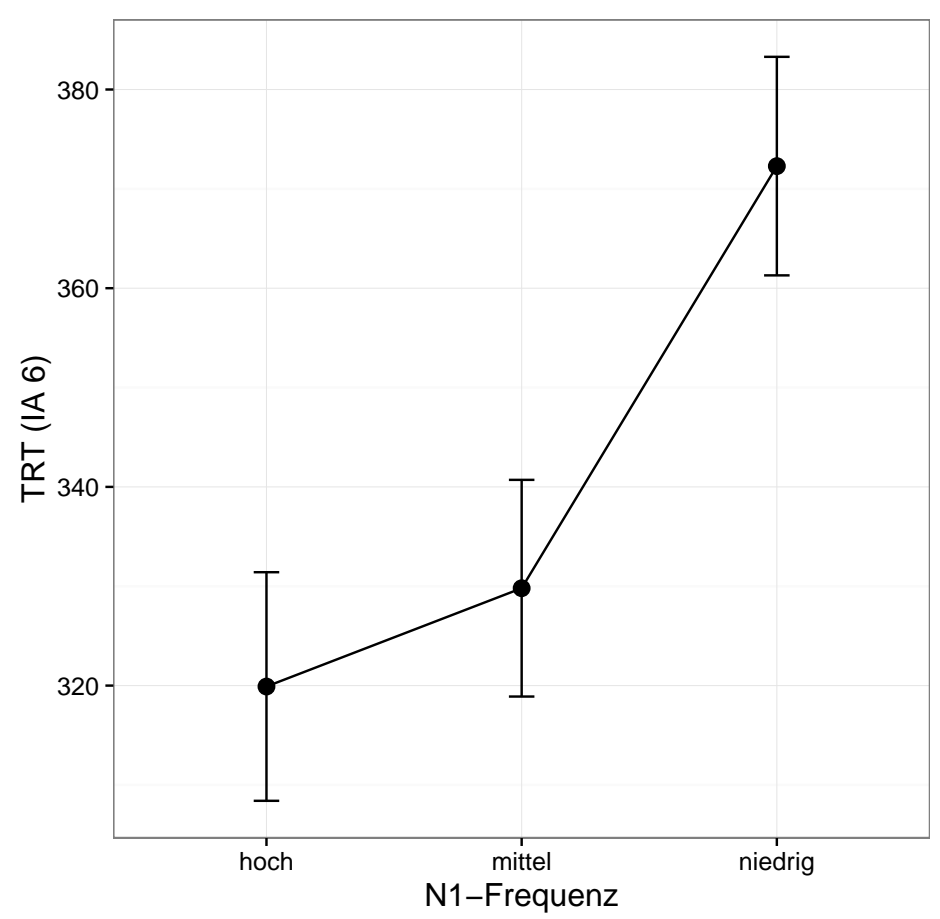

Abbildung 6.37: Plot der Mittelwerte der TRT der Anaphern-Region (IA 6) für den Faktor N1-FREQUENZ

\section{Interpretation}

In Schritt 1 konnte anhand der FPFD der Ankerausdrücke gezeigt werden, dass für frequentere Ausdrücke im ersten Lesedurchlauf tendenziell kürzere Fixationszeiten vorliegen. Da die Inferenzstatistik jedoch keine reliablen Ergebnisse liefert, kann entsprechender Verarbeitungsvorteil nicht sicher bestätigt werden. In Schritt 2 zeigt sich anhand der TRT der Anaphern-Region (entgegen der Ergebnisse aus Exp. 3a), dass für frequentere N1 kürzere Gesamtfixationszeiten vorliegen, diese demnach zugänglicher sind. Tendenziell scheint also ein Zusammenhang zwischen hoher Frequenz, höherer Aktiviertheit und besserer Zugänglichkeit zu bestehen.

Allerdings sind die Ergebnisse in ihrer Aussagekraft einzuschränken, da es sich bei der Frequenz um einen Pseudofaktor handelt, der nicht sauber zwischen den Items ausbalanciert wurde. Zudem liegen die Frequenzwerte oft relativ nah beieinander.

\section{NN-Frequenz}

Wie bereits in Kapitel 2.3.4 und 6.2.6 diskutiert, gibt es verschiedene Möglichkeiten, wie sich die NN-Frequenz auf die N1-Zugänglichkeit auswirken kann. Eine mögliche Hypothese ist, dass mit Zunahme der Frequenz Bedeutungskonsolidierung erfolgt, der Grad an Lexikalisiertheit des Kompositums somit zu- und die N1-Zugänglichkeit abnimmt. Eine andere Hypothese ist, dass Frequenz und Lexikalisiertheit und somit Frequenz und N1-Zugänglichkeit an sich nicht relatiert sind. Wie bereits in Experiment 2 sollen auch die Daten von Experiment 3 zur Analyse eines möglichen Zusammenhangs herangezogen werden. 


\section{Hypothesen}

Sollte zwischen Lexikalisiertheit und Frequenz ein direkter Zusammenhang bestehen, müsste ein NN mit zunehmendem Grad an Lexikalisiertheit über eine entsprechend höhere Frequenz verfügen. Je weniger lexikalisiert es ist, desto niedriger wäre dann seine Frequenz und desto besser müsste die N1-Zugänglichkeit sein. Dies sollte sich wiederum in einer entsprechend kürzeren TRT der Anapher widerspiegeln. Um dies zu prüfen, wurde die Frequenz der Komposita 19 (Bedingungen RK und WK) als Faktor für die TRT der Anaphern-Region überprüft. Die Hypothese dazu lautet TRT(niedrig) $<$ TRT(mittel) ${ }^{20}$ Eine Gegenhypothese dazu wäre, dass es keinen direkten Zusammenhang zwischen Lexikalisiertheit und Frequenz gibt. Darüber hinaus bleibt die Frage, ob zunehmende Lexikalisiertheit notwendigerweise mit abnehmender Transparenz einhergeht (vgl. Diskussion in Kap. 2.3.4.

\section{Ergebnisse}

Die Ergebnisse sind in Tabelle 6.87 dargestellt. Deskriptiv verhalten sich die Mittelwerte entsprechend der Hypothese, allerdings sind die ANOVAs nicht signifikant (vgl. Tab. 6.88).

\begin{tabular}{cccccc}
\hline \hline Faktor & AV & Bed. & MW & Std. Abw. & n \\
\hline \multirow{2}{*}{ NN-FREQUENZ } & \multirow{2}{*}{ TRT } & mittel & 377.74 & 292.32 & 225 \\
& & niedrig & 364.49 & 265.66 & 207 \\
\hline
\end{tabular}

Tabelle 6.87: Deskriptive Statistik für die TRT der Anaphern-Region (IA 6) für den Faktor NN-FREQUENZ (Bed. RK und WK)

\begin{tabular}{c|cc|cc}
\hline \hline AV & $F_{1}(1,26)$ & $p$ & $F_{2}(1,22)$ & $p$ \\
\hline TRT & 1.703 & 0.203 & 0.012 & 0.915 \\
\hline \hline
\end{tabular}

Tabelle 6.88: Inferenzstatistik für die TRT der Anaphern-Region (IA 6) für den Faktor NN-FREQUENZ (Bed. RK und WK)

\section{Interpretation}

Der aufgezeigte Trend für eine bessere N1-Zugänglichkeit bei weniger frequenten N1 spiegelt sich wie bereits in Experiment 3a in den Daten für Experiment 3c wider. Die Ergebnisse sind jedoch in beiden Teilexperimenten nicht signifikant.

Dies kann dem experimentellen Material geschuldet sein, da die hier verwendeten Komposita gezielt über eine kompositionale Bedeutung verfügen (siehe Materialerstellung Kap. 6.1.2). Beim Test mit Komposita mit höherem Lexikalisierungsgrad (z.B. Apfelmännchen) wäre mit aussagekräftigeren Ergebnisse zu rechnen.

Andererseits kann das Ausbleiben eines Effekts bei der Überprüfung mit zwei verschiedenen Paradigmen auch darauf hindeuten, dass hier tatsächlich kein nachweisbarer Zusammenhang besteht. Dies würde für die Hypothese sprechen, dass der Zusammenhang zwischen Frequenz, Transparenz und Lexikalisiertheit weitaus komplexer ist.

\footnotetext{
${ }^{19}$ Hierfür wurden analog zu den Untersuchungen zur N1-FREQUENZ die Frequenzklassen des Leipziger Wortschatzes genutzt.

${ }^{20}$ In der Gruppe ,hoch` befinden sich nur Monolexeme.
} 


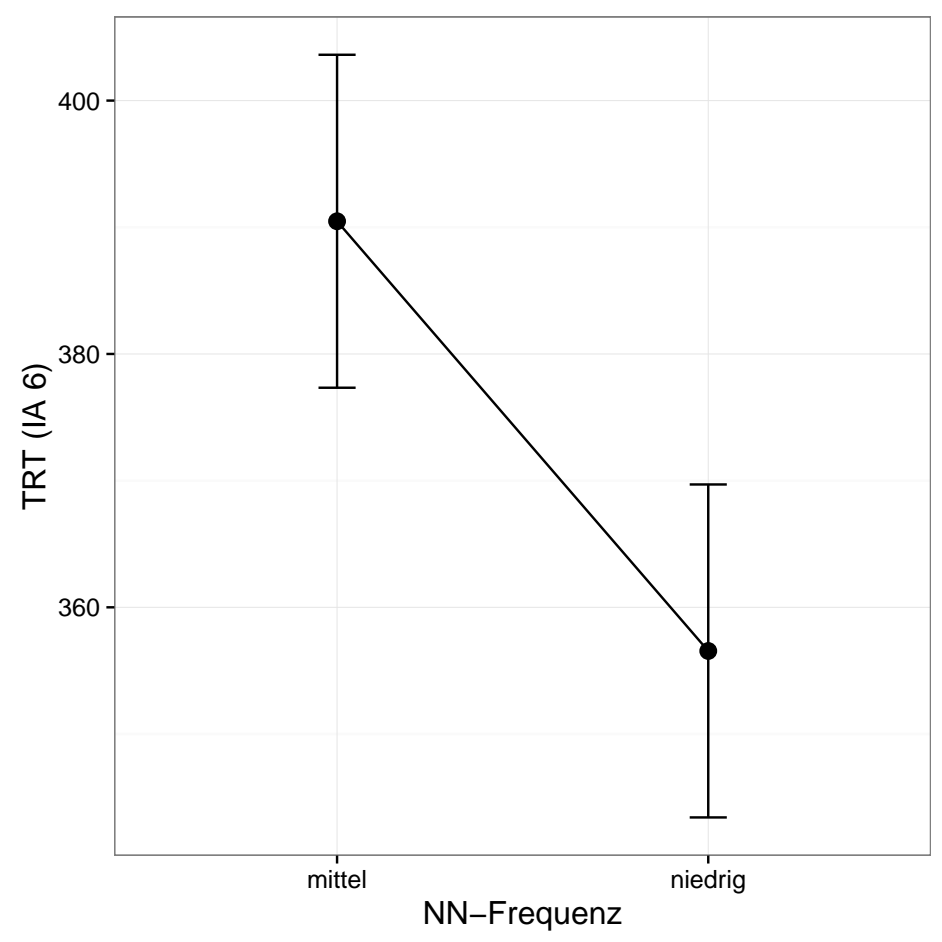

Abbildung 6.38: Plot der Mittelwerte der TRT der Anaphern-Region (IA 6) für den Faktor NN-FREQUENZ (Bed. RK und WK)

\section{Reaktionszeiten der Verständnisfragen}

In Experiment 3c schließt sich bei jedem Item an die Leseaufgabe eine Verständnisfrage an. Bei einem Viertel der experimentellen Items (sechs Fälle) zielt diese Verständnisfrage direkt auf die Anapher ab. Dadurch lässt sich zusätzlich prüfen, ob sich der Grad an N1-Zugänglichkeit auch im Erinnern des Anaphern-Kontexts widerspiegelt. In Bezug auf das Beispiel-Item (6) wäre (7) die entsprechende Verständnisfrage. Um Unterschiede im Erinnern der Anapher anhand derartiger Fragen messbar zu machen, wurde zusätzlich zur gegebenen Antwort die Reaktionszeit (RT) für die Entscheidung aufgezeichnet. Dabei wurde der Zeitabstand zwischen dem Erscheinen der Frage und der Entscheidung per Tastendruck gemessen. Die VPn wurden gebeten, während des gesamten Experiments den Kopf in der Kopfstütze und die Finger auf den benötigten Tasten zu belassen. Dies sollte zum einen die Genauigkeit der Kalibrierung konstant halten und zum anderen sollte die Verzögerung durch eine Suche der entsprechenden Tasten $\left(, 0^{6}\right.$ und, $\left.1^{6}\right)$ minimiert werden.

(6) Marlene ist immer sehr unternehmungslustig.

Dieses Jahr haben Freunde sie zu einem Aktivurlaub

eingeladen und ohne große Nachfragen ist sie mitgefahren.

RK: Die Bergbesteigung ist dann unerwartet anstrengend. Er soll

WK: Die Bergetappe ist dann unerwartet anstrengend. Er soll

Mono: Der Berg ist dann unerwartet anspruchsvoll. Er soll auch für einige schwierige Passagen bekannt sein.

Beim nächsten Mal will sich Marlene vorher besser informieren.

(7) Soll der Berg für einige schwierige Passagen bekannt sein? 


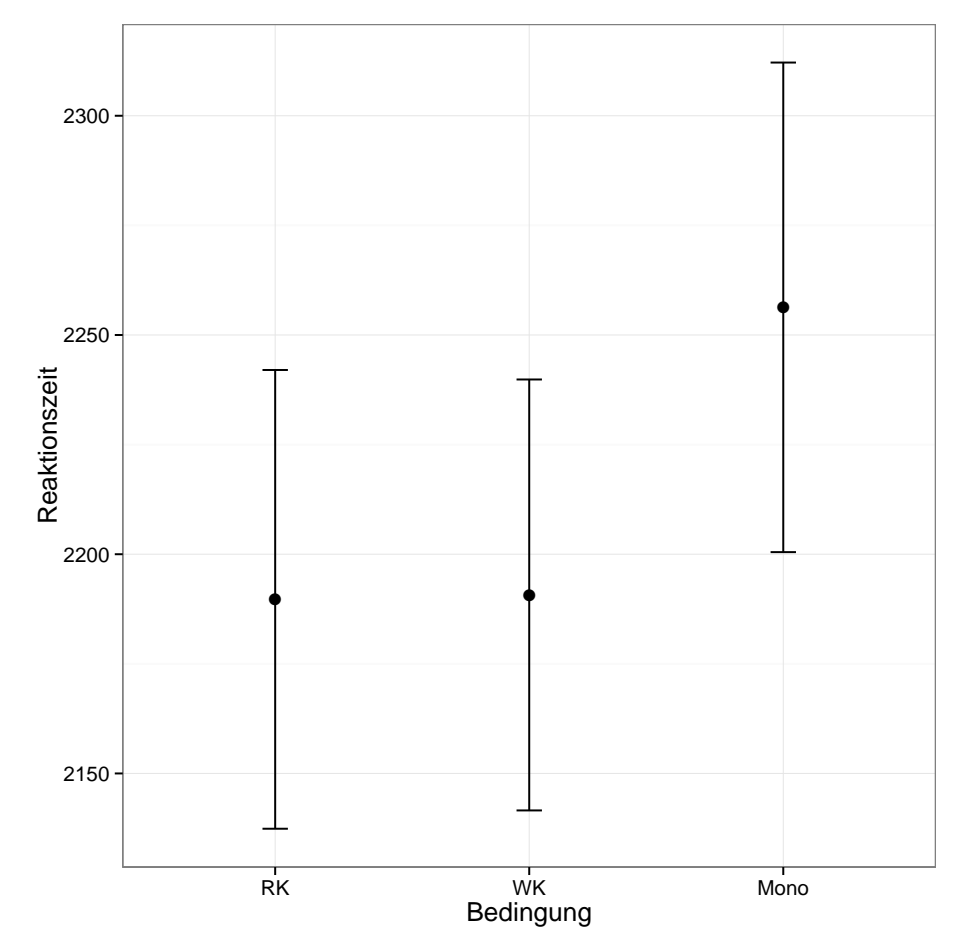

Abbildung 6.39: Plot der Mittelwerte der Reaktionszeiten der auf die Anaphernresolution abzielenden Verständnisfragen

\section{Hypothesen}

Die Hypothese, der im Rahmen der Arbeitshypothese anhand der Verständnisfragen nachgegangen wird, lautet: Je zugänglicher das Antezedens ist, desto stärker ist es aktiviert und desto besser wird es erinnert. Daraus ergibt sich folgende Prognose für die RT: $\mathrm{RT}($ Mono $)<\mathrm{RT}(\mathrm{RK})<\mathrm{RT}(\mathrm{WK})$. Diese Hypothese leitet sich nicht zuletzt daraus ab, dass die Frequenzanalysen gegen einen Verarbeitungsvortiel durch ,deep processing“ sprechen.

\section{Ergebnisse}

Die Reaktionszeiten sind annähernd normalverteilt nach Logarithmieren. Die deskriptive Verteilung der Reaktionszeiten auf die einzelnen Bedingungen ist in Tabelle 6.89 wiedergegeben.

\begin{tabular}{ccccccc}
\hline \hline Faktor & AV & Bed. & MW & Std. Abw. & \% korrekt & n \\
\hline \multirow{4}{*}{ STRUKTUR } & \multirow{2}{*}{ RT } & RK & 2189.70 & 600.01 & 83.33 & 54 \\
& & MK & 2190.71 & 608.26 & 83.33 & 54 \\
& & Mono & 2237.99 & 633.85 & 66.67 & 53 \\
\hline \hline
\end{tabular}

Tabelle 6.89: Deskriptive Statistik der Reaktionszeiten (RT) der auf die Anaphernresolution abzielenden Verständnisfragen (Werte logarithmiert und bereinigt um Werte > 5000) 


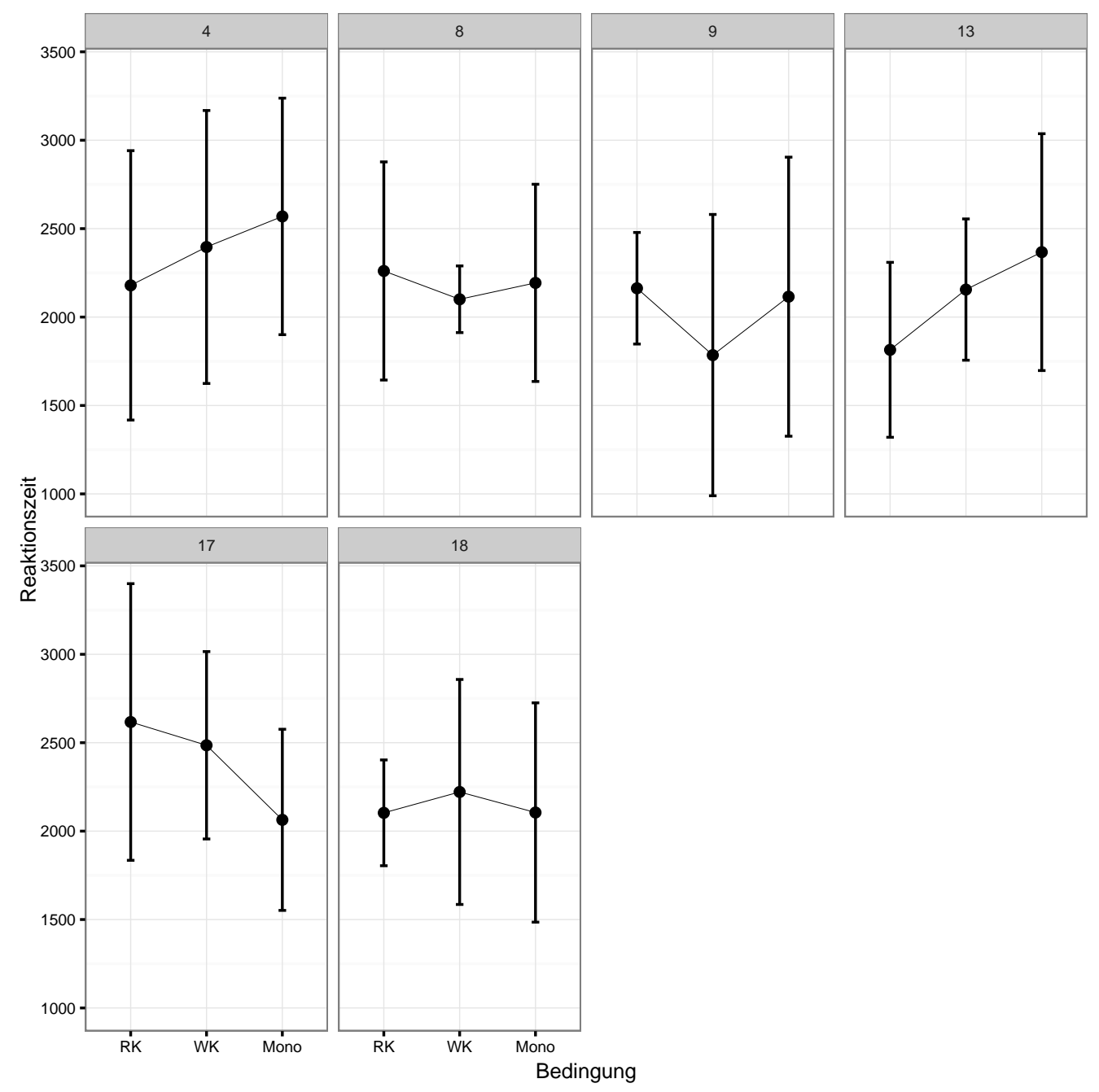

Abbildung 6.40: Plot der Mittelwerte der Reaktionszeiten der auf die Anaphernresolution abzielenden Verständnisfragen für die einzelnen Items

Das Muster der mittleren Reaktionszeiten ist $\mathrm{RT}(\mathrm{RK})<\mathrm{RT}(\mathrm{WK})<\mathrm{RT}($ Mono) und entspricht nicht der Hypothese. Die Reaktionszeiten zwischen den beiden Kompositabedingungen unterscheiden sich kaum, in der Bedingung Mono liegen größere Reaktionszeiten vor. Untermauert wird dieses Ergebnis dadurch, dass auch die Zahl falscher Antworten in der Bedingung Mono am größten ist.

Da es sich insgesamt nur um wenige Datenpunkte handelt, soll ein Blick auf die Ausprägung des Musters für die einzelnen Items geworfen werden (vgl. Abb. 6.40). Hierbei zeigt sich, dass für die verschiedenen Items verschiedene Muster und große Standardfehler vorliegen.

\section{Interpretation}

Die höchste mittlere Reaktionszeit und die höchste Fehlerquote liegen in der Bedingung Mono vor. Dieses unerwartete Verhalten der Kontrollbedingung lässt darauf schließen, dass das Ergebnis der Reaktionszeiten für die Verständnisfragen nicht die Schwierigkeiten des Resolutionsprozesses widerspiegelt. Möglicherweise wurde die Frage nach dem direkten Antezedens als zu einfach empfunden und verunsicherte die VPn. 
Ein weiterer Punkt ist die geringe Zahl an Datenpunkten: Da nur Daten für sechs Items vorliegen, ist die Stichprobe nicht repräsentativ. Die Methode ist eventuell auch ungeeignet. Es ist denkbar, dass sich der Unterschied nicht auf die Offline-Verarbeitung auswirkt und dass die Abbildung des gesamten Lese- und Entscheidungsprozesses in einer AV zu ungenau ist. Die Betrachtung der einzelnen Items zeigt zudem eine große Varianz innerhalb und zwischen den Items. Anhand der vorliegenden Daten kann somit nicht von einem Zusammenhang zwischen N1-Zugänglichkeit und Reaktionszeit für die Verständnisfrage ausgegangen werden.

\section{Zusammenfassung der weiteren Analysen}

Im Eye-Tracking-Experiment wurden neben dem eigentlichen Faktor STRUKTUR auch weitere mögliche Eigenschaften der Items annotiert und ausgewertet.

Der Faktor RzK kann hier in seinem Einfluss bestätigt werden. Wie auch schon in Experiment 2 zeigt sich ein Zugänglichkeitsvorteil für die Faktorstufe +RZK gegenüber der Faktorstufe -RZK. Entgegen der Ergebnisse aus Experiment 2 wirkt die RZK hier unabhängig vom Faktor STRUKTUR.

Auch die Hypothese zur N2-KONKRETHEIT konnte bestätigt werden. Ein konkretes N1 erweist sich (deskriptiv) als zugänglicher, wenn das N2 abstrakt und nicht ebenfalls konkret ist. Verstärkt wird dieser Eindruck durch die Auswertung der NOMINALISIERUNGSLESART. Hier zeigt sich innerhalb der Rektionskomposita eine signifikant kürzere Gesamtfixationszeit im Fall von (abstrakten) Prozessnominalisierungen, was für einen höheren Grad an N1-Zugänglichkeit spricht. Die Ergebnisse aus den Experimenten 3a und 3c für die Faktoren N2-KONKRETHEIT und NOMINALISIERUNGSLESART ergeben zusammen starke Indizien für die Idee des konzeptuellen Fokus'.

Weniger klar ist das Bild für die N1-ZÄHLBARKEIT. Hier wurden entgegen der Erwartungen kürzere Gesamtlesezeiten für Massenomen gefunden. Im Zusammenhang mit den Ergebnissen der anderen Experimente stellt sich diese Eigenschaft somit nicht als Einflussgröße für die N1-Zugänglichkeit dar.

Aus den Ergebnissen von Experiment 2 wurde ein Einfluss des GEnUs des N1 und somit des Pronomens abgeleitet. Die Ergebnisse des Eye-Trackings lassen die Interpretation zu, dass das Neutrum-Pronomen als Vorfeld-es oder Komplexanapher interpretiert wird und diese Fehlinterpretation während der Verarbeitung der Satzergänzung revidiert wird. Insgesamt hat das GENUs jedoch im Gegensatz zu den Satzergänzungsexperimenten nur geringen Einfluss auf das Ergebnis des Eye-Trackings.

Die Auswertung der N1-Frequenzen liefert Indizien für einen höheren Grad an Zugänglichkeit für frequente Antezedenten, für die auch ein allgemeiner Verarbeitungsvorteil zu bestehen scheint. Dieses Ergebnis deutet auf einen Zusammenhang zwischen Verarbeitungsvorteil durch Frequenz und Zugänglichkeit für ein Antezedens hin. Darüber hinaus wurde anhand der NN-Frequenzen dem Zusammenhang zwischen Frequenz und Lexikalisiertheit nachgegangen. Geht man davon aus, dass das N1 lexikalisierter Komposita schlechter zugänglich ist und diese Ausdrücke gleichzeitig eine höhere Frequenz haben, müssten niedrig frequente NN über eine bessere N1-Zugänglichkeit verfügen. Ein solcher Zusammenhang wurde deskriptiv gefunden, bleibt aber kritisch zu hinterfragen.

Bei allen Analysen unter Kapitel 6.4.7 ist der spekulative Charakter der Interpretation zu bedenken, da die entsprechenden Faktoren nicht optimal ausbalanciert und das Material nicht darauf ausgerichtet ist. Außerdem basiert die Annotation der verschiedenen Eigenschaften mitunter auf einer subjektiven Einschätzung von Denotatsqualitäten. 


\subsection{Zusammenfassung der drei Experimente (3a, b, c)}

\subsubsection{Zusammenfassung der Ergebnisse}

Nachdem die 24 Komposita-Items in Experiment 3 in drei verschiedenen Paradigmen Satzergänzung (3a), Kohärenzrating (3b) und Eye-Tracking (3c) - getestet wurden, soll nun eine Zusammenschau der Ergebnisse erfolgen.

Das wichtigste Ergebnis ist, dass sowohl die Ergebnisse des Satzergänzungsexperiments 3a als auch des Eye-Tracking-Experiments 3c für eine bessere Zugänglichkeit des N1 in Rektions- als in Wurzelkomposita sprechen. Die Hypothese zum Einfluss der KompositaStruktur auf den referenziellen Status des N1 und somit auf die N1-Zugänglichkeit kann somit bestätigt werden (vgl. Kap. 4). In den Eye-Tracking-Daten konnte der Unterschied zwischen Wurzel- und Rektionskomposita für die FPFD und die ANOVA-by-subjects der TRT der Anaphern-Region als signifikant bestätigt werden. Die Auswertung der übrigen $\mathrm{AVn}$ widersprechen diesem Ergebnis nicht, sondern untermauern die Interpretation als Zugänglichkeitsvorteil. So konnte kein genereller Verarbeitungsunterschied zwischen den Komposita-Arten gefunden werden, dafür aber eine Effekt für die Regressionen in die Anaphern-Region sowie ein Effekt für die Gesamtfixationszeit der Satzeinbettung der Anapher. Der Einfluss der experimentellen Manipulation auf die FPFD der AnaphernRegion weist darüber hinaus auf einen frühen Effekt hin, d.h. auf einen Einfluss der Kompositums-Struktur und damit auch der N1-Zugänglichkeit bereits während lexikalischer und morphosyntaktischer Verarbeitungsschritte. Dieses Ergebnis spricht für eine Einordnung des N1-Bezugs auf der Skala zwischen direkter und indirekter Anapher mit größerer Nähe zur direkten Anapher.

Für die Satzergänzungsaufgabe und das Eye-Tracking wurden zusätzlich weitere mögliche Einflussgrößen analysiert. Eine Gegenüberstellung der Ergebnisse der beiden Experimente ist in Tabelle 6.90 dargestellt. Da das experimentelle Design auf den Faktor STRUKTUR ausgerichtet ist, sind alle zusätzlichen Analysen eher explorativer Natur und können maximal als Tendenzen betrachtet werden.

Bezüglich der RZK sprechen beide Experimente für eine bessere N1-Zugänglichkeit in der Bedingung +RZK. Als weitere Faktoren zum Einfluss der Konzeptkombination wurden die N2-KONKRETHEIT und die NOMINALISIERUNGSLESART betrachtet. Vergleicht man die Ergebnisse der beiden Faktoren in beiden Teilexperimenten, zeichnet sich deutlich ein Zugänglichkeitsvorteil für konkrete N1 im Fall abstrakter N2 ab. Dieser Unterschied in der Konkretheit scheint einen Einfluss auf den konzeptuellen Fokus des Kompositums und damit auf die N1-Zugänglichkeit auszuüben. Denkbar ist, dass es sich hierbei um einen Einfluss von Visualisierungstendenzen handelt, wie er auch der RZK zugeschrieben wird (vgl. Kap. 3.3.4).

Anders stellen sich die Ergebnisse zur N1-ZÄHLBARKEIT dar. Dieser Faktor kontrastiert grammatische und konzeptuelle Einflüsse, da Massenomen determiniererlos referenzfähig sind, Individualnomen hingegen über einen Unikalitätsvorteil verfügen. In Experiment 3a konnte kein Unterschied zwischen Masse- und Individualnomen gefunden werden, in Experiment 3c liegt ein leichter Vorteil für Massenomen vor. Im Gegensatz dazu konnte in Experiment 2 ein Vorteil für Individualnomen gefunden werden. Diese heterogenen Resultate sprechen gegen die Einstufung der N1-ZÄHLBARKEIT als Einflussfaktor für die N1-Zugänglichkeit.

Eine Eigenschaft, deren Art von Einfluss vom Paradigma abhängt, ist das GENUs. Hierbei 


\begin{tabular}{r|ll}
\hline \hline Faktor & Exp. 3a & Exp. 3c \\
\hline STRUKTUR & RK $>$ WK ${ }^{*}$ & RK $>$ WK ${ }^{*}$ \\
\hline weitere Analysen: & & \\
RZK & + RZK $>-$ RZK & + RZK $>-$ RZK $^{*}$ \\
N2-KONK. & - konk. $>+$ konk. $*$ & - konk. $>+$ konk. \\
NOM.-LESART & Prozess $>$ Resultat & Prozess $>$ Resultat $*$ \\
N1-ZÄHLBARKEIT & Masse $=$ Individual & Masse $>$ Individual $*$ \\
GENUS & mask $>$ neutr $*$ & IA $6:$ neutr $>$ mask \\
& & IA $7:$ mask $>$ neutr $*$ \\
N1-FREQUENZ & niedrig $>$ mittel $>$ hoch & IA 6: hoch $>$ mittel $>$ niedrig $*$ \\
NN-FREQUENZ & niedrig $>$ mittel & IA 6: niedrig $>$ mittel \\
\hline \hline
\end{tabular}

Tabelle 6.90: Gegenüberstellung der Ergebnisse von Experiment 3a (Satzergänzungsexperiment) und 3c (Eye-Tracking); Vergleich der N1-Zugänglichkeit in Abhängigkeit von den jeweiligen Bedingungen; Angabe eines inferenzstatistischen Effekts durch , *،

handelt es sich nicht um einen Faktor, der direkt die Zugänglichkeit beeinflusst. Vielmehr beeinflusst das Genus des Pronomens die zur Verfügung stehenden Resolutionsmöglichkeiten und -präferenzen und dadurch auch indirekt das Ergebnis der Experimente. Die Ergebnisse des Eye-Trackings stützen die Hypothese der präferierten Interpretation des Neutrum-Pronomens als Vorfeld-es oder Komplexanapher.

Die Analyse der N1-Frequenz anhand der Eye-Tracking-Daten bestätigt (entgegen der deskriptiven Ergebnisse aus Experiment 3a) einen Zusammenhang zwischen Verarbeitungsvorteil durch höhere Frequenz eines Ausdrucks und seiner anaphorischen Zugänglichkeit. Ebenso deutet sich ein Zugänglichkeitsvorteil bei niedrigeren NN-Frequenzen an. Für eine verlässliche Analyse des Zusammenhangs zwischen Konstituenten- und KompositaFrequenzen sowie Verarbeitungsunterschieden und Lexikalisiertheitsgraden sind jedoch genauere Analysen und ein darauf ausgerichtetes experimentelles Design unerlässlich.

\subsubsection{Evaluation des Materials}

Um methodenunabhängige Ergebnisse zu erzielen, wurde das experimentelle Material in drei verschiedenen Paradigmen getestet und der Hauptfaktor STRUKTUR mit Hilfe von zwei verschiedenen Paradigmen (Satzergänzung und Eye-Tracking) überprüft. Die Ergebnisse von Experiment 3a und 3c für den Faktor STRUKTUR weisen in dieselbe Richtung. Die Vergleichbarkeit der Items wurde im Kohärenzrating in Experiment 3b geprüft. Sollte der Effekt des Faktors STRUKTUR dennoch primär durch einzelne Items getragen werden, müsste dies in allen drei Teilexperimenten sichtbar werden. Die geplotteten Mittelwerte der AVn auf Itemebene sind im Anhang in den Abbildungen C.1, C.4 und C.6 abgebildet. Bei der Zusammenschau der Experimente auf Item-Ebene fällt auf, dass sich über die Experimente hinweg nicht stets dieselben Items hypothesenkonform oder -gegenläufig verhalten. Lediglich drei von 24 Items verhalten sich in allen Paradigmen hypothesenkonform: Item 8 (Müllbeseitgung vs. -tonne), 20 (Dachbegrünung vs. -garten), 23 (Waldrettung vs. -straße). Gleichsam in allen Experimenten gegenläufige Items gibt es nicht, jedoch in 3a und 3c: Item 10 (Salzgewinnung vs. -konzentration), 15 (Panzerbekämpfung vs. -kette), 21 (Gartenbeleuchtung vs. -licht). Offensichtliche lexikalische Gemeinsamkeiten zwischen den hypothesenkonformen Items lassen sich nicht beobachten. Insgesamt lässt sich daraus 
schließen, dass das Gesamtergebnis nicht von einzelnen Items getragen wird, sondern im Mittel von allen Items und allen Paradigmen. Beim Vergleich der Item-Analysen fällt zudem auf, dass der Standardfehler in den meisten Fällen in der Bedingung WK am größten und in der Bedingung Mono am kleinsten ist.

Eine geordnete Gegenüberstellung der Ergebnisse der einzelnen Items in ihren Bedingungen für die Experimente 3a und 3c gibt ein detaillierteres Bild (vgl. Anhang Abb. C.2 und C.7): Die meisten Bezüge auf das avisierte Antezedens (N1) in Experiment 3a liegen für die Monolexeme vor, ebenfalls im oberen Bereich liegen Waldrettung und Tabakverarbeitung. Der mittlere Bereich ist in Bezug auf die Bedingungen gemischt. Bei den niedrigen Werten liegen vorrangig, aber nicht ausschließlich Wurzelkomposita vor, so z.B. Ölwanne, Buchpreis und sowohl Autowerkstatt als auch Autoanfertigung. Für Experiment 3c ergibt sich ein ähnliches Bild: Die kürzesten Gesamtlesezeiten der Anaphern-Region liegen v.a. bei den Monolexemen vor. Rektionskomposita mit relativ kurzen Gesamtlesezeiten sind Dachbegrünung und Waldrettung; Tabakverarbeitung erschient bei Experiment 3c erst im Mittelfeld. Unter den Items mit den längsten Lesezeiten sind sowohl Wurzel- als auch Rektionskomposita zu finden. Neben Dachgarten und Mülltonne ist auch in Experiment 3c hier Autowerkstatt zu finden. Autoanfertigung liegt jedoch eher im mittleren Bereich. Insgesamt scheint es auf Itemebene einige Gemeinsamkeiten, aber auch deutliche Unterschiede zwischen den Ergebnissen der beiden Experimente zu geben, sodass nicht ausschließlich die spezifische Lexik einzelner Items für den Effekt verantwortlich sein kann. Da das Gesamtmuster über alle Items hinweg von beiden Experimenten getragen wird, kann das Material als geeignete Stichprobe gewertet werden. Die starken Schwankungen zwischen den Items illustrieren den Einfluss spezifischer Lexik. Die statistisch ermittelten Effekte bilden einen generellen, lexik-unabhängigen Trend ab. 



\section{7. Übergreifende Diskussion}

\subsection{Lexikalische Integrität revisited}

Die lexikalische Integrität ist nach wie vor ein klassisches Wortkriterium. Gemäß der LIH darf kein syntaktischer Prozess ausschließlich lexikalische Strukturen involvieren (vgl. u.a. Lapointe 1979). Demnach müsste es sich bei Komposita, die nach gängiger Auffassung als Wörter gelten, um anaphorische Inseln handeln. Eine Vielzahl von Autor*inn*en schlussfolgert daraus, dass Komposita-Erstglieder nicht für pronominale Anaphern zugänglich sind (vgl. Kap. 2.1).

Diese Arbeit konnte anhand vielfältiger Gegenbeispiele und mit Hilfe von theoretischen und empirischen Untersuchungen zeigen, dass diese Annahme nicht allgemeingültig ist. Die wichtigste Erkenntnis dabei ist, dass es sich bei der Unzugänglichkeit von Erstgliedern nicht um ein kategorisches, sondern um ein graduelles Phänomen handelt. Um eine grammatische Beschränkung kann es sich bei Unzugänglichkeit demnach nicht handeln. Dies wiederum bedeutet, dass Komposita nicht per se anaphorische Inseln sind. Eine Vielzahl an Faktoren wurde identifiziert, die dazu beitragen können, dass das Erstglied in unterschiedlichen Graden zugänglich sein kann, bzw. Erstglied-Bezüge graduell akzeptabel sein können. Dabei stellt sich die Frage, ob anaphorische Zugänglichkeit überhaupt ein grammatisches Prinzip ist. Plausibler scheint, dass es keine kategorische Unterscheidung zwischen anaphorischen Inseln und Nicht-Inseln gibt, sondern es sich immer um eine graduelle Abstufung handelt. Ist eine anaphorische Beziehung nicht grammatisch, liegt dies meines Erachtens nicht an der Unzugänglichkeit des Antezedens, sondern daran, dass darüber hinaus grammatische Beschränkungen verletzt werden, z.B. Bindungsprinzipien.

Diese Annahmen bringen die Frage mit sich, welche Rückschlüsse für die Gültigkeit des Prinzips der lexikalischen Integrität gezogen werden müssen. Versteht man die LIH wie Lapointe (1979) so, dass kein syntaktischer Prozess ausschließlich lexikalische Strukturen involvieren darf, bedeutet dies im Umkehrschluss, dass ein anaphorischer Bezug kein (ausschließlich) syntaktischer Prozess ist, bzw. eine anaphorische Beziehung keine syntaktische Beziehung ist.

Bereits Wunderlich (1986 219) deutete als Konsequenz für die Anaphorik an, dass, sollten Erstglied-Bezüge möglich sein, eine Anaphernbeziehung kein primär syntaktischer Prozess sein könne. Vielmehr handelt es sich um einen Prozess auf diskursstruktureller Ebene, bei dem nicht auf einen linguistischen Ausdruck Bezug genommen wird, sondern auf einen durch einen linguistischen Ausdruck etablierten Diskursreferenten.

In Bezug auf grammatische Prozesse spielt morphosyntaktische Kongruenz bei pronominalen Anaphern eine Rolle. Die explorative Auswertung verschiedener Satzergänzungsexperimente zeigt jedoch, dass diese nicht zwingend und ausschlaggebend ist. Wichtigstes Kriterium ist und bleibt die pragmatische Plausibilität. 
In diesem Zusammenhang sei die Diskussion um Andersons (1992) Definition zur lexikalischen Integrität wieder aufgegriffen: Die Aussage, dass die Syntax keinen Zugang zur internen Struktur von Wörtern hat, konnte widerlegt werden. Ein Erstglied muss für die Syntax sichtbar sein, denn: (i) die Klammerparadoxie stellte sich als produktives Phänomen dar, bei dem ein Adjektiv morphosyntaktisch mit dem Erstglied kongruiert; (ii) konnten Koester et al. (2004) einen Effekt für ein Mismatch des N1-Genus finden, d.h. das Genus des Wortteils muss zugänglich sein; und (iii) konnten im Rahmen dieser Arbeit pronominale Erstglied-Bezüge als produktive Form der Sprachverwendung belegt werden. In diesem Sinne ist Booijs (2009b) Reinterpretation der lexikalischen Integrität beizupflichten. Komplexe Wörter haben eine interne Struktur und diese muss (zumindest in einem gewissen Grad) für die Syntax (oder die Grammatik allgemein) zugänglich sein.1 1

Wie in Kapitel 2.2.2 gezeigt wurde, stellen verschiedene Arten von Komposita die meisten Kriterien zur Abgrenzung zwischen Wörtern und Phrasen infrage. Aus diesem Grund stimme ich auch hier Booijs Vorschlag zu, die Abgrenzung am Kohäsionsgrad zwischen den Konstituenten festzumachen. Die relative Kohäsion zwischen Komposita-Konstituenten ist nun stärker als zwischen Konstituenten einer Phrase. Wie auch immer diese Eigenschaften im Speziellen auszulegen sind, im Vergleich zu Konstituenten einer Phrase sind Konstituenten von Komposita weniger gut manipulierbar und i.d.R. auch weniger gut zugänglich. Ich stimme Booij zu, dass es eine Art von Prinzip wie dem der lexikalischen Integrität geben muss, da es einen intuitiven Unterschied zwischen Wörtern und Phrasen gibt. Allerdings handelt es sich dabei nicht um eine Universalie, sondern eher um einen unscharfen Ausdruck von Kohäsion.

Einhergehend mit der Aufweichung des Prinzips der lexikalischen Integrität gilt es zu diskutieren, inwiefern lexikalistische Grammatikansätze noch zu rechtfertigen sind. Im Rahmen der morphosyntaktischen Analyse von verschiedenen Komposita-Arten in Kapitel 4 fiel die Wahl auf einen syntaktischen Ansatz: Die Distribuierte Morphologie.

Auch innerhalb der syntaktischen Ansätze bildet die Morphologie eine notwendige Ebene. Nur handelt es sich nicht um ein isoliertes Modul mit eigenen Prinzipien, sondern das syntaktische Instrumentarium findet universellen Einsatz bis auf die Morphemebene. Eine einheitliche Beschreibung der Konstruktion von komplexen Ausdrücken - seien es Wörter oder Phrasen - mit einem einheitlichen Instrumentarium erscheint mir sinnvoll und erstrebenswert. In dieses System ließe sich auch ein Prinzip wie die lexikalische Integrität im Sinne von Kohäsionsunterschieden integrieren. Als Ursache wäre dann jedoch nicht eine strenge Bildungsabfolge von Morphologie und Syntax anzunehmen. Im Fall von Komposita wäre eine Ursache, dass ein Erstglied einen anderen syntaktischen Status hat als ein selbstständiges Wort, nämlich keine NP ist. Diese Annahme ist tief verwurzelt in den syntaktischen Ansätzen. So konstatierte bereits Sproat (1985), dass ein Erstglied nicht maximal, ergo nicht referenziell sein könne. Dies ist allerdings nicht universell gültig, wie auch die lexikalische Integrität nicht universell gültig ist. Schließlich können auch Phrasen abhängig von ihrer Komplexität unterschiedliche grammatische Status haben.

Diese Arbeit vertritt die Idee einer Skala mit zunehmender Zugänglichkeit vom typischen Erstglied mit dem Status $N^{\circ}$ hin zu typischem Nomen mit dem Status NP/D. Jeder Ausdruck auf dieser Skala kann als erste Konstituente in einem Kompositum fungieren. In diesem Sinne ist die lexikalische Integrität nicht Ausdruck einer Grenze zwischen Morphologie und Syntax, sondern davon, dass Konstituenten von Wörtern einen anderen gram-

${ }^{1}$ Zur Auffassung der Restriktion hinsichtlich der generellen Unterbrechbarkeit der Wortstruktur will diese Arbeit keine Aussagen treffen. 
matischen Status haben als Konstituenten von Phrasen, was wiederum in der relativen Kohäsion zwischen den Konstituenten zum Ausdruck kommt.

\subsection{Einflussfaktoren für die N1-Zugänglichkeit}

\subsubsection{Arten von Faktoren}

Die im Rahmen dieser Arbeit durchgeführten Experimente bestätigen, dass es sich bei der (Un-)Zugänglichkeit von Komposita-Erstgliedern um eine graduelle Eigenschaft handelt, die von einer Vielzahl unterschiedlicher Faktoren in ihrer Ausprägung beeinflusst wird und die durch die verschiedenen Akzeptabilitätsgrade pronominaler Erstglied-Bezüge in Erscheinung tritt. Der Einfluss einiger dieser Faktoren auf die Zugänglichkeit eines Erstglieds konnte im Rahmen von drei Experimenten empirisch nachgewiesen werden. Dabei zeigt sich, dass auch zwischen den Faktoren und ihren Faktorstufen graduelle Abstufungen im Einflussgrad bestehen. Als entscheidende Eigenschaften der Komposita, die durch die verschiedenen Faktoren beeinflusst werden, treten dabei v.a. die Salienz und die Referenzialität des Erstglieds in den Vordergrund.

In früheren Studien wurden vorrangig pragmatische Faktoren betrachtet, die z.B. durch Eigenschaften des Kontexts sowie der Informationsstruktur die Salienz eines N1 erhöhen (vgl. Kap.2.6.1). In Bezug auf die Komposita selbst wurden v.a. die Transparenz der Konstituenten und der Grad an Kompositionalität der Bildung analysiert (vgl. Kap. 2.3.4 und 2.6.2). Diese Arbeit konzentrierte sich nun auf diskursunabhängige, ausdrucks-inhärente Faktoren bei transparenten endozentrischen NN-Komposita.

Kapitel 3 setzte sich zunächst mit konzeptuellen Faktoren auseinander, die verschiedene Wissensaspekte zu Komposita umfassen. Dabei standen zunächst Faktoren des Wissens über das Erstglied im Vordergrund (vgl. Kap. 3.2). Dazu zählen Denotateigenschaften, die in einem Lebenswelteffekt resultieren, z.B. Animatheit und emotionale Valenz; aber auch Eigenschaften, die in einem Wahrnehmungseffekt resultieren, z.B. Konkretheit und Zählbarkeit. Insgesamt kristallisierte sich heraus, dass ein Erstglied umso zugänglicher ist, je eindeutiger sein Denotat zu identifizieren ist, was mit dem Schlagwort ,Unikalität' umrissen wurde. Am deutlichsten zeigte sich dies im Fall von Eigennamen. Diese Ergebnisse sind nicht überraschend, da es sich um Eigenschaften handelt, die generell für eine höhere Aktiviertheit und damit bessere Zugänglichkeit eines Ausdrucks sorgen.

Zur empirischen Überprüfung wurde exemplarisch in Experiment 1 in einer Satzergänzungsstudie der Faktor ANIMATHEIT als Beispiel für einen Faktor des Lebenswelteffekts untersucht. Dabei stellte sich heraus, dass mehr Bezüge zu tierisch-animaten Erstgliedern hergestellt werden als zu inanimaten. In diesem Sinne kann die Animatheit als Faktor der N1-Zugänglichkeit bestätigt werden. Als Beispiel für einen Faktor des Wahrnehmungseffekts wurde in den Experimenten 2 und 3 der Unterschied zwischen Individualnomen und Massenomen an der N1-Position als Subfaktor analysiert. Während sich in der Satzergänzungsstudie in Experiment 2 ein leichter Vorteil für Individualnomen abzeichnete, wird in der Eye-Tracking-Studie in Experiment 3c ein Vorteil für Massenomen sichtbar. Aufgrund dieser Divergenzen kann die Zählbarkeit des N1 nicht als Faktor bestätigt werden. Gleichzeitig zeigt sich, wie anfällig manche Eigenschaften für Paradigmenkonfundierung sind. 
Kapitel 3.3 setzte sich mit konzeptuellen Faktoren auseinander, die erst innerhalb einer Konzeptkombination wirksam werden. Dabei handelt es sich um Faktoren, die die Art und Weise der Interaktion der Konzepte von N1 und N2 bei der Interpretation eines NNKonzepts beeinflussen.

Ein Determinativkompositum ist morphosyntaktisch und semantisch rechtsköpfig, z.B. denotiert der Ausdruck Kuchenteller einen Teller. Allerdings ist dies nicht notwendigerweise der Fall, z.B. denotiert Heuhaufen sowohl Heu als auch einen Haufen. Aufbauend auf dieser Beobachtung wurde postuliert, dass ein N1 umso zugänglicher ist, je zentraler es für das NN-Konzept ist, d.h. je stärker der konzeptuelle Fokus auf dem N1 liegt. Hierbei spielt u.a. die semantische Relation zwischen den beiden Konstituenten eine Rolle. Als Abstraktion über die semantischen Relationen wurde die Idee der raum-zeitlichen Kontiguität (RZK) der Konstituenten formuliert: Ist die Repräsentation des N1 für das NN-Konzept notwendig, ist es auch zugänglicher. Schließlich wurden Interaktionen von N1- und N2-Eigenschaften untersucht. So ist anzunehmen, dass ein N1 zugänglicher ist, wenn es sich bei dem entsprechenden Ausdruck um ein Konkretum handelt, während das N2 abstrakt ist. Wie bereits bei der Idee der RZK spielen hier neben dem Einfluss des konzeptuellen Fokus auch Visualisierungstendenzen eine Rolle: Konstituenten, die bei der Visualisierung eine wichtige Rolle spielen, erscheinen auch zugänglicher zu sein.

Der Einfluss der semantischen Relation auf die N1-Zugänglichkeit wurde in Experiment 1 überprüft. Dabei wurde die Relation HAVE als Beispiel für eine Relation mit dem konzeptuellen Fokus auf dem N1 mit der Relation FOR verglichen, bei der der konzeptuelle Fokus in Richtung N2 verlagert ist. Deskriptiv zeichnete sich eine bessere N1-Zugänglichkeit für N1 in Komposita mit einer HAVE-Relation ab, allerdings lässt sich dieser Effekt inferenzstatistisch nicht belegen. Darüber hinaus erwies sich die semantische Relation nicht als geeigneter Faktor für experimentelle Untersuchungen, da das Set an Relationen nicht objektivierbar ist und sich Komposita nicht trennscharf auf die Relationen aufteilen lassen. In der Satzergänzungsstudie in Experiment 2 wurde daher der kategoriale Faktor der RZK nutzbar gemacht. Dieser zeitigt dahingehend einen signifikanten Effekt, dass N1 in Komposita mit obligatorischer RZK mehr N1-Bezüge aufweisen und damit zugänglicher sind als N1 in Komposita ohne RZK. In Experiment 3 wurde die RZK als Subfaktor ebenfalls ausgewertet und die Ergebnisse aus Experiment 2 ließen sich bestätigen. Schließlich wurde in Experiment 3 der Einfluss der N2-Konkretheit und der Unterschied zwischen Prozess- und Resultatsnominalisierungen als Subfaktor ausgewertet. Über verschiedene Teilexperimente hinweg zeigte sich, dass die N1-Zugänglichkeit erhöht ist, wenn das N1 konkret und das N2 abstrakt ist, d.h. der konzeptuelle Fokus in Richtung N1 verschoben ist.

Die Eye-Tracking-Studie in Experiment 3c deutet außerdem an, dass eine höhere Frequenz eines Ausdrucks einen Verarbeitungsvorteil bewirkt und eine höhere N1-Frequenz eine bessere N1-Zugänglichkeit mit sich bringt. Gleichzeitig wird mit Hofmeister (2011) dafür argumentiert, dass Verarbeitungsunterschiede zwischen Ausdrücken nicht per se in Unterschieden in der Zugänglichkeit resultieren. Wichtiger ist der relative Aktiviertheitsvorteil eines Ausdrucks an der N1-Position unter Berücksichtigung der Konzeptkombination.

Ein Faktor, der sich außerdem bestätigen lässt, ist das Genus des N1. Hier zeigen sich in den Satzergänzungsstudien in Experiment 2 und 3a unterschiedliche Resolutionspräferenzen für Neutrum- und Maskulinum-/Femininum-Pronomen (vgl. z.B. Kap. 5.7.2). Dies liegt allerdings nicht in der Zugänglichkeit des N1 begründet, sondern darin, dass für Neutrum-Pronomen mehr Resolutionsalternativen (z.B. als Komplexanpher oder Vorfeld- 
es) zur Verfügung stehen als für die Pronomen der übrigen Genera. Auch in der EyeTracking-Studie in Experiment 3c zeigte sich ein Einfluss des Genus auf die Blickbewegungen der Versuchspersonen. Ein Konfundierung der Ergebnisse durch das N1-Genus wurde jedoch durch das experimentelle Design ausgeschlossen.

Die Generizität des N1, die oftmals als Ursache für die Unzugänglichkeit benannt wird, wurde in Kapitel 3.5 analysiert. Hierbei zeigte sich, dass es sich bei der beobachteten Einflussgröße eher um eine Form der Spezifizität als um Generizität i.e.S. handelt. Da es sich bei dieser Eigenschaft um einen Faktor handelt, der allgemein Einfluss auf die Akzeptabilität anaphorischer Bezüge ausübt, ist auch von einem Einfluss auf die N1Zugänglichkeit auszugehen. In Experiment 2 wurde der Einfluss nachgewiesen: Für Fälle mit spezifischen N1 wie Filmbewertung konnten signifikant mehr N1-Bezüge verzeichnet werden als für Fälle mit unspezifischem N1 wie Filmrecht.

Kapitel 4 setzte sich mit der morphosyntaktischen Struktur verschiedener KompositaArten auseinander. Dabei wurde postuliert, dass verschiedene Arten von Komposita auch über verschiedene Arten der Strukturbildung verfügen, da die Konstituenten in unterschiedlichen Verhältnissen zueinander stehen können, z.B. in einem Modifikations- oder in einem Subkategorisierungsverhältnis. Diese Unterschiede können wiederum in verschiedenen referenziellen Status des N1 resultieren, die mit verschiedenen Zugänglichkeitsgraden assoziiert werden. Für Wurzel- und Rektionskomposita wurden Analysemöglichkeiten im Framework der Distribuierten Morphologie aufgezeigt. Bei Wurzelkomposita besteht ein reines Modifikationsverhältnis; das N1 verbleibt im Wurzelstatus und ist somit unzugänglich. Bei Rektionskomposita hingegen besteht ein Subkategorisierungsverhältnis; das N1 ist nominal kategorisiert, verfügt über Argumentstatus und kann bspw. im Fall von Eigennamen sogar eine maximale Projektion (D) sein. Der Faktor STRUKTUR kann somit als Ausdruck des referenziellen Status des N1 angesehen werden, der ebenfalls graduell auf die Zugänglichkeit des N1 einwirkt. Die Annahme besteht darin, dass die erste Konstituente sich auf einer Skala von einem typischen N1 wie in Dachgarten (kategorielose Wurzel, nicht referenziell) bis zu einer DP wie in Der-schöne-Rheingau-Laberei (nominal, maximal, referenziell) bewegen kann. Dazwischen liegen verschieden Abstufungen (z.B. nominal ohne Argumentstatus wie in Schrankseite).

In Experiment 2 zeigte sich entgegen der Hypothese ein Effekt für eine bessere Zugänglichkeit des N1 in Wurzelkomposita im Gegensatz zu Rektionskomposita. Hier konnte allerdings eine Interaktion mit dem Faktor RZK nachgewiesen werden. In Experiment 3 wurde dies kontrolliert und sowohl in der Satzergänzungsaufgabe 3a als auch in der EyeTracking-Studie 3c tritt ein deutlicher Effekt für den Faktor STRUKTUR in prognostizierter Richtung zutage: Das N1 verfügt in Rektionskomposita über einen höheren Grad an Zugänglichkeit als in Wurzelkomposita. Beim Eye-Tracking-Experiment zeigen sich darüber hinaus im Blickverhalten sowohl frühe als auch späte Effekte der Anaphernresolution. Daraus ist zu schlussfolgern, dass die Möglichkeit der Etablierung eines N1-Bezugs nicht ausschließlich auf pragmatische Prozesse wie Bridging auf Grundlage des Kontexts reduziert werden kann. Vielmehr scheinen tatsächlich morphosyntaktische Eigenschaften des Erstglieds erhalten zu bleiben, verarbeitet zu werden und einen Beitrag zur Resolution der Anapher zu leisten.

Insgesamt zeigt sich, dass es sich nicht nur bei der Unzugänglichkeit des N1 um ein graduelles Phänomen handelt, sondern auch die identifizierten Faktoren an sich nicht notwendigerweise kategorial sind. So verfügt z.B. die Animatheit gemäß Aissen (2003) über die Abstufungen unter (1). 


$$
\text { inanimat }<\text { animal-animat }<\text { human-animat }
$$

Ebenso ist für die raum-zeitliche Kontiguität neben ,präsent ' und ,absent' die Zwischenstufe ,fakultativ‘ plausibel (Skala entsprechend (2)).

$$
\text { absent }<\text { fakultativ }<\text { präsent }
$$

Auch die Untersuchungen zur Struktur von Komposita legen, wie oben angedeutet, ein Stufenmodell wie unter (3) nahe.

(3) Wurzelkompositum mit underiviertem N1 < Wurzelkompositum mit relationalem N2 $<$ Rektionskompositum $<$ Phrasenkompositum

Die hier vorgestellte Liste an Faktoren und ihren Abstufungen ist nicht exhaustiv, sondern spannt einen Rahmen für detailliertere Modellierungen auf. Es zeigt sich, dass auf verschiedenen Ebenen der Linguistik nach Faktoren gesucht werden muss: Sowohl im Bereich der Grammatik als auch im Bereich der Pragmatik. Die bereits gewonnenen Erkenntnisse machen deutlich, dass bei der Erstellung von Verarbeitungsmodellen verschiedene Arten von Faktoren integriert werden müssen.

\subsubsection{Interaktion von Faktoren}

Neben der Benennung verschiedener Faktoren ist auch die Art ihrer Interaktion aufschlussreich für das Verständnis der Komposita-Verarbeitung. Im Rahmen der empirischen Untersuchungen stellte sich heraus, dass die verschiedenen Faktoren keinesfalls gleichrangig wirken, sondern auf vielfältige Art interagieren können. Dies wird v.a. daran deutlich, dass sich manche Faktoren überlagern.

In Experiment 1 wurde die Animatheit als Faktor des N1-Denotatwissens der semantischen Relation als Faktor der Konzeptkombination gegenübergestellt. Dabei zeigte sich ein Haupteffekt für die Animatheit, für die semantische Relation allerdings nicht. Beim Modellvergleich zeigte sich allerdings, dass die semantische Relation einen Effekt zeitigt, wenn das N1 inanimat ist. In diesem Sinne scheinen animate N1 über einen derart starken Aktiviertheitsvorteil zu verfügen, dass dies den Einfluss der semantischen Relation überlagert. Die beiden Faktoren sind demnach in Hinblick auf die Stärke ihrer Einflussnahme gewichtet.

In Experiment 2 wurde die RZK als Faktor der Konzeptkombination mit der morphosyntaktischen Struktur verknüpft. Dabei stellte sich heraus, dass die RZK einen derart starken Einfluss auf die N1-Zugänglichkeit hat, dass der Effekt für die Struktur umgekehrt wird. In einer Post-hoc-Analyse zeigte sich hier ein Wechselspiel zwischen konzeptuellen und morphosyntaktischen Eigenschaften: Komposita, die sich aufgrund ihrer speziellen Konzeptkombination als Schnittmengenbildungen beschreiben lassen, verfügen über eine besonders gute N1-Zugänglichkeit und sind gleichzeitig strukturell Wurzelkomposita.

Bei der Item-Erstellung und -Auswertung für die verschiedenen Experimente stellte es sich als eine besondere Herausforderung dar, die Vielzahl möglicher Einflussfaktoren zu kontrollieren oder auszubalancieren. Experiment 3 wurde schließlich im Design speziell 
auf die Untersuchung des Struktureinflusses ausgerichtet und tatsächlich zeitigte dieser darin einen Effekt.

Insgesamt verfestigt sich der Eindruck, dass konzeptuelle Faktoren ein stärkeres Gewicht haben als grammatische. Dies zeigt sich besonders in Experiment 2 durch die Überlagerung des strukturellen Einflusses durch die RZK. Darüber hinaus wurden bereits in Kapitel 3.2 Faktoren untersucht, die zusätzlich über grammatische Reflexe verfügen, also auch als linguistische Faktoren zu behandeln sind. Im Fall von Eigennamen wirken der grammatische Aspekt (direkte Referenzialität) und der konzeptuelle (Unikalität in der externen Welt) in die gleiche Richtung, nämlich zur Steigerung der Zugänglichkeit, was sich empirisch plausibilisieren lässt. Im Fall von Massenomen verhält es sich anders: Der grammatische Aspekt (Möglichkeit der determiniererlosen Referenz) prognostiziert bessere Zugänglichkeit, der konzeptuelle (unspezifisches Denotat) wirkt in die Gegenrichtung. In Experiment 2 zeigte sich deskriptiv ein Nachteil für Massenomen, was für einen stärkeren Einfluss konzeptueller Aspekte spricht. Im Satzergänzungs-Experiment 3a zeigte sich kein Einfluss und im Eye-Tracking-Experiment 3c ein Vorteil für Massenomen. Eventuell handelt es sich hier tatsächlich um einen grammatischen Effekt, der nur durch die feinkörnigen Messungen in Erscheinung tritt. Derartige Analysen sind allerdings spekulativ, sodass der Einfluss bislang nicht bestätigt werden kann.

Letztlich zeigte auch die explorative Auswertung verschiedener Satzergänzungen, dass grammatische Faktoren nicht ausschlaggebend sind, sondern immer Plausibilität und Weltwissen die obersten Kriterien bei der Anaphernresolution bilden. Zugunsten der Plausibilität wurden z.T. sogar die morphosyntaktischen Eigenschaften der Pronomen außer Acht gelassen und Kongruenzbeschränkungen verletzt, um die gewünschte Satzergänzung bilden zu können (vgl. Kap. 5.7.6).

\subsection{Implikationen für die Grammatiktheorie}

\subsubsection{Morphosyntax von Komposita}

Eine zentrale Frage aus dem Bereich der Morphosyntax von Komposita, der sich über die Untersuchung der N1-Zugänglichkeit genähert wird, ist die nach dem kategorialen Status von Komposita-Konstituenten. Hierfür muss zunächst eine einheitliche Analyse von Komposita-Strukturen die Grundlage bilden. Diese Arbeit folgt dem Trend zu syntaktischen Ansätzen der Wortbildung. Das Hauptargument für einen syntaktischen Ansatz ist, dass bei einem sukzessiven Durchlaufen von Morphologie und Syntax die Wortstruktur opak und unzugänglich sein müsste. Vielmehr stellen sich Morphologie und Syntax allerdings als interagierende Ebenen dar. Die Morphologie ist dabei kein separates Modul, sondern eher ein spezielles Regelset. Die grundlegenden Bausteine und Prinzipien wirken jedoch gleichsam auf beiden Ebenen. Die Konstituenten für die Wortbildung sind gleichermaßen syntaktische Formen wie die Konstituenten phrasaler Strukturen. Bei der Lexik-Syntax-Schnittstelle handelt es sich demnach nicht um einen Übergang zwischen zwei Modulen, sondern um den Berührungspunkt zweier Ebenen.

Beruft man sich auf die ganze Bandbreite des syntaktischen Instrumentariums, lassen sich so auch Unterschiede zwischen Rektions- und Wurzelkomposita modellieren. Ein Analysevorschlag, der auch den Unterschieden in der N1-Zugänglichkeit gerecht wird, wurde im Framework der Distribuierten Morphologie in Kapitel 4 vorgestellt. Wie bereits oben 
erwähnt, wird dabei keine einheitliche Struktur stipuliert, sondern je nach spezifischen Eigenschaften des Kompositums kann die erste Konstituente eines Kompositums verschiedene Status inne haben; von einer kategorielosen Wurzel bis zu einer maximalen Projektion. Der kategoriale Status eines Erstglieds - wenn nicht gar einer Kompositums-Konstituente im Allgemeinen - ist demnach nicht einheitlich bestimmbar. Gleichzeitig belegen die Ergebnisse aus Experiment 3, dass die verschiedenen kategorialen Status eines Erstglieds Reflexe auf die Verarbeitung eines Kompositums haben können, was durch Unterschiede in der N1-Zugänglichkeit zum Ausdruck kommt.

\subsubsection{Semantik von Komposita}

Aus dem Bereich der Semantik ist eine zentrale Frage an das Phänomen, ob für alle Komposita einheitliche Verrechnungsprinzipien angenommen werden können. Hier zeigt sich, dass zwischen verschiedenen Arten von Komposita, allein schon zwischen Wurzelund Rektionskomposita, unterschiedliche semantische Verhältnisse vorliegen. Während Wurzelkomposita über eine unterspezifizierte semantische Relation ihre Bedeutung konstituieren, erfolgt bei Rektionskomposita die semantische Interpretation anhand der Argumentstruktur der verbalen Konstituente. Dieser Unterschied verdeutlicht, dass ein einheitliches Kompositumstemplate der Bildungsvielfalt in der Komposition nicht gerecht werden kann.

Für eine semantische Modellierung ist interessant, ob ein N1 generell einen Diskursreferenten etabliert und falls ja, wie sich im Fall von Ambiguität Präferenzen und Gradienz in der Zugänglichkeit abbilden lassen.

Diese Arbeit konnte zeigen, dass ein N1 unter dem Einfluss eines bestimmten Sets an sprachlichen und konzeptuellen Faktoren zugänglich sein kann und dass es keine grammatische Beschränkung gibt, die diese Zugänglichkeit kategorisch verhindert. In diesem Sinne ist anzunehmen, dass ein N1 einen Diskursreferenten etablieren kann. Dieser kann allerdings je nach konkretem Ko- und Kontext verschieden gut zugänglich sein. Diesem Umstand und vor allem der Gradienz in der Zugänglichkeit muss ein geeignetes semantisches Framework Rechnung tragen.

Bewegt man sich im Rahmen der DRT und der $\mathrm{DRT}_{\text {lex }}$ wie in Kapitel 2.2.4 vorgestellt, ist zunächst eine Integration der beiden DRSn notwendig. Zunächst unterscheidet sich ein Diskurs mit einem einfachen Nomen (vgl. (4) in seinem Grundaufbau nicht von einem Diskurs mit einem komplexen Nomen (vgl. (5) ). Allerdings kann in (5) dem Antezedens keine Variable zugeordnet werden, wodurch die Anapher nicht aufgelöst werden kann.

(4) Leon gießt die Rosen. Sie blühen.

$$
\begin{array}{|c|}
\hline x y \\
\hline \operatorname{Leon}(\mathrm{x}) \\
\operatorname{rosen}(\mathrm{y}) \\
\mathrm{x} \text { gießt y } \\
\mathrm{u} \text { blüht } \\
\mathrm{u}=\mathrm{y} \\
\hline
\end{array}
$$

Leon gießt das Rosenbeet. Sie blühen.

$$
\begin{array}{|c|}
\hline x y \\
\hline \text { Leon }(\mathrm{x}) \\
\text { rosenbeet }(\mathrm{y}) \\
\mathrm{x} \text { gießt } \mathrm{y} \\
\mathrm{u} \text { blüht } \\
\mathrm{u}=? \\
\hline
\end{array}
$$


Fügt man für das Kompositum eine DRS $_{l e x}$ nach (Meyer 1993) ein, ergibt sich eine DRS wie in (6). Verschiedene Faktoren können nun beeinflussen, bis zu welcher Ebene das N1 (v) gelangt und welche Position es im Diskursuniversum einnimmt. Will man sich in (6) mit der Variablen u auf das N1 (v) beziehen, müssen Blockaden überwunden werden. Das Referieren in eine subordinierte Box ist jedoch nicht zulässig, wodurch das Prinzip der lexikalischen Integrität abgebildet wäre. Unterschiede in der Unzugänglichkeit könnten sich nun z.B. über den Abbau bestehender oder den Aufbau zusätzlicher Blockaden modellieren lassen. Für Eigennamen als N1 wurde ein hoher Grad an Zugänglichkeit festgestellt. Dies lässt sich damit begründen, dass Eigennamen direkt referieren. Dies könnte bedeuten, dass sie automatisch einen Diskursreferenten im äußersten Diskursuniversum etablieren und somit zugänglich sind.

(6) Leon gießt das Rosenbeet. Sie blühen.

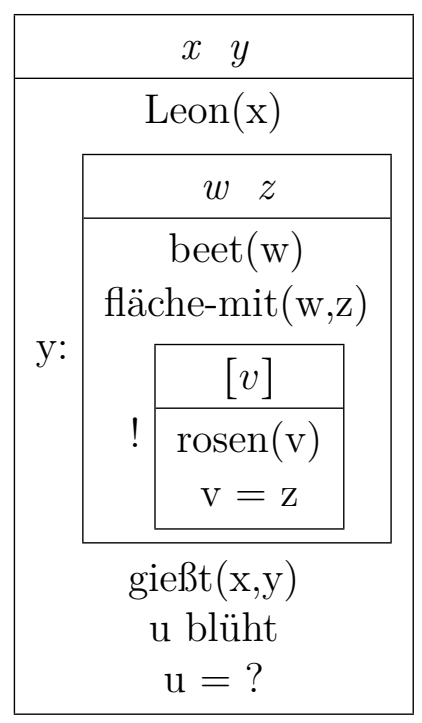

Ein Unterschied im Grad der Unzugänglichkeit könnte auch durch An-/Abwesenheit des Generizitätsoperators modelliert werden. Nimmt man an, dass, wie in Kapitel 3.5 diskutiert, ein N1 eine unspezifische oder eine spezifische Entität denotieren kann, und möchte man diese Eigenschaft durch den Generizitätsoperator ausdrücken, steht das N1 nur im ersten Fall im Skopus des Operators (vgl. (7) ). Im Falle eines spezifischen N1 muss durch das Fehlen des Operators bei einer anaphorischen Bezugnahme eine Blockade weniger überwunden werden, das N1 ist also zugänglicher (vgl. (8)]).

Rosenbeet

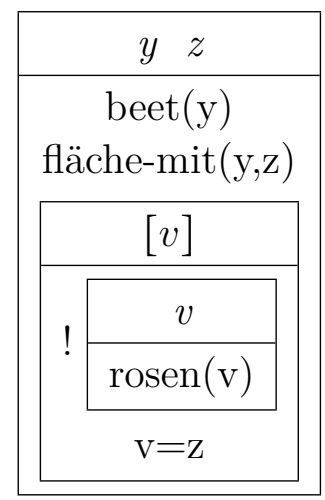

(8) Raketenstart

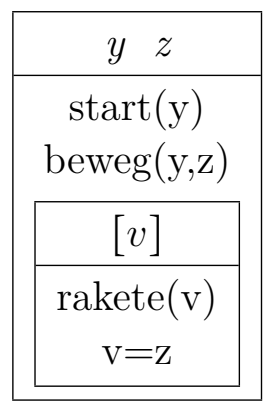


An dieser Stelle stößt die Modellierung an ihre Grenzen. Die konzeptuellen Faktoren aus Kapitel 3 lassen sich mit dem vorhandenen Instrumentarium nicht abbilden. Aber auch die Abhängigkeit von Spezifizität/Generizität zwischen Anapher und Antezedens kann nicht ohne Weiteres ausbuchstabiert werden. Letztlich ist auch der Generizitätsoperator als probates Mittel in Zweifel zu ziehen. Wie in Kapitel 3.5 thematisiert, denotiert ein N1 i.d.R. unspezifisch, jedoch nicht generisch. Dies wird noch einmal deutlich, wenn man generische Sätze als wenn-dann-Konstruktion modelliert. Für generisch verwendete Ausdrücke lassen sich wahre Paraphrasen entwickeln (vgl. (9-a)), für Komposita nicht (vgl. $(9-b)$.

a. Rosen haben Dornen. $\Rightarrow$ Wenn eine Entität eine Rose ist, hat sie i.d.R. Dornen.

b. Rosenbeet $\not$ Wenn eine Entität eine Rose ist, befindet sie sich i.d.R. in einem/diesem Beet.

Insgesamt zeigt sich, dass die DRT bei Fragen gradueller N1-Zugänglichkeit an ihre Grenzen stößt und aus sich heraus keine neuen Erkenntnisse zu den Faktoren der N1Zugänglichkeit liefern kann. Vielmehr kann sie als Mittel zur Illustration bereits etablierter Befunde genutzt und entsprechend modifiziert werden.

Wünschenswert wäre ein Modell, das die Darstellung verschiedener Faktoren unter Berücksichtigung ihrer Gewichtung und Interaktion ermöglicht. Eine Möglichkeit wäre, die Diskursreferenten im Diskursuniversum nicht nach der Reihenfolge ihres Auftretens abzubilden, sondern nach ihrer Salienz. Dies würde den Unterschieden in der anaphorischen Zugänglichkeit Rechnung tragen und gleichzeitig Präferenzen widerspiegeln. Hierfür eignet sich eventuell die SDRT, da diese die Einbeziehung von Distanz-Informationen ermöglicht (vgl. Lascarides/Asher 2007). Um ein solches Modell ausbauen zu können, muss allerdings zunächst das Set an Faktoren und Präferenzen final analysiert sein.

Eine weitere Modellierungsmöglichkeit liegt in einer gewichteten Faktorrangordnung. In Bezug auf die Interaktion verschiedener Faktoren ist auch ein Venn-Digramm mit den einzelnen Faktoren denkbar, ähnlich wie es Crystal (1967) für die Eigenschaften von Nomen vorschlägt. Hierin liegt ein Anknüpfungspunkt für nachfolgende Arbeiten.

\subsection{Implikationen für die Verarbeitungstheorie}

An einigen Stellen ergeben sich aus den Ergebnissen dieser Arbeit Implikationen für Theorien zur Verarbeitung von Komposita.

Eine Frage aus diesem Bereich ist die nach den bei der Komposita-Verarbeitung involvierten Wissenstypen. In Experiment 1 wird deutlich, dass verschiedene Arten von konzeptuellem Wissen eine Rolle spielen müssen, da sie andernfalls keinen Einfluss auf die N1-Zugänglichkeit haben könnten. Gleichzeitig scheinen verschiedene Elemente des Konzeptwissens zu interagieren und auch die Interaktion von Wissensaspekten von Einzelausdruck und Konzeptkombination scheint eine Rolle zu spielen.

Darüber hinaus belegt Experiment 3 den Einfluss sprachlichen Wissens auf die KompositaVerarbeitung. Die frühen, morphosyntaktischen Effekte der Eye-Tracking-Studie belegen, dass das Erstglied morphosyntaktisch verarbeitet wurde. 
Im Kontext zu Fragen des lexikalischen Zugriffs lassen sich Argumente gegen die ÖkonomieHypothese und für Libbens (1998) Idee der Maximization of Opportunity ableiten. So spricht die generelle Möglichkeit der Zugänglichkeit von Komposita-Erstgliedern dafür, dass auch Wortteile einzeln aktiviert werden. Werden Bedeutungsteile für die Interpretation nicht benötigt, werden diese wieder inhibiert, z.B. weil ihr Beitrag zur Bedeutung des Gesamtworts opak ist und von der Bedeutung als Simplex abweicht. Dies führt dazu, dass die Zugänglichkeit nachlässt. Aus diesem Grund kann Butterblume nicht als Anker für eine Anapher fungieren, die auf die Bedeutung von Butter als Nahrungsmittel abzielt (vgl. (10)).

Was für schöne Butterblumen du hast! *Da fällt mir ein, die muss ich dringen nachkaufen.

Ebenso wird die Idee eines obligatorischen, automatischen Parsers (vgl. Libben 1998) durch den Einfluss von Fugenelementen gestützt. Obwohl diese keinen morphosyntaktischen Gehalt haben, können sie die N1-Zugänglichkeit beeinflussen, wenn sie als Pluralmarker interpretiert werden (vgl. Kap. 2.2.2).

Ein diffuses Gebiet, auf dem experimentelle Befunde unerlässlich sind, ist das komplexe Zusammenspiel von Transparenz, Lexikalisiertheit und Frequenz. Der Einfluss der Transparenz der Komposita-Konstituenten und der Kompositionalität auf die N1-Zugänglichkeit kann als gesichert angesehen werden. In welchem Zusammenhang dies allerdings mit den verschiedenen Frequenzen und dem Grad an Lexikalisiertheit steht, ist nach wie vor unklar. In Experiment 3 deutet sich an, dass die Frequenz eines Erstglieds Einfluss auf dessen Aktiviertheit und darüber auf die Zugänglichkeit hat. Ob allerdings die Frequenz eines Kompositums Auskunft über seinen Grad an Kompositionalität geben kann, wird angezweifelt (vgl. Kap. 6.4.7). Auch der Zusammenhang zwischen Kompositionalität und Lexikalisiertheit ist nach wie vor nicht ausbuchstabiert. Für eine aussagekräftigere Bestimmung des Lexikalisiertheitsgrads halte ich computerlinguistische Ansätze für einen geeigneten Weg, z.B. Schäfer/Bell (2016).

\subsection{Implikationen für die Anaphorik}

Anhand der Ergebnisse dieser Arbeit lässt sich eine Einordnung des Phänomens des pronominalen N1-Bezugs in das Spannungsfeld zwischen direkter und indirekter Anapher neu diskutieren (vgl. dazu Schwarz (2000) und Kap. 2.4.2).

Nach Averintseva-Klisch (2013) signalisiert die Akzeptabilität von Demonstrativpronomen als Anapher, dass das Antezedens aktiviert ist, und die Akzeptabilität von Personalpronomen, dass das Antezedens fokussiert ist. Im Rahmen eines Vortests zu Experiment 2 (vgl. Kap. 5.3.2) konnte gezeigt werden, dass Demonstrativa tendenziell geeigneter sind, um einen N1-Bezug zu realisieren. Daraus lässt sich zunächst schließen, dass Erstglieder durchaus über eine Einzelaktivierung verfügen können.

Allerdings belegen alle im Rahmen dieser Arbeit durchgeführten Satzergänzungsstudien, dass der Bezug auch mittels Personalpronomen möglich ist. Gemäß dem Mehrspeichermodell ist der Anker einer indirekten Anapher nach Schwarz (2000) fokussiert und Elemente der kognitiven Domäne, auf die durch die Anapher Bezug genommen wird, sind aktiviert. Die Verwendung einer Anapher in Form eines Personalpronomens setzt nun vorraus, dass 
das Antezedens fokussiert ist. Dies ist bei indirekten Anaphern nicht gegeben. In diesem Sinne müsste es sich bei einem pronominalen N1-Bezug um eine direkte Anapher handeln.

Ein weiteres wichtiges Kriterium bei der Entscheidung zwischen direkter und indirekter Anapher ist die Frage nach der Etablierung eines Diskursreferenten. Während bei direkten Anaphern die Anapher den durch das Antezedens etablierten Diskursreferenten lediglich wieder aufnimmt, etablieren indirekte Anaphern selbst einen. Hierin liegt auch der Grund, warum Pronomen i.d.R. nicht als indirekte Anaphern fungieren können: Sie verfügen über zu geringen semantischen Gehalt um einen Diskursreferenten etablieren zu können, bzw. präsupponieren sie Vorerwähntheit. Ein N1 ist an der Textoberfläche sichtbar und kann mit einem Pronomen morphosyntaktisch kongruent sein. Da Koreferenz nicht als notwendiges Kriterium direkter Anaphern angesehen wird (vgl. z.B. ,sloppy identity ${ }^{6}$ ), ist dies auch bei N1-Bezügen nicht obligatorisch. Die Frage ist nun, ob jedes N1 von vornherein einen eigenen Diskursreferenten etabliert, der je nach speziellen Eigenschaften des Kompositums mehr oder weniger salient ist, oder ob der Diskursreferent eventuell erst während eines Reanalyseprozesses nachträglich etabliert wird. Es lässt sich jedoch festhalten, dass die Möglichkeit der Realisierung pronominaler Anaphern die Möglichkeit der Etablierung eines Diskursreferenten voraussetzt.

Während die Präsenz des Antezedens an der Textoberfläche und die Möglichkeit der pronominalen Bezugnahme für die Einordnung des N1-Bezugs als direkte Anapher sprechen, spricht die Kontextsensitivität und die allgemeine Tendenz zur N1-Unzugänglichkeit für die Einordnung als indirekte Anapher. Darüber hinaus wurde syntagmatische Distanz als förderlich für die Akzeptabilität von N1-Bezügen ausgewiesen. Dies äußert sich nicht zuletzt darin, dass Diskursanaphern für Erstglieder im Allgemeinen akzeptabler sind als intrasententiale. Dieser Befund spricht für eine Einordnung als indirekte Anapher, da direkte Anaphern aufgrund der zu memorierenden Kongruenzinformationen tendeziell eher über kurze Distanzen etabliert werden.

Weiteren Aufschluss kann die Frage nach Verarbeitungsunterschieden geben. Die Etablierung einer indirekten Anapher wird als kognitiv aufwändiger beschrieben und die Etablierung einer direkten Anapher gilt als die primäre Prozedur (vgl. Schwarz 2000).

Anhand der Kontroll-Items in Experiment 2 (vgl. Kap. 5.7.6) wird ein Vergleich zwischen pronominalen Bezügen auf direkt und indirekt eingeführte Antezedenten unternommen. Dabei bestätigt sich zunächst die Präferenz für direkte Antezedenten. Die mittlere Zahl der N1-Bezüge liegt zwischen den Mittelwerten für direkte und indirekte Anaphern mit größerer Nähe zu den indirekten Fälle. Dies legt eine Einordnung in Richtung indirekter Anapher nahe.

Aussagekräftiger als die Auswertung der Satzergänzungen sind allerdings die Ergebnisse der Eye-Tracking-Studie (vgl. Kap. 6.4.5). Hierbei zeigt sich zunächst ein klarer Verarbeitungsvorteil für direkte Anaphern; die Bedingung Mono verfügt auf allen relevanten AVn über die kürzesten Fixationszeiten. Darüber hinaus liefert die FPFD der AnaphernRegion, die lediglich das Pronomen und ein Modalverb enthält, wichtige Erkenntnisse: Die FPFD zeigt nicht nur einen signifikanten Verarbeitungsvorteil für die direkte Bezugnahme, sondern v.a. einen signifikanten Unterschied zwischen den beiden Kompositabedingungen (RK und WK). Da Fixationen in spätere Satzteile nicht in der FPFD enthalten sind, ist Bridging auf Basis von Kontextinformationen an dieser Stelle auszuschließen. Da Pronomen und N1 zwischen den Bedingungen identisch sind, können Unterschiede in der 
FPFD der Anapher nur aus Unterschieden in der experimentellen Manipulation, nämlich aus der Kompositums-Struktur resultieren. Das Kompositum muss also beim Lesen des Pronomens reaktiviert worden sein. Dass ein Pronomen eine Reaktivierung bewirkt, setzt voraus, dass ein morphosyntaktisch kongruenter Ausdruck identifiziert wurde. Dabei kann es sich nur um das N1 handeln. Dies wiederum bedeutet, dass die entsprechenden morphosyntaktischen Eigenschaften des N1 wahrgenommen wurden und durch das N1 ein Diskursreferent etabliert wurde. Dabei handelt es sich um typisches Verhalten bei der Resolution einer direkten Anapher.

Die Ergebnisse der Untersuchungen bestätigen, dass es sich bei der Etablierung einer direkten Anapher um die primäre Prozedur handelt und diese weniger Verarbeitungskosten verursacht als die Etablierung einer indirekten Anapher. Es zeigt sich allerdings auch, dass es sich beim Übergang zwischen direkten und indirekten Anaphern um ein Kontinuum handelt, in dem pronominale N1-Bezüge eine Zwischenposition besetzen. Die Ergebnisse der Eye-Tracking-Studie sowie die theoretische Analyse der Aktiviertheitsgrade sprechen allerdings gegen eine Verortung in der Mitte, sondern für eine größere Nähe zur direkten Anapher. In jedem Fall scheint das N1 einen Diskrusreferenten etablieren zu können, der allerdings in Salienz und Zugänglichkeit variiert.

\subsection{Ausblick}

Diese Arbeit konnte einige grundlegende Fragen zum Phänomen der Erstglied-Zugänglichkeit bei NN-Komposita klären. Komposita-Erstglieder können für pronominale Anaphern zugänglich sein. Die Akzeptabilität dieser Anaphern ist nicht ausschließlich von pragmatischen Faktoren abhängig, sondern auch von einer Vielzahl ausdrucks-inhärenter und sogar grammatischer Eigenschaften. Trotz allem bleiben viele Fragen ungeklärt und ein breites Spektrum von Anknüpfungspunkten für weitere Forschung eröffnet sich.

Vor dem Hintergrund, dass in dieser Arbeit eine Vielzahl unterschiedlicher Faktoren identifiziert wurde, die einen Einfluss auf den Grad der Akzeptabilität von N1-Bezügen nehmen können und diese darüber hinaus in ihrer Einflussstärke gewichtet zu sein scheinen, ist eine einheitliche Klassifizierung und Formalisierung dieser Faktoren erstrebenswert. Versuche der semantischen Formalisierung im Rahmen der DRT stießen schnell an ihre Grenzen. In die morphosyntaktische Formalisierung im Rahmen der DM wiederum scheinen semantische Faktoren kaum integrierbarer. Ein Framework zur Abbildung eines breiten Spektrums an Faktoren konnte noch nicht identifiziert werden. Hinzu kommt, dass die hier zusammengetragene Liste an Faktoren nicht erschöpfend ist und auch im Bereich der Interaktionen noch Analysearbeit zu leisten ist, die die Voraussetzung für eine konkrete Modellierung bildet.

Für eine einheitliche und umfassende Abbildung der involvierten Faktoren scheint zum jetzigen Stand eine gewichtete Faktorenhierarchie ein probates Mittel zu sein. Dabei muss jeder Faktor in sich wiederum über eine Skala mit verschiedenen Faktorstufen verfügen. Die daraus abzuleitende Prognose wäre, dass ein N1 umso zugänglicher ist, je mehr höherrangige Faktoren auf einer hohen Faktorstufe vorliegen. Für die drei empirisch bestätigten Faktoren wäre bspw. eine Hierarchie wie in Tabelle 7.1 denkbar. 


\begin{tabular}{crlll}
\hline \hline Rang & Faktor & Stufe 1 & Stufe 2 & Stufe 3 \\
\hline 3 & ANIMATHEIT & inanimat & animal & human \\
2 & RZK & N1 absent & N1 fakultativ & N1 präsent \\
1 & STRUKTUR & Wurzel & $\mathrm{N}^{o}$ & $\mathrm{D}^{o}$ \\
\hline \hline
\end{tabular}

Tabelle 7.1: Modell einer Faktorenhierarchie nach Effektstärke für verschiedene N1Eigenschaften

Eine Vorhersage anhand dieser Ordnung wäre, dass ein N1 mit der Kombination \{animal, N1 präsent, Wurzel \} zugänglicher ist als ein N1 mit der Faktorstufenkombination \{inanimat, $\mathrm{N} 1$ absent, $\left.\mathrm{D}^{\circ}\right\}$. Auf konkrete Beispiele übertragen, würde dies wiederum vorhersagen, dass (11-a) akzeptabler ist als (11-b). Meiner Intuition zufolge trifft diese Vorhersage $\mathrm{zu}$.

a. Paula hat im Fernsehen eine niedliche Vogelmutter gesehen. Jetzt möchte sie auch gern einen haben.

b. Paula hat im Fernsehen einen stylischen Wasserkocher gesehen. Jetzt möchte sie auch gern welches haben.

Eine solche Rangordnung müsste nun in aller Richtungen ausgebaut werden. Die generelle Möglichkeit, Faktoren zu benennen und zu gewichten, lässt eventuell auch eine Modellierung im Rahmen der Optimalitätstheorie oder nicht-klassischer Varianten dieser zu (z.B. stochastische OT oder lineare OT nach Keller (2006)).

Eine dritte Modellierungsoption liegt in der Beschreibung der graduellen N1-Zugänglichkeit als prototypenbasiertes Konzept mit den einzelnen Faktoren als gewichtete Dimensionen.

In der Auswahl eines Modellierungsmodells und der konkreten Ausgestaltung mit einem gesicherten Faktorenset liegt Potenzial für nachfolgende Arbeiten.

In Bezug auf die Gültigkeit des Prinzips der lexikalischen Integrität gilt es neben Aussagen zur Zugänglichkeit von Wortkonstituenten auch den Forschungsstand hinsichtlich der Unterbrechbarkeit von Wortstrukturen auszubauen, d.h. zum Manipulationsverbot von Wortteilen (vgl. Booij 2009b). Hier wären empirische Arbeiten denkbar, die die morphosyntaktische Bedeutung von Fugenelementen systematisch beleuchten. Generell stellen Fugenelemente nach wie vor ein fruchtbares Forschungsgebiet dar, das sowohl theoretisch als auch empirisch einen wichtigen Beitrag zum Verständnis der Komposita-Produktion und -verarbeitung leisten kann.

Diese Arbeit ist in erster Linie auf endozentrische NN-Komposita des Deutschen beschränkt. Davon ausgehend sind Ausdehnungen des Untersuchungsgegenstandes in verschiedene Richtungen denkbar. Eine Richtung ist die Übertragbarkeit auf andere Sprachen mit ähnlichen Kompositionsregeln. Hierbei gilt es jedoch die experimentellen Paradigmen anzupassen, da z.B. im Englischen das Genus des Pronomens nicht zur Disambiguierung des Bezugs genutzt werden kann. Darüber hinaus stellt sich die Frage, wie es sich mit der N2-Zugänglichkeit bei Sprachen mit linksköpfigen Komposita wie einigen romanischen Sprachen verhält. Schließlich liefern Sprachen mit speziellen Formen der Komposition wie das Chinesische und das Hebräische (vgl. Kap. 4.3.4) vielfältige Anknüpfungspunkte.

In Bezug auf das Deutsche gilt es die Untersuchungen auf andere Arten von Komposita auszudehnen. Bspw. wäre eine Vermutung, dass transparente exozentrische Komposita 
über eine gute N1-Zugänglichkeit verfügen, da keine der Konstituenten den semantischen Kopf kennzeichnet. Gleichzeitig ist eine Trennung der Konstituenten-Denotate schwieriger. Eine Ausdehnung auf andere Wortarten ist ebenfalls naheliegend, z.B. verbale oder adjektivische Konstituenten.

Neben den Komposita sind weitere Typen sog. anaphorischer Inseln zu betrachten. Allen voran sind hierbei die Derivate ein naheliegender Untersuchungsgegenstand (vgl. (12)) .

(12) $\quad{ }^{*}$ The guitarist thought that it was a beautiful instrument. (vgl. Lakoff/Ross 1972:121)

Aber auch die Forschung zu Idiomen hat Schnittmengen mit der hier betriebenen Forschung, z.B. den Einfluss von metaphorischer und eigentlicher Lesart. Eine Übertragung der hier vorgestellten Analysen auf die verschiedenen Forschungsgegenstände kann wiederum einen Betrag dazu leisten, die Gültigkeit dieser Analysen zu prüfen. 



\section{Schlussbemerkung}

Bei dieser Arbeit handelt es sich in besonderem Maße um eine Arbeit, die Brücken schlägt. Zum einen stellen Komposita an sich bereits eine Brücke zwischen Morphologie und Syntax dar. Gerade die vielfältigen Einflussgrößen, aber auch die Beschränkungen bei N1Bezügen erschweren die Zuordnung zu einer der beiden linguistischen Domänen, wenn nicht gar deren Abgrenzung an sich. Zum anderen versucht diese Arbeit Brücken zwischen Grammatik und Verarbeitung zu schlagen. Es besteht die Annahme, dass die spezifische Konstitution verschiedener Strukturen die Eigenschaften der involvierten Teile beeinflusst und dadurch wiederum Einfluss auf die Verarbeitung der Teile und der Gesamtstruktur nimmt. So werden in Kapitel 4 strukturspezifische Unterschiede im referenziellen Status von Erstgliedern postuliert, die wiederum mit verschiedenen Zugänglichkeitsgraden assoziiert werden. Einen empirischen Beleg für diese Annahmen versucht Experiment $3 \mathrm{zu}$ erbringen (vgl. Kap. 6) und damit im Umkehrschluss die vorgeschlagenen Strukturunterschiede zu plausibilisieren.

Das Phänomen der graduellen N1-Unzugänglichkeit wurde als komplexes Zusammenspiel unterschiedlicher und in sich skalarer Einflussfaktoren beschrieben. Dabei wurden Faktoren aus ganz unterschiedlichen Bereichen zusammengetragen: Denotatwissen über das N1 (z.B. Animatheit), Aspekte der Konzeptkombination (z.B. der konzeptuelle Fokus), Strukturaspekte (z.B. Rektions- und Wurzelkomposita), aber natürlich auch pragmatische Aspekte sowie Transparenz und Kompositionalität u.a. Darüber hinaus wurden komplexe Interaktionen zwischen den Faktoren identifiziert. Über alle Einflussgrößen hinweg herrscht jedoch das Primat der Plausibilität für Welt- und Kontextwissen.

Vor dem Hintergrund der in dieser Arbeit identifizierten Einflussgrößen sollen abschließend die in der Einleitung angeführten authentischen Fälle von N1-Bezügen analysiert werden. Dabei soll geprüft werden, ob sich diese Fälle hoher Akzeptabilität auch mit Hilfe der identifizierten Einflussgrößen erklären lassen.

In (1) liegt ein Rektionskompositum vor. Darüber hinaus wird das N1 durch bestimmte konzeptuelle Eigenschaften aktiviert: Rosen sind im mitteleuropäischen Kulturkreis eine Pflanzenart mit Symbolkraft und daher von hoher emotionaler Valenz. Zudem handelt es sich um ein Konkretum. Zwischen Antezedens und Anapher wirkt das Parallelitätsprinzip: Beide Ausdrücke liegen an der Oberfläche im Plural vor und beide referieren unspezifisch. In Bezug auf die Informationsstruktur ist der Ausdruck Rosenhasser durch die elliptische Satzeinbettung fokussiert.

(1) Kira Dorn: Lupo, kannst du dir vorstellen, dass jemand die Bombe in deinem Garten vergraben hat?

Lupo: $\quad$ Ein Rosenhasser? Die tun doch niemandem was.

(Tatort vom 05.02.2017 (Min. 09:27)) 
In (2) wird die N1-Zugänglichkeit besonders durch die Konzeptkombination gefördert: Beim N1 handelt es sich um ein Konkretum, während das N2 abstrakt ist. In diesem Sinne verfügt das N1 über einen repräsentationalen Vorteil. Auch der semantische Kopf des Kompositums ist tendenziell eher beim N1 zu verorten. Auch hier sind Antezedens und Anapher beide unspezifisch. Konzeptuell kann man beim N1 eventuell von einem (schwachen) Lebenswelteffekt sprechen, da Croissants als Lebensmittel Teil der menschlichen Lebenswelt sind; aufgrund der guten Vorstellbarkeit von Croissant auch von einem Wahrnehmungseffekt.

(2) $[\ldots]$ das Fenster ist offen und es ziehen die ganze Zeit Croissant-Gerüche von dem Bäcker unter uns zu mir hoch. Ich mag die ja nicht mal, aber appetitanregend ist es halt trotzdem.

(Hörbeleg)

In (3) handelt es sich beim N1 um einen Ausdruck, dessen Denotat unikal in der externen Welt ist. Letztlich sind Bezeichnungen für Kontinente auch Eigennamen, die generell über eine hohe Zugänglichkeit verfügen. Anapher und Antezedens sind in diesem Fall beide spezifisch.

(3) [...] ich möchte mich bei Ihnen ganz herzlich für das Afrikabuch bedanken. Es ist wunderschön, genau so, wie ich es in Erinnerung habe.

(private Postkarte)

In (4) liegt wiederum unspezifische Referenz und ein Rektionskompositum vor. Ein weiterer Zugänglichkeitsvorteil liegt auch hier in der Konzeptkombination: Das N1 ist konkret, während das N2 abstrakt ist. In diesem Fall geht dies sogar mit der raum-zeitlichen Kontiguität einher: Ohne die Anwesenheit von Berlinern kann auch kein entsprechendes Angebot bestehen.

(4) Verkäuferin: Wir haben diese Woche ein Berliner-Angebot!

Kundin: Oh ja, dann nehme ich davon noch zwei.

(Hörbeleg beim Bäcker)

Die Analyse der beobachteten Fälle zeigt, dass die identifizierten Einflussfaktoren durchaus zur Aufklärung von akzeptablen N1-Bezügen beitragen können.

Sind Komposita-Erstglieder auch in den meisten Fällen unzugänglich, konnte diese Arbeit doch zeigen, dass es sich hierbei nicht um eine kategorische Eigenschaft handelt. Vielmehr zeigt sich, dass es sich bei der N1-Unzugänglichkeit um ein graduelles Phänomen handelt, welches in seiner Charakteristik mit den Akzeptabilitätsgraden zwischen verschiedenen Arten von ungrammatischen Sätzen verglichen werden kann.

Das Phänomen der Erstglied-Zugänglichkeit präsentiert sich als vielschichtiger Untersuchungsgegenstand, bei dem ein komplexes Zusammenspiel zahlreicher, vielfältiger und interagierender Einflussfaktoren zum Tragen kommt. Dabei zeigt sich zum einen, dass die Grammatik Reflexe auf die Verarbeitung haben kann. Zum anderen entwickelten sich Anaphern im Rahmen dieser Arbeit vom Werkzeug zu einem zusätzlichen Forschungsgegenstand. Waren Anaphern anfangs das Mittel, um Zugänglichkeit durch Akzeptabilität sichtbar zu machen, wurden durch die experimentellen Befunde auch Erkenntnisse zum 
Anaphernresolutions-Prozess an sich gewonnen.

NN-Komposita stellen sich als Phänomenbereich dar, bei dem lexikalische, morphosyntaktische, konzeptuelle und Diskursfaktoren in ihrem Wechselspiel zu beobachten sind. Die vielen verschiedenen dabei wirksamen Faktoren und ihre Interaktion belegen anschaulich, dass es sich um einen komplexen und herausfordernden Untersuchungsgegenstand handelt, der noch viel Potenzial für weitere Untersuchungen bereit hält und Anknüpfungspunkte an die verschiedenen linguistischen Disziplinen ermöglicht. 



\section{Literaturverzeichnis}

Aarts, Baas (2007): Syntactic Gradience: The Nature of Grammatical Indeterminacy. New York: OUP.

Ackema, Peter (1995): Syntax below Zero. Phd dissertation, Utrecht University, Utrecht.

Ackema, Peter; Neeleman, Ad (2004): Beyond Morphology: Interface Conditions on Word Formation. New York: OUP.

Ackema, Peter; Neeleman, Ad (2010): The role of syntax and morphology in compounding. In: Scalise, Sergio; Vogel, Irene (Hg.), Cross-Disciplinary Issues in Compounding, Amsterdam, Philadelphia: Benjamins, Current Issues in Linguistic Theory, 21-36.

Aissen, Judith (2003): Differential Object Marking: Iconicity vs. Economy. In: Natural Language and Linguistic Theory 21.3, 435-483.

Alexiadou, Artemis (2001): Functional structure in nominals: Nominalization and ergativity: Habil-Schr.-Potsdam, 1999, LA, Band 42. Amsterdam: Benjamins.

Alexiadou, Artemis (2014): Nominal Derivation. In: Lieber, Rochelle; Štekauer, Pavol (Hg.), The Oxford Handbook of Derivational Morphology, Oxford: OUP, 235-256.

Alexiadou, Artemis; Schäfer, Florian (2010): On the syntax of episodic vs. dispositional -er nominals. In: Alexiadou, Artemis; Rathert, Monika (Hg.), The Syntax of Nominalizations across Languages and Frameworks, Berlin, New York: de Gruyter, Interface explorations, 9-38.

Anderson, Stephen R. (1992): A-morphous Morphology. Cambridge: CUP.

Ariel, Mira (1988): Referring and accessibility. In: Journal of Linguistics 24, 65-87.

Ariel, Mira (2001): Accessibility theory: an overview. In: Sanders, Ted; Schilperoord, Joost; Spooren, Wilbert (Hg.), Text representation, Amsterdam, Philadelphia: Benjamins, Human cognitive processing, 29-87.

Aronoff, Mark (1994): Morphology by Itself. Cambridge, Mass: MIT Press.

Asher, Nicholas (1993): Reference to Abstract Objects in Discourse. Dordrecht: Kluwer.

Averintseva-Klisch, Maria (2013): Textkohärenz, KEGLI, Band 14. Heidelberg: Winter.

Baayen, Harald (2014): Experimental and Psycholinguistic Approaches. In: Lieber, Rochelle; Štekauer, Pavol (Hg.), The Oxford Handbook of Derivational Morphology, Oxford: OUP, 95-117.

Baayen, Harald; Schreuder, Robert (1999): War and Peace: Morphemes and Full Forms in a Noninteractive Activation Dual-Route Model. In: Brain and Language 68, 27-32. 
Baayen, Harald; Dijkstra, Ton; Schreuder, Robert (1997): Singulars and Plurals in Dutch: Evidence for a Parallel Dual-Route Model. In: Journal of Memory and Language 37.1, 94-117.

Baker, Mark C. (1988): Incorporation: A theory of grammatical function changing. A Chicago original paperback, Chicago: University of Chicago Press.

Bard, Ellen G.; Robertson, Dan; Sorace, Antonella (1996): Magnitude estimation of linguistic acceptability. In: Language 72.1, 32-68.

Barr, Dale J.; Levy, Roger; Scheepers, Christoph; Tily, Harry J. (2013): Random effects structure for confirmatory hypothesis testing: Keep it maximal. In: Journal of Memory and Language 68, 255-278.

Bauer, Laurie (2003): Introducing Linguistic Morphology. Washington DC: GUP, 2. Auflage.

Behrens, Leila (2005): Genericity from a cross-linguistic perspective. In: Linguistics 43.2, 275-344.

Bloomer, Robert K. (1996): Die pleonastischen Zusammensetzungen der deutschen Gegenwartssprache. In: American Journal of Germanic Linguistics and Literatures 8.1, 69-90.

Boase-Beier, Jean; Toman, Jindřich (1986): On Theta-role assignment in German compounds. In: Folia Linguistica 20.3/4, 319-340.

Bobaljik, Jonathan D. (2012): Universals in comparative morphology: Suppletion, superlatives, and the structure of words. Cambridge, Mass: MIT Press.

Böer, Katja; Kotowski, Sven; Härtl, Holden (2012): Nominal Composition and the Demarcation between Morphology and Syntax: Grammatical, Variational and Cognitive Factors. In: Röder, Katrin; Wischer, Ilse (Hg.), Proceedings/Anglistentag 2012 Potsdam, Trier: Wiss. Verl. Trier, Proceedings of the conference of the German Association of University Teachers of English, 63-74.

Booij, Geert (2009a): Compounding and Construction Morphology. In: Lieber, Rochelle; Štekauer, Pavol (Hg.), The Oxford Handbook of Compounding, Oxford, New York: OUP, 201-216.

Booij, Geert (2009b): Lexical integrity as a formal universal. A constructionist view. In: Scalise, Sergio; Magni, E.; Bisetto, Antonietta (Hg.), Universals of language today, Berlin: Springer, 83-100.

Booij, Geert; van Haaften, Ton (1988): On the external syntax of derived words: evidence from Dutch. In: YM 1, 29-44.

Borer, Hagit (1993): Parallel morphology. Ms., University of Massachusetts at Amherst: MIT Press.

Borer, Hagit (2009): Afro-Asiatic, Semitic: Hebrew. In: Lieber, Rochelle; Štekauer, Pavol (Hg.), The Oxford Handbook of Compounding, Oxford, New York: OUP, 491-511.

Borer, Hagit (2013): Structuring Sense: Vol. III: Taking Form. Oxford u.a.: OUP, 3. Auflage. 
Bosch, Peter; Katz, Graham; Umbach, Carla (2007): The Non-Subject Bias of German Demonstrative Pronouns. In: Schwarz-Friesel, Monika; Consten, Manfred; Knees, Mareile (Hg.), Anaphors in Text, Amsterdam u. a.: Benjamins, Studies in language companion series, 145-164.

Brandt, Patrick; Dettmer, Daniel; Dietrich, Rolf-Albert (1999): Sprachwissenschaft: Ein roter Faden für das Studium. Böhlau Studienbücher Grundlagen des Studiums, Köln: Böhlau.

Bücking, Sebastian (2010): German Nominal Compounds as Underspecified Names for Kinds. In: Susan Olsen (Hg.), New Impulses in Word-Formation, Hamburg: Buske, LB Sonderheft 17, 253-281.

Burkhardt, Petra (2006): Inferential bridging relations reveal distinct neural mechanisms: Evidence from event-related brain potentials. In: Brain and Language 98, 159-168.

Chomsky, Noam (1970): Remarks on Nominalization. In: R. Jacobs \& P. Rosenbaum (Hg.), Readings in English Transformational Grammar, Waltham, MA: Ginn, 184-221.

Chomsky, Noam (1981): Lectures on government and binding. Dordrecht u.a.: Foris.

Chomsky, Noam (1995): Bare Phrase Structure. In: Campos, Héctor; Kempchinsky, Paula (Hg.), Evolution and Revolution in Linguistic Theory, Washington DC: GUP, 51-109.

Clahsen, Harald; Gerth, Sabrina; Heyer, Vera; Schott, Esther (2015): Morphology constrains native and non-native word formation in different ways: Evidence from plural inside compounds. In: The Mental Lexicon 10, 53-87.

Compton, Richard; Pittman, Christine (2010): Word-formation by phase in Inuit. In: Lingua 120, 2167-2192.

Consten, Manfred (2004): Anaphorisch oder deiktisch? Zu einem integrativen Modell domänengebundener Refenrez, Linguistische Arbeiten, Band 484. Tübingen: Niemeyer.

Coolen, Riet; van Jaarsveld, Henk J.; Schreuder, Robert (1993): Processing novel compounds: Evidence for interactive meaning activation of ambiguous nouns. In: Memory \& Cognition 21.2, 235-246.

Cornish, Francis; Garnham, Alan; Cowles, H. Wind; Fossard, Marion; André, Virginie (2005): Indirect anaphora in English and French: A cross-linguistic study of pronoun resolution. In: Journal of Memory and Language 52, 363-376.

Coulmas, Florian (1988): Wörter, Komposita und anaphorische Inseln. In: Folia Linguistica $22,315-336$.

Craik, Fergus I. M. (2002): Levels of processing: Past, present ... and future? In: Memory $10.5 / 6,305-318$.

Craik, Fergus I. M.; Lockhart, Robert S. (1972): Levels of Processing: A Framework for Memory Research. In: Journal of Verbal Learning and Verbal Behavior 11, 671-684.

Crocker, Matthew W.; Keller, Frank (2006): Probabilistic Grammars as Models of Gradience in Language Processing. In: Fanselow, Gisbert; Féry, Caroline; Vogel, Ralf; Schlesewsky, Matthias (Hg.), Gradience in Grammar, Oxford: OUP, 227-245.

Crystal, David (1967): English. In: Lingua 17, 24-56. 
Di Sciullo, Anna-Maria; Williams, Edwin (1987): On the Definition of Word. Cambridge, Mass: MIT Press.

Dietrich, Rainer (2007): Psycholinguistik, Sammlung Metzler, Band 342. Stuttgart: Metzler, 2., aktualisierte und erw. Auflage.

Downing, Pamela (1977): On the Creation and Use of English Compound Nouns. In: Language and Cognitive Processes 53.4, 810-842.

Dressler, Wolfgang U. (2007): Compound Types. In: Libben, Gary; Jarema, Gonia (Hg.), The representation and processing of compound words, Oxford: OUP, 23-44.

Ehrich, Veronika (1991): Nominalisierungen (Nominalizations). In: von Stechow, Arnim; Wunderlich, Dieter (Hg.), Semantik. Semantics. Ein internationales Handbuch der zeitgenössischen Forschung. An International Handbook of Contemporary Research, Berlin, New York: de Gruyter, Handbücher zur Sprach- und Kommunikationswissenschaft (HSK), 441-458.

Ehrich, Veronika; Rapp, Irene (2000): Sortale Bedeutung und Argumentstruktur: ungNominalisierungen im Deutschen. In: Zeitschrift für Sprachwissenschaft 19.2, 245-303.

Ehrlich, Kate (1983): Eye Movements in Pronoun Assignment: A Study of Sentence Integration. In: Rayner, Keith (Hg.), Eye Movements in Reading, Oxford: Elsevier Science, Perspectives in neurolinguistics, neuropsychology, and psycholinguistics, 253-268.

Ellert, Miriam; Holler, Anke (2011): Semantic and Structural Constraints on the Resolution of Ambiguous Personal Pronouns - A Psycholinguistic Study. In: Hendrickx, Iris; Devi, Sobha Lalitha; Branco, Antónia; Mitkov, Ruslan (Hg.), Anaphora Processing and Applications, Berlin, Heidelberg: Springer, LNAI, 157-170.

Embick, David (2010): Localism versus globalism in morphology and phonology. Cambridge, Mass. u.a.: MIT Press.

Embick, David (2015): The Morpheme: A Theoretical Introduction. Boston, Berlin: de Gruyter.

Embick, David; Noyer, Rolf (2001): Movement Operations after Syntax. In: Linguistic Inquiry $32.4,555-595$.

Embick, David; Noyer, Rolf (2007): Distributed Morphology and the Syntax/Morphology Interface. In: Ramchand, Gillian; Reiss, Charles (Hg.), The Oxford Handbook of Linguistic Interfaces, Oxford u.a.: OUP, 289-324.

Epstein, Samuel D.; Kitahara, H.; Seely, D. (2016): Phase Cancellation by External PairMerge of Heads. In: The Linguistic Review 33.1, 87-102.

Erben, Johannes (2000): Einführung in die deutsche Wortbildungslehre, Grundlagen der Germanistik, Band 17. Berlin: E. Schmidt, 4., aktualisierte und erg. Auflage.

Erkü, Feride; Gundel, Jeanette (1987): The Pragmatics of Indirect Anaphors. In: Verschueren, Jef; Bertuccelli-Papi, Marcella (Hg.), The Pragmatic Perspective, Amsterdam, Philadelphia: Benjamins, Pragmatics \& Beyond, 533-545.

Estes, Zachary; Glucksberg, Sam (2000): Interactive property attribution in concept combination. In: Memory \& Cognition 28.1, 28-34. 
Fabb, Nigel (1984): Syntactic affixation. Phd dissertation, MIT, Cambridge, Mass.

Fabricius-Hansen, Cathrine (1993): Nominalphrasen mit Kompositum als Kern. In: Beiträge zur Geschichte der deutschen Sprache und Literatur 115.2, 193-243.

Fandrych, Christian; Thurmair, Maria (1994): Ein Interpretationsmodell für Nominalkomposita: linguistische und didaktische Überlegungen. In: Deutsch als Fremdsprache $31,34-45$.

Fanselow, Gisbert (1981): Zur Syntax und Semantik der Nominalkomposition: Ein Versuch praktischer Anwendung der Montague-Grammatik auf die Wortbildung im Deutschen, Linguistische Arbeiten, Band 107. Tübingen: Niemeyer.

Fanselow, Gisbert (1988): 'Word syntax' and semantic principles. In: Booij, Geert; van Marle, Jaap (Hg.), Yearbook of Morphology 1988, Boston, Mass. u.a.: Kluwer, 95-122.

Fanselow, Gisbert; Frisch, Stefan (2006): Effects of Processing Difficulty on Judgements of Acceptability. In: Fanselow, Gisbert; Féry, Caroline; Vogel, Ralf; Schlesewsky, Matthias (Hg.), Gradience in Grammar, Oxford: OUP, 291-316.

Fanselow, Gisbert; Féry, Caroline; Vogel, Ralf; Schlesewsky, Matthias (2006): Gradience in Grammar. In: Fanselow, Gisbert; Féry, Caroline; Vogel, Ralf; Schlesewsky, Matthias (Hg.), Gradience in Grammar, Oxford: OUP, 1-22.

Fanselow, Gisbert; Schlesewsky, Matthias; Vogel, Ralf; Weskott, Thomas (2011): Animacy effects on crossing wh-movement in German. In: Linguistics 49.4, 657-683.

Featherston, Sam (2007): Reply. In: Theoretical Linguistics 33.3, 407-413.

Ferreira, Fernanda; Patson, Nikole D. (2007): The 'Good Enough' Approach to Language Comprehension. In: Language and Linguistics Compass 1.1-2, 71-83.

Ferreira, Fernanda; Bailey, Karl G. D.; Ferraro, Vittoria (2002): Good-enough representations in language comprehension. In: Current Directions in Psychological Science 11, $11-15$.

Frege, Gottlob (1892): Über Sinn und Bedeutung. In: Zeitschrift für Philosophie und philosophische Kritik 100, 25-50. Abdruck in: Frege: Funktion, Begriff, Bedeutung. Fünf logische Studien. Göttingen: Vandenhoeck \& Ruprecht 2011, 40-65.

Frisson, Steven; Niswander-Klement, Elizabeth; Pollatsek, Alexander (2008): The role of semantic transparency in the processing of English compound words. In: British Journal of Psychology 99, 87-107.

Fu, Jingqui; Roeper, Thomas; Borer, Hagit (2001): The VP within Process Nominals: Evidence from Adverbs and the VP Anaphor do-so. In: Natural Language and Linguistic Theory 19, 549-582.

Fukumura, Kumiko; van Gompel, Roger P.G (2011): The effect of animacy on the choice of referring expressions. In: Language and Cognitive Processes 26, 1472-1504.

Gaeta, Livio (2010): Synthetic compounds: With special reference to German. In: Scalise, Sergio; Vogel, Irene (Hg.), Cross-Disciplinary Issues in Compounding, Amsterdam, Philadelphia: Benjamins, Current Issues in Linguistic Theory, 219-235. 
Gagné, Christina L. (2001): Relation and lexical priming during the interpretation of noun-noun combinations. In: Journal of Experimental Psychology: Learning, Memory, and Cognition 27.1, 236-254.

Gagné, Christina L. (2009): Psycholinguistic Perspectives. In: Lieber, Rochelle; Štekauer, Pavol (Hg.), The Oxford Handbook of Compounding, Oxford, New York: OUP, 255271.

Gagné, Christina L.; Shoben, Edward J. (1997): Influence of Thematic Relations on the Comprehension of Modifier-Noun Combinations. In: Journal of Experimental Psychology: Learning, Memory, and Cognition 23.1, 71-87.

Gagné, Christina L.; Spalding, Thomas L. (2007): Conceptual Combination: Implications for the mental lexicon. In: Libben, Gary; Jarema, Gonia (Hg.), The representation and processing of compound words, Oxford: OUP, 145-168.

Gallmann, Peter (1990): Kategoriell komplexe Wortformen. Tübingen: Niemeyer.

Gallmann, Peter (1999): Wortbegriff und Nomen-Verb-Verbindungen. In: Zeitschrift für Sprachwissenschaft 18.2, 269-304.

Garnham, Alan; Garrod, Simon; Sanford, Anthony (2006): Observations on the Past and Future of Psycholinguistics. In: Traxler, Matthew J.; Gernsbacher, Morton A. (Hg.), Handbook of Psycholinguistics, Elsevier, 1-18.

Garvey, Catherine; Caramazza, Alfonso (1974): Implicit causality in verbs. In: Linguistic Inquiry 5, 459-464.

Gernsbacher, Morton A. (1991): Comprehending conceptual anaphors. In: Language and Cognitive Processes 6.2, 81-105.

Giegerich, Heinz J. (2006): Attribution in English and the distinction between phrases and compounds. In: Rösel, Petr (Hg.), English in Space and Time, Trier: WVT, 10-27.

Grewendorf, Günther (1988): Aspekte der deutschen Syntax: Eine Rektions-BindungsAnalyse, Studien zur deutschen Grammatik, Band 33. Tübingen: Narr.

Grimshaw, Jane B. (1992): Argument Structure, Linguistic inquiry monographs, Band 18. Cambridge, Mass: MIT Press.

Gundel, Jeanette; Hedberg, Nancy.; Zacharski, Ron (1993): Referring Expressions in Discourse. In: Language 69, 274-307.

Gundel, Jeanette K. (1996): Relevance Theory Meets the Givenness Hierarchy: An Account of Inferrables. In: Fretheim, Thorstein; Gundel, Jeanette K. (Hg.), Reference and referent accessibility, Amsterdam: Benjamins, Pragmatics \& Beyond, 141-153.

Halle, Morris; Marantz, Alec (1993): Distributed Morphology and the Pieces of Inflection. In: Hale, Kenneth Locke; Keyser, Samuel Jay (Hg.), The view from building 20, Cambridge, Mass: MIT Press, Current studies in linguistics, 111-176.

Harley, Heidi (2009): Compounding in Distributed Morphology. In: Lieber, Rochelle; Štekauer, Pavol (Hg.), The Oxford Handbook of Compounding, Oxford, New York: OUP, $129-144$.

Harley, Heidi (2014): On the identity of roots. In: Theoretical Linguistics 40.3/4, 225-276. 
Harley, Heidi; Noyer, Rolf (1999): State-of-the-Article: Distributed Morphology. In: GLOT $4.4,3-9$.

Härtl, Holden (2013): Arguments of non-heads. In: Interfaces of Morphology, Berlin: Akademie, 163-177.

Härtl, Holden (2015): Situationsargumente von Nicht-Köpfen: Verb-Nomen-Komposita im Zusammenspiel von Morphologie, Syntax und Pragmatik. In: Fortmann, Christian; Lübbe, Anja; Rapp, Irene (Hg.), Situations-Argumente im Nominalbereich, Berlin, Boston: de Gruyter, 159-184.

Haspelmath, Martin (2011): The indeterminacy of word segmentation and the nature of morphology and syntax. In: Folia Linguistica 45.1, 31-80.

Hegarty, Michael; Gundel, Jeanette K.; Borthen, Kaja (2001): Information structure and the accessibility of clausally introduced referents. In: Theoretical Linguistics 27, 163186.

Henrich, Verena; Hinrichs, Erhard (2011): Determining Immediate Constituents of Compounds in GermaNet. In: Popescu, M. (Hg.), Proceedings of Recent Advances in Natural Language Processing, 420-426.

Higginbotham, James (1985): On semantics. In: Linguistic Inquiry 16, 547-594.

Hofmeister, Philip (2011): Representational complexity and memory retrieval in language comprehension. In: Language and Cognitive Processes 26.3, 376-405.

Holler-Feldhaus, Anke (2004): Koreferenz in Hypertexten: Anforderungen an die Annotation. In: Osnabrücker Beiträge zur Sprachtheorie 68, 9-29.

Jarema, Gonia (2007): Compound Representation and Processing: A cross-language perspective. In: Libben, Gary; Jarema, Gonia (Hg.), The representation and processing of compound words, Oxford: OUP, 45-70.

Kadmon, Nirit (2001): Formal pragmatics: Semantics, pragmatics, presupposition, and focus. Malden: Blackwell.

Kaiser, Elsi; Li, David Cheng-Huan; Holsinger, Edward (2011): Exploring the lexical and acoustic consequences of referential predictability. In: Hendrickx, Iris; Devi, Sobha Lalitha; Branco, Antónia; Mitkov, Ruslan (Hg.), Anaphora Processing and Applications, Berlin, Heidelberg: Springer, LNAI, 171-183.

Kamp, Hans; Reyle, Uwe (1993): From discourse to logic: Introduction to modeltheoretic semantics of natural language, formal logic and discourse representation theory, Studies in linguistics and philosophy, Band 42. Dordrecht, Boston, London: Kluwer.

Karttunen, Lauri (1976): Discourse Referents. In: McCawley, J. D. (Hg.), Syntax and Semantics 7: Notes from the Linguistic Underground, New York: Academic Press, 363385 .

Keller, Frank (2006): Linear Optimality Theory as a Model of Gradience in Grammar. In: Fanselow, Gisbert; Féry, Caroline; Vogel, Ralf; Schlesewsky, Matthias (Hg.), Gradience in Grammar, Oxford: OUP, 270-287. 
Kiparsky, Paul (1982): From Cyclic Phonology to Lexical Phonology. In: H. van der Hulst \& N. Smith (Hg.), The Structure of Phonological Representation, Dordrecht: Foris, $131-175$.

Klos, Verena (2011): Komposition und Kompositionalität: Möglichkeiten und Grenzen der semantischen Dekodierung von Substantivkomposita, Reihe Germanistische Linguistik, Band 292. Berlin: de Gruyter.

Koester, Dirk; Gunter, Thomas C.; Wagner, Susanne; Friederici, Angela D. (2004): Morphosyntax, Prosody, and Linking Elements: The Auditory Processing of German Nominal Compounds. In: Journal of Cognitive Neuroscience 16.9, 1647-1668.

Krifka, Manfred; Pelletier, Francis J.; Carlson, Greg N.; ter Meulen, Alice; Link, Godehard; Chierchia, Gennaro (1995): Genericity: An Introduction. In: Carlson, Greg N.; Pelletier, Francis Jeffry (Hg.), The Generic Book, Chicago: University of Chicago Press, $1-124$.

Kripke, Saul A. (1980): Naming and Necessity. Oxford: Blackwell.

Kuiper, Koenraad (1999): Compounding by adjunction and its empirical consequences. In: Language Sciences 21, 407-422.

Lakoff, George; Ross, John R. (1972): A Note on Anaphoric Islands and Causatives. In: Linguistic Inquiry 3.1, 121-125.

Langacker, Ronald W. (1991): Cognitive Grammar. In: Droste, Flip G.; Joseph, John E. (Hg.), Linguistic Theory and Grammatical Description, Amsterdam, Philadelphia: Benjamins, 275-306.

Lapointe, Stephen (1979): A Theory of Grammatical Agreement. Phd dissertation, University of Massachusetts, Amherst.

Lascarides, Alex; Asher, Nicholas (2007): Segmented discourse representation theory: Dynamic semantics with discourse structure. In: Bunt, Harry; Muskens, Reinhard (Hg.), Computing meaning, Dordrecht u.a.: Springer, Studies in linguistics and philosophy, $87-124$.

Lees, Robert B. (1960): The Grammar of English Nominalizations. The Hague: Mouton.

Levi, Judith N. (1977): The Constituent Structure of Complex Nominals: Or, that's Funny, you don't Look Like a Noun. In: Beach, Woodford A. (Hg.), Papers from the thirtennth regional meeting of the Chicago Linguistic Society, Chicago, Band 13, 325-351.

Levi, Judith N. (1978): The syntax and semantics of complex nominals. New York: Academic Press.

Levin, Beth; Rappaport Hovav, Malka (1995): Unaccusativity: At the syntax-lexical semantics interface, Linguistic inquiry monographs, Band 26. Cambridge, Mass: MIT Press.

Libben, Gary (1998): Semantic Transparency in the Processing of Compounds: Consequences for Representation, Processing and Impairment. In: Brain and Language 61, $30-44$. 
Libben, Gary (2005): Everything is Psycholinguistics: Material and Methodological Considerations in the Study of Compound Processing. In: Canadian Journal of Linguistics $50,267-283$.

Libben, Gary (2007): Why Study Compound Processing? An overview of the issues. In: Libben, Gary; Jarema, Gonia (Hg.), The representation and processing of compound words, Oxford: OUP, 1-22.

Libben, Gary; Gibson, Martha; Yoon, Yea Bom; Sandra, Dominiek (2003): Compound fracture: The role of semantic transparency and morphological headedness. In: Brain and Language 84, 50-64.

Lieber, Rochelle (1982): Allomorphy. In: Linguistic Analysis 10.1, 27-52.

Lieber, Rochelle (1983): Argument linking and compounds in English. In: Linguistic Inquiry 14, 251-285.

Lieber, Rochelle (1988): Phrasal compounds in English and the morphology-syntax interface. In: Brentari, D.; Larson, G. L MacLeod (Hg.), CLS-24-II, Chicago: Chicago Linguistic Society, 202-222.

Lieber, Rochelle (1992): Deconstruction Morphology: Word formation in syntactic theory. Chicago and London: The University of Chicago Press.

Lieber, Rochelle (2004): Morphology and Lexical Semantics. Cambridge: CUP.

Lieber, Rochelle (2009): IE, Germanic: English. In: Lieber, Rochelle; Štekauer, Pavol (Hg.), The Oxford Handbook of Compounding, Oxford, New York: OUP, 357-369.

Lieber, Rochelle (2010): On the lexical semantics of compounds: Non-affixal (de)verbal compounds. In: Scalise, Sergio; Vogel, Irene (Hg.), Cross-Disciplinary Issues in Compounding, Amsterdam, Philadelphia: Benjamins, Current Issues in Linguistic Theory, $127-144$.

Lieber, Rochelle (2014): Methodological Issues in Studying Derivation. In: Lieber, Rochelle; Stekauer, Pavol (Hg.), The Oxford Handbook of Derivational Morphology, Oxford: OUP, 84-94.

Lieber, Rochelle; Štekauer, Pavol (2009): Introduction: status and definition of compounding. In: Lieber, Rochelle; Štekauer, Pavol (Hg.), The Oxford Handbook of Compounding, Oxford, New York: OUP, 3-18.

Lockhart, Robert S.; Craik, Fergus I. M. (1990): Levels of processing: A Retrospective Commentary on a Framework for Memory Research. In: Canadian Journal of Psycho$\log y 44.1,87-112$.

Longobardi, Giuseppe (1994): Reference and Proper Names: A Theory of N-Movement in Syntax and Logical Form. In: Linguistic Inquiry 25.4, 609-665.

López, Luis (2015): Parallel Computation in Word Formation. In: Linguistic Inquiry 46.4, $657-701$.

Marantz, Alec (1984): On the Nature of Grammatical Relations. Linguistic Inquiry Monograph 10, Cambridge, Mass: MIT Press. 
Marantz, Alec (1996): 'Cat' as a Phrasal Idiom: Consequences of Late Insertion in Distributed Morphology. Ms., MIT, Cambridge Mass.

Marantz, Alec (1997): No Escape from Syntax: Don't Try Morphological Analysis in the Privacy of Your Own Lexicon. In: University of Pennsylvania Working Papers in Linguistics 4.2, 201-225.

Marantz, Alec (2013a): Locality domains for contextual allomorphy across the interfaces. In: Matushansky, Ora; Marantz, Alec (Hg.), Distributed morphology today, Cambridge, Mass: MIT Press, 95-115.

Marantz, Alec (2013b): No escape from morphemes in morphological processing. In: Language and Cognitive Processes 28.7, 905-916.

Marantz, Alec (2016): Linguistics as Cognitive Science: Back to our Roots. Vortrag, Harvard University, Cambridge Mass (11.04.2016).

McKoon, Gail; Ward, Gregory; Ratcliff, Roger; Sproat, Richard (1993): Morphosyntactic and Pragmatic Factors Affecting the Accessibilty of Discourse Entities. In: Journal of Memory and Language 32, 56-75.

Meibauer, Jörg (2003): Phrasenkomposita zwischen Wortsyntax und Lexikon. In: Zeitschrift für Sprachwissenschaft 22.2, 153-188.

Meibauer, Jörg (2007): Einführung in die germanistische Linguistik. Stuttgart: Metzler, 2. Auflage.

Meineke, Eckhard (1991): Springlebendige Tradition: Kern und Grenzen des Kompositums. In: Sprachwissenschaft 16, 27-88.

Meyer, Ralf (1993): Compound comprehension in isolation and in context: The contribution of conceptual and discourse knowledge to the comprehension of German novel noun-noun compounds, Linguistische Arbeiten, Band 299. Tübingen: Niemeyer.

Mithun, Marianne (2010): Constraints on compounds and incorporation. In: Scalise, Sergio; Vogel, Irene (Hg.), Cross-Disciplinary Issues in Compounding, Amsterdam, Philadelphia: Benjamins, Current Issues in Linguistic Theory, 37-56.

Neef, Martin (2009): IE, Germanic: German. In: Lieber, Rochelle; Štekauer, Pavol (Hg.), The Oxford Handbook of Compounding, Oxford, New York: OUP, 386-399.

Olsen, Susan (2000): Composition. In: Booij, Geert et al. (Hg.), Morphologie/Morphology: HSK 17, Berlin, New York: de Gruyter, 897-916.

Olsen, Susan (2012): Der Einfluss des Mentalen Lexikons auf die Interpretation von Komposita. In: Gaeta, Livio; Schlücker, Barbara (Hg.), Das Deutsche als kompositionsfreudige Sprache, Berlin: de Gruyter, 135-170.

Olsen, Susan (2014): Delineating Derivation and Compounding. In: Lieber, Rochelle; Štekauer, Pavol (Hg.), The Oxford Handbook of Derivational Morphology, Oxford: OUP, 26-49.

Ortner, Hanspeter; Ortner, Lorelies (1984): Zur Theorie und Praxis der Kompositaforschung: Mit einer ausführlichen Bibliographie, Forschungsberichte (Institut für Deutsche Sprache), Band 55. Tübingen: Narr. 
Partee, Barbara H. (2003): Are there privative adjectives. Vortrag, University of Massachusetts, Amherst (07./08.02.2003).

Pause, Peter E. (1991): Anaphern im Text. In: von Stechow, Arnim; Wunderlich, Dieter (Hg.), Semantik. Semantics. Ein internationales Handbuch der zeitgenössischen Forschung. An International Handbook of Contemporary Research, Berlin, New York: de Gruyter, Handbücher zur Sprach- und Kommunikationswissenschaft (HSK), 548-559.

Pesetsky, David (1979): Russian morphology and lexical phonology. Ms., MIT, Cambridge Mass.

Pollatsek, Alexander; Hyönä, Jukka; Bertram, Raymond (2000): The Role of Morphological Constituents in Reading Finnish Compound Words. In: Journal of Experimental Psychology: Human Perception and Performance 26.2, 820-833.

Postal, Paul M. (1969): Anaphoric Islands. In: Binnick, Robert I. et al (Hg.), Papers from the fifth regional meeting of the Chicago Linguistic Society, Chicago, Band 5, 205-239.

Punske, Jeffrey (2016): Compounding. In: Language and Linguistics Compass 10.8, 382393.

Radach, Ralph; Heller, Dieter; Huestegge, Lynn (2002): Blickbewegungen beim Lesen: neueste Entwicklungen und Ansatzpunkte für die Legasthenieforschung. In: SchulteKörne, Gerd (Hg.), Legasthenie, Winkler, 61-87.

Rayner, Keith (1998): Eye Movements in Reading and Information Processing: 20 Years of Research. In: Psychological Bulletin 124.3, 372-422.

Reinhard, Sabine (2001): Deverbale Komposita an der Deverbale Komposita an der Morphologie-Syntax-Semantik-Schnittstelle: ein HPSG-Ansatz. Dissertation, Universität Tübingen, Tübingen.

Reinhart, Tanya (1991): Pronouns. In: von Stechow, Arnim; Wunderlich, Dieter (Hg.), Semantik. Semantics. Ein internationales Handbuch der zeitgenössischen Forschung. An International Handbook of Contemporary Research, Berlin, New York: de Gruyter, Handbücher zur Sprach- und Kommunikationswissenschaft (HSK), 535-547.

Reuland, Eric (2006): Gradedness: Interpretive Dependencies and Beyond. In: Fanselow, Gisbert; Féry, Caroline; Vogel, Ralf; Schlesewsky, Matthias (Hg.), Gradience in Grammar, Oxford: OUP, 45-69.

Richardson, Daniel C.; Spivey, Michael J. (2004): Eye tracking: Characteristics and methods. In: Lee, S.; Peng Yuan; Robinson, Michael R. (Hg.), Encyclopedia of biomaterials and biomedical engineering, New York: Marcel Dekker, 568-572.

Rivet, Anne (1999): Rektionskomposita und Inkorporationstheorie. In: Linguistische Berichte $179,307-342$.

Roberts, Craige (2002): Demonstratives as definites. In: von Deemter, Kees; Kibble, Roger (Hg.), Information sharing, CSLI Publications, 89-196.

Roberts, Craige (2005): Pronouns as Definites. In: Reimer, Marga; Bezuidenhout, Anne (Hg.), Descriptions and beyond, Oxford: OUP, 503-543.

Roeper, Tom (1988): Compound syntax and head movement. In: G. Booij, J. van Marle (Hg.), Yearbook of Morhology 1988, Dordrecht: Foris, 187-228. 
Roeper, Tom; Siegel, Muffy E. A. (1978): A lexical transformation for verbal compounds. In: Linguistic Inquiry 9, 199-260.

Sadock, Jerrold M. (1998): On the Autonomy of Compounding Morphology. In: Lapointe, Steven; Brentari, Diane; Farrell, Patrick (Hg.), Morpholgy and its Relation to Phonology and Syntax, Stanford, Calif: CSLI Publications, 161-187.

Sandra, Dominiek (1990): On the Representation and Processing of Compound Words: Automatic Access to Constituent Morphemes Does Not Occur. In: The Quarterly Journal of Experimental Psychology 42.3, 529-567.

Sanford, Anthony J.; Garrod, Simon; Lucas, A.; Henderson, Robert (1983): Pronouns without explicit Antecedents? In: Journal of Semnatics 2.3-4, 303-318.

Scalise, Sergio; Bisetto, Antonietta (2009): The Classification of Compounds. In: Lieber, Rochelle; Štekauer, Pavol (Hg.), The Oxford Handbook of Compounding, Oxford, New York: OUP, 34-53.

Scalise, Sergio; Fábregas, Antonio (2010): The head in compounding. In: Scalise, Sergio; Vogel, Irene (Hg.), Cross-Disciplinary Issues in Compounding, Amsterdam, Philadelphia: Benjamins, Current Issues in Linguistic Theory, 109-125.

Scalise, Sergio; Vogel, Irene (2010): Why compounding? In: Scalise, Sergio; Vogel, Irene (Hg.), Cross-Disciplinary Issues in Compounding, Amsterdam, Philadelphia: Benjamins, Current Issues in Linguistic Theory, 1-18.

Schäfer, Martin (2013): Semantic Transparency and Anaphoric Islands. In: ten Hacken, Pius; Thomas, Claire (Hg.), The Semantics of Word Formation and Lexicalization, Edinburgh: EUP, 140-160.

Schäfer, Martin; Bell, Melanie J. (2016): Modelling semantic transparency. In: Morphology $26.2,157-199$.

Schlesewsky, Matthias; Bornkessel, Ina; McElree, Brian (2006): Decomposing Gradience: Quantitative versus Qualitative Distinctions. In: Fanselow, Gisbert; Féry, Caroline; Vogel, Ralf; Schlesewsky, Matthias (Hg.), Gradience in Grammar, Oxford: OUP, 124-142.

Schrauf, Judith (2011): Vom Konkreten im Abstrakten: Eine kognitionslinguistische Analyse zu Konkreta und Abstrakta. Dissertation, Philipps-Universität Marburg, Marburg.

Schreuder, Robert; Baayen, Harald (1995): Modelling morphological processing. In: Feldman, Laurie B. (Hg.), Morphological aspects of language processing, Hove: Erlbaum, $131-156$.

Schulte im Walde, Sabine; Hätty, Anna; Bott, Stefan (2016): The Role of Modifier and Head Properties in Predicting the Compositionality of English and German Noun-Noun Compounds: A Vector-Space Persepctive. In: Proceedings of the Fifth Joint Conference on Lexical and Computaional Semantics (SEM 2016), 148-158.

Schwarz, Monika (2000): Indirekte Anaphern in Texten: Studien zur domänengebundenen Referenz und Kohärenz im Deutschen: Univ., Habil.-Schr.-Köln, 1998, Linguistische Arbeiten, Band 413. Tübingen: Niemeyer.

Selkirk, Elisabeth O. (1982): The Syntax of Words. Cambridge, Mass: MIT Press. 
Selkirk, Elisabeth O. (1984): Phonology and syntax: The relation between sound and structure, Current studies in linguistics series, Band 10. Cambridge, Mass: MIT Press.

Sereno, Sara C. (1992): Early Lexical Effects when Fixating a Word in Reading. In: Rayner, Keith (Hg.), Eye movements and visual cognition, New York: Springer, Springer series in neuropsychology, 304-316.

Shoben, Edward J. (1991): Predicating and nonpredicating combinations. In: Schwanenflugel, Paula J. (Hg.), The psychology of word meanings, Hillsdale, NJ: Erlbaum, 117135.

Siebert, Susann (1999): Wortbildung und Grammatik: Syntaktische Restriktionen in der Struktur komplexer Wörter. Tübingen: Niemeyer.

Smyth, Mary M.; Scholey, Keith A. (1994): Interference in immediate spatial memory. In: Memory \& Cognition 22.1, 1-13.

Soehn, Jan-Philipp (2005): Über Bärendienste und erstaunte Bauklötze: Idiome ohne freie Lesart in der HPSG. Dissertation, Friedrich-Schiller-Universität, Jena.

Solstad, Torgrim (2010): Post-nominal genitives and prepositional phrases in German: A uniform analysis. In: Alexiadou, Artemis; Rathert, Monika (Hg.), The syntax of Nominalizations across Languages and Frameworks, Berlin, New York: de Gruyter, Interface explorations, 219-252.

Spencer, Andrew (1991): Morphological theory: An introduction to word structure in generative grammar. Oxford, Cambridge Mass.: Blackwell.

Spencer, Andrew (2011): What's in a compound? In: Journal of Linguistics 47.2, 481-507.

Sproat, Richard (2005): Current morphological theory. In: SKASE Journal of Theoretical Linguistics 2.2, 63-75.

Sproat, Richard William (1985): On deriving the lexicon. Phd dissertation, MIT, Cambridge, Mass.

Sprouse, Jon (2007): A Program for Experimental Syntax: Finding the Relationship Between Acceptability and Grammatical Knowledge. Phd dissertation, University of Maryland, College Park.

SR Research Ltd. (2002-2007): EyeLink Data Viewer: User's Manual: Document Version 1.8.221. Mississauga, Canada.

Štekauer, Pavol (1998): An Onomasiological Theory of English Word-Formation. Amsterdam, Philadelphia: Benjamins.

Sternefeld, Wolfgang (2003): Klammerparadoxien, gibt es sie wirklich? Vortrag, Universität Leipzig (07.11.2003).

Sternefeld, Wolfgang (2008): Syntax, Stauffenburg-Linguistik, Band 31,1. Tübingen: Stauffenburg, 3., überarb. Auflage.

Stevenson, Roesmary J.; Crawley, Rosalind A.; Kleinman, David (1994): Thematic roles, focus and the representation of events. In: Language and Cognitive Processes 9.4, 519548.

Strawson, Peter F. (1950): On Referring. In: Mind 59, 320-344. 
Stump, Gregory (2001): Inflectional Morphology: A Theory of Paradigm Structure. Cambridge: CUP.

Toman, Jindřich (1990): Issues in the Theory of Inheritance. In: Grewendorf, Günther; Sternefeld, Wolfgang (Hg.), Scrambling and Barriers, Amsterdam, Philadelphia: Benjamins, LA, 423-442.

Twain, Mark (2009): The awful German language: Die schreckliche deutsche Sprache. Übers. von Ana Maria Brock. Waltrop: Manuscriptum, 10. Auflage.

Ward, Gregory; Sproat, Richard; McKoon, Gail (1991): A Pragmatic Analysis of So-Called Anaphoric Islands. In: Language and Cognitive Processes 67.3, 439-474.

Wermke, Matthias; Kunkel-Razum, Kathrin; Scholze-Stubenrecht, Werner (Hg.) (2009): Duden. Die Grammatik. Mannheim: Dudenverlag, 8. Auflage.

Wiese, Richard (1996): Phrasal Compounds and the Theory of Word Syntax. In: Linguistic Inquiry 27, 183-193.

Williams, Edwin (1981): Argument Structure and Morphology. In: Linguistic Review 1, $81-114$.

Wunderlich, Dieter (1986): Probleme der Wortstruktur. In: Zeitschrift für Sprachwissenschaft 5.2, 209-252.

Zhang, Niina Ning (2007): Root Merger in Chinese Compounds. In: Studia Linguistica $61.2,170-184$.

Zwitserlood, Pinie (1994): The Role of Semantic Transparency in the Processing and Representation of Dutch Compounds. In: Language and Cognitive Processes 9.3, 341368. 


\section{Weitere Quellen}

\section{Software}

Bates, Douglas; Maechler, Martin; Bolker, Ben; Walker, Steven (2013): R Package 'lme4': Linear mixed-effects models using Eigen and S4 (Version 1.0-5). Verfügbar unter: http://lme4.r-forge.r-project.org/.

Bates, Douglas; Maechler, Martin; Bolker, Ben; Walker, Steve (2015): Fitting Linear Mixed-Effects Models Using lme4. Journal of Statistical Software, 67(1), 1-48. doi:10.18637/jss.v067.i01.

COSMAS II (Corpus Search, Management and Analysis System): (C) 1991-2016 Institut für Deutsche Sprache, Mannheim. www.ids-mannheim.de/cosmas2/ (23.10.2014).

Experiment Builder (2010): (Version 1.10.165). Mississauga, Canada: SR Research Ltd.

Leiner, D. J. (2013): SoSci Survey (Version 2.3.05-i). Verfügbar unter:

https://www.soscisurvey.de.

Onea, Edgar G. (2012): OnExp: Software for Online Questionnaires (Versionen 1.2 und 1.3.1). Göttingen: Courant Forschungszentrum „Textstrukturen“ (Universität Göttingen). Verfügbar unter https://onexp.textstrukturen.uni-goettingen.de/.

R Core Team (2013-2015): R: A Language and Environment for Statistical Computing (Version 3.2). Wien: R Foundation for Statistical Computing. Verfügbar unter:

http://www.R-project.org/

Wickham, Hadley (2009): ggplot2: Elegant Graphics for Data Analysis. New York: SpringerVerlag.

\section{Korpora}

DWDS-KK: DWDS-Kernkorpus des 20. Jahrhunderts (Digitales Wörterbuch der deutschen Sprache). Berlin-Brandenburgische Akademie der Wissenschaften.

http://www.dwds.de/r/ (23.10.2014).

Institut für Deutsche Sprache (2014b): Das Deutsche Refernzkorpus DeReKo/Archiv der Korpora geschriebener Gegenwartssprache 2014-II (Release vom 11.09.2014). Mannheim: Institut für Deutsche Sprache. www.ids-mannheim.de/DeReKo.

LWS: German newspaper corpus based on material crawled in 2011 (deu_newscrawl_2011). (C) 2017 Abteilung Automatische Sprachverarbeitung, Universität Leipzig.

www.wortschatz.uni-leipzig.de/ (23.10.2014). 



\section{Tabellenverzeichnis}

2.1. Semantische Relationen nach Levi (1978:76f)] . . . . . . . . . . . . . . 27

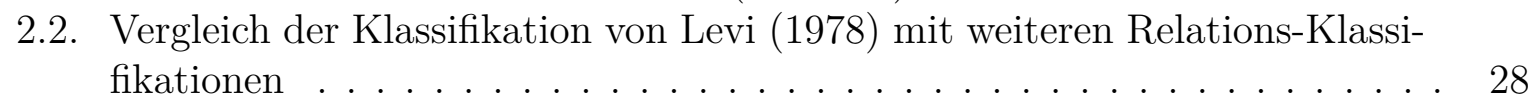

2.3. Kombinationen von transparenten und opaken Kompositagliedern . . . . 68

3.1. Beispiele für eine Einteilung von Komposita nach raum-zeitlicher Kontiguität (RZK) der Konstituenten . . . . . . . . . . . . . . . . 85

3.2. Bedingungen und Beispiele für die in Experiment 1 verwendeten Komposita 91

3.3. Beispiel-Stimuli für alle Bedingungen in Experiment 1 . . . . . . . . . . . 92

3.4. Deskriptive Statistik der relativen Häufigkeit der N1-Bezüge für den Faktor

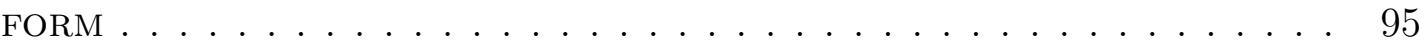

3.5. Deskriptive Statistik der relativen Häufigkeit der N1-Bezüge für den Faktor ANIMATHEIT für die Kompositabedingung . . . . . . . . . . . . . . . . 95

3.6. Deskriptive Statistik der relativen Häufigkeit der N1-Bezüge für den Faktor RELATION für die Kompositabedingung . . . . . . . . . . . . . . . 95

3.7. Deskriptive Statistik der relativen Häufigkeit der N1-Bezüge für die Faktoren ANIMATHEIT und RELATION für die Kompositabedingung . . . . . . . . 96

3.8. Deskriptive Statistik der relativen Häufigkeit der N1-Bezüge für den Faktor ANIMATHEIT für die phrasale Bedingung . . . . . . . . . . . . . . . 96

3.9. Deskriptive Statistik der relativen Häufigkeit der N1-Bezüge für den Faktor RELATION für die phrasale Bedingung . . . . . . . . . . . . . . . . 97

3.10. Deskriptive Statistik der relativen Häufigkeit der N1-Bezüge für die Faktoren ANIMATHEIT und RELATION für die phrasale Bedingung . . . . . . . . . 97

3.11. Deskriptive Statistik der relativen Häufigkeit der N1-Bezüge für die Faktoren FORM, ANIMATHEIT und RELATION . . . . . . . . . . . . 97

3.12. Inferenzstatistik (LMEM) für die N1-Bezüge für den Faktor FORM, kom-

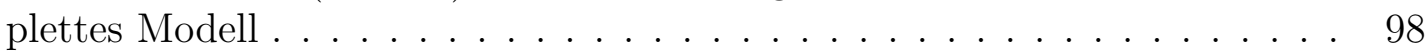

3.13. Inferenzstatistik (LMEM) für die N1-Bezüge für den Faktor ANIMATHEIT für die Kompositabedingung . . . . . . . . . . . . . . . . . . . . . 99

3.14. Inferenzstatistik (LMEM) für die N1-Bezüge für den Faktor RELATION für die Kompositabedingung . . . . . . . . . . . . . . . . . . . . . . . 99

3.15. Inferenzstatistik (LMEM) für die N1-Bezüge für die Faktoren ANIMATHEIT

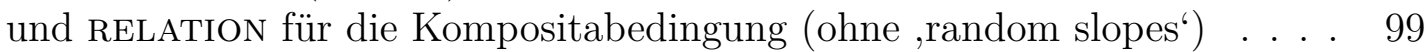

3.16. Inferenzstatistik (Modellvergleich) für den Faktor ANIMATHEIT in Abhängigkeit des Faktors RELATION für die Kompositabedingung. . . . . . . . . . 100

3.17. Inferenzstatistik (Modellvergleich) für den Faktor RELATION in Abhängigkeit des Faktors ANIMATHEIT für die Kompositabedingung . . . . . . . . . . 100

3.18. Inferenzstatistik (LMEM) für die N1-Bezüge für die Faktoren ANIMATHEIT und RELATION für die phrasale Bedingung (Interaktion ohne, random slopes $\left.{ }^{6}\right) 100$ 
5.1. Beispiele für die in Experiment 2 verwendeten Komposita in den vier Bedingungen mit den Faktoren RZK und STRUKTUR . . . . . . . . . . . . . 153

5.2. Deskriptive Statistik der relativen Häufigkeit der N1-Bezüge für den Faktor

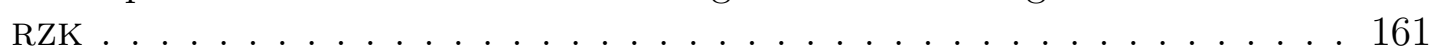

5.3. Deskriptive Statistik der relativen Häufigkeit der N1-Bezüge für den Faktor

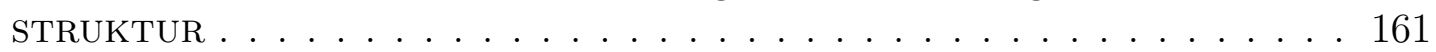

5.4. Deskriptive Statistik der relativen Häufigkeit der N1-Bezüge für die Faktoren RZK und STRUKTUR.

5.5. Deskriptive Statistik der relativen Häufigkeit der N1-Bezüge für den Faktor

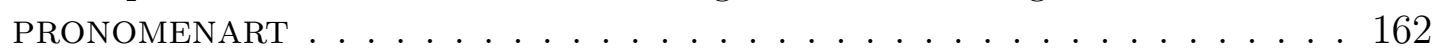

5.6. Deskriptive Statistik der relativen Häufigkeit der N1-Bezüge für die Faktoren RZK, STRUKTUR und PRONOMENART . . . . . . . . . . . . . . 163

5.7. Inferenzstatistik (LMEM) für die N1-Bezüge für den Faktor RZK mit 24 Items, 56 VPn und Item und VP als, random factors ${ }^{6} \ldots$. . . . . . . . . . . 164

5.8. Inferenzstatistik (LMEM) für die N1-Bezüge für den Faktor STRUKTUR mit 24 Items, 56 VPn und Item und VP als, random factors ${ }^{6}$. . . . . . . . . . . 164

5.9. Inferenzstatistik (LMEM) für die N1-Bezüge für die Faktoren RZK und STRUKTUR mit 24 Items und 54 VPn, ohne, random slopes ${ }^{6}$. . . . . . . . . . 164

5.10. Inferenzstatistik (Modellvergleich) für den Faktor RZK in Abhängigkeit des Faktors STRUKTUR mit 24 Items und 54 VPn ohne, random slopes ${ }^{6}$. . . . 165

5.11. Inferenzstatistik (Modellvergleich) für den Faktor STRUKTUR in Abhängigkeit des Faktors RZK mit 24 Items, 54 VPn und Item und VP als ,random

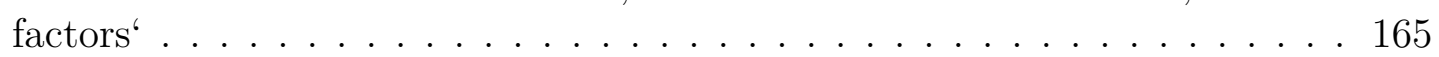

5.12. Inferenzstatistik (LMEM) für die N1-Bezüge für den Faktor PRONOMENART mit 24 Items, 56 VPn und Item und VP als ,random factors ${ }^{2}$. . . . . 166

5.13. Deskriptive Statistik der relativen Häufigkeit der N1-Bezüge für den Faktor SCHNITTMENGENBILDUNG . . . . . . . . . . . . . . . 167

5.14. Inferenzstatistik (LMEM) für die N1-Bezüge für den Faktor SCHNITTMENGENBILDUNG mit 27 Items, 56 VPn und Item und VP als ,random factors ${ }^{9} 167$

5.15. Deskriptive Statistik der relativen Häufigkeit der N1-Bezüge für die Faktoren RZK, STRUKTUR und SCHNITTMENGENBILDUNG . . . . . . . . . . . . . . 167

5.16. Deskriptive Statistik der relativen Häufigkeit der N1-Bezüge für den Faktor

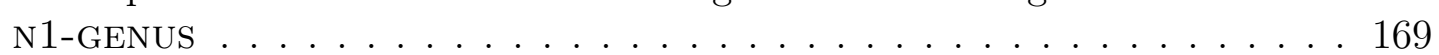

5.17. Inferenzstatistik (LMEM) für die N1-Bezüge für den Faktor N1-GENUS mit 24 Items, 56 VPn und Item und VP als, random factors $6 . . . . .169$

5.18. Deskriptive Statistik der relativen Häufigkeit der N1-Bezüge für den Faktor N1-SPEZIFIZITÄT . . . . . . . . . . . . . . . . . . . . . . 169

5.19. Inferenzstatistik (LMEM) für die N1-Bezüge für den Faktor N1-SPEZIFIZITÄT mit 27 Items, 56 VPn und Item und VP als, random factors ${ }^{6}$. . . . . . . . 170

5.20. Deskriptive Statistik der relativen Häufigkeit der N1-Bezüge für den Faktor N1-Z̈̈HLBARKEIT . . . . . . . . . . . . . . . . 170

5.21. Inferenzstatistik (LMEM) für die N1-Bezüge für den Faktor N1-ZÄHLBARKEIT

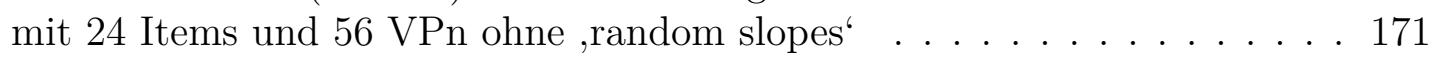

5.22. Deskriptive Statistik der relativen Häufigkeiten der N1-Bezüge für den Faktor NOMINALISIERUNGSART für Rektionskomposita . . . . . . . . . . . . . . 171

5.23. Deskriptive Statistik der relativen Häufigkeit der N1-Bezüge für die verschiedenen semantischen Relationen . . . . . . . . . . . . . . . . . 172 
5.24. Deskriptive Statistik der relativen Häufigkeit der anaphorischen Bezüge auf das implizit oder explizit erwähnte Antezedens in den Kontroll-Items . . . 174

5.25. Inferenzstatistik (LMEM) für die Bezüge auf das Instrument-Antezedens in den Kontroll-Items mit 12 Items, 56 VPn und Item und VP als ,random

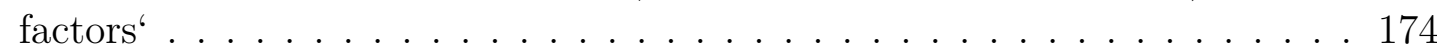

6.1. Beispiele für die in Experiment 3 verwendeten Komposita in den drei Bedingungen des Faktors STRUKTUR . . . . . . . . . . . . . . 180

6.2. Deskriptive Statistik der relativen Häufigkeit der N1-Bezüge auf dem gesamten Datensatz für den Faktor STRUKTUR . . . . . . . . . . . . . . . . 185

6.3. Deskriptive Statistik der relativen Häufigkeit der N1-Bezüge auf dem ge-

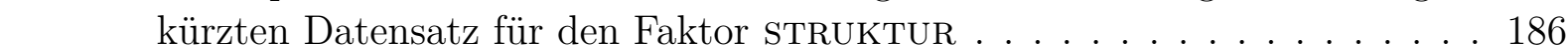

6.4. Inferenzstatistik (LMEM) für die N1-Bezüge auf dem gekürzten Datensatz für den Faktor STRUKTUR, Kontraste (RK)/(Mono) und (WK)/(Mono) . . 187

6.5. Inferenzstatistik (Modellvergleich) auf dem gekürzten Datensatz für den

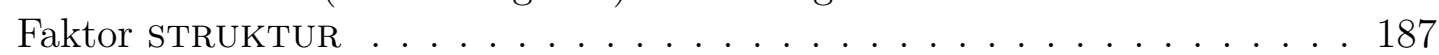

6.6. Inferenzstatistik (LMEM) für die N1-Bezüge auf dem gekürzten Datensatz für den Faktor STRUKTUR ohne Bed. Mono . . . . . . . . . . . . . . . . . . . 188

6.7. Inferenzstatistik (Modellvergleich) für die N1-Bezüge auf dem gekürzten Datensatz für den Faktor STRUKTUR ohne Bed. Mono . . . . . . . . . . . 188

6.8. Deskriptive Statistik der relativen Häufigkeit der N1-Bezüge auf dem ge-

kürzten Datensatz für den Faktor RZK . . . . . . . . . . . . . . . . . . . 189

6.9. Inferenzstatistik (LMEM) für die N1-Bezüge auf dem gekürzten Datensatz für den Faktor RZK . . . . . . . . . . . . . . . . . . . . . . . . . . . . 189

6.10. Deskriptive Statistik der relativen Häufigkeit der N1-Bezüge auf dem gekürzten Datensatz für den Faktor N2-KONKRETHEIT . . . . . . . . . . . . . 190

6.11. Inferenzstatistik für die N1-Bezüge auf dem gekürzten Datensatz für den Faktor N2-KONKRETHEIT . . . . . . . . . . . . . . . . . . . . . 191

6.12. Deskriptive Statistik der relativen Häufigkeit der N1-Bezüge auf dem gekürzten Datensatz für den Faktor NOMINALISIERUNGSLESART . . . . . . . . 192

6.13. Inferenzstatistik für die N1-Bezüge auf dem gekürzten Datensatz für den

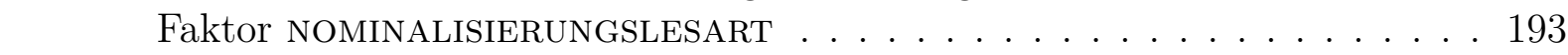

6.14. Deskriptive Statistik der relativen Häufigkeit der N1-Bezüge auf dem gekürzten Datensatz für den Faktor N1-ZÄHLBARKEIT . . . . . . . . . . . . . . 193

6.15. Inferenzstatistik für die N1-Bezüge auf dem gekürzten Datensatz für den Faktor N1-ZÄHLBARKEIT . . . . . . . . . . . . . . . . . . . . . 194

6.16. Deskriptive Statistik der relativen Häufigkeit der N1-Bezüge auf dem gekürzten Datensatz für den Faktor GENUS . . . . . . . . . . . . . . . . . 195

6.17. Inferenzstatistik für die N1-Bezüge auf dem gekürzten Datensatz für den Faktor GENUS . . . . . . . . . . . . . . . . . . . 195

6.18. Deskriptive Statistik der relativen Häufigkeit der N1-Bezüge auf dem gekürzten Datensatz für den Faktor N1-FREQUENZ . . . . . . . . . . . . . . . . 197

6.19. Inferenzstatistik für die N1-Bezüge auf dem gekürzten Datensatz für den Faktor N1-FREQUENZ . . . . . . . . . . . . . . . . . 197

6.20. Deskriptive Statistik der relativen Häufigkeit der N1-Bezüge auf dem gekürzten Datensatz für den Faktor NN-FREQUENZ . . . . . . . . . . . . . . . . 198

6.21. Inferenzstatistik für die N1-Bezüge auf dem gekürzten Datensatz für den Faktor NN-FREQUENZ . . . . . . . . . . . . . . . . . . 198

6.22. Deskriptive Statistik des Kohärenzratings für den Faktor STRUKTUR . . . . 206 
6.23. Inferenzstatistik (ANOVA omnibus-by-items, ANOVA omnibus-by-subjects, minF') des Kohärenzratings für den Faktor STRUKTUR . . . . . . . . . . . . 207

6.24. Liste verwendeter AVn (vgl. SR Research Ltd.|2002-2007:64ff)]. . . . . . . . . 211

6.25. Zusammenfassung der Hypothesen für die verschiedenen Interest Areas (IAs) und abhängigen Variablen $(\mathrm{AVn})$. . . . . . . . . . . . . . . . . . . 213

6.26. Deskriptive Statistik für die FFD der Anaphern-Region (IA 6) für den

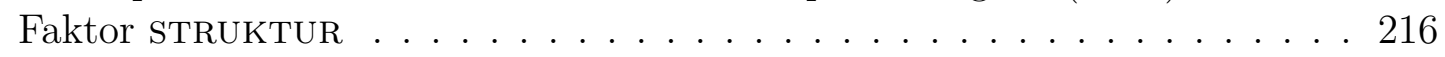

6.27. Inferenzstatistik (ANOVA omnibus-by-subjects, ANOVA omnibus-by-items, minF') für die FFD der Anaphern-Region (IA 6) für den Faktor STRUKTUR 216

6.28. Deskriptive Statistik für die FPFD der Anaphern-Region (IA 6) . . . . . . 217

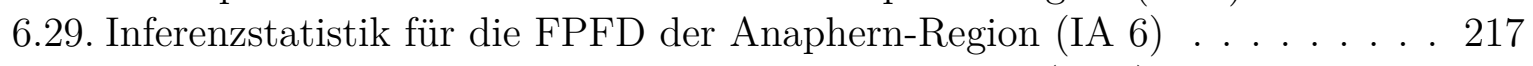

6.30. Inferenzstatistik für die FPFD der Anaphern-Region (IA 6) ohne Bed. Mo-

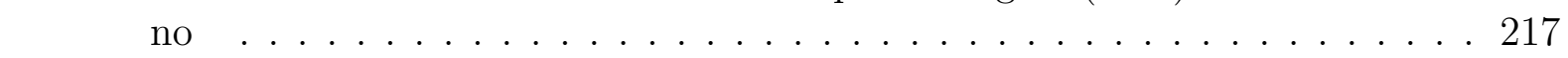

6.31. Deskriptive Statistik für die SPFD der Anaphern-Region (IA 6) . . . . . . . 218

6.32. Deskriptive Statistik für die RPD der Anaphern-Region (IA 6) . . . . . . . 218

6.33. Inferenzstatistik für die RPD der Anaphern-Region (IA 6) $\ldots$. . . . . . . . . 218

6.34. Deskriptive Statistik für die TRT der Anaphern-Region (IA 6) . . . . . . . 219

6.35. Inferenzstatistik für die TRT der Anaphern-Region (IA 6) $\quad$. . . . . . . . . . 219

6.36. Inferenzstatistik für die TRT der Anaphern-Region (IA 6) ohne Bed. Mono 219

6.37. Deskriptive Statistik für die ROCs der Anaphern-Region (IA 6) . . . . . . . 221

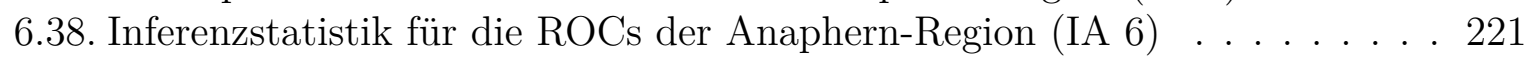

6.39. Deskriptive Statistik für die RICs der Anaphern-Region (IA 6) . . . . . . . 221

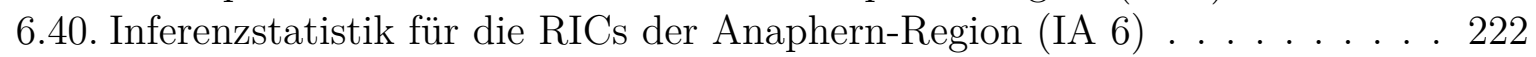

6.41. Inferenzstatistik für die RICs der Anaphern-Region (IA 6) ohne Bed. Mono 223

6.42. Deskriptive Statistik für die FPFD der Satzergänzung (IA 7) . . . . . . . . 224

6.43. Inferenzstatistik für die FPFD der Satzergänzung (IA 7) . . . . . . . . . . . . 225

6.44. Deskriptive Statistik für die SPFD der Satzergänzung (IA 7) . . . . . . . . 225

6.45. Deskriptive Statistik für die RPD der Satzergänzung (IA 7) . . . . . . . . . 225

6.46. Inferenzstatistik für die RPD der Satzergänzung (IA 7) . . . . . . . . . . . . 225

6.47. Inferenzstatistik für die RPD der Satzergänzung (IA 7) ohne Bed. Mono . 225

6.48. Deskriptive Statistik für die TRT der Satzergänzung (IA 7) $\ldots$. . . . . . . . 226

6.49. Inferenzstatistik für die TRT der Satzergänzung (IA 7) . . . . . . . . . . . 226

6.50. Inferenzstatistik für die TRT der Satzergänzung (IA 7) ohne Bed. Mono . 226

6.51. Deskriptive Statistik für die ROCs der Satzergänzung (IA 7) . . . . . . . 228

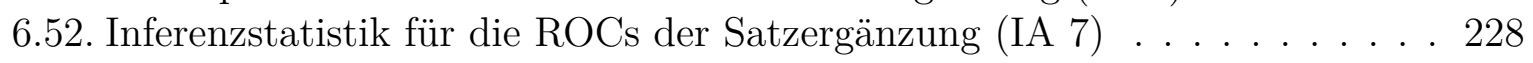

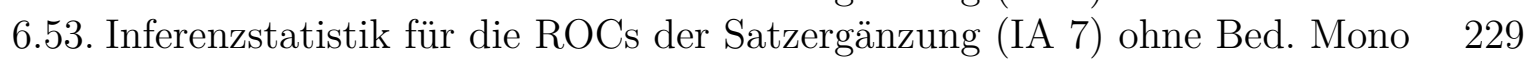

6.54. Deskriptive Statistik für die FPFD der Antezedens-Region (IA 4) mit Berücksichtigung der Längen- und Frequenzresiduen . . . . . . . . . . . . . 230

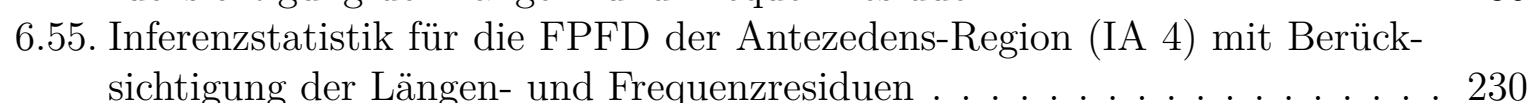

6.56. Deskriptive Statistik für die RPD der Antezedens-Region (IA 4) mit Berücksichtigung der Längen- und Frequenzresiduen . . . . . . . . . . . . . 231

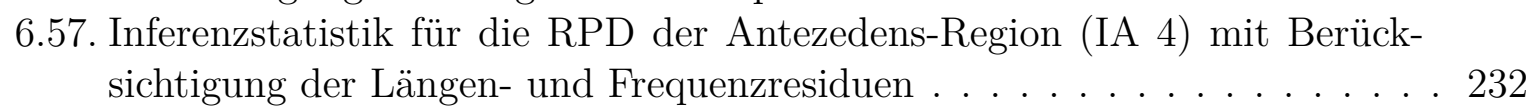

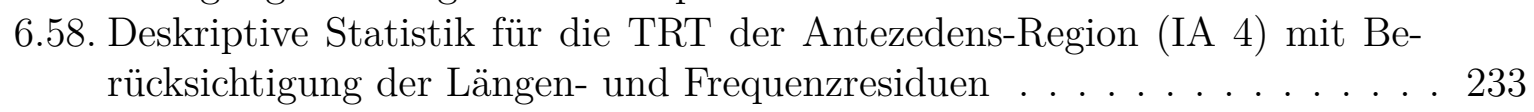

6.59. Inferenzstatistik für die TRT der Antezedens-Region (IA 4) mit Berücksichtigung der Längen- und Frequenzresiduen . . . . . . . . . . . . . . . 234 
6.60. Deskriptive Statistik für die RICs der Antezedens-Region (IA 4) mit Berücksichtigung der Längen- und Frequenzresiduen . . . . . . . . . . . . . 235

6.61. Inferenzstatistik für die RICs der Antezedens-Region (IA 4) mit Berücksichtigung der Längen- und Frequenzresiduen . . . . . . . . . . . . . . . 236

6.62. Deskriptive Statistik für die FPFD der Spillover-Region (IA 5) mit Berücksichtigung der Längenresiduen . . . . . . . . . . . . . . . . . . . . 237

6.63. Inferenzstatistik für die FPFD der Spillover-Region (IA 5) mit Berücksichtigung der Längenresiduen . . . . . . . . . . . . . . . . . . . 237

6.64. Deskriptive Statistik für die RPD der Spillover-Region (IA 5) mit Berücksichtigung der Längenresiduen . . . . . . . . . . . . . . . . . 238

6.65. Inferenzstatistik für die RPD der Spillover-Region (IA 5) mit Berücksichtigung der Längenresiduen . . . . . . . . . . . . . . . . . . . . . . 238

6.66. Deskriptive Statistik für die TRT der Spillover-Region (IA 5) mit Berücksichtigung der Längenresiduen . . . . . . . . . . . . . . . . . . . 239

6.67. Inferenzstatistik für die TRT der Spillover-Region (IA 5) mit Berücksichtigung der Längenresiduen . . . . . . . . . . . . . . . . . 239

6.68. Zusammenfassung der Hypothesen und deskriptiven Ergebnisse für die verschiedenen Interest Areas (IAs) und abhängigen Variablen (AVn) . . . . . . 242

6.69. Deskriptive Statistik für die TRT der Anaphern-Region (IA 6) für den Faktor RZK (ohne Bed. Mono) $\ldots \ldots \ldots \ldots . \ldots \ldots 243$

6.70. Inferenzstatistik für die TRT der Anaphern-Region (IA 6) für den Faktor

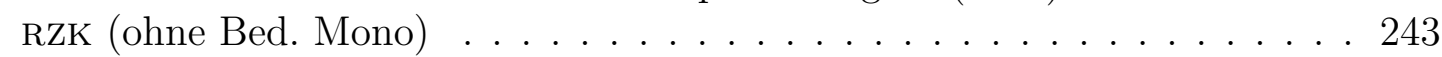

6.71. Deskriptive Statistik für die TRT der Anaphern-Region (IA 6) für den \begin{tabular}{|l|l|l}
\hline Faktor RZK in Abhängigkeit vom Faktor STRUKTUR (ohne Bed. Mono) & . . 244 \\
\hline
\end{tabular}

6.72. Inferenzstatistik für die TRT der Anaphern-Region (IA 6) für den Faktor RZK in Abhängigkeit vom Faktor STRUKTUR (ohne Bed. Mono)

6.73. Deskriptive Statistik für die TRT der Anaphern-Region (IA 6) für den Faktor N2-KONKRETHEIT (ohne Bed. Mono) . . . . . . . . . . . . . . 246

6.74. Inferenzstatistik für die TRT der Anaphern-Region (IA 6) für den Faktor N2-KOnKRETHEIT (ohne Bed. Mono) . . . . . . . . . . . . . 246

6.75. Deskriptive Statistik für die TRT der Anaphern-Region (IA 6) für den Faktor NOMINALISIERUNGSLESART (nur Bed. RK)] . . . . . . . . . . . . 247

6.76. Inferenzstatistik für die TRT der Anaphern-Region (IA 6) für den Faktor NOMINALISIERUNGSLESART (nur Bed. RK) . . . . . . . . . . . . . 248

6.77. Deskriptive Statistik für die TRT der Anaphern-Region (IA 6) für den

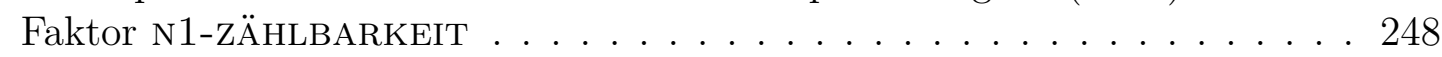

6.78. Inferenzstatistik für die TRT der Anaphern-Region (IA 6) für den Faktor

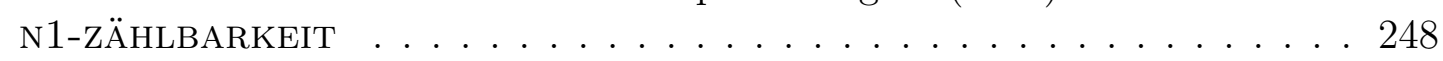

6.79. Deskriptive Statistik für die Anaphern-Region (IA 6) für den Faktor GENUS 250 6.80. Inferenzstatistik für die Anaphern-Region (IA 6) für den Faktor GENUS . . 250

6.81. Deskriptive Statistik für die TRT der Satzergänzung (IA 7) für den Faktor

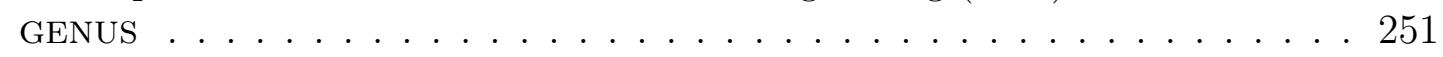

6.82. Inferenzstatistik für die TRT der Satzergänzung (IA 7) für den Faktor GENUS251

6.83. Deskriptive Statistik für die Längenresiduen der FPFD der AntezedensRegion (IA 4) für den Faktor NN-FREQUENZ . . . . . . . . . . . . . . . . 253

6.84. Inferenzstatistik für die Längenresiduen der FPFD der Antezedens-Region

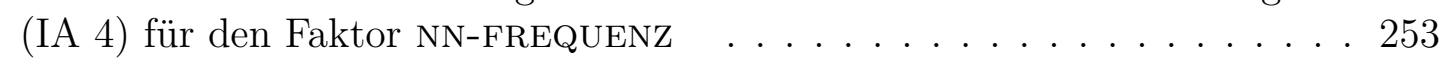


6.85. Deskriptive Statistik für die TRT der Anaphern-Region (IA 6) für den Faktor N1-FREQUENZ . . . . . . . . . . . . . . . . 254

6.86. Inferenzstatistik für die TRT der Anaphern-Region (IA 6) für den Faktor

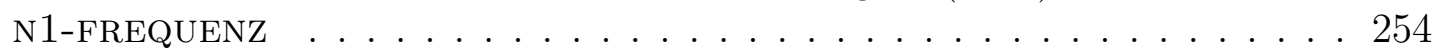

6.87. Deskriptive Statistik für die TRT der Anaphern-Region (IA 6) für den

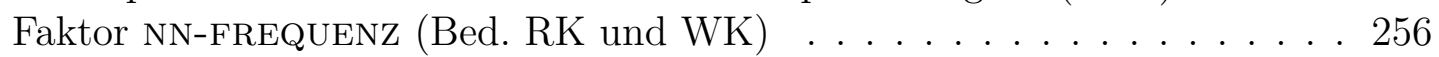

6.88. Inferenzstatistik für die TRT der Anaphern-Region (IA 6) für den Faktor NN-FREQUENZ (Bed. RK und WK) . . . . . . . . . . . . . . 256

6.89. Deskriptive Statistik der Reaktionszeiten (RT) der auf die Anaphernresolution abzielenden Verständnisfragen (Werte logarithmiert und bereinigt um

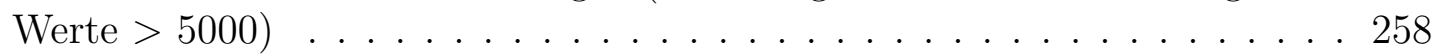

6.90. Gegenüberstellung der Ergebnisse von Experiment 3a (Satzergänzungsexperiment) und 3c (Eye-Tracking); Vergleich der N1-Zugänglichkeit in Abhängigkeit von den jeweiligen Bedingungen; Angabe eines inferenzstatisti-

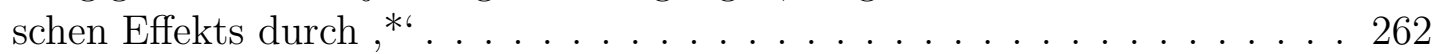

7.1. Modell einer Faktorenhierarchie nach Effektstärke für verschiedene N1Eigenschaften . . . . . . . . . . . . . . . . . 278

C.2. Verteilung möglicher Einflussgrößen zwischen Items und Bedingungen . . . 331

C.5. Mittelwerte und Standardabweichungen des Kohärenzratings für alle Items und Bedingungen des Faktors STRUKTUR . . . . . . . . . . . . . . . 350 


\section{Abbildungsverzeichnis}

3.1. Screenshot aus Experiment 1 (Sosci Survey 2.3) . . . . . . . . . . . . . . . . 94

3.2. Plot der um die Zwischensubjektvarianz korrigierten Mittelwerte der N1Bezüge (y-Achse) in Abhängigkeit von den Faktoren ANIMATHEIT und RELATION für die Kompositabedingung; die Fehlerbalken repräsentieren +/-1 Standardfehler des Mittelwerts . . . . . . . . . . . . . . . . . . 96

3.3. Plot der um die Zwischensubjektvarianz korrigierten Mittelwerte der N1Bezüge (y-Achse) in Abhängigkeit von den Faktoren FORM, ANIMATHEIT

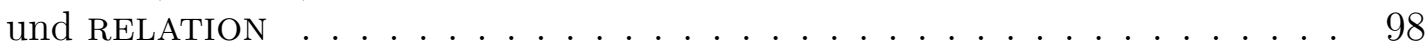

4.1. Verschiedene kategoriale Status von N1 angeordnet auf einer Skala mit steigender Zugänglichkeit . . . . . . . . . . . . . . . . . . . . . . . 150

5.1. Screenshot aus Experiment $2($ OnExp 1.2$)$. . . . . . . . . . . . . . . 160

5.2. Plot der um die Zwischensubjektvarianz korrigierten Mittelwerte der N1Bezüge (y-Achse) in Abhängigkeit von den Faktoren RZK und STRUKTUR; die Fehlerbalken repräsentieren $+/-1$ Standardfehler des Mittelwerts $\ldots 162$

5.3. Plot der um die Zwischensubjektvarianz korrigierten Mittelwerte der N1Bezüge (y-Achse) in Abhängigkeit von den Faktoren RZK, STRUKTUR und

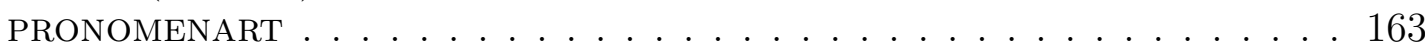

6.1. Plot der um die Zwischensubjektvarianz korrigierten Mittelwerte der N1Bezüge (y-Achse) in Abhängigkeit vom Faktor sTRUKTUR (x-Achse) auf dem gesamten Datensatz; die Fehlerbalken repräsentieren +/-1 Standardfehler des Mittelwerts . . . . . . . . . . . . . . . . . . . . . 186

6.2. Plot der Mittelwerte der relativen Häufigkeit der N1-Bezüge auf dem gekürzten Datensatz für den Faktor STRUKTUR . . . . . . . . . . . . . . . . . 187

6.3. Plot der Mittelwerte der relativen Häufigkeit der N1-Bezüge auf dem gekürzten Datensatz für den Faktor RZK . . . . . . . . . . . . . . . . . . . . 190

6.4. Plot der Mittelwerte der relativen Häufigkeit der N1-Bezüge auf dem gekürzten Datensatz für den Faktor N2-KONKRETHEIT . . . . . . . . . . . . . 191

6.5. Plot der Mittelwerte der relativen Häufigkeit der N1-Bezüge auf dem gekürzten Datensatz für den Faktor NOMINALISIERUNGSLESART . . . . . . . . 192

6.6. Plot der Mittelwerte der relativen Häufigkeit der N1-Bezüge auf dem gekürzten Datensatz für den Faktor N1-zÄHLBARKEIT . . . . . . . . . . . . . . 194

6.7. Plot der Mittelwerte der relativen Häufigkeit der N1-Bezüge auf dem gekürzten Datensatz für den Faktor GENUS . . . . . . . . . . . . . . . . . . 195

6.8. Plot der relativen Häufigkeit der N1-Bezüge auf dem gekürzten Datensatz für den Faktor N1-FREQUENZ . . . . . . . . . . . . . . . . . . . . . . . . 197

6.9. Plot der Mittelwerte der relativen Häufigkeit der N1-Bezüge auf dem gekürzten Datensatz für den Faktor NN-FREQUENZ . . . . . . . . . . . . . . . . 199

6.10. Plot der Mittelwerte des Kohärenzratings für die 24 Items . . . . . . . . . . 205 
6.11. Plot der Mittelwerte des Kohärenzratings (y-Achse) der 24 Filler-Items für die $24 \mathrm{VPn}(\mathrm{x}$-Achse $) \ldots \ldots \ldots \ldots \ldots \ldots$

6.12. Plot der um die Zwischensubjektvarianz korrigierten Mittelwerte des Kohärenzratings (y-Achse) in Abhängigkeit vom Faktor STRUKTUR (x-Achse); die Fehlerbalken repräsentieren +/-1 Standardfehler des Mittelwerts . . . 207

6.13. Beispiel für mögliches Blickverhalten . . . . . . . . . . . . . . . 211

6.14. Plot der um die Zwischensubjektvarianz korrigierten Mittelwerte der FFD der Anaphern-Region (IA 6) (y-Achse) in Abhängigkeit vom Faktor STRUKTUR (x-Achse); die Fehlerbalken repräsentieren $+/-1$ Standardfehler des

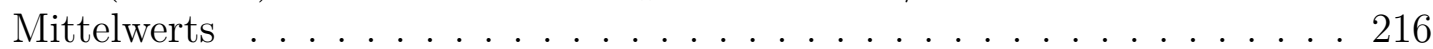

6.15. Plot der Mittelwerte der FPFD der Anaphern-Region (IA 6) für den Faktor

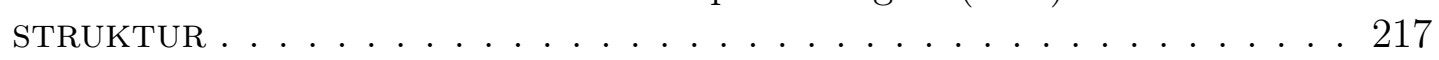

6.16. Plot der Mittelwerte der RPD der Anaphern-Region (IA 6) . . . . . . . . . 219

6.17. Plot der Mittelwerte der TRT der Anaphern-Region (IA 6) . . . . . . . . . 220

6.18. Plot der Mittelwerte der ROCs der Anaphern-Region (IA 6) . . . . . . . . 222

6.19. Plot der Mittelwerte der RICs der Anaphern-Region (IA 6) . . . . . . . . . 222

6.20. Plot der Mittelwerte der FPFD der Satzergänzung (IA 7) . . . . . . . . . . . 224

6.21. Plot der Mittelwerte der RPD der Satzergänzung (IA 7) . . . . . . . . . . . 226

6.22. Plot der Mittelwerte der TRT für die Satzergänzung (IA 7) . . . . . . . . . 227

6.23. Plot der Mittelwerte der ROCs der Satzergänzung (IA 7) . . . . . . . . . . . 228

6.24. Plot der Mittelwerte der FPFD der Antezedens-Region (IA 4) mit Berücksichtigung der Längen- und Frequenzresiduen . . . . . . . . . . . . . . . 231

6.25. Plot der Mittelwerte der RPD der Antezedens-Region (IA 4) mit Berücksichtigung der Längen- und Frequenzresiduen . . . . . . . . . . . . . . . . . 232

6.26. Plot der Mittelwerte der TRT der Antezedens-Region (IA 4) mit Berücksichtigung der Längen- und Frequenzresiduen . . . . . . . . . . . . . . . . 233

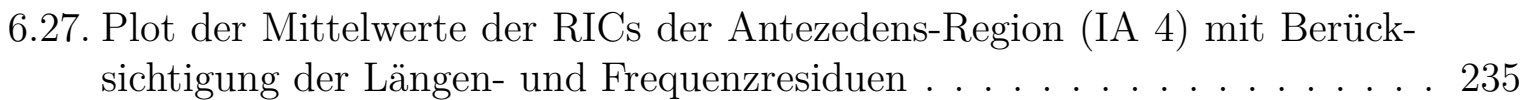

6.28. Plot der Mittelwerte der FPFD der Spillover-Region (IA 5) mit Berücksichtigung der Längenresiduen . . . . . . . . . . . . . . . . . . 237

6.29. Plot der Mittelwerte der RPD der Spillover-Region (IA 5) mit Berücksich-

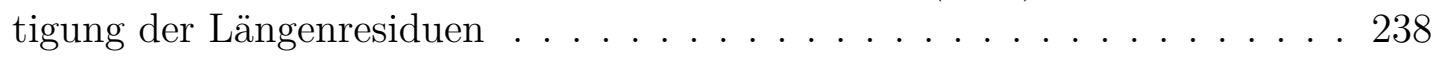

6.30. Plot der Mittelwerte der TRT der Spillover-Region (IA 5) mit Berücksichtigung der Längenresiduen . . . . . . . . . . . . . . . . . . . . . 239

6.31. Plot der Mittelwerte der TRT der Anaphern-Region (IA 6) für den Faktor RZK (ohne Bed. Mono) . . . . . . . . . . . . . . . . . . 243

6.32. Plot der Mittelwerte der TRT der Anaphern-Region (IA 6) für den Faktor RZK in Abhängigkeit vom Faktor STRUKTUR (ohne Bed. Mono) $]$. . . . . . . 244

6.33. Plot der Mittelwerte der TRT der Anaphern-Region (IA 6) für den Faktor N2-KONKRETHEIT (ohne Bed. Mono) . . . . . . . . . . . . . . 246

6.34. Plot der Mittelwerte der TRT der Anaphern-Region (IA 6) für den Faktor NOMINALISIERUNGSLESART (nur Bed. RK) $\ldots \ldots \ldots$. . . . . . . . 247

6.35. Plot der Mittelwerte der TRT der Anaphern-Region (IA 6) für den Faktor

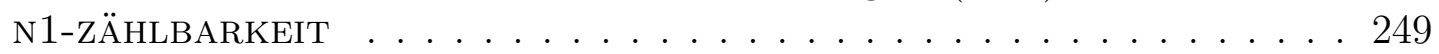

6.36. Plot der Mittelwerte der Längenresiduen der FPFD der Antezedens-Region 2 (IA 4) für den Faktor NN-FREQUENZ . . . . . . . . . . . . . . . . . . . . . 254

6.37. Plot der Mittelwerte der TRT der Anaphern-Region (IA 6) für den Faktor

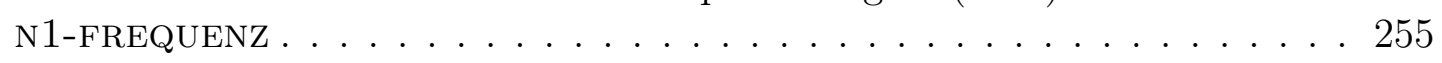


6.38. Plot der Mittelwerte der TRT der Anaphern-Region (IA 6) für den Faktor

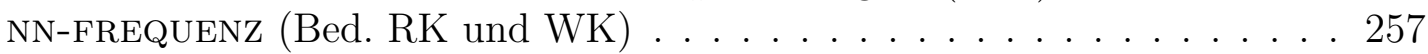

6.39. Plot der Mittelwerte der Reaktionszeiten der auf die Anaphernresolution abzielenden Verständnisfragen . . . . . . . . . . . . . . . . . . 258

6.40. Plot der Mittelwerte der Reaktionszeiten der auf die Anaphernresolution abzielenden Verständnisfragen für die einzelnen Items . . . . . . . . . . . . . 259

B.1. Plot der Mittelwerte der N1-Bezüge in Abhängigkeit von den Faktoren RZK und STRUKTUR auf Item-Ebene . . . . . . . . . . . . . . . . . . . 326

B.2. Plot der Mittelwerte der N1-Bezüge in Abhängigkeit von den Faktoren RZK und STRUKTUR auf VP-Ebene . . . . . . . . . . . . . . . . . . . 327

C.1. Plot der Mittelwerte der relativen Häufigkeit der N1-Bezüge für den Faktor STRUKUTR für die einzelnen Items (gesamter Datensatz) . . . . . . . . . . . 335

C.2. Plot der Mittelwerte der relativen Häufigkeit der N1-Bezüge für die einzelnen Items in allen Bedingungen (gekürzter Datensatz), geordnet nach steigendem Wert . . . . . . . . . . . . . . . . . . . 336

C.3. Plot der Mittelwerte der relativen Häufigkeit der N1-Bezüge für den Faktor STRUKTUR für die einzelnen VPn (gesamter Datensatz) . . . . . . . . . . . 337

C.4. Plot der Mittelwerte des Kohärenzratings für den Faktor STRUKTUR für die einzelnen Items . . . . . . . . . . . . . . . . . . . . 351

C.5. Plot der Mittelwerte des Kohärenzratings für den Faktor STRUKTUR für

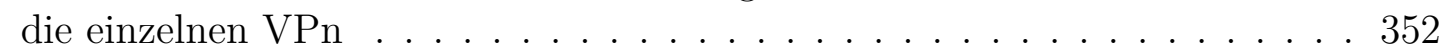

C.6. Plot der Mittelwerte der TRT der Anaphern-Region (IA 6) für den Faktor

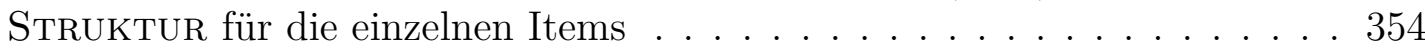

C.7. Plot der Mittelwerte der TRT der Anaphern-Region (IA 6) für die einzelnen Items in allen Bedingungen (gekürzter Datensatz), geordnet nach steigendem Wert . . . . . . . . . . . . . . . . . . . 355

C.8. Plot der Mittelwerte der TRT der Anaphern-Region (IA 6) für den Faktor STRUKTUR für die einzelnen $\mathrm{VPn} \ldots \ldots \ldots$. . . . . . . . . . . 356 



\section{Abkürzungen}

$\begin{array}{ll}\text { A } & \text { Adjektiv } \\ \text { ANOVA } & \text { Analysis of Variance } \\ \text { AP } & \text { Adjektivphrase } \\ \text { AV } & \text { Abhängige Variable } \\ \text { DM } & \text { Distribuierte Morphologie } \\ \text { DP } & \text { Determiniererphrase } \\ \text { DRS } & \text { Diskursrepräsentationsstruktur } \\ \text { DRT } & \text { Diskursrepräsentationstheorie } \\ \text { FFD } & \text { First Fixation Duration } \\ \text { FPFD } & \text { First Pass Fixation Duration } \\ \text { GB } & \text { Government and Binding } \\ \text { IA } & \text { Interest Area } \\ \text { KZG } & \text { Kurzzeitgedächtnis } \\ \text { LF } & \text { Logische Form } \\ \text { LIH } & \text { Lexical Integrity Hypothesis } \\ \text { LMEMs } & \text { Linear Mixed-Effects Models } \\ \text { LZG } & \text { Langzeitgedächtnis } \\ \text { Mono } & \text { Monolexem/monomorphematisches Wort } \\ \text { MW } & \text { Mittelwert } \\ \text { N } & \text { Nomen } \\ \text { N1 } & \text { Nominales Erstglied eines Kompositums } \\ \text { N2 } & \text { Nominales Zweitglied eines Kompositums } \\ \text { NN } & \text { Kompositum mit zwei nominalen Konstituenten } \\ \text { NP } & \text { Nominalphrase } \\ \text { OO } & \text { Kompositum mit opakem Erst- und Zweitglied } \\ \text { OS } & \text { Oberflächenstruktur } \\ \text { OT } & \text { Optimalitätstheorie/Kompositum mit opakem Erst- und trans- } \\ \text { P } & \text { parentem Zweitglied } \\ \text { PF } & \text { Präposition } \\ \text { RIC } & \text { Phonologische Form } \\ \text { RK } & \text { Regression In Count } \\ \text { RN } & \text { Rektionskompositum } \\ \text { ROC } & \text { Randnummer } \\ \text { RPD } & \text { Regression Out Count } \\ \text { RT } & \text { Regression Path Duration } \\ \text { RZK } & \text { Reaktionszeit (reaction time) } \\ \text { SPFD } & \text { Raum-zeitliche Kontiguität } \\ \text { Std. Abw. } & \text { Standardabweichung } \\ \text { TO } & \text { Kompositum mit transparentem Erst- und opakem Zweitglied } \\ & \end{array}$




$\begin{array}{ll}\text { TRT } & \text { Total Reading Time } \\ \text { TS } & \text { Tiefenstruktur } \\ \text { TT } & \text { Kompositum mit transparentem Erst- und Zweitglied } \\ \text { UV } & \text { Unabhängige Variable } \\ \text { V } & \text { Verb } \\ \text { VP } & \text { Versuchsperson/Verbalphrase } \\ \text { WK } & \text { Wurzelkompositum }\end{array}$




\section{A. Anhang zu Experiment 1}

\section{A.1. Komposita}

\begin{tabular}{llll}
\hline Item Nr. & ANIMATHEIT & RELATION & Kompositum \\
\hline 1 & -animat & HAVE & Rosenbeet \\
2 & -animat & HAVE & Bücherregal \\
3 & -animat & HAVE & Kleiderschrank \\
4 & -animat & HAVE & Rosinenbrötchen \\
5 & -animat & HAVE & Früchtekuchen \\
6 & -animat & HAVE & Stickeralbum \\
7 & -animat & HAVE & Kräutertee \\
8 & -animat & HAVE & Säulengang \\
\hline 9 & -animat & FOR & Dosenöffner \\
10 & -animat & FOR & Kettenöl \\
11 & -animat & FOR & Kerzenständer \\
12 & -animat & FOR & Nervengift \\
13 & -animat & FOR & Zigarrenmesser \\
14 & -animat & FOR & Gurkenschäler \\
15 & -animat & FOR & Flusensieb \\
16 & -animat & FOR & Getränkewagen \\
\hline 17 & +animat & HAVE & Hühnerstall \\
18 & +animat & HAVE & Ziegengehege \\
19 & +animat & HAVE & Mäusezirkus \\
20 & +animat & HAVE & Schwalbennest \\
21 & +animat & HAVE & Schweinetransporter \\
22 & +animat & HAVE & Tigerkäfig \\
23 & +animat & HAVE & Elefantenreservat \\
24 & +animat & HAVE & Forellenbecken \\
\hline 25 & +animat & FOR & Hundenapf \\
26 & +animat & FOR & Mückenspray \\
27 & +animat & FOR & Läusekamm \\
28 & +animat & FOR & Meisenknödel \\
29 & +animat & FOR & Rattengift \\
30 & +animat & FOR & Hamsterrad \\
31 & +animat & FOR & Katzenfutter \\
32 & +animat & FOR & Krötenzaun \\
\hline & & &
\end{tabular}




\section{A.2. Items}

\begin{tabular}{|c|c|c|c|c|}
\hline $\begin{array}{l}\text { Item } \\
\text { Nr. }\end{array}$ & $\begin{array}{l}\text { ANIMAT- } \\
\text { HEIT }\end{array}$ & RELATION & $\begin{array}{l}\text { Ante- } \\
\text { zedens }\end{array}$ & Stimulus \\
\hline 1 & -animat & HAVE & N1 & $\begin{array}{l}\text { Leon hat in seinem Vorgarten das bunte Rosen- } \\
\text { beet angelegt. Sie ... }\end{array}$ \\
\hline 1 & - animat & HAVE & NP & $\begin{array}{l}\text { Leon hat in seinem Vorgarten das bunte Beet } \\
\text { mit den Rosen angelegt. Sie ... }\end{array}$ \\
\hline 2 & -animat & HAVE & N1 & $\begin{array}{l}\text { Justus hat in seinem Wohnzimmer das rustikale } \\
\text { Bücherregal aufgestellt. Sie ... }\end{array}$ \\
\hline 2 & -animat & HAVE & $\mathrm{NP}$ & $\begin{array}{l}\text { Justus hat in seinem Wohnzimmer das rustikale } \\
\text { Regal mit den Büchern aufgestellt. Sie ... }\end{array}$ \\
\hline 3 & -animat & HAVE & N1 & $\begin{array}{l}\text { Jonas hat für einen guten Zweck den vollgestopf- } \\
\text { ten Kleiderschrank ausgemistet. Sie ... }\end{array}$ \\
\hline 3 & -animat & HAVE & NP & $\begin{array}{l}\text { Jonas hat für einen guten Zweck den vollgestopf- } \\
\text { ten Schrank mit den Kleidern ausgemistet. Sie } \\
\text {.. }\end{array}$ \\
\hline 4 & -animat & HAVE & N1 & $\begin{array}{l}\text { Lukas hat zum Frühstück das warme Rosinen- } \\
\text { brötchen gegessen. Sie ... }\end{array}$ \\
\hline 4 & -animat & HAVE & NP & $\begin{array}{l}\text { Lukas hat zum Frühstück das warme Brötchen } \\
\text { mit den Rosinen gegessen. Sie ... }\end{array}$ \\
\hline 5 & -animat & HAVE & N1 & $\begin{array}{l}\text { Der Bäcker hat heute Morgen den beliebten } \\
\text { Früchtekuchen gebacken. Sie... }\end{array}$ \\
\hline 5 & - animat & HAVE & NP & $\begin{array}{l}\text { Der Bäcker hat heute Morgen den beliebten Ku- } \\
\text { chen mit den Früchten gebacken. Sie ... }\end{array}$ \\
\hline 6 & -animat & HAVE & N1 & $\begin{array}{l}\text { Der Neffe hat mit viel Sorgfalt das umfangreiche } \\
\text { Stickeralbum gepflegt. Sie ... }\end{array}$ \\
\hline 6 & -animat & HAVE & $\mathrm{NP}$ & $\begin{array}{l}\text { Der Neffe hat mit viel Sorgfalt das umfangreiche } \\
\text { Album mit den Stickern gepflegt. Sie... }\end{array}$ \\
\hline 7 & -animat & HAVE & N1 & $\begin{array}{l}\text { Der Opa hat zur Beruhigung den wohltuenden } \\
\text { Kräutertee aufgebrüht. Sie ... }\end{array}$ \\
\hline 7 & -animat & HAVE & NP & $\begin{array}{l}\text { Der Opa hat zur Beruhigung den wohltuenden } \\
\text { Tee mit den Kräutern aufgebrüht. Sie ... }\end{array}$ \\
\hline 8 & -animat & HAVE & N1 & $\begin{array}{l}\text { Der Vater hat im Urlaub den restaurierten Säu- } \\
\text { lengang bewundert. Sie ... }\end{array}$ \\
\hline 8 & -animat & HAVE & $\mathrm{NP}$ & $\begin{array}{l}\text { Der Vater hat im Urlaub den restaurierten Gang } \\
\text { mit den Säulen bewundert. Sie ... }\end{array}$ \\
\hline 9 & -animat & FOR & N1 & $\begin{array}{l}\text { Mirko hat zum Campen den praktischen Dosen- } \\
\text { öffner mitgenommen. Sie ... }\end{array}$ \\
\hline 9 & -animat & FOR & NP & $\begin{array}{l}\text { Mirko hat zum Campen den praktischen Öffner } \\
\text { für die Dosen mitgenommen. Sie ... }\end{array}$ \\
\hline 10 & -animat & FOR & N1 & $\begin{array}{l}\text { Hannes hat seit der Lehre auf das rückfettende } \\
\text { Kettenöl geschworen. Sie ... }\end{array}$ \\
\hline 10 & -animat & FOR & $\mathrm{NP}$ & $\begin{array}{l}\text { Hannes hat seit der Lehre auf das rückfettende } \\
\text { Öl für die Ketten geschworen. Sie ... }\end{array}$ \\
\hline 11 & -animat & FOR & N1 & $\begin{array}{l}\text { Sebastian hat für das Ambiente den gewaltigen } \\
\text { Kerzenständer aufgestellt. Sie ... }\end{array}$ \\
\hline
\end{tabular}




\begin{tabular}{|c|c|c|c|c|}
\hline 11 & -animat & FOR & NP & $\begin{array}{l}\text { Sebastian hat für das Ambiente den gewaltigen } \\
\text { Ständer für die Kerzen aufgestellt. Sie ... }\end{array}$ \\
\hline 12 & -animat & FOR & N1 & $\begin{array}{l}\text { Oliver hat für die Biologieprüfung viel über das } \\
\text { tödliche Nervengift gelernt. Sie ... }\end{array}$ \\
\hline 12 & -animat & FOR & NP & $\begin{array}{l}\text { Oliver hat für die Biologieprüfung viel über das } \\
\text { tödliche Gift für die Nerven gelernt. Sie ... }\end{array}$ \\
\hline 13 & -animat & $\overline{\text { FOR }}$ & N1 & $\begin{array}{l}\text { Der Professor hat jeden Sonntag das silberne Zi- } \\
\text { garrenmesser benutzt. Sie ... }\end{array}$ \\
\hline 13 & -animat & FOR & NP & $\begin{array}{l}\text { Der Professor hat jeden Sonntag das silberne } \\
\text { Messer für die Zigarren benutzt. Sie ... }\end{array}$ \\
\hline 14 & -animat & FOR & N1 & $\begin{array}{l}\text { Der Kassierer hat aufgrund eines Angebots den } \\
\text { handlichen Gurkenschäler gekauft. Sie ... }\end{array}$ \\
\hline 14 & -animat & FOR & NP & $\begin{array}{l}\text { Der Kassierer hat aufgrund eines Angebots den } \\
\text { handlichen Schäler für die Gurken gekauft. Sie } \\
\text {.. }\end{array}$ \\
\hline 15 & -animat & FOR & N1 & $\begin{array}{l}\text { Der Installateur hat am Trockner letztlich nur } \\
\text { das defekte Flusensieb gewechselt. Sie ... }\end{array}$ \\
\hline 15 & -animat & FOR & NP & $\begin{array}{l}\text { Der Installateur hat am Trockner letztlich nur } \\
\text { das defekte Sieb für die Flusen gewechselt. Sie } \\
\text {.. }\end{array}$ \\
\hline 16 & -animat & FOR & N1 & $\begin{array}{l}\text { Der Kellner hat im neuen Lokal kaum Platz für } \\
\text { den praktischen Getränkewagen gefunden. Sie }\end{array}$ \\
\hline 16 & -animat & FOR & NP & $\begin{array}{l}\text { Der Kellner hat im neuen Lokal kaum Platz für } \\
\text { den praktischen Wagen für die Getränke gefun- } \\
\text { den. Sie ... }\end{array}$ \\
\hline 17 & + animat & HAVE & N1 & $\begin{array}{l}\text { Günther hat heute Morgen den alten Hühner- } \\
\text { stall ausgemistet. Sie ... }\end{array}$ \\
\hline 17 & + animat & HAVE & NP & $\begin{array}{l}\text { Günther hat heute Morgen den alten Stall mit } \\
\text { den Hühnern ausgemistet. Sie ... }\end{array}$ \\
\hline 18 & + animat & HAVE & N1 & $\begin{array}{l}\text { Joseph hat mit großer Mühe das heruntergekom- } \\
\text { mene Ziegengehege repariert. Sie ... }\end{array}$ \\
\hline 18 & +animat & HAVE & NP & $\begin{array}{l}\text { Joseph hat mit großer Mühe das heruntergekom- } \\
\text { mene Gehege mit den Ziegen repariert. Sie . . }\end{array}$ \\
\hline 19 & +animat & HAVE & N1 & $\begin{array}{l}\text { Ingo hat mit viel Liebe den lustigen Mäusezirkus } \\
\text { aufgebaut. Sie ... }\end{array}$ \\
\hline 19 & +animat & HAVE & NP & $\begin{array}{l}\text { Ingo hat mit viel Liebe den lustigen Zirkus mit } \\
\text { den Mäusen aufgebaut. Sie ... }\end{array}$ \\
\hline 20 & +animat & HAVE & N1 & $\begin{array}{l}\text { Michael hat am Dachgiebel das neue Schwalben- } \\
\text { nest entdeckt. Sie ... }\end{array}$ \\
\hline 20 & + animat & HAVE & NP & $\begin{array}{l}\text { Michael hat am Dachgiebel das neue Nest mit } \\
\text { den Schwalben entdeckt. Sie ... }\end{array}$ \\
\hline 21 & +animat & HAVE & N1 & $\begin{array}{l}\text { Der LKW-Fahrer hat zum wiederholten Mal } \\
\text { den stinkenden Schweinetransporter gefahren. } \\
\text { Sie ... }\end{array}$ \\
\hline 21 & +animat & HAVE & NP & $\begin{array}{l}\text { Der LKW-Fahrer hat zum wiederholten Mal den } \\
\text { stinkenden Transporter mit den Schweinen ge- } \\
\text { fahren. Sie ... }\end{array}$ \\
\hline
\end{tabular}




\begin{tabular}{|c|c|c|c|c|}
\hline 22 & +animat & HAVE & N1 & $\begin{array}{l}\text { Der Tierpfleger hat mit großer Vorsicht den lan- } \\
\text { gen Tigerkäfig betreten. Sie ... }\end{array}$ \\
\hline 22 & + animat & HAVE & $\mathrm{NP}$ & $\begin{array}{l}\text { Der Tierpfleger hat mit großer Vorsicht den lan- } \\
\text { gen Käfig mit den Tigern betreten. Sie ... }\end{array}$ \\
\hline 23 & +animat & HAVE & N1 & $\begin{array}{l}\text { Der Tourist hat aus der Ferne das beeindrucken- } \\
\text { de Elefantenreservat fotografiert. Sie ... }\end{array}$ \\
\hline 23 & +animat & HAVE & $\mathrm{NP}$ & $\begin{array}{l}\text { Der Tourist hat aus der Ferne das beeindrucken- } \\
\text { de Reservat mit den Elefanten fotografiert. Sie } \\
\text {... }\end{array}$ \\
\hline 24 & +animat & HAVE & N1 & $\begin{array}{l}\text { Der Nachbar hat im Frühjahr das überfüllte Fo- } \\
\text { rellenbecken vergrößert. Sie . . }\end{array}$ \\
\hline 24 & +animate & HAVE & NP & $\begin{array}{l}\text { Der Nachbar hat im Frühjahr das überfüllte Be- } \\
\text { cken mit den Forellen vergrößert. Sie ... }\end{array}$ \\
\hline 25 & +animate & FOR & N1 & $\begin{array}{l}\text { Florian hat im Auslauf den großen Hundenapf } \\
\text { aufgefüllt. Sie ... }\end{array}$ \\
\hline 25 & +animat & FOR & NP & $\begin{array}{l}\text { Florian hat im Auslauf den großen Napf für die } \\
\text { Hunde aufgefüllt. Sie ... }\end{array}$ \\
\hline 26 & +animat & FOR & N1 & $\begin{array}{l}\text { Matthias hat für den Urlaub das altbewährte } \\
\text { Mückenspray eingepackt. Sie ... }\end{array}$ \\
\hline 26 & +animat & FOR & $\mathrm{NP}$ & $\begin{array}{l}\text { Matthias hat für den Urlaub das altbewährte } \\
\text { Spray für die Mücken eingepackt. Sie ... }\end{array}$ \\
\hline 27 & +animat & FOR & N1 & $\begin{array}{l}\text { Philipp hat dreimal am Tag den elektrischen } \\
\text { Läusekamm benutzt. Sie ... }\end{array}$ \\
\hline 27 & +animat & FOR & NP & $\begin{array}{l}\text { Philipp hat dreimal am Tag den elektrischen } \\
\text { Kamm für die Läuse benutzt. Sie ... }\end{array}$ \\
\hline 28 & +animat & FOR & N1 & $\begin{array}{l}\text { Christoph hat im tiefen Winter den nahrhaften } \\
\text { Meisenknödel aufgehängt. Sie ... }\end{array}$ \\
\hline 28 & +animat & FOR & $\mathrm{NP}$ & $\begin{array}{l}\text { Christoph hat im tiefen Winter den nahrhaften } \\
\text { Knödel für die Meisen aufgehängt. Sie ... }\end{array}$ \\
\hline 29 & +animat & FOR & N1 & $\begin{array}{l}\text { Der Kleingärtner hat um den Geräteschuppen } \\
\text { das aggressive Rattengift ausgestreut. Sie ... }\end{array}$ \\
\hline 29 & + animat & FOR & $\mathrm{NP}$ & $\begin{array}{l}\text { Der Kleingärtner hat um den Geräteschuppen } \\
\text { das aggressive Gift für die Ratten ausgestreut. } \\
\text { Sie ... }\end{array}$ \\
\hline 30 & +animat & FOR & N1 & $\begin{array}{l}\text { Der Schüler hat in den Sommerferien das gelbe } \\
\text { Hamsterrad geputzt. Sie ... }\end{array}$ \\
\hline 30 & +animat & FOR & $\mathrm{NP}$ & $\begin{array}{l}\text { Der Schüler hat in den Sommerferien das gelbe } \\
\text { Rad für die Hamster geputzt. Sie ... }\end{array}$ \\
\hline 31 & +animat & FOR & N1 & $\begin{array}{l}\text { Der Praktikant hat in bester Absicht das teure } \\
\text { Katzenfutter bestellt. Sie ... }\end{array}$ \\
\hline 31 & +animat & FOR & NP & $\begin{array}{l}\text { Der Praktikant hat in bester Absicht das teure } \\
\text { Futter für die Katzen bestellt. Sie ... }\end{array}$ \\
\hline 32 & +animat & FOR & N1 & $\begin{array}{l}\text { Der Tierschützer hat an der Bundesstraße den } \\
\text { provisorischen Krötenzaun aufgespannt. Sie ... }\end{array}$ \\
\hline 32 & +animat & FOR & $\mathrm{NP}$ & $\begin{array}{l}\text { Der Tierschützer hat an der Bundesstraße den } \\
\text { provisorischen Zaun für die Kröten aufgespannt. } \\
\text { Sie ... }\end{array}$ \\
\hline
\end{tabular}




\section{B. Anhang zu Experiment 2}

\section{B.1. Komposita}

\begin{tabular}{|c|c|c|c|}
\hline Item Nr. & STRUKTUR & RZK & Kompositum \\
\hline 1 & RK & -RZK & Filmbewertung \\
\hline 1 & WK & $+\mathrm{RZK}$ & Filmbeispiel \\
\hline 1 & RK & $+\mathrm{RZK}$ & Filmbearbeitung \\
\hline 1 & WK & $-\mathrm{RZK}$ & Filmrecht \\
\hline 2 & RK & -RZK & Autoversicherung \\
\hline 2 & WK & $+\mathrm{RZK}$ & Autopanne \\
\hline 2 & RK & $+\mathrm{RZK}$ & Autovermietung \\
\hline 2 & WK & $-\mathrm{RZK}$ & Autofähre \\
\hline 3 & RK & -RZK & Fußbekleidung \\
\hline 3 & WK & $+\mathrm{RZK}$ & Fußspitze \\
\hline 3 & RK & $+\mathrm{RZK}$ & Fußwaschung \\
\hline 3 & WK & $-\mathrm{RZK}$ & Fußmatte \\
\hline 4 & RK & $-\mathrm{RZK}$ & Ölpumpe \\
\hline 4 & WK & $+\mathrm{RZK}$ & Ölreserve \\
\hline 4 & RK & $+\mathrm{RZK}$ & Ölgewinnung \\
\hline 4 & WK & $-\mathrm{RZK}$ & Öltank \\
\hline 5 & RK & - RZK & Geldtransporter \\
\hline 5 & WK & $+\mathrm{RZK}$ & Geldmünze \\
\hline 5 & RK & $+\mathrm{RZK}$ & Geldtransport \\
\hline 5 & WK & $-\mathrm{RZK}$ & Geldautomat \\
\hline 6 & RK & -RZK & Fensterreiniger \\
\hline 6 & WK & $+\mathrm{RZK}$ & Fensterspalt \\
\hline 6 & RK & $+\mathrm{RZK}$ & Fensteröffnung \\
\hline 6 & WK & $-\mathrm{RZK}$ & Fensterladen \\
\hline 7 & RK & - RZK & Wasserkocher \\
\hline 7 & WK & $+\mathrm{RZK}$ & Wasserfontäne \\
\hline 7 & RK & $+\mathrm{RZK}$ & Wasserzufuhr \\
\hline 7 & WK & $-\mathrm{RZK}$ & Wasserpfeife \\
\hline 8 & RK & - RZK & Hausverwaltung \\
\hline 8 & WK & $+\mathrm{RZK}$ & Hausfassade \\
\hline 8 & RK & $+\mathrm{RZK}$ & Hausputz \\
\hline 8 & WK & $-\mathrm{RZK}$ & Hausschlüssel \\
\hline 9 & RK & $-\mathrm{RZK}$ & Kabelbinder \\
\hline 9 & WK & $+\mathrm{RZK}$ & Kabelsalat \\
\hline 9 & $\mathrm{RK}$ & $+\mathrm{RZK}$ & Kabelverbindung \\
\hline 9 & WK & $-\mathrm{RZK}$ & Kabelkanal \\
\hline
\end{tabular}




\begin{tabular}{|c|c|c|c|}
\hline 10 & RK & - RZK & Fleischschneider \\
\hline 10 & WK & $+\mathrm{RZK}$ & Fleischsorte \\
\hline 10 & RK & $+\mathrm{RZK}$ & Fleischgenuss \\
\hline 10 & WK & $-\mathrm{RZK}$ & Fleischtopf \\
\hline 11 & RK & $-\mathrm{RZK}$ & Metallgießerei \\
\hline 11 & WK & $+\mathrm{RZK}$ & Metallscheibe \\
\hline 11 & RK & $+\mathrm{RZK}$ & Metallgestaltung \\
\hline 11 & WK & $-\mathrm{RZK}$ & Metallwerkstatt \\
\hline 12 & RK & $-\mathrm{RZK}$ & Gastanker \\
\hline 12 & WK & $+\mathrm{RZK}$ & Gaswolke \\
\hline 12 & RK & $+\mathrm{RZK}$ & Gasaustausch \\
\hline 12 & WK & $-\mathrm{RZK}$ & Gaslaterne \\
\hline 13 & RK & $-\mathrm{RZK}$ & Müllvermeidung \\
\hline 13 & WK & $+\mathrm{RZK}$ & Müllmenge \\
\hline 13 & RK & $+\mathrm{RZK}$ & Müllverwertung \\
\hline 13 & WK & $-\mathrm{RZK}$ & Müllauto \\
\hline 14 & RK & $-\mathrm{RZK}$ & Haarbürste \\
\hline 14 & WK & $+\mathrm{RZK}$ & Haarstrang \\
\hline 14 & RK & $+\mathrm{RZK}$ & Haarpflege \\
\hline 14 & WK & $-\mathrm{RZK}$ & Haarspange \\
\hline 15 & RK & $-\mathrm{RZK}$ & Programmbeschreibung \\
\hline 15 & WK & $+\mathrm{RZK}$ & Programmdatei \\
\hline 15 & RK & $+\mathrm{RZK}$ & Programmentwicklung \\
\hline 15 & WK & $-\mathrm{RZK}$ & Programmname \\
\hline 16 & RK & $-\mathrm{RZK}$ & Kopfstütze \\
\hline 16 & WK & $+\mathrm{RZK}$ & Kopfform \\
\hline 16 & RK & $+\mathrm{RZK}$ & Kopfhaltung \\
\hline 16 & WK & $-\mathrm{RZK}$ & Kopfhöhe \\
\hline 17 & RK & $-\mathrm{RZK}$ & Salzstreuer \\
\hline 17 & WK & $+\mathrm{RZK}$ & Salzkonzentration \\
\hline 17 & RK & $+\mathrm{RZK}$ & Salzgewinnung \\
\hline 17 & WK & $-\mathrm{RZK}$ & Salzstraße \\
\hline 18 & RK & $-\mathrm{RZK}$ & Wasserzähler \\
\hline 18 & WK & $+\mathrm{RZK}$ & Wassersäule \\
\hline 18 & RK & $+\mathrm{RZK}$ & Wasserverschmutzung \\
\hline 18 & WK & $-\mathrm{RZK}$ & Wasserflasche \\
\hline 19 & RK & $-\mathrm{RZK}$ & Tabakvermarktung \\
\hline 19 & WK & $+\mathrm{RZK}$ & Tabakblatt \\
\hline 19 & RK & $+\mathrm{RZK}$ & Tabakverarbeitung \\
\hline 19 & WK & $-\mathrm{RZK}$ & Tabakdose \\
\hline 20 & RK & $-\mathrm{RZK}$ & Panzerabwehr \\
\hline 20 & WK & $+\mathrm{RZK}$ & Panzergruppe \\
\hline 20 & RK & $+\mathrm{RZK}$ & Panzerbekämpfung \\
\hline 20 & WK & $-\mathrm{RZK}$ & Panzerkette \\
\hline 21 & RK & $-\mathrm{RZK}$ & Texterkennung \\
\hline 21 & WK & $+\mathrm{RZK}$ & Textzeile \\
\hline 21 & RK & $+\mathrm{RZK}$ & Textsammlung \\
\hline
\end{tabular}




\begin{tabular}{llll}
21 & WK & - RZK & Textfeld \\
\hline 22 & RK & - RZK & Papiermühle \\
22 & WK & +RZK & Papiertüte \\
22 & RK & +RZK & Papierherstellung \\
22 & WK & - RZK & Papiermaschine \\
\hline 23 & RK & - RZK & Theaterförderung \\
23 & WK & +RZK & Theaterpremiere \\
23 & RK & +RZK & Theatervorführung \\
23 & WK & - RZK & Theaterschminke \\
\hline 24 & RK & - RZK & Videoprojektor \\
24 & WK & +RZK & Videokassette \\
24 & RK & +RZK & Videoverleih \\
24 & WK & - RZK & Videorekorder \\
\hline 25 & RK & - RZK & Geldforderung \\
25 & WK & +RZK & Geldschein \\
25 & RK & +RZK & Geldverschwendung \\
25 & WK & - RZK & Geldschrank \\
\hline 26 & RK & - RZK & Ölfilter \\
26 & WK & +RZK & Ölfilm \\
26 & RK & +RZK & Ölwechsel \\
26 & WK & - RZK & Öllampe \\
\hline 27 & RK & - RZK & Bettbezug \\
27 & WK & +RZK & Bettpfosten \\
27 & RK & +RZK & Bettreinigung \\
27 & WK & - RZK & Bettpfanne \\
\hline & & &
\end{tabular}

\section{B.2. Items}

\section{B.2.1. Stimuli}

\begin{tabular}{|c|c|c|c|}
\hline $\begin{array}{l}\text { Item } \\
\text { Nr. }\end{array}$ & STRUKTUR & RZK & Stimulus \\
\hline 1 & RK & $-\mathrm{RZK}$ & $\begin{array}{l}\text { Paula hat neulich im Seminar eine umstrittene Filmbe- } \\
\text { wertung gelesen. Diesen/Jenen hat sie ... }\end{array}$ \\
\hline 1 & WK & $+\mathrm{RZK}$ & $\begin{array}{l}\text { Paula hat neulich im Seminar ein umstrittenes Filmbei- } \\
\text { spiel gesehen. Diesen/Jenen hat sie ... }\end{array}$ \\
\hline 1 & $\mathrm{RK}$ & $+\mathrm{RZK}$ & $\begin{array}{l}\text { Paula hat neulich im Seminar eine umstrittene Filmbear- } \\
\text { beitung vorgestellt. Diesen/Jenen hat sie ... }\end{array}$ \\
\hline 1 & WK & $-\mathrm{RZK}$ & $\begin{array}{l}\text { Paula hat neulich im Seminar ein umstrittenes Filmrecht } \\
\text { kennengelernt. Diesen/Jenen hat sie ... }\end{array}$ \\
\hline 2 & RK & $-\mathrm{RZK}$ & $\begin{array}{l}\text { Daniel hat schon vor Wintereinbruch eine teure Autover- } \\
\text { sicherung abgeschlossen. Jenes hat er ... }\end{array}$ \\
\hline 2 & WK & $+\mathrm{RZK}$ & $\begin{array}{l}\text { Daniel hat schon vor Wintereinbruch eine teure Autopan- } \\
\text { ne verursacht. Jenes hat er ... }\end{array}$ \\
\hline 2 & RK & $+\mathrm{RZK}$ & $\begin{array}{l}\text { Daniel hat schon vor Wintereinbruch eine teure Autover- } \\
\text { mietung durchgeführt. Jenes hat er ... }\end{array}$ \\
\hline
\end{tabular}




\begin{tabular}{|c|c|c|c|}
\hline 2 & WK & $-\mathrm{RZK}$ & $\begin{array}{l}\text { Daniel hat schon vor Wintereinbruch eine teure Autofähre } \\
\text { gebucht. Jenes hat er ... }\end{array}$ \\
\hline 3 & RK & $-\mathrm{RZK}$ & $\begin{array}{l}\text { Tina hat nach der Operation eine spezielle Fußbekleidung } \\
\text { bekommen. Diesen hat sie ... }\end{array}$ \\
\hline 3 & WK & $+\mathrm{RZK}$ & $\begin{array}{l}\text { Tina hat nach der Operation eine spezielle Fußspitze be- } \\
\text { kommen. Diesen hat sie ... }\end{array}$ \\
\hline 3 & RK & $+\mathrm{RZK}$ & $\begin{array}{l}\text { Tina hat nach der Operation eine spezielle Fußwaschung } \\
\text { bekommen. Diesen hat sie ... }\end{array}$ \\
\hline 3 & WK & $-\mathrm{RZK}$ & $\begin{array}{l}\text { Tina hat nach der Operation eine spezielle Fußmatte be- } \\
\text { kommen. Diesen hat sie ... }\end{array}$ \\
\hline 4 & RK & $-\mathrm{RZK}$ & $\begin{array}{l}\text { Sarah hat für die Polarexpedition vorsichtshalber eine ein- } \\
\text { fache Ölpumpe gekauft. Jenes hat sie ... }\end{array}$ \\
\hline 4 & WK & $+\mathrm{RZK}$ & $\begin{array}{l}\text { Sarah hat für die Polarexpedition vorsichtshalber eine ein- } \\
\text { fache Ölreserve angelegt. Jenes hat sie ... }\end{array}$ \\
\hline 4 & RK & $+\mathrm{RZK}$ & $\begin{array}{l}\text { Sarah hat für die Polarexpedition vorsichtshalber eine ein- } \\
\text { fache Ölgewinnung erlernt. Jenes hat sie ... }\end{array}$ \\
\hline 4 & WK & $-\mathrm{RZK}$ & $\begin{array}{l}\text { Sarah hat für die Polarexpedition vorsichtshalber einen } \\
\text { einfachen Öltank gekauft. Jenes hat sie ... }\end{array}$ \\
\hline 5 & RK & $-\mathrm{RZK}$ & $\begin{array}{l}\text { Armin hat in der Hafengegend einen verdächtigen } \\
\text { Geldtransporter beobachtet. Dieses hat er ... }\end{array}$ \\
\hline 5 & WK & $+\mathrm{RZK}$ & $\begin{array}{l}\text { Armin hat in der Hafengegend eine verdächtige Geldmün- } \\
\text { ze gefunden. Dieses hat er ... }\end{array}$ \\
\hline 5 & RK & $+\mathrm{RZK}$ & $\begin{array}{l}\text { Armin hat in der Hafengegend einen verdächtigen } \\
\text { Geldtransport beobachtet. Dieses hat er ... }\end{array}$ \\
\hline 5 & WK & $-\mathrm{RZK}$ & $\begin{array}{l}\text { Armin hat in der Hafengegend einen verdächtigen Geld- } \\
\text { automaten gesehen. Dieses hat er ... }\end{array}$ \\
\hline 6 & RK & $-\mathrm{RZK}$ & $\begin{array}{l}\text { Thomas hat wegen der nervigen Insekten einen alten } \\
\text { Fensterreiniger ausprobiert. Jenes hat er ... }\end{array}$ \\
\hline 6 & WK & $+\mathrm{RZK}$ & $\begin{array}{l}\text { Thomas hat wegen der nervigen Insekten einen alten } \\
\text { Fensterspalt abgedichtet. Jenes hat er ... }\end{array}$ \\
\hline 6 & RK & $+\mathrm{RZK}$ & $\begin{array}{l}\text { Thomas hat wegen der nervigen Insekten eine alte Fens- } \\
\text { teröffnung abgedichtet. Jenes hat er ... }\end{array}$ \\
\hline 6 & WK & $-\mathrm{RZK}$ & $\begin{array}{l}\text { Thomas hat wegen der nervigen Insekten einen alten } \\
\text { Fensterladen angeschraubt. Jenes hat er ... }\end{array}$ \\
\hline 7 & RK & $-\mathrm{RZK}$ & $\begin{array}{l}\text { Mareike hat am vergangenen Montag einen auffälligen } \\
\text { Wasserkocher überprüft. Dieses hat sie ... }\end{array}$ \\
\hline 7 & WK & $+\mathrm{RZK}$ & $\begin{array}{l}\text { Mareike hat am vergangenen Montag eine auffällige Was- } \\
\text { serfontäne gefilmt. Dieses hat sie ... }\end{array}$ \\
\hline 7 & RK & $+\mathrm{RZK}$ & $\begin{array}{l}\text { Mareike hat am vergangenen Montag eine auffällige Was- } \\
\text { serzufuhr überprüft. Dieses hat sie ... }\end{array}$ \\
\hline 7 & WK & $-\mathrm{RZK}$ & $\begin{array}{l}\text { Mareike hat am vergangenen Montag eine auffällige Was- } \\
\text { serpfeife gekauft. Dieses hat sie ... }\end{array}$ \\
\hline 8 & RK & $-\mathrm{RZK}$ & $\begin{array}{l}\text { Ruth hat trotz mehrerer Zwischenfälle eine weitere Haus- } \\
\text { verwaltung angenommen. Jenes hat sie ... }\end{array}$ \\
\hline 8 & WK & $+\mathrm{RZK}$ & $\begin{array}{l}\text { Ruth hat trotz mehrerer Zwischenfälle eine weitere Haus- } \\
\text { fassade verputzt. Jenes hat sie ... }\end{array}$ \\
\hline 8 & RK & $+\mathrm{RZK}$ & $\begin{array}{l}\text { Ruth hat trotz mehrerer Zwischenfälle einen weiteren } \\
\text { Hausputz gemeistert. Jenes hat sie ... }\end{array}$ \\
\hline
\end{tabular}




\begin{tabular}{|c|c|c|c|}
\hline 8 & WK & $-\mathrm{RZK}$ & $\begin{array}{l}\text { Ruth hat trotz mehrerer Zwischenfälle einen weiteren } \\
\text { Hausschlüssel hinterlegt. Jenes hat sie ... }\end{array}$ \\
\hline 9 & RK & $-\mathrm{RZK}$ & $\begin{array}{l}\text { Konrad hat letztens hinter dem Fernseher einen unnöti- } \\
\text { gen Kabelbinder angebracht. Dieses hat er ... }\end{array}$ \\
\hline 9 & WK & $+\mathrm{RZK}$ & $\begin{array}{l}\text { Konrad hat letztens hinter dem Fernseher einen unnöti- } \\
\text { gen Kabelsalat angerichtet. Dieses hat er ... }\end{array}$ \\
\hline 9 & $\mathrm{RK}$ & $+\mathrm{RZK}$ & $\begin{array}{l}\text { Konrad hat letztens hinter dem Fernseher eine unnötige } \\
\text { Kabelverbindung eingerichtet. Dieses hat er ... }\end{array}$ \\
\hline 9 & WK & -RZK & $\begin{array}{l}\text { Konrad hat letztens hinter dem Fernseher einen unnöti- } \\
\text { gen Kabelkanal angebracht. Dieses hat er ... }\end{array}$ \\
\hline 10 & RK & $-\mathrm{RZK}$ & $\begin{array}{l}\text { Anja hat im Feinkostladen einen exquisiten Fleischschnei- } \\
\text { der getestet. Jenes hat sie ... }\end{array}$ \\
\hline 10 & WK & $+\mathrm{RZK}$ & $\begin{array}{l}\text { Anja hat im Feinkostladen eine exquisite Fleischsorte aus- } \\
\text { gewählt. Jenes hat sie ... }\end{array}$ \\
\hline 10 & RK & $+\mathrm{RZK}$ & $\begin{array}{l}\text { Anja hat im Feinkostladen einen exquisiten Fleischgenuss } \\
\text { erlebt. Jenes hat sie... }\end{array}$ \\
\hline 10 & WK & $-\mathrm{RZK}$ & $\begin{array}{l}\text { Anja hat im Feinkostladen einen exquisiten Fleischtopf } \\
\text { poliert. Jenes hat sie... }\end{array}$ \\
\hline 11 & RK & $-\mathrm{RZK}$ & $\begin{array}{l}\text { Hannah hat an der Kunstakademie eine anspruchsvolle } \\
\text { Metallgießerei betreut. Dieses hat sie ... }\end{array}$ \\
\hline 11 & WK & $+\mathrm{RZK}$ & $\begin{array}{l}\text { Hannah hat an der Kunstakademie eine anspruchsvolle } \\
\text { Metallscheibe bearbeitet. Dieses hat sie ... }\end{array}$ \\
\hline 11 & RK & $+\mathrm{RZK}$ & $\begin{array}{l}\text { Hannah hat an der Kunstakademie eine anspruchsvolle } \\
\text { Metallgestaltung betreut. Dieses hat sie ... }\end{array}$ \\
\hline 11 & WK & $-\mathrm{RZK}$ & $\begin{array}{l}\text { Hannah hat an der Kunstakademie eine anspruchsvolle } \\
\text { Metallwerkstatt betreut. Dieses hat sie ... }\end{array}$ \\
\hline 12 & RK & -RZK & $\begin{array}{l}\text { Jürgen hat für die Stadt einen ungewöhnlichen Gastanker } \\
\text { begutachtet. Jenes hat er ... }\end{array}$ \\
\hline 12 & WK & $+\mathrm{RZK}$ & $\begin{array}{l}\text { Jürgen hat für die Stadt eine ungewöhnliche Gaswolke } \\
\text { begutachtet. Jenes hat er ... }\end{array}$ \\
\hline 12 & RK & $+\mathrm{RZK}$ & $\begin{array}{l}\text { Jürgen hat für die Stadt einen ungewöhnlichen Gasaus- } \\
\text { tausch durchgeführt. Jenes hat er ... }\end{array}$ \\
\hline 12 & WK & $-\mathrm{RZK}$ & $\begin{array}{l}\text { Jürgen hat für die Stadt eine ungewöhnliche Gaslaterne } \\
\text { begutachtet. Jenes hat er ... }\end{array}$ \\
\hline 13 & RK & $-\mathrm{RZK}$ & $\begin{array}{l}\text { Silke hat beim Entsorgungswerk eine umweltschonende } \\
\text { Müllvermeidung erlernt. Diesen hat sie ... }\end{array}$ \\
\hline 13 & WK & $+\mathrm{RZK}$ & $\begin{array}{l}\text { Silke hat beim Entsorgungswerk eine umweltschonende } \\
\text { Müllmenge produziert. Diesen hat sie ... }\end{array}$ \\
\hline 13 & RK & $+\mathrm{RZK}$ & $\begin{array}{l}\text { Silke hat beim Entsorgungswerk eine umweltschonende } \\
\text { Müllverwertung erlernt. Diesen hat sie ... }\end{array}$ \\
\hline 13 & WK & $-\mathrm{RZK}$ & $\begin{array}{l}\text { Silke hat beim Entsorgungswerk ein umweltschonendes } \\
\text { Müllauto bestellt. Diesen hat sie ... }\end{array}$ \\
\hline 14 & RK & $-\mathrm{RZK}$ & $\begin{array}{l}\text { Johanna hat für den Abschlussball eine glamouröse Haar- } \\
\text { bürste eingepackt. Jenes hat sie ... }\end{array}$ \\
\hline 14 & WK & $+\mathrm{RZK}$ & $\begin{array}{l}\text { Johanna hat für den Abschlussball einen glamourösen } \\
\text { Haarstrang geflochten. Jenes hat sie ... }\end{array}$ \\
\hline 14 & RK & $+\mathrm{RZK}$ & $\begin{array}{l}\text { Johanna hat für den Abschlussball eine glamouröse Haar- } \\
\text { pflege betrieben. Jenes hat sie ... }\end{array}$ \\
\hline
\end{tabular}




\begin{tabular}{|c|c|c|c|}
\hline 14 & WK & $-\mathrm{RZK}$ & $\begin{array}{l}\text { Johanna hat für den Abschlussball eine glamouröse Haar- } \\
\text { spange eingepackt. Jenes hat sie ... }\end{array}$ \\
\hline 15 & RK & $-\mathrm{RZK}$ & $\begin{array}{l}\text { Steffen hat für die Grafiksoftware eine komplizierte Pro- } \\
\text { grammbeschreibung gelesen. Dieses hat er ... }\end{array}$ \\
\hline 15 & WK & $+\mathrm{RZK}$ & $\begin{array}{l}\text { Steffen hat für die Grafiksoftware eine komplizierte Pro- } \\
\text { grammdatei geladen. Dieses hat er ... }\end{array}$ \\
\hline 15 & RK & $+\mathrm{RZK}$ & $\begin{array}{l}\text { Steffen hat für die Grafiksoftware eine komplizierte Pro- } \\
\text { grammentwicklung betrieben. Dieses hat er ... }\end{array}$ \\
\hline 15 & WK & $-\mathrm{RZK}$ & $\begin{array}{l}\text { Steffen hat für die Grafiksoftware einen komplizierten } \\
\text { Programmnamen festgelegt. Dieses hat er ... }\end{array}$ \\
\hline 16 & RK & $-\mathrm{RZK}$ & $\begin{array}{l}\text { Sophie hat im Anatomiepraktikum eine ideale Kopfstütze } \\
\text { benutzt. Jenen hat sie ... }\end{array}$ \\
\hline 16 & WK & $+\mathrm{RZK}$ & $\begin{array}{l}\text { Sophie hat im Anatomiepraktikum eine ideale Kopfform } \\
\text { erkannt. Jenen hat sie ... }\end{array}$ \\
\hline 16 & RK & $+\mathrm{RZK}$ & $\begin{array}{l}\text { Sophie hat im Anatomiepraktikum eine ideale Kopfhal- } \\
\text { tung vorgeführt. Jenen hat sie ... }\end{array}$ \\
\hline 16 & WK & $-\mathrm{RZK}$ & $\begin{array}{l}\text { Sophie hat im Anatomiepraktikum eine ideale Kopfhöhe } \\
\text { angezeichnet. Jenen hat sie ... }\end{array}$ \\
\hline 17 & RK & $-\mathrm{RZK}$ & $\begin{array}{l}\text { Julia hat im Urlaub an der Adria einen besonderen Salz- } \\
\text { streuer gekauft. Dieses hat sie ... }\end{array}$ \\
\hline 17 & WK & $+\mathrm{RZK}$ & $\begin{array}{l}\text { Julia hat im Urlaub an der Adria eine besondere Salzkon- } \\
\text { zentration inhaliert. Dieses hat sie ... }\end{array}$ \\
\hline 17 & RK & $+\mathrm{RZK}$ & $\begin{array}{l}\text { Julia hat im Urlaub an der Adria eine besondere Salzge- } \\
\text { winnung beobachtet. Dieses hat sie ... }\end{array}$ \\
\hline 17 & WK & $-\mathrm{RZK}$ & $\begin{array}{l}\text { Julia hat im Urlaub an der Adria eine besondere Salzstra- } \\
\text { ße besichtigt. Dieses hat sie ... }\end{array}$ \\
\hline 18 & RK & $-\mathrm{RZK}$ & $\begin{array}{l}\text { Guido hat mit der Feuerwehr einen bedenklichen Wasser- } \\
\text { zähler entdeckt. Jenes hat er ... }\end{array}$ \\
\hline 18 & WK & $+\mathrm{RZK}$ & $\begin{array}{l}\text { Guido hat mit der Feuerwehr eine bedenkliche Wasser- } \\
\text { säule entdeckt. Jenes hat er ... }\end{array}$ \\
\hline 18 & RK & $+\mathrm{RZK}$ & $\begin{array}{l}\text { Guido hat mit der Feuerwehr eine bedenkliche Wasser- } \\
\text { verschmutzung entdeckt. Jenes hat er ... }\end{array}$ \\
\hline 18 & WK & $-\mathrm{RZK}$ & $\begin{array}{l}\text { Guido hat mit der Feuerwehr eine bedenkliche Wasserfla- } \\
\text { sche entdeckt. Jenes hat er ... }\end{array}$ \\
\hline 19 & RK & $-\mathrm{RZK}$ & $\begin{array}{l}\text { Marie hat in Kolumbien eine interessante Tabakvermark- } \\
\text { tung kennengelernt. Diesen hat sie ... }\end{array}$ \\
\hline 19 & WK & $+\mathrm{RZK}$ & $\begin{array}{l}\text { Marie hat in Kolumbien ein interessantes Tabakblatt ge- } \\
\text { pflückt. Diesen hat sie ... }\end{array}$ \\
\hline 19 & RK & $+\mathrm{RZK}$ & $\begin{array}{l}\text { Marie hat in Kolumbien eine interessante Tabakverarbei- } \\
\text { tung kennengelernt. Diesen hat sie ... }\end{array}$ \\
\hline 19 & WK & $-\mathrm{RZK}$ & $\begin{array}{l}\text { Marie hat in Kolumbien eine interessante Tabakdose auf- } \\
\text { gestöbert. Diesen hat sie ... }\end{array}$ \\
\hline 20 & RK & $-\mathrm{RZK}$ & $\begin{array}{l}\text { Britt hat auf dem Truppengelände eine massive Panzer- } \\
\text { abwehr befehligt. Jenen hat sie ... }\end{array}$ \\
\hline 20 & WK & $+\mathrm{RZK}$ & $\begin{array}{l}\text { Britt hat auf dem Truppengelände eine massive Panzer- } \\
\text { gruppe befehligt. Jenen hat sie ... }\end{array}$ \\
\hline 20 & RK & $+\mathrm{RZK}$ & $\begin{array}{l}\text { Britt hat auf dem Truppengelände eine massive Panzer- } \\
\text { bekämpfung befehligt. Jenen hat sie ... }\end{array}$ \\
\hline
\end{tabular}




\begin{tabular}{|c|c|c|c|}
\hline 20 & WK & $-\mathrm{RZK}$ & $\begin{array}{l}\text { Britt hat auf dem Truppengelände eine massive Panzer- } \\
\text { kette getragen. Jenen hat sie ... }\end{array}$ \\
\hline 21 & RK & $-\mathrm{RZK}$ & $\begin{array}{l}\text { Elise hat auf der geliehenen Festplatte eine pfiffige Tex- } \\
\text { terkennung installiert. Diesen hat sie ... }\end{array}$ \\
\hline 21 & WK & $+\mathrm{RZK}$ & $\begin{array}{l}\text { Elise hat auf der geliehenen Festplatte eine pfiffige Text- } \\
\text { zeile gefunden. Diesen hat sie ... }\end{array}$ \\
\hline 21 & RK & $+\mathrm{RZK}$ & $\begin{array}{l}\text { Elise hat auf der geliehenen Festplatte eine pfiffige Text- } \\
\text { sammlung gefunden. Diesen hat sie ... }\end{array}$ \\
\hline 21 & WK & -RZK & $\begin{array}{l}\text { Elise hat auf der geliehenen Festplatte ein pfiffiges Text- } \\
\text { feld gefunden. Diesen hat sie ... }\end{array}$ \\
\hline 22 & RK & $-\mathrm{RZK}$ & $\begin{array}{l}\text { Mattis hat auf der Klassenfahrt eine typische Papiermüh- } \\
\text { le besichtigt. Jenes hat er ... }\end{array}$ \\
\hline 22 & WK & $+\mathrm{RZK}$ & $\begin{array}{l}\text { Mattis hat auf der Klassenfahrt eine typische Papiertüte } \\
\text { angemalt. Jenes hat er ... }\end{array}$ \\
\hline 22 & RK & $+\mathrm{RZK}$ & $\begin{array}{l}\text { Mattis hat auf der Klassenfahrt eine typische Papierher- } \\
\text { stellung ausprobiert. Jenes hat er ... }\end{array}$ \\
\hline 22 & WK & $-\mathrm{RZK}$ & $\begin{array}{l}\text { Mattis hat auf der Klassenfahrt eine typische Papierma- } \\
\text { schine besichtigt. Jenes hat er ... }\end{array}$ \\
\hline 23 & RK & $-\mathrm{RZK}$ & $\begin{array}{l}\text { Jonathan hat trotz vieler Bedenken eine professionelle } \\
\text { Theaterförderung durchgesetzt. Dieses hat er ... }\end{array}$ \\
\hline 23 & WK & $+\mathrm{RZK}$ & $\begin{array}{l}\text { Jonathan hat trotz vieler Bedenken eine professionelle } \\
\text { Theaterpremiere aufgeführt. Dieses hat er ... }\end{array}$ \\
\hline 23 & $\mathrm{RK}$ & $+\mathrm{RZK}$ & $\begin{array}{l}\text { Jonathan hat trotz vieler Bedenken eine professionelle } \\
\text { Theatervorführung durchgesetzt. Dieses hat er ... }\end{array}$ \\
\hline 23 & WK & $-\mathrm{RZK}$ & $\begin{array}{l}\text { Jonathan hat trotz vieler Bedenken eine professionelle } \\
\text { Theaterschminke eingeführt. Dieses hat er ... }\end{array}$ \\
\hline 24 & RK & $-\mathrm{RZK}$ & $\begin{array}{l}\text { Ida hat noch kurz vor Ladenschluss einen brauchbaren } \\
\text { Videoprojektor aufgetrieben. Jenes hat sie ... }\end{array}$ \\
\hline 24 & WK & $+\mathrm{RZK}$ & $\begin{array}{l}\text { Ida hat noch kurz vor Ladenschluss eine brauchbare Vi- } \\
\text { deokassette ausgeliehen. Jenes hat sie ... }\end{array}$ \\
\hline 24 & RK & $+\mathrm{RZK}$ & $\begin{array}{l}\text { Ida hat noch kurz vor Ladenschluss einen brauchbaren } \\
\text { Videoverleih abgewickelt. Jenes hat sie ... }\end{array}$ \\
\hline 24 & WK & $-\mathrm{RZK}$ & $\begin{array}{l}\text { Ida hat noch kurz vor Ladenschluss einen brauchbaren } \\
\text { Videorekorder aufgetrieben. Jenes hat sie ... }\end{array}$ \\
\hline 25 & RK & $-\mathrm{RZK}$ & $\begin{array}{l}\text { Emma hat vor ein paar Tagen eine exklusive Geldforde- } \\
\text { rung bearbeitet. Dieses hat sie ... }\end{array}$ \\
\hline 25 & WK & $+\mathrm{RZK}$ & $\begin{array}{l}\text { Emma hat vor ein paar Tagen einen exklusiven Geld- } \\
\text { schein gefunden. Dieses hat sie ... }\end{array}$ \\
\hline 25 & RK & $+\mathrm{RZK}$ & $\begin{array}{l}\text { Emma hat vor ein paar Tagen eine exklusive Geldver- } \\
\text { schwendung betrieben. Dieses hat sie ... }\end{array}$ \\
\hline 25 & WK & -RZK & $\begin{array}{l}\text { Emma hat vor ein paar Tagen einen exklusiven Geld- } \\
\text { schrank aufgestellt. Dieses hat sie ... }\end{array}$ \\
\hline 26 & RK & $-\mathrm{RZK}$ & $\begin{array}{l}\text { Jochen hat im Herbst trotz schlechter Sicht einen schmie- } \\
\text { rigen Ölfilter ausgewechselt. Jenes hat er ... }\end{array}$ \\
\hline 26 & WK & $+\mathrm{RZK}$ & $\begin{array}{l}\text { Jochen hat im Herbst trotz schlechter Sicht einen schmie- } \\
\text { rigen Ölfilm beseitigt. Jenes hat er ... }\end{array}$ \\
\hline 26 & RK & $+\mathrm{RZK}$ & $\begin{array}{l}\text { Jochen hat im Herbst trotz schlechter Sicht einen schmie- } \\
\text { rigen Ölwechsel vorgenommen. Jenes hat er ... }\end{array}$ \\
\hline
\end{tabular}




\begin{tabular}{llll}
26 & WK & - RZK & $\begin{array}{l}\text { Jochen hat im Herbst trotz schlechter Sicht eine schmie- } \\
\text { rige Öllampe ausgewechselt. Jenes hat er . . }\end{array}$ \\
\hline 27 & RK & - RZK & $\begin{array}{l}\text { Pia hat während der Ausbildung einen ekligen Bettbezug } \\
\text { desinfiziert. Dieses hat sie . . }\end{array}$ \\
27 & WK & + RZK & $\begin{array}{l}\text { Pia hat während der Ausbildung einen ekligen Bettpfos- } \\
\text { ten desinfiziert. Dieses hat sie ... }\end{array}$ \\
27 & RK & + RZK & $\begin{array}{l}\text { Pia hat während der Ausbildung einen ekligen Bettpfos- } \\
\text { ten desinfiziert. Dieses hat sie ... }\end{array}$ \\
27 & WK & - RZK & $\begin{array}{l}\text { Pia hat während der Ausbildung einen ekligen Bettpfos- } \\
\text { ten desinfiziert. Dieses hat sie . . }\end{array}$ \\
\hline
\end{tabular}

\section{B.2.2. Kontroll-Items}

\begin{tabular}{|c|c|c|}
\hline $\begin{array}{l}\text { Item } \\
\text { Nr. }\end{array}$ & $\begin{array}{l}\text { ANAPHER- } \\
\text { ART }\end{array}$ & Stimulus \\
\hline 1 & indirekt & Klara ist in den Ferien jede freie Minute gerodelt. Nun hat sie ihn \\
\hline 1 & direkt & $\begin{array}{l}\text { Klara ist in den Ferien jede freie Minute mit dem Schlitten gero- } \\
\text { delt. Nun hat sie ihn ... }\end{array}$ \\
\hline 2 & indirekt & $\begin{array}{l}\text { Caro ist für das Vorstellungsgespräch in die vierte Etage gefahren. } \\
\text { Vorher hat sie ihn ... }\end{array}$ \\
\hline 2 & direkt & $\begin{array}{l}\text { Caro ist für das Vorstellungsgespräch in die vierte Etage mit dem } \\
\text { Aufzug gefahren. Vorher hat sie ihn ... }\end{array}$ \\
\hline 3 & indirekt & $\begin{array}{l}\text { Claudia hat nach der Kneipentour ganz leise die Tür aufgeschlos- } \\
\text { sen. Dann hat sie ihn ... }\end{array}$ \\
\hline 3 & direkt & $\begin{array}{l}\text { Claudia hat nach der Kneipentour ganz leise die Tür mit dem } \\
\text { Schlüssel aufgeschlossen. Dann hat sie ihn ... }\end{array}$ \\
\hline 4 & indirekt & $\begin{array}{l}\text { Anne hat beim Essen lustlos in der Suppe herumgerührt. Dabei } \\
\text { hat sie ihn ... }\end{array}$ \\
\hline 4 & direkt & $\begin{array}{l}\text { Anne hat beim Essen lustlos in der Suppe mit dem Löffel herum- } \\
\text { gerührt. Dabei hat sie ihn ... }\end{array}$ \\
\hline 5 & indirekt & $\begin{array}{l}\text { Josef hat nach Feierabend auf der Treppe vor dem Laden eine } \\
\text { Zigarette angezündet. Danach hat er es ... }\end{array}$ \\
\hline 5 & direkt & $\begin{array}{l}\text { Josef hat nach Feierabend auf der Treppe vor dem Laden eine } \\
\text { Zigarette mit einem Feuerzeug angezündet. Danach hat er es ... }\end{array}$ \\
\hline 6 & indirekt & $\begin{array}{l}\text { Robin hat mangels Schredder den Papierberg Stück für Stück zer- } \\
\text { schnitten. Schließlich hat er sie ... }\end{array}$ \\
\hline 6 & direkt & $\begin{array}{l}\text { Robin hat mangels Schredder den Papierberg Stück für Stück mit } \\
\text { einer Schere zerschnitten. Schließlich hat er sie ... }\end{array}$ \\
\hline 7 & indirekt & $\begin{array}{l}\text { Matilda hat heute in der Kita zum ersten Mal mit Wasserfarben } \\
\text { gemalt. Dafür hat sie ihn ... }\end{array}$ \\
\hline 7 & direkt & $\begin{array}{l}\text { Matilda hat heute in der Kita zum ersten Mal mit einem Pinsel } \\
\text { gemalt. Dafür hat sie ihn ... }\end{array}$ \\
\hline 8 & indirekt & $\begin{array}{l}\text { Sven hat vor dem Urlaub noch den Bonsai gegosssen. Danach hat } \\
\text { er sie ... }\end{array}$ \\
\hline 8 & direkt & $\begin{array}{l}\text { Sven hat vor dem Urlaub noch den Bonsai mit der Gießkanne } \\
\text { gegosssen. Danach hat er sie ... }\end{array}$ \\
\hline
\end{tabular}




\begin{tabular}{|c|c|c|}
\hline 9 & indirekt & Alexander ist am Freitag zur Konferenz gefahren. Dafür hat er es \\
\hline 9 & direkt & $\begin{array}{l}\text { Alexander ist am Freitag zur Konferenz mit dem Auto gefahren. } \\
\text { Dafür hat er es ... }\end{array}$ \\
\hline 10 & indirekt & $\begin{array}{l}\text { Mario hat nach dem Spaziergang den Hund ordentlich gekämmt. } \\
\text { Schließlich hat er sie ... }\end{array}$ \\
\hline 10 & direkt & $\begin{array}{l}\text { Mario hat nach dem Spaziergang den Hund ordentlich mit einer } \\
\text { Bürste gekämmt. Schließlich hat er sie ... }\end{array}$ \\
\hline 11 & indirekt & $\begin{array}{l}\text { Sebastian hat am nächsten Morgen wie versprochen abgewaschen. } \\
\text { Dabei hat er es ... }\end{array}$ \\
\hline 11 & direkt & $\begin{array}{l}\text { Sebastian hat am nächsten Morgen wie versprochen mit Spülmit- } \\
\text { tel abgewaschen. Dabei hat er es ... }\end{array}$ \\
\hline 12 & indirekt & $\begin{array}{l}\text { Hendrik hat im Sommer geduldig nach einem Hecht gefischt. Vor- } \\
\text { her hat er sie ... }\end{array}$ \\
\hline 12 & direkt & $\begin{array}{l}\text { Hendrik hat im Sommer geduldig nach einem Hecht mit einer } \\
\text { Angel gefischt. Vorher hat er sie ... }\end{array}$ \\
\hline
\end{tabular}




\section{B.3. Weitere Plots}
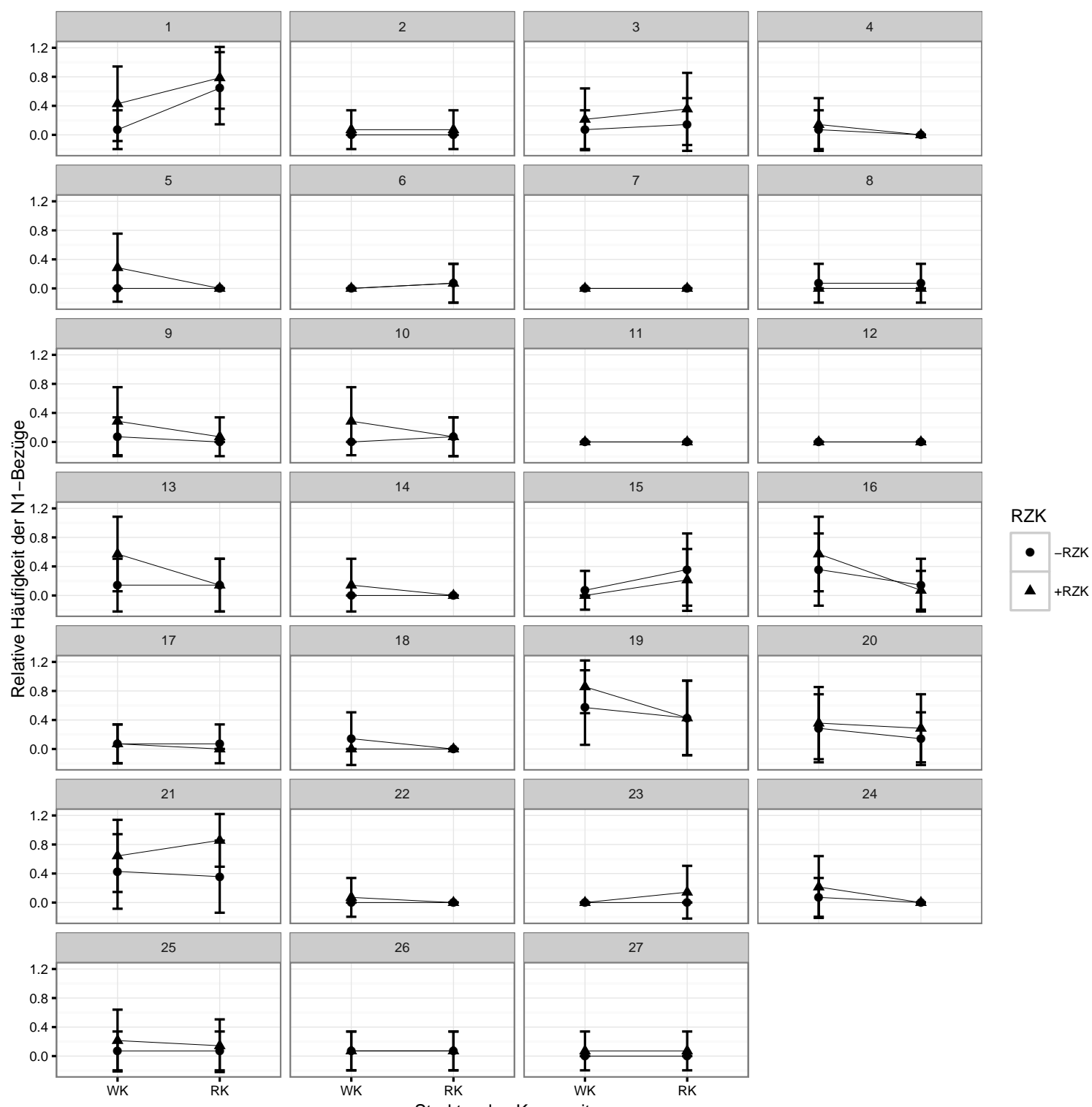

$\Delta+\mathrm{RZK}$
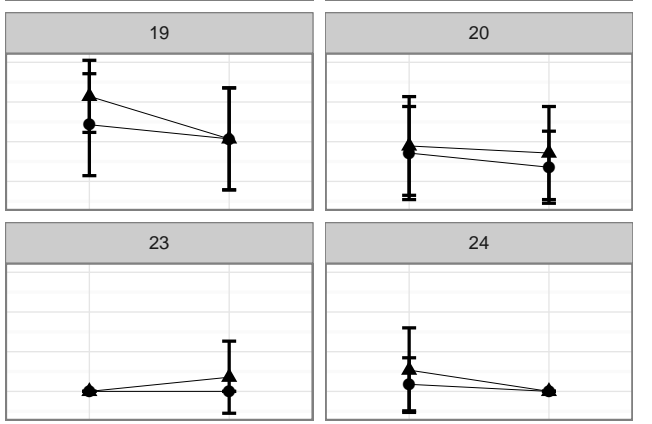

26

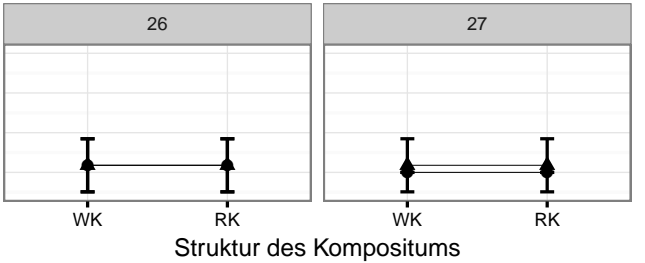

Abbildung B.1: Plot der Mittelwerte der N1-Bezüge in Abhängigkeit von den Faktoren RZK und STRUKTUR auf Item-Ebene 


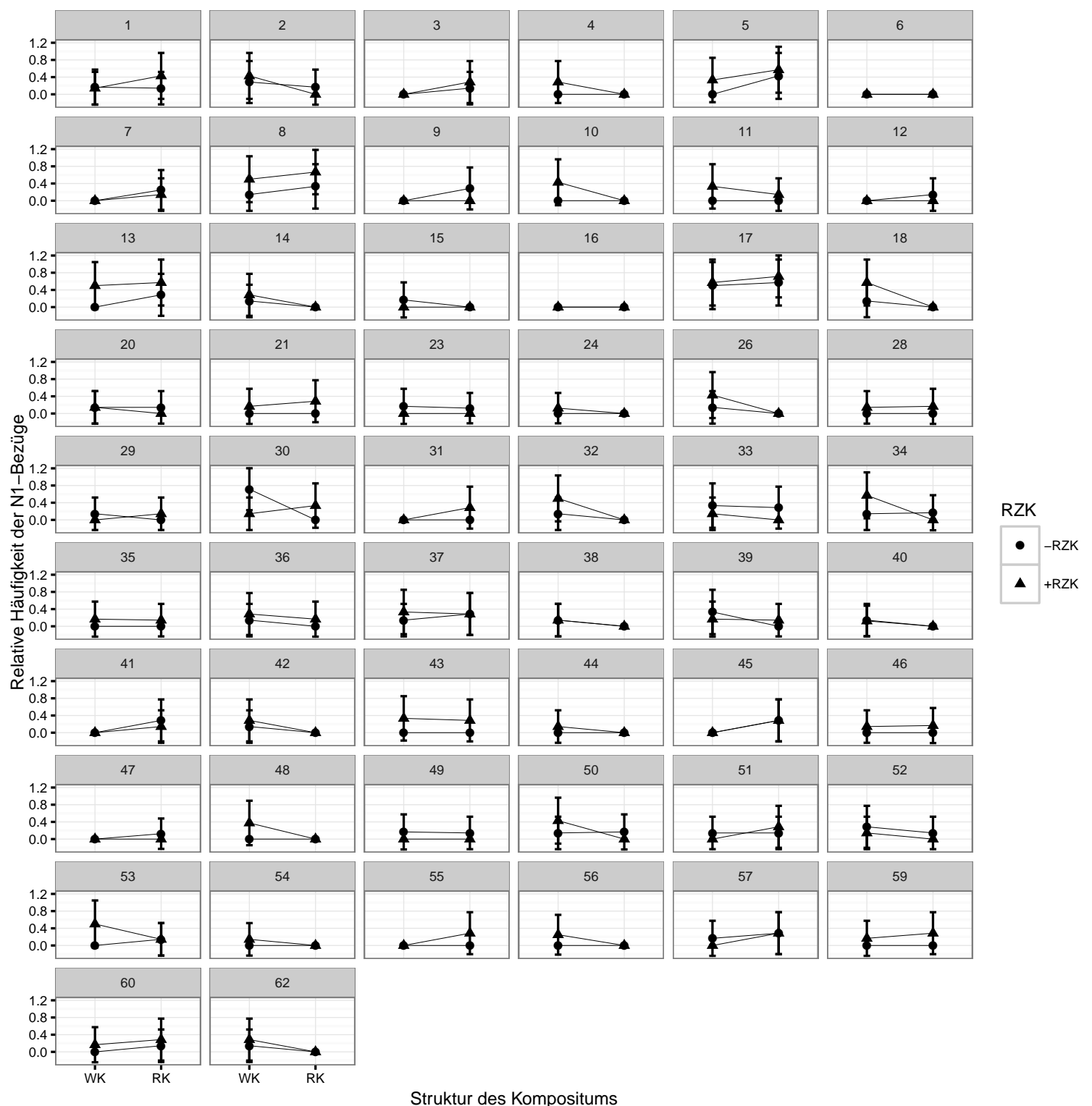

Abbildung B.2: Plot der Mittelwerte der N1-Bezüge in Abhängigkeit von den Faktoren RZK und STRUKTUR auf VP-Ebene 



\section{Anhang zu Experiment 3}

\section{C.1. Komposita}

\begin{tabular}{|c|c|c|}
\hline Item $\mathrm{Nr}$. & STRUKTUR & Kompositum \\
\hline 1 & RK & Tabakverarbeitung \\
\hline 1 & WK & Tabakfabrik \\
\hline 1 & Mono & Tabak \\
\hline 2 & RK & Geldsendung \\
\hline 2 & WK & Geldbrief \\
\hline 2 & Mono & Geld \\
\hline 3 & RK & Videovorführung \\
\hline 3 & WK & Videorekorder \\
\hline 3 & Mono & Video \\
\hline 4 & RK & Fleischuntersuchung \\
\hline 4 & WK & Fleischsaft \\
\hline 4 & Mono & Fleisch \\
\hline 5 & RK & Kabelbefestigung \\
\hline 5 & WK & Kabelkanal \\
\hline 5 & Mono & Kabel \\
\hline 6 & RK & Kopfpolsterung \\
\hline 6 & WK & Kopfteil \\
\hline 6 & Mono & Kopf \\
\hline 7 & RK & Metallgestaltung \\
\hline 7 & WK & Metallkunde \\
\hline 7 & Mono & Metall \\
\hline 8 & RK & Müllbeseitigung \\
\hline 8 & WK & Mülltonne \\
\hline 8 & Mono & Müll \\
\hline 9 & RK & Zugverbindung \\
\hline 9 & WK & Zugstrecke \\
\hline 9 & Mono & Zug \\
\hline 10 & RK & Salzgewinnung \\
\hline 10 & WK & Salzkonzentration \\
\hline 10 & Mono & Salz \\
\hline 11 & RK & Papierherstellung \\
\hline 11 & WK & Papiermaschine \\
\hline 11 & Mono & Papier \\
\hline 12 & RK & Bettreinigung \\
\hline 12 & WK & Bettpfosten \\
\hline 12 & Mono & Bett \\
\hline
\end{tabular}




\begin{tabular}{|c|c|c|}
\hline 13 & RK & Ölverteilung \\
\hline 13 & WK & Ölwanne \\
\hline 13 & Mono & Öl \\
\hline 14 & RK & Wasseraufbereitung \\
\hline 14 & WK & Wasserqualität \\
\hline 14 & Mono & Wasser \\
\hline 15 & RK & Panzerbekämpfung \\
\hline 15 & WK & Panzerkette \\
\hline 15 & Mono & Panzer \\
\hline 16 & RK & Texterkennung \\
\hline 16 & WK & Textfeld \\
\hline 16 & Mono & Text \\
\hline 17 & RK & Autoanfertigung \\
\hline 17 & WK & Autowerkstatt \\
\hline 17 & Mono & Auto \\
\hline 18 & RK & Bergbesteigung \\
\hline 18 & WK & Bergetappe \\
\hline 18 & Mono & Berg \\
\hline 19 & RK & Buchveröffentlichung \\
\hline 19 & WK & Buchpreis \\
\hline 19 & Mono & Buch \\
\hline 20 & RK & Dachbegrünung \\
\hline 20 & WK & Dachgarten \\
\hline 20 & Mono & Dach \\
\hline 21 & RK & Gartenbeleuchtung \\
\hline 21 & WK & Gartenlicht \\
\hline 21 & Mono & Garten \\
\hline 22 & RK & Kreditgewährung \\
\hline 22 & WK & Kreditvolumen \\
\hline 22 & Mono & Kredit \\
\hline 23 & RK & Waldrettung \\
\hline 23 & WK & Waldstraße \\
\hline 23 & Mono & Wald \\
\hline 24 & RK & Wegmarkierung \\
\hline 24 & WK & Wegzeichen \\
\hline 24 & Mono & Weg \\
\hline
\end{tabular}




\begin{tabular}{llll}
\hline \hline N1 ist Massenomen & 10 ja & 14 nein & \\
\hline Genus des N1 & $11 \mathrm{~m}$ & $13 \mathrm{n}$ & \\
\hline RZK ohne Kontext (ohne Bed. Mono) & $23 \mathrm{ja}$ & $25 \mathrm{nein}$ & \\
... nach Bedingung (nur ja) & $17 \mathrm{RK}$ & $6 \mathrm{WK}$ & \\
... nach Bedingung (nur nein) & $7 \mathrm{RK}$ & $18 \mathrm{WK}$ & \\
\hline RZK mit Kontext (mit Bed. Mono) & 41 ja & $31 \mathrm{nein}$ & \\
$\ldots$.. nach Bedingung (nur ja) & $15 \mathrm{RK}$ & $13 \mathrm{WK}$ & 14 Mono \\
$\ldots$.. nach Bedingung (nur nein) & $9 \mathrm{RK}$ & $11 \mathrm{WK}$ & 10 Mono \\
\hline Konkretheit des N2 & $24 \mathrm{ja}$ & $24 \mathrm{nein}$ & \\
... nach Bedingung (nur ja) & $7 \mathrm{RK}$ & $17 \mathrm{WK}$ & \\
$\ldots$.. nach Bedingung (nur nein) & $17 \mathrm{RK}$ & $7 \mathrm{WK}$ & \\
\hline Prozesslesart (Bed. RK) & 13 ja & $11 \mathrm{nein}$ & \\
\hline \hline
\end{tabular}

Tabelle C.2: Verteilung möglicher Einflussgrößen zwischen Items und Bedingungen

\section{C.2. Anhang zu Experiment 3a}

\section{C.2.1. Items}

\begin{tabular}{|c|c|c|}
\hline $\begin{array}{l}\text { Item } \\
\text { Nr. }\end{array}$ & $\begin{array}{l}\text { STRUK- } \\
\text { TUR }\end{array}$ & Stimulus \\
\hline 1 & RK & $\begin{array}{l}\text { Susanna hat in den Semesterferien in Kolumbien eine Plantage be- } \\
\text { sucht. Die Tabakfabrik erscheint ziemlich modern. Er soll ... }\end{array}$ \\
\hline 1 & WK & $\begin{array}{l}\text { Susanna hat in den Semesterferien in Kolumbien eine Plantage be- } \\
\text { sucht. Die Tabakverarbeitung erscheint ziemlich aufwändig. Er soll }\end{array}$ \\
\hline 1 & Mono & $\begin{array}{l}\text { Susanna hat in den Semesterferien in Kolumbien eine Plantage be- } \\
\text { sucht. Der Tabak wird ziemlich aufwändig verarbeitet. Er soll ... }\end{array}$ \\
\hline 2 & RK & $\begin{array}{l}\text { Armin hat eine Mahnung von seinem Vermieter bekommen. Die } \\
\text { Geldsendung kommt gerade noch rechtzeitig. Es muss ... }\end{array}$ \\
\hline 2 & WK & $\begin{array}{l}\text { Armin hat eine Mahnung von seinem Vermieter bekommen. Der } \\
\text { Geldbrief kommt gerade noch rechtzeitig. Es muss ... }\end{array}$ \\
\hline 2 & Mono & $\begin{array}{l}\text { Armin hat eine Mahnung von seinem Vermieter bekommen. Das } \\
\text { Geld kommt gerade noch rechtzeitig. Es muss ... }\end{array}$ \\
\hline 3 & RK & $\begin{array}{l}\text { Ida hat für das Wochenende einen Bollywood-Abend geplant. Die } \\
\text { Videovorführung findet im Gemeinschaftsraum statt. Es muss ... }\end{array}$ \\
\hline 3 & WK & $\begin{array}{l}\text { Ida hat für das Wochenende einen Bollywood-Abend geplant. Der } \\
\text { Videorekorder steht im Gemeinschaftsraum. Es muss ... }\end{array}$ \\
\hline 3 & Mono & $\begin{array}{l}\text { Ida hat für das Wochenende einen Bollywood-Abend geplant. Das } \\
\text { Video wird im Gemeinschaftsraum gezeigt. Es muss ... }\end{array}$ \\
\hline 4 & RK & $\begin{array}{l}\text { Anja hat eine Zeit lang bei der Lebensmittelkontrolle gearbei- } \\
\text { tet. Die Fleischuntersuchung wird sehr gründlich vorgenommen. Es } \\
\text { muss ... }\end{array}$ \\
\hline 4 & WK & $\begin{array}{l}\text { Anja hat eine Zeit lang bei der Lebensmittelkontrolle gearbeitet. } \\
\text { Der Fleischsaft wird sehr gründlich untersucht. Es muss ... }\end{array}$ \\
\hline 4 & Mono & $\begin{array}{l}\text { Anja hat eine Zeit lang bei der Lebensmittelkontrolle gearbeitet. } \\
\text { Das Fleisch wird sehr gründlich untersucht. Es muss ... }\end{array}$ \\
\hline
\end{tabular}




\begin{tabular}{|c|c|c|}
\hline 5 & RK & $\begin{array}{l}\text { Konrad muss seinen neuen Arbeitsplatz zuerst neu einrichten. Die } \\
\text { Kabelbefestigung liegt mitten im Raum. Es soll ... }\end{array}$ \\
\hline 5 & WK & $\begin{array}{l}\text { Konrad muss seinen neuen Arbeitsplatz zuerst neu einrichten. Der } \\
\text { Kabelkanal liegt mitten im Raum. Es soll ... }\end{array}$ \\
\hline 5 & Mono & $\begin{array}{l}\text { Konrad muss seinen neuen Arbeitsplatz zuerst neu einrichten. Das } \\
\text { Kabel liegt mitten im Raum. Es soll ... }\end{array}$ \\
\hline 6 & RK & $\begin{array}{l}\text { Mareike hat in ihrer Zahnarztpraxis einen neuen Behandlungsstuhl. } \\
\text { Die Kopfpolsterung ist besonders komfortabel. Er soll ... }\end{array}$ \\
\hline 6 & WK & $\begin{array}{l}\text { Mareike hat in ihrer Zahnarztpraxis einen neuen Behandlungsstuhl. } \\
\text { Das Kopfteil ist besonders komfortabel. Er soll ... }\end{array}$ \\
\hline 6 & Mono & $\begin{array}{l}\text { Mareike hat in ihrer Zahnarztpraxis einen neuen Behandlungsstuhl. } \\
\text { Der Kopf bekommt eine speziell geformte Stütze. Er soll ... }\end{array}$ \\
\hline 7 & RK & $\begin{array}{l}\text { Hannah hat einige Zeit an der Kunstakademie verbracht. Die Me- } \\
\text { tallgestaltung ist besonders faszinierend.Es muss ... }\end{array}$ \\
\hline 7 & WK & $\begin{array}{l}\text { Hannah hat einige Zeit an der Kunstakademie verbracht. Die Me- } \\
\text { tallkunde ist besonders faszinierend. Es muss ... }\end{array}$ \\
\hline 7 & Mono & $\begin{array}{l}\text { Hannah hat einige Zeit an der Kunstakademie verbracht. Das Me- } \\
\text { tall ist ein faszinierender Werkstoff. Es muss ... }\end{array}$ \\
\hline 8 & RK & $\begin{array}{l}\text { Silke hat ein Praktikum auf einem Recyclinghof gemacht. Die Müll- } \\
\text { beseitigung ist ein viel diskutiertes Thema. Er muss ... }\end{array}$ \\
\hline 8 & WK & $\begin{array}{l}\text { Silke hat ein Praktikum auf einem Recyclinghof gemacht. Die Müll- } \\
\text { tonne ist ein viel diskutiertes Thema. Er muss ... }\end{array}$ \\
\hline 8 & Mono & $\begin{array}{l}\text { Silke hat ein Praktikum auf einem Recyclinghof gemacht. Der Müll } \\
\text { ist ein viel diskutiertes Thema. Er muss ... }\end{array}$ \\
\hline 9 & RK & $\begin{array}{l}\text { Johanna studiert jetzt in einer anderen Stadt. Die Zugverbindung } \\
\text { ist eigentlich ganz gut. Er soll ... }\end{array}$ \\
\hline 9 & WK & $\begin{array}{l}\text { Johanna studiert jetzt in einer anderen Stadt. Die Zugstrecke ist } \\
\text { eigentlich machbar. Er soll ... }\end{array}$ \\
\hline 9 & Mono & $\begin{array}{l}\text { Johanna studiert jetzt in einer anderen Stadt. Der Zug ist eigentlich } \\
\text { sogar recht günstig. Er soll ... }\end{array}$ \\
\hline 10 & RK & $\begin{array}{l}\text { Julia hat ihren Urlaub in den bayerischen Alpen verbracht. Die } \\
\text { Salzgewinnung ist typisch für diese Gegend. Es soll ... }\end{array}$ \\
\hline 10 & WK & $\begin{array}{l}\text { Julia hat ihren Urlaub in den bayerischen Alpen verbracht. Die } \\
\text { Salzkonzentration ist typisch für diese Gegend. Es soll ... }\end{array}$ \\
\hline 10 & Mono & $\begin{array}{l}\text { Julia hat ihren Urlaub in den bayerischen Alpen verbracht. Das } \\
\text { Salz ist typisch für diese Gegend. Es soll ... }\end{array}$ \\
\hline 11 & RK & $\begin{array}{l}\text { Mattis besucht seine Schwester in der Verpackungsfabrik. Die Pa- } \\
\text { pierherstellung wird nicht mehr per Hand gemacht. Es soll ... }\end{array}$ \\
\hline 11 & WK & $\begin{array}{l}\text { Mattis besucht seine Schwester in der Verpackungsfabrik. Die Pa- } \\
\text { piermaschine wird nicht mehr per Hand bedient. Es soll ... }\end{array}$ \\
\hline 11 & Mono & $\begin{array}{l}\text { Mattis besucht seine Schwester in der Verpackungsfabrik. Das Pa- } \\
\text { pier wird nicht mehr per Hand hergestellt. Es soll ... }\end{array}$ \\
\hline 12 & RK & $\begin{array}{l}\text { Pia ist Stationsschwester im Kreiskrankenhaus und für vier Auszu- } \\
\text { bildende verantwortlich. Die Bettreinigung wird oft vernachlässigt. } \\
\text { Es muss ... }\end{array}$ \\
\hline 12 & WK & $\begin{array}{l}\text { Pia ist Stationsschwester im Kreiskrankenhaus und für vier Aus- } \\
\text { zubildende verantwortlich. Der Bettpfosten wird oft vernachlässigt. } \\
\text { Es muss ... }\end{array}$ \\
\hline
\end{tabular}


12 Mono Pia ist Stationsschwester im Kreiskrankenhaus und für vier Auszubildende verantwortlich. Das Bett wird oft vernachlässigt. Es muss

\begin{tabular}{lll} 
& $\ldots$ \\
\hline 13 & RK & Stefan hat in seiner kleine Autowerkstatt viele treue Kunden. Die
\end{tabular} Ölverteilung wird oft als Fehlerquelle übersehen. Es muss ...

13 WK Stefan hat in seiner kleine Autowerkstatt viele treue Kunden. Die Ölwanne wird oft als Fehlerquelle übersehen. Es muss ...

13 Mono Stefan hat in seiner kleine Autowerkstatt viele treue Kunden. Das Öl wird oft als Fehlerquelle übersehen. Es muss ...

\begin{tabular}{|c|c|c|}
\hline 14 & RK & $\begin{array}{l}\text { Tanja hat einen neuen Job bei den Stadtwerken. Die Wasseraufbe- } \\
\text { reitung ist für die Gemeinde sehr wichtig. Es muss ... }\end{array}$ \\
\hline 14 & WK & $\begin{array}{l}\text { Tanja hat einen neuen Job bei den Stadtwerken. Die Wasserqualität } \\
\text { ist für die Gemeinde sehr wichtig. Es muss ... }\end{array}$ \\
\hline 14 & Mono & $\begin{array}{l}\text { Tanja hat einen neuen Job bei den Stadtwerken. Das Wasser wird } \\
\text { für die Gemeinde aufbereitet. Es muss ... }\end{array}$ \\
\hline 15 & RK & $\begin{array}{l}\text { Britta besucht mit ihrer Schulklasse ein Museum über den Zweiten } \\
\text { Weltkrieg. Die Panzerbekämpfung wirkt ziemlich brutal. Er muss }\end{array}$ \\
\hline
\end{tabular}

15 WK Britta besucht mit ihrer Schulklasse ein Museum über den Zweiten Weltkrieg. Die Panzerkette wirkt ziemlich monströs. Er muss ...

15 Mono Britta besucht mit ihrer Schulklasse ein Museum über den Zweiten Weltkrieg. Der Panzer wirkt ziemlich monströs. Er muss ...

\begin{tabular}{|c|c|c|}
\hline 16 & RK & $\begin{array}{l}\text { Elise arbeitet als Webdesignerin bei einem Online-Journal. Die Tex- } \\
\text { terkennung erleichtert das Arbeiten sehr. Er muss ... }\end{array}$ \\
\hline 16 & WK & $\begin{array}{l}\text { Elise arbeitet als Webdesignerin bei einem Online-Journal. Das } \\
\text { Textfeld erleichtert das Arbeiten sehr. Er muss ... }\end{array}$ \\
\hline 16 & Mono & $\begin{array}{l}\text { Elise arbeitet als Webdesignerin bei einem Online-Journal. Der Text } \\
\text { kann digital eingelesen werden. Er muss ... }\end{array}$ \\
\hline 17 & RK & $\begin{array}{l}\text { Bernd hat einen extravaganten Geschmack. Die Autoanfertigung } \\
\text { dauert länger als erwartet. Es kann ... }\end{array}$ \\
\hline 17 & WK & $\begin{array}{l}\text { Bernd hat einen extravaganten Geschmack. Die Autowerkstatt ist } \\
\text { am Wochenende geschlossen. Es kann ... }\end{array}$ \\
\hline 17 & Mono & $\begin{array}{l}\text { Bernd hat einen extravaganten Geschmack. Das Auto ist aber leider } \\
\text { noch nicht ganz fertig. Es kann ... }\end{array}$ \\
\hline 18 & RK & $\begin{array}{l}\text { Marlene bricht kurzentschlossen mit Freunden zu einem Aktivur- } \\
\text { laub auf. Die Bergbesteigung ist dann unerwartet anstrengend. Er } \\
\text { soll ... }\end{array}$ \\
\hline 18 & WK & $\begin{array}{l}\text { Marlene bricht kurzentschlossen mit Freunden zu einem Aktivur- } \\
\text { laub auf. Die Bergetappe ist dann unerwartet anstrengend. Er soll }\end{array}$ \\
\hline 18 & Mono & $\begin{array}{l}\text { Marlene bricht kurzentschlossen mit Freunden zu einem Aktivur- } \\
\text { laub auf. Der Berg ist dann unerwartet anspruchsvoll. Er soll ... }\end{array}$ \\
\hline 19 & RK & $\begin{array}{l}\text { David ist ein richtiges Arbeitstier. Die Buchveröffentlichung steht } \\
\text { kurz bevor. Es kann ... }\end{array}$ \\
\hline 19 & WK & $\begin{array}{l}\text { David ist ein richtiges Arbeitstier. Der Buchpreis steht kurz bevor. } \\
\text { Es kann ... }\end{array}$ \\
\hline 19 & Mono & $\begin{array}{l}\text { David ist ein richtiges Arbeitstier. Das Buch steht kurz vor der } \\
\text { Veröffentlichung. Es kann ... }\end{array}$ \\
\hline
\end{tabular}




\begin{tabular}{|c|c|c|}
\hline 20 & RK & $\begin{array}{l}\text { Karl ist ein richtiger Heimwerker geworden. Die Dachbegrünung ist } \\
\text { wirklich gut gelungen. Es kann ... }\end{array}$ \\
\hline 20 & WK & $\begin{array}{l}\text { Karl ist ein richtiger Heimwerker geworden. Der Dachgarten ist } \\
\text { wirklich gut gelungen. Es kann ... }\end{array}$ \\
\hline 20 & Mono & $\begin{array}{l}\text { Karl ist ein richtiger Heimwerker geworden. Das Dach ist wirklich } \\
\text { gut gestaltet. Es kann ... }\end{array}$ \\
\hline 21 & RK & $\begin{array}{l}\text { Barbara hat dieses Jahr große Schwierigkeiten mit dem Sommerfest. } \\
\text { Die Gartenbeleuchtung ist auch noch ausgefallen. Er muss ... }\end{array}$ \\
\hline 21 & WK & $\begin{array}{l}\text { Barbara hat dieses Jahr große Schwierigkeiten mit dem Sommerfest. } \\
\text { Das Gartenlicht ist auch noch ausgefallen. Er muss ... }\end{array}$ \\
\hline 21 & Mono & $\begin{array}{l}\text { Barbara hat dieses Jahr große Schwierigkeiten mit dem Sommerfest. } \\
\text { Der Garten liegt plötzlich auch noch im Dunkeln. Er muss ... }\end{array}$ \\
\hline 22 & RK & $\begin{array}{l}\text { Wiebke wird seit Tagen von einer nervigen Kundin belästigt. Die } \\
\text { Kreditgewährung unterliegt strengen Vorschriften. Er kann ... }\end{array}$ \\
\hline 22 & WK & $\begin{array}{l}\text { Wiebke wird seit Tagen von einer nervigen Kundin belästigt. Das } \\
\text { Kreditvolumen unterliegt strengen Vorschriften. Er kann ... }\end{array}$ \\
\hline 22 & Mono & $\begin{array}{l}\text { Wiebke wird seit Tagen von einer nervigen Kundin belästigt. Der } \\
\text { Kredit unterliegt strengen Vorschriften. Er kann ... }\end{array}$ \\
\hline 23 & RK & $\begin{array}{l}\text { Franziska steckt viel Zeit in ihr Ehrenamt beim Umweltschutz. Die } \\
\text { Waldrettung ist nach großer Aufregung geglückt. Er soll ... }\end{array}$ \\
\hline 23 & WK & $\begin{array}{l}\text { Franziska steckt viel Zeit in ihr Ehrenamt beim Umweltschutz. Die } \\
\text { Waldstraße wird nun doch nicht gebaut. Er soll ... }\end{array}$ \\
\hline 23 & Mono & $\begin{array}{l}\text { Franziska steckt viel Zeit in ihr Ehrenamt beim Umweltschutz. Der } \\
\text { Wald wird nun doch nicht gerodet. Er soll ... }\end{array}$ \\
\hline 24 & RK & $\begin{array}{l}\text { Melanie unternimmt mit ihren Eltern eine Wanderung zu einer Aus- } \\
\text { sichtsplattform. Die Wegmarkierung ist völlig zugewuchert. Er kann }\end{array}$ \\
\hline 24 & WK & $\begin{array}{l}\text { Melanie unternimmt mit ihren Eltern eine Wanderung zu einer Aus- } \\
\text { sichtsplattform. Das Wegzeichen ist völlig zugewuchert. Er kann ... }\end{array}$ \\
\hline 24 & Mono & $\begin{array}{l}\text { Melanie unternimmt mit ihren Eltern eine Wanderung zu einer Aus- } \\
\text { sichtsplattform. Der Weg ist völlig zugewuchert. Er kann ... }\end{array}$ \\
\hline
\end{tabular}




\section{C.2.2. Weitere Plots}
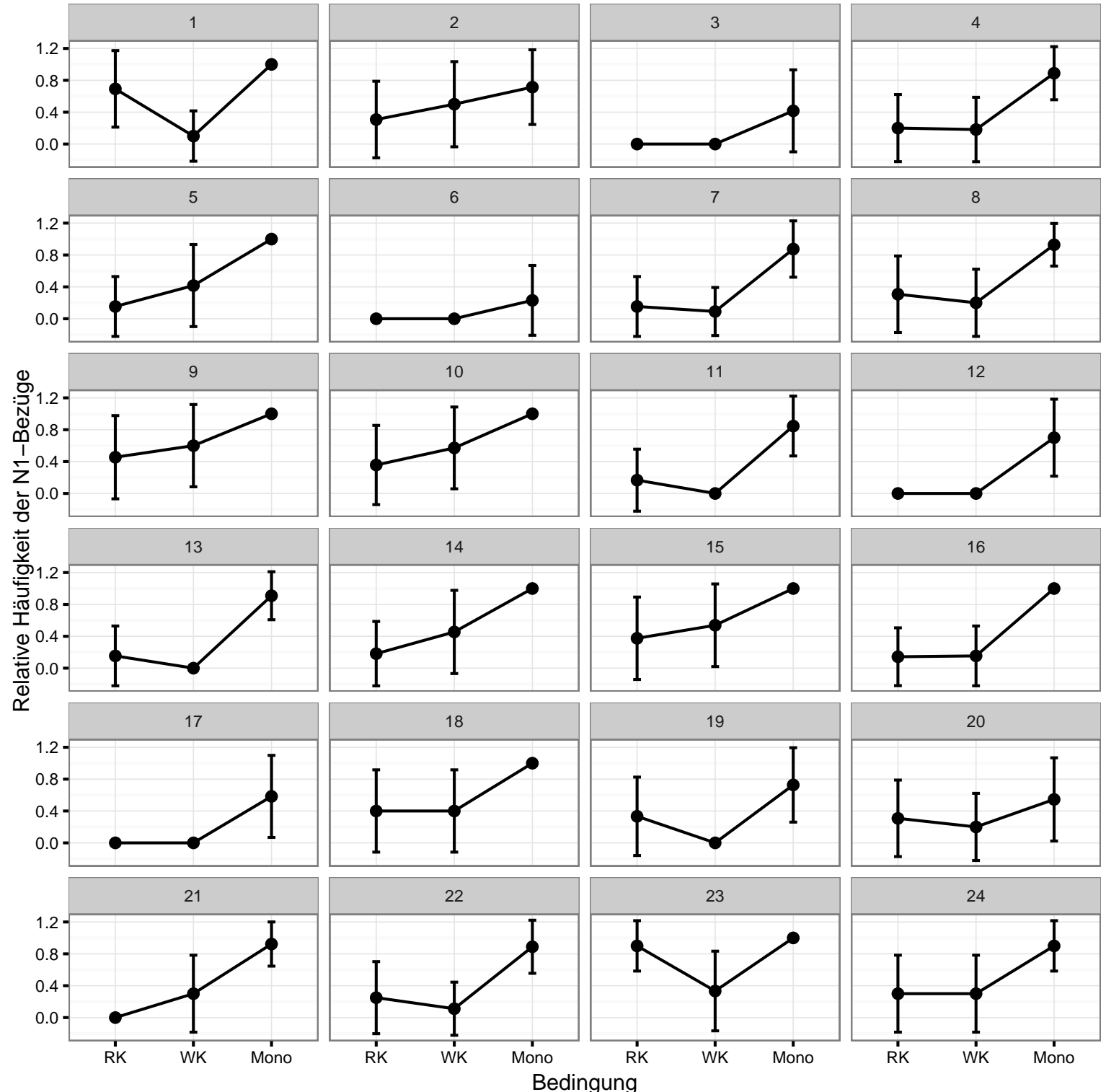

Abbildung C.1: Plot der Mittelwerte der relativen Häufigkeit der N1-Bezüge für den Faktor StRukutr für die einzelnen Items (gesamter Datensatz) 


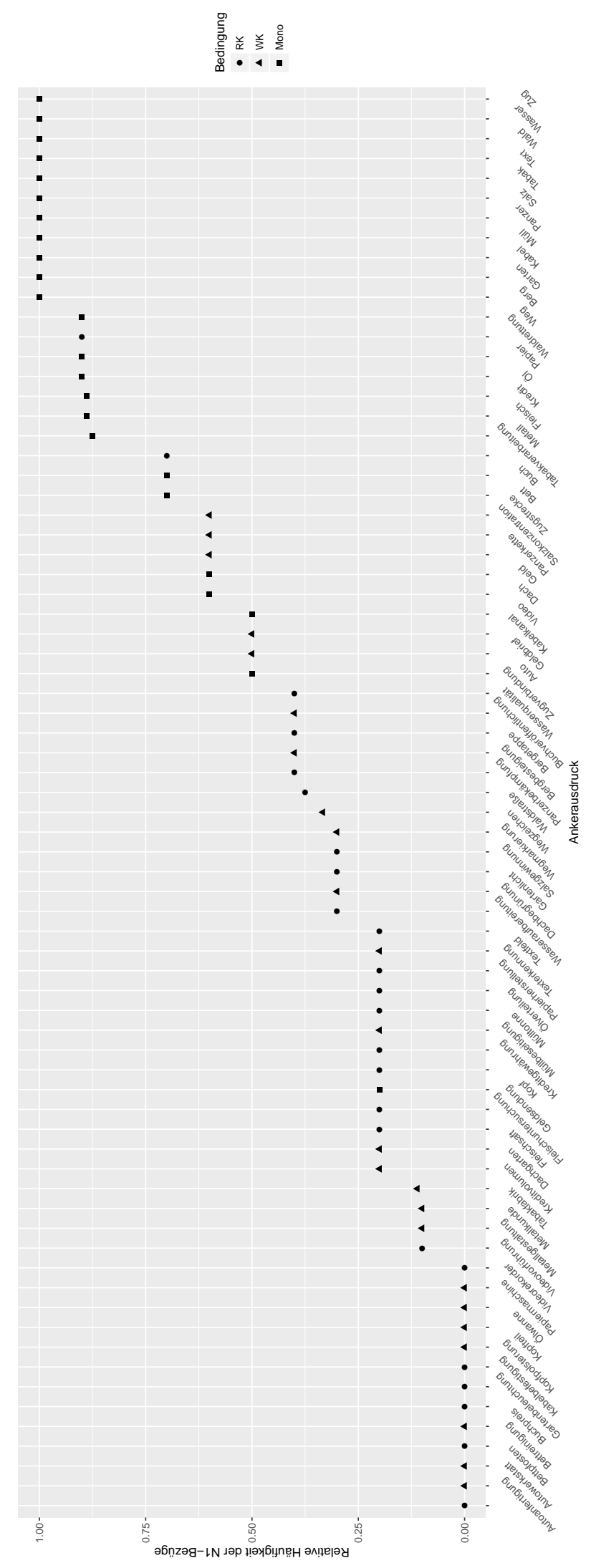

Abbildung C.2: Plot der Mittelwerte der relativen Häufigkeit der N1-Bezüge für die einzelnen Items in allen Bedingungen (gekürzter Datensatz), geordnet nach steigendem Wert 


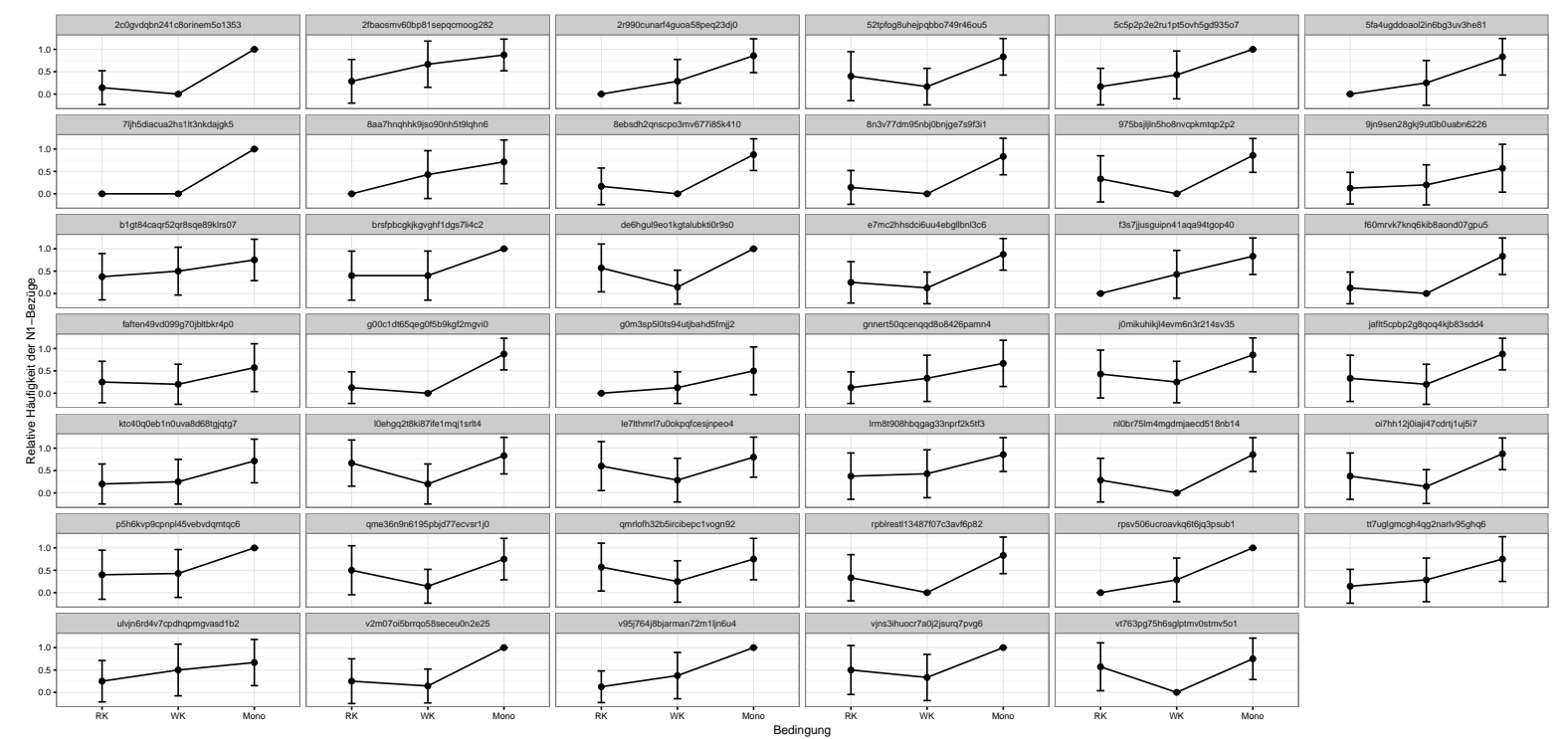

Abbildung C.3: Plot der Mittelwerte der relativen Häufigkeit der N1-Bezüge für den Faktor STRUKTUR für die einzelnen VPn (gesamter Datensatz)

\section{C.2.3. Auswertungs-Output: Bsp. aus R 3.2.0}

LMEMs für den Faktor STRUKTUR auf dem gekürzten Datensatz:

$>\operatorname{print}(\mathrm{m} 1<-$ glmer $($ rating $\sim$ bed $+(1 \mid$ subject $)+(1 \mid$ item $)$, data=d, family= +binomial (link=logit)), REML=FALSE)

Generalized linear mixed model fit by maximum likelihood (Laplace Approximation) ['glmerMod']

Family: binomial ( logit)

Formula: rating $\sim$ bed $+(1 \mid$ subject $)+(1 \mid$ item $)$

Data: d

$\begin{array}{rrrrr}\text { AIC } & \text { BIC } & \text { logLik } & \text { deviance } & \text { df.resid } \\ 664.5363 & 687.3485 & -327.2681 & 654.5363 & 703\end{array}$

Random effects:

Groups Name Std.Dev.

subject (Intercept) 0.456

item (Intercept) 1.189

Number of obs: 708, groups: subject, 41; item, 24

$\begin{array}{lrr}\text { Fixed Effects: } & & \\ \text { (Intercept) } & \text { bed1 } & \text { bed2 } \\ -0.2825 & 1.1778 & 1.2554\end{array}$

$>\operatorname{summary}(\mathrm{m} 1)$

Generalized linear mixed model fit by maximum likelihood (Laplace Approximation) ['glmerMod']

Family: binomial ( logit)

Formula: rating bed $+(1 \mid$ subject $)+(1 \mid$ item $)$

Data: d 


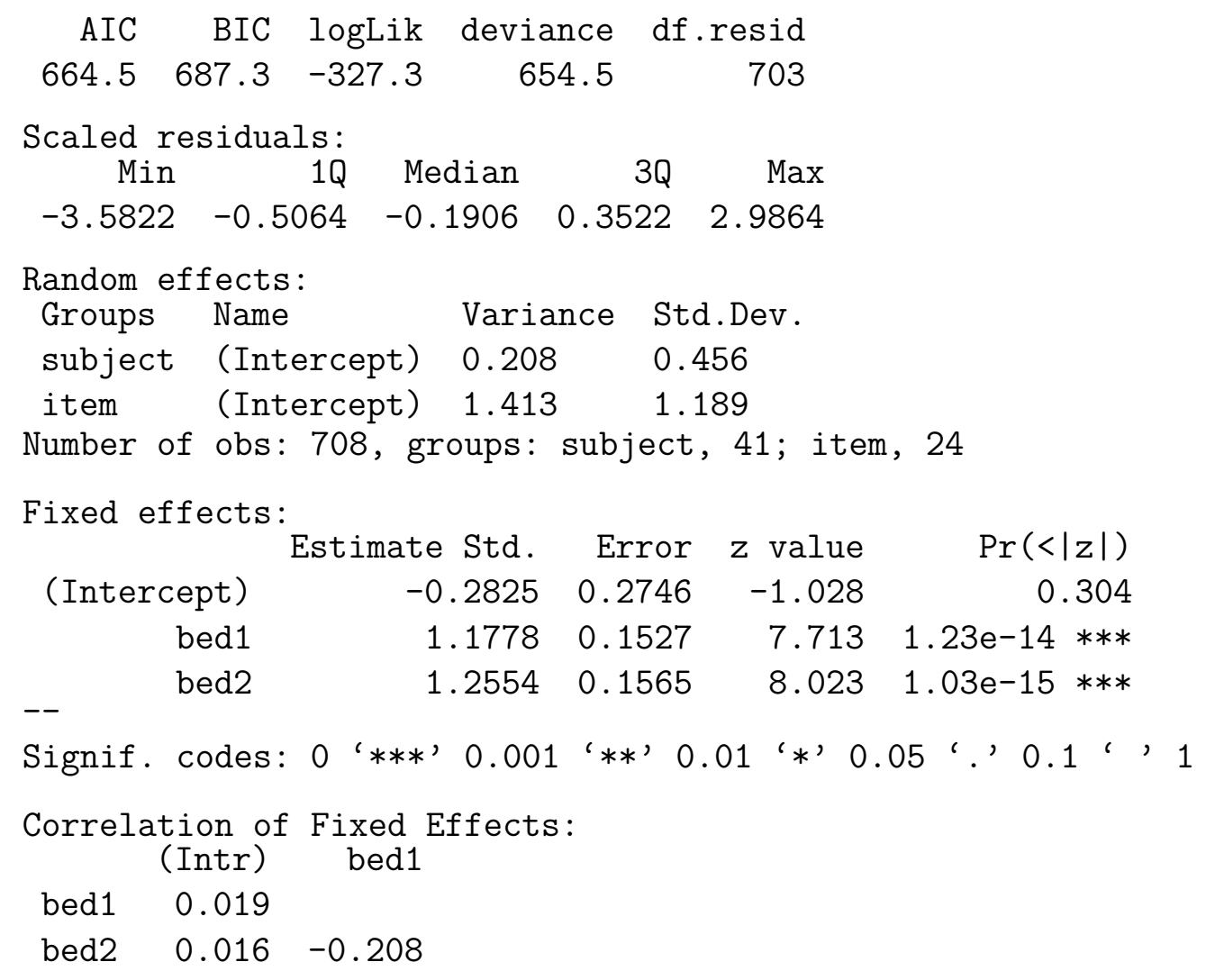

\section{C.3. Anhang zu Experiment 3b}

\section{C.3.1. Items}

\begin{tabular}{|c|c|c|c|}
\hline $\begin{array}{l}\text { Item } \\
\text { Nr. }\end{array}$ & $\begin{array}{l}\text { STRUK- } \\
\text { TUR }\end{array}$ & Zeile & Stimulus \\
\hline \multirow{6}{*}{1} & \multirow{6}{*}{ RK } & 1 & Susanne hat in den Semesterferien eine Fernreise unternommen. \\
\hline & & 2 & In Kolumbien hat sie die Gelegenheit genutzt und \\
\hline & & 3 & mit einer Freundin eine Plantage besucht. \\
\hline & & 4 & Die Tabakverarbeitung erscheint ziemlich aufwändig. Er soll \\
\hline & & 5 & qualitativ auch wirklich herausragend sein. \\
\hline & & 6 & Die Atmosphäre beeindruckt Susanne schon ein wenig. \\
\hline \multirow{6}{*}{1} & \multirow{6}{*}{ WK } & 1 & Susanne hat in den Semesterferien eine Fernreise unternommen. \\
\hline & & 2 & In Kolumbien hat sie die Gelegenheit genutzt und \\
\hline & & 3 & mit einer Freundin eine Plantage besucht. \\
\hline & & 4 & Die Tabakfabrik erscheint ziemlich modern. Er soll \\
\hline & & 5 & qualitativ auch wirklich herausragend sein. \\
\hline & & 6 & Die Atmosphäre beeindruckt Susanne schon ein wenig. \\
\hline \multirow{6}{*}{1} & \multirow{6}{*}{ Mono } & 1 & Susanne hat in den Semesterferien eine Fernreise unternommen. \\
\hline & & 2 & In Kolumbien hat sie die Gelegenheit genutzt und \\
\hline & & 3 & mit einer Freundin eine Plantage besucht. \\
\hline & & 4 & Der Tabak wird ziemlich aufwändig verarbeitet. Er soll \\
\hline & & 5 & qualitativ auch wirklich herausragend sein. \\
\hline & & 6 & Die Atmosphäre beeindruckt Susanne schon ein wenig. \\
\hline
\end{tabular}




\begin{tabular}{|c|c|c|c|}
\hline \multirow{6}{*}{2} & \multirow{6}{*}{ RK } & 1 & Armin hat eine Mahnung von seinem Vermieter bekommen. \\
\hline & & 2 & Er ist mit seiner Miete monatelang in Verzug und muss \\
\hline & & 3 & nun notgedrungen seine Eltern um Hilfe bitten. \\
\hline & & 4 & Die Geldsendung kommt gerade noch rechtzeitig. Es muss \\
\hline & & 5 & jetzt nur noch schnell eingezahlt werden. \\
\hline & & 6 & Das wird Armin so schnell nicht wieder passieren. \\
\hline \multirow{6}{*}{2} & \multirow{6}{*}{ WK } & 1 & Armin hat eine Mahnung von seinem Vermieter bekommen. \\
\hline & & 2 & Er ist mit seiner Miete monatelang in Verzug und muss \\
\hline & & 3 & nun notgedrungen seine Eltern um Hilfe bitten. \\
\hline & & 4 & Der Geldbrief kommt gerade noch rechtzeitig. Es muss \\
\hline & & 5 & jetzt nur noch schnell eingezahlt werden. \\
\hline & & 6 & Das wird Armin so schnell nicht wieder passieren. \\
\hline \multirow{6}{*}{2} & \multirow{6}{*}{ Mono } & 1 & Armin hat eine Mahnung von seinem Vermieter bekommen. \\
\hline & & 2 & Er ist mit seiner Miete monatelang in Verzug und muss \\
\hline & & 3 & nun notgedrungen seine Eltern um Hilfe bitten. \\
\hline & & 4 & Das Geld kommt gerade noch rechtzeitig. Es muss \\
\hline & & 5 & jetzt nur noch schnell eingezahlt werden. \\
\hline & & 6 & Das wird Armin so schnell nicht wieder passieren. \\
\hline \multirow{6}{*}{3} & \multirow{6}{*}{ RK } & 1 & Ida hat für das Wochenende einen Bollywood-Abend geplant. \\
\hline & & 2 & Sie hat viele Freunde eingeladen und allerlei \\
\hline & & 3 & indisches Fingerfood und Süßigkeiten besorgt. \\
\hline & & 4 & $\begin{array}{l}\text { Die Videovorführung findet im Gemeinschaftsraum statt. Es } \\
\text { muss }\end{array}$ \\
\hline & & 5 & jetzt nur noch abgespielt werden. \\
\hline & & 6 & Filmpartys sind Idas Leidenschaft. \\
\hline \multirow{6}{*}{3} & \multirow{6}{*}{ WK } & 1 & Ida hat für das Wochenende einen Bollywood-Abend geplant. \\
\hline & & 2 & Sie hat viele Freunde eingeladen und allerlei \\
\hline & & 3 & indisches Fingerfood und Süßigkeiten besorgt. \\
\hline & & 4 & Der Videorekorder steht im Gemeinschaftsraum. Es muss \\
\hline & & 5 & jetzt nur noch abgespielt werden. \\
\hline & & 6 & Filmpartys sind Idas Leidenschaft. \\
\hline \multirow{6}{*}{3} & \multirow{6}{*}{ Mono } & 1 & Ida hat für das Wochenende einen Bollywood-Abend geplant. \\
\hline & & 2 & Sie hat viele Freunde eingeladen und allerlei \\
\hline & & 3 & indisches Fingerfood und Süßigkeiten besorgt. \\
\hline & & 4 & Das Video wird im Gemeinschaftsraum gezeigt. Es muss \\
\hline & & 5 & jetzt nur noch abgespielt werden. \\
\hline & & 6 & Filmpartys sind Idas Leidenschaft. \\
\hline \multirow{6}{*}{4} & \multirow{6}{*}{ RK } & 1 & Anja hat die Einladung zum Grillabend angenommen. \\
\hline & & 2 & Seitdem sie bei der Lebensmittelkontrolle arbeitet, \\
\hline & & 3 & hat sie wieder Vertrauen in die regionalen Produzenten. \\
\hline & & 4 & $\begin{array}{l}\text { Die Fleischuntersuchung wird sehr gründlich vorgenommen. Es } \\
\text { muss }\end{array}$ \\
\hline & & 5 & allen vorgeschriebenen Kriterien entsprechen. \\
\hline & & 6 & Auf ein saftiges Steak freut sich Anja deshalb sehr. \\
\hline
\end{tabular}




\begin{tabular}{|c|c|c|c|}
\hline \multirow{6}{*}{4} & \multirow{6}{*}{ WK } & 1 & Anja hat die Einladung zum Grillabend angenommen. \\
\hline & & 2 & Seitdem sie bei der Lebensmittelkontrolle arbeitet, \\
\hline & & 3 & hat sie wieder Vertrauen in die regionalen Produzenten. \\
\hline & & 4 & Der Fleischsaft wird sehr gründlich untersucht. Es muss \\
\hline & & 5 & allen vorgeschriebenen Kriterien entsprechen. \\
\hline & & 6 & Auf ein saftiges Steak freut sich Anja deshalb sehr. \\
\hline \multirow{6}{*}{4} & \multirow{6}{*}{ Mono } & 1 & Anja hat die Einladung zum Grillabend angenommen. \\
\hline & & 2 & Seitdem sie bei der Lebensmittelkontrolle arbeitet, \\
\hline & & 3 & hat sie wieder Vertrauen in die regionalen Produzenten. \\
\hline & & 4 & Das Fleisch wird sehr gründlich untersucht. Es muss \\
\hline & & 5 & allen vorgeschriebenen Kriterien entsprechen. \\
\hline & & 6 & Auf ein saftiges Steak freut sich Anja deshalb sehr. \\
\hline \multirow{6}{*}{5} & \multirow{6}{*}{ RK } & 1 & Konrad muss seinen neuen Arbeitsplatz zuerst neu einrichten. \\
\hline & & 2 & Leider hat sein Vorgänger ein ziemliches Chaos hinterlassen \\
\hline & & 3 & und den Drucker sehr ungünstig aufgestellt. \\
\hline & & 4 & Die Kabelbefestigung liegt mitten im Raum. Es soll \\
\hline & & 5 & eigentlich immer an der Wand entlang verlaufen. \\
\hline & & 6 & Mit so viel Mehraufwand hatte Konrad nicht gerechnet. \\
\hline \multirow{6}{*}{5} & \multirow{6}{*}{ WK } & 1 & Konrad muss seinen neuen Arbeitsplatz zuerst neu einrichten. \\
\hline & & 2 & Leider hat sein Vorgänger ein ziemliches Chaos hinterlassen \\
\hline & & 3 & und den Drucker sehr ungünstig aufgestellt. \\
\hline & & 4 & Der Kabelkanal liegt mitten im Raum. Es soll \\
\hline & & 5 & eigentlich immer an der Wand entlang verlaufen. \\
\hline & & 6 & Mit so viel Mehraufwand hatte Konrad nicht gerechnet. \\
\hline \multirow{6}{*}{5} & \multirow{6}{*}{ Mono } & 1 & Konrad muss seinen neuen Arbeitsplatz zuerst neu einrichten. \\
\hline & & 2 & Leider hat sein Vorgänger ein ziemliches Chaos hinterlassen \\
\hline & & 3 & und den Drucker sehr ungünstig aufgestellt. \\
\hline & & 4 & Das Kabel liegt mitten im Raum. Es soll \\
\hline & & 5 & eigentlich immer an der Wand entlang verlaufen. \\
\hline & & 6 & Mit so viel Mehraufwand hatte Konrad nicht gerechnet. \\
\hline \multirow{6}{*}{6} & \multirow{6}{*}{ RK } & 1 & Mareike hat für ihre Zahnarztpraxis neue Möbel bekommen. \\
\hline & & 2 & Im Behandlungszimmer begutachtet sie die \\
\hline & & 3 & neue und größere Zahnarztliege. \\
\hline & & 4 & Die Kopfpolsterung ist besonders komfortabel. Er soll \\
\hline & & 5 & möglichst bequem abgelegt werden können. \\
\hline & & 6 & Hoffentlich sind die Patienten so begeistert wie Mareike. \\
\hline \multirow{6}{*}{6} & \multirow{6}{*}{ WK } & 1 & Mareike hat für ihre Zahnarztpraxis neue Möbel bekommen. \\
\hline & & 2 & Im Behandlungszimmer begutachtet sie die \\
\hline & & 3 & neue und größere Zahnarztliege. \\
\hline & & 4 & Das Kopfteil ist besonders komfortabel. Er soll \\
\hline & & 5 & möglichst bequem abgelegt werden können. \\
\hline & & 6 & Hoffentlich sind die Patienten so begeistert wie Mareike. \\
\hline
\end{tabular}




\begin{tabular}{|c|c|c|c|}
\hline \multirow{6}{*}{6} & \multirow{6}{*}{ Mono } & 1 & Mareike hat für ihre Zahnarztpraxis neue Möbel bekommen. \\
\hline & & 2 & Im Behandlungszimmer begutachtet sie die \\
\hline & & 3 & neue und größere Zahnarztliege. \\
\hline & & 4 & Der Kopf bekommt eine speziell geformte Stütze. Er soll \\
\hline & & 5 & möglichst bequem abgelegt werden können. \\
\hline & & 6 & Hoffentlich sind die Patienten so begeistert wie Mareike. \\
\hline \multirow{6}{*}{7} & \multirow{6}{*}{ RK } & 1 & Hannah hat einige Zeit an der Kunstakademie verbracht. \\
\hline & & 2 & Sie konnte sich in verschiedenen Werkstätten ausprobieren \\
\hline & & 3 & und hat letztlich auch eine neue Leidenschaft entdeckt. \\
\hline & & 4 & Die Metallgestaltung ist besonders faszinierend. Es muss \\
\hline & & 5 & $\begin{array}{l}\text { bei hohen Temperaturen und mit viel Geschick bearbeitet wer- } \\
\text { den. }\end{array}$ \\
\hline & & 6 & Langfristig will Hannah sich als Goldschmiedin verwirklichen. \\
\hline \multirow{6}{*}{7} & \multirow{6}{*}{ WK } & 1 & Hannah hat einige Zeit an der Kunstakademie verbracht. \\
\hline & & 2 & Sie konnte sich in verschiedenen Werkstätten ausprobieren \\
\hline & & 3 & und hat letztlich auch eine neue Leidenschaft entdeckt. \\
\hline & & 4 & Die Metallkunde ist besonders faszinierend. Es muss \\
\hline & & 5 & $\begin{array}{l}\text { bei hohen Temperaturen und mit viel Geschick bearbeitet wer- } \\
\text { den. }\end{array}$ \\
\hline & & 6 & Langfristig will Hannah sich als Goldschmiedin verwirklichen. \\
\hline \multirow{6}{*}{7} & \multirow{6}{*}{ Mono } & 1 & Hannah hat einige Zeit an der Kunstakademie verbracht. \\
\hline & & 2 & Sie konnte sich in verschiedenen Werkstätten ausprobieren \\
\hline & & 3 & und hat letztlich auch eine neue Leidenschaft entdeckt. \\
\hline & & 4 & Das Metall ist ein faszinierender Werkstoff. Es muss \\
\hline & & 5 & $\begin{array}{l}\text { bei hohen Temperaturen und mit viel Geschick bearbeitet wer- } \\
\text { den. }\end{array}$ \\
\hline & & 6 & Langfristig will Hannah sich als Goldschmiedin verwirklichen. \\
\hline \multirow{6}{*}{8} & \multirow{6}{*}{ RK } & 1 & Silke hat ein Praktikum auf einem Recyclinghof gemacht. \\
\hline & & 2 & Die Arbeit dort ist nicht immer angenehm, aber die ablaufenden \\
\hline & & 3 & Phasen und Prozesse sind mitunter schon interessant. \\
\hline & & 4 & Die Müllbeseitigung ist ein viel diskutiertes Thema. Er muss \\
\hline & & 5 & sinnvoll getrennt und abtransportiert werden. \\
\hline & & 6 & Eventuell will Silke nun etwas auf diesem Gebiet studieren. \\
\hline \multirow{6}{*}{8} & \multirow{6}{*}{ WK } & 1 & Silke hat ein Praktikum auf einem Recyclinghof gemacht. \\
\hline & & 2 & Die Arbeit dort ist nicht immer angenehm, aber die ablaufenden \\
\hline & & 3 & Phasen und Prozesse sind mitunter auch sehr interessant. \\
\hline & & 4 & Die Mülltonne ist ein viel diskutiertes Thema. Er muss \\
\hline & & 5 & sinnvoll getrennt und abtransportiert werden. \\
\hline & & 6 & Eventuell will Silke nun etwas auf diesem Gebiet studieren. \\
\hline \multirow{6}{*}{8} & \multirow{6}{*}{ Mono } & 1 & Silke hat ein Praktikum auf einem Recyclinghof gemacht. \\
\hline & & 2 & Die Arbeit dort ist nicht immer angenehm, aber die ablaufenden \\
\hline & & 3 & Phasen und Prozesse sind mitunter auch sehr interessant. \\
\hline & & 4 & Der Müll ist ein viel diskutiertes Thema. Er muss \\
\hline & & 5 & sinnvoll getrennt und verarbeitet werden. \\
\hline & & 6 & Eventuell will Silke nun etwas auf diesem Gebiet studieren. \\
\hline
\end{tabular}




\begin{tabular}{|c|c|c|c|}
\hline \multirow{6}{*}{9} & \multirow{6}{*}{ RK } & 1 & Johanna muss sich von ihrer Freundin verabschieden. \\
\hline & & 2 & Sie studiert jetzt in einer anderen Stadt, aber sie \\
\hline & & 3 & wollen sich regelmäßig besuchen. \\
\hline & & 4 & Die Zugverbindung ist eigentlich ganz gut. Er soll \\
\hline & & 5 & zweimal täglich ohne viele Zwischenstopps durchfahren. \\
\hline & & 6 & Schon bald will Johanna einmal vorbeischauen. \\
\hline \multirow{6}{*}{9} & \multirow{6}{*}{ WK } & 1 & Johanna muss sich von ihrer Freundin verabschieden. \\
\hline & & 2 & Sie studiert jetzt in einer anderen Stadt, aber sie \\
\hline & & 3 & wollen sich regelmäßig besuchen. \\
\hline & & 4 & Die Zugstrecke ist eigentlich machbar. Er soll \\
\hline & & 5 & zweimal täglich ohne viele Zwischenstopps durchfahren. \\
\hline & & 6 & Schon bald will Johanna einmal vorbeischauen. \\
\hline \multirow{6}{*}{9} & \multirow{6}{*}{ Mono } & 1 & Johanna muss sich von ihrer Freundin verabschieden. \\
\hline & & 2 & Sie studiert jetzt in einer anderen Stadt, aber sie \\
\hline & & 3 & wollen sich regelmäßig besuchen. \\
\hline & & 4 & Der Zug ist eigentlich sogar recht günstig. Er soll \\
\hline & & 5 & zweimal täglich ohne viele Zwischenstopps durchfahren. \\
\hline & & 6 & Schon bald will Johanna einmal vorbeischauen. \\
\hline \multirow{6}{*}{10} & \multirow{6}{*}{ RK } & 1 & Julia hat ihren Urlaub in den bayerischen Alpen verbracht. \\
\hline & & 2 & Dort ist sie viel gewandert und hat außerdem \\
\hline & & 3 & ein sehr traditionelles Bergwerk besichtigt. \\
\hline & & 4 & Die Salzgewinnung ist typisch für diese Gegend. Es soll \\
\hline & & 5 & vor allem für die Atemwege heilende Kräfte haben. \\
\hline & & 6 & Nach dem Urlaub fühlt sich Julia gesund wie nie. \\
\hline \multirow{6}{*}{10} & \multirow{6}{*}{ WK } & 1 & Julia hat ihren Urlaub in den bayerischen Alpen verbracht. \\
\hline & & 2 & Dort ist sie viel gewandert und hat außerdem \\
\hline & & 3 & ein sehr traditionelles Bergwerk besichtigt. \\
\hline & & 4 & Die Salzkonzentration ist typisch für diese Gegend. Es soll \\
\hline & & 5 & vor allem für die Atemwege heilende Kräfte haben. \\
\hline & & 6 & Nach dem Urlaub fühlt sich Julia gesund wie nie. \\
\hline \multirow{6}{*}{10} & \multirow{6}{*}{ Mono } & 1 & Julia hat ihren Urlaub in den bayerischen Alpen verbracht. \\
\hline & & 2 & Dort ist sie viel gewandert und hat außerdem \\
\hline & & 3 & ein sehr traditionelles Bergwerk besichtigt. \\
\hline & & 4 & Das Salz ist typisch für diese Gegend. Es soll \\
\hline & & 5 & vor allem für die Atemwege heilende Kräfte haben. \\
\hline & & 6 & Nach dem Urlaub fühlt sich Julia gesund wie nie. \\
\hline \multirow{6}{*}{11} & \multirow{6}{*}{ RK } & 1 & Mattis besucht seine Schwester in der Verpackungsfabrik. \\
\hline & & 2 & Sie hat ihn schon überall herumgeführt und viel erklärt, \\
\hline & & 3 & aber Mattis ist sehr neugierig und will alles ganz genau wissen. \\
\hline & & 4 & $\begin{array}{l}\text { Die Papierherstellung wird nicht mehr per Hand gemacht. Es } \\
\text { soll }\end{array}$ \\
\hline & & 5 & schließlich eine gleichmäßige Faserung erhalten. \\
\hline & & 6 & Irgendwie hat sich Mattis das alles spannender vorgestellt. \\
\hline
\end{tabular}




\begin{tabular}{|c|c|c|c|}
\hline \multirow{6}{*}{11} & \multirow{6}{*}{ WK } & 1 & Mattis besucht seine Schwester in der Verpackungsfabrik. \\
\hline & & 2 & Sie hat ihn schon überall herumgeführt und viel erklärt, \\
\hline & & 3 & aber Mattis ist sehr neugierig und will alles ganz genau wissen. \\
\hline & & 4 & Die Papiermaschine wird nicht mehr per Hand bedient. Es soll \\
\hline & & 5 & schließlich eine gleichmäßige Faserung erhalten. \\
\hline & & 6 & Irgendwie hat sich Mattis das alles spannender vorgestellt. \\
\hline \multirow{6}{*}{11} & \multirow{6}{*}{ Mono } & 1 & Mattis besucht seine Schwester in der Verpackungsfabrik. \\
\hline & & 2 & Sie hat ihn schon überall herumgeführt und viel erklärt, \\
\hline & & 3 & aber Mattis ist sehr neugierig und will alles ganz genau wissen. \\
\hline & & 4 & Das Papier wird nicht mehr per Hand hergestellt. Es soll \\
\hline & & 5 & schließlich eine gleichmäßige Faserung erhalten. \\
\hline & & 6 & Irgendwie hat sich Mattis das alles spannender vorgestellt. \\
\hline \multirow{6}{*}{12} & \multirow{6}{*}{ RK } & 1 & Pia ist Stationsschwester im Kreiskrankenhaus. \\
\hline & & 2 & Manchmal muss sie Auszubildenden auch Pflege- \\
\hline & & 3 & und Hygienevorschriften vermitteln. \\
\hline & & 4 & Die Bettreinigung gehört zu den oft vernachlässigten Aufgaben. \\
\hline & & 5 & $\begin{array}{l}\text { Es muss } \\
\text { wirklich von allen Seiten eine gründliche Desinfektion bekom- } \\
\text { men. }\end{array}$ \\
\hline & & 6 & Zum Glück muss Pia aber selbst nur noch selten Hand anlegen. \\
\hline \multirow{6}{*}{12} & \multirow{6}{*}{ WK } & 1 & Pia ist Stationsschwester im Kreiskrankenhaus. \\
\hline & & 2 & Manchmal muss sie Auszubildenden auch Pflege- \\
\hline & & 3 & und Hygienevorschriften vermitteln. \\
\hline & & 4 & $\begin{array}{l}\text { Der Bettpfosten gehört zu den oft vernachlässigten Teilen. Es } \\
\text { muss }\end{array}$ \\
\hline & & 5 & $\begin{array}{l}\text { wirklich von allen Seiten eine gründliche Desinfektion bekom- } \\
\text { men. }\end{array}$ \\
\hline & & 6 & Zum Glück muss Pia aber selbst nur noch selten Hand anlegen. \\
\hline \multirow{6}{*}{12} & \multirow{6}{*}{ Mono } & 1 & Pia ist Stationsschwester im Kreiskrankenhaus. \\
\hline & & 2 & Manchmal muss sie Auszubildenden auch Pflege- \\
\hline & & 3 & und Hygienevorschriften vermitteln. \\
\hline & & 4 & Das Bett gehört zu den oft vernachlässigten Teilen. Es muss \\
\hline & & 5 & $\begin{array}{l}\text { wirklich von allen Seiten eine gründliche Desinfektion bekom- } \\
\text { men. }\end{array}$ \\
\hline & & 6 & Zum Glück muss Pia aber selbst nur noch selten Hand anlegen. \\
\hline \multirow{6}{*}{13} & \multirow{6}{*}{ RK } & 1 & Stefan hat eine eigene kleine Autowerkstatt. \\
\hline & & 2 & Wegen seiner herzlichen Art hat er viele treue Kunden, \\
\hline & & 3 & die manchmal spontan vor der Tür stehen und Rat suchen. \\
\hline & & 4 & Die Ölverteilung wird oft als Fehlerquelle übersehen. Es muss \\
\hline & & 5 & zur genauen Überprüfung zunächst abgelassen werden. \\
\hline & & 6 & Meistens hat Stefan eine schnelle Lösung parat. \\
\hline \multirow{6}{*}{13} & \multirow{6}{*}{ WK } & 1 & Stefan hat eine eigene kleine Autowerkstatt. \\
\hline & & 2 & Wegen seiner herzlichen Art hat er viele treue Kunden, \\
\hline & & 3 & die manchmal spontan vor der Tür stehen und Rat suchen. \\
\hline & & 4 & Die Ölwanne wird oft als Fehlerquelle übersehen. Es muss \\
\hline & & 5 & zur genauen Überprüfung zunächst abgelassen werden. \\
\hline & & 6 & Meistens hat Stefan eine schnelle Lösung parat. \\
\hline
\end{tabular}




\begin{tabular}{|c|c|c|c|}
\hline \multirow{6}{*}{13} & \multirow{6}{*}{ Mono } & 1 & Stefan hat eine eigene kleine Autowerkstatt. \\
\hline & & 2 & Wegen seiner herzlichen Art hat er viele treue Kunden, \\
\hline & & 3 & die manchmal spontan vor der Tür stehen und Rat suchen. \\
\hline & & 4 & Das Öl wird oft als Fehlerquelle übersehen. Es muss \\
\hline & & 5 & zur genauen Überprüfung zunächst abgelassen werden. \\
\hline & & 6 & Meistens hat Stefan eine schnelle Lösung parat. \\
\hline \multirow{6}{*}{14} & \multirow{6}{*}{ RK } & 1 & Tanja hat einen neuen Job bei den Stadtwerken. \\
\hline & & 2 & Nach dem Chemiestudium wollte sie gern etwas \\
\hline & & 3 & Angewandtes machen und bekommt nun die Gelegenheit dazu. \\
\hline & & 4 & $\begin{array}{l}\text { Die Wasseraufbereitung ist für die Gemeinde sehr wichtig. Es } \\
\text { muss }\end{array}$ \\
\hline & & 5 & den geltenden Standards entsprechen und chemisch rein sein. \\
\hline & & 6 & Über diese verantwortungsvolle Arbeit freut sich Tanja sehr. \\
\hline \multirow{6}{*}{14} & \multirow{6}{*}{ WK } & 1 & Tanja hat einen neuen Job bei den Stadtwerken. \\
\hline & & 2 & Nach dem Chemiestudium wollte sie gern etwas \\
\hline & & 3 & Angewandtes machen und bekommt nun die Gelegenheit dazu. \\
\hline & & 4 & Die Wasserqualität ist für die Gemeinde sehr wichtig. Es muss \\
\hline & & 5 & den geltenden Standards entsprechen und chemisch rein sein. \\
\hline & & 6 & Über diese verantwortungsvolle Arbeit freut sich Tanja sehr. \\
\hline \multirow{6}{*}{14} & \multirow{6}{*}{ Mono } & 1 & Tanja hat einen neuen Job bei den Stadtwerken. \\
\hline & & 2 & Nach dem Chemiestudium wollte sie gern etwas \\
\hline & & 3 & Angewandtes machen und bekommt nun die Gelegenheit dazu. \\
\hline & & 4 & Das Wasser wird für die Gemeinde aufbereitet. Es muss \\
\hline & & 5 & den geltenden Standards entsprechen und chemisch rein sein. \\
\hline & & 6 & Über diese verantwortungsvolle Arbeit freut sich Tanja sehr. \\
\hline \multirow{6}{*}{15} & \multirow{6}{*}{ RK } & 1 & Britta macht mit ihrer Schulklasse einen Ausflug. \\
\hline & & 2 & In einem Museum über den Zweiten Weltkrieg \\
\hline & & 3 & bekommen sie alte Fotos von Gefechtsstellungen zu sehen. \\
\hline & & 4 & Die Panzerbekämpfung wirkt ziemlich brutal. Er muss \\
\hline & & 5 & damals Angst und Schrecken verbreitet haben. \\
\hline & & 6 & Den Kindern und Britta wird es richtig mulmig. \\
\hline \multirow{6}{*}{15} & \multirow{6}{*}{ WK } & 1 & Britta macht mit ihrer Schulklasse einen Ausflug. \\
\hline & & 2 & In einem Museum über den Zweiten Weltkrieg \\
\hline & & 3 & bekommen sie alte Fotos von Gefechtsstellungen zu sehen. \\
\hline & & 4 & Die Panzerkette wirkt ziemlich monströs. Er muss \\
\hline & & 5 & damals Angst und Schrecken verbreitet haben. \\
\hline & & 6 & Den Kindern und Britta wird es richtig mulmig. \\
\hline \multirow{6}{*}{15} & \multirow{6}{*}{ Mono } & 1 & Britta macht mit ihrer Schulklasse einen Ausflug. \\
\hline & & 2 & In einem Museum über den Zweiten Weltkrieg \\
\hline & & 3 & bekommen sie alte Fotos von Gefechtsstellungen zu sehen. \\
\hline & & 4 & Der Panzer wirkt ziemlich monströs. Er muss \\
\hline & & 5 & damals Angst und Schrecken verbreitet haben. \\
\hline & & 6 & Den Kindern und Britta wird es richtig mulmig. \\
\hline
\end{tabular}




\begin{tabular}{|c|c|c|c|}
\hline \multirow{6}{*}{16} & \multirow{6}{*}{ RK } & 1 & Elise arbeitet als Webdesignerin bei einem Online-Journal. \\
\hline & & 2 & Vor einigen Tagen wurden ganz neue Programme eingeführt, \\
\hline & & 3 & $\begin{array}{l}\text { an einige neue Softwarekomponenten muss sie sich erst gewöh- } \\
\text { nen. }\end{array}$ \\
\hline & & 4 & Die Texterkennung erleichtert das Arbeiten sehr. Er muss \\
\hline & & 5 & dann nur noch per Hand formatiert werden. \\
\hline & & 6 & Nach ein bisschen Übung ist Elise ganz begeistert. \\
\hline \multirow{6}{*}{16} & \multirow{6}{*}{ WK } & 1 & Elise arbeitet als Webdesignerin bei einem Online-Journal. \\
\hline & & 2 & Vor einigen Tagen wurden ganz neue Programme eingeführt, \\
\hline & & 3 & $\begin{array}{l}\text { an einige neue Softwarekomponenten muss sie sich erst gewöh- } \\
\text { nen. }\end{array}$ \\
\hline & & 4 & Das Textfeld erleichtert das Arbeiten sehr. Er muss \\
\hline & & 5 & dann nur noch per Hand formatiert werden. \\
\hline & & 6 & Nach ein bisschen Übung ist Elise ganz begeistert. \\
\hline \multirow{6}{*}{16} & \multirow{6}{*}{ Mono } & 1 & Elise arbeitet als Webdesignerin bei einem Online-Journal. \\
\hline & & 2 & Vor einigen Tagen wurden ganz neue Programme eingeführt, \\
\hline & & 3 & $\begin{array}{l}\text { an einige neue Softwarekomponenten muss sie sich erst gewöh- } \\
\text { nen. }\end{array}$ \\
\hline & & 4 & Der Text kann digital eingelesen werden. Er muss \\
\hline & & 5 & dann nur noch per Hand formatiert werden. \\
\hline & & 6 & Nach ein bisschen Übung ist Elise ganz begeistert. \\
\hline \multirow{6}{*}{17} & \multirow{6}{*}{ RK } & 1 & Bernd hat einen extravaganten Geschmack. \\
\hline & & 2 & Design und Stil sind für ihn enorm wichtig \\
\hline & & 3 & und endlich erfüllt er sich einen großen Traum. \\
\hline & & 4 & Die Autoanfertigung dauert länger als erwartet. Es kann \\
\hline & & 5 & die gewünschte Lackierung erst am Montag erhalten. \\
\hline & & 6 & Aber in wenigen Tagen wird Bernd das neue Cabrio abholen. \\
\hline \multirow{6}{*}{17} & \multirow{6}{*}{ WK } & 1 & Bernd hat einen extravaganten Geschmack. \\
\hline & & 2 & Design und Stil sind für ihn enorm wichtig \\
\hline & & 3 & und endlich erfüllt er sich einen großen Traum. \\
\hline & & 4 & Die Autowerkstatt ist am Wochenende geschlossen. Es kann \\
\hline & & 5 & die gewünschte Lackierung erst am Montag erhalten. \\
\hline & & 6 & Aber in wenigen Tagen wird Bernd das neue Cabrio abholen. \\
\hline \multirow{6}{*}{17} & \multirow{6}{*}{ Mono } & 1 & Bernd hat einen extravaganten Geschmack. \\
\hline & & 2 & Design und Stil sind für ihn enorm wichtig \\
\hline & & 3 & und endlich erfüllt er sich einen großen Traum. \\
\hline & & 4 & Das Auto ist aber leider noch nicht ganz fertig. Es kann \\
\hline & & 5 & die gewünschte Lackierung erst am Montag erhalten. \\
\hline & & 6 & Aber in wenigen Tagen wird Bernd das neue Cabrio abholen. \\
\hline \multirow{6}{*}{18} & \multirow{6}{*}{ RK } & 1 & Marlene ist immer sehr unternehmungslustig. \\
\hline & & 2 & Dieses Jahr haben Freunde sie zu einem Aktivurlaub \\
\hline & & 3 & eingeladen und ohne große Nachfragen ist sie mitgefahren. \\
\hline & & 4 & Die Bergbesteigung ist dann unerwartet anstrengend. Er soll \\
\hline & & 5 & auch für einige schwierige Passagen bekannt sein. \\
\hline & & 6 & Beim nächsten Mal will sich Marlene vorher besser informieren. \\
\hline
\end{tabular}




\begin{tabular}{|c|c|c|c|}
\hline \multirow{6}{*}{18} & \multirow{6}{*}{ WK } & 1 & Marlene ist immer sehr unternehmungslustig. \\
\hline & & 2 & Dieses Jahr haben Freunde sie zu einem Aktivurlaub \\
\hline & & 3 & eingeladen und ohne große Nachfragen ist sie mitgefahren. \\
\hline & & 4 & Die Bergetappe ist dann unerwartet anstrengend. Er soll \\
\hline & & 5 & auch für einige schwierige Passagen bekannt sein. \\
\hline & & 6 & Beim nächsten Mal will sich Marlene vorher besser informieren. \\
\hline \multirow{6}{*}{18} & \multirow{6}{*}{ Mono } & 1 & Marlene ist immer sehr unternehmungslustig. \\
\hline & & 2 & Dieses Jahr haben Freunde sie zu einem Aktivurlaub \\
\hline & & 3 & eingeladen und ohne große Nachfragen ist sie mitgefahren. \\
\hline & & 4 & Der Berg ist dann unerwartet anspruchsvoll. Er soll \\
\hline & & 5 & auch für einige schwierige Passagen bekannt sein. \\
\hline & & 6 & Beim nächsten Mal will sich Marlene vorher besser informieren. \\
\hline \multirow{6}{*}{19} & \multirow{6}{*}{ RK } & 1 & David ist ein richtiges Arbeitstier. \\
\hline & & 2 & Tag und Nacht sitzt er an seinem neusten Projekt, \\
\hline & & 3 & diesmal aber mit tatkräftiger Unterstützung. \\
\hline & & 4 & Die Buchveröffentlichung steht kurz bevor. Es kann \\
\hline & & 5 & mit etwas Glück der neue Bestseller werden. \\
\hline & & 6 & So nah war David seinem Traum noch nie. \\
\hline \multirow{6}{*}{19} & \multirow{6}{*}{ WK } & 1 & David ist ein richtiges Arbeitstier. \\
\hline & & 2 & Tag und Nacht sitzt er an seinem neusten Projekt, \\
\hline & & 3 & diesmal aber mit tatkräftiger Unterstützung. \\
\hline & & 4 & Der Buchpreis steht kurz bevor. Es kann \\
\hline & & 5 & mit ewas Glück der neue Bestseller werden. \\
\hline & & 6 & So nah war David seinem Traum noch nie. \\
\hline \multirow{6}{*}{19} & \multirow{6}{*}{ Mono } & 1 & David ist ein richtiges Arbeitstier. \\
\hline & & 2 & Tag und Nacht sitzt er an seinem neusten Projekt, \\
\hline & & 3 & diesmal aber mit tatkräftiger Unterstützung. \\
\hline & & 4 & Das Buch steht kurz vor der Veröffentlichung. Es kann \\
\hline & & 5 & mit etwas Glück der neue Bestseller werden. \\
\hline & & 6 & So nah war David seinem Traum noch nie. \\
\hline \multirow{6}{*}{20} & \multirow{6}{*}{ RK } & 1 & Karl ist ein richtiger Heimwerker geworden. \\
\hline & & 2 & Seit die Kinder ausgezogen sind, \\
\hline & & 3 & ist er nur noch am Bauen und Buddeln. \\
\hline & & 4 & Die Dachbegrünung ist wirklich gut gelungen. Es kann \\
\hline & & 5 & $\begin{array}{l}\text { jetzt bei gutem Wetter wunderbar als Rückzugsort genutzt wer- } \\
\text { den. }\end{array}$ \\
\hline & & 6 & Als nächstes will sich Karl den Keller vornehmen. \\
\hline \multirow{6}{*}{20} & \multirow{6}{*}{ WK } & 1 & Karl ist ein richtiger Heimwerker geworden. \\
\hline & & 2 & Seit die Kinder ausgezogen sind, \\
\hline & & 3 & ist er nur noch am Bauen und Buddeln. \\
\hline & & 4 & Der Dachgarten ist wirklich gut gelungen. Es kann \\
\hline & & 5 & $\begin{array}{l}\text { jetzt bei gutem Wetter wunderbar als Rückzugsort genutzt wer- } \\
\text { den. }\end{array}$ \\
\hline & & 6 & Als nächstes will sich Karl den Keller vornehmen. \\
\hline
\end{tabular}




\begin{tabular}{|c|c|c|c|}
\hline \multirow{6}{*}{20} & \multirow{6}{*}{ Mono } & 1 & Karl ist ein richtiger Heimwerker geworden. \\
\hline & & 2 & Seit die Kinder ausgezogen sind, \\
\hline & & 3 & ist er nur noch am Bauen und Buddeln. \\
\hline & & 4 & Das Dach ist wirklich gut gestaltet. Es kann \\
\hline & & 5 & $\begin{array}{l}\text { jetzt bei gutem Wetter wunderbar als Rückzugsort genutzt wer- } \\
\text { den. }\end{array}$ \\
\hline & & 6 & Als nächstes will sich Karl den Keller vornehmen. \\
\hline \multirow{6}{*}{21} & \multirow{6}{*}{ RK } & 1 & Barbara hat dieses Jahr ein Sommerfest organisiert. \\
\hline & & 2 & Leider ist bisher alles schief gelaufen: Der Partyservice \\
\hline & & 3 & hat sich im Datum vertan und die Band steckt im Stau. \\
\hline & & 4 & Die Gartenbeleuchtung ist auch noch ausgefallen. Er muss \\
\hline & & 5 & jetzt schnell noch mit Kerzen dekoriert werden. \\
\hline & & 6 & Den ganzen Tag telefoniert Barbara nun schon umher. \\
\hline \multirow{6}{*}{21} & \multirow{6}{*}{ WK } & 1 & Barbara hat dieses Jahr ein Sommerfest organisiert. \\
\hline & & 2 & Leider ist bisher alles schief gelaufen: Der Partyservice \\
\hline & & 3 & hat sich im Datum vertan und die Band steckt im Stau. \\
\hline & & 4 & Das Gartenlicht ist auch noch ausgefallen. Er muss \\
\hline & & 5 & jetzt schnell noch mit Kerzen dekoriert werden. \\
\hline & & 6 & Den ganzen Tag telefoniert Barbara nun schon umher. \\
\hline \multirow{6}{*}{21} & \multirow{6}{*}{ Mono } & 1 & Barbara hat dieses Jahr ein Sommerfest organisiert. \\
\hline & & 2 & Leider ist bisher alles schief gelaufen: Der Partyservice \\
\hline & & 3 & hat sich im Datum vertan und die Band steckt im Stau. \\
\hline & & 4 & Der Garten liegt plötzlich auch noch im Dunkeln. Er muss \\
\hline & & 5 & jetzt schnell noch mit Kerzen dekoriert werden. \\
\hline & & 6 & Den ganzen Tag telefoniert Barbara nun schon umher. \\
\hline \multirow{6}{*}{22} & \multirow{6}{*}{ RK } & 1 & Wiebke wird seit Tagen von einer Kundin belästigt. \\
\hline & & 2 & Sie geht ihr richtig auf die Nerven und ruft ständig an, \\
\hline & & 3 & dabei kann Wiebke ihr wirklich nicht helfen. \\
\hline & & 4 & Die Kreditgewährung unterliegt strengen Vorschriften. Er kann \\
\hline & & 5 & nicht ohne Weiteres erhöht werden. \\
\hline & & 6 & Beim nächsten Anruf will Wiebke dem Chef Bescheid geben. \\
\hline \multirow{6}{*}{22} & \multirow{6}{*}{ WK } & 1 & Wiebke wird seit Tagen von einer Kundin belästigt. \\
\hline & & 2 & Sie geht ihr richtig auf die Nerven und ruft ständig an, \\
\hline & & 3 & dabei kann Wiebke ihr wirklich nicht helfen. \\
\hline & & 4 & Das Kreditvolumen unterliegt strengen Vorschriften. Er kann \\
\hline & & 5 & nicht ohne Weiteres erhöht werden. \\
\hline & & 6 & Beim nächsten Anruf will Wiebke dem Chef Bescheid geben. \\
\hline \multirow{6}{*}{22} & \multirow{6}{*}{ Mono } & 1 & Wiebke wird seit Tagen von einer Kundin belästigt. \\
\hline & & 2 & Sie geht ihr richtig auf die Nerven und ruft ständig an, \\
\hline & & 3 & dabei kann Wiebke ihr wirklich nicht helfen. \\
\hline & & 4 & Der Kredit unterliegt strengen Vorschriften. Er kann \\
\hline & & 5 & nicht ohne Weiteres erhöht werden. \\
\hline & & 6 & Beim nächsten Anruf will Wiebke dem Chef Bescheid geben. \\
\hline
\end{tabular}




\begin{tabular}{|c|c|c|c|}
\hline \multirow{6}{*}{23} & \multirow{6}{*}{ RK } & 1 & Franziska steckt viel Zeit in ihr Ehrenamt. \\
\hline & & 2 & Zusammen mit anderen Aktivisten engagiert sie sich \\
\hline & & 3 & auf lokaler Ebene für die Umwelt. \\
\hline & & 4 & Die Waldrettung ist nach großer Aufregung geglückt. Er soll \\
\hline & & 5 & als Erholungsgebiet bestehen bleiben. \\
\hline & & 6 & Auf diesen Erfolg ist Franziska sehr stolz. \\
\hline \multirow{6}{*}{23} & \multirow{6}{*}{ WK } & 1 & Franziska steckt viel Zeit in ihr Ehrenamt. \\
\hline & & 2 & Zusammen mit anderen Aktivisten engagiert sie sich \\
\hline & & 3 & auf lokaler Ebene für die Umwelt. \\
\hline & & 4 & Die Waldstraße wird nun doch nicht gebaut. Er soll \\
\hline & & 5 & als Erholungsgebiet bestehen bleiben. \\
\hline & & 6 & Auf diesen Erfolg ist Franziska sehr stolz. \\
\hline \multirow{6}{*}{23} & \multirow{6}{*}{ Mono } & 1 & Franziska steckt viel Zeit in ihr Ehrenamt. \\
\hline & & 2 & Zusammen mit anderen Aktivisten engagiert sie sich \\
\hline & & 3 & auf lokaler Ebene für die Umwelt. \\
\hline & & 4 & Der Wald wird nun doch nicht gerodet. Er soll \\
\hline & & 5 & als Erholungsgebiet bestehen bleiben. \\
\hline & & 6 & Auf diesen Erfolg ist Franziska sehr stolz. \\
\hline \multirow{6}{*}{24} & \multirow{6}{*}{ RK } & 1 & Melanie geht mit ihren Eltern wandern. \\
\hline & & 2 & Leider ist die Karte etwas veraltet und sie \\
\hline & & 3 & haben Mühe die Aussichtsplattform zu finden. \\
\hline & & 4 & Die Wegmarkierung ist völlig zugewuchert. Er kann \\
\hline & & 5 & nur bei genauem Hinsehen gefunden werden. \\
\hline & & 6 & Langsam hat Melanie wirklich keine Lust mehr. \\
\hline \multirow{6}{*}{24} & \multirow{6}{*}{ WK } & 1 & Melanie geht mit ihren Eltern wandern. \\
\hline & & 2 & Leider ist die Karte etwas veraltet und sie \\
\hline & & 3 & haben Mühe die Aussichtsplattform zu finden. \\
\hline & & 4 & Das Wegzeichen ist völlig zugewuchert. Er kann \\
\hline & & 5 & nur bei genauem Hinsehen gefunden werden. \\
\hline & & 6 & Langsam hat Melanie wirklich keine Lust mehr. \\
\hline \multirow{6}{*}{24} & \multirow{6}{*}{ Mono } & 1 & Melanie geht mit ihren Eltern wandern. \\
\hline & & 2 & Leider ist die Karte etwas veraltet und sie \\
\hline & & 3 & haben Mühe die Aussichtsplattform zu finden. \\
\hline & & 4 & Der Weg ist völlig zugewuchert. Er kann \\
\hline & & 5 & nur bei genauem Hinsehen gefunden werden. \\
\hline & & 6 & Langsam hat Melanie wirklich keine Lust mehr. \\
\hline
\end{tabular}

\section{C.3.2. Weiteres Material}

\section{Aufgabenstellung}

Die nachfolgenden Texte sollen bezüglich ihrer Textkohärenz, also ihres inhaltlichen Zusammenhangs bewertet werden. Es geht darum, ob die einzelnen Sätze im textuellen Kontext passen und der Text als Ganzes sowie in seinen Teilen sinnvoll ist. Auch die Textkohäsion, also der ausdrucksseitige Zusammenhang soll hierbei mit einbezogen werden. 
Die Bewertung erfolgt mittels einer Skala von 1 (=absolut inkohärent) bis 7 (= völlig kohärent). Beispielsweise wäre der folgende Text mit $7 \mathrm{zu}$ bewerten:

Der Chirurg hat die OP erfolgreich durchgeführt. Sein Patient wird bald wieder gesund sein und nach einer Physiotherapie auch wieder ohne Hilfe laufen können. Das hätte er vor ein paar Monaten noch nicht gedacht. Er ist dem Arzt sehr dankbar und überlegt, was er ihm schenken könnte.

Der folgende Text wäre hingegen mit 1 zu bewerten:

Der Chirurg hat die Blume erfolgreich durchgeführt. Ihr Patient wird bald gesund sein und nach einer Seilbahn auch wieder ohne Hilfe laufen können. Das hätte er in ein paar Monaten noch nicht gedacht. Er ist dem Astronauten sehr dankbar und überlegt, was er ihm rufen könnte.

Bei der Beurteilung sollten Sie Ihr Urteil SPONTAN und INTUITIV fällen.

Bitte versuchen Sie, beim Bewerten der Sätze den ganzen Bereich der Skala auszunutzen und lassen Sie keinen der Texte aus.

Vielen Dank für Ihre Mitarbeit!

\section{Einzelergebnisse}




\begin{tabular}{l|l|l|l}
\hline \hline Item & RK & WK & Mono \\
\hline 01 & $4.71(1.6)$ & $4.62(1.41)$ & $5.44(0.88)$ \\
02 & $6.33(0.71)$ & $6.43(0.79)$ & $6.5(0.76)$ \\
03 & $5.62(0.92)$ & $4.89(1.27)$ & $5.86(1.46)$ \\
04 & $5.71(1.38)$ & $4.12(1.55)$ & $4.33(1.58)$ \\
05 & $6.00(1.22)$ & $5.86(0.69)$ & $6.12(0.99)$ \\
06 & $5.62(1.3)$ & $6.22(0.67)$ & $5.86(2.04)$ \\
07 & $5.86(1.35)$ & $6.12(0.99)$ & $5.67(0.87)$ \\
08 & $5.89(1.17)$ & $5.29(1.5)$ & $6.25(0.89)$ \\
09 & $6.25(0.46)$ & $6.00(1.12)$ & $6.43(0.79)$ \\
10 & $6.29(0.76)$ & $6.25(0.46)$ & $5.89(1.17)$ \\
11 & $5.56(1.13)$ & $5.57(1.27)$ & $5.38(1.19)$ \\
12 & $5.88(1.25)$ & $5.22(1.39)$ & $5.86(0.9)$ \\
13 & $5.00(1.83)$ & $5.12(1.25)$ & $4.89(1.96)$ \\
14 & $6.44(0.73)$ & $6.14(0.9)$ & $5.38(1.41)$ \\
15 & $5.50(1.2)$ & $5.44(1.01)$ & $6.29(1.11)$ \\
16 & $6.00(0.82)$ & $5.38(1.19)$ & $5.78(0.97)$ \\
17 & $4.33(1.58)$ & $5.00(1.29)$ & $4.62(1.41)$ \\
18 & $5.25(1.67)$ & $4.89(0.93)$ & $5.57(0.98)$ \\
19 & $5.71(1.38)$ & $5.5(0.76)$ & $5.44(1.01)$ \\
20 & $6.11(1.05)$ & $5.14(1.77)$ & $5.38(1.41)$ \\
21 & $6.38(0.74)$ & $5.44(1.88)$ & $6.57(0.53)$ \\
22 & $5.43(1.27)$ & $5.12(1.25)$ & $6.00(1.32)$ \\
23 & $6.00(1.5)$ & $4.43(1.51)$ & $5.38(0.74)$ \\
24 & $5.75(1.16)$ & $6.22(0.67)$ & $6.00(1.53)$ \\
\hline \hline
\end{tabular}

Tabelle C.5: Mittelwerte und Standardabweichungen des Kohärenzratings für alle Items und Bedingungen des Faktors STRukTur 


\section{C.3.3. Weitere Plots}

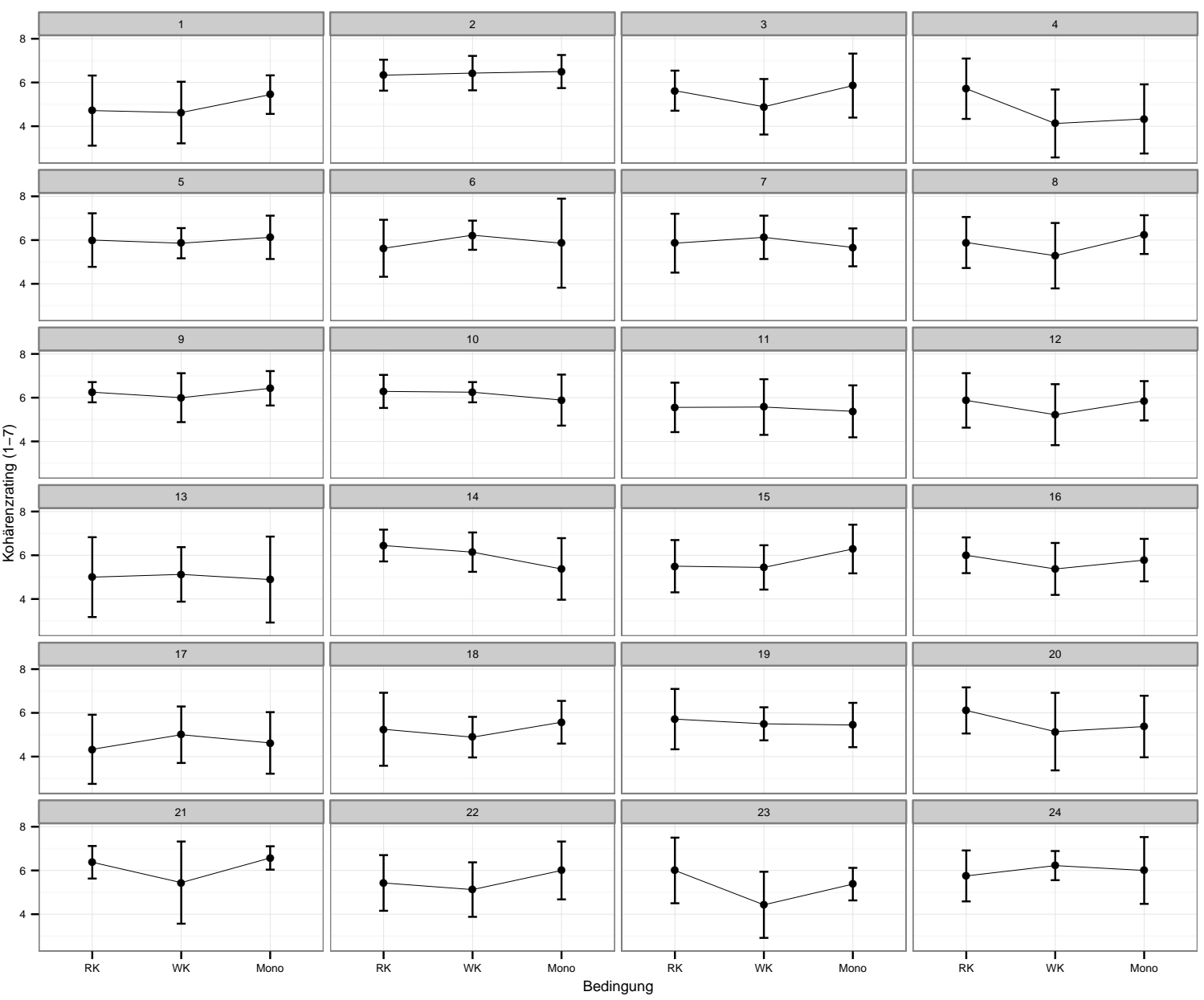

Abbildung C.4: Plot der Mittelwerte des Kohärenzratings für den Faktor sTRUKTuR für die einzelnen Items 


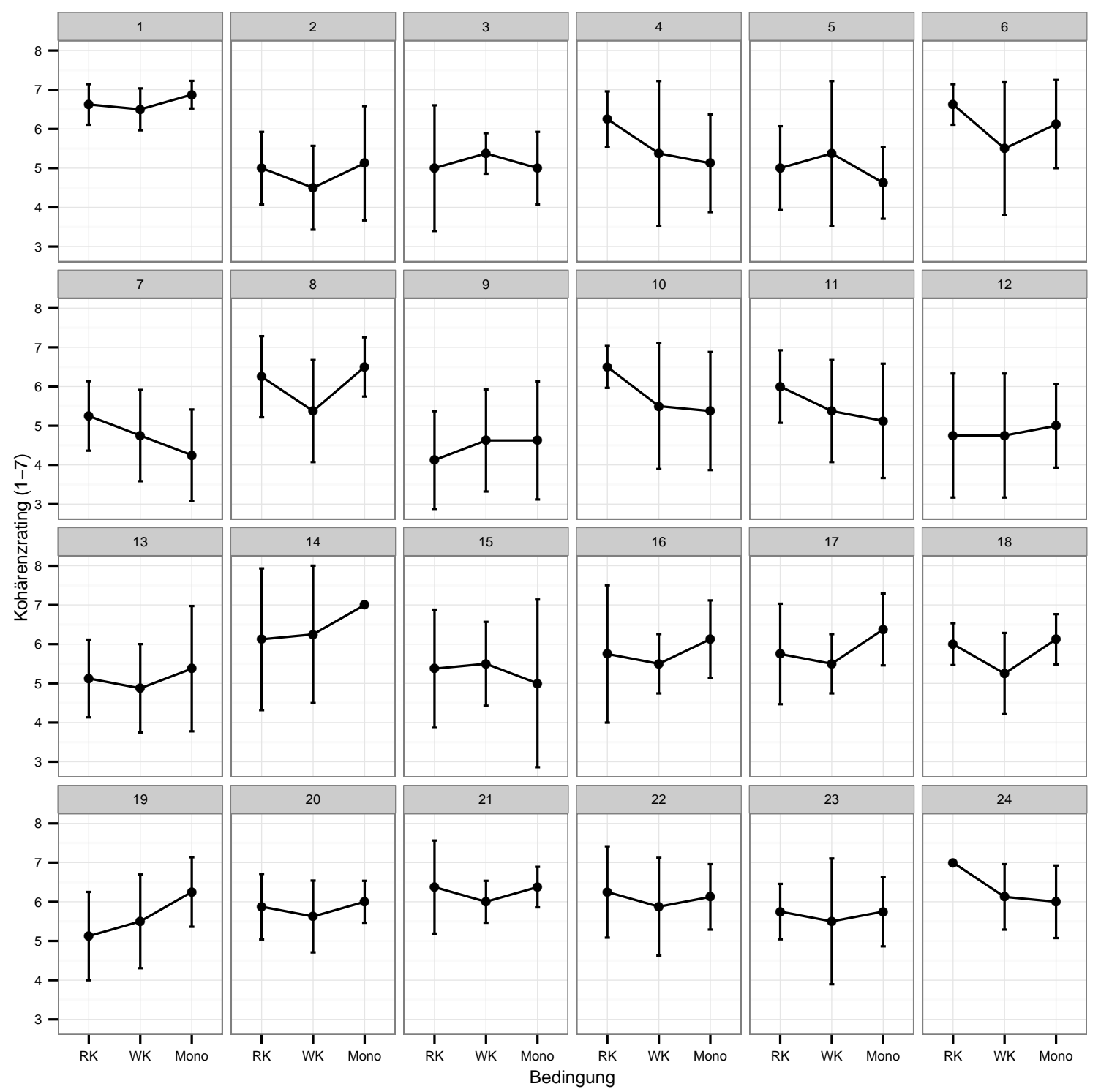

Abbildung C.5: Plot der Mittelwerte des Kohärenzratings für den Faktor STRUKTuR für die einzelnen $V P n$

\section{C.3.4. Auswertungs-Output: Bsp. aus R 3.2.0}

Inferenzstatistik für den Faktor STRUKTUR:

ANOVA omnibus-by-subjects

$>$ summary(aov(judgement $\sim$ bedingung + Error (subject/bedingung), data=d1))

Error: subject

$\begin{array}{rrrrrr} & \text { Df } & \text { Sum Sq } & \text { Mean Sq } & F \text { value } & \operatorname{Pr}(>F) \\ \text { Residuals } & 23 & 188.4 & 8.193 & & \end{array}$

Error: subject: bedingung 


$\begin{array}{lrrrrc} & \text { Df } & \text { Sum Sq } & \text { Mean Sq } & \text { F value } & \operatorname{Pr}(>F) \\ \text { bedingung } & 2 & 10.01 & 5.005 & 4.352 & 0.0186 * \\ \text { Residuals } & 46 & 52.91 & 1.150 & & \\ -- & & & & \\ \text { Signif. codes: } 0 \text { '***' } & 0.001 \text { '**' } 0.01 \text { '*' } 0.05 \text { '?' } 0.1 \text { ' ' } 1\end{array}$

Error: Within

$\begin{array}{rrrrrr} & \text { Df } & \text { Sum Sq } & \text { Mean Sq } & \text { F value } & \operatorname{Pr}(>F) \\ \text { Residuals } & 504 & 678.4 & 1.346 & & \end{array}$

ANOVA omnibus-by-items

$>$ summary(aov(judgement $\sim$ bedingung $+\operatorname{Error}($ item/bedingung), data=d1))

Error: item

$\begin{array}{lrrrrr} & \text { Df } & \text { Sum Sq } & \text { Mean Sq } & F \text { value } & \operatorname{Pr}(>F) \\ \text { bedingung } & 2 & 11.84 & 5.922 & 1.115 & 0.347 \\ \text { Residuals } & 21 & 111.52 & 5.310 & & \end{array}$

Error: item:bedingung

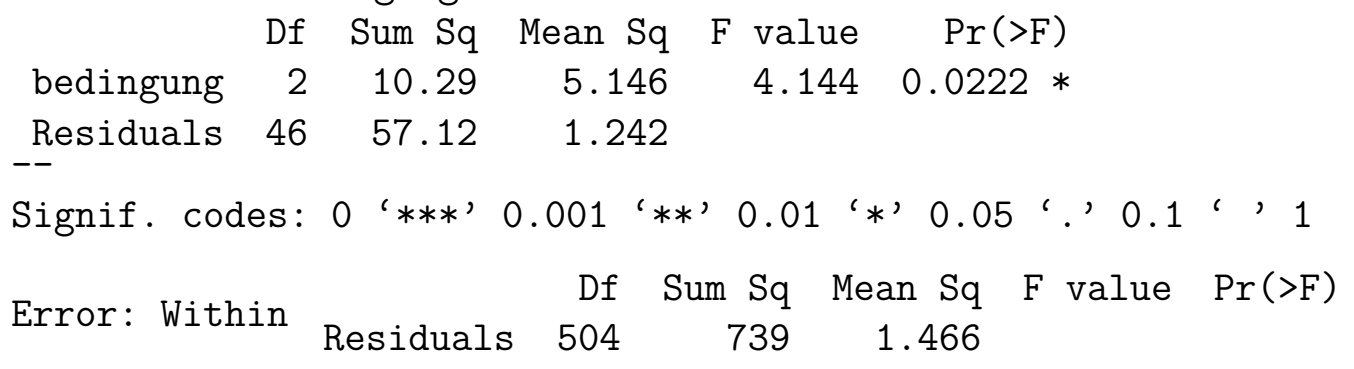

$\operatorname{minF}{ }^{\prime}$

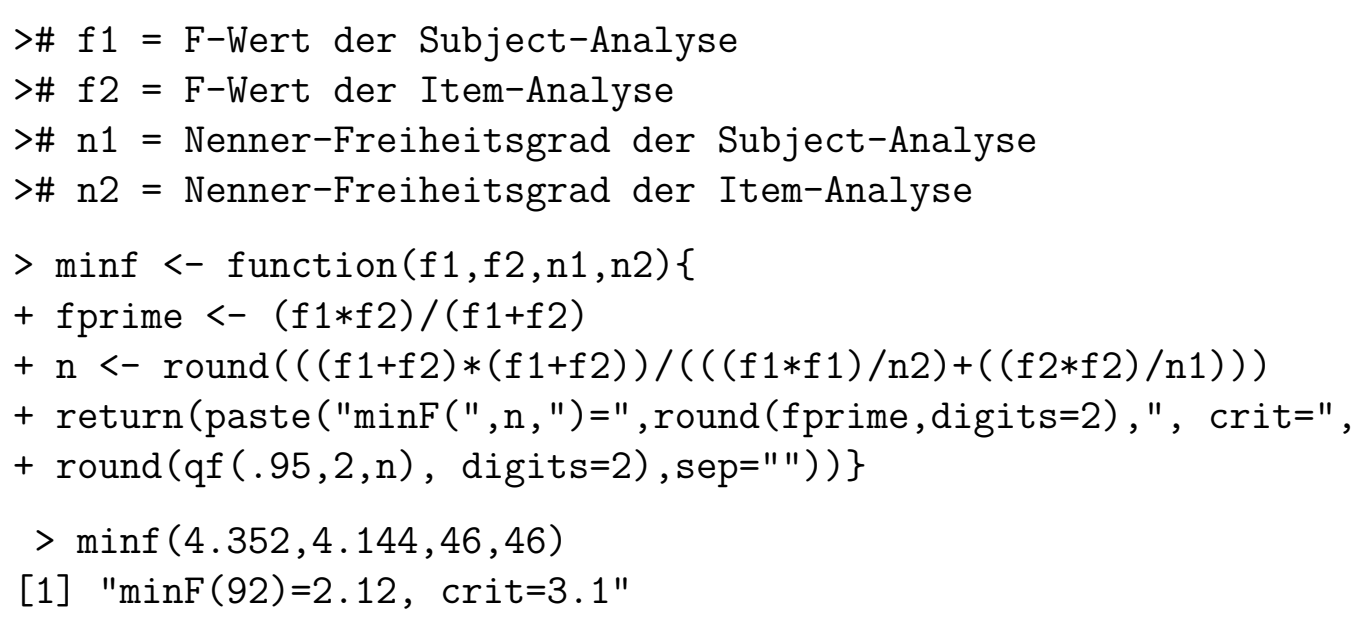

\section{C.4. Anhang zu Experiment 3c}

\section{C.4.1. Items}

Die Items von Experiment 3c sind identisch mit den Items aus Experiment 3b (siehe C.3.1. 


\section{C.4.2. Weitere Plots}

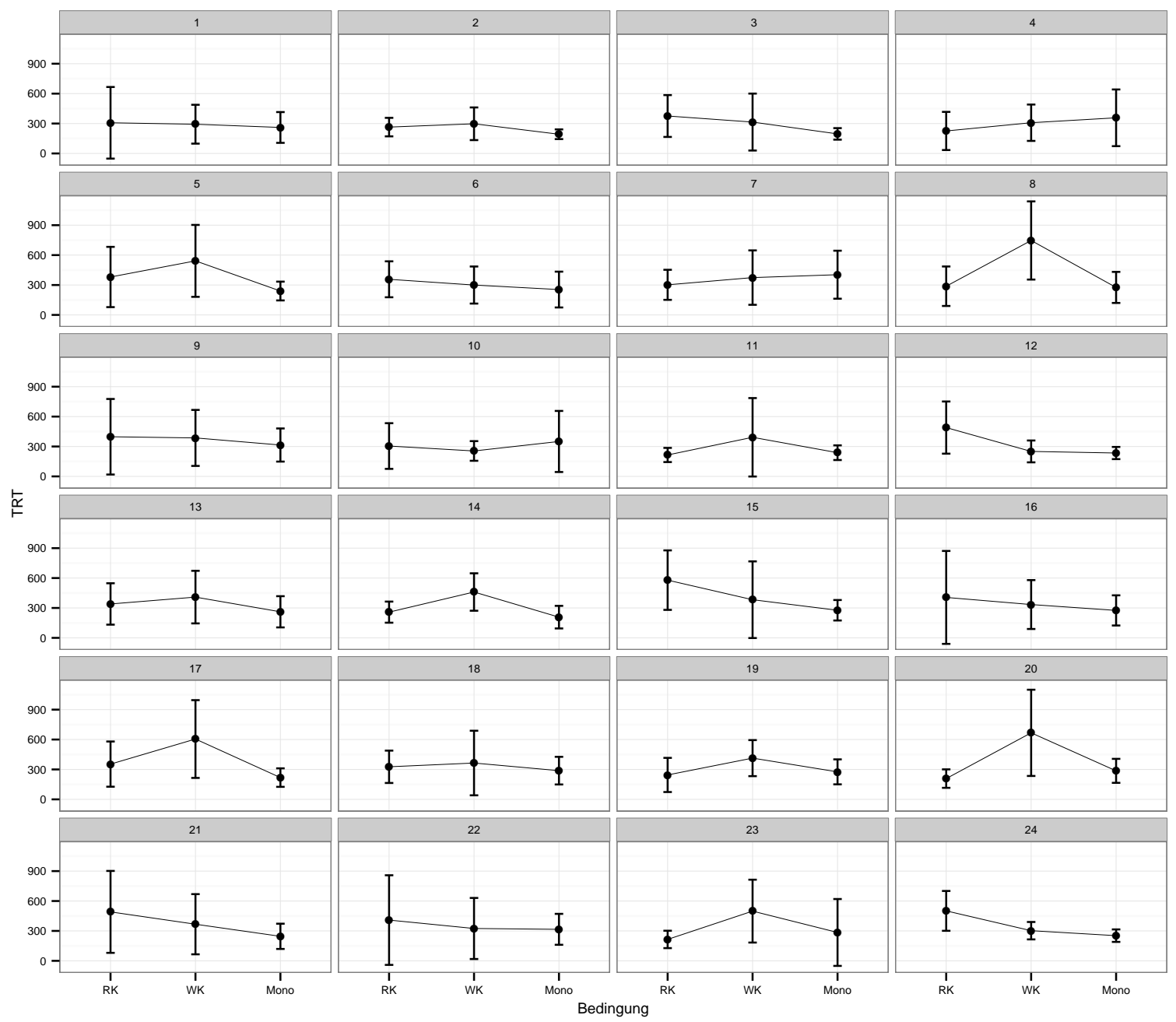

Abbildung C.6: Plot der Mittelwerte der TRT der Anaphern-Region (IA 6) für den Faktor StrukTur für die einzelnen Items 


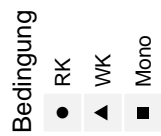

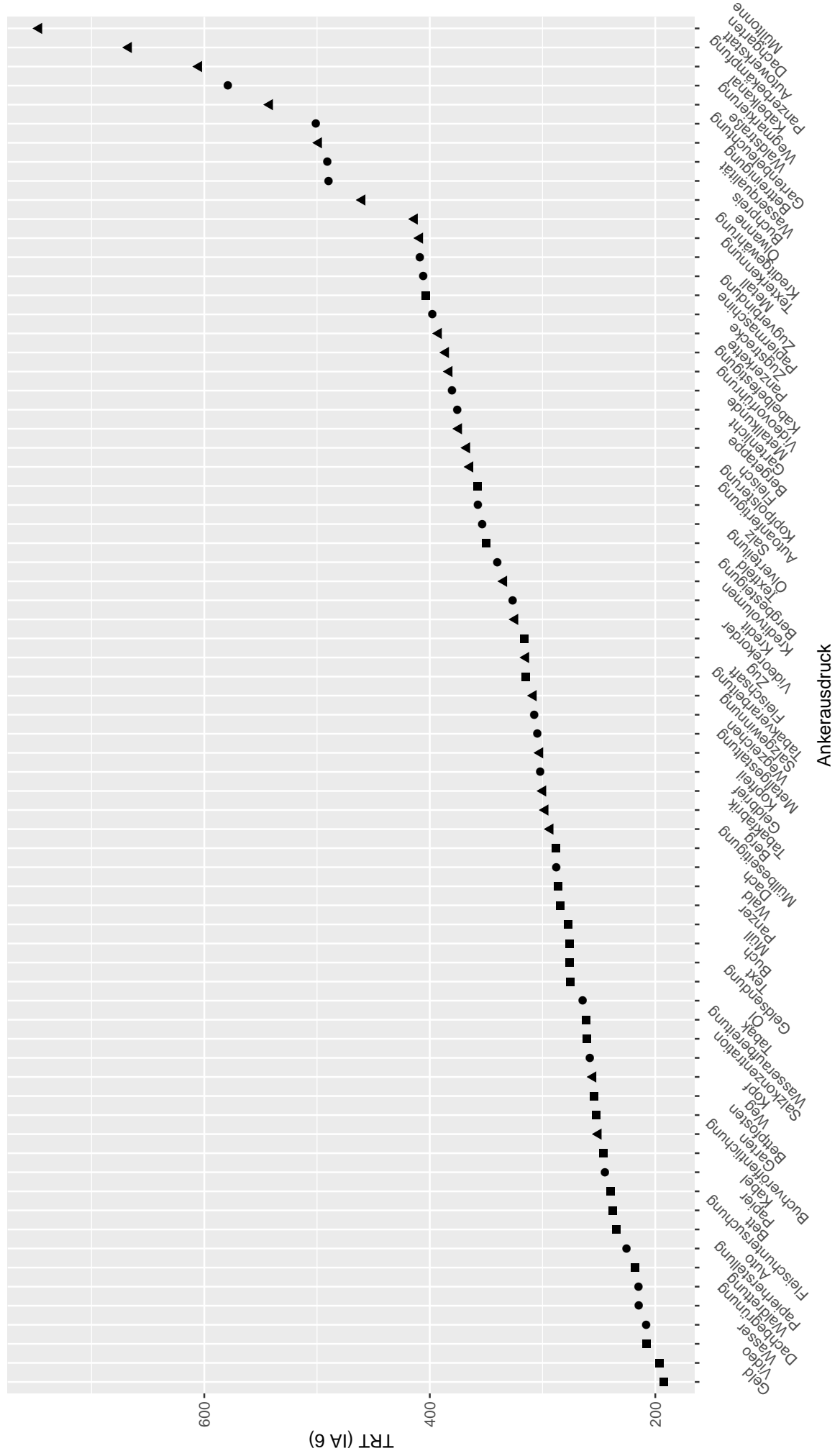

Abbildung C.7: Plot der Mittelwerte der TRT der Anaphern-Region (IA 6) für die einzelnen Items in allen Bedingungen (gekürzter Datensatz), geordnet nach steigendem Wert 


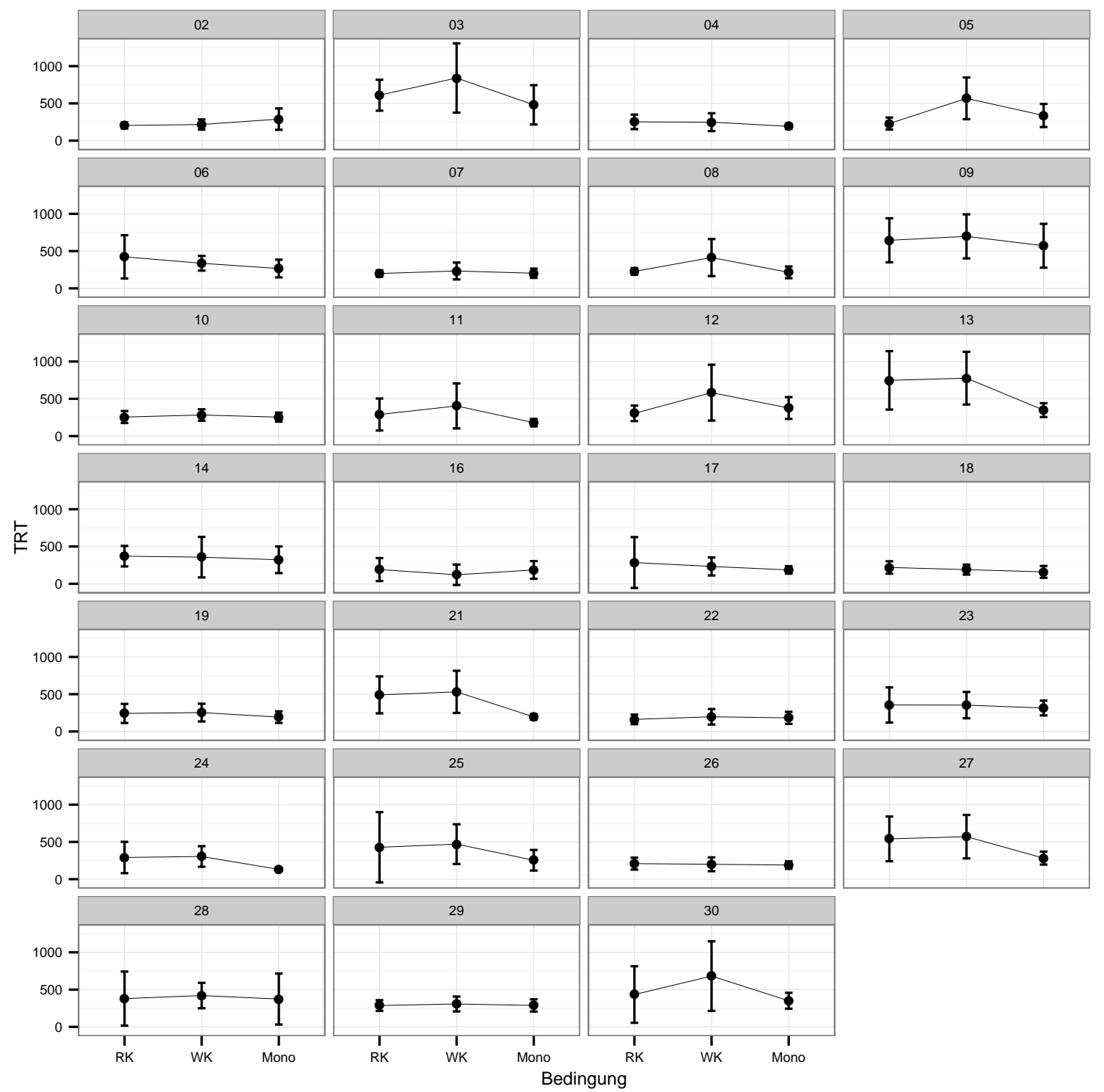

Abbildung C.8: Plot der Mittelwerte der TRT der Anaphern-Region (IA 6) für den Faktor STRUKTUR für die einzelnen VPn

\section{C.4.3. Auswertungs-Output: Bsp. aus R 3.2.0}

Auswertung der FFD der Anaphern-Region (IA 6)

ANOVA omnibus-by-subjects

$>\operatorname{summary}(\operatorname{aov}($ IA_FIRST_FIXATION_DURATION $\sim$ bed + Error (vp/bed), d3.ia.ana))

Error: vp

$\begin{array}{lrrrrr} & \text { Df } & \text { Sum Sq } & \text { Mean Sq } & \text { F value } & \operatorname{Pr}(>F) \\ \text { bed } & 2 & 100925 & 50463 & 1.367 & 0.274 \\ \text { Residuals } & 24 & 886015 & 36917 & & \end{array}$


Error: vp:bed

Df Sum Sq Mean Sq F value $\operatorname{Pr}(>F)$

$\begin{array}{llllll}\text { bed } & 2 & 20642 & 10321 & 1.77 & 0.18\end{array}$

Residuals $52 \quad 303154 \quad 5830$

Error: Within

Df Sum Sq Mean Sq $F$ value $\operatorname{Pr}(>F)$

Residuals $558 \quad 3515819 \quad 6301$

ANOVA omnibus-by-subjects

$>$ summary(aov(IA_FIRST_FIXATION_DURATION $\sim$ bed +

+ Error(item/bed), d3.ia.ana))

Error: item

$\begin{array}{lrrrrr} & \text { Df } & \text { Sum Sq } & \text { Mean Sq } & F \text { value } & \operatorname{Pr}(>F) \\ \text { bed } & 2 & 20649 & 10324 & 1.295 & 0.295 \\ \text { Residuals } & 21 & 167408 & 7972 & & \end{array}$

Error: item:bed

$\begin{array}{lrrrrr} & \text { Df } & \text { Sum Sq } & \text { Mean Sq } & F \text { value } & \operatorname{Pr}(>F) \\ \text { bed } & 2 & 21649 & 10824 & 1.365 & 0.266 \\ \text { Residuals } & 46 & 364863 & 7932 & & \end{array}$

Error: Within

Df Sum Sq Mean Sq F value $\operatorname{Pr}(>F)$

Residuals $567 \quad 4251987 \quad 7499$

$\min F^{\prime}$

$>\operatorname{minf}<-$ function $(\mathrm{f} 1, \mathrm{f} 2, \mathrm{n} 1, \mathrm{n} 2)\{$

+ fprime $<-(f 1 * f 2) /(f 1+f 2)$

$+\mathrm{n}<-\operatorname{round}(((\mathrm{f} 1+\mathrm{f} 2) *(\mathrm{f} 1+\mathrm{f} 2)) /(((\mathrm{f} 1 * \mathrm{f} 1) / \mathrm{n} 2)+((\mathrm{f} 2 * \mathrm{f} 2) / \mathrm{n} 1)))$

$+\operatorname{return}($ paste $(" \operatorname{minF}(", \mathrm{n}, ")=$ ", round (fprime, digits=2), ", crit=" ,

$+\operatorname{round}(\mathrm{qf}(.95,2, \mathrm{n})$, digits=2), sep=""))\}

$>\operatorname{minf}(1.77,1.365,52,46)$

[1] $\operatorname{minF}(95)=0.77$, crit=3.09" 



\section{Danksagung}

Während der Arbeit an dieser Dissertation konnte ich auf die Unterstützung vieler Menschen bauen, bei denen ich mich an dieser Stelle ganz herzlich bedanken möchte:

... bei meiner Doktormutter Prof. Dr. Anke Holler, die mich stets unterstützt hat und mir alle Freiräume für die Verwirklichung meiner Ideen gelassen hat. Neben der wissenschaftlichen Begleitung danke ich ihr für die institutionelle Einbindung und die Unterstützung bei eigenen Schritten im Wissenschaftsbetrieb. Außerdem danke ich meinem Zweitbetreuer Prof. Dr. Markus Steinbach für seine stetige Unterstützung und kompetente Beratung. ... bei Dr. Thomas Weskott, dessen methodische Unterstützung und Beratung für das Entstehen dieser Arbeit unerlässlich waren. Besonders danke ich ihm für die vielen Diskussionen und Gedankenexperimente, die mir wichtige Impulse gegeben haben.

... bei Dr. Andreas Blümel, der mich mit seinem breiten syntaktischen Wissen unterstützt und die Überlegungen im Bereich der Distribuierten Morphologie vorangetrieben hat.

... bei meinen Göttinger Kolleginnen und Kollegen, die mich durch fachlichen Input, anregende Diskussionen, praktische Hilfe bei den Experimenten und ein kollegiales Arbeitsklima unterstützt haben: Dr. Mailin Antomo, Jun.-Prof. Dr. Marco Coniglio, Simone Gerle, Melanie Göbel-Hoffmann, Prof. Dr. Annika Herrmann, Cheryl Hodgkinson, Dr. Jana Hosemann, Annika Hübl, Johanna Klages, Dr. Joost Kremers, Anja Müller, Derya Nuhbalaoglu, Markus Paluch, Liona Paulus, Nina-Kristin Pendzich, Stefanie Pietsch, Sina Proske, Tanja Recke, Susanna Salem, Svenja Sepúlveda, Philipp Siebenthaler, Julia Steinmetz, Dr. Katja Suckow, Heinke Syring, Elisabeth Volk, Anne Wienholz, Dr. Sarah Zobel und das gesamte Psychlab-Team.

... bei meinen Kollegen außerhalb Göttingens, die mir mit Rat, Tat und Korrekturlesen zur Seite gestanden haben, v.a. Dr. Martin Schäfer, Judith Schlenter und Philipp Rauth.

... bei allen Studentinnen und Studenten, die mich als Versuchspersonen unterstützt haben und sich geduldig durch unzählige Items gearbeitet haben.

... beim Courant Forschungszentrum „Textstrukturen“ für das Zurverfügungstellen von wissenschaftlicher Literatur und Infrastruktur.

... bei der Graduiertenschule für Geisteswissenschaften Göttingen (GSGG) für ihre vielfältige Unterstützung und im Besonderen für die Gewährung eines Abschlussstipendiums, das mir die Fertigstellung der Arbeit ermöglicht hat.

Ein besonderer Dank gilt meiner Familie und meinen Freunden. Ich danke meiner Mutter Lydia Rößler und meinem Vater Dr. Udo Rößler sowie Angelika und Martin Langmann für ihr unerschütterliches Vertrauen in mich. Außerdem Johanna Klages und Steffen Klemer, auf deren Freundschaft und Hilfe ich zu jeder Tages- und Nachtzeit zählen konnte. Und schließlich richtet sich mein Dank an Tim Langmann, nicht nur fürs Debugging, sondern v.a. für seine Liebe und Fürsorge, die mir immer wieder den nötigen Auftrieb gegeben haben. 
